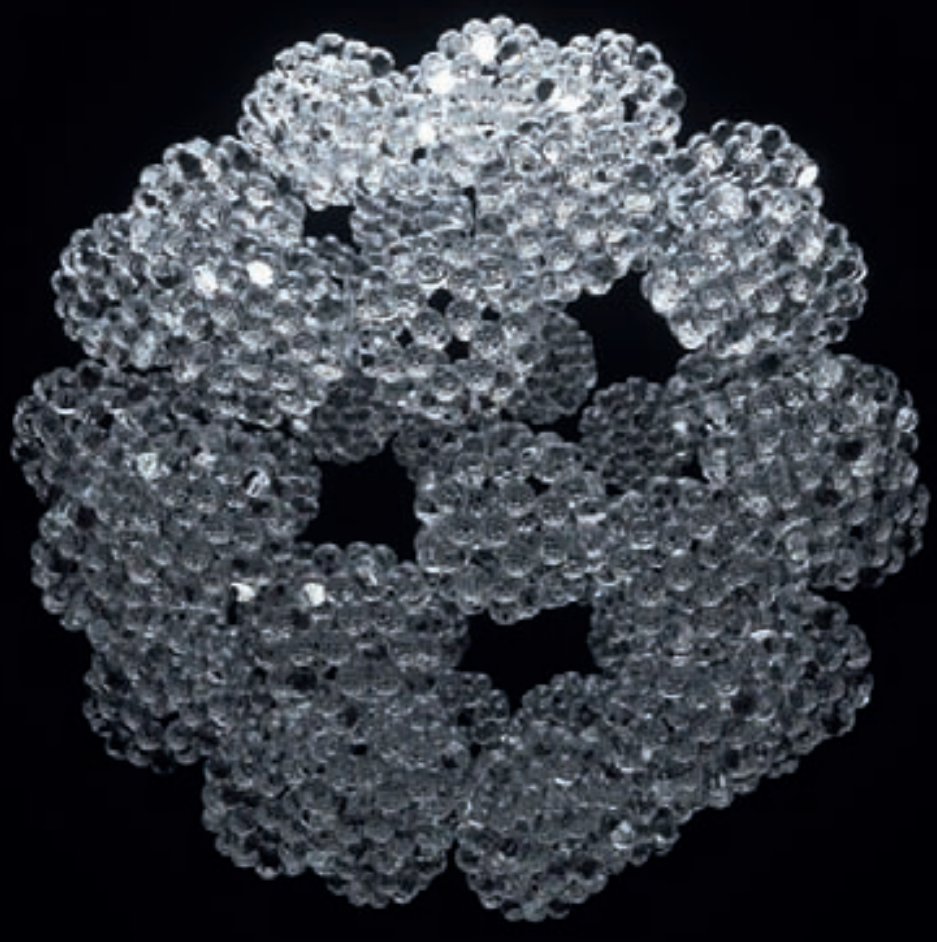

Edited by Vincent Blay, Luis F. Bobadilla, Alejandro Cabrera-García

Zeolites and

Metal-Organic Frameworks

From Lab to Industry 
Zeolites and Metal-Organic Frameworks 


\section{Atlantis Advances in Nanotechnology, Material Science AND ENERGY TECHNOLOGIES}

\section{Series Editor: Dr. Christian Ngô}

ISSN: 2352-0655

The aim of "Atlantis Advances in Nanotechnology, Material Science and Energy Technologies" is to publish high quality manuscripts giving an upto date and clear view on topical scientific contents in nanotechnology, material sciences and energy technologies.

These three fields evolve rapidly and their understanding is essential with regard to contemporary science and as well as in the context of everyday life. Nanotechnology is a fast growing science and a technological field with applications in numerous areas such as materials, health, electronics, information processing, defence and security, catalysis, sensors, food, cosmetics and many more. The results of material sciences are the basis for any object around us, they are omnipresent in human life. Mastering materials and processes is therefore crucial. In particular, research on microscopic understanding is essential to develop models predicting the properties of new materials and structures. The final goal is to be able to predict macroscopic properties of materials from their microscopic properties. Finally, energy technologies enfold a complex area where each technological advance has to be weighed against economical, environmental, political and sociological constraints. Energy is closely linked to economic development and, more generally speaking, to everyday life.

As nanotechnology, materials science and energy technologies are closely interconnected, this series offers the reader both, highly specialized monographs as well as easy-to-grab overviews. Each publication focuses on one of the fields. At the same time, it is highly relevant to explore their interconnections and to include interdisciplinary approaches.

All book proposals submitted to this series are being reviewed by key experts before publication and each book focuses on a certain field putting it into perspective with its implications at the economic and societal level. 


\title{
Zeolites and Metal-Organic Frameworks
}

From lab to industry

\author{
Edited by
}

Vincent Blay, Luis F. Bobadilla and Alejandro Cabrera García 
Cover illustration: (C) 2010 James Coleman

Cover design: Coördesign, Leiden

Lay-out: Konvertus/Newgen

Amsterdam University Press English-language titles are distributed in the US and Canada by the University of Chicago Press.

$\begin{array}{ll}\text { ISBN } & 9789462985568 \\ \text { e-ISBN } & 9789048536719 \\ \text { DOI } & 10.5117 / 9789462985568 \\ \text { NUR } & 971\end{array}$

Creative Commons License CC BY NC ND

(http://creativecommons.org/licenses/by-nc-nd/3.0)

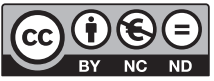

Cᄃ The authors / Atlantis Press B.V. / Amsterdam University Press B.V., Amsterdam 2018

All rights reserved. Without limiting the rights under copyright reserved above, no part of this book may be reproduced, stored in or introduced into a retrieval system, or transmitted, in any form or by any means (electronic, mechanical, photocopying, recording or otherwise) without the written permission of both the copyright owner and the author of the book.

Every effort has been made to obtain permission to use all copyrighted illustrations reproduced in this book. Nonetheless, whosoever believes to have rights to this material is advised to contact the publisher. 


\section{Contents}

Prologue

Vincent Blay and Suk Bong Hong

1. Zeolites and MOFs? Dare to Know Them!

Valentin Valtchev and Svetlana Mintova

2. Synthesis and Identification Methods for Zeolites and MOFs

L. Marcela Martínez T., Svetlana Ivanova, Benoit Louis and José Antonio Odriozola

3. Spectroscopic Methods of Characterization for Zeolites and MOFs

Luis F. Bobadilla, Laetitia Oliviero, Francisca Romero-Sarria and Marco Daturi

4. Oil Refining and Petrochemistry: Use of Zeolites and Opportunities for MOFs

María Ángeles Romero, Jesús Lázaro and Juana Frontela

5. Biomass Transformation into Chemicals Using Zeolites and MOFs

Óscar Hernando Laguna Espita, Simona M. Coman, Miguel Ángel Centeno Gallego and Vasile I. Pârvulescu

6. Biocatalysis on Porous Materials

Isabel Díaz, Rosa María Blanco, Manuel Sánchez-Sánchez and Carlos Márquez-Álvarez

7. Adsorption Processes on Zeolites and Metal-Organic

Frameworks for Industrial and Environmental Applications

Elena López-Maya, Carmen Montoro, L. Marleny Rodriguez-Albelo and Carmen R. Maldonado

8. Membrane Technology: how, where, and why

Motomu Sakai, Masahiro Seshimo and Masahiko Matsukata 
9. Computational Chemistry Experiment Possibilities

Barttomiej M. Szyja and Danny Vanpoucke

10. Zeolites and Metal-Organic Frameworks as Biomedical Nanodevices

Alejandro Cabrera-García, Zeneida Díaz-Betancor and Eva Rivero-Buceta

11. Zeolites and MOFs as Catalysts in Fine Chemical Reactions 289 Francisco G. Cirujano and Anna Nowacka

12. The Future of Zeolite and MOF Materials

Eduardo Falabella Sousa-Aguiar, Pedro Augusto Arroyo,

Maria Angélica Simões Dornellas de Barros and

Jussara Lopes de Miranda 


\section{Prologue}

\section{Vincent Blay ${ }^{1}$ and Suk Bong Hong ${ }^{2}$}

'Departamento de Ingeniería Química, Universitat de València, Av. de la Universitat s/n, 46100 Burjassot, Spain.

${ }^{2}$ Center for Ordered Nanoporous Materials Synthesis, School of Environmental Science and Engineering, POSTECH, Pohang 37673 , South Korea.

It is a pleasure for us to introduce this book Zeolites and Metal-Organic Frameworks - From lab to industry. The editors are distinguished alumni from the Instituto de Tecnología Química (ITQ), in Valencia, Spain. ITQ is a joint research centre founded in 1990 by the Spanish National Research Council (CSIC) and the Polytechnic University of Valencia (UPV). Catalysis by zeolites has been the most emblematic passion of ITQ, to which many other fields of research added over the years. ITQ has become an iconic research centre worldwide, embracing organic, inorganic, photo-, electroand computational chemistry, materials science, pollution control, chemical engineering, and health care.

Our Center for Ordered Nanoporous Materials (CONS) was established at POSTECH, Pohang, Korea, in 2012. CONS is the National Creative Research Initiative Program supported by the National Research Foundation of Korea. Analogously to ITQ, CONS aims to become a leader in the field of ordered nanoporous materials science. The main objectives of CONS are to demonstrate new and innovative synthesis strategies and to create unique zeolite framework structures and/or compositions that could devise new processes and applications in environmentally sustainable catalysis and gas (mainly $\mathrm{H}_{2}$ and $\mathrm{CO}_{2}$ ) separation.

As researchers, we strive to push forward the boundaries of scientific knowledge for the benefit of the society and, to this end, we must be aware of the need of extensive collaboration for progress and mutual enrichment. This book is one example, to which experienced and promising young researchers have equally contributed to bringing you their knowledge and findings acquired over the years.

The history of catalysis dates back to the dawn of civilization, when alcohol started to be produced by fermentation. It was not until 1835 that Berzelius initiated systematic studies of catalysts. These studies started to 
be implemented as industrial processes mainly at the end of the $19^{\text {th }}$ century. In the 1930s, Houdry developed the catalytic cracking of petroleum for Sun Oil and, in 1939, Chambers, Lewis and Gilliland, at MIT, worked out the basis of the fluid-bed reactor for the Catalytic Research Associates consortium (led by Standard Oil). In the 195os, Milton and Breck discovered zeolites A, X and Y at Linde, Union Carbide. Zeolite A started to be used as an adsorbent, but it was not until the 1970s that Na-A zeolite was used in detergents, replacing phosphate builders, thanks to scientists at Henkel, and Procter and Gamble. In the 196os, zeolite X revolutionized cracking in the petroleum industry at Mobil Oil. This work on zeolites definitely spurred the development of metal-organic frameworks, which took off in the mid199os after Kitagawa (Kyoto University) and Yaghi (University of California, Berkeley) observed permanent microporosity in metal-organic materials. Since those days, incredible progress has been made in both classes of materials. And their range of applications is being extended to undreamed-of levels, as this book manifests.

The proposal of a book targeting zeolites and metal-organic frameworks (MOFs) is interesting, as they share common features, which are also paralleled by remarkable differences. These are evidenced throughout the book. By addressing both materials in a synergic text, the reader will see opportunities for shifting and contributing to both fields of research, the commonalities and differences in terms of concepts, synthesis, characterization, properties, and applications.

In this sense, the book does a remarkable job. It is a book coordinated by, written by, and designed for young scholars and researchers, ranging from those in undergraduate and graduate university courses to PhDs and early-stage researchers. Moreover, the book provides an overview wide enough for most professionals to find it of interest and learn something new. It includes the participation of renowned experts and invited industry practitioners, ensuring rigor and a balance between new research, progress in the field, and a didactic approach that only experience bestows. By the end of the book, the readers will be excellently equipped to delve further into other important topics such as electrocatalysis, photocatalysis or sensing. The same applies to important characterization techniques, such as solid-state nuclear magnetic resonance or X-ray photoelectron spectroscopy, to name but a couple.

In Chapter 1, Professors Valtchev and Mintova provide a knowledgeable introduction to zeolites and metal-organic frameworks - what they are, how they are classified, why they are so important -, as well as some other matters that are expanded on later in the book. 
Chapter 2 overviews the different synthesis methods available for zeolites and metal-organic frameworks: from classical hydrothermal syntheses to non-conventional sono- or mechanochemical methods in the case of zeolites. An interesting account of the knowledge on the crystallization mechanism of zeolites is also provided, which is one of the most complex and fundamental questions open to discussion in this field. The chapter also provides a brief presentation of some standard characterization methods of porous materials for phase identification and textural analysis.

Characterization is a topic of great importance in porous materials, as it bridges the knowledge gap between the preparation of the material and its performance in the real application. We can correlate this performance (for instance, in catalysis, adsorption, drug delivery, etc.) with properties of the material and then alter or tune the synthesis protocols to affect the properties in the desired manner. Based largely on the experience of Professor Daturi's group in the last decades, Chapter 3 presents a general, infrared spectroscopy methodology to observe the potential active site directly or by adsorption of probe molecules, as well as several examples that illustrate the qualitative and quantitative analyses of the physicochemical vibrational properties of the entities on the surface of the material.

Having prepared and characterized the materials, the book moves on to present some relevant applications. Petroleum refining is the field which fostered the development of zeolites and the one in which they add the most value. Thus, Chapter 4 presents an industrial overview of the many oil \& gas processes in which zeolites are involved. These include separation operations, fluid catalytic cracking, hydrocracking, isomerization, alkylation of benzene, dewaxing, etc. However, the stability of metal-organic frameworks prevents their consideration for many of these processes, although the expert authors from CEPSA also identify some promising niches for MOFs.

Chapter 5 presents how zeolites and MOFs could contribute to the biorefineries of tomorrow, which would use renewable biomass residues as feedstocks instead of fossil resources. Many of these transformations are carried out in aqueous media, therefore the stability of both zeolites and MOFs is involved. Nevertheless, some of these materials turn out remarkably suitable, and great progress has been achieved in the conversion of cellulose, hemicellulose and lignin polymers present in biomass over some zeolites and MOFs. The platform molecules resulting from the refining could then be used by the chemical industry in other processes.

As a counterpoint to the previous chapters, in Chapter 6 zeolites and MOFs do not act as catalysts themselves but they are used to host enzymes, which are sophisticated biocatalysts developed by nature, fine-tuned by 
the biotechnologists and protein engineers. The advantages of heterogeneization of catalysts into periodic structures also apply to biocatalysts. In this case, given the large molecular size of enzymes, the larger pores of MOFs are especially well suited. In addition to describing the immobilization of important enzymes such as lipases and laccases, the authors, one of the leading groups in this field, also discuss the characterization techniques involved.

In Chapter 7, the applications of MOFs and zeolites as adsorbents are addressed. In addition to the design of materials, other strategies to adjust their behaviour as adsorbents are presented, such as ion exchange, post-synthesis modification or, very promising for the case of MOFs, the presence of defects. An ample review of applications in both gas and liquid phases is presented, including research and uses as diverse as hydrogen storage, removal of heavy metal ions or radionuclides, and $\mathrm{CO}_{2}$ capture, which evidence the possibilities for tailoring these materials to any objective.

Adsorption can be further improved by a suitable design of the material at the mesoscale. Membranes are a remarkable example of a technical presentation of zeolites and MOFs. Membranes hold promise to improve many industrial processes. For instance, separations could be carried out in a continuous steady operation and reactions could be performed without worrying about equilibrium limitations and/or with higher selectivity, as highly pure products would be withdrawn as they form. In Chapter 8, Prof. Matsukata and his group expand on these ideas and tell us how membranes are prepared, characterized and applied for separations, as well as the hard challenges in this field.

Adsorption and diffusion are key phenomena in porous materials. These are very suitable for study in computational chemistry experiments, and so are chemical reactions. Chapter 9 starts with the fundamentals of computational chemistry and the different methods available, which condition the level of exactness achievable and the computation time required. It teaches us how the computational models are designed and how the experiments are run and exploited by means of algorithms, concluding with the results of applications to current questions.

Chapter 10 presents the state of the art of biomedical applications of zeolites and especially MOFs, a young but promising field, which includes their use in drug delivery, contrast agents, in diagnosis and therapy, as well as multifunctional materials for combination therapy.

The application of heterogeneous catalysts in the fine chemical industry (pharmaceutical, cosmetic, phytosanitary, etc.) has been traditionally low. Their comparatively low production volumes allowed the generation 
of high proportions of residues, which can no longer be tolerated in our current efforts for a sustainable industry. Nowadays, in addition to high product yields, chemo-, region- and stereoselectivity are the main goals in organic syntheses given the importance of avoiding by-products and additional purification steps. Thus, in Chapter 11 the applicability of zeolites and MOFs as catalysts for this industry is also demonstrated.

Lastly, in Chapter 12, Professor Falabella and his colleagues provide a commercially oriented, high-level overview of most applications of zeolites and MOFs, including adsorption, ion exchange, catalysis, composites and other devices. Importantly, they provide their knowledgeable forecast on the future of these materials in the coming years.

Overall, this book is a demonstration of liveliness in the world of ordered porous materials, in general, and of zeolites and MOFs, in particular, both showing ever-growing opportunities in traditional and in emerging fields of application. One can speculate whether our predecessors in the field would ever have dreamt of the extensive benefits that these materials have brought to the society. On the other hand, the book evidences, through all the contributors, that the next generations are going to be deserving successors to face the major scientific challenges of the decades ahead. They will bring greater benefits to the society through ordered porous materials that we cannot even dream of today. 



\title{
1. Zeolites and MOFs? Dare to Know Them!
}

\author{
Valentin Valtchev and Svetlana Mintova \\ Laboratoire Catalyse et Spectrochimie, Normandie Univ- \\ ENSICAEN-CNRS, 6 Bd Maréchal Juin, 14000 Caen, France.
}

Keywords: Zeolites, MOFs, porous materials, catalysis, separation

\section{Introduction}

A porous material is a solid matter permeable to fluids due to the presence of pores. Behind this short definition are hidden thousands of porous solids with different pore characteristics, chemical natures and structures. Although not visible, the impact of porous solids on our lives is extremely large. It is difficult to conceive modern society without the extensive use of different types of porous solids for industrial and household needs.

The International Union of Pure and Applied Chemistry (IUPAC) divides porous solids into three groups: microporous, with pore size below $2 \mathrm{~nm}$, mesoporous, with pores between 2 and $50 \mathrm{~nm}$, and macroporous, with pores between 50 and $1000 \mathrm{~nm}[1]$. This classification does not reflect the chemical nature and structural ordering of porous solids that have a great impact on their properties. In the present book two families of crystalline porous solids, zeolites and metal-organic frameworks (MOFs) are reviewed. While zeolites are classical microporous materials with pores below $2 \mathrm{~nm}$ and in most cases below $1 \mathrm{~nm}$, some MOF materials exceed the micropore range. However, both classes of materials exhibit crystalline structures and thus pores with well-defined dimensions.

The porous materials first used by mankind were porous carbon and clay-type minerals. However, zeolites were the ones introduced on a large scale with highly technological processes. Among these processes we find fluid catalytic cracking (FCC) and hydrocracking processes, which convert heavy oil fractions to transportation fuels, as well as different separation processes like selective adsorption of aromatic isomers in petrochemistry. Zeolites have been the backbone of the petrochemical industry for over fifty years and their application as heterogeneous catalysts and separation 
materials continue to expand $[2,3]$. Today, the application of crystalline microporous materials extends far beyond the petrochemical industry, including areas like optics, electronics, medical diagnosis and chemical sensing [4]. New technological challenges, governmental regulations and environmental issues require new materials that can perform better, faster and are environmentally friendly. Hence, the development of new materials in terms of structure types, framework compositions, size and morphology is a continuous task. A steady growth of new zeolite structures is discovered every year, and their number is already 233 [5].

The classical definition of zeolite, which reflects the particularities of zeolite minerals, is "a crystalline porous aluminosilicate built of adjacent $\mathrm{SiO}_{4}$ and $\mathrm{AlO}_{4}$ tetrahedra" [6]. The quest for porous solids with new properties resulted in materials virtually identical to zeolite minerals but with compositions substantially different from the natural counterparts. Starting in the sixties a new family of zeolite materials has been discovered almost every decade. The $\mathrm{Si} / \mathrm{Al}$ ratio, which varies between 1 and 5 in natural zeolites, was extended to synthetic zeolites much richer in silica. The first high silica zeolite, named Beta, was synthesized in the late sixties [7], and in the seventies all-silica zeolite materials were produced [8]. The early eighties were marked by the discovery of aluminophosphate molecular sieves, which were the first silica-free zeolitic materials [9]. Zeolites exhibit well-defined pores, which for industrially relevant zeolites is below $0.8 \mathrm{~nm}$. This confers them shape selectivity, that is, the ability to select which molecules can be adsorbed or reacted inside their pores. However, a relatively small pore diameter is a drawback in the processing of bulky molecules, like those present in heavy oil fractions. The efforts of synthetic chemists to extend the pore dimension of zeolites led to a family of porous materials with pores larger than $2 \mathrm{~nm}$, called ordered mesoporous materials (OMMs) [10]. Ordered mesoporous materials exhibit well-defined channels as their size can be varied between 2 and $50 \mathrm{~nm}[11,12]$. However, the walls building the pore system are amorphous, which is the reason for a low acid strength compared to that of zeolites. Consequently, these materials did not fulfil the great expectations for processing heavy oil fractions.

The latest big family of crystalline porous solids, discovered in the late eighties are metal-organic framework materials (MOFs) [13]. A MOF framework is built of metallic atoms connected by organic linkers. The flexibility available to build such structures is substantially higher than that for crystalline inorganic materials [14]. In just a decade thousands of MOFs were published in open literature. The absence of a strict classification makes the determination of their exact number difficult. 
The objective of this chapter is to introduce these two important families of crystalline porous solids: zeolites, which have been used in the chemical process industry for over fifty years, and MOFs, which are promising for applications that might be different from those typical of zeolite molecular sieves. More detail information of their properties and applications can be found in this book.

\section{Zeolites}

A zeolite is formed under hydrothermal conditions from an alkali-rich aluminosilicate hydrogel system. In such a system, water is a major component playing two roles, a reactant and reaction medium. The alkaline aqueous solutions dissolve and transport material from the polymerized aluminoslilicate gel to growing crystals. The dissolution and transport of aluminosilicate precursors leads to supersaturation and nucleation in certain zones of the system $[2,6]$. Zeolite nucleation is a spontaneous process, which can be controlled to some extent under laboratory conditions. For example, by controlling the number of nuclei in the system, the ultimate crystal size can be tuned (since, after exhausting the nutrient pool, crystal growth stops) [15]. The key parameters controlling zeolite formation are gel composition and crystallization temperature. The previous history of the reactants, their purity and their mixing may also influence the zeolite synthesis. The aging time of the precursor gel is another important parameter that appears critical for some types of zeolites. The crystallization temperatures vary between 80 and $180^{\circ} \mathrm{C}$, but syntheses at ambient conditions or at temperature much higher than $180^{\circ} \mathrm{C}$ are also reported [6]. The crystallization of different types of zeolites may last from several minutes to several weeks. The crystallization time is important not only for obtaining a highly crystalline zeolite material but also to avoid the transformation of a desired product in another phase. Zeolites are metastable phases that may transform into more stable and denser crystals upon long contact with the mother liquor.

The general chemical composition of a zeolite is $\mathrm{Me}_{2 / n} \mathrm{O}: \mathrm{Al}_{2} \mathrm{O}_{3}: x \mathrm{SiO}_{2}$ : $y \mathrm{H}_{2} \mathrm{O}$, where Me is the metal cation (usually alkali or alkaline earth), $n$ is the charge of the cation, and $x$ and $y$ depend on the zeolite type. The framework silicon/aluminum ratio ( $\mathrm{Si} / \mathrm{Al})$ is always greater than 1 since Löwenstein's rule prohibits two neighboring aluminum tetrahedra, i.e. Al-O-Al bonds are forbidden due to unfavourable electrostatic interactions [16]. 


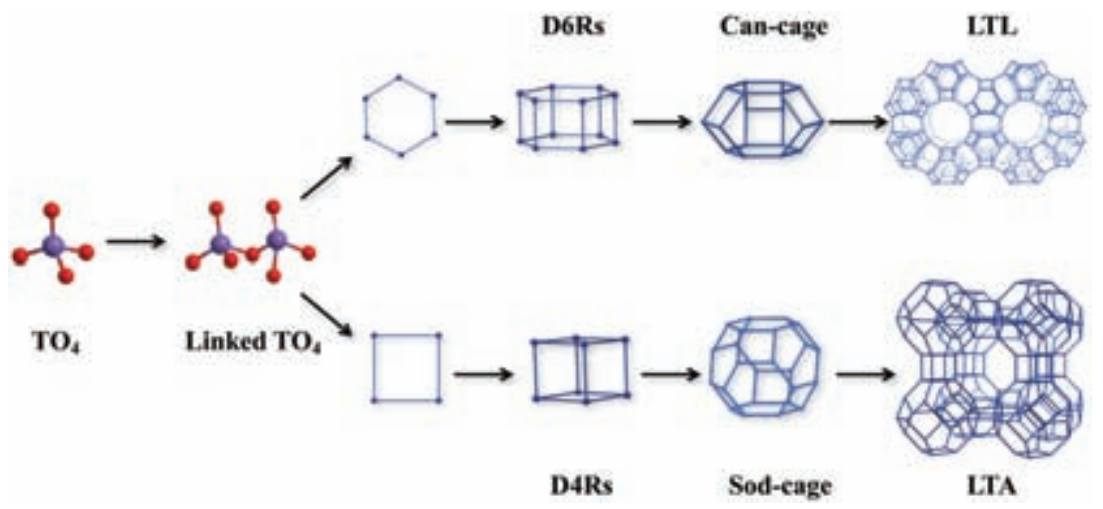

Figure 1. Schematic representation of the formation of zeolite building units and the resultant zeolite structures.

The network of pores and cavities in the zeolite framework is structured by templating species, which can be removed after the zeolite formation. In natural zeolites, the role of the template is played by alkali metal-water clusters. Barrer and Denny firstly introduced tetraalkylammonium cations in zeolite synthesis, which resulted in the formation of silica- richer zeolites [17]. This approach was further extended to the synthesis of very high silica and all-silica zeolite structures [18]. Besides extending the Si/Al ratio to infinity, the use of organic templates provided more flexibility for the incorporation of transition metals in zeolite frameworks. Zeolites with certain amounts of framework Ti, B, P, Fe, Ga or Ge were obtained, and some of them, like Ti-silicate-1 (TS-1), attained great commercial success [19]. It is worth noting the impact of $\mathrm{Ge}$ on the synthesis of extra-large pore zeolites. Owing to the smaller angle of Si-O-Ge and Ge-O-Ge compared to Si-O-Si bonds, Ge-rich initial systems form small (four- and three-membered) ring units and thus zeolites with lower framework density. Some of these materials exhibited pores with size between 1 and $2 \mathrm{~nm}$ and bridged the gap between classical zeolites and mesoporous materials [20]. Unfortunately, none of these materials reached the stage of industrial use due to cost and thermal stability issues.

Zeolites are often conceptually classified by the units that compose the zeolite structure [5]. The so-called "secondary building units" (SBUs) are simple polyhedra that, arranged in different ways by symmetrical operations, result in the different framework types (Figure 1).

The number of T-atoms (that is, the atoms in "tetrahedral" coordination, namely $\mathrm{Si}$ and $\mathrm{Al}$ ) forming a zeolite channel determine to a great extent the size of the pore opening, which is an important characteristic of 
microporous molecular sieves. Consequently, zeolites are classified according to the number of T-atoms building the ring (n-MR):

- small pore zeolites: 8-MR, with diameters $c a .4 \AA$

- medium pore zeolites: $10-\mathrm{MR}$, with diameters $c a .5 .5 \AA$

- large pore zeolites: 12-MR, with diameters $c a .7 .5 \AA$

- extra-large pore zeolites: >14-MR, with diameters over $8 \AA$

The replacement of $\mathrm{Si}$ by $\mathrm{Al}$ in a zeolite structure introduces a negative charge on the framework, which is compensated by cations situated in the channels. These charge-balancing cations are exchangeable and extensively used to modify the properties of zeolites. The replacement of an alkali cation by proton $\left(\mathrm{H}^{+}\right)$converts a zeolite into a solid acid, which is largely exploited for preparation of heterogeneous catalysts. Ion exchange properties of zeolites are also intensively used for solving environmental issues, such as the capture of $\mathrm{Cs}^{+}, \mathrm{Sr}^{2+}$ or $\mathrm{NH}_{4}^{+}$, or the purification or softening of water for domestic, agriculture and industrial necessities.

Physicochemical properties of zeolites are addressed in a number of books and specialized reviews. The most important properties that make zeolites exceptional heterogeneous catalysts, molecular sieves and ion exchangers are mentioned next:

- Well-defined crystalline porous structure with variable dimensions, connectivity and geometry of the pores [21]. The pore system of zeolites is the basis of shape selectivity in catalytic and separation processes. The zeolite channel system is able to separate molecules with differences smaller than 1 Å. The pore system of zeolites also determines:

- The micropore volume (up to $0.35 \mathrm{~cm}^{3} \mathrm{~g}^{-1}$ ).

- The specific surface area (up to $850 \mathrm{~m}^{2} \mathrm{~g}^{-1}$ ).

- Variable chemical composition. Zeolite chemistry can be tuned in very wide ranges, first by varying the $\mathrm{Si} / \mathrm{Al}$ ratio from 1 to infinity and second by the incorporation of heteroatoms into the zeolite framework. The ion exchange properties of zeolites offer an additional level of flexibility in tuning zeolite chemistry. Important properties related to the chemical composition of zeolites are:

- Chemical and thermal stability

- Hydrophilic/hydrophobic interactions

- Number, strength and distribution of Brønsted and Lewis acid sites

- Environmentally friendly materials. 
- Acid/base properties of zeolites combined with exceptional shape selectivity, thermal and mechanical stability make zeolites ideal heterogeneous catalysts [22]. The catalytic application of zeolites is demonstrated in several chapters in this book. Shape selectivity and dipolar interactions between guest species and zeolite structures are the basis of the wide use of zeolite as adsorption and separation materials. These applications strongly depend on the pore characteristics, the nature of the charge-balancing cations and on the hydrophobic/hydrophilic character of the zeolite framework [23]. It is worth noting that zeolite applications are not limited to large-scale chemical processes. Recently zeolitic materials have also been used in electronic, optical and medical applications [24].

\section{Metal-organic Frameworks}

Metal-organic frameworks are hybrid crystalline porous materials with frameworks consisting of inorganic building units (metal ions or clusters) and organic linkers connected by coordination bonds of moderate strength (Figure 2) [25]. The metal ions employed in the synthesis of MOFs could be mono $(\mathrm{Cu})$, bi $(\mathrm{Zn}, \mathrm{Mn}, \mathrm{Co}, \mathrm{Cu})$, tri $(\mathrm{Cr}, \mathrm{Fe}, \mathrm{Al})$ and tetravalent $(\mathrm{Zr})$. More complex clusters such as $\mathrm{Cr}_{3} \mathrm{O}\left(\mathrm{H}_{2} \mathrm{O}\right)_{3}(\mathrm{COO})_{6}, \mathrm{Zr}_{6} \mathrm{O}_{4}(\mathrm{OH})_{10}\left(\mathrm{H}_{2} \mathrm{O}\right)_{6}$ $(\mathrm{COO})_{6}, \mathrm{Zn}_{4} \mathrm{O}(\mathrm{COO})_{6}$ or $\mathrm{Cu}_{2}(\mathrm{COO})_{4}$ are also used [26]. There are different approaches for the synthesis of stable MOFs, including modulated synthesis, isoreticular expansion and topology-guided design [27]. The

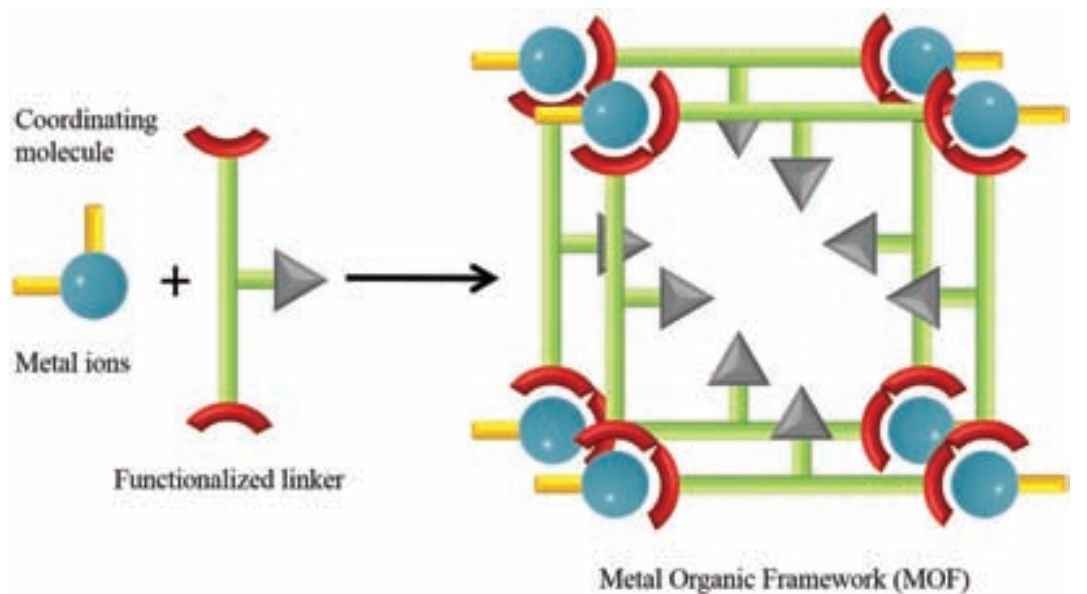

Figure 2. Schematic representation of the formation of a MOF structure. 
presence of diverse inorganic and organic components in MOFs provides a large number of structures with various properties, which will be described briefly following the sequence followed above for zeolites.

The crystalline nature of MOF materials permits a precise structural determination by classical experimental methods such as XRD, TEM and spectroscopic techniques such as NMR, UV-vis and Raman. In addition, computational chemistry is widely applied to predict new materials [28]. In general, MOFs exhibit much lower framework density than zeolites, which can be as low as $0.13 \mathrm{~g} \mathrm{~cm}^{-3}$. Consequently, some MOF materials exhibit $90 \%$ free volume and specific surface areas up to $7000 \mathrm{~m}^{2} \mathrm{~g}^{-1}$.

The stability of any crystalline material is defined as the resistance of the structure to degradation upon heating, cooling or exposure to chemically aggressive environments. The stability of MOFs is governed by multiple factors, including $\mathrm{pK}_{\mathrm{a}}$ of ligands, oxidation state, reduction potential and ionic radius of the metal ions, metal-ligand coordination geometry, and hydrophobicity of the structure [29]. The chemical weak spot of MOFs is usually the metal-linker bond that hydrolyses in aqueous medium, yielding a protonated linker and a de-ligated inorganic moiety. Many examples have demonstrated that both acidic and basic solutions accelerate the dissolution of MOF structures.

Although the general perception is that the stability of MOFs is limited, there are examples of fairly stable structures. For instance zeolitic imidazolate frameworks (ZIFs), metal-azolate (MAF, ZIF-57), and 'zeolitelike MOFs' (ZMOFs) combining azolate and carboxylate coordination capabilities on a single linker show substantially improved chemical and hydrothermal stability [30-32]. ZIF-8 is one of the materials exhibiting a high hydrothermal stability due to the fact that its pore apertures lack polar groups and, thus, the material is hydrophobic. MIL-type MOFs are based on trivalent metals $(\mathrm{Cr}, \mathrm{Al}, \mathrm{Fe})$ or lanthanides $(\mathrm{Eu}, \mathrm{Tb}, \mathrm{Y})$, which form strong bonds with oxygen-anion-terminated linkers and exhibit high chemical stability [33,34]. The hydrothermal stability of various MOFs under steaming at different pressures and temperatures was studied. The most stable under hydrothermal conditions MOFs are zirconium-based UiO-66, MIL-14 and hydrophobic ZIFs [35]. The mechanical stability of MOFs has been found to depend on the valence of the inorganic component and on there structural features. For example, MOFs based on divalent metals have higher mechanical stability as a result of the high strength of their inorganic-linker bond and are therefore more resistant to crystal deformation and damage [36]. 
MOF materials have a number of exceptional properties that make them very promising for future applications in fields where high specific surface area and pore volume are required. In addition, an exceptionally high pore volume and specific surface area combined with a diverse surface chemistry, might extend the application of porous materials beyond that of zeolites. At present, however, the applications considered for MOFs are mainly conceptual because their performance has only been assessed under ideal conditions and collected data is not sufficient to ensure an economically successful application.

MOFs exhibit isolated, well-defined active sites including open metal sites, metalloporphyrins and reactive functional (inorganic or organic) groups, which can serve different types of reactions. Consequently, MOFs have been intensively studied as heterogeneous catalysts. Although there has been no breakthrough resulting from MOF catalysis so far, a number of examples demonstrate the potential of these materials. The vanadium-based MOFs (MIL-47, MOF-48) have been shown to be stable, catalytically active and highly selective in the conversion of $\mathrm{CH}_{4}$ and $\mathrm{CO}$ to acetic acid [37]. Another example is the $\mathrm{Ce}^{4+}$-driven water oxidation reactions catalysed by MOF [38]. Gas-phase catalysis with MOFs materials has been studied as well [39]. An important prospective of MOFs was recently reviewed, in which several single-site catalytic functions can be combined within the framework and used as powerful enzyme-mimicking materials [40].

Certain MOF materials are exceptional adsorbents in terms of the available pore volume [41]. Water-stable MOFs could possibly be applied to effectively uptake gases under moist conditions. There are an increasing number of studies on the use of stable MOFs for wastewater remediation. Targeted compounds in water systems include $\mathrm{SO}_{\mathrm{x}}, \mathrm{NO}_{\mathrm{x}}$, greenhouse gases, VOCs, dyes, drugs, pharmaceuticals, organic chemicals, metal ions, etc. Experimental results have revealed that MOFs have been efficient in adsorbing toxic gases under dry conditions but their adsorption ability was reduced in a humid atmosphere due to the competitive adsorption of water. One exception is $\mathrm{NH}_{3}$, where the decrease in adsorption capacity was negligible, suggesting that ammonia could be removed by a MOF sorbent under both dry and humid conditions. MOFs have also been extensively studied for the past decade as promising hydrogen storage materials [42]. However, many technical problems still remain to be encountered in order to reach practical application.

Water-stable MOFs are extensively employed in membrane separation applications, such as pervaporation, steam separation, desalination, 
and wastewater treatment. Whether MOFs can fully maintain their functions and structure across multi-cycle applications remains a questionable challenge.

Potential applications of MOFs as chemical sensors and adsorbents for the detection and removal of heavy-metal ions have been envisaged [43]. The wide variety of organic linkers and metal nodes that can be incorporated into MOFs could be selected in a way to increase the sensitivity and selectivity towards particular analytes. MOFs could be used as a functional sensing element or as an auxiliary filtering element, while the real-time gas sensing response could be monitored through various methods. For example, electrical property-based sensors were used to monitor the adsorption of analytes within the pores by measuring changes in the conductivity, impedance or resistance of the sensing material. The potential of MOFs in this field is fairly high since the stability of the materials is not a primary concern.

MOFs have also been targeted for biological applications mainly for the controlled delivery of active ingredients. The requirements for MOFs in this field are not only relevant functionalities but also good biocompatibility and degradation under physiological conditions, as they might lead to potential toxicity issues. Considering that a large number of therapeutic molecules possess polycomplex groups in their structure, there are continuous efforts to use active ingredients for the construction of bio-MOFs [44]. Although significant progress has been made in the preparation of bio-MOFs, the possible bio-related application is still in its infancy.

\section{Summary and outlook}

Zeolites and metal-organic frameworks, two important families of crystalline molecular sieve materials, were reviewed, introducing information on the preparation, physicochemical properties and applications that are expanded on later in this book. The two families exhibit a multitude of similarities, like crystalline structure and well-defined pore system, surface functionality and chemical reactivity. On the other hand, the two families differ substantially in terms of chemical nature, pore size and available pore volume, thermal and chemical stability.

Zeolites are inorganic materials with robust frameworks that withstand elevated temperatures and harsh chemical environments. Owing to their high stability, strong acid/base properties and exceptional selectivity, aluminosilicate zeolites are the most successful catalysts in the chemical 
process industry. Zeolite molecular sieves have been actively used more than half a century and new processes based on these exceptional materials continue to be reported. On the other hand, the pore size, volume, and specific surface area of zeolites are substantially lower than that of MOF molecular sieves. The latter exhibit much higher available pore volume and often much larger (meso)pores. The surface chemistry of MOFs can be modulated by in situ or post-synthesis methods and thus adapted to different applications. In the last two decades, MOFs have been the most actively studied family of porous materials and great advances in their preparation, modification and stability have been achieved. It seems, however, that MOF molecular sieves cannot afford the industrial requirements of stability, long lifetime and moderate production cost, yet. Thus, zeolites remain unbeatable as heterogeneous catalysts, adsorbents and ion exchangers. Nevertheless, MOF materials will certainly be used in the future, most probably in areas where they don't compete with zeolites in terms of stability and production price. The fact that these promising porous materials are still not industrially employed may lie in the traditional thinking of applied chemists, which is always biased towards applications where zeolites already have commercial success. We believe that it will not be long until the first industrial use of a MOF material is reported.

\section{Acknowledgements}

The authors acknowledge the financial support from ANR project 15-CEo6ooo4.

\section{References}

[1] Thommes, M., Kaneko, K., Neimark, A.V., Olivier, J.P., Rodriguez-Reinoso, F., Rouquerol, J., Sing, K.S.W. Pure Appl. Chem. 2015, 87, 1051.

[2] Breck, D. Zeolite Molecular Sieves, John Wiley \& Sons, 1974.

[3] Martínez, C., Corma, A. Coord. Chem. Rev. 2011, 255, $155^{8}$.

[4] Mintova, S., Jaber, M., Valtchev, V. Chem. Soc. Rev. 2015, 44, 7207.

[5] http://www.iza-structure.org/databases/

[6] Barrer, R.M. Hydrothermal Chemistry of Zeolites, Academic Press, 1982.

[7] Wadlinger, R.L., Kerr, G.T., Rossinski, E.J. US Patent 3,308,o69, 1967.

[8] Flanigen, E.M., Bennet, J.M., Grose, R.W., Cohen, J.P., Kirchner, R.L., Smith, J.V. Nature 1978, 271,512 .

[9] Wilson, S.T., Lok, B.M., Messina, C.A., Cannan, T.R., Flanigen, E.M.J. Am. Chem. Soc. 1982, $104,1146$. 
[10] Kresge, C.T., Leonowicz, M.E., Roth, W.J., Vartuli, J.C., Beck, J.S. Nature 1992, 359, 710.

[11] Beck, J.S., Vartuli, J.C., Roth, W.J., Leonowicz, M.E., Kresge, C.T., Schmitt, K.D., Chu, C.T.W., Olson, D.H., Sheppard, E.W., McCullen, S.B., Higgins, J.B., Schlenker, J.L. J. Am. Chem. Soc. 1992, 114, 10834.

[12] Zhao, D., Feng, J., Huo, Q., Melosh, N., Frederickson, G.H., Chmelka, B.F., Stucky, G.D. Science 1998, 279, 548.

[13] Li, H., Eddaoudi, M., O'Keeffe, M., Yaghi, O.M. Nature 1999, 402, 276.

[14] Chae, H.K., Siberio-Perez, D.Y., Kim, J., Go, Y., Eddaoudi, M., Matzger, A.J., O’Keeffe, M., Yaghi, O.M. Nature 2004, 427, 276.

[15] Tosheva, L., Valtchev, V. Chem. Mater. 2005, 17, 2494.

[16] Loewenstein, W. Am. Mineral. 1954, 39, 92.

[17] Barrer, R.M., Denny, P.J.J. Chem. Soc. 1961, 983.

[18] Lobo, R.F., Zones, S.I., Davis, M.E.J. Incl. Phenom. Mol. Recogn. Chem. 1995, 21, 47.

[19] Bellussi, G., Carati, A., Clerici, M.G., Esposito, A. Stud. Surf. Sci. Catal. 1991, 63, 421.

[20] Jiang, J., Yu, J., Corma, A. Angew. Chem. Int. Ed. 2010, 49, 3120.

[21] Dyer, A. An introduction to zeolite molecular sieves, John Wiley \& Sons, 1988.

[22] Weitkamp, J., Puppe, L. Catalysis and Zeolites: Fundamentals and Applications, Springer, 1999 .

[23] Di Renzo, F., Fajula, F. Stud. Surf. Sci. Catal. 2005, 157, 1-12.

[24] Sherman, J.D. PNAS 1999, 96, 3471.

[25] O'Keeffe, M., Yaghi, O.M. Chem. Rev. 2012, 112, 675 .

[26] Stock, N., Biswas, S. Chem. Rev. 2012, 112, 933.

[27] Bai, Y., Dou, Y., Xie, L.H., Rutledge, W., Li, J.R., Zhou, H.C. Chem. Soc. Rev. 2016, 45, 2327.

[28] Colon, Y.J., Snurr, R.Q. Chem. Soc. Rev. 2014, 43, 5735.

[29] Chapman, K.W., Halder, G.J., Chupas, P.J.J. Am. Chem. Soc. 2009, 131, 17546.

[3o] Eddaoudi, M., Sava, D.F., Eubank, J.F., Adil, K., Guillerm, V. Chem. Soc. Rev. 2015, 44, 228.

[31] Zhang, J.P., Zhang, Y.B., Lin, J.B., Chen, X.M. Chem. Rev. 2012, 112, 1001.

[32] Liu, Y., Kravtsov, V.C., Eddaoudi, M. Angew. Chem. Int. Ed. 2008, 47, 8446.

[33] Férey, G., Mellot-Draznieks, C., Serre, C., Millange, F., Dutour, J., Surblé, S., Margiolaki, I. Science 2005, 309, 2040.

[34] Alezi, D., Peedikakkal, A.M.P., Weseliński, L.J., Wojtas, R., Eddaoudi M. J. Am. Chem. Soc. $2015,137,5421$.

[35] Nguyen, N.T.T., Furukawa, H., Gándara, F., Nguyen, H.T., Cordova, K.E., Yaghi, O.M. Angew. Chem. Int. Ed. 2014, 53, 10645 .

[36] Wu, H., Yildirim, T., Zhou, W.J. Phys. Chem. Lett. 2013, 4, 925.

[37] Phan, A., Czaja, A.U., Gándara, F., Knobler, C.B., Yaghi, O. Inorg. Chem. 2011, 50, 7388.

[38] Wang, C., Wang, J.L., Lin, W.J. Am. Chem. Soc. 2012, 134, 19895.

[39] Nguyen, H.G.T., Schweitzer, N.M., Chang, C.Y., Drake, T.L., So, M.C., Stair, P.C., Farha, O.K., Hupp, J.T., Nguyen, S.B.T. ACS Catal. 2014, 4, 2496.

[40] Rogge, S.M.J., Bavykina, A., Hajek, J., Garcia, H., Olivos-Suarez, A.I., Sepúlveda-Escribano, A., Vimont, A., Clet, G., Bazin, P., Kapteijn, F., Daturi, M., Ramos-Fernandez, E.V., Llabrés i Xamena, F.X., Van Speybroeck, V., Gascon, J. Chem. Soc. Rev. 2017, 46, 3134.

[41] Wang, C., Liu, X., Demir, N.K., Chenb, J.P., Li, K. Chem. Soc. Rev. 2016, 45, 5107.

[42] Suh, M.P., Park, H.J., Prasad, T.K., Lim, D.W. Chem. Rev. 2012, 112, 782.

[43] Wales, D.J., Grand, J., Ting, V.P., Burke, R.D., Edler, K.J., Bowen, C.R, Mintova, S., Burrow, A.D. Chem. Soc. Rev. 2015, 44, 4290.

[44] Gimenez-Marques, M., Hidalgo, T., Serre, C., Horcajada, P. Coord. Chem. Rev. 2015, 307, 342. 



\title{
2. Synthesis and Identification Methods for Zeolites and MOFs
}

\author{
L. Marcela Martínez T., ${ }^{1}$ Svetlana Ivanova, ${ }^{1}$ Benoît Louis, ${ }^{2}$ \\ José Antonio Odriozola
}

${ }^{1}$ Departamento de Química Inorgánica e Instituto de Ciencia de Materiales de Sevilla, Universidad de Sevilla - CSIC, Avda Américo Vespucio 49, 41092 Sevilla, Spain.

${ }^{2}$ CNRS Laboratoire de Synthèse, Réactivité Organiques et Catalyse (LaSyROC), UMR 7177, Université de Strasbourg (UDS), France.

Keywords: Synthesis, crystallization, X-ray diffraction, surface area measurement, electron microscopy

\section{Introduction}

Conceptually, there are no important differences between classical inorganic porous solids as zeolites and hybrid porous solids as metal-organic frameworks (MOFs) [1]. Based only on their porosity, both groups are porous solids with modular structure offering important structural diversity and, even more importantly, the possibility to synthesize them on-demand, with tailored properties for targeted applications. The difference between them lies in the units composing the structure, only inorganic for the case of zeolites, and of hybrid inorganic-organic nature for MOFs. Although MOFs are often considered hybrid materials mimicking zeolites, in the last decade they started to surpass the versatility of purely inorganic zeolites, also benefiting from permanent porosity. MOFs outstand by their large surface area and by the wide assortment of pore sizes and pore specific volumes that can be synthesized, both converting these materials in promising alternative to the zeolites even in their traditional fields of application such as gas separation and purification.

Both materials, by virtue of their structure (i.e. porosity, surface area, pore shape and functionalities), have found useful applications in almost all fields of human life where chemical, biochemical or physicochemical processes take place. They are used for the purification of gases or liquid mixtures by sorption, sieving, and filtering. More specifically, zeolites are 
used as ion exchangers (they have replaced polyphosphates as water softeners for laundry purposes), as catalysts in refining and petrochemistry or, more recently, for the synthesis of fine chemicals [2-5]. MOFs are used for the production of optically and magnetically active materials, drug delivery, proton conduction, fine chemistry and catalysis, etc. [6-9]. Some of these applications are discussed in detail in subsequent chapters of this book.

As far as synthesis is concerned, synthetic strategies should be easy to apply, simple to reproduce and based on a careful choice of parameters that influence the structure of the material and its properties. Although zeolites can be found in nature, nowadays many zeolites are synthesized in laboratories without having a known natural counterpart. Today, more than 230 different zeolites are known and classified according to their structural parameters. By contrast, MOF materials are all synthetic, and more than 50,000 structures are registered in the Cambridge Crystal Database [10]. In general, the synthetic strategies concerning zeolites are based on mimicking the conditions under which natural zeolites of volcanic origin are presumably formed, i.e. under elevated temperatures, high salt concentrations and autogenous pressure in hydrothermal conditions [11]. The diversity of existing MOF structures, on the other hand, suggests a wider variety of preparation methods. However, as will be discussed later, the majority of synthetic strategies are based on precipitating the solid from solution. Hence, the aim of this chapter is to survey the synthetic strategies for zeolites and MOFs. This will be accompanied by some examples selected from the most known and used materials of each class. Finally, a brief description of some routine characterization methods applicable to zeolite and MOF materials after their synthesis is presented.

\section{Zeolites}

\subsection{Structure and nomenclature}

Zeolites are crystalline aluminosilicates belonging to the group of tectosilicate molecular sieves. They are porous solids with ordered, interconnected microporous channels with diameters ranging from 0.2 to $2 \mathrm{~nm}$, corresponding to the size of many organic molecules. They possess a well-defined three-dimensional porous structure arising from a framework of $\left[\mathrm{SiO}_{4}\right]^{4-}$ and $\left[\mathrm{AlO}_{4}\right]^{5-}$ tetrahedra $\left(\mathrm{TO}_{4}\right.$, where $\mathrm{T}$ represents $\mathrm{Si}$ or $\left.\mathrm{Al}\right)$ linked together by all their corners, as depicted in Figure 1 a). These primary 
building units (PBU) or blocks are assembled so that each $\mathrm{O}$ is shared by two identical tetrahedra, thus creating a lattice made by identical building blocks, which extends infinitely as it is the case in all crystalline materials. A structure directing agent (named $\mathrm{X}$ in Figure 1) is usually used to guide the self-assembly of $\mathrm{SiO}_{4}$ (red) and $\mathrm{AlO}_{4}$ (blue) units. The nature of $\mathrm{X}$ depends on the zeolite that we aim to prepare and on the synthesis conditions, and can be of organic (amine, alkylammonium or phosphonium ions) or inorganic $\left(\mathrm{Na}^{+}\right)$.

Each Si ion has its 4+ charge balanced by the four surrounding $\mathrm{O}$ (each with a 2 - charge), so the silica tetrahedron in the lattice is electrically neutral. Since the trivalent $\mathrm{Al}$ is also bonded to four shared oxygen anions, the residual charge on each $\mathrm{AlO}_{4}$ unit is 1-. Therefore, each alumina tetrahedron requires a $1+$ charge from a cation (mono- or divalent) located into the channels or cavities in order to keep the electrical neutrality throughout the structure. After the synthesis of the zeolites these cations are usually sodium or ammonium, but they can be readily replaced by ion exchange. In addition, water molecules are present in the void volume of the cavities and channels.

The unit cell formula of zeolites is usually written as:

$$
M_{x / n}\left[\left(\mathrm{AlO}_{2}\right)_{x}\left(\mathrm{SiO}_{2}\right)_{y}\right] z \mathrm{H}_{2} \mathrm{O}
$$

where $\mathrm{M}$ represents the cation (alkali or alkaline earth metal) counterbalancing the negative charge associated with the framework aluminium ions, $n$ is the charge of the cation, $x$ is the number of Al per unit cell, $y$ represents the number of $\mathrm{Si}$, and $z$ is the number of water molecules entrapped

a

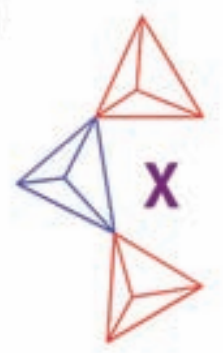

b
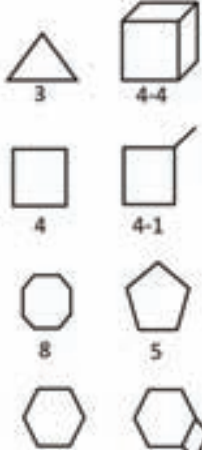

6
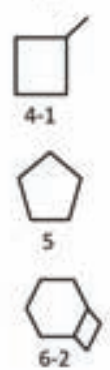
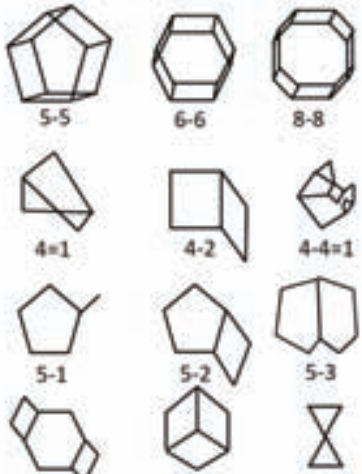

6-1
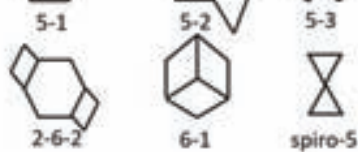

Figure 1. a) $\mathrm{TO}_{4}$ tetrahedra linked together to generate the $3 \mathrm{D}$ structure. b) Main secondary Building Units (SBUs). 
(physisorbed) in the channel system. The $\mathrm{Si} / \mathrm{Al}$ mole ratio is always larger than (or equal to) one, since a direct linkage between two $\mathrm{AlO}_{4}$ entities is forbidden, according to Löwenstein's rule [12].

The tetrahedral PBUs are combined in a more sophisticated way to generate repeating structural sub-units. These recurring units are called secondary building units (SBUs). The system of SBUs is quite simple and was therefore adopted as a convenient way for zeolite classification. Figure 1 b) represents sixteen SBUs that actually describe most of known zeolite structures. These SBUs only reflect the aluminosilicate skeleton (i.e. the relative positions of $\mathrm{Si}, \mathrm{Al}$, and $\mathrm{O}$ in space) but exclude the consideration of the cations and water moieties present within the cavities and channels. When SBUs are joined to form infinite lattices, they can define larger rings, containing $6,8,10,12$ and up to 20 linked tetrahedra (rings of $\mathrm{T}$ atoms).

The International Zeolite Association (IZA) adopted a classification in which it was assumed that all known frameworks could be described by a combination of one or more SBUs [13-14]. According to the IUPAC, the structure type codes of zeolites consist of three capital letters derived from their original names [15]. These codes have been developed to create a short nomenclature that does not depend on the composition, nature of T-atoms, cell dimensions, or symmetry.

Additionally, zeolites can also be classified according to the size of their pores [16]:

- small pores: six- or eight-membered ring pores (6 to $8 \mathrm{~T}$-atoms) having a pore diameter between $3-4.5 \AA$ (LTA zeolite)

- medium pores: ten-membered ring pores, diameter between 4.5-6 (such as ZSM-5 or ZSM-11, where ZSM stands for Zeolite Socony Mobil)

- large pores: twelve-membered ring or larger pores, diameter between 8 and $20 \AA$ (BEA, Cloverite)

Pore systems may differ considerably between zeolites, depending on the presence of interconnections between the different pore types that may be found in a single structure, ranging from parallel and independent in LTL zeolite (12-membered rings), to $3 \mathrm{D}$ interconnected cage systems, as in FAU (12-membered rings).

\subsection{Synthesis of zeolites}

Systematic synthesis of zeolites started with the pioneering work of Barrer and Milton in the 1950s. Despite hundreds of patents and many papers 
Table 1. Chemical Ingredients Involved In The Zeolite Synthesis.

\begin{tabular}{ll}
\hline Source & Function \\
\hline $\mathrm{SiO}_{2}$ & Building the primary units of the framework \\
$\mathrm{AlO}_{2}^{-}$ & Creating the negative charge \\
$\mathrm{OH}^{-}, \mathrm{F}^{-}$ & Mineralizer \\
Alkali cation: $\mathrm{Na}^{+}, \mathrm{K}^{+}$ & Compensating the charge while avoiding Si polymerization \\
Template: $\mathrm{TPA}^{+}, \mathrm{TEA}^{+}$ & Directing crystallization \\
Water & Solvent, guest molecule \\
\hline
\end{tabular}

published in this field during the last decades, the number of zeolite structures actually known remains low compared to the several million (potentially) stable zeolite structures predicted by computers [17].

Zeolites are traditionally prepared via sol-gel hydrothermal synthesis under autogenous pressure. The chemical sources and their function during the synthesis are listed in Table 1.

The main point of zeolite synthesis is its crystallization from an inhomogeneous gel formed from silica and alumina sources, which were combined with water under high $\mathrm{pH}$ conditions generated by $\mathrm{OH}^{-}$ions. Many parameters need to be controlled, which render the understanding of the zeolite growth mechanisms and crystallization control relatively complex [16].

Little variations of the zeolite synthetic procedures are observed during the last 50 years and all are based on sol-gel hydrothermal syntheses. The application of microwave heating or sonochemistry influences the temperature, time and synthesis duration, although the zeolite formation still occurs under traditional hydrothermal conditions.

$\mathrm{SiO}_{2}$ and $\mathrm{AlO}_{2}$ sources

The silica source is a key parameter, as its dissolution may favour crystallization towards a particular zeolite type. Soluble silicates and their hydrates are widely used, as they can exist in a monomeric form to establish Si-O-Al or Si-O-Si associations [18].

The aluminium source is not so crucial, and most of the laboratories use metal aluminates, commonly sodium aluminate. Zeolite formation is enhanced by the presence of $\left[\mathrm{Al}(\mathrm{OH})_{4}\right]^{-}$moieties at $\mathrm{pH}>10$.

\section{Alkali cations and templates}

The alkali cation acts as a core around which the silica structure is built up. It also interferes in $\mathrm{SiO}_{2}$ polymerization. The template or, more correctly, 
Table 2. Templates reported for the synthesis of ZSM-5 zeolite.

\begin{tabular}{ll}
\hline Tetrapropylammonium halide & Methylquinuclidine \\
Tetraethylammonium halide & Morpholine \\
Tripropylamine & Ethylenediamine \\
Dipropylamine & Diethylenetriamine \\
Propylamine & Triethylenetetraamine \\
1,6-diaminohexane & Ethanol + ammonia \\
1,5-diaminopentane & Ethanol \\
1,6-hexanediol & Ethanolamine \\
Propanolamine & Glycerol \\
\hline
\end{tabular}

the structure-directing agent (SDA), is usually an organic cation containing a functional group able to bind with silicate and aluminate species. The synthesis of "high-silica" zeolites usually requires the presence of an organic structure-directing agent to achieve the desired structure. For example, a wide range of templates can be used to synthesize ZSM-5 zeolite (Table 2).

The template is believed to have two functions when directing the synthesis. Firstly, it promotes the formation of the desired building blocks in the gel. Secondly, it acts as a hydrophobic pore filler, preventing the dissolution and recrystallization of the crystals already formed. In the case of alkylamine salts, the template must be removed after synthesis via Hofmann elimination by calcination at high temperatures $\left(>50{ }^{\circ} \mathrm{C}\right)$.

Figure 2 presents the mechanism of auto-assembly that occurs through van der Waals, hydrogen-bond, and ionic interactions between the hydrophobic silicate species and the template $[19,20]$. The driving force of the process is the replacement of water molecules by Si and $\mathrm{Al}$ moieties around the organic cation. This mechanism is quite different from any mechanism encountered in classical organic chemistry, since no other reactants and other pathways are available to reach the desired product. The structuredirecting agent acts as a template for the zeolite cavities and can be chosen to tailor the pore openings.

In addition to the SDA, co-templates can also be added in the synthesis. One remarkable example is the synthesis of mesoporous LTA zeolite by Choi et al. [21] with the incorporation of a surfactant in the synthesis gel. Sophisticated morphologies have also been obtained by Valtchev et al. [22], who added a leaf of Equisetum arvense to the gel, thus enabling replication of the plant's surface morphology. Likewise, sugar cane bagasse 

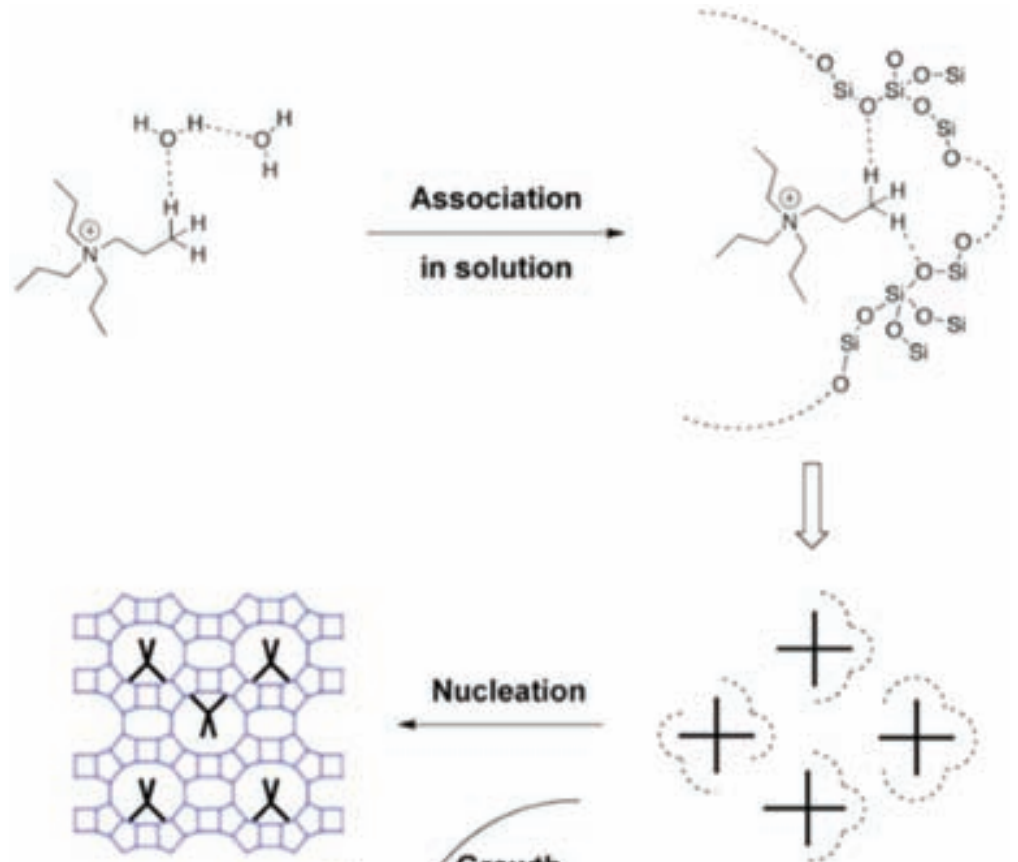

\section{Nucleation}
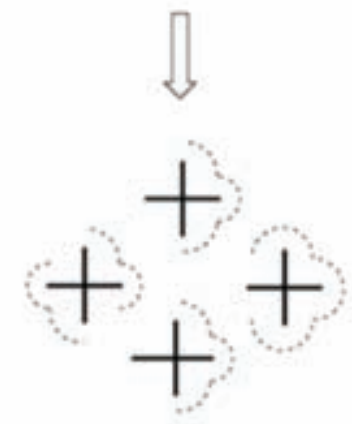

Growth

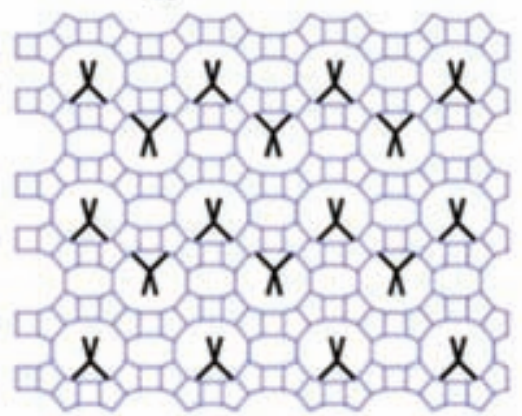

Figure 2. Mechanism of structure-directing action and crystal growth involving combined inorganic-organic species in ZSM-5 zeolite synthesis reprinted with permission from [19]. Copyright 1995 American Chemical Society.

residues were successfully used to produce MFI zeolite crystals exhibiting a nano-"French fries" morphology [23].

\section{$\mathrm{OH}^{-}$}

Since the $\mathrm{pH}$ is often higher than 10, hydroxyl anions act as the mineralizer, a nucleophile that influences the crystallization time. In general, crystallization 
of zeolites happens from an inhomogeneous hydrogel, formed by the T-sources ( $\mathrm{Si}$ and $\mathrm{Al}$ ), the mineralizing agent, and the SDA via a supersaturated solution, favouring the nucleation of initial nuclei that then grow to form larger crystallites [24]. Once, the concentration shifts below the supersaturation limit, no new nuclei are formed and the present crystallites continue to grow as long as T-sources are available. It is important to note that complex dissolution-recrystallization processes occur parallel to crystal growth [25].

\section{Fluoride media}

$\mathrm{F}^{-}$ions can be used instead of $\mathrm{OH}^{-}$anions as the mineralizer and it thus becomes possible to synthesize zeolites at $\mathrm{pH}$ values in the acidic range $[26-32]$. These conditions are particularly appropriate for the synthesis of high-silica materials. The fluoride route is the best strategy to produce highly crystalline materials exempt from defects [32]. The crystallinity of the materials produced in fluoride media is generally higher than that for materials obtained by the hydroxyl-mediated route. In addition, [F]-ZSM-5 zeolites exhibit a regular and narrow crystal size distribution in the 1 to $100 \mu \mathrm{m}$ range. This larger crystal size as compared to that arising in the alkaline route is due to the growth in a less supersaturated solution. Here, fewer metastable phases are formed, which implies a certain ease of preparation of the desired zeolite.

Another important advantage is the direct formation of the ammonium form $\mathrm{NH}_{4}-\mathrm{ZSM}-5$ form, rather than Na-ZSM-5, which has to be subjected to repeated ion-exchange steps. In this procedure, a single calcination step is needed to burn the template and to generate the catalytically active acidic $\mathrm{H}-\mathrm{ZSM}-5_{5}$ form.

This "unconventional" procedure also offers the possibility for an incorporation of doping elements such as $\mathrm{Fe}^{3+}, \mathrm{Co}^{2+}$ or lanthanides, which are sparingly soluble in alkaline media.

Reaction variables: concentration, temperature, pressure and time

Not only the presence of all components in the reaction mixture is important, but also their relative proportion. A required $\mathrm{Si} / \mathrm{Al}$ zeolite composition can be obtained only by using an appropriate template and/or an adequate $\mathrm{OH}^{-} /$ $\mathrm{SiO}_{2}$ ratio. If the latter increases, more silicate remains in solution and lower $\mathrm{Si} / \mathrm{Al}$ products are formed. Additionally, the $\mathrm{H}_{2} \mathrm{O} / \mathrm{SiO}_{2}$ ratio impacts the formation of any zeolite structure, affecting the degree of polymerization. The proper combination of all these parameters is not trivial [25,33]. Figure 3 shows a typical phase transition diagram as a function of composition.

Several studies highlighted the influence of synthesis duration and temperature over the metastability of the zeolites $[34,35]$. Zeolites crystallize from alkaline aqueous gels at temperatures ranging from room temperature 


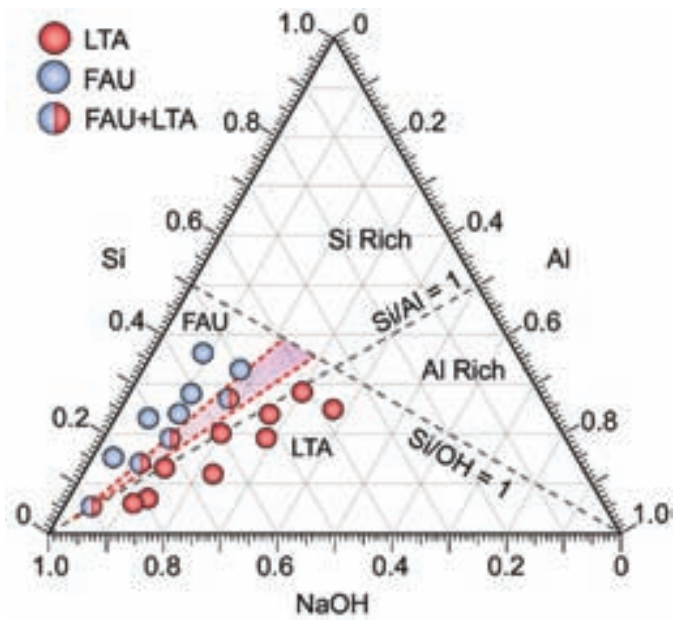

Figure 3 .Typical zeolite crystallization domain showing the phases formed from gels with different composition at a given temperature and synthesis time reprinted with permission from [25]. Copyright 2013 American Chemical Society.

to $300{ }^{\circ} \mathrm{C}$. Therefore, the syntheses are most often conducted in sealed vessels under autogenous pressure, i.e. that generated by the vapour phase when the temperature is raised.

Time is another key variable. It is important in two ways: i) an induction period during which the mixture is held at ambient temperature (gel ageing) favours the synthesis yield, ii) in some cases, different metastable structures can be formed depending on time (e.g. Mordenite vs. analcime). Ostwald's law states that transformations have to occur in the direction of more highly stabilized phases [36].

Despite a long debate regarding the mechanism(s) of crystallization, significant advances have been achieved, thanks to contributions like those of Valtchev and Mintova [16,37-39]. On a general consensus, zeolites are synthesized from aluminosilicate gels in which structural and chemical rearrangements occur, allowing (under suitable conditions) nucleation and crystallization into highly organized structures $[16,40]$.

A typical S-shaped curve usually is observed when plotting the yield of crystalline material against synthesis time [36]. When the synthesis mixture reaches the temperature necessary for zeolite formation, crystallization can only be expected after an induction period during which nucleation occurs. In fact, the state of nucleating solutions at times prior to the development of periodicity has become accessible by spectroscopic techniques such as NMR and X-ray scattering [40-42]. It was shown that at this stage, the SBUs later forming the crystal still are species in solution [43]. The gel 
and the species in solution thus continuously rearrange, changing phases from monomers to clusters of silicates and aluminosilicates. These clusters form and disappear by condensation and hydrolysis under the influence of temperature. In this re-organization process some particles become stable and small nuclei (in the nanometer range) are formed before crystallization (and crystal growth) can start. All these features suggest that crystal growth is preceded by a slow nucleation step initiated by local concentration fluctuations inside the mixture and followed by a spontaneous growth of nuclei exceeding a critical size [40]. Pope has explained this phenomenon in terms of a decrease in the Gibbs free energy, which favours crystal growth [44].

According to Ostwald's law of successive transformations, the first product to appear in the course of a zeolite synthesis may not be the more stable [37]. In other words, a non-stable system does not necessarily transform into the most stable state but rather into one close to its own, and so on. It means that in zeolite synthesis, a series of kinetic products may form as transient meta-stable phases, prior to the formation of the most stable thermodynamic product [45]. The corollary is that a minor modification in the gel, which represents a large panel of variables, may allow shifting from one structure to another. Likewise, the possibilities for tailoring any zeolite intrinsic properties are nearly infinite, since the set of synthesis parameters is wide [46]. Quite often, a "seeding" technique is applied to guide the crystallization towards the desired material, i.e. a few milligram of the desired zeolite are added to the synthesis mixture.

The synthesis of a zeolite occurs through weak and strong interactions between building units, forming covalently bonded framework stabilized by extra-framework species. The mineralizer and the organic template (and co-template) play a role in determining the size of the precursor particles in the gel. Subotic et al. [47-51] have demonstrated that different kinds of solid particles were present in the hydrogel before and after hydrothermal treatment: aggregates of amorphous gel particles, partially ordered particles, aggregates of fully ordered particles. During the initial (alumino) silica polymerization stages, small randomly aggregated gel particles were shown to form [16]. The composition of the gel particles approaches the stoichiometric zeolite composition once a chemical equilibrium between solid and liquid phases achieved. In summary, the zeolite organization proceeds through: i) formation of primary units (precursor species), ii) aggregation of those species and their densification resulting in amorphous particles, whose size and morphology are indicative for the preparation of zeolites with similar properties, iii) further nucleation occurs on the amorphous particles and the crystal growth continues within the particles followed by the addition of primary units (or aggregation of nanoparticles) to the 


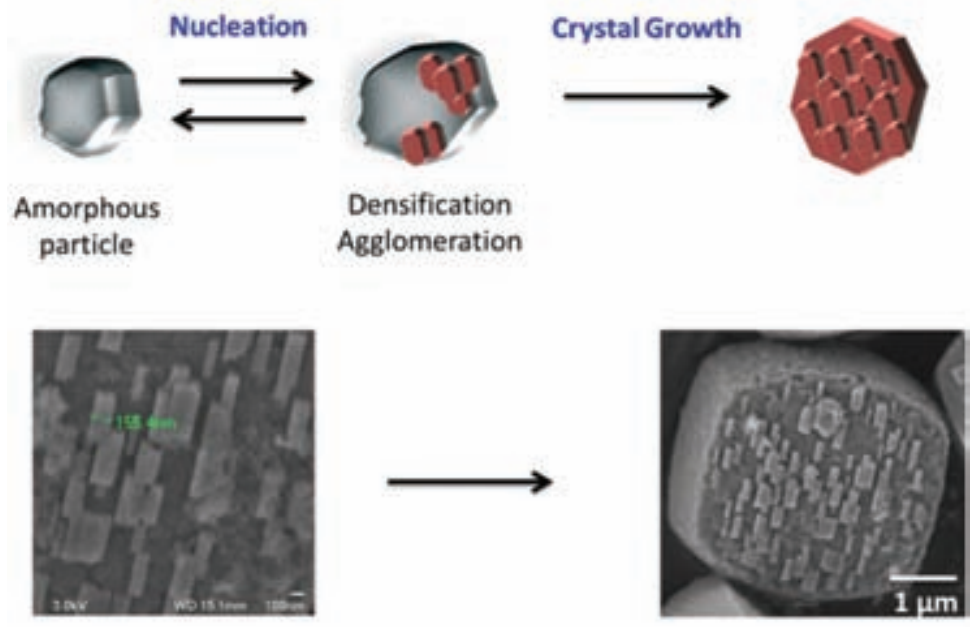

Figure 4. Scheme of an aggregation mechanism for zeolite crystal growth (top) illustrated by a homemade zeolite synthesis (bottom).

growing crystal with time. Figure 4 provides a schematic overview of an aggregation mechanism inspired by Davis' studies [52]. SEM images of a homemade zeolite illustrates suggestions from Mintova and Davis $\left[16,5^{2}\right]$.

In view of the numerous structures, compositions and synthesis conditions that may exist, a universal mechanism that can explain all zeolite crystallization modes does not exist. Whilst a solution-mediated process has been discussed for many years, the existence of solid-solid transformations in the gel represents nowadays a well-established route. Still a proper control of the structure at the nanoscale remains the key for tailoring zeolite properties and structure at the molecular and microscopic levels.

\section{Metal-organic frameworks}

\subsection{Structure and nomenclature}

In the development of the metal-organic frameworks the know-how gained from zeolites served, with no doubt, as inspiration. Since the discovery of zeolites, and especially since the mastery of their synthesis and application in shape selective catalysis, chemists dream of well-defined porous structures. This quest led to a constant emergence of novel materials with potential catalytic applications and other fields of porous materials evolved, such as mesoporous materials, hierarchical systems, and metal-organic-frameworks (MOFs). 


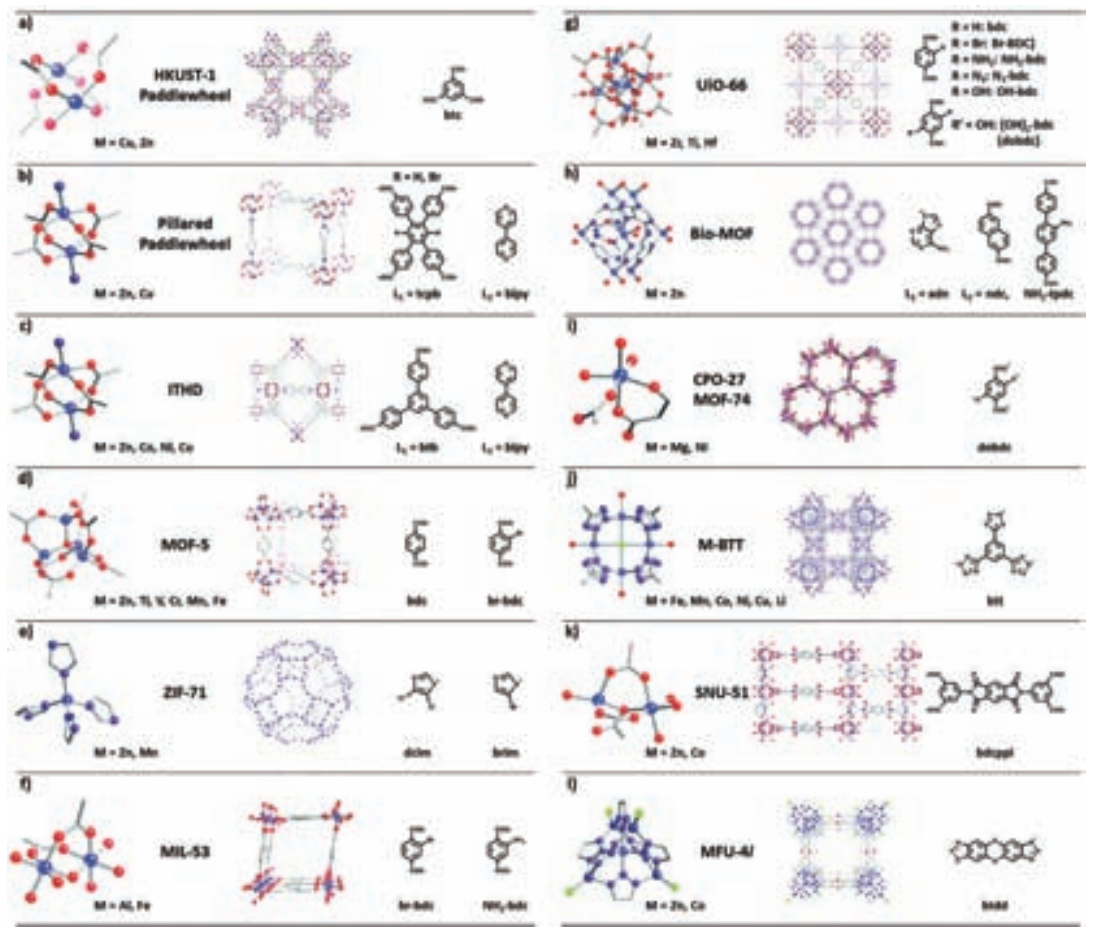

Figure 5. Lattice structures (middle) and corresponding SBUs (metal nodes (left), and organic linkers (right) of some MOFs. Atom definition: blue - metal, red - oxygen, purple - nitrogen, grey - carbon, green - chlorine). [53] - Published by The Royal Society of Chemistry.

Metal-organic frameworks, also called coordination polymers (CPs), are a type of hybrid organic-inorganic solid built up by an extended network of metal ions (or clusters) coordinated to multidentate organic molecules. This definition englobes materials with different crystallinity, chemical nature and porosity [8]. As for zeolites, metal-organic components form extended $3^{D}$-skeletons by association of secondary building units (SBU). Whereas zeolites contained only inorganic parts (namely $\mathrm{SiO}_{4}$ and $\mathrm{AlO}_{4}$ tetrahedra), a MOF SBU contains organic linkers bonded to a metal centre through covalent bonds. Figure 5 present some examples of MOF SBU.

The abbreviation MOF is used as a general term of this class of compounds, although it also designates a subclass of different compounds when combined with a number (MOF-74, MOF-101, MOF-177, etc.). Analogously to zeolites, existing MOFs are grouped in families of compounds, designated by a name and a number. The families are organized either by similarity in structure and symmetry, like IRMOFs (IsoReticular Metal-Organic Frameworks) and F-MOF-1 (Fluorinated Metal-Organic Frameworks), by similarity to zeolite topology, like 
ZIF (Zeolite Imidazole Framework), or by abbreviations corresponding to the place of their discovery, like MIL-53, MIL-101 (Materials of Institute Lavoisier) or HKUST (Honk Kong University of Science and Technology).

MOFs are generally sought for their porosity, therefore, the type and size of the SBU employed is the predominant parameter to be controlled. The openness of the framework is ensured by the use of organic ligands that confer a rigidity and allow the control of the pores such that "the larger the brick, the larger the pores" [1] as illustrated in Figure 6. However,

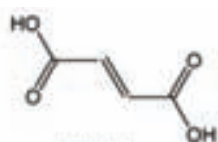

FMA

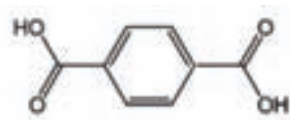

BDC

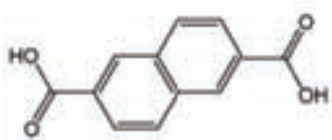

NDC

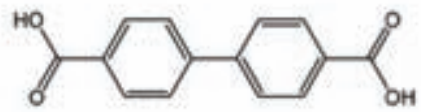

BPDC

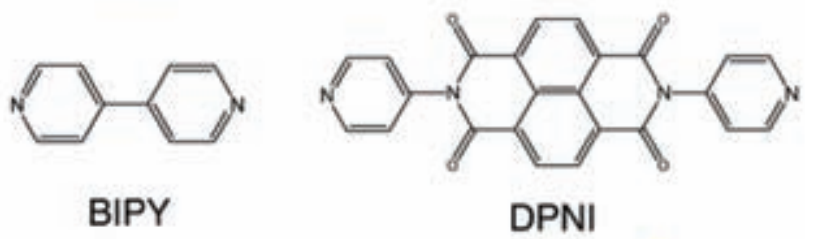

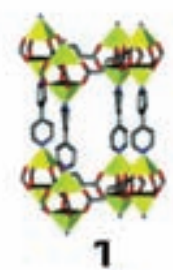

1

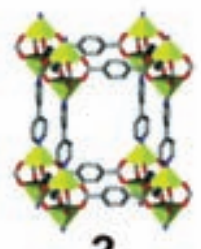

2

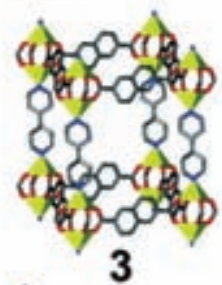

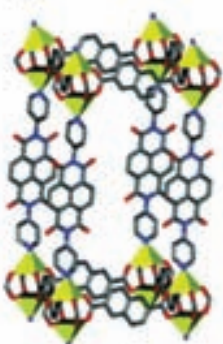

4

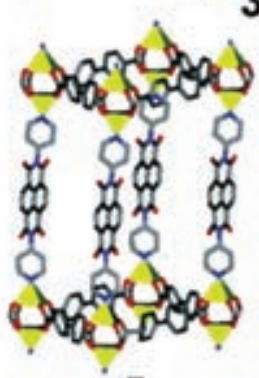

5

Figure 6. Single network units for compounds 1-5. The yellow polyhedral represent the zinc ions. Carbon: gray. Oxygen: red. Nitrogen: blue. Reprinted from ref [56] with permission. Copyright 2005 American Chemical Society. 
large organic linkers could weaken the structure or diminish its porosity through lattice self-interpenetration [54,55]. To palliate those problems the employed synthetic procedure plays a key role.

\subsection{Synthesis of MOFs}

The different preparation methods available for MOFs can be divided into two groups: traditional synthesis, usually referring to classical precipitation

and hydro/solvothermal method, and non-traditional methods, including microwave and ultrasound-assisted syntheses, mechanochemical and microemulsion methods.

\section{Traditional methods}

The precipitation from solution, also called one-pot, occurs at room temperature or upon heating from the mixture of metallic precursor and organic linkers in adequate solvent at $\mathrm{pH}$ and saturation concentrations adequate for MOF precipitation.

The easiest synthetic approach is performed at ambient pressure and temperature, although it sometimes results in poor crystallinity and reproducibility. Nevertheless, it has the advantage of allowing rapid MOF isolation and avoiding energy consumption. Almost all existing MOFs structures are prepared at least once using this method.

The second method in this group, the solvothermal method, has similar parameters to those in the sol-gel hydrothermal synthesis of zeolites. It implies the use of solvent above its normal boiling point in a hermetically sealed chemical reactor. The method results in high product yields and crystallinity and surpasses by far the reproducibility of the one-pot synthesis. Similarly to zeolite synthesis, a successful application of the solvothermal method to the MOF synthesis depends on reaction variables (temperature, time...), concentration and solubility of the precursor in the solvent, etc. The framework's self-assembly is greatly influenced by the characteristics of organic ligand, the coordination nature of the metal ions and the behaviour of the solvent, the $\mathrm{pH}$ of the solution, and by the presence or not of the template.

Unlike zeolite synthesis in which a directing agent is usually added to the precursors mixture, in the solvothermal approach this role can be assumed directly by the solvent. The organic molecules present in the synthesis (solvents, $\mathrm{pH}$ controllers like amines, templates if any, etc.) can play different roles in the MOF structure formation: i) as a solvent or deprotonation 
agent, ii) as a template (with a functions similar to the zeolite templates), and iii) as a coordinating ligand to the metal ion [57]. Not only the crystallinity but also the type of structure obtained can be influenced by changing the operational parameters, like temperature and duration. The latter is considered to be a disadvantage of the traditional methods of synthesis, in which several days are sometimes necessary to achieve acceptable product qualities. Long synthesis duration also involves higher expense, since highly energy-consuming processes and equipment (pressure-sealed vessels) are required.

\section{Microwave- and ultrasound-assisted processes}

Though quite different in nature, both methods provide ways to accelerate MOF's formation rate in comparison to traditional methods. Microwave (MW) heating offers a reduction of the synthesis duration by increasing the efficiency of heating. It has been reported that MW heating provides energy to overcome the activation energy barrier for the framework assembling. MW also influences the metastability of the system and its reactivity in a way that, the less stable the system, the higher the reactivity of the components and the faster the rate of MOF formation [58]. For example, Zou et al. [59] reported an unusually short time for HKUST-1synthesis by microwave irradiation (20 minutes rather than 8 hours in traditional hydrothermal synthesis), which was due to a faster nucleation rate rather than faster crystal growth. Schlesinger et al. [6o] evaluated several synthetic methods for $\left[\mathrm{Cu}_{2}(\mathrm{btc})_{3}\left(\mathrm{H}_{2} \mathrm{O}\right)_{3}\right]$ and $\left[\mathrm{Cu}_{2}(\mathrm{btc})\right.$ $(\mathrm{OH})\left(\mathrm{H}_{2} \mathrm{O}\right)$ ] formation. The microwave-assisted solvothermal method was the fastest one for pure $\left[\mathrm{Cu}_{3}(\mathrm{btc})_{2}\right]$ with high BET surface area and specific pore volume when DMF was used as the solvent. The choice of solvent is probably the most important variable to be controlled in this method. The solvent should absorb the microwave radiation and convert it to heat unless a metastable point of the system can be achieved at near ambient temperature.

Microwave-assisted MOF synthesis is considered a very simple and energy-efficient strategy to obtain these materials. It is easy to control, reduces crystallization times and increases the yield to solids. This method also promotes nanocrystals formation through a rapid and uniform nucleation, which is a consequence of the formation of local hot-spots and a fast heat transfer within the reaction media [61].

The ultrasound-assisted process (sonochemical synthesis), on the other hand, increases the area of contact between the reactants and, as a consequence, increases the rate of nucleation and crystal formation. In addition, 
the release of air bubbles formed by the decrease of the pressure in the liquid under condition of acoustic wave propagation disintegrates the particle agglomerates and results in an homogeneous particle size produced in short reaction times. Armstrong et al. [62] studied the crystallization mechanism of HKUST-1 under sonochemical conditions and found that crystallization initiates in spontaneous super-saturation points along the shockwaves generated by the implosion of the bubbles. Crystal growth followed a simple solid-on-solid model at high reactant concentrations and relatively low energy levels, thus shortening the time of synthesis, currently to less than 30 minutes for the formation of Zn-HKUST-1 and Cu-HKUST-1 nanorods at room temperature and ambient pressure [63]. It is clear that both microwave- and ultrasound-assisted methods are very suitable for the preparation of nano-MOF materials [64].

\section{Mecanochemical synthesis}

As a solvent-free method, mecanochemical synthesis has successfully found its place within the recently reported methods for MOF preparation $\left[61,65^{-67}\right]$. This method consists of a solid-solid reaction initiated by mechanical energy, usually ball milling. The rise of pressure or temperature is not required but sometimes a secondary phase can be obtained making difficult the isolation of the products. However, the presence of a small proportion of liquid component (not a solvent) could offer some benefits, such as an easier crystallization and higher yield of the desired product due to the improved mobility of the species and their homogenization [10]. Klimakow et al. [67] synthesized significant amounts of high quality HKUST-1 through a mechanochemical approach, proving that the procedure was reliable, effective and very fast ( 25 minutes). Nevertheless, they found some pore blocking effect from unreacted molecules, which can be removed in an adequate activation treatment. Still, this method is limited to MOFs whose synthesis is normally made under mild conditions and excludes interesting systems based on $\mathrm{Fe}, \mathrm{Cr}, \mathrm{Al}$ or $\mathrm{Ti}$ [64].

\section{Microemulsion synthesis}

This approach is based on the microemulsion formation, i.e. thermodynamically stable dispersions of immiscible liquids (water and oil phases) and the presence of emulsifiers or surfactants. The emulsifier is a molecule presenting both polar and non-polar parts. In very diluted water or oil solutions, the emulsifier exists as monomer, but when its concentration exceeds a certain concentration, called critical micelle concentration (CMC), the emulsifier molecules associate to form aggregates, that is, micelles [68]. 
These micelles are considered as nanoreactors wherein MOF formation reaction takes place. Thus, the size and the morphology of the resulting MOF particles are greatly influenced by the water/oil phase ratio and the surfactant concentration [69-71].

Other synthetic strategies are also available in the literature and may offer different advantages depending on the MOFs desired structure. It is worth mentioning the electrochemical method, in which metal ions are homogeneously supplied by the dissolution of an anode. It is reported as a very clean and fast method but it is generally restricted to $\mathrm{Cu}$-containing MOFs, like HKUST [72,73]. The continuous flow production method should be noted as the first one applied for large-scale industrial preparation. It is based on the induced crystallization from a stream of dissolved precursors in organic solvents. Finally, we should also mention the developing field of post-synthetic transformation, which is based on the transformation of already prepared MOFs into a novel structures, some of which cannot be accessed using the previous methods [74].

No matter the time or the energy involved in the synthesis, before starting one must remember the basic requirements: i) the adequate selection of salts and linkers, ii) the manner in which they connect together to obtain a possible porous structure, and iii) the necessity of a purification or activation step after the preparation. These steps are as important as the choice of the synthetic strategy.

All changes produced in a function of synthetic parameters variations should be controlled and this control is usually assured by the use of sample characterization techniques. This characterization should include structure identification, by X-ray diffraction analysis, and morphological and elemental analysis, which can be obtained by electron microscopy.

\section{Standard characterization for structure, texture and morphology}

As mentioned above the study and comprehension of physicochemical properties are of paramount importance to modulating the synthesis protocols in order to obtain solids with the adequate morphology, texture and structure. Standard characterization methods for the structure, texture and morphology of MOFs and zeolites include: powder X-ray diffraction (XRD), Brunauer-Emmett-Teller (BET) surface area measurement, and electron microscopy techniques. It should be emphasized that the use of a single method is insufficient for the analysis of a MOF or zeolite. Therefore, 
elemental analysis, IR spectroscopy, NMR and others are normally used in combination with the above methods.

\section{Powder X-Ray diffraction}

Zeolites and metal-organic frameworks are, by definition, crystalline solids, that is, they have a long-range atomic or molecular order. Thus, powder $\mathrm{X}$-ray diffraction (XRD) is typically the first method used for their identification, as each crystalline solid phase has its own characteristic XRD pattern as a "fingerprint". XRD is a routine, non-destructive characterization technique with high sensitivity, reliability, and easy sample preparation and data interpretation $\left[75^{-78}\right]$.

XRD measurements give information on the solid structural organization, proportion and size of the crystalline structures, spacing between the lattice planes, preferential order and growth of the crystallites $[78,79]$. For zeolites and metal-organic frameworks, this also includes framework topology and extra-framework cations and/or adsorbed molecules positions. The presence of the latter influences the adsorption and catalytic properties of the materials through their interactions with the guest species [8o-82]. The purity of the sample can also be revealed by considering the proportions and compositions of other phases, if present. Figure 7 shows some powder XRD patterns of different materials and their corresponding electron microscopy images.

Zeolite families present very distinct patterns $[83,84]$. To identify the precise structure, XRD patterns are compared to reference patterns of known zeolite structures. The unit cell composition of a zeolite can be directly determined and its reference patterns can be found in the Atlas of
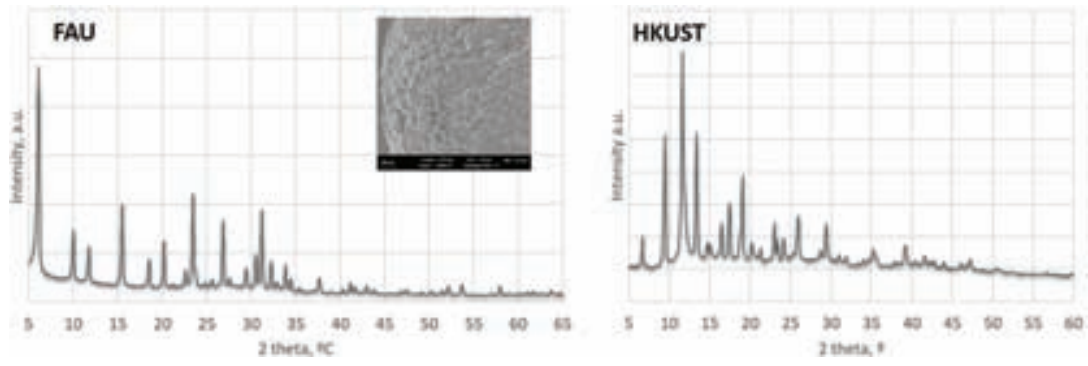

Figure 7. Examples of basic structural and morphological characterization. Simulated XRD analysis for zeolite FAU and H-KUST MOF. Insert: 'French fries' MFI structure. 
Zeolite Framework Types [85] or in the Collection of Simulated XRD Patterns for Zeolites [86]. The powder diffraction data and simulated patterns for the reference structures are listed alphabetically according to the respective framework type code [87]. Materials with the same framework code (i.e. framework topology) but different composition will have more or less different diffraction patterns; therefore, several reference materials may be found.

On the other hand, the width and shape of the diffraction peaks is also significant and are a convolution of effects due to crystallite size, strain, and instrumental broadening. The Scherrer equation normally relates the diffraction peaks widths to the average (by mass or volume) crystallite dimension [76]. Certain zeolites often present highly isotropic crystals, especially those with cubic symmetry like zeolites A, X, or Y [88]. However, other zeolites present highly anisotropic dimensions. Many one-dimensional pore zeolites tend to possess needle- or rod-shaped crystals. In catalytic or adsorptive applications, it may be desirable to have crystallites that are short in a particular direction in order to create the most efficient access within the pores of the solid. Characterizing the crystallite dimensions is therefore relevant in the interpretation of their behaviour.

The powder XRD diffraction analysis applied to MOFs allows for the drawing of conclusions concerning the reproducibility of the synthetic procedures and could explain structural differences between samples of the same MOF prepared by different methods [89]. Small variations in the synthetic conditions often influence MOF crystallization but may also result in the appearance of other polymorphic phases. Combined experimental and computational studies can be used to identify the driving forces in the formation of the different crystal phases [9o]. It is usual to employ simulated powder XRD patterns calculated from model crystal data [91] and to compare them with experimental MOF patterns [92,93] directly or by Rietveld refinement analysis $[94,95]$. Using this method, a reference pattern for the model crystal is calculated and then compared to the experimental data. A fitting algorithm allows us to refine the model and obtain precise values for the lattice parameters, crystal densities, degree of crystallinity, and crystallite size. Nevertheless, MOFs crystal structures are not always unambiguously determined. Although XRD is widely employed and well understood, the structure elucidation is not always a straightforward process. The large unit cell parameters of MOFs and the high electron density on the metal sites can make it challenging to determine its structure by X-ray diffraction.

The thermal stability, an important characteristic of MOFs, [96] can be also analyzed by X-ray diffraction analysis as temperature is increased. 
Heating MOFs induces evaporation of the solvent, hosted in the porous structure of the solid. As a consequence, the MOF structure changes: structure shrinks and metal-containing secondary building units convert to metal oxides. A complementary method for studying the thermal stability of MOFs is thermogravimetric analysis (TGA) [97], which measures the mass of an analysed sample as a function of temperature to determine the thermal decomposition of the sample. It also allows for the establishment of a purification and activation protocol. Moreover, if the thermogravimetric analysis is combined with a mass spectrometer it is possible to determine the temperature at which the sample changes and to quantify the solvent removal and the stability of the structure under heating.

\section{Adsorption and surface area measurement}

Properties, such as surface area, pore volume and pore-size distribution are essential parameters, especially in shape selective catalysis and adsorption. The reactants must run across the porous system to reach the catalytically active sites and the resulting products have to leave the sites. These mass transfer processes depend on the pore size, i.e. bulk diffusion in large macropores (diameter $>50 \mathrm{~nm}$ ), Knudsen diffusion in mesopores (diameter $2-50 \mathrm{~nm}$ ) and configurational diffusion in the micropores (diameter $<2 \mathrm{~nm}$ ) [98]. Generally, the physical adsorption of a gas [99-101] is routinely used to characterize porous materials. This technique accurately determines the amount of gas adsorbed by the solid material, an indirect measure of the pore properties and structure. The starting point of the BET analysis is a determination of the adsorption and desorption isotherms, which are represented as the amount of probe gas adsorbed as a function of its relative pressure (the ratio of its partial pressure to its vapour pressure) at a fixed temperature. Different probe gases including $\mathrm{N}_{2}, \mathrm{Ar}$, and $\mathrm{CO}_{2}$ are frequently used as probe gases, depending on the nature of the material (adsorbent) and on the information required [102-104]. $\mathrm{N}_{2}$ adsorption at $77 \mathrm{~K}$ at sub-atmospheric pressures is frequently used as routine quality control. If applied over a wide range of relative pressures, $\mathrm{N}_{2}$ adsorption isotherms can provide information on the pore size distributions in the whole range of porosity of the material: micro-, meso- and macroporosity (approximately $0.5^{-200 ~} \mathrm{~nm}$ ) [105].

Valuable information can be deduced from the shape of the nitrogen adsorption/desorption isotherms. According to IUPAC classification, six types can 
be distinguished, although only four are usually found in routine characterization [105]. Type I isotherms are characteristic of microporous adsorbents, such as zeolites, and Type IV are typical of mesoporous solids. The latter is characterized by an increase in the adsorbed gas volume at high $\mathrm{p} / \mathrm{p}_{\circ}$ and by the existence of a hysteresis loop. The presence of hysteresis in the isotherms indicates the presence of mesopores and its shape is related to the shape of the mesopores [106]. Roughly, a vertical loop indicates cylindrical mesopores, whereas a more horizontal one indicates ink bottle-type mesopores. The increase of the adsorbate volume at low $\mathrm{p} / \mathrm{p}$ 。 pressures in the type IV isotherms indicates the presence of micropores along with the mesopores. Experimentally, micropores can be analysed by adsorption calorimetry coupled with isotherm measurements or by pre-adsorption of large molecules [99]. The determination of the micropore size distribution in zeolites is preferentially performed using $\mathrm{Ar}$ adsorption at $87 \mathrm{~K}$ (or $77 \mathrm{~K}$ ). The use of $\mathrm{Ar}$ is justified as $\mathrm{N}_{2}$ presents quadrupolar moment causing an enhanced interaction with the zeolite framework and hindering discrimination of different pore sizes [101]. In addition, $\mathrm{N}_{2}$ adsorption in micropores occurs at lower $\mathrm{p} / \mathrm{p}$ o values than $\mathrm{Ar}$, the latter being thus more favourable for accurate measurements of smaller micropores [107].

BET area is a measure of the total area of the solid (micro-, meso-, and macropores). It is based on the multilayer adsorption model proposed by Brunauer, Emmet and Tellet (BET), applied to adsorption data in a suitable range of low pressures. If the cross-section area of the adsorbate is known (e.g. $0.162 \mathrm{~nm}^{2}$ for $\mathrm{N}_{2}$ ), the area of the solid can be estimated.

MOFs surface areas can be also calculated geometrically from the corresponding crystal structures [108]. In this sense Walton and Snurr [109] simulated nitrogen isotherms in a series of MOFs and showed that the accessible surface areas agree very well with the BET surface areas obtained from the simulated isotherms, thus demonstrating that the use of this method is physically meaningful for MOFs characterization [110].

The t-plot and s-plot are empirical methods that allow a semi-quantitative analysis of the micropore surface area [111]. Both methods are based on the comparison of adsorption isotherm data of porous sample to a nonporous one (type II) of comparable chemical composition and surface properties. The $t$-plot is the representation of the amount of gas adsorbed $v s$. the statistical thickness that the adsorbate would have at the same relative pressure on the nonporous sample. By fitting it to a straight line, estimates of the mesopore surface area and of micropore volume can be obtained from the slope and intercept, respectively [112].

Several methods to calculate the pore-size distribution from adsorption/desorption isotherms have been developed. The Barrett, Joyner and 
Halenda $(\mathrm{BJH})$ model is commonly used for the mesopore range and part of the macropore range $[105,100]$. It uses the desorption branch of the isotherms to calculate the pore size distribution and the adsorbed volume. It is designed as an ASTM standard method D 4641/87, and based on the modified Kelvin equation for capillary condensation. The method gives a reasonably good pore size distribution up to $c a .4 \mathrm{~nm}$ diameter. However, below a pore diameter of $2 \mathrm{~nm}$ the Kelvin equation is not valid [113-114].

Due to the size and regular nature of their pores and apertures, one of the most important zeolite functions is to serve as a molecular sieve. Depending on the pore system of the zeolite, molecules can penetrate into the channel network or can be excluded from it. However, sometimes the transport of reactants to the active sites or the counter-diffusion of products in the channels is difficult and leads to intracrystalline transport limitations [115]. Development of more open structures by creating additional porosity, e.g. by combining micro- and mesopores is a well-known approach to improving zeolite difusional properties [115-116].

MOFs are characterized by the diversity of their structures, different symmetries and pore sizes. The chain length of the linker conditions their pore size, while the introduction of substituents and functional groups into the linker provides additional selectivity and chemical properties in the pores [117]. MOFs are one of the known materials with the highest specific surface area, some of them presenting BET specific surface area values close to $6000 \mathrm{~m}^{2} \mathrm{~g}^{-1}$ [118-119]. The post synthesis treatment of the frameworks (catenation) and the presence of organic or inorganic species hosted in the pores can greatly reduce these surface areas [89,102]. For instance, Kaye et al. [120] obtained extremely porous $\mathrm{MOF}-5$ by minimizing its exposure to atmospheric or solvent molecules during the preparation. They also report as a possible reason for $\mathrm{MOF}-5$ surface loss: the framework decomposition under exposure to water and humid air during and after the synthesis procedure.

\section{Electron microscopy}

Zeolites may have different dimensional channels and may exist in a variety of morphologies [121-123]. They can exist as relatively large crystals of ca. $1 \mu \mathrm{m}$ [124] and, in this case, scanning electron microscopy (SEM) can provide useful information about morphology, defect presence and growth mechanism, as presented in Figures 4 and 7. For both zeolites and MOFs SEM can be used to monitor the presence of mesopores and the quality of the crystals [125]. However, when the features are around 1-3 nm, the use of 
TEM microscopy is better suited. The first works to describe the usefulness of transmission electron microscopy (TEM) for extracting morphological and structural information date from the 1940s [126-127]. Nowadays TEM is considered a powerful characterization technique providing fundamental information on morphology and microstructure [126]. The structures of defects (e.g. dislocations, stacking faults, twins, etc.) can be also characterized [124]. TEM can also show the effects of dealumination of the zeolite structure [128] or reveal the porosity in three dimensions by means of rotation and image reconstruction (tomography) [129] and even locate positions of metal particles within the pores [130].

Microstructure characterization is crucial for the understanding and optimization of zeolites and MOF synthesis. For multicomponent solids, in general, proper mixing of the components is of crucial importance. The presence of phases other than the desired ones can influence in a great manner the properties of the material and therefore its applications. On a macroscopic scale the control of "mixing" is usually obtained by using "bulk" techniques such as X-ray diffraction or vibrational spectroscopy [92]. In many cases, however, local information at the nanometer scale is desired. TEM is the method used for that purpose, providing direct imaging, electron diffraction patterns and X-ray fluorescence spectroscopy of specific sample points with high spatial resolution [131]. When high resolution TEM (HRTEM) imaging is used, the critical pore structures, lattice planes, and atomic arrangements within the solid can be observed [132]. In HRTEM analysis, the crystallographic information is directly related to its position in the image: the crystal structure is "imaged". This allows the direct characterization of local features like surface facets or interface identification.

However the electron beam of HRTEM may damage the microstructure of zeolites and MOFs [133]. Depending on the accelerating voltage, different electron damage mechanisms become important. In general, electron beam damage occurs more rapidly in zeolites having a higher water concentration [134] and decreased silica to alumina ratio. As another example, the sintering of small Pt particles supported on zeolite during a TEM study was also reported [135]. The scanning mode of TEM (STEM) can reduce electron beam damage produced by the continuous static illumination in HRTEM imaging [136]. Moreover, STEM images are particularly sensitive to a variation of the average atomic number and give rise to an exceptionally high signal-to-noise ratio, which is very suitable for observing metal particles in zeolites and MOFs [137]. Few TEM/STEM studies of MOFs are available in the literature. The direct imaging of intact MOF crystals by TEM was first reported for MIL-101(Cr) [138]. Díaz-García et al. [139] also 
studied nanosized MOF-74 materials in STEM mode. The image quality was sufficient to resolve the channel structure of the crystals, although the sensitivity of the MOF to the electron beam made it difficult to achieve more information on the intact materials.

\section{Concluding remarks}

Both zeolites and metal-organic frameworks are well-defined porous materials with varying structures and morphology depending on their building units. As they present very similar characteristics, the synthetic methods employed are usually similar, the hydro/solvothermal method being the one most reported. Variations of the preparation methods are guided by application requirements: smaller crystal can be obtained by ultrasound and microwave assisted methods but sometimes in detriment to the crystallinity. No matter what the synthesis procedure, a basic characterization of the structure, porosity and morphology is required to guide the posterior change of synthetic parameters or additional treatments, or to assess the suitability of the materials for the intended use.

\section{References}

[1] Férey, G. Chem. Soc. Rev. 2008, 37, 191.

[2] Olmos, A., Louis, B., Pale, P. Chem. Eur. J. 2012, 18, 4894.

[3] Bernardon, C., Louis, B., Beneteau, V., Pale, P. ChemPlusChem. 2013, 78, 1134.

[4] Chassaing, S., Beneteau, V., Louis, B., Pale, P. Curr. Org. Chem. 2016, 20, 1.

[5] Losch, P., Kolb, J.F., Astafan, A., Daou, T.J., Pinard, L., Pale, P., Louis, B. Green. Chem. 2016, 18, 4714 .

[6] Karmakar, A., Desai, A.V., Ghosh, S.K., Coord. Chem. Rev. 2016, 307, 313.

[7] Wang, L., Han, Y., Feng, X., Zhou, J., Qi, P., Wang, B. Coord. Chem. Rev. 2016, 307, 361.

[8] Corma, A., García, H., Llabrés i Xamena, F.X., Chem. Rev. 2010, 110, 4606.

[9] Liu, J., Chen, L., Cui, H., Zhang, J., Zhang, L., Su, C.Y. Chem. Soc. Rev. 2014, 43, 6011.

[10] Butova, V.V., Soldatov, M.A., Guda, A.A., Lomachenko, K.A, Lamberti, C. Russian Chem. Rev. 2016, 85, 280 .

[11] Milton, R.M. in Zeolite Synthesis, ACS Symposium Series 398, 1, Am. Chem. Soc., 1989.

[12] Loewenstein, W. Am. Miner. 1954, 39, 92.

[13] Morris, R.J. Mater. Chem. 2005, 15, 931.

[14] Barrer, R.M. Pure Appl. Chem. 1979, 51, 1091.

[15] Hölderich, W.F., van. Bekkum, H. Stud. Surf. Sci. Catal. 1991, 58, 631.

[16] Grand, J., Awala, H., Mintova, S. Cryst. Eng. Comm. 2016, 18, 650.

[17] Dyer, A. Introduction to Zeolite Molecular Sieves, John Wiley \& Sons, 1988.

[18] Harvey, G., Dent Glasser, L.S. in Zeolite synthesis, ACS Symposium Series 398, 49, Am. Chem. Soc., 1989. 
[19] Burkett, S.L., Davis, M.E. Chem. Mater. 1995, 7, 920.

[20] Davis, M.E. Stud. Surf. Sci. Catal. 1995, 97, 35.

[21] Choi, M., Cho, H.S., Srivastava, R., Venkatesan, C., Choi, D.H., Ryoo, R. Nat. Mater. 2006, 5, 718.

[22] Valtchev, V., Smaihi, M., Faust, A.C., Vidal, L. Angew. Chem. Int. Ed. 2003, 42, 2782.

[23] Ocampo, F., Cunha, J.A., de Lima Santos, M.R., Tessonnier, J.P., Pereira, M.M., Louis, B. Appl. Catal., A 2010, 390, 102.

[24] Kirschhock, C.E.A., Feijen, E.J.P., Jacobs, P.A., Martens J.A. in Handbook of Heterogeneous Catalysis, Vol 1, 160, Ertl, G., Knozinger, H., Schüth, F., Weitkamp, J. (Eds.), 2008.

[25] Maldonado, M., Oleksiak, M.D., Chinta, S.J., Rimer, J.D. J. Am. Chem. Soc. 2013, 135, 2641.

[26] Kessler, H., Patarin, J., Schott-Darie, C. Stud. Surf. Sci. Catal. 1994, 85, 75.

[27] Camblor, M.A., Villaescusa, L.A., Diaz-Cabanas, M.J. Top. Catal. 1999, 9, 59.

[28] Guth, J.L., Kessler, H., Higel, J.M., Lamblin, J.M., Patarin, J., Seive, A., Chezeau, J.M., Wey, R. in Zeolite Synthesis, Am Chem Soc. Symposium Series 398, Washington DC (1989) 176-195.

[29] Guth, J.L., Delmotte, L., Soulard, M., Brunard, N., Joly, J.F., Espinat, D. Zeolites 1992, 12, 929.

[30] Louis, B., Kiwi-Minsker, L. Microp. Mesop. Mater. 2004, 74, 171.

[31] Arichi, J., Louis, B., Cryst. Growth Des. 2008, 8, 3999.

[32] Losch, P., Pinar, A.B., Willinger, M.G., Soukup, K., Chavan, S., Vincent, B., Pale, P., Louis, B. J. Catal. 2017, 345, 11.

[33] Sefcik, J., McCormick, A.V. Chem. Eng. Sci. 1999, 54, 3513.

[34] Oleksiak, M.D., Rimer, J.D. Rev. Chem. Eng. 2013, 30, 1.

[35] Navrotsky, A., Trofymluk, O., Levchenko, A.A. Chem. Rev. 2009, 109, 3885.

[36] Barrer, R.M. in: Zeolite Synthesis, ACS Symposium Series 398, 11, Am Chem Soc., 1989.

[37] Ostwald, W. in Lehrbuch der Allgemeinen Chemie, vol 2, 444, Engelmann, 1896.

[38] Qin, Z., Melinte, G., Gilson, J.P., Jaber, M., Bozhilov, K., Boullay, P., Mintova, S., Ersen, O., Valtchev, V. Angew. Chem. Int. Ed. 2016, 55, 1.

[39] Larlus, O., Valtchev, V. Chem. Mater. 2004, 16, 3381.

[40] Schüth, F. Solid State Mater. Sci. 2001, 5, 389.

[41] Kirschhock, C.E.A., Kremer, S.P.B., Grobet, P.J., Jacobs, P.A., Martens, J.A. J. Phys. Chem. B 2002, 106, 4897 .

[42] Kirschhock, C.E.A., Ravishankar, R., Jacobs, P.A., Martens, J.A.J. Phys. Chem. B 1999, 103, 11021.

[43] Taulelle, F., Haouas, M., Gerardin, C., Estournes, C., Loiseau, T., Ferey, G. Colloids Surf. A 1999, 158, 299.

[44] Pope, C.G. Microp. Mesop. Mater. 1998, 21, 333.

[45] Cundy, C.S., Cox, P.A. Microp. Mesop. Mater. 2005, 82, 1.

[46] Mintova, S., Jaber, V., Valtchev, V. Chem. Soc. Rev. 2015, 44, 7207.

[47] Kosanovic, C., Bosnar, S., Subotic, B., Svetlicic, V., Misic, T., Drazic, G., Havancsak, K. Microp. Mesop. Mater. 2008, 110, 177 .

[48] Kosanovic, C., Havancsak, K., Subotic, B., Svetlicic, V., Misic Radic T., Cziraki, A., Huhn, G., Buljan, I., Smrecki, V. Microp. Mesop. Mater. 2011, 142, 139.

[49] Kosanovic, C., Havenscak, K., Subotic, B., Svetlicic, V., Misic, T., Cziraki, A., Huhn, G. Microp. Mesop. Mater. 2009, 123, 15 o.

[50] Ren, N., Subotic, B., Bronic, J., Tang, Y., Dutour Sikiric, M., Misic Radic, M., Svetlicic, V., Bosnar, S., Antonic Jelic, T. Chem. Mater. 2012, 24, 1726.

[51] Ren, N., Bosnar, S., Bronic, J., Dutour Sikiric, M., Misic, T., Svetlicic, V., Mao, J.J., Antonic Jelic, T., Hadjiza, M., Subotic, B. Langmuir 2014, 30, 8570.

[52] Davis, T.M., Drews, T.O., Ramanan, H., He, C., Dong, J., Schnablegger, H., Katsoulakis, M.A., Kokkoli, E., Mccormick, A.V., Penn, R.L., Tsapatsis, M. Nat. Mater. 2006, 5, 400.

[53] Deria, P., Mondloch, J.E., Karagiaridi, O., Bury, W., Hupp, J.T., Farha, O.K. Chem. Soc. Rev. 2014, 43, 5896 . 
[54] Schnobrich, J.K., Koh, K., Sura, K.N., Matzger, A.J. Langmuir 2010, 26, 5808.

[55] Chen, B., Eddaoudi, M., Hyde, S.T., O'Keeffe, M., Yaghi, O.M. Science 2001, 291, 1021.

[56] Ma, B.Q., Mulfort, K.L., Hupp, J.T. Inorg. Chem. 2005, 44, 4912.

[57] Zhu, Y.J., Chen, F. Chem. Rev. 2014, 114, 6462.

[58] Hayes, B.L. Microwave Synthesis: Chemistry at the Speed of Light, CEM Publish., 2002.

[59] Zou, F., Yu, F., Li, R., Li, W. ChemPhysChem. 2013, 14, 2825.

[6o] Schlesinger, M., Schulze, S., Hietschold, M., Mehring, M. Microp. Mesop. Mater. 2010, 132, 121.

[61] Pichon, A., Lazuen-Garay, A., James, S.L. Cryst. Eng. Comm. 2006, 8, 211.

[62] Armstrong, M.R., Senthilnathan, S., Balzer, C.J., Shan, B., Chen, L., Mua, B. Ultrason. Sonochem. 2017, 34, 365 .

[63] Azhdari Tehrania, A., Safarifarda, V., Morsalia, A., Bruno, G., Amiri Rudbari, H. Inorg. Chem. Comm. 2015, 59, 41.

[64] Giménez-Marqués, M., Hidalgo, T., Serre, C., Horcajada, P. Coord. Chem. Rev. 2016, 307, 342.

[65] Braga, D., Giaffreda, S.L., Grepioni, F., Pettersen, A., Maini, L., Curzi, M., Polito, M., Dalton Trans. 2006, 1249.

[66] Klimakow, M., Klobes, P., Rademann, K., Emmerling, F. Microp. Mesop. Mater. 2012, 154, 113.

[67] Klimakow, M., Klobes, P., Thunemann, A.F., Rademann, K., Emmerling, F. Chem. Mater. 2010, 22, 5216 .

[68] Capek, I. Adv. Colloid Interf. Sci. 2004, 110, 49.

[69] Rieter, W.J., Taylor, K.M.L., An, H., Lin, W., Lin, W.J. Am. Chem. Soc. 2006, 128, 9024.

[70] Liu, Q., Jin, L.N., Sun, W.Y. Chem. Commun. 2012, 48, 8814.

[71] Liu, Y., Zhang, Y., Chen, J., Pang, H. Nanoscale 2014, 6, 10989.

[72] Jiang, L.L., Zeng, X., Li, M., Wang, M.Q., Su, T.Y., Tian, X.C., Tang, J. RSC Adv. 2017, 7, 9316.

[73] Albo, J., Vallejo, D., Beobide, G., Castillo, O., Castaño, P., Irabien, A. ChemSusChem 2017, 10, 1100.

[74] Qiu, S., Zhu, G. Coordin. Chem. Rev. 2009, 253, 2891.

[75] Kittel, C. Introduction to Solid State Physics, 5th edition, John Wiley \& Sons, 1976.

[76] Cullity, B.D. Elements of X-ray Diffraction, 2nd edition, Addison Wesley, 1978.

[77] West, A.R. Solid State Chemistry and its Applications, John Wiley \& Sons, 1984.

[78] Rohrer, G.S. Structure and Bonding in Crystalline Materials, Cambridge University Press, 2001.

[79] Baur, W.H., Fischer, R.X., Shannon, R.D. Relations and Correlations in Zeolite RHO and Computer Simulations of its Crystal Structure. In Innovation in Zeolite Material Science, Grobet, P.J. et al. (Eds.), Elsevier, 1988.

[8o] Furukawa, H., Cordova, K., O'Keeffe, M., Yaghi, O. Science 2013, 341, 974.

[81] Mason, J.A., Veenstra, M., Long, J.R. Chem. Sci. 2014, 5, 32.

[82] Phan, A., Czaja, A.U., Gándara, F., Knobler, C.B., Yaghi, O.M. Inorg. Chem. 2011, 50, 7388.

[83] McCusker, L.B. Acta Cryst. A 1991, 47, 297.

[84] Baerlocher, C., McCusker, L.B. in Advanced Zeolite Science and Applications. Stud. Surf. Sci. Catal. Vol 85, Elsevier, 1994.

[85] Baerlocher, C., Meier, W.M., Olson, D.H. Atlas of Zeolite Framework Types. 5th Edition, Elsevier, 2001.

[86] Treacy, M.M.J., Higgins, J.B. Collection of Simulated XRD Powder Patterns for Zeolites (5th Edition), Elsevier, 2001.

[87] http://www.iza-online.org/

[88] Chester, W.A., Derouane, E.G. Zeolite Characterization and Catalysis: A Tutorial, Springer, 2009.

[89] Hafizovic, J., Bjorgen, M., Olsbye, U., Dietzel, P.D.C., Bordiga, S., Prestipino, C., Lamberti, C., Lillerud, K.P. J. Am. Chem. Soc. 2007, 129, 3612. 
[9o] Platero-Prats, A.E., de la Peña-O'Shea, V.A., Proserpio, D.M., Snejko, N., Gutiérrez-Puebla, E., Monge, A.J. Am. Chem. Soc. 2012, 134, 4762.

[91] Abednatanzi, S., Abbasi, A., Masteri-Farahani, M.J. Mol. Catal. A Chem. 2015, 399, 2015.

[92] Tranchemontagne, D.J., Hunt, J.R., Yaghi, O.M. Tetrahedron 2008, 64, 8553.

[93] Kraus, W., Nolze, G. J. Appl. Crystallogr. 1996, 29, 301.

[94] Herrmann, M., Kempa, P.B., Fietzek, H., Altenburg, T., Polyzoidis, A., Piscopo, C.G., Lobbecke, S. Chem. Ing. Tech. 2016, 88, 967.

[95] Young, R.A. (Ed.) The Rietveld Method, Oxford University Press, 1993.

[96] Botas, J.A., Calleja, G., Sánchez-Sánchez, M., Orcajo, M.G. Int. J. Hydrogen Energy 2011, 36, 10834.

[97] Raoof, J.B., Hosseini, S.R., Ojani, R., Mandegarzad, S. Energy 2015, 90, 1075.

[98] Gregg, S.J., Sing, K.S.W. Adsorption, Surface Area and Porosity, Academic Press, 1982.

[99] Seifert, J., Emig, G. Chem. Ing. Tech. 1987, 59, 475.

[100] Groen, J.C., Peter, L.A.A., Pérez-Ramírez, J. Microp. Mesop. Mater. 2003, 6o, 1.

[101] Storck, S., Bretinger, H., Maier, W.F. Appl. Catal., A 1998, 174, 137.

[102] Tsao, C.S., Yu, M.S., Chung, T.Y., Wu, H.C., Wang, C.Y., Chang, K.S., Chen, H.L. J. Am. Chem. Soc. $2007,129,15997$.

[103] Webb, S.W., Cconner, W.C. Sorption of Gases on Microporous Solids: Pore Size Characterization by Gas Sorption. In Characterization of Porous Solids II, F. Rodriguez-Reinoso et al. (Eds.), Elsevier, 1991.

[104] Reichert. H. Sorption of argon and nitrogen on network types of zeolites and aluminophosphates. In Characterization of Porous Solids II, F. Rodriguez-Reinoso et al. (Eds.), Elsevier, 1991.

[105] Leofanti, G., Padovan, M., Tozzola, G., Venturelli, B. Catal. Today 1998, 41, 207.

[106] Kaneko, K.J. Membr. Science 1994, 96, 59.

[107] Ravikovitch, P.I., Vishnyakov, A., Russo, R., Neimark, A.V. Langmuir 2000, 16, 2311.

[108] Leach, A.R. Molecular Modelling: Principles and Applications, 2nd Ed., Prentice Hall, 2001.

[109] Walton, K.S., Snurr, R.Q.J.J. Am. Chem. Soc. 2007, 129, 8552.

[110] Sang Bae, Y., Dubbeldam, D., Nelson, A., Walton, K.S., Hupp, J.T., Snurr, R.Q. Chem. Mater. 2009, 21, 4768.

[111] Sing, K.S.W. Chem. Ind. 1968, 1520.

[112] Barrett, E.P., Joyner, L.G., Halenda, P.P.J. Amer. Chem. Soc. 1951, 61, 373.

[113] Lynch, J., Raatz, F., Dufresne, P. Zeolites 1987, 7, 333.

[114] Ogura, M., Shinomiya, S.Y., Tateno, J., Nara, Y., Nomura, M., Kikuchi, E., Matsukata, M. Appl. Catal., A 2001, 219, 33

[115] Pérez-Ramírez, J., Mitchell, S., Verboekend, D., Milina, M., Michels, N.L., Krumeich, F., Marti, N., Erdmann, M. ChemCatChem 2011, 3, 1731.

[116] Pérez-Ramírez, J., Verboekend, D., Bonilla, A., Abelló, S. Adv. Func. Mater. 2009, 19, 3972.

[117] Lillerud, K.P., Olsbye, U., Tilset, M. Top. Catal. 2010, 53, 859.

[118] Furukawa, H., Ko, N., Go, Y.B., Aratani, N., Choi, S.B., Choi, E., Yazaydin, A.O., Snurr, R.Q., O'Keeffe, M., Kim, J., Yaghi, O.M. Science 2010, 329, 424.

[119] Farha, O.K., Eryazici, I., Jeong, N.C., Hauser, B.G., Wilmer, C.E., Sarjeant, A.A., Snurr, R.Q., Nguyen, S.T., Yazaydın, A.O., Hupp, J.T.J. Am. Chem. Soc. 2012, 134, 9860.

[120] Kaye, S.S., Dailly, A., Yaghi, O.M., Long, J.R.J.J. Am. Chem. Soc. 2007, 129, 14176.

[121] Chan, I.Y., Labun, P.A., Pan, M., Zones, S.I. Microp. Mesop. Mater. 1995, 3, 409.

[122] Meriaudeau, P., Tuan, V.A., Nghiem, V.T., Lai, S.Y., Hung, L.N., Naccache, C.J. Catal. 1997, 169, 55.

[123] Sakamoto, Y., Kaneda, M., Terasaki, O., Zhao, D.Y., Kim, J.M., Stucky, G., Shin, H.J., Ryoo, R. Nature 2000, 408, 449.

[124] Terasaki, O. J. Electron Microsc. 1994, 43, 337.

[125] Liangkui, Z., Daliang Z., Ming, X., Huan, L., Shilun, Q. Cryst. Eng. Comm. 2013, 15, 9356. 
[126] Ardenne, M., Beischer, D. Angew. Chem. Int. Ed. 1940, 53, 103.

[127] Turkevich, J.J. Chem. Phys. 1945, 13, 235.

[128] Choi-Feng, C., Hall, J.B., Huggins, B.J., Beyerlein, R.A.J. Catal. 1993, 140, 395.

[129] Koster, A.J., Ziese, U., Verklij, A.J., Janssen, A.H., de Jong, K.P.J. Phys. Chem. B 2000, 104, 9368.

[130] Janssen, A.H., Yang, C.M., Wang, Y., Schuth, F., Koster, A.J., de Jong, K.P.J. Phys. Chem. B 2003, $107,1055^{2}$.

[131] Cowley, J.M. (Ed.) Electron Diffraction Techniques vol. I and II, Oxford University Press, 1992.

[132] Terasaki, O.J. Solid. State Chem. 1993, 106, 190.

[133] Hren, J.J., Goldstein, J.J., Joy, D.C. Introduction to Analytical Electron Microscopy, Plenum, 1979 .

[134] Bursill, L.A., Lodge, E.A., Thomas, J.M.J. Phys. Chem. 1980, 286, 111.

[135] Jentoft, R.E., Tsapatsis, M., Davis, M.E., Gates, B.C.J. Catal. 1998, 179, 565.

[136] Williams, D.B., Carter, C.B. Transmission Electron Microscopy - A Textbook for Materials Science, Plenum, 1996.

[137] Borisevich, A.Y., Wang, S., Rashkeev, S.N., Pantelides, S.T., Sohlberg, K., Pennycook, S.J. Microsc. Microanal. Proc. 2004, 10, 460.

[138] Lebedev, O.I., Millange, F., Serre, C., Tendeloo, V., Férey, G. Chem. Mat. 2005, 17, 6525.

[139] Díaz-García, M., Mayoral, A., Díaz, I., Sánchez-Sánchez, M. Cryst. Growth. Des. 2014, 14, 2479 . 


\title{
3. Spectroscopic Methods of Characterization for Zeolites and MOFs
}

\author{
Luis F. Bobadilla, ${ }^{1}$ Laetitia Oliviero, ${ }^{2}$ \\ Francisca Romero-Sarria ${ }^{1}$ and Marco Daturi ${ }^{2}$ \\ ${ }^{1}$ Instituto de Ciencia de Materiales de Sevilla, Centro Mixto \\ CSIC-Universidad de Sevilla. Av. Américo Vespucio 49, 41092 \\ Sevilla, Spain. \\ ${ }^{2}$ Laboratoire Catalyse et Spectrochimie, ENSICAEN, Université \\ de Caen, CNRS, Bd. Maréchal Juin 6, F-14050 Caen, France.
}

\begin{abstract}
Keywords: Acidity, basicity, vibrational spectroscopy, IR spectrum, probe molecule
\end{abstract}

\section{Fundamentals}

Generally speaking, spectroscopic methods of analysis aim to determine the chemical composition of a sample through the interaction of an electromagnetic beam $(\mathrm{EMB})$ with the matter. This beam is characterised by its intensity $\left(I_{\circ}\right)$ and its wavelength $\left(\lambda_{\circ}\right)$. The different beam-matter interaction modes involved in spectroscopic techniques are schematically described in Figure 1. The incident beam can be:

1) absorbed by the matter at the atomic, molecular or at a higher level, and the analysed beam is the transmitted one, which characterised by $\left(\mathrm{I}<\mathrm{I}, \lambda_{\circ}\right)$,

2 ) absorbed by the matter and lead to emission of electrons (photoelectric effect) or photons (fluorescence or phosphorescence phenomena with $\left.\lambda>\lambda_{\circ}\right)$,

3) diffused by diffraction in ordered matter leading to constructive and destructive interferences, in this case the analysis focuses on the angle of diffraction,

4) diffused by reflection, the resulting beam having same $\lambda_{\circ}$ but lower intensity $\left(\mathrm{I}^{\prime}<\mathrm{I}_{\mathrm{o}}\right)$. 


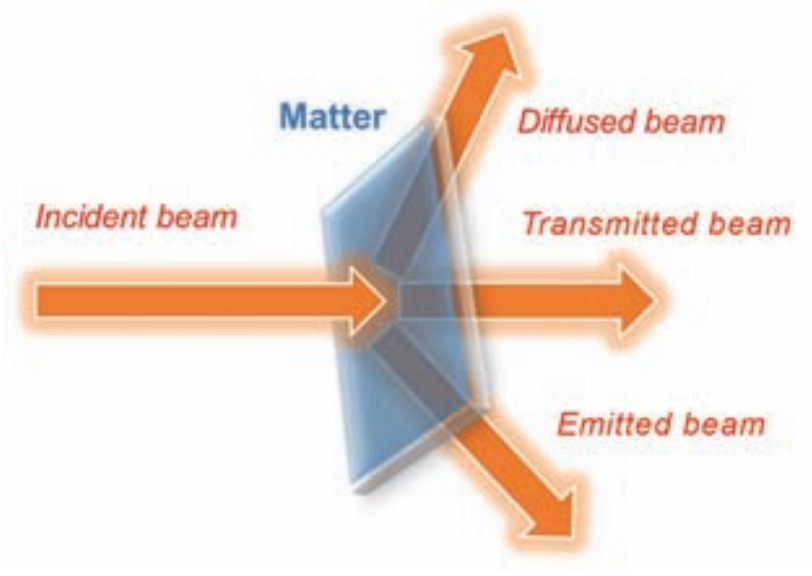

Figure 1. Interaction EMB-matter.

Table 1. Energy range and obtained information of the main spectroscopic methods.

\begin{tabular}{|c|c|c|}
\hline $\begin{array}{l}\text { EMB (energy } \\
\text { range, eV) }\end{array}$ & $\begin{array}{l}\text { Spectroscopy name } \\
\text { (phenomena) }\end{array}$ & Information \\
\hline $\begin{array}{l}\text { Y-ray } \\
\left(1.210^{9}-1.210^{6}\right)\end{array}$ & Mössbauer (absorption/emission) & Nuclear atomic transition \\
\hline \multirow[t]{2}{*}{$X$-ray $\left(10^{5}-10^{2}\right)$} & $\begin{array}{l}\text { EXAFS/XANES (absorption) } \\
\text { EDX (emission) } \\
\text { XPS (electron emission) }\end{array}$ & $\begin{array}{l}\text { Internal electrons } \\
\text { Atomic composition } \\
\text { Atomic composition, oxidation } \\
\text { number }\end{array}$ \\
\hline & XRD (diffraction) & Atomic arrangement, structure \\
\hline UV-visible (1-10) & UV-visible (absorption) & $\begin{array}{l}\text { External electrons, molecular } \\
\text { bonds, oxidation degree }\end{array}$ \\
\hline $\operatorname{IR}(0.1-1)$ & IR (absorption) Raman (diffusion) & Molecule vibration/rotation \\
\hline
\end{tabular}

EXAFS: extended X-ray absorption fine structure, XANES: X-ray absorption near edge structure, EDX: energy-dispersive X-ray spectroscopy, XPS: X-ray photoelectron spectroscopy.

According to the energy associated with the incident or emitted beam, a range of information can be obtained, as summarised in Table 1, giving rise to the usual spectroscopic techniques.

The objective of the characterization of materials in heterogeneous catalysis is to understand the activity and selectivity of solid catalysts in a given reaction. Heterogeneous catalysis relies on the solid-gas or solid-liquid interface but also on the accessibility of active sites. Because of the wide 
range of energies associated with spectroscopic methods, complementary information about the material as well as the reaction mechanism can be obtained by a proper combination of techniques. Bulk characterization information must be distinguished from surface information, even if they are related. Also, an important distinction has to be made between techniques that characterise the material as prepared and the ones that allow the characterization of the catalyst during its operation, an intermediate one being in situ characterization. Note that, in addition to the study of the activity/selectivity of the catalytic reaction, these operando and in situ approaches, also allow for studying formation (crystallization) of the material during its synthesis, particularly in the case of zeolitic and hybrid materials.

In the case of zeolite and MOF characterization, the determination of the atomic arrangement composing the $3 \mathrm{D}$ structure is mainly performed by X-ray diffraction and NMR spectroscopy. EXAFS and XANES spectroscopies could complete the description about the order/disorder at a more local level. Such information is relevant in order to verify the synthesis and purity of the material but also to understand the transport phenomena of reactants and products inside the porous material. In the case of zeolites, the main structures are described in the Database of the International Zeolite Association [1].

$\mathrm{X}$-ray photoelectron spectroscopy is used to discern the global chemical composition of the external layer of a solid $(<2-3 \mathrm{~nm})$ and, more specifically, the distribution of the oxidation number for each element. Note that this technique requires low pressure and is mainly used as an ex-situ technique. Also, in the case of highly porous materials like zeolites and MOFs, a great deal of the area inside the (micro)pores may not be analysed, even though it can contribute to activity. For specific atoms such as iron or cobalt, Mössbauer spectroscopy allows following the oxidation state and coordination number in in situ conditions. Energy-dispersive $\mathrm{X}$-ray spectroscopy, EDX, is performed in an electron microscope and also allows determination of the chemical composition but in a more localised region (usually in a volume of around $1 \mu \mathrm{m}^{3}$ ). UV-visible spectroscopy allows identifying the elements and their oxidation number as well as their local arrangements. The use of this technique in in situ conditions can also give information on the adsorbed species and on the modification of the coordination of transition metal ions in zeolites. However, the resolution of this technique is low since the coexistence of different species makes their distinction difficult. 
Vibrational spectroscopies (IR, Raman) can be used first to get information on the structure of zeolites and MOF. Vibrational spectroscopies are especially useful to establish structure-activity relationships since they also allow distinguishing various surface species according to their local environment. The ideal characterization of the catalyst is indeed the one able to reach the concentration of individual, independent sites in order to determine their intrinsic activity. Vibrational spectroscopies are also informative for studying the reaction mechanism: they can allow the identification of intermediates, poisons or spectators species, even if the distinction between these various species is not always straightforward. The main limitation in this regard is that reactive intermediates have a short lifetime on the catalyst surface. As a result, new fast spectroscopic devices are under development. Since vibrational spectroscopies have a specifically wide application domain in the field of zeolite and MOFs, their common fundamental basis will be shortly described next.

Vibrational spectroscopy involves transitions between discrete vibrational energy levels of polyatomic species, which are associated with their different vibration modes. Such modes can be described, as a first approximation, considering a chemical bond in a molecule or solid lattice as a harmonic oscillator (like two masses $\mathrm{m}_{1}$ and $\mathrm{m}_{2}$ bound by an ideal spring). The corresponding allowed transitions between equidistant vibrational energy levels are the ones with a one-unit increase in the vibrational quantum number (v), the so-called fundamental vibrations. A more realistic model is provided by the anharmonic oscillator, which is particularly relevant for explaining overtones (vibrational transitions with $\Delta v>1$ ). For the description of this model, the reader is referred to reference [2]. The frequency of the vibration according to the harmonic oscillator model is given by the following relation:

$$
\nu=\frac{1}{2 \pi} \sqrt{{ }^{k} / \mu}
$$

where $\mathrm{k}$ is the bond force constant and $\mu$ is the reduced mass, $\mu=\mathrm{m}_{1} \cdot \mathrm{m}_{2} /$ $\left(m_{1}+m_{2}\right)$. In this model, the higher the frequency (or the wavenumber), the stronger the bond.

For a given molecule of $\mathrm{N}$ atoms, there are $3 \mathrm{~N}-6$ fundamental vibrations $\left({ }_{3} \mathrm{~N}-5\right.$ in the case of a linear molecule). The nomenclature of these fundamental vibrations is shown in Figure 2. The stretching $(v)$ vibrations lead to a change in bond length and can be either symmetric or asymmetric. Bending vibrations $(\delta)$ correspond to a variation of angles in the plane of the atoms involved and include the rocking vibration of the tetrahedral unit. Variation of angles out of the plane of the atoms is named either bending 


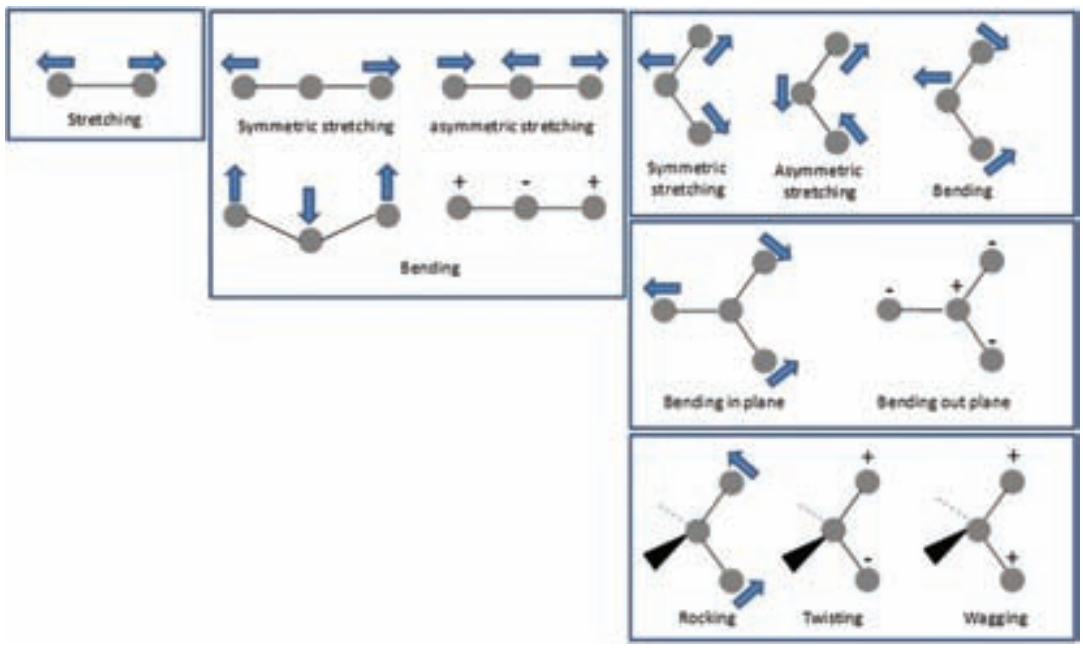

Figure 2. Representation of fundamental vibration modes.

out of plane or $\gamma$ vibration and includes the wagging mode of the tetrahedral unit. Finally, torsion $(\tau)$ vibration, such as the twisting of a tetrahedral unit, changes the angle between two atomic planes.

In IR spectroscopy, absorption of the incident beam occurs if its frequency corresponds to the frequency of the vibration and if during the vibration the dipole moment of the molecule is changed. In Raman spectroscopy, the vibration frequency appears as a Raman shift of the incident frequency due to scattering and implies that the polarisability of the molecule is changed during the vibration.

\section{General characterization}

To characterise the structure of zeolitic materials, the main vibrations of the primary building units $\left(\mathrm{TO}_{4}\right.$ tetrahedra) are reported in Table 2 according to references [3] and [4]. Raman spectra can be measured directly, whereas for IR spectra a dilution of the sample in a transparent media (such as $\mathrm{KBr}$ ) is often necessary, due to the strong absorptivity of the materials.

The position of the cations counterbalancing the lattice charge can also be characterised by vibrational spectroscopy. These vibrations occur in the $5^{0}-250 \mathrm{~cm}^{-1}$ domain. It has been shown that the frequency vibration depends on the charge and mass of the counter ion and also on the position inside the porous structure [5]. 
Table 2. Main vibration domains of internal and external linkages in zeolites.

\begin{tabular}{lllc}
\hline Internal tetrahedra & Vibrations $\left(\mathbf{c m}^{-1}\right)$ & External linkages & Vibrations $\left(\mathbf{c m}^{-1}\right)$ \\
\hline Asym. stretch & $1250-920$ & Double ring & $650-500$ \\
Sym.stretch & $720-650$ & Pore opening & $420-300$ \\
T-O bend & $500-420$ & Asym. stretch & $1150-1050$ \\
& & Sym. stretch & $870-750$ \\
\hline
\end{tabular}

In the case of organic-inorganic materials, the skeletal vibrations can be found below $1000 \mathrm{~cm}^{-1}$ for the transition metal-oxygen bonds, and between 1700 and $1200 \mathrm{~cm}^{-1}$ for the organic $\mathrm{C}-\mathrm{O}, \mathrm{N}-\mathrm{O}$ or $\delta(\mathrm{CH})$ vibrational modes.

On the other hand, the stretching vibration of the surface hydroxyl groups gives rise to several bands characterizing their position in the zeolite structure [6]. In the case of zeolite $Y$ for instance, the band at $3745 \mathrm{~cm}^{-1}$ is attributed to silanol groups (terminal or extra lattice), the one at $3655 \mathrm{~cm}^{-}$ ${ }^{1}$ to $\mathrm{OH}$ of extraframework aluminium, the ones at 3630 and $3560 \mathrm{~cm}^{-1}$ to bridging SiOHAl groups in the supercage and in smaller cavities, respectively $[7,8]$. MOFs also present $\mathrm{OH}$ vibrations in the $375^{0-3600 ~ \mathrm{~cm}^{-1}}$ range, when the cations are coordinatively unsaturated $[9,10]$.

Zeolite materials can present different sites, such as Brønsted or Lewis acid sites, basic sites and redox sites. To understand the catalysis on the material, these have to be distinguished and quantified in concentration and strength, taking into account their accessibility. To this end, adsorption of probe molecules followed by IR spectroscopy is an informative method. Indeed, the selection of molecules with variable basic strength allows for probing the acid strength of the solid. In the same manner, varying the size of the probe molecule allows for characterization of the accessibility of such sites. Ideally, the expected reactant can also be used as a probe in order to get information on its adsorption mode, which is related to its activation on the surface. In the following section, a description of the main probes used to characterise Brønsted and Lewis acidity and redox properties of a zeolite is presented.

\subsection{Acidity analysis}

Acidity is classically described by the Brønsted and Lewis concepts. Brønsted sites are proton donors, which can be directly detected by their $\mathrm{O}-\mathrm{H}$ vibrations, notably in the $3800-3500 \mathrm{~cm}^{-1}$ stretching interval. From a 
general point of view, the position of the vibration provides an approximate indication of its strength: acidity increases when $v(\mathrm{OH})$ decreases. This is true for a set of bands on the same sample, but cannot be used as a rule to compare different materials due to the structural and topological parameters influencing such vibrations. For example, linear hydroxyls are generally weakly acidic, whereas bridged hydroxyls are more acidic, due to the fact that the oxygen bond to the surface is stronger, so that the $\mathrm{O}-\mathrm{H}$ bond must be weaker. However, the amount of information that can be gathered from the bare hydroxyl IR spectrum is limited and the use of probe molecules is necessary, notably to separate the bands in a heterogeneous massif, to compare the acid strength or to verify the accessibility of the sites. In this case, three complexes can be formed between the basic probe (B) and the hydroxyl proton: i) a weak H-bond (B-...H-O), ii) a strong H-bond, giving rise to a quasi-symmetrical complex $(\mathrm{B} \cdots \cdot \mathrm{H} \cdot \cdots \mathrm{O})$, and iii) protonation, i.e. the formation of an ion pair with more or less dissociate ions $\left(\mathrm{BH}^{+} \ldots \mathrm{O}^{-}\right)$.

In the case of Lewis acid sites, the absence of a direct IR signal makes the use of probe molecules compulsory to characterise the acidity. This is obtained by analysing the molecular interactions between the surface and the basic probe, considering the band frequency and intensity in the spectrum of the perturbed probe with respect to the spectrum of the isolated molecule [11,12]. Upon different calibrations, this indicates the kind of sites and their surface concentration. Therefore an appropriate probe has to be chosen in order to obtain qualitative and quantitative information. The ideal probe molecule should have an optimum basic strength (sufficient to bond with the acid site, not too high to perturb it), an adequate size to access the porosity of the sample, a good selectivity towards the different sites, a satisfactory spectral response (in terms of intensity and shift proportional to the interaction strength), a good stability on the catalyst (no decomposition) and a sufficient vapour pressure to be introduced into the experimental cell.

$\mathrm{H}_{2} \mathrm{O}$

An efficient way to test Lewis sites is to transform them into Brønsted sites by a reversible adsorption of water. Each water molecule coordinated onto an activated sample induces the creation of two Brønsted sites presenting bands in the region $3700-3580 \mathrm{~cm}^{-1}[9,10]$. It is worth remarking that over oxidic compounds presenting Lewis acid-base pair water molecules are dissociated and form two hydroxyls as well. Other protic molecules (such as alcohols) can coordinate on Lewis sites and transform them into Brønsted acid sites whose acid strength is proportional to the acidity of the adsorptive [10]. 
CO

Based on the aforementioned concepts, $\mathrm{CO}$ is often considered as the ideal probe, being small in size, having very weak interactions, and with a spectral shift of more than $90 \mathrm{~cm}^{-1}$ depending on the strength of the acid site [13]. Being a very weak and soft base is very suitable for the characterization of strongly acidic surfaces. The wavenumber of the linear $\nu(\mathrm{CO})$ stretching vibration of molecules adsorbed at acidic centres without electron back donation is shifted to higher wavenumbers than gaseous CO $\left(2143 \mathrm{~cm}^{-1}\right)$, giving rise to bands between 2150 and $2180 \mathrm{~cm}^{-1}$ when interacting with Brønsted acid sites, or up to $2240 \mathrm{~cm}^{-1}$ for Lewis sites $[14,15]$. This shift can be explained in simple terms by the Blyholder model [16], due to the interaction of the filled $5 \sigma$-orbital of $\mathrm{CO}$ (HOMO) with an electron acceptor centre at the surface. This orbital is slightly antibonding between $\mathrm{C}$ and $\mathrm{O}$. Withdrawing of electrons from this orbital strengthens the $\mathrm{CO}$ bond. Electron $\pi$-backdonation from d-orbitals of surface transition metals atoms to the $2 \pi *$ - orbital of CO (LUMO) would partially weaken the CO bond. But globally the $\mathrm{CO}$ bond is strengthened, which results in a blueshift (higher wavenumber) of the corresponding $\nu(\mathrm{CO})$ vibration [17]. The higher the blueshift, the higher the Lewis acidity. To a certain extent, Brønsted acid sites can also be characterised with $\mathrm{CO}$ due to weak hydrogen bonding to surface $\mathrm{OH}$ groups. Accordingly, adsorption being weak, it should be favoured by low temperatures ranging down to the boiling point of nitrogen $(77 \mathrm{~K})$. In this case, $\mathrm{CO}$ and $\mathrm{OH}$ wavenumbers are shifted due to mutual interaction [18]. A linear correlation can be observed between the $\nu(\mathrm{OH})$ shift, the $\mathrm{H}_{\mathrm{o}}$ (Hammet acidity function) value and the $v(\mathrm{CO})$ band position, which allows ranking of the solid acidity among a large panel of compounds [10].

\section{(Substituted) pyridine}

Pyridine is certainly the most employed molecule to probe acidity. It has a strong base $\left(\mathrm{PA}=93 \mathrm{O} \mathrm{kJ} \mathrm{mol}^{-1}, \mathrm{pK}_{\mathrm{B}}=8.75\right)$ and it easily gives rise to the formation of H-bonded and pyridinium ion species on weak and strong Brønsted acid sites, respectively, and to coordinated species on Lewis acid sites [19]. Spectral analyses are generally performed over the $1400-1700 \mathrm{~cm}^{-1}$ $v(\mathrm{C}=\mathrm{C})$ ring vibrations range, in which four fundamental modes occur, accounting for the nature of the species formed. Among them, pyridinium species (Brønsted acid sites) are characterised by bands at 1640 and $1545 \mathrm{~cm}^{-1}$, whereas coordinated pyridine species give rise to bands at about

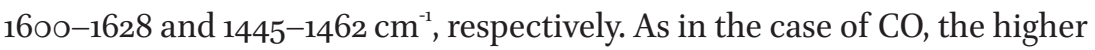
these frequencies are, the stronger the Lewis acidity is. Concerning H-bonded species, the corresponding bands are less sensitive and show vibrations at 

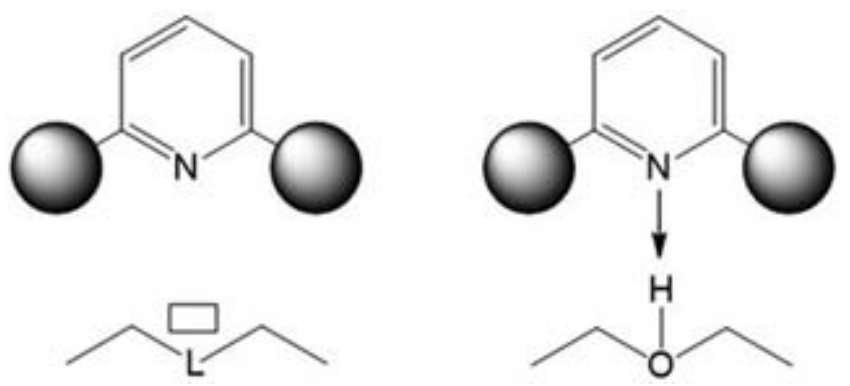

Figure 3. Interaction of substituted pyridine with the surface sites.

wavenumbers closer to the ones observed in the liquid phase (1596 and $1445 \mathrm{~cm}^{-1}$ ). Such small shifts can make assignments ambiguous when both weak Lewis and Brønsted acid sites are expected on the surface. Therefore, the only way to prove the formation of H-bonded species is the concomitant observation of a broad $v(\mathrm{OH})$ band shifted to a lower wavenumber than that corresponding to the free $\mathrm{OH}$ groups. Moreover, these species, hold relatively weakly on the surface hydroxyl groups, are easily removed by pumping off at $423 \mathrm{~K}$.

Pyridine is frequently used in quantitative studies by introducing successive aliquots of the probe on an activated surface. The progressive growing of the band intensity allows quantifying the concentration of the accessible acid sites on the solid. Then, operating a thermal desorption after surface saturation permits distinguishing the sites by acid strength. However, DMP (dimethylpyridine or lutidine) is more convenient than pyridine to detect weak Brønsted acidity due to its higher basicity $\left(\mathrm{pK}_{\mathrm{a}}=6.7\right.$ compared with 5.2 for pyridine) and also due to the interaction of the methyl groups with the surface favouring DMP adsorption on Brønsted acid sites, as schematised in Figure 3 [20]. Various adsorption modes of DMP with the surface of a solid give rise to specific spectral features in the $166 \mathrm{o}-158 \mathrm{o} \mathrm{cm}^{-1}$ range [21-24]. In particular, weakly adsorbed species (H-bonded or $\pi$-coordinated) produce absorption bands at $\sim 1600-1580 \mathrm{~cm}^{-1}, \sigma$-coordinated species on coordinatively unsaturated cations lead to bands around 1615-16oo and $158 \mathrm{o} \mathrm{cm}^{-1}$, the protonated species $\mathrm{DMPH}^{+}$absorbs near $165^{\mathrm{O}-1645}$ and $1625 \mathrm{~cm}^{-1}$.

\section{Ammonia}

Ammonia can be very useful as an acidity probe, although in some cases it is too reactive and gives rise to dissociative adsorption [25], disproportionation reaction, or is transformed into amide, imide and hydrazine 
species, as well as oxidised to $\mathrm{N}_{2}, \mathrm{~N}_{2}^{-}$and $\mathrm{N}_{3}^{-}[26]$. It is a good probe for acidic non-oxidizing or poorly oxidizing surfaces [27].

\section{Acetonitrile}

Acetonitrile is a probe with medium basicity that can interact with Lewis and Brønsted sites to form, depending on the acid strength of the site, protonated species or species strongly bound to the electrophilic site via a hydrogen bond in the case of $\mathrm{OH}$ groups. The $\mathrm{pK}_{\mathrm{a}}$ of $\mathrm{CH}_{3} \mathrm{CNH}^{+}$is 11.8, and protonation of acetonitrile only takes place with acids approaching the limit of superacidity. $\mathrm{CD}_{3} \mathrm{CN}$ is generally used in place of $\mathrm{CH}_{3} \mathrm{CN}$ because the latter molecule displays not only one but two bands in the $v(\mathrm{C} \equiv \mathrm{N})$ region used to characterise the acidity. This is due to a Fermi resonance between the fundamental $v(\mathrm{C} \equiv \mathrm{N})$ vibration and the combination mode $\delta_{\mathrm{S}}\left(\mathrm{CH}_{3}\right)+$ $v(\mathrm{C}-\mathrm{C})$. This interaction does not exist in the case of $\mathrm{CD}_{3} \mathrm{CN}$, which presents in the liquid state only one $v(\mathrm{C} \equiv \mathrm{N})$ band at $2263 \mathrm{~cm}^{-1}$, which shifts to a higher wavenumber upon interaction with acid sites [28].

Different nitriles can be used as acidity probe molecules, such as pivalonitrile, benzonitrile or $o$-toluonitrile [29,30]. The reference bands corresponding to the $v(\mathrm{C} \equiv \mathrm{N})$ vibration are close to that of acetonitrile, and they also undergo a blueshift proportional to the site acidity. The main difference consists in the steric hindrance of the molecule, which can be selectively used to probe sites inside the channels of porous materials, as described below.

\subsection{Site accessibility}

The description of the position of the sites inside the porosity of a zeolite or a MOF is of paramount importance, notably to correlate the site properties with their reactivity. For this purpose, different strategies have been elaborated to analyse the type, the concentration of sites and their strength inside the channels and the pockets of zeolitic compounds. The majority of the molecules mentioned above (CO, ammonia, acetonitrile, etc.) are small enough to enter the totality of the cavities. On the contrary, molecules with a greater steric hindrance (larger nitriles, substituted pyridine, etc.) can only enter large cavities. This has been largely applied to FAU and MOR structures, made of big and small cavities, in which some acidic hydroxyls are out of reach of basic molecules such as pyridine. Therefore, by operating co-adsorption of molecules presenting different sizes and basicity it is possible to determine the populations of the sites and their location inside 
the porosity. For example, the co-adsorption of the strongly basic trimethylamine and $\mathrm{NH}_{3}$ has provided, for the first time, an infrared evidence of four distinct acidic hydroxyls in defect-free HY. These sites have different acidic strengths and provide therefore various coordination environments to the ammonia and ammonium species inside the sample [31,32].

Pivalonitrile is too large a molecule to enter the side pockets of Mordenites, so the adsorption sites present there can be distinguished from those inside the main cavities by using various nitriles [29]. $O$-toluonitrile is larger than the channels of MOR, MIF and FER zeolites, thus allowing selective probing of their external surface and determination of the role of sites outside the porosity [30,33].

Accessibility can also be derived from infrared spectroscopy of substituted alkylpyridines with different sizes (pyridine: $0.57 \mathrm{~nm}, 2,6$-lutidine: $0.67 \mathrm{~nm}$, collidine: $0.74 \mathrm{~nm}$ ). For example, the enhanced accessibility of hierarchical ZSM- 5 crystals containing different degrees of intracrystalline mesoporosity was demonstrated this way. A relatively bulky molecule such as collidine, which probes practically no acid sites of the parent medium pore MFI structure, could access up to $40 \%$ of the Brønsted sites in the hierarchical sample [34].

\subsection{Basicity}

Basicity is a property more difficult to test than acidity, due to the intrinsic characteristics of the basic site [35]. $\mathrm{CO}_{2}$ is often used as probe molecules for the characterization of basic oxides: the subsequent formation of carbonates provides reliable indications on the kind and strength of basic sites on the material surface, considering both the symmetry of the formed species and their thermal stability [36]. However, carbonates strongly modify the probed solid and in the case of zeolitic compounds the framework oxygen basicity is generally too low to induce their formation. Thus, molecule alternatives to $\mathrm{CO}_{2}$ (or $\mathrm{NO}_{2}$ and $\mathrm{SO}_{2}$, which acting in the same way will form nitrates and sulphates, demonstrating the presence of basic sites) have to be used. Some years ago, Lavalley reviewed the infrared spectroscopic studies of the surface basicity of metal oxides and zeolites using adsorbed probe molecules [37]. Results obtained from carbon monoxide, carbon dioxide, sulphur dioxide, pyrrole, chloroform, acetonitrile, alkanes, thiols, boric acid, trimethyl ether, ammonia, and pyridine were discussed and their drawbacks were highlighted. Even if it was clearly stated that no probe could be used universally, pyrrole appeared to be quite suitable in 
the case of alkaline zeolites. A pioneering review on the basic properties of zeolites was conducted by Barthomeuf [38].

More recently, $\mathrm{NO}_{2}$ disproportionation on alkaline zeolites was used to generate nitrosonium $\left(\mathrm{NO}^{+}\right)$and nitrate ions whose infrared vibrations are shown to be very sensitive to the chemical hardness of the cation and the basicity of zeolitic oxygen atoms [39]. In general, the best probe molecule for IR measurements would be an H-donating $\mathrm{HX}$ molecule able to adsorb on surface centres such as $-\mathrm{O}^{2-}$ or $-\mathrm{OH}^{-}$sites through a hydrogen bond interaction. From the $v(\mathrm{H}-\mathrm{X})$ shift, a scale of surface basicity could be established [40]. Knözinger proposed a way to obtain a relative ranking of the basic strength of a series of catalyst materials by FT-IR spectroscopy using methylacetylene and tert-butylacetylene as probe molecules [41].

Michalska et al. pointed out that propyne is an excellent probe for the study of oxygen basicity in mesoporous molecular sieves [42]. Additionally, they verified that probe dissociation does not depend on the site strength but can be due to the presence of Lewis-acid sites coupled with basic sites: the formation of the hydrogen bond weakens the bond between $\equiv \mathrm{C}$ and $\mathrm{H}$. In the presence of an acid site, which can host the $\mathrm{CH}_{3}-\mathrm{C} \equiv \mathrm{C}$ moiety, the surface protonation is easily achieved. Therefore, propyne dissociation is a good probe for the presence of acid-base pairs on a surface as well [42]. In a similar way, propyne highlighted the presence of acid-base pairs in MIL$53(\mathrm{Fe})-\mathrm{CH}_{3}$. The bridging hydroxyl group $\mu_{2}-\mathrm{OH}$ acts as a proton donor toward the $\mathrm{C} \equiv \mathrm{C}$ triple bond of propyne and as proton acceptor toward the $\equiv \mathrm{C}-\mathrm{H}$ group, thanks to the simultaneous presence of an acid and a basic function in the propyne molecule, which is not the case for other probe molecules [43]. This approach also allowed the correlation of basic properties in solid catalysts (such as lanthanum oxides) with their performances for the synthesis of phytosterol esters from transesterification of a fatty methyl ester (dodecanoate) with b-sitosterol. Using the shift of the $v(C \equiv C)$ stretching mode for the adsorbed propyne species it was possible to determine the strength of the basic sites at the surface of the carbonated oxide: the lower the position of that vibration, the greater the basicity of the corresponding site. A ranking of the basic strength of the surface carbonate species of the lanthanum oxycarbonate samples was thus possible and it was correlated with the catalytic activity: the lower the basicity of carbonates, the higher the phytosterol ester yield [44].

Another protic molecule used to probe basicity is methanol when adsorbed molecularly. Upon accurate spectroscopic analyses (notably by coupling volumetric/IR and gravimetric/IR methods), it enables discrimination 
between molecular and dissociative adsorption of methanol and quantifying the basic sites exposed by a surface [45] $\mathrm{H}_{2} \mathrm{~S}$ has also been used as a hydrogen donor to characterize basicity. For example, infrared analyses on MIL-47 $(\mathrm{V})$ have shown that adsorption of $\mathrm{H}_{2} \mathrm{~S}$ preferentially occurs on $\mu_{2}-\mathrm{O}$ atoms of the $\mathrm{V}=\mathrm{O}$...V entities through hydrogen bonded species. The interaction is weak, with a calculated adsorption enthalpy of $27-29 \mathrm{~kJ} \mathrm{~mol}^{-1}$, revealing that the basicity of these entities is consequently weak [46]. In the case of a NaY zeolite, the high basicity of a few of the oxygen atoms of the supercage was shown by $\mathrm{H}_{2} \mathrm{~S}$ dissociation, leading to the creation of some $\mathrm{OH}$ groups in the supercage only, whereas sodalite cavities (although accessible) remained unaffected [47].

\subsection{Cationic and redox sites}

Hydroxyls complete the coordinative unsaturation of cations. They are therefore, intrinsic probes of the environment and oxydation degree of the moieties bearing them. In some cases, $\mathrm{OH}$ groups can indicate the presence of surface defects and the redox behaviour of a solid. This is particularly true for cerium-based compounds, in which the hydroxyl bands shift according to the presence of $\mathrm{Ce}^{3+} / \mathrm{Ce}^{4+}$ sites and/or oxygen vacancies [48]. The best parallelism to this evidence is provided by methoxy species formed on the activated surface by methanol dissociative adsorption. Methoxys are sensitive to the local environment of cations, so they present specific bands for each cationic species they are coordinated to, and help to describe the surface composition of a material. Moreover, they unravel the oxidation degree of cations and enable a quantification of the concentration of different species present on the surface, as well as the fraction, which can be reversibly transformed by a redox cycle [49-52]. This allows for the identification of mechanistic steps in catalytic reactions, as well as the active sites taking part in them [53].

$\mathrm{CO}$ and $\mathrm{NO}$ are invaluable tools for the characterization of cationic species inside porous compounds. For example, CO adsorption in Rh-ZSM- 5 led to the discovery of a new kind of rhodium gem-dicarbonyls and made possible to understand the mechanism of different catalytic reactions. The shift of the $v(\mathrm{CO})$ vibration showed the Rh position in the porous structure, its oxidation state and the capacity to host different chemical species having different stability, especially in the presence of water [54]. According to this methodology, $\mathrm{CO}$ and $\mathrm{NO}$ adsorption on zeolite-supported $\mathrm{Rh}$ 
nanoparticles containing different promoter elements permitted both characterizing the effect of the additive and the catalytic activity of the noble metal [55].

However, the best application of this methodology is certainly the characterization of copper and iron in porous compounds, since NO probes the $\mathrm{Cu}^{2+}$ and $\mathrm{Fe}^{2+}$ states, while $\mathrm{CO}$ adsorption is more specific to $\mathrm{Cu}^{+}$and, sometimes, $\mathrm{Fe}^{3+}$ states [56-58]. Thanks to the properties of these probe molecules it was possible to study the iron distribution and oxidation state in ZSM-5 [59,6o], FER [61] and Y [62] zeolites, which is of paramount importance for their application in environmental chemistry and petrochemistry. These studies showed that both the oxidation and the coordination states of $\mathrm{Fe}^{2+}$ confined in Ferrierites may change easily, which makes them excellent candidates for active redox sites [61]. Combining $\mathrm{CO}$ and $\mathrm{NO}$ as molecular probes, it was possible to go into the characterization of Fe-FER in very fine detail, identifying the position of iron on three distinct sites inside the crystalline structure [63]. Similarly, the $\mathrm{Fe}^{2+} / \mathrm{Fe}^{3+}$ ratio and distribution in MIL-10o(Fe) were evaluated by combining $\mathrm{CO}$ and NO probes [64] while their role in gas separation was highlighted $[65,66]$.

\subsection{Quantification of sites: coupling IR spectroscopy with thermogravimetry (TGA)}

The quantification of sites by IR spectroscopy is a key point that generally requires the determination of molar absorption coefficients $(\varepsilon)$, which can be measured by progressively adsorbing measured amounts of the probe molecule. However, the discrepancies between the values reported in the literature are worth noting due to a lack of precise control of the amount of probe adsorbed on the sample [67]. One way to overcome this problem is to couple IR spectroscopy and thermogravimetric analysis. In a setup developed in Caen, qualitative as well as quantitative information is obtained simultaneously by combining thermogravimetry and operando IR spectroscopy with online mass spectrometry. The weight (and therefore the number of adsorbed probe molecules) and IR spectra of a solid sample can be analysed simultaneously in real-time operando conditions in a gas flow between room temperature and $773 \mathrm{~K}$. Integrated molar absorbance coefficients can consequently be obtained directly [68]. 


\section{Characterization of zeolites using spectroscopic methods: examples}

\subsection{Acidity in zeolites}

Brønsted acidity in zeolites

It is generally accepted that Brønsted sites are the most reactive species in the channels and cavities of the zeolites in protonic form. According to Knözinger et al. [69], three wavenumber ranges can be observed in the $v(\mathrm{OH})$ stretching region of $\mathrm{H}$-forms of zeolites in the dehydrated state, namely $3745^{-} 3750$, 36oo-3650 and $3530-35^{\circ} \mathrm{o} \mathrm{cm}^{-1}$. The high frequency region has its origin in the presence of terminal silanol groups, which are located at the external surface of zeolite crystallites. The central frequency range $3600-3650 \mathrm{~cm}^{-1}$ is typical of the bridging hydroxyl groups $\mathrm{Si}-\mathrm{OH}-\mathrm{Al}$, responsible of the Brønsted acidity, which are located in large cavities or sufficiently wide pores, where they are unperturbed by interactions with their local environment and accessible to reactant or probe molecules. Finally, the low frequency feature, which typically appears in faujasite-type zeolites, is attributed to perturbed $\mathrm{OH}$ groups which are located in sodalite cages where they are inaccessible to even small probe molecules, such as $\mathrm{CO}, \mathrm{H}_{2}$ or $\mathrm{N}_{2}$.

The concentration and strength of the surface hydroxyl groups responsible for Brønsted acidity in zeolites (that is, both silanols and bridged $\mathrm{Si}(\mathrm{OH}$ ) Al groups) can be determined by adsorption of basic probe molecules monitored by IR spectroscopy. The interaction of the probe basic molecule with the $\mathrm{OH}$ forms an adduct through the acid-base reaction illustrated in Figure 4. The $\mathrm{OH}$ vibrations modes thus differ from the original Brønsted group because the hydrogen bonding perturbation is usually associated with modifications of the hydroxyl vibrational frequencies and minor changes of the internal modes of the bases B.

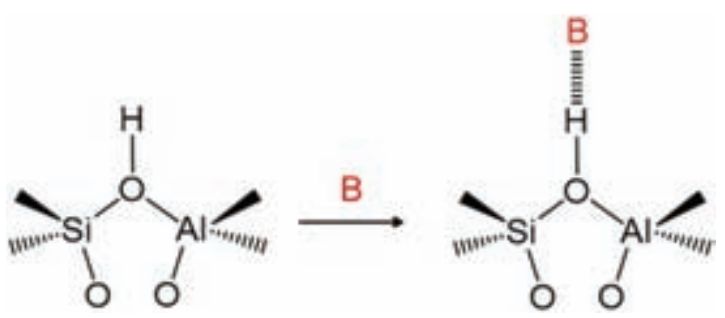

Figure 4. Formation of $\mathrm{ZH} \cdot . . . \mathrm{B}$ adducts between a Brønsted acid $\mathrm{OH}$ hosted inside a zeolite channel and a base $B$ via a classical acid-base reaction. 
An illustrative example was reported by Zecchina et al. [70] in which $\mathrm{N}_{2}$ probe molecule induced modifications on the $\mathrm{OH}$ vibration modes of a H-ZSM- 5 zeolite. Upon dosage of $\mathrm{N}_{2}$ (base B), the intensity of the $v(\mathrm{OH})$ mode of the unperturbed Brønsted groups associated with $\mathrm{Si}(\mathrm{OH}) \mathrm{Al}$ gradually decreases while that of the $v(\mathrm{ZH} \cdot \cdots \cdot \mathrm{B})$ vibration simultaneously increases. The observation of an isosbestic point evidences that the $\mathrm{ZH} \cdot \cdots \cdot \mathrm{B}$ interaction is really taking place in a stoichiometric manner. At higher pressures, the $\mathrm{Al}-\mathrm{OH}$ groups and finally the silanols groups are also perturbed because of the formation of 1:1 adducts with dinitrogen. Although the interaction of $\mathrm{N}_{2}$ with the structural Brønsted groups is very weak, the induced polarization is sufficient to make the $v(\mathrm{~N}-\mathrm{N})$ modes IR active. The Brønsted acidity of H-ZSM- 5 can be correlated with the magnitude of the redshift of the $\mathrm{OH}$ stretching vibration upon interaction with dinitrogen at low temperature by hydrogen bonding. The shift induced on the $v(\mathrm{OH})$ stretching frequency of $\mathrm{AlOH}$ and of silanols is significantly lower than that on the $\mathrm{Si}(\mathrm{Al}) \mathrm{OH}$ groups, this reflects the fact that hydroxyl groups of structural 

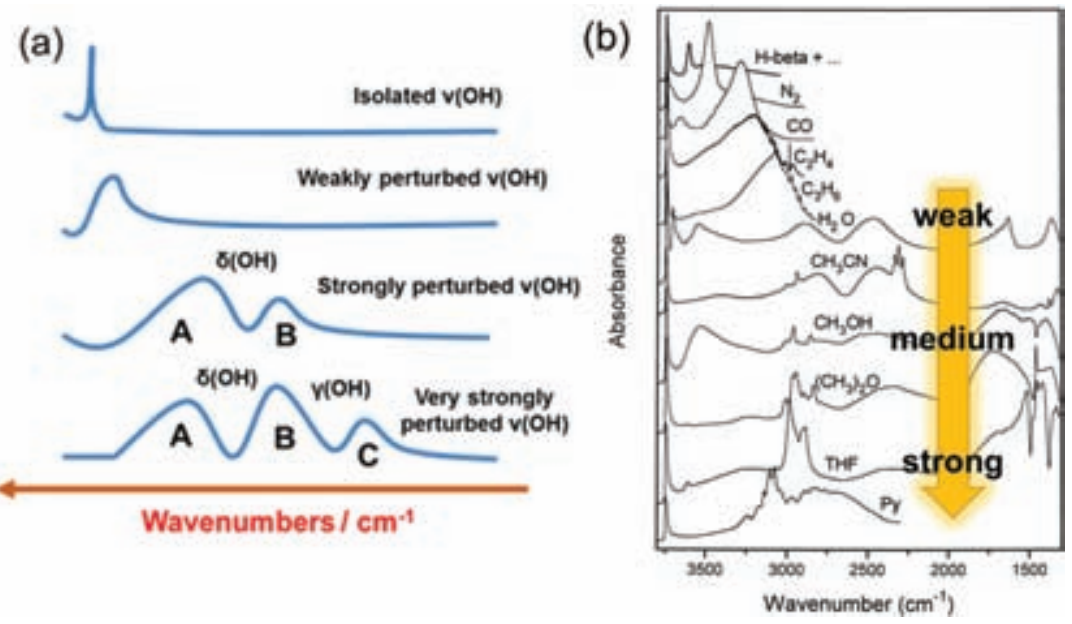

Figure 5. a) Evans' window on a $v(\mathrm{OH})$ vibration in the cases of free $v(\mathrm{OH})$ and $v(\mathrm{OH})$ perturbed by a $\mathrm{H}$-bond, strong $\mathrm{H}$-bond, and very strong $\mathrm{H}$-bond. b) Comparison of the background subtracted IR spectra of H-Beta/B adducts. Adapted with permission from [74]. Copyright 1997, American Chemical Society

with the two $\delta(\mathrm{OH})$ and two $\gamma(\mathrm{OH})$ harmonics, themselves shift to higher wavenumbers by the $\mathrm{H}$-bonding. The band structure observed is denoted as an A, B, C system and is indicative of a very strong H-bonding [76]. This reveals that the interaction has become sufficiently strong to shift the $\delta$ vibration modes out of the range of the framework vibrations. On the basis of these considerations we can now understand the sequence of spectra in Figure 5 b, which correspond to the interaction of a series of bases (increasing the basic character from $\mathrm{N}_{2}$ to pyridine) with zeolite $\mathrm{H}-\beta[74,77]$. A gradual shift to lower frequencies of the broad absorption associated with the perturbed $\nu(\mathrm{OH})$ as well as the formation and evolution of $\mathrm{A}, \mathrm{B}, \mathrm{C}$ features as a function of the basic character of the probe molecule emerges. It can also be noted that for THF and pyridine the shift is slightly smaller because the proton was transferred to the nucleophilic probe molecule. Similar observations have been obtained for other zeolites such as H-MOR or ZSM-5 [78].

The choice of probe molecule is crucial to obtain an overall view of the acidity. Its size has to be small enough to interact with all available sites and to avoid confinement effects but its basic strength has to be strong enough to interact even with the weakest acidic sites. Ammonia seems to be a good candidate for this but due to the high polarity of the NH bonds, hydrogen bonding with basic entities governs the coordination of adsorbed species and direct conclusions about acid strength are not straightforward. That is 

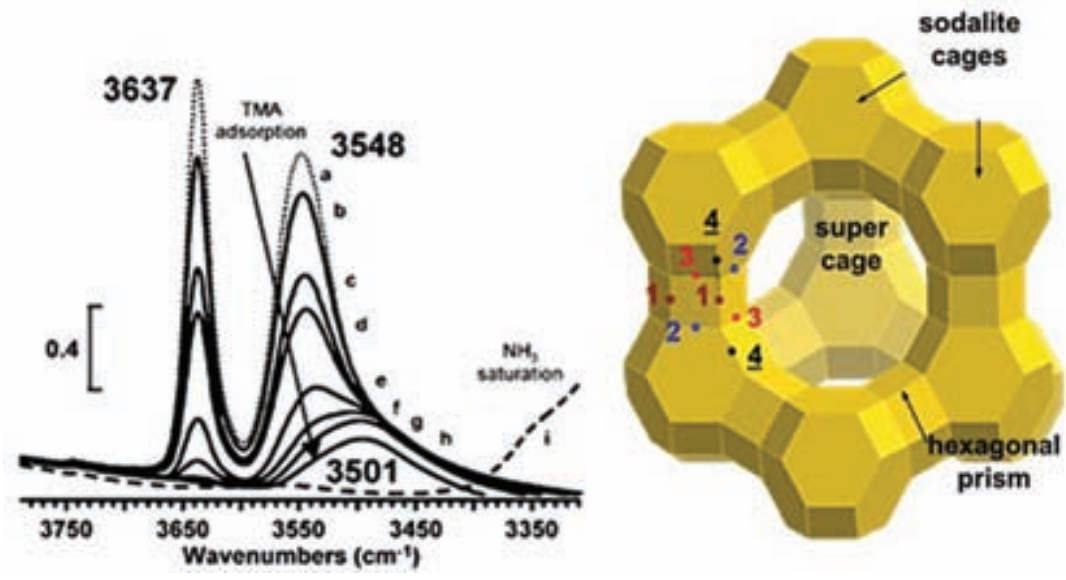

Figure 6. IR spectra of the $\mathrm{HY}$ zeolite upon TMA adsorption and $\mathrm{NH}_{3}$ saturation evidencing hydroxyls in the supercages at $3637 \mathrm{~cm}^{-1}$, in the sodalite units at $3548 \mathrm{~cm}^{-1}$ and in the hexagonal prism at $3501 \mathrm{~cm}^{-1}$. Adapted with permission from [32]. Copyright 2005, American Chemical Society

why the adsorption of several probe molecules is often required [35]. As an example, the FAU and the MOR structures are made of larger and small cavities in which some acidic hydroxyls are out of reach for basic molecules such as pyridine. The co-adsorption of the strongly basic trimethylamine (TMA) and ammonia (Figure 6) give an IR evidence of three kinds of acidic hydroxyls in defect-free HY zeolites [31,32]. Moreover, the TMA desorption is associated with the recovery of hydroxyls at 3656 and $3638 \mathrm{~cm}^{-1}$. The two corresponding $v(\mathrm{~N}-\mathrm{H})$ bands reveal the presence of at least two distinct acidic strengths for the hydroxyls located inside the supercages. For the same site location, the local chemical factor should then play a role: aluminium distribution in the framework is not necessarily homogeneous, and the number of $\mathrm{Al}$ next-nearest neighbours influences the acidic strength of a given site. Another explanation for the unusual $3656 \mathrm{~cm}^{-1}$ component could be that part of the $\mathrm{O}_{4}$ crystallographic site is a proton holder for this $\mathrm{HY}$ zeolite with low $\mathrm{Si} / \mathrm{Al}$ ratio. In such cases, all the four theoretically forecasted sites in the zeolitic FAU structure (Figure 6) would have been observed by IR spectroscopy [32]. The combined use of these two molecules also helped us to better characterize the various coordinated $\mathrm{NH}_{4}^{+}$and to determine the activity ranking between ammonium species and coordinated ammonia over Lewis sites during $\mathrm{NO}_{\mathrm{x}}$ selective reduction.

\section{Lewis acidity in zeolites}

The origins of Lewis acidity in zeolites are diverse and depend on the structure and chemical composition of the zeolite material under investigation 
[79,80]. From a general point of view, three types of Lewis acid centres in zeolites can be distinguished: i) charge-balancing extraframework alkali cations $[69,73,81,82]$, ii) extraframework aluminum species located in defect centres [83-85], and iii) heteroatoms isomorphically substituted in the framework [86-91]. No direct observation of any vibration band by IR spectroscopy for Lewis acid sites is possible. Nevertheless, the influence of the acid site on a probe molecule will provide information on the Lewis acid site by IR spectroscopy [67]. CO is the probe molecule most widely used for characterizing Lewis acidity in zeolites [92], although other basic molecules such as methanol, amines, or pyridine also give relevant information [93-96].

Charge-balancing cations such as the monovalent alkali cations, other than $\mathrm{H}^{+}$, may be considered Lewis acid sites [81]. These cations generate strong electric fields within the zeolite cages or channels and are capable to polarize the probe molecule admitted when they are located in accessible sites. For instance, $\mathrm{CO}$ can interact with the exchangeable cations in zeolites shifting the $\mathrm{C}-\mathrm{O}$ stretching frequency to higher values than that in the gas phase $\left(2143 \mathrm{~cm}^{-1}\right)$ through an interaction via the carbon atom [97]. Knözinger et al. [69] reported a linear correlation between the ionic radius of the alkali metal exchanged and the shift of $v(\mathrm{CO})$ for the series of zeolites LiY, NaY, KY, RbY and CsY. As illustrated in Figure 7, the frequency shift $\Delta v(\mathrm{CO})$ clearly decreases when increasing the cation radius, LiY showing the strongest Lewis acidity. This suggests an electrostatic interaction with

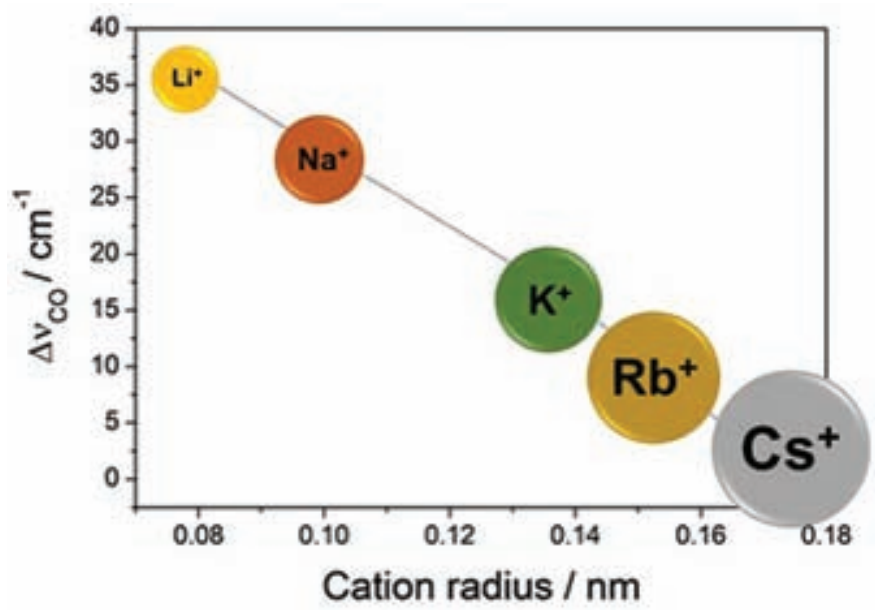

Figure 7. Interaction of CO with exchangeable alkali metal cations in Y-zeolite: correlation of C-O stretching frequency shift with cation radius. 
the electric field strength $(\mathrm{F})$ determining the frequency shift. In a similar study, Otero-Areán et al. [92] reported that, besides the fundamental $\mathrm{C}-\mathrm{O}$ stretching mode, weaker band appears at $90-140 \mathrm{~cm}^{-1}$. This band can be assigned to the combination mode between the frequency of $v(\mathrm{CO})$ and the cation-carbon bond vibration. The authors observed this band (at $139 \mathrm{~cm}^{-1}$ ) for CO adsorbed on Na-Y by using far IR radiation from a synchrotron source. Observation of the combination mode is relevant to zeolite characterization by IR spectroscopy, since the characteristic cation-carbon stretching vibration is very sensitive to the specific cation present in the zeolite.

The presence of Lewis acidity in protonic zeolites is usually attributed to the presence of extraframework Al-containing species (EFAL) [98-101]. The structure of a true Lewis acid site is still controversial although it could be associated with trigonal $\mathrm{Al}$ atoms formed as a result of zeolite dehydroxylation by thermal treatment or leached from the zeolite framework during chemical treatment [101-105]. In fact, the presence of Lewis acidity for zeolites rich in extraframework $\mathrm{Al}$ species is well detectable by IR spectroscopy of adsorbed probe molecules. Catana et al. [79] identified three types of Lewis acid sites by FTIR of CO adsorbed at low temperature and the relative intensities of these peaks were correlated with the structure type and extraframework $\mathrm{Al}$ amount. In this study they used different methods to create Lewis acid sites in a controlled way. Alumination with $\mathrm{AlCl}_{3}$ leads to well-distributed extraframework $\mathrm{Al}$ species with a highly distorted geometry, which act as strong Lewis acid sites. A mild steaming procedure leads to similar sites and a high concentration of extraframework Al. Although high, the amount of $\mathrm{Al}$ extracted from the framework with a severe steaming procedure is less effective in creating strong Lewis acid sites, probably because the clustered $\mathrm{Al}$ species block the access of the probe molecules to the active sites.

The incorporation of trivalent ion such as $\mathrm{Al}^{3+}, \mathrm{B}^{3+}, \mathrm{Ga}^{3+}, \mathrm{Fe}^{3+}$ or $\mathrm{In}^{3+}$ in the silica framework creates one negative charge in the zeolite lattice, which is balanced by a counter-ion, usually a proton [106]. As was mentioned in the previous section for the case of $\mathrm{Al}$, the bridging hydroxyls associated to the $\mathrm{Si}(\mathrm{OH}) \mathrm{M}^{3+}$ group are responsible of the Brønsted acidity. Many works subsituting aluminum by B, Fe or Ga have been published and this practice affects directly the properties of the bridging hydroxyls. On the other hand, when a tetravalent cation $\left(\mathrm{Ge}^{4+}, \mathrm{Sn}^{4+}, \mathrm{Ti}^{4+}\right)$ substitutes a Si atom of the framework, the zeolite lattice remains neutral due to the identical charges and no Brønsted acid sites are generated, although this substitution could directly affect the properties of an $\mathrm{OH}$ group connected to the heteroatom [107]. 
By contrast, strong acid Lewis sites can be formed upon thermal induced migration of heteroatoms $\left(\mathrm{Al}^{3+}, \mathrm{B}^{3+}, \mathrm{Ga}^{3+}, \mathrm{Ti}^{4+}, \mathrm{Sn}^{4+}\right)$ from the framework into partial or total extraframework positions, generating "defect sites". The formation of these centres is favoured by the presence of water vapour in the gas phase during the thermal treatments at high temperature $[70,75]$. In a few cases, "defect sites" are detected in the IR spectrum of activated samples by specific IR bands, mainly in the low frequency range: bands situated at $3782 / 880 \mathrm{~cm}^{-1}$ on $\beta$-zeolite [108] and around $960 \mathrm{~cm}^{-1}$ on Ti-silicalite [109], that can be considered as a fingerprint of Lewis-acid sites.

\subsection{Basicity in zeolites}

Zeolites intrinsically possess Lewis basic sites linked to the framework oxygen atoms bearing negative charge, which increase as the framework $\mathrm{Al}$ content increases [110]. For a given $\mathrm{Si} / \mathrm{Al}$ ratio, the negative charge on the oxygen atoms is higher the more electropositive, (or the less electronegative) the charge-balancing extraframework cations are. For instance, considering the series of alkaline cations the basicity of the zeolite increases in the order: $\mathrm{Li}^{+}<\mathrm{Na}^{+}<\mathrm{K}^{+}<\mathrm{Rb}^{+}<\mathrm{Cs}^{+}[110,111]$. Although the framework oxygen atoms are the most characteristic basic sites in zeolites, other basic centres can be found in zeolites as well, such as hydroxyl groups resulting from the dissociation of water in hydrated extraframework cations [112] or basic oxygen atoms in oxide clusters in the pores [113]. In alkaline forms of zeolites, only the centres associated with regular or strained oxygen bridges of the $\mathrm{Si}-\mathrm{O}-\mathrm{Al}$ type are expected to exist.

The determination of the basicity of a zeolite involves estimating the number and strength of these basic centres. As in the case of acid sites, basic sites can be identified adsorbing probe molecules, although in this case acid probe molecules, such as $\mathrm{CO}_{2}$, pyrrole, methanol, acetonitrile, acetylene, halogenated alkanes, $\mathrm{H}_{2} \mathrm{~S}, \mathrm{NO}$ and $\mathrm{N}_{2} \mathrm{O}_{4}[37,110,114,115]$ are required. An ideal acidic probe molecule should interact exclusively with the zeolite basic lattice oxygen. However, the compensating alkaline cations act as Lewis acid sites and form conjugated acid-base pairs, which can interact with any molecule. An ideal probe molecule for zeolite basicity should adsorb selectively on basic lattice oxygen atoms and not induce any modifications in the solid. In this sense, the best molecules are those which disproportionate into cations and anions. The anions immobilize the exchangeable cations. The cations adsorb onto the lattice oxygen of the zeolitic framework and probe the basicity of this oxygen. 
Acetylene and its derivatives also turned out to be suitable probemolecules for basic centres in zeolites. Acetylene exhibits shifts in $v(\mathrm{C}-\mathrm{H})$ frequency, which are sensitive to the strength of basic sites of alkaliexchanged zeolites. Uranova et al. [115] studied the adsorption of acetylene on $\mathrm{NaX}, \mathrm{Cs} / \mathrm{NaX}$, and $\mathrm{Na} / \mathrm{Y}$ zeolites. Two types of complexes with acetylene may be formed: i) complexes with metal cations (complex 1) and ii) complexes with basic oxygen atoms of the framework (complex 2). One can expect that the frequency of the stretching vibrations will slightly increase in the case of complex 1, whereas the opposite shift of this band may be anticipated in the case of complex 2. The decrease in frequency of the stretching vibrations in such complexes compared to the gas phase $\left(3287 \mathrm{~cm}^{-1}\right)$ may be accounted for by the weakening of the $\mathrm{C}-\mathrm{H}$ bond in the complex involving basic oxygen atoms. Therefore, this frequency may be used as a tool for estimating the basic strength of surface oxygens. Thus, the $v(\mathrm{C}-\mathrm{H})$ frequency decreases from $3216 \mathrm{~cm}^{-1}$ for NaM and $3205 \mathrm{~cm}^{-1}$ for $\mathrm{NaY}$ zeolites, to $3175^{-} 3185 \mathrm{~cm}^{-1}$ for $\mathrm{NaX}$ and $\mathrm{Cs} / \mathrm{NaX}$ zeolites, indicating a strengthening of the basic centres in X-type zeolites. Lavalley et al. [116] proposed the use of but-1-yne and showed that this probe could be used for zeolites with moderate basicity such as $\mathrm{Na}-\mathrm{Y}$ or Na-X zeolites and could be more sensitive to the heterogeneity of basic sites than other probe molecules.

Other probe molecules have also been used for the characterization of basicity in zeolites. For example, $\mathrm{H}_{2} \mathrm{~S}$ was used for characterising faujasite type zeolites, such as $\mathrm{NaX}$ and $\mathrm{NaY}$. The adsorption depends on their Si/Al ratio. Protons, which were generated via dissociation of $\mathrm{H}_{2} \mathrm{~S}$, attacked the zeolite lattice and formed new $\mathrm{OH}$ groups. On $\mathrm{NaY}$ with a $\mathrm{Si} / \mathrm{Al}$ ratio of $c a$. 2.5 or higher, no $\mathrm{H}_{2} \mathrm{~S}$ dissociation occurred, suggesting a weak basicity $[37,117,118]$. More recently, the disproportionation reaction of $\mathrm{NO}_{2}$, leading to a nitrate anion and a $\mathrm{NO}^{+}$cation (nitrosonium), has been used to characterize the basicity of alkali-exchanged FAU zeolites. The nitrate ions are stabilized by extraframework cations, whereas $\mathrm{NO}^{+}$directly interacts with framework oxygen atoms. The $\nu\left(\mathrm{NO}^{+}\right)$stretching frequency shift is sensitive to the electron density of the oxygen atom framework and, consequently, provides information about the basicity of the zeolite [39].

\subsection{Redox properties: metal cation exchanged zeolites}

Numerous metal transitions, noble metals and rare-earth cations have been extensively used as charge-balancing metals in zeolites. Significant efforts have been dedicated to the spectroscopic characterization of these 
materials aimed at understanding the nature (oxidation state, location, coordination environment, etc.) of the active metal sites in different zeolite structures. The redox properties of these materials are very important for their catalytic performance in numerous reactions like the selective catalytic reduction (SCR) of nitrogen oxides with hydrocarbons [35]. Below, we discuss as case study where a Co-containing zeolite shows the potential of IR spectroscopy for the characterization of metal transition sites in zeolites.

The co-adsorption of $o$-toluonitrile (oTN) and nitrile (NO) allowed the identification of different $\mathrm{Co}^{\mathrm{n}+}$ species and their location in a CoH-MFI zeolite [33]. Significant amounts of cobalt species were located on the external surface, mostly in the form of divalent cobalt, whereas on the internal surface the predominant Co species were trivalent and divalent ions. These observations were very valuable for explaining the reactivity of methane-SCR reaction. The $\mathrm{Co}^{3+}$ species active sites were located in the cavities, although probably in non-classical cation positions, characterized by a nitrosyl $\nu(\mathrm{NO})$ band at $1930 \mathrm{~cm}^{-1}$. These are able to convert $\mathrm{NO}$ to an adsorbed bridging nitrate species, which can be later decomposed to yield gas phase $\mathrm{NO}_{2}$ [119]. The cavity may contribute to the stabilization of aggregates containing trivalent cobalt. At the same time, the presence of cobalt-isocyanates involved in the SCR suggests that a possible route for the reaction implies the reduction of nitrate-like species by methane, forming water and isocyanates, which could later react with $\mathrm{NO}$ producing $\mathrm{N}_{2}$ and carbon dioxide. On the contrary, it seemed that substitutional $\mathrm{Co}^{2+}$ ions did not play a key role in the reaction, being almost certainly "redox-inactive". $\mathrm{Co}^{2+}$ dinitrosyls formed on them being decomposed well below the reaction temperature, they did not seem to be involved in the reaction [119]. These considerations link the active site with the reactivity of the species coordinated on it and the possible intermediates for the SCR reaction.

\section{Characterization of MOFs using spectroscopic methods: examples}

Metal-organic frameworks (MOFs) are characterization porous materials consisting of metal ions or clusters linked by polydentate organic linkers forming $3 \mathrm{D}$ structures with very high porosity and specific surface area $[120,121]$. The diversity of metals and organic ligands that can be combined to form MOFs is huge, with more than 20000 compounds of this class already reported in the literature [122]. Modifications of metal and/or organic linkers allows for changing the properties, size and shape of the pores. 
Moreover, the introduction of guest species into the pores, which also modify the MOF properties and the type of active sites, is possible. These factors make MOFs very versatile compounds that may be potentially applied in different fields such as gas adsorption [123-125], molecular separation $[126,127]$, or catalysis $[128,129]$, among others $[130,131]$, as will be discussed later on in this book.

The basic techniques used for MOF characterization are similar to those used for other materials, namely, XRD, $\mathrm{N}_{2}$ adsorption/desorption, thermogravimetric analyses, NMR and SEM. XRD, which allow us to determine the crystallinity and phase of the material. The surface area and porosity are calculated from the $\mathrm{N}_{2}$ adsorption/desorption isotherms and thermogravimetric. Analyses inform on the thermal stability of the compound. Studies by SEM are useful to determine crystal size and morphology of the solid particles. As stated in the introduction, EXAFS and XANES spectroscopies are also used to determine the local environment of the sites. This data may be completed with information about the oxidation state of the metal obtained from UV-visible spectroscopy. Thanks to the development of infrared spectroscopy and its use in the characterization of other materials, IR occupies a relevant place in the study of active sites in MOF structures. In addition, this technique is used to determine their behaviour when exposed to certain reactive molecules. This is especially important in the field of catalysis. Hence, in this part we will focus on infrared spectroscopy, commenting on some of the most relevant works on MOF characterization using this technique.

The active sites in MOFs can be the metallic ions or clusters, the functional groups in the organic linkers or guest species into the pores. The aspects most studied by IR spectroscopy are thus: i) the organic linkers, ii) the metallic ions or clusters, and iii) the acid-base properties. The organic linkers, structural components in MOFs, are characterized by analysis of the direct IR spectra, while the study of metallic centres and acid base properties require the adsorption of probe molecules in a similar way to the metallic oxides or zeolites. Accordingly, the discussion of examples has been divided into "direct IR analysis" and "adsorption of probe molecules".

\subsection{Direct IR analysis}

The direct analysis of the IR spectra permits observation of the presence of organic molecules interacting (or not) with the metallic sites and hydroxyl groups in the MOF structure [132]. Therefore, this technique is widely used 
in the characterization of MOF compounds and it allows confirmation of the synthesis of the desired compound. Furthermore, the potential of this technique makes it adequate for application in other cases.

For instance, Xue et al. [132] were interested in developing catalysts for the cycloaddition of $\mathrm{CO}_{2}$ and prepared a gadolinium-based MOF with PMDA (pyromellitic dianhydride) as an organic linker instead of the aromatic carboxylic acid usually used in this synthesis. The product obtained was characterized by conventional techniques (DRX, $\mathrm{N}_{2}$ adsorption, TEM...) and the IR spectra of the pure organic linker and prepared MOF were compared. The spectrum of PMDA revealed two bands at 1563 and $1368 \mathrm{~cm}^{-1}$, attributed to the asymmetric and symmetric stretching vibrations of carboxylate groups in the linker used. The formation of chemical bonds between the carboxylate groups in the PMDA and Gd(III) sites implies a modification of the carboxylate symmetry. Consequently, the shift of these bands to closer wavenumbers in the synthesized MOF confirmed the interaction metal-linker in the synthesized compound.

Among the most studied metal-organic frameworks, we find UiO, MIL-53 and MIL-10o families. Zr-based UiO are particularly interesting for their high thermal stability, which is of great importance as catalysts or absorbents in applications. These potential applications motivated the study of different aspects of the compounds. One such example is the work by Liang et al. on UiO-66 $\left(\mathrm{NH}_{2}\right)$ [133]. UiO-66 shows good activity in photocatalysis, comparable to inorganic standard photocatalysts. However, an improvement on its absorbance is required. In order to obtain an improved photocatalyst, the combination of UiO-66- $\mathrm{NH}_{2}$ and zinc phthalocyanine (a typical organic conductor) by a condensation reaction was studied in this work. The metallic clusters were octahedral $\mathrm{Zr}_{6} \mathrm{O}_{4}(\mathrm{OH})_{4}$ units, with the edges of the octahedron bridged by carboxylates from dicarboxylic acid BDC-NH (2-amino-1,4-benzenedicarboxylic acid), which acted as an organic linker in this compound [134] (Figure 8). The reaction between UiO-66 $\left(\mathrm{NH}_{2}\right)$ and phtalocyanine was followed by IR spectroscopy. After reaction, the disappearance of the IR band typical of $-\mathrm{NH}_{2}$ groups (1030 and $1130 \mathrm{~cm}^{-1}$ ) was observed and the appearance of new bands at 1620 and $3344 \mathrm{~cm}^{-1}$ attributed to $\mathrm{CO}-\mathrm{NH}_{2}$ and N-H stretched respectively, confirming the incorporation of the phtalocyanine to the MOF structure by an amide bond to the linker.

Continuing with the UiO family, we analyse the behaviour of hydroxyl groups using direct IR spectroscopy next. Besides a high thermal stability, the creation of coordination vacancies, which act as Lewis acid sites, during dehydration, makes the $\mathrm{UiO}$ family a very good candidate for use 


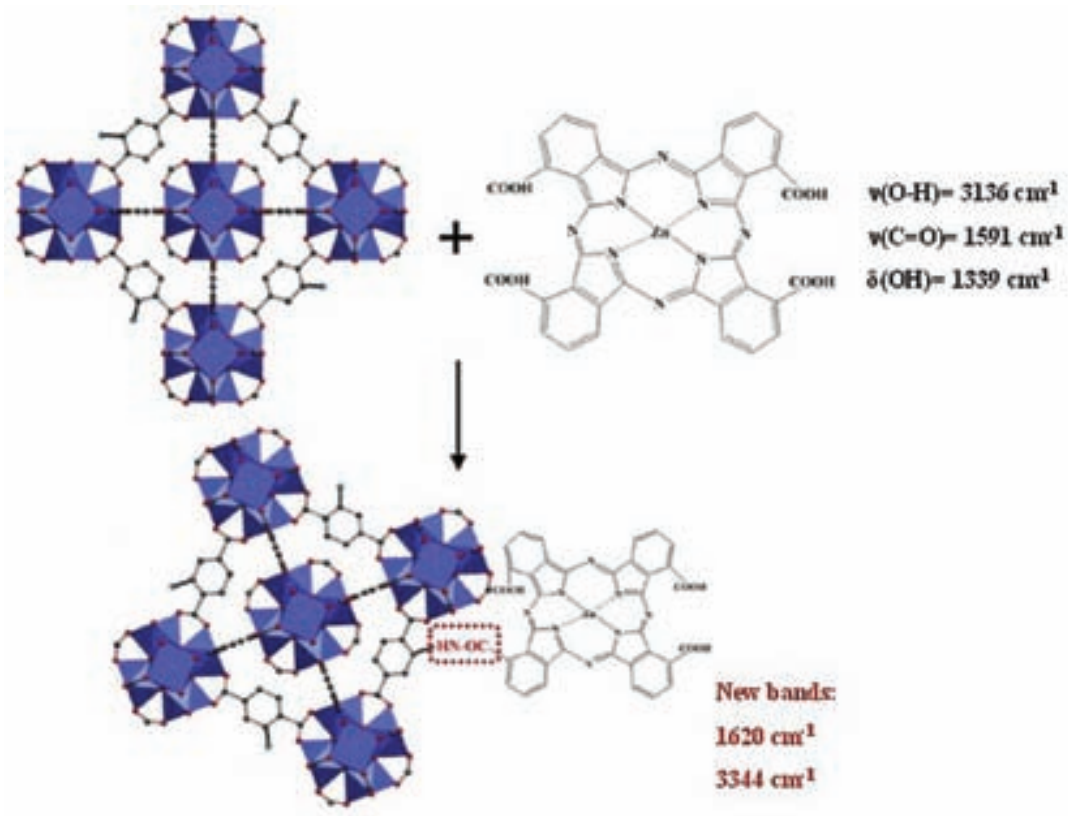

Figure 8. Combination of UiO-66- $\mathrm{NH}_{2}$ and zinc phthalocyanine. Adapted with permission from [134]. Copyright 2008 American Chemical Society.

in catalysis. This motivated the study by Shearer et al. [135]. In this work, a combined DRIFTS-TG analysis permitted the determination of the $\mathrm{OH}$ species implied in the dehydration process of UiO-66 and UiO-67. The authors detected only one band at $3681 \mathrm{~cm}^{-1}$ in the dehydrated UiO- 67 sample, in agreement with the structure of the $\mathrm{Zr}_{6} \mathrm{O}_{4}(\mathrm{OH})_{4}$ units and the symmetrical $\mathrm{OH}$ distribution in the cluster. However, in the sample UiO-66, constituted by similar cornerstones, the hydroxyl region of the spectrum shows a high complexity and at least six $\mathrm{OH}$ bands appear during the dehydration process. The authors explain the observed differences between both samples by the presence of $\mathrm{Cl}^{-}$ions coming from the synthesis. The $\mathrm{Cl}^{-}$may replace some $\mathrm{OH}$ groups resulting in a lower symmetry of hydroxyls. These results point to an influence of the organic linker on the behaviour of the resulting compound.

\subsection{Adsorption of probe molecules}

CO adsorption monitored by IR spectroscopy is the most widely used method to obtain information on the acid sites strength of metallic oxides, 
zeolites, and MOFs [12,136,137]. The interaction of a weakly acid molecule like $\mathrm{CO}$ with the $\mathrm{H}$-atom from the hydroxyl group results in an elongation of the $\mathrm{O}-\mathrm{H}$ bond and, consequently, a shift of the $\mathrm{v}(\mathrm{OH})$ vibration mode to lower wavenumbers. The extent of the shift is indicative of the $\mathrm{OH}$ acidity [138]. $\mathrm{N}_{2}$ has frequently been used as probe molecule because it is an IR inactive molecule (has no dipolar moment) and it does not interact with the active site, so its perturbation is only attributed to electrostatic interactions, especially important in the channels of porous materials such as zeolites [139].

Among the most widely studied MOFs we find M-MIL-53, where M is a trivalent metallic cation. The structure of this compound is constituted by octahedral $\mathrm{MO}_{6}$ units whose corners are connected (via $\mathrm{OH}$ ) by 1,4-benzenedicarboxylate (Figure 9) [140-142]. Mihaylov et al. [143] studied in detail the hydroxyls region of the IR spectra of a MIL-53 (Al) series by CO and ${ }^{15} \mathrm{~N}_{2}$ adsorption. Structural $\mu_{2}-\mathrm{OH}$ species absorbing at $3707 \mathrm{~cm}^{-1}\left(3704 \mathrm{~cm}^{-1}\right.$ by Ravon et al. [144]) were observed. Two shoulders at lower frequencies were assigned to $\mathrm{OH}$ interacting via weak $\mathrm{H}$-bond with the framework or

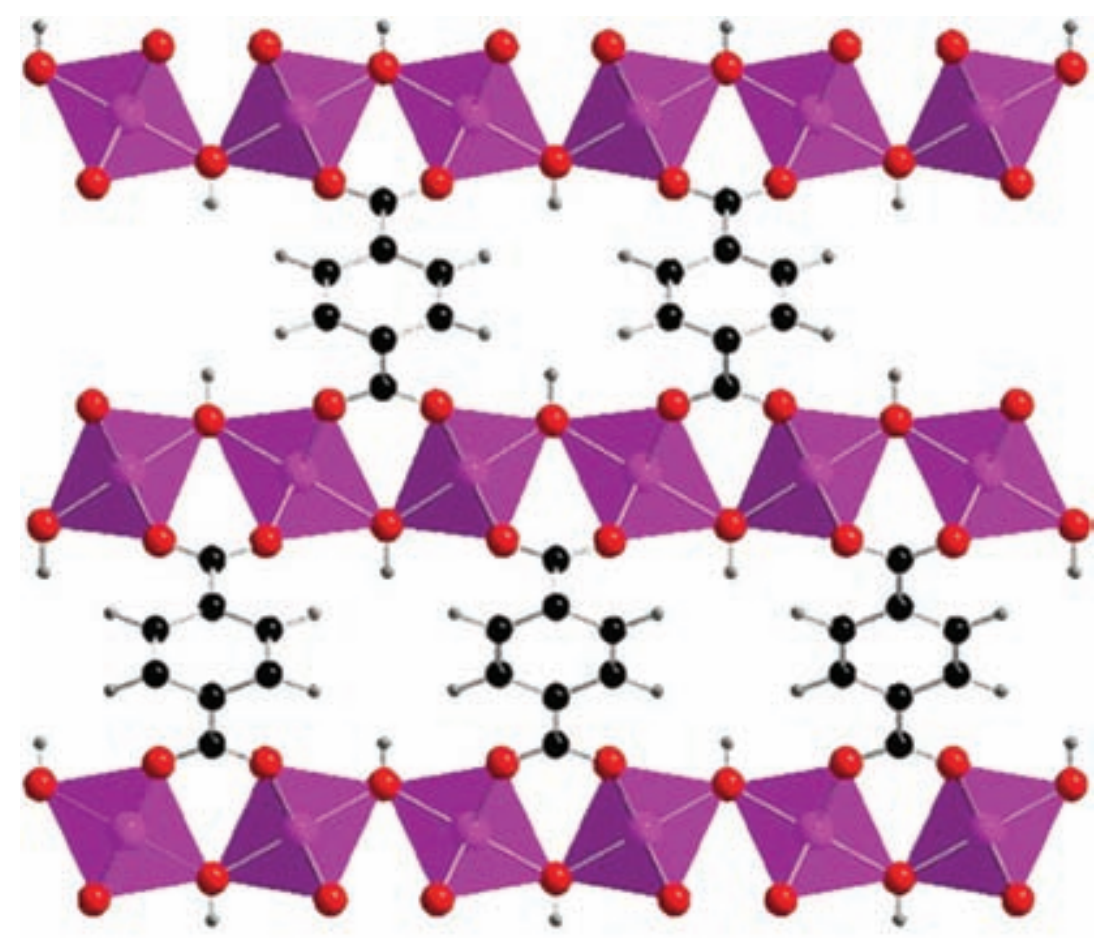

Figure 9. The local M environment within MIL-53. Adapted with permission from [94b]. Copyright 2013 American Chemical Society. 
with the rest of the acid used in the synthesis. These authors demonstrated the huge importance of this situation on the quantification of $\mathrm{OH}$ acid strength. The interaction via H-bond of hydroxyl groups with the framework provokes a shift to lower frequencies in the initial spectrum of the sample and the measured shift after $\mathrm{CO}$ adsorption does not correspond to the real situation. The so-called H-bond method, used in this work, permits calculation of the proton affinity $(\mathrm{PA})$ from the $\Delta v(\mathrm{OH})$ measured after $\mathrm{CO}$ adsorption at $100 \mathrm{~K}$ according to the following equation:

$$
P A^{O H}=P A^{S i O H}-442.5 \log \left(\frac{\Delta v(O H)}{\Delta v(S i O H)}\right)
$$

where $\mathrm{PA}^{\mathrm{OH}}$ and $\mathrm{PA}^{\mathrm{SiOH}}$ are the proton affinities of a specific $\mathrm{OH}$ in the sample, corresponding to a silanol group (taken as reference), respectively. The interaction via $\mathrm{H}$-bond observed in the initial spectrum of the sample, provokes a deviation of the measured values from the real ones. Therefore, it is very important in this type of solids to calculate the intrinsic $\mathrm{OH}$ frequency to obtain the correct values of the induced CO shift. In this way, calculation of proton affinities using the equation above will provide the correct values of this parameter.

The influence of the metal on the acid properties of MIL-53 was also studied by $\mathrm{CO}$ adsorption at low temperature on MIL-53 (Al) and MIL-53 (Ga) [144]. The experimental observations showed a small shift to lower frequencies of $\nu(\mathrm{OH})$ after $\mathrm{CO}$ adsorption at $100 \mathrm{~K}: 30-50 \mathrm{~cm}^{-1}$ for MIL-

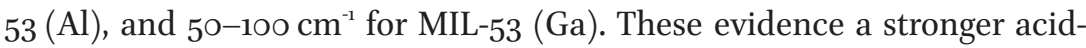
ity of the Ga-containing MOF. The authors also detected a difference between the measured shift of $v(\mathrm{OH})$ vibration mode and the one calculated by DFT. Moreover, molecular modelling evidenced that $\mathrm{OH}$ groups in the Al-containing MOF are straight, while tilted $\mathrm{OH}$ are present in MIL-53 (Ga). This characterization was used to explain the observed activity in the Friedel-Crafts alkylation reaction. The low activity observed using MIL-53 (Al) was related to its low acidity. However, the acid strength alone cannot explain the high activity of MIL-53 (Ga). The authors attributed the high catalytic activity of this compound to a strong stabilization of the reaction intermediate by the tilted $\mathrm{OH}$ groups in the Ga-MOF supported by theoretical calculations.

The flexibility of MOF structures is among the most important properties of these solids. It depends on several factors but the most important one is the nature of the metal [145]. Hence, Nouar et al. [145] studied MIL-53 (Fe-Cr), and the results were compared to those for MIL-53 (Fe) and MIL-53 (Cr). The analyses of the $950-800 \mathrm{~cm}^{-1}$ region evidenced the 
presence of bands at $847 \mathrm{~cm}^{-1}$ and $928 \mathrm{~cm}^{-1}$ for the compounds containing $\mathrm{Fe}$ and $\mathrm{Cr}$, respectively. These bands, sensitive to $\mathrm{H}_{2} / \mathrm{D}_{2}$ exchange, were attributed to $\delta(\mathrm{OH})$ modes. In the bimetallic sample, besides these bands, a new band at $885 \mathrm{~cm}^{-1}$ was observed. Through comparison of the spectra, this new band was attributed to $\mathrm{OH}$ bridged to both metals $(\mathrm{Cr}$ and $\mathrm{Fe}$ ), demonstrating the interaction between both metals. $\mathrm{CO}_{2}$ adsorption isotherms evidenced that the presence of Fe-Cr interaction strongly modifies the behaviour of the solid to the point that it does not match the expected behaviour for a theoretical mixture of monometallic compounds. Additionally, MIL-53 ( $\mathrm{Fe})-\mathrm{X}\left(\mathrm{X}=\mathrm{CH}_{3}, \mathrm{Cl}, \mathrm{Br}\right)$ were characterized by propyne adsorption. This probe molecule, containing acid and base functions, may be used to detect the presence of acid-base pairs in the solid. The $\mathrm{C} \equiv \mathrm{C}$ bond acts as proton acceptor and the function $\equiv \mathrm{C}-\mathrm{H}$ group as proton donor.

In the case of the MIL-10o family characterized by CO adsorption, we note the work by Vimont et al. [9]. The hydroxyls region of MIL-10o at different states of hydration was analysed. After a treatment at high temperature only one band at $3585 \mathrm{~cm}^{-1}$ was observed. It shows $\mathrm{OH}$ bonded to the $\mathrm{Cr}^{3+}$ sites and demonstrates that this type of $\mathrm{OH}$ is present in the MOF structure. At a higher hydration degree (lower temperature of treatment), this $\mathrm{OH}$ is perturbed, which points to some Brønsted acidity in this $\mathrm{OH}$. Accordingly, measurements of Brønsted acidity by $\mathrm{CO}$ adsorption were carried out. The results show a shift of the hydroxyl band from $3585 \mathrm{~cm}^{-}$ ${ }^{1}$ to $3495 \mathrm{~cm}^{-1}\left(\Delta v=90 \mathrm{~cm}^{-1}\right)$ after interaction with the $\mathrm{CO}$ molecules, thus concluding that $\mathrm{Cr}-\mathrm{OH}$ sites have an acid strength similar to that of $\mathrm{Si}-\mathrm{OH}$ groups. The $\mathrm{CO}$ adsorption at higher hydration degree indicated a weaker acidity of the water molecules interacting with the $\mathrm{Cr}-\mathrm{OH}$. Moreover, the $\mathrm{CO}$ adsorption permitted characterization of the Lewis acid sites. Three bands at 2192, 2200 and $2184 \mathrm{~cm}^{-1}$ were detected, which suggested a high heterogeneity of Lewis acid sites in this solid.

Gas separation and storage are among the most studied applications of MOFs. In this sense, it is worth noting the work about $\mathrm{H}_{2}$ storage and $\mathrm{CO}_{2}$ adsorption on M-MOF-74 (M=Mg, Mn, Fe, Co, Zn) [123] by Fitzgerald et al. CPO-27-M. Spectra recorded after $\mathrm{CO}_{2}$ adsorption on every solid at different pressures and temperatures were compared. The $\nu_{3}$ vibration (symmetric stretching mode of $\mathrm{CO}_{2}$ ) seems to be the most sensitive to the gas-solid interaction and was related to the interaction strength due to electrostatic and charge transfer effects, which were different for each metal. This study evidences one more time versatility in tuning the properties of MOFs, in this case, the interaction with $\mathrm{CO}_{2}$. 
With regard to the importance of operando techniques in spectroscopy, Wuttke et al. used operando IR spectroscopy to clarify the separation mechanism of propane/propene mixtures [65]. Observation of the surface material during the separation of both hydrocarbons evidenced that $\mathrm{Fe}(\mathrm{II})$ sites, thanks to their ability to interact with the double bond of the olefin, hold the main responsible for the separation. Propane adsorption on the MOF generated two bands at 2868 and $2958 \mathrm{~cm}^{-1}$, attributed to $2 \delta\left(\mathrm{CH}_{3}\right)$ and $v\left(\mathrm{CH}_{3}\right)$, respectively, while the interaction of propene with the solid was characterized by the presence of $\nu\left(\mathrm{CH}_{3}\right)$ vibration mode at $2930 \mathrm{~cm}^{-1}$ and $\nu(\mathrm{C}=\mathrm{C})+\delta\left(\mathrm{CH}_{3}\right)$ at $306 \mathrm{~cm}^{-1}$ (if adsorbed on $\mathrm{Fe}^{\mathrm{III}}$ ) or $3048 \mathrm{~cm}^{-1}$ (if the olefin interacts with $\mathrm{Fe}^{\mathrm{II}}$ sites). These differences distinguished the adsorption of both molecules on the surface, made possible following up the separation process and even permitted quantitative analysis to be carried out.

\section{Concluding remarks}

IR spectroscopy can provide valuable and useful information on the physical-chemical properties of zeolites and MOFs, both directly and via the adsorption of adequate probe molecules. The acidity, basicity and redox sites can be identified and quantified in strength and concentration. This information is crucial for developing more efficient materials in their applications. From the point of view of catalysis, operando spectroscopic techniques can visualize, which sites play a role in the catalytic reaction behaving as active sites or hosting reacting agents or products. Vibrational spectroscopy has a special relevance in the development of structure-activity relationships for heterogeneous catalysis since it provides detailed molecular insights on the adsorbed species over the catalyst surface, including reaction intermediates, spectator species and deactivating products. In this sense, it is always useful to ask "what can we see by IR spectroscopy in this reaction process?" As shown in this chapter, IR spectroscopy is of great interest in characterizing acid-basic and redox active centres in zeolites and MOFs as it can often provide valuable information to better understand and improve the operation of these materials.

\section{References}

[1] http://www.iza-structure.org/databases/

[2] Niemantsverdriet, J.W. In Spectroscopy in Catalysis, Wiley-VCH: 2007, pp 217-249. 
[3] Auerbach, S.M., Carrado, K.A., Dutta, P.K. Handbook of zeolites Science and Technology. 2003.

[4] Karge, H.G. Micropor. Mesopor. Mater. 1998, 22, 547.

[5] Esemann, H., Forster, H., Geidel, E., Krause, K. Micropor. Mater. 1996, 6, 321.

[6] Hadjiivanov, K., Friederike, C.J. Chapter Two - Identification and Characterization of Surface Hydroxyl Groups by Infrared Spectroscopy. in Adv. Catal., Academic Press: 2014, Vol. 57, pp 99-318.

[7] Khabtou, S., Chevreau, T., Lavalley, J.C. Micropor. Mater. 1994, 3, 133.

[8] Cairon, O., Thomas, K., Chevreau, T. Micropor. Mesopor. Mater. 2001, 46, 327.

[9] Vimont, A., Goupil, J.M., Lavalley, J.C., Daturi, M., Surble, S., Serre, C., Millange, F., Ferey, G., Audebrand, N. J. Am. Chem. Soc. 2006, 128, 3218.

[10] Vimont, A., Leclerc, H., Mauge, F., Daturi, M., Lavalley, J.C., Surble, S., Serre, C., Ferey, G. J. Phys. Chem. C 2007, 111, 383 .

[11] Busca, G. Catal. Today 1998, 41, 191.

[12] Busca, G. Phys. Chem. Chem. Phys. 1999, 1, 723.

[13] Hadjiivanov, K.I., Vayssilov, G.N. Characterization of oxide surfaces and zeolites by carbon monoxide as an IR probe molecule. in Adv. Catal., 2002, Vol. 47, pp 307-511.

[14] Krahl, T., Vimont, A., Eltanany, G., Daturi, M., Kemnitz, E. J. Phys. Chem. C 2007, 111, 18317.

[15] Vimont, A., Daturi, M., Winfield, J.M. Investigation of Surface Acidity using a Range of Probe Molecules. in Functionalized Inorganic Fluorides: Synthesis, Characterization \& Properties of Nanostructured Solids, Tressssaud, A. (Ed.), John Wiley \& Sons, 2010, 101-139.

[16] Blyholder, G.J. Phys. Chem. 1964, 68, 2772.

[17] Lercher, J.A., Grundling, C., EderMirth, G. Catal. Today 1996, 27, 353.

[18] Morterra, C., Magnacca, G. Catal. Today 1996, 27, 497.

[19] Travert, A., Vimont, A., Sahibed-Dine, A., Daturi, M., Lavalley, J.C. Appl. Catal., A 20o6, 307,98 .

[20] Knözinger, H., Stolz, H. Berichte Der Bunsen-Gesellschaft Fur Physikalische Chemie 1971, 75,1055 .

[21] Lahousse, C., Aboulayt, A., Mauge, F., Bachelier, J., Lavalley, J.C. J. Mol. Catal. 1993, 84, 283.

[22] Petit, C., Mauge, F., Lavalley, J.C. Stud. Surf. Sci. Catal. 1997, 106, 157.

[23] Jacobs, P.A., Heylen, C.F. J. Catal. 1974, 34, 267.

[24] Corma, A., Rodellas, C., Fornes, V.J. Catal. 1984, 88, 374.

[25] Tsyganenko, A.A., Pozdnyakov, D.V., Filimonov, V.N. J. Mol. Struct. 1975, 29, 299.

[26] Ramis, G., Yi, L., Busca, G., Turco, M., Kotur, E., Willey, R.J.J. Catal. 1995, 157, 523.

[27] Ramis, G., Busca, G., Cristiani, C., Lietti, L., Forzatti, P., Bregani, F. Langmuir 1992, 8, 1744.

[28] Thibault-Starzyk, F., Travert, A., Saussey, J., Lavalley, J.C. Top. Catal. 1998, 6, 111.

[29] Marie, O., Thibault-Starzyk, F., Lavalley, J.C. Phys. Chem. Chem. Phys. 2000, 2, 5341.

[30] Montanari, T., Bevilacqua, M., Resini, C., Busca, G.J. Phys. Chem. B 2004, 108, 2120.

[31] Romero-Sarria, F., Marie, O., Saussey, J., Daturi, M.J. Phys. Chem. B 2005, 109, 1660.

[32] Romero-Sarria, F., Blasin-Aubé, V., Saussey, J., Marie, O., Daturi, M.J. Phys. Chem. B 20o6, 110, 13130.

[33] Montanari, T., Marie, O., Daturi, M., Busca, G. Catal. Today 2005, 110, 339.

[34] Thibault-Starzyk, F., Stan, I., Abelló, S., Bonilla, A., Thomas, K., Fernandez, C., Gilson, J.P., Pérez-Ramírez, J.J. Catal. 2009, 264, 11.

[35] Vimont, A., Thibault-Starzyk, F., Daturi, M. Chem. Soc. Rev. 2010, 39, 4928.

[36] Daturi, M., Binet, C., Lavalley, J.C., Galtayries, A., Sporken, R. Phys. Chem. Chem. Phys. 1999, 1, 5717-5724.

[37] Lavalley, J.C. Catal. Today 1996, 27, 377.

[38] Barthomeuf, D. Catal. Rev. Sci. Eng. 1996, 38, 521. 
[39] Marie, O., Malicki, N., Pommier, C., Massiani, P., Vos, A., Schoonheydt, R., Geerlings, P., Henriques, C., Thibault-Starzyk, F. Chem. Commun. 2005, 1049.

[40] Payen, E., Grimblot, J., Lavalley, J.C., Daturi, M., Maugé, F. Application of Vibrational Spectroscopy in the Characterization of Oxides and Sulfides Catalysts. In Handbook of Vibrational Spectroscopy, Chalmers, J.M., Griffith, P.R., eds. Wiley: 2001, Vol. 4, pp 3005-3041.

[41] Mordenti, D., Grotz, P., Knözinger, H. Catal. Today 2001, 70, 83.

[42] Michalska, A., Daturi, M., Saussey, J., Nowak, I., Ziolek, M.T Micropor. Mesopor. Mater. 2006, 90, 362 .

[43] Moulin, B., Salles, F., Bourrelly, S., Llewellyn, P.L., Devic, T., Horcajada, P., Serre, C., Clet, G., Lavalley, J.C., Daturi, M., Maurin, G., Vimont, A. Micropor. Mesopor. Mater. 2014, 195, 197.

[44] Valange, S., Beauchaud, A., Barrault, J., Gabelica, Z., Daturi, M., Can, F. J. Catal. 2007, 251,113 .

[45] Moulin, B., Oliviero, L., Bazin, P., Daturi, M., Costentin, G., Mauge, Phys. Chem. Chem. Phys. 2011, 13, 10797.

[46] Hamon, L., Leclerc, H., Ghoufi, A., Oliviero, L., Travert, A., Lavalley, J.C., Devic, T., Serre, C., Ferey, G., De Weireld, G., Vimont, A., Maurin, G.J. Phys. Chem. C 2011, 115, 2047.

[47] Mauge, F., Sahibed-Dine, A., Gaillard, M., Ziolek, M.J. Catal. 2002, 207, 353.

[48] Daturi, M., Finocchio, E., Binet, C., Lavalley, J.C., Fally, F., Perrichon, V.J. Phys. Chem. B 1999, $103,4884$.

[49] Fally, F., Perrichon, V., Vidal, H., Kaspar, J., Blanco, G., Pintado, J.M., Bernal, S., Colon, G., Daturi, M., Lavalley, J.C. Catal. Today 2000, 59, 373.

[5o] Daturi, M., Finocchio, E., Binet, C., Lavalley, J.C. Fally, F., Perrichon, V., Vidal, H., Hickey, N., Kaspar, J.J. Phys.Chem. B 2000, 104, 9186.

[51] Finocchio, E., Busca, G. Catal. Today 2001, 70, 213.

[52] Binet, C., Daturi, M. Catal. Today 2001, 70, 155 .

[53] Nouar, F., Breeze, M.I., Campo, B.C., Vimont, A., Clet, G., Daturi, M., Devic, T., Walton, R.I., Serre, C. Chem. Commun. 2015, 51, 14458.

[54] Ivanova, E., Mihaylov, M., Thibault-Starzyk, F., Daturi, M., Hadjiivanov, K.J. Catal. 2005, 236, 168.

[55] Lepage, M., Visser, T., Soulimani, F., Iglesias-Juez, A., Weckhuysen, B.M. J. Phys. Chem. C 2010, 114, 2282.

[56] Strauss, S.H. Dalton Trans. 2000, 1-6.

[57] Zecchina, A., Bordiga, S., Palomino, G.T., Scarano, D., Lamberti, C., Salvalaggio, M. J. Phys. Chem. B 1999, 103, 3833 .

[58] Xamena, F., Fisicaro, P., Berlier, G., Zecchina, A., Palomino, G.T., Prestipino, C., Bordiga, S., Giamello, E., Lamberti, C. J. Phys. Chem. B 2003, 107, 7036.

[59] Joyner, R., Stockenhuber, M.J. Phys. Chem. B 1999, 103, 5963.

[6o] Chen, H.Y., El-Malki, E.M., Wang, X., van Santen, R.A., Sachtler, W.M.H. J. Mol. Catal. A Chem. 2000, 162, 159 .

[61] Blasin-Aube, V., Marie, O., Saussey, J., Plesniar, A., Daturi, M., Nguyen, N., Hamon, C., Mihaylov, M., Ivanova, E., Hadjiivanov, K.J. Phys. Chem. C 2009, 113, 8387.

[62] Novakova, J., Kubelkova, L., Wichterlova, B., Juska, T., Dolejsek, Z. Zeolites 1982, 2, 17.

[63] Ivanova, E., Mihaylov, M., Hadjiivanov, K., Blasin-Aubé, V., Marie, O., Plesniar, A., Daturi, M. Appl. Catal., B 2010, 93, 325.

[64] Yoon, J.W., Seo, Y.K., Hwang, Y.K., Chang, J.S., Leclerc, H., Wuttke, S., Bazin, P., Vimont, A., Daturi, M., Bloch, E., Llewellyn, P.L., Serre, C., Horcajada, P., Greneche, J.M., Rodrigues, A.E., Ferey, G. Angew. Chem. Int. Ed. 2010, 49, 5949.

[65] Wuttke, S., Bazin, P., Vimont, A., Serre, C., Seo, Y.K., Hwang, Y.K., Chang, J.S., Ferey, G., Daturi, M. Chem. Eur.J. 2012, 18, 11959. 
[66] Yoon, J.W., Lee, J.S., Lee, S., Cho, K.H., Hwang, Y.K., Daturi, M., Jun, C.H., Krishna, R., Chang, J.S. Chem. Eur.J. 2015, 21, 18431.

[67] Thibault-Starzyk, F., Maugé, F. Infrared Spectroscopy. in Characterization of Solid Materials and Heterogeneous Catalysts, Wiley-VCH Verlag GmbH \& Co. KGaA: 2012, pp 1-48.

[68] Bazin, P., Alenda, A., Thibault-Starzyk, F. Dalton Trans. 2010, 39, 8432.

[69] Knözinger, H., Huber, S. J. Chem. Soc. Faraday Trans. 1998, 94, 2047.

[70] Zecchina, A., Spoto, G., Bordiga, S. Phys. Chem. Chem. Phys. 2005, 7, 1627.

[71] Delgado, M.R., Bulánek, R., Chlubná, P., Arean, C.O. Catal. Today 2014, 227, 45.

[72] Arean, C.O., Delgado, M.R., Nachtigall, P., Thang, H.V., Rubes, M., Bulanek, R., Chlubna-Eliasova, P. Phys. Chem. Chem. Phys. 2014, 16, 10129.

[73] Bordiga, S., Lamberti, C., Bonino, F., Travert, A., Thibault-Starzyk, F. Chem. Soc. Rev. 2015, 44, 7262 .

[74] Zecchina, A., Spoto, G., Ricchiardi, G., Bordiga, S., Bonino, F., Prestipino, C., Lamberti, C. Stud. Surf. Sci. Catal. 2002, 142, 3.

[75] Zecchina, A., Spoto, G., Bordiga, S. Vibrational Spectroscopy of Zeolites. in Handbook of Vibrational Spectroscopy, John Wiley \& Sons, Ltd: 2006, 3042-3071.

[76] Evans, J.C., Wright, N. Spectrochim. Acta Part A 1960, 16, 352-357.

[77] Pazé, C., Bordiga, S., Lamberti, C., Salvalaggio, M., Zecchina, A., Bellussi, G. J. Phys. Chem. B 1997, 101, 4740.

[78] Buzzoni, R., Bordiga, S., Ricchiardi, G., Lamberti, C., Zecchina, A., Bellussi, G. Langmuir $1996,12,930$.

[79] Catana, G., Baetens, D., Mommaerts, T., Schoonheydt, R.A., Weckhuysen, B.M. J. Phys. Chem. B 2001, 105, 4904.

[80] Sandoval-Díaz, L.E., González-Amaya, J.A., Trujillo, C.A. Micropor. Mesopor. Mater. 2015, 215, 229 .

[81] Ward, J.W.J. Catal. 1968, 10, 34.

[82] Bulánek, R., Koudelková, E. Catal. Today 2015, 243, 62.

[83] Baran, R., Millot, Y., Onfroy, T., Krafft, J.M., Dzwigaj, S. Micropor. Mesopor. Mater. 2012, 163, 122.

[84] Janiszewska, E., Macario, A., Wilk, J., Aloise, A., Kowalak, S., Nagy, J.B., Giordano, G. Micropor. Mesopor. Mater. 2013, 182, 220.

[85] Serrano, D.P., García, R.A., Vicente, G., Linares, M., Procházková, D., Čejka, J. J. Catal. 2011, 279, 366 .

[86] Damin, A., Ricchiardi, G., Bordiga, S., Zecchina, A., Ricci, F., Spanò, G., Lamberti, C. Stud. Surf. Sci. Catal. 2001, 140, 195.

[87] Dapsens, P.Y., Mondelli, C., Pérez-Ramírez, J. Chem. Soc. Rev. 2015, 44, 7025.

[88] Sushkevich, V.L., Vimont, A., Travert, A., Ivanova, I.I.J. Phys. Chem. C 2015, 119, 17633.

[89] Dapsens, P.Y., Kusema, B.T., Mondelli, C., Pérez-Ramírez, J. J. Mol. Catal. A Chem. 2014, 388-389, 141-147.

[9o] Dapsens, P.Y., Mondelli, C., Pérez-Ramírez, J. ChemSusChem 2013, 6, 831.

[91] Kulkarni, B.S., Krishnamurty, S., Pal, S. J. Mol. Catal. A Chem. 2010, 329, 36.

[92] Otero-Areán, C., Turnes Palomino, G., Zecchina, A., Spoto, G., Bordiga, S., Roy, P. Phys. Chem. Chem. Phys. 1999, 1, 4139.

[93] Rep, M., Palomares, A.E., Eder-Mirth, G., Van Ommen, J.G., Rösch, N., Lercher, J.A. J. Phys. Chem. B 2000, 104, 8624 .

[94] Docquir, F., Toufar, H., Su, B.L. Langmuir 2001, 17, 6282.

[95] Gil, B., Zones, S.I., Hwang, S.J., Bejblová, M., Čejka, J.J. Phys. Chem. C 2008, 112, 2997.

[96] Daniell, W., Topsøe, N.Y., Knözinger, H. Langmuir 2001, 17, 6233.

[97] Hush, N.S., Williams, M.L.J. Mol. Spectrosc. 1974, 50, 349. 
[98] Phung, T.K., Busca, G. Appl. Catal., A 2015, 504, 151.

[99] Marques, J.P., Gener, I., Ayrault, P., Bordado, J.C., Lopes, J.M., Ribeiro, F.R., Guisnet, M. Compt. Rend. Chem. 2005, 8, 399.

[10o] Wichterlová, B., Bortnovsky, O., Sobalík, Z., Bastl, Z.J. Catal. 2002, 210, 171.

[101] Trombetta, M., Busca, G., Storaro, L., Lenarda, M., Casagrande, M., Zambon, A. Phys. Chem. Chem. Phys. 2000, 2, 3529 .

[102] Jiang, M., Karge, H.G.J. Chem. Soc. Faraday Trans. 1996, 92, 2641.

[103] Matias, P., Sá Couto, C., Graa, I., Lopes, J.M., Carvalho, A.P., Ramôa Ribeiro, F., Guisnet, M. Appl. Catal., A 2011, 399, 100.

[104] Ordóñez, S., Díaz, E. Basic zeolites: Structure, preparation and environmental applications. in Handbook of Zeolites: Structure, Properties and Applications, 2009, pp 51-66.

[105] Wu, P., Komatsu, T., Yashima, T. J. Chem. Soc. Faraday Trans. 1996, 92, 861.

[106] van Bokhoven, J.A., Lamberti, C. Coordin. Chem. Rev. 2014, 277-278, 275.

[107] Chakarova, K., Andonova, S., Dimitrov, L., Hadjiivanov, K. Micropor. Mesopor. Mater. 2016, $220,188$.

[108] Vimont, A., Thibault-Starzyk, F., Lavalley, J.C.J. Phys. Chem. B 2000, 104, 286.

[109] Ricchiardi, G., Damin, A., Bordiga, S., Lamberti, C., Spanò, G., Rivetti, F., Zecchina, A. J. Am. Chem. Soc. 2001, 123, 11409 .

[110] Sánchez-Sánchez, M., Blasco, T. Catal. Today 2009, 143, 293.

[111] Hattori, H. Chem. Rev. 1995, 95, 537.

[112] Siporin, S.E., McClaine, B.C., Davis, R.J. Langmuir 2003, 19, 4707.

[113] Belin, T., Mve Mfoumou, C., Mignard, S., Pouilloux, Y. Micropor. Mesopor. Mater. 2013, 182, 109.

[114] Schoonheydt, R.A., Geerlings, P., Pidko, E.A., van Santen, R.A.J. Mater. Chem. 2012, 22, 18705 .

[115] Uvarova, E.B., Kustov, L.M., Kazansky, V.B. Stud. Surf. Sci. Catal. 1995, 94, 254.

[116] Lavalley, J.C., Lamotte, J., Travert, A., Czyzniewska, J., Ziolek, M.J. Chem. Soc. Faraday Trans. 1998, 94, 331 .

[117] Karge, H.G., Raskó, J.J. Colloid Interface Sci. 1978, 64, 522.

[118] Karge, H.G., Ziółek, M., Łaniecki, M. Zeolites 1987, 7, 197.

[119] Montanari, T., Marie, O., Daturi, M., Busca, G. Appl. Catal. B 2007, 71, 216.

[120] Howarth, A.J., Peters, A.W., Vermeulen, N.A., Wang, T.C., Hupp, J.T., Farha, O.K. Chem. Mater. 2017, 29, 26.

[121] Zhou, H.J., Kitagawa, S. Chem. Soc. Rev. 2014, 43, 5415.

[122] Furukawa, H., Cordova, K.E., O'Keeffe, M., Yaghi, O.M. Science 2013, 341.

[123] Fitzgerald, S.A., Schloss, J.M., Pierce, C.J., Thompson, B., Rowsell, J.L.C., Yu, K., Schmidt, J.R. J. Phys. Chem. C 2015, 119, 5293.

[124] Chen, Y., Wang, H., Li, J., Lockard, J.V.J. Mater. Chem. A 2015, 3, 4945.

[125] Vitillo, J.G., Regli, L., Chavan, S., Ricchiardi, G., Spoto, G., Dietzel, P.D.C., Bordiga, S., Zecchina, A. J. Am. Chem. Soc. 2008, 130, 8386.

[126] Tan, K., Zuluaga, S., Gong, Q., Gao, Y., Nijem, N., Li, J., Thonhauser, T., Chabal, Y.J. Chem. Mater. 2015, 27, 2203.

[127] Valenzano, L., Civalleri, B., Chavan, S., Palomino, G.T., Areán, C.O., Bordiga, S. J. Phys. Chem. C 2010, 114, 11185 .

[128] Bhattacharjee, S., Lee, Y.-R., Puthiaraj, P., Cho, S.-M., Ahn, W.-S. Catal. Surv. Asia 2015, 19, 203.

[129] Dhakshinamoorthy, A., Alvaro, M., Garcia, H. Chem. Commun. 2012, 48, 11275.

[130] Szeto, K.C., Lillerud, K.P., Tilset, M., Bjørgen, M., Prestipino, C., Zecchina, A., Lamberti, C., Bordiga, S.J. Phys. Chem. B 2006, 110, 21509.

[131] Huang, Y.-B., Liang, J., Wang, X.-S., Cao, R. Chem. Soc. Rev. 2017, 46, 126.

[132] Xue, Z., Jiang, J., Ma, M.-G., Li, M.-F., Mu, T. ACS Sustain. Chem. Eng. 2017, 5, 2623. 
[133] Liang, Q., Zhang, M., Zhang, Z., Liu, C., Xu, S., Li, Z.J. Alloys Compd. 2017, 690, 123.

[134] Cavka, J.H., Jakobsen, S., Olsbye, U., Guillou, N., Lamberti, C., Bordiga, S., Lillerud, K.P. J. Am. Chem. Soc. 2008, 130, 13850 .

[135] Shearer, G.C., Forselv, S., Chavan, S., Bordiga, S., Mathisen, K., Bjørgen, M., Svelle, S., Lillerud, K.P. Top. Catal. 2013, 56, 770.

[136] Morterra, C., Bolis, V., Magnacca, G.J. Chem. Soc. Faraday Trans. 1996, 92, 1991.

[137] Chakarova, K., Hadjiivanov, K. Micropor. Mesopor. Mater. 2013, 177, 59.

[138] Cairon, O., Chevreau, T., Lavalley, J.C.J. Chem. Soc. Faraday Trans. 1998, 94, 3039.

[139] Geobaldo, F., Lamberti, C., Ricchiardi, G., Bordiga, S., Zecchina, A., Palomino, G.T., Arean, C.O.J. Phys. Chem. 1995, 99, 11167.

[140] Zhang, Y., Lucier, B.E.G., Terskikh, V.V., Zheng, R., Huang, Y. Solid State Nucl. Magn. Reson. $2017,84,118$.

[141] Wack, J., Siegel, R., Ahnfeldt, T., Stock, N., Mafra, L., Senker, J.J. Phys. Chem. C 2013, 117, 19991.

[142] Loiseau, T., Serre, C., Huguenard, C., Fink, G., Taulelle, F., Henry, M., Bataille, T., Férey, G. Chem. Eur. J. 2004, 10, 1373.

[143] Mihaylov, M., Andonova, S., Chakarova, K., Vimont, A., Ivanova, E., Drenchev, N., Hadjiivanov, K. Phys. Chem. Chem. Phys. 2015, 17, 24304.

[144] Ravon, U., Chaplais, G., Chizallet, C., Seyyedi, B., Bonino, F., Bordiga, S., Bats, N., Farrusseng, D. ChemCatChem 2010, 2, 1235 .

[145] Nouar, F., Devic, T., Chevreau, H., Guillou, N., Gibson, E., Clet, G., Daturi, M., Vimont, A., Greneche, J.M., Breeze, M.I., Walton, R.I., Llewellyn, P.L., Serre, C. Chem. Commun. 2012, 48, 10237 . 



\title{
4. Oil Refining and Petrochemistry: Use of Zeolites and Opportunities for MOFs
}

\author{
María Ángeles Romero, Jesús Lázaro and Juana Frontela \\ Cepsa Research Center, Avenida Punto Com, $\mathrm{n}^{\circ}$ 1, 28805 Alcalá de \\ Henares (Madrid), Spain.
}

Keywords: Refining, petrochemical, oil\&gas, process, adsorption, catalysis, shape selectivity

\section{Introduction}

Compañía Española de Petróleos S.A.U. (Cepsa) is an integrated energy service company that is active along the entire petroleum value chain. Cepsa performs commercial activities in the field of exploration and production of crude petroleum, refinery, petrochemical processes, gas and electricity, and is also present in the distribution and commercialization of all its products. Cepsa has actively participated in the energy service sector since 1929, when it was constituted as the first privately owned petroleum company in Spain. Since then, it has focused on research activities related to production processes, as well as developing innovative products. In August 2011, the International Petroleum Investment Company (IPIC), an investment group established by the Abu Dhabi government in 1984 - which had been a stakeholder for 26 years - completely acquired Cepsa. At present, Cepsa has a worldwide international presence in Europe, America, Africa and Asia, and has over 10,700 employees.

Cepsa refining activity is centred in Spain, where we own three refineries that account for $34 \%$ of our total refining capacity of 27 million tons per year. Our refining operation is presently concentrated in three refineries focused on delivering fuel and petrochemical commodities, as well as a fourth one (co-owned at $50 \%$ ), which, produces asphalts. On the other hand, our petrochemical business is based on aromatics. Most of Cepsa's chemical products start from BTX (Benzene, Toluene and Xylenes). Cepsa carries out the basic petrochemicals operations at the refineries, obtaining raw materials, intermediate and final products. These have a multitude of 
uses. For instance, the raw materials are used to produce detergents, polyesters, resins, electronic components, insecticides, synthetic fibres, and pharmaceutical products.

In this chapter, we will survey the roles that zeolites and metal-organic frameworks have in these sectors today and the ones they could play in the years to come, from our viewpoint of a research-intensive industrial company. Zeolites, as analysed in previous chapters, are defined as crystalline aluminosilicates with porous structure and ion exchange capability whilst they have been widely used as adsorbents and catalysts in the refining and petrochemical industry for the last 5 o years. The international zeolite market was assessed at around USD 3.5 billion and a volume over $3000 \mathrm{kt}$. The compound annual growth rate (CAGR) is predicted to grow at around $3.5 \%$ from 2015 to 2020 [1-3]. Metal-organic frameworks (MOFs) are also crystalline porous materials, but their three-dimensional framework is composed of metal ions (clusters) linked to multidentate organic molecules. Thus, they are coordination polymers. MOFs can have very high specific surface areas, up to $5900 \mathrm{~m}^{2} \mathrm{~g}^{-1}$, and specific volumes, up to $2 \mathrm{~cm}^{3} \mathrm{~g}^{-1}\left[3^{-4}\right]$.

Most of the refining and petrochemical processes require a high temperature at several steps due to thermodynamic or kinetic limitations. Some processes, like cracking, hydrotreating, etc., operate at temperatures higher than $400{ }^{\circ} \mathrm{C}$. This high severity prevents the extensive use of MOFs in such operations due to their limited thermal stability. Moreover, several streams of the refining and petrochemical industry are highly polar, and MOFs are not as stable as desired either. Acid and polluted streams from industrial operations need to be treated or purified for downstream units or to fulfil legal specifications. In these cases, chemical stability is a limitation for the industrial application of MOFs, too [3-6]. As a result, there are no important industrial processes based on MOFs in the current refining and petrochemical industry at present. Zeolites, however, are extensively used in many applications in this industry, as demonstrated in the following sections. These are divided into adsorbents, refining catalysts, and petrochemical catalysts. Table 1 at the end of the chapter provides an overview of the different catalytic refining and petrochemical processes in which zeolites participate.

\section{Zeolites as adsorbents in the refining and petrochemical industry}

The use of zeolites as adsorbents in the refining and petrochemical industry is mainly linked to the production of lineal paraffins and aromatics, in 
which shape selectivity plays a key role [7]. The operating temperature for adsorption processes is normally below $200^{\circ} \mathrm{c}$. Adsorption processes in the refining and petrochemical industry are made possible by the development of adsorbents exhibiting very specific selectivity, in particular, synthetic zeolites. Thus, shape selectivity of zeolites becomes crucial in many separation steps. Adsorption is usually applied in two cases: in the ultrapurification of feed to other processes, and in the fractionating of mixtures that are very difficult to separate by distillation [8].

\subsection{Linear paraffin production}

One of the most widely used tensioactives in the detergent industry is the LABSA (sulphonated linear alkylbenzene). This LABSA precursor, the linear alkylbenzene or $\mathrm{LAB}$, is produced by alkylation of benzene with long chain olefins $\left(\mathrm{C}_{9}\right.$ to $\left.\mathrm{C}_{14}\right)$. LAB's current global demand exceeds $3000 \mathrm{kt}$ per year. These olefins are produced through dehydrogenation of linear paraffins from kerosene. Straight run kerosene contains not only linear paraffins but also isoparaffins, naphthenes, and aromatics. After a hydrotreating step to remove impurities, kerosene is sent to a separation process. This step uses a $5 \mathrm{~A}$ zeolite molecular sieve to produce a kerosene raffinate with very few linear paraffins and a kerosene extract containing mostly linear paraffins.

In 1945, Barrer et al. discovered the adsorption capacity of linear paraffins over natural zeolites. They conducted a deep study on molecule sizes and operating conditions of the adsorption phenomena. Since then, the importance of the use of zeolites as adsorbents in separations processes has continued to grown until today [9].

There are two industrial processes employing molecular sieves: Molex ${ }^{\mathrm{TM}}$ (UOP) and Eluxyl ${ }^{\mathrm{TM}}$ (IFP) [8]. The world global demand of normal paraffins in 2016 exceeded $3000 \mathrm{kt}$ and it is expected to increase growth by about $4 \%$ in the coming years. Molex ${ }^{\mathrm{TM}}$ is the process most used for this application. It was developed in the sixties by Universal Oil Products (UOP). The process consists of three steps: feed pretreatment, adsorption, and $n$-paraffin recovery. During the feed pretreatment, a hydrotreatment process takes place in order to remove mainly sulphur, nitrogen, olefins and oxygen from the kerosene, which are poisons to the molecular sieve. The adsorption step mimics a countercurrent flow between the feed and the $n$-paraffins, while the zeolite remains packed (simulated moving bed). This simulation occurs thanks to a rotary valve that feeds kerosene and desorbent and yields raffinate and extract at different points in the adsorption column. Eluxyl ${ }^{\mathrm{TM}}$ is 
also based on a simulated moving bed but it employs several valves instead of the rotary valve of the UOP system [8].

\subsection{Xylenes production: $m$-xylene and $p$-xylene}

There is another application of zeolites in separation processes closely related to the $n$-paraffins separation process. In this case, the desired molecule is the $p$-xylene, which is the raw material for the production of PET (polyethylene terephthalate). Shape selectivity in this case allows the separation of $p$-xylene from a mixture of $p$-, $o$ - and, $m$-xylenes.

In 1964 Eberly and Arey used 13X molecular sieves for xylenes separation [10]. In 1981 Rosback proposed the used of Ba- and K-faujasite type zeolites for the same process [11]. In 1969, UOP launched the Parex ${ }^{\mathrm{TM}}$ process, which consists of a simulated moving bed, while in 1997 IFP introduced the Eluxy $^{\mathrm{TM}}$ technology for this same application [8]. UOP has introduced MX Sorbex $^{\mathrm{TM}}$ for the separation of $m$-xylene, a process very similar to Parex ${ }^{\mathrm{TM}}$ but with a different molecular sieve, more selective towards adsorption of $m$-xylene.

\subsection{Olefins production}

Zeolites exhibit an adsorption selectivity towards olefins over paraffins of approximately 10:1. Based on this, there are several industrial applications with this objective $[8,12-13]$ :

- UOP Olex Process: for separation of $\mathrm{C}_{10}-\mathrm{C}_{14}$ olefins on a modified X zeolite

- UOP Sorbutene process: for 1-butene separation from a $\mathrm{C}_{4}$ cut

- Union Carbide Olefinsiv process: for separation of $n-\mathrm{C}_{4} /$ isobutene on a 5A zeolite

\subsection{Liquid and gas purification}

Other applications of zeolites in the petrochemical industry are related to stream purification by adsorption, as in the LAB production for detergents. Along the LAB production scheme, the $n$-paraffins obtained through the Molex $^{\mathrm{TM}}$ process are dehydrogenated to produce $n$-olefins. This is done in the UOP Pacol ${ }^{\mathrm{TM}}$ process (paraffin conversion to olefins), which produces 
not only olefins but also a small amount of undesired alkylaromatics as these can be dialkylated in subsequent steps producing a further loss of yields. UOP PEP ${ }^{\mathrm{TM}}$ (Pacol $^{\mathrm{TM}}$ Enhanced Process) uses an adsorbent to remove these aromatics [14].

Zeolites are also used in the refining and petrochemical industry for PSA (pressure swing adsorption). This technology is widely used for hydrogen $\left(\mathrm{H}_{2}\right)$ purification. Hydrogen is produced and consumed in many processes in the oil and gas industry. Depending on the process, several contaminants can be present along with $\mathrm{H}_{2}$, such as $\mathrm{N}_{2}, \mathrm{CH}_{4}, \mathrm{C}_{2} \mathrm{H}_{6}, \mathrm{CO}$, $\mathrm{CO}_{2}, \mathrm{H}_{2} \mathrm{~S}, \mathrm{H}_{2} \mathrm{O}$ and $\mathrm{NH}_{3}$. When $\mathrm{H}_{2}$ purity is over 60 vol.\%, PSA is the best option for $\mathrm{H}_{2}$ purification compared to cryogenic or gaseous permeation processes. PSA technology is based on the use of activated carbon and/or zeolitic molecular sieve and alternating pressure to effect the $\mathrm{H}_{2}$ separation from the impurities [8].

\section{Zeolites as catalysts in the refining industry}

Current refining processes have three main objectives:

- Separateing the lighter and more valuable fractions (gasoline, kerosene, diesel) from the crude oil.

- Converting the remaining heavy fractions into lighter ones through conversion processes. These processes require severe conditions (temperature and/or pressure).

- Fine-tuning the obtained light fractions in order to fulfil the legal requirements for fuels and petrochemical needs.

As shown in Figure 1, zeolites are key elements of refinery operations due to their excellent properties for the selective chemical conversion of several hydrocarbons families into more valuable ones.

\subsection{Fluid catalytic cracking}

Cracking units are used to convert vacuum gasoil and other low valuable cuts into more valuable ones such as gasoline, jet fuel or diesel. The fluid catalytic cracking (FCC) process involves a series of simultaneous reactions, both exothermic and endothermic. Nevertheless, the overall process is endothermic and therefore requires a continuous energy supply in order to reach reaction temperatures above $45^{\circ}{ }^{\circ} \mathrm{C}\left[15^{-16}\right]$. Catalytic cracking is 


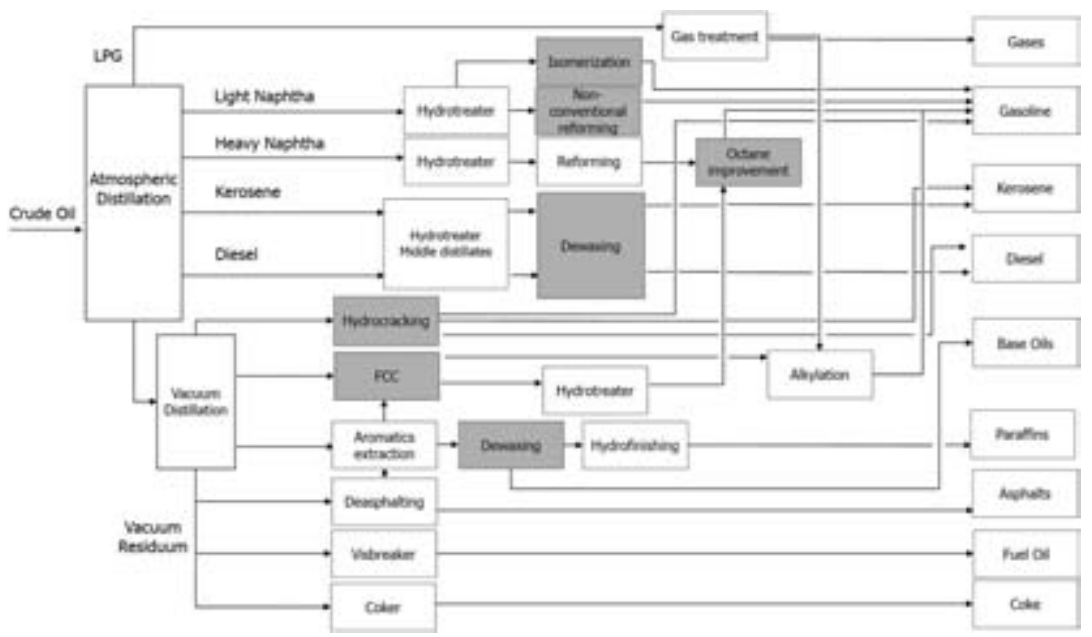

Figure 1. Refinery scheme (zeolite-based processes are highlighted).

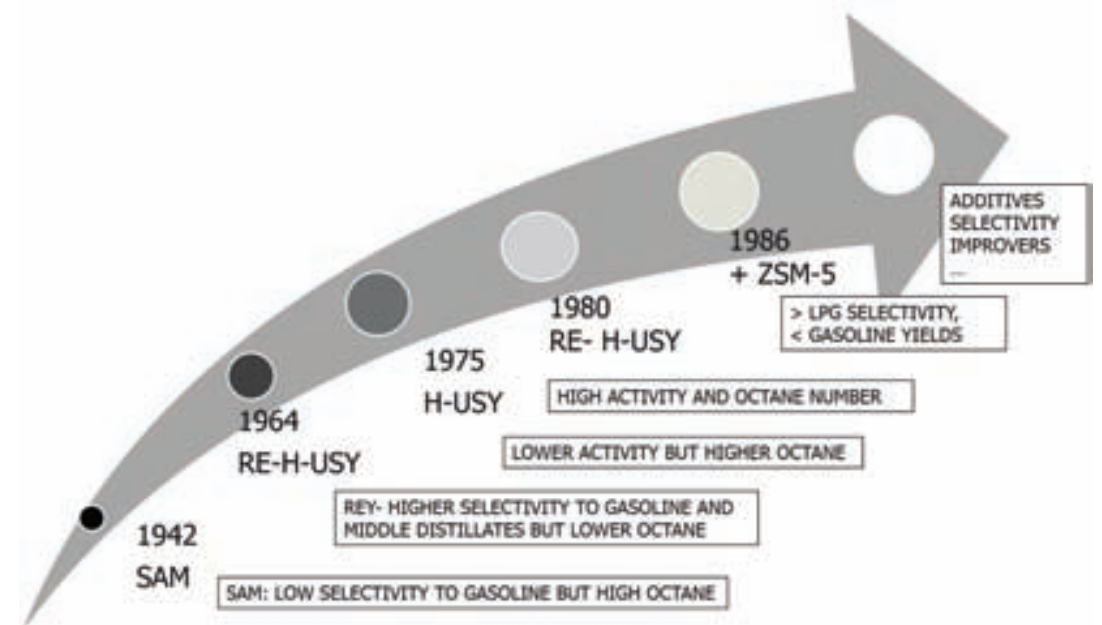

Figure 2. The evolution of FCC catalysts [15-19].

the second-largest zeolite consumer, representing more than $95 \%$ of the total volume of zeolites used as catalysts. The highest consumption of zeolites is as detergent builders, which accounts for more than two thirds of the total market [17].

Regarding the catalysts used in the FCC, Figure 2 shows its historic evolution. Since the first developments, when amorphous catalysts were used 
(selective active matrix or SAM), to the 6os, when Mobil invented a spraydried catalyst based on ultra-stable Y zeolite (USY), there has been a clear evolution aligned with product requirements. Since then, zeolite Y with different modifications and additives has been the base of FCC catalyst. Nowadays, the incorporation of ZSM- 5 into the catalyst in order to increase light olefin yields and to boost gasoline octane is widespread [15-19].

The Resid FCC process (RFCC) converts heavier feedstock compared to the conventional FCC, with a residue content between 1 to $6 \mathrm{wt} . \%$. This feedstock contains more organic nitrogen and organic metals (vanadium, iron, nickel, sodium and calcium) than those of the conventional FCC. The two most common technologies are Axens RFCC $\left(\mathrm{R}_{2} \mathrm{R}^{\mathrm{TM}}\right.$ technology) and UOP RFCC. In both cases, a two-stage catalyst regeneration is used in order to minimize catalyst deactivation due to vanadium [15]. The USY requires a balanced concentration of acid sites of proper acid strength in order to minimize coke formation and maximize yields [18].

There are several commercial suppliers which commercialize FCC catalysts, such as GRACE, BASF, Albemarle, SINOPEC and Rive in partnership with GRACE. A review of the latest patents demonstrates that relevant research is developing in this area, in particular in catalyst additives [20] and new zeolite preparation methods for improved stability and mesoporosity $[21]$.

\subsection{Hydrocracking}

The hydrocracking process allows the transformation of certain heavy streams (vacuum gas oil, deasphalted oils (DAO), coker gas oil) and lowvalue aromatic streams (such as light cycle oil (LCO) from FCC unit and residues from the vacuum tower) into lighter and higher added value products (such as gasoline or diesel) through a catalytic cracking process in the presence of hydrogen. The reaction system requires high pressures between $80-200$ bar and temperatures between $35^{\circ}-45^{\circ}{ }^{\circ} \mathrm{C}$ [19]. There are several possible configurations for the hydrocracking process comprising one or two catalytic fixed beds, depending on the conversion requirements. The general scheme is shown in Figure 3.

In both configurations, there is a pretreatment step to remove metals, heterocompounds (sulphur, nitrogen, oxygenates), and some aromatics. The main catalyst for the first reactor is usually nickel-molybdenum supported on alumina $\left(\mathrm{NiMo} / \mathrm{Al}_{2} \mathrm{O}_{3}\right)$. The second step, in which the cracking process takes place, uses an acid catalyst. Several reactions to the same 


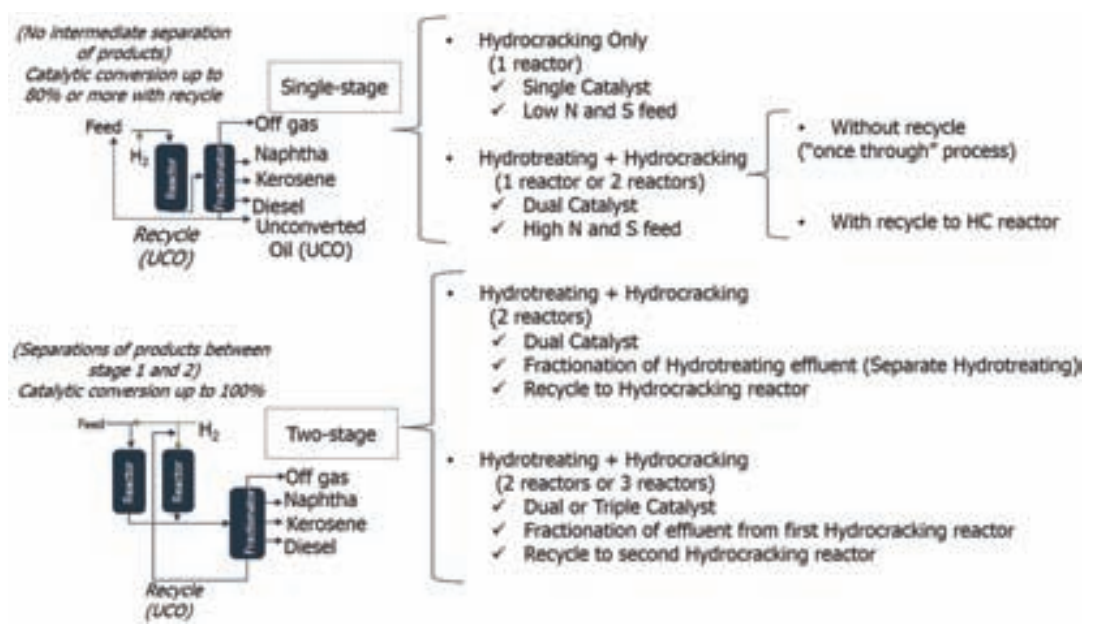

Figure 3. Hydrocracker configurations [22].

catalyst occur at the same time (aromatics hydrogenation, ring opening and isomerization).

The first hydrocracking supports were amorphous silica-alumina (ASA). Subsequently, Y zeolite-based catalyst was developed. Zeolites show higher cracking activity but lower $\mathrm{NH}_{3}$ and organic nitrogen tolerance. The zeolite can be exchanged with rare earth or dealuminated. The catalyst is bifunctional, containing a zeolite-based acid function and a metallic phase. Depending on the situation different metals can be used: NiW mixed sulphides provide hydrogenation activity, NiMo mixed sulphides favour nitrogen removal. Even, in some two-stage configurations, noble metals such as platinum or palladium are used if sulphur concentrations are low $[19,23]$. The modification of a Y zeolite via dealumination has a marked influence on the product's distribution obtained from hydrocracking. The reduction of the unit cell size and the modification of the strength of the acid centres and their distribution improves the selectivity to middle distillates $[22,24]$.

In addition to the standard hydrocracking process, there are two different variations:

- Mild hydrocracking (MHC), which works under much less severe conditions than hydrocracking (30-70 bar, $35^{\circ}-440^{\circ} \mathrm{C}$ ) and is used to convert vacuum gas oil into medium distillates and low sulphur fuel [25]. Depending on the nitrogen content of the feedstock, loading amorphous or zeolite-based catalysts may not provide a gain of conversion compared to the conventional Co-Mo supported on alumina. 
- Resid hydrocracker for vacuum residue gas oils (boiling point $>560^{\circ} \mathrm{C}$ ), which works at maximum pressures of 200 bar and temperatures up to $450^{\circ} \mathrm{C}$. Y zeolite can be used for this purpose. There are several industrial technologies [26]:

- Fixed bed processes licensed by Chevron Lummus Global, UOP, Axens and ExxonMobil.

- Ebullated bed licensed by Axens/IFP (H-Oil $\left.{ }^{\mathrm{TM}}\right)$ and Chevron Lummus Global (LC-Fining ${ }^{\mathrm{TM}}$ and LC-MAX ${ }^{\mathrm{TM}}$ ).

- Hybrid/moving bed licensed by Chevron Lummus Global (called On Stream Catalyst Replacement, OCR) and Shell technology.

Depending on each case the active phase can be Mo, Co-Mo, Ni-Mo or Ni-V. The catalysts have a large specific surface area with a low metal retention capacity and low acidity.

Nowadays the main suppliers that commercialize hydrocracking catalysts are UOP, Criterion, ART-Grace, Albemarle, Haldor-Topsoe, Axens, and SINOPEC.

\subsection{Linear paraffin isomerization}

The stream commonly called "light naphtha" comes from the atmospheric distillation tower and is composed of linear and branched hydrocarbons with five and six carbon atoms. The isomerization of this stream is intended to maximize the octane number by increasing its branching degree. This isomerized stream is then added to the gasoline pool $[15,27]$.

The catalysts used in this process are chlorinated aluminas, zeolites, and sulphated zirconias. Chlorinated aluminas work at a lower temperature $\left(180^{\circ} \mathrm{C}\right)$ than zeolites $\left(250^{\circ} \mathrm{C}\right)$ because of their higher acidity. However, the need for a continuous chlorine input to maintain activity produces an effluent with small amounts of $\mathrm{HCl}$, which results in corrosion problems. In this regard, chlorinated aluminas also have low water tolerance. As for zeolites, the most commonly used is Mordenite, which is dealuminated to obtain the required acidity. It is also impregnated with a noble metal (mainly platinum), which prevents coke formation by hydrogenating coke precursors. The first zeolitic process was developed by Shell in the 1960s under the name of Hysomer using Pt-Mordenite as a catalyst. Subsequently, UOP introduced the Penex ${ }^{\mathrm{TM}}$ process, similar to the previous one, in which the isomerization was integrated with a separation over a molecular 
sieve and subsequent recycling [15]. In the late 1980s, Cepsa developed its own version of the isomerization catalyst, which Süd-Chemie commercializes under the name Hysopar. This catalyst is based on a Mordenite with better sulphur and coke resistance than the conventional Mordenite [27-29].

Subsequently, Süd-Chemie launched a new catalyst version, called HYSOPAR-SA (super acid SA). The introduction of superacid catalysts based on sulphated zirconias, which allow working at lower temperatures (around $60^{\circ} \mathrm{C}$ ), suggests that zeolites could be displaced by this type of catalysts if zirconia resistance to poisons like water and sulphur is improved in the future [28]. There are other suppliers that commercialize these catalysts, namely Shell and UOP.

\subsection{Post-treatment of reformate}

The octane number of naphtha (from six to ten carbon atoms) produced in the reforming process can be further increased by converting the residual linear paraffins (with very low octane numbers) to aromatics and branched alkanes through a zeolite-based hydrocracking process. ZSM-5 catalysts are preferred because their shape selectivity only allows linear paraffins to access their active sites.

In the 1960s Mobil introduced the Selectoforming process, using an erionite-based catalyst, which was modified in the 70 os by the M-forming process, employing a more shape selective $\mathrm{ZSM}-5$ type zeolite. Gulf also commercialized a similar process using Ferrierite as a catalyst. The commercial success of these processes is limited by the loss of gasoline yield compared to the octane gain and the by the low added value of the products. After that, Mobil developed a process named Mobil Reformate Upgrading (MRU), in which the zeolite is located at the bottom of the last fixed-bed reactor of the reforming process. In 1996 ExxonMobil commercialized a process based on the old Selectoforming called BTXtra ${ }^{\mathrm{TM}}$, in which zeolite is used in the last reactor of a semi-regenerative catalytic reforming plant to increase the production of toluene and xylenes $[15,24,27-28,30]$.

\subsection{Naphtha post-treatment}

Heavy stream naphtha produced in the FCC unit requires a deep hydrotreating step in order to fulfil sulphur requirements while minimizing 
the loss of the gasoline octane number and avoiding olefin hydrogenation. The current industrial processes include: Axens Prime $\mathrm{G}^{\mathrm{TM}}{ }^{\mathrm{T}}$, ExxonMobil SCANfining $^{\mathrm{TM}}$, CD Tech CD Hydro ${ }^{\mathrm{TM}}$ and CD HDS $^{\mathrm{TM}}$, UOP ISAL ${ }^{\mathrm{TM}}$, ExxonMobil OCTgain $^{\mathrm{TM}}$, and Phillips S Zorb SRT ${ }^{\mathrm{TM}}$. Although almost all these processes use catalysts based on Ni or Co-Mo on alumina, ISAL ${ }^{\mathrm{TM}}$ and OCTgain $^{\mathrm{TM}}$ use zeolite due to its reactant shape selectivity. In the case of the ISAL ${ }^{\mathrm{TM}}$ process, it uses a CoMo- $\mathrm{P} / \mathrm{Al}_{2} \mathrm{O}_{3}$ associated to a $\mathrm{Ga}-\mathrm{Cr} / \mathrm{HZSM}-5$ zeolite $[28,31-32]$.

\subsection{Non-conventional reforming}

Conventional catalytic reforming process has limitations in the aromatization of molecules with six and seven carbon atoms. Other catalysts based on zeolites appear to cover these deficiencies. Zeolite $\mathrm{L}$ exchanged with $\mathrm{Ba}$ and $\mathrm{K}$ and impregnated with Pt shows a very high selectivity to aromatics compared to the conventional reforming catalyst (Pt-Re on chlorinated alumina), although it exhibits extreme sensitivity to sulphur in the feed [33]. The current industrial process' that use L zeolite are Chevron Aromax and UOP RZ-Platforming ${ }^{\mathrm{TM}}$, both operating at temperatures up to around $500{ }^{\circ} \mathrm{C}[34-35]$.

\subsection{Dewaxing and isodewaxing of middle distillates and lubricants}

Dewaxing and isodewaxing processes can be applied to both medium distillates (kerosene and diesel) and lubricants. These processes operate at high temperatures $\left(28 \mathrm{O}-400{ }^{\circ} \mathrm{C}\right)$ and pressures $(2 \mathrm{O}-110 \mathrm{bar})$. They are intended to improve "cold properties" such as pour point, cloud point, cold filter clogging point, etc. by eliminating or modifying linear paraffins. This dewaxing process can be carried out in several ways: extraction with solvents, selective cracking of paraffins or isomerization of paraffins. In the case of distillates, the latter two are most commonly used [1].

ZSM-5 is the catalyst most widely used in dewaxing by catalytic cracking. It was introduced by Mobil (MDDW ${ }^{\mathrm{TM}}$, Mobil Distillate Dewaxing) in 1978. In the late 1980s, Akzo-Fina improved the process by adding a desulphurising function to the ZSM-5. In 1992, an alliance between Mobil, Akzo and Kellogg, (MAK) improved desulphurization catalyst properties in a new process called MDDW-CFI ${ }^{\mathrm{TM}}$ (Mobil Distillate Dewaxing Process). This process is able to treat middle distillate feedstock with 


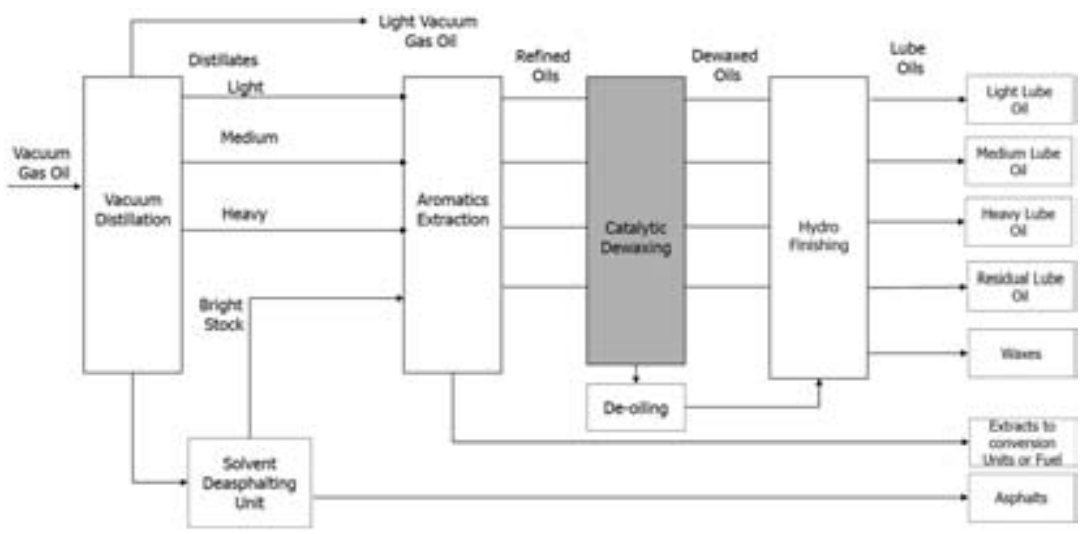

Figure 4. Lube oils production scheme (zeolite-based processes are highlighted) [37].

higher $n$-paraffins content in order to produce better quality kerosene $[1,28,36]$.

Dewaxing by isomerization employs a bifunctional catalyst of a metal supported on a ZSM-5 zeolite or SAPO-11. The first process of this type was introduced in 1990 and commercially tested by ExxonMobil in 1996 (MIDW-Mobil Isomerization Dewaxing) to produce low pour point and very low sulphur diesel. The use of Beta zeolite in a first step followed by a medium pore zeolite (like zeolite ZSM-22 or ZSM-23) is described for this process with benefits in shape selectivity for linear paraffins [28].

As for dewaxing and isodewaxing of lubricants, the process scheme is illustrated in Figure 4. The different cuts of vacuum gas oil from the vacuum distillation tower are subjected to a furfural extraction process to extract the aromatics. The raffinate, from which the aromatics have been removed, requires a dewaxing or isodewaxing process to improve its cold flow properties. Subsequently, the dewaxed stream can be treated in the hydrofinishing unit to improve the colour (removal of aromatics) and remove sulphur [37].

Catalytic dewaxing for lube oils uses the following zeolites $[1,28,36-39]$ :

- British Petroleum (BP) developed the first catalytic dewaxing process in 1972. The catalyst was Pt on Mordenite. However, the relatively large pore size of this zeolite also caused cracking on some of the isoparaffins, causing a bad viscosity index (VI) in the final lubricant.

- In the 1980s Mobil introduced the most widely used lubricant dewaxing process: MLDWTM (Mobil Lube Dewaxing). This process is based on a modified ZSM-5 that presents a greater selectivity to the cracking of 
linear paraffins. However, it also presents some problems regarding the VI when it is compared to products obtained by solvent dewaxing.

Catalytic dewaxing by isomerization for lube oils uses the following zeolites $[1,28,36-39]$ :

- Exxon Mobil has a lubricant dewaxing process MSDWTM (Mobil Selective Dewaxing) based on a medium pore zeolite with a metal function that compensates the cracking activity of the zeolite (for example Pt on SAPO-11).

- Chevron developed its process using SAPO-11 zeolite. The onedimensional nature of SAPO-11 and its pore size makes it very selective in producing highly branched products so the pour point is also improved.

\section{Zeolites as catalysts in the petrochemical industry}

The use of zeolites as catalysts in petrochemistry is closely connected to aromatic hydrocarbons. Many schemes have been proposed to optimise the production of aromatics depending on the products that one wants to maximize. Some technology licensors offer comprehensive solutions known as the 'xylenes loop', in which technologies from several companies can be combined. ParamaX ${ }^{\mathrm{TM}}$ from Axens is an example of this [40]. Figure 5 shows a possible scheme of a petrochemical complex, highlighting those processes that use zeolites.

Commercial sources of aromatics include catalytic reforming, pyrolysis gasoline, and coal pyrolysis. In crude oil refining, reforming naphtha

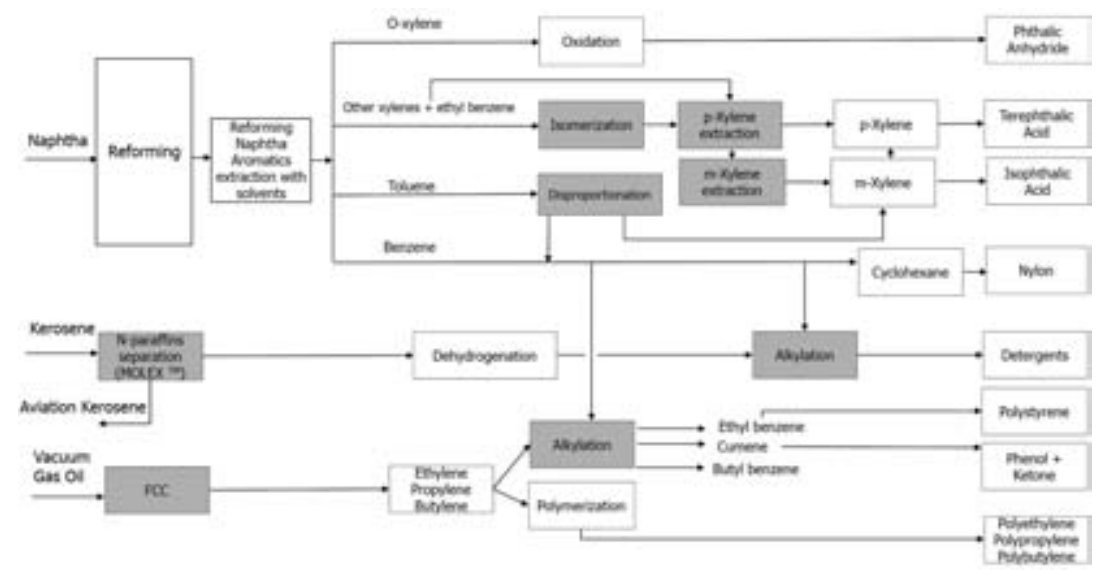

Figure 5. Petrochemical scheme (zeolite-based processes are highlighted). 
or reformate is the product obtained from the catalytic reforming process, in which a low octane naphtha is converted to high octane aromatics including benzene, toluene and xylenes. The amount of aromatics contained in the reforming naphtha is typically in the range of 18 to $33 \mathrm{vol} . \%$. Pyrolysis gasoline is a co-product of steam cracking of a hydrocarbon feed, whereas light oil from coal pyrolysis is a co-product of the manufacture of coke for the steel industry, and both processes produce certain amounts of aromatics.

\subsection{Benzene alkylation with light olefins: cumene and ethylbenzene}

Approximately $90 \%$ of all ethylbenzene consumed in the styrenepolystyrene industry is produced by the alkylation of benzene with ethylene. This reaction is carried out by acid catalysis over $\mathrm{AlCl}_{3}, \mathrm{BF}_{3} / \mathrm{Al}_{2} \mathrm{O}_{3}$ or zeolites. The usual temperature range for these reactions is between 100$180{ }^{\circ} \mathrm{C}$. The Mobil-Badger process, marketed in the 1970s, was the first commercial process that employed zeolite $\left(\mathrm{ZSM}_{-5}\right)$ as a catalyst. The shape selectivity of the zeolite allowed a reduction of coke formation by condensation and hydrogen transfer reactions (transition state shape selectivity). Substantial amounts of diethylbenzene were produced in the process. These were recycled to the reactor and converted to ethylbenzene via transalkylation with benzene. The process had an overall yield of $99 \%$ to ethylbenzene. The Mobil-Badger EBMax (1995) process employs a zeolite, which produces fewer polyalkylates and oligomers, thanks to its particular porous system, which is capable of carrying out the alkylation reaction in liquid phase, thanks to its higher intrinsic activity [41]. Lummus/UOP has developed the EBone process, also a liquid phase alkylation process using a zeolite catalyst, which according to the supplier, can work during long cycles [42].

The alkylation of benzene with propylene yields cumene (isopropylbenzene), is another important product in petrochemistry as it is the precursor of phenol. For this process, larger pore zeolites such as Mordenite, Beta or HY are usually used, since the narrower ZSM- 5 leads to higher yields of undesired $n$-propylbenzene. The process developed by Mobil/Badger has also been applied to the cumene process and is actually the most commonly used today. UOP developed the QMax ${ }^{\mathrm{TM}}$ process using an internally developed zeolitic catalyst, which allows (always according to the manufacturer) high cumene yields and a longer lifetime [43]. 
Other reactions related to the alkylation of aromatics with short olefins using proprietary zeolites are the alkylation of toluene with methanol to yield xylenes [44], and the synthesis of $p$-ethyltoluene in the production of $p$-methylstyrene [45].

\subsection{Benzene alkylation with heavy olefins: linear alkylbenzene (LAB)}

Long chain alkylaromatic compounds are used as raw materials in many industrial fields. Among the most important applications is the production of surfactants for detergency and enhanced oil recovery. The usual process to produce these compounds in industry is the dehydrogenation of linear paraffins in the range $\mathrm{C}_{9}$ to $\mathrm{C}_{16}$ (see section 2.1) and the alkylation with benzene of these olefins. Linear alkylbenzene sulphonate (LABSA) is produced by sulphonation of the linear alkylbenzene (LAB) and subsequent neutralization of the corresponding sulphonic acids. The range of temperatures for the alkylation process is around $120-170{ }^{\circ} \mathrm{C}$. For the alkylation reaction, acid catalysts such as hydrofluoric acid are usually employed [46]. The use of hydrofluoric acid has the disadvantage of being a corrosive, difficult to handle with a lot of health and environment concerns.

Solid catalysts such as fluorinated silica-alumina and zeolites have been successfully developed in recent decades to replace hydrofluoric acid. Regarding zeolites, the use of zeolite Y, Beta, ZSM-5, ZSM-18, ZSM-2O, Mordenite and offretite have been described as valid for this process. In practice, when the aromatic compound is alkylated over acid catalysts, undesired dialkylated products are formed in addition to the monoalkylated species, especially when the reaction proceeds at a high conversion and low benzene/olefin ratio. Thanks to the shape selectivity of the zeolites, the formation of these compounds is greatly minimised. This effect is very beneficial since decreasing the production of by-products greatly improves the economy of the process at the same time. Regarding the use of zeolite Y, it is possible to modify its acidic properties and stability by an exchange with rare earth cations ( $\mathrm{La}, \mathrm{Ce}, \mathrm{Nd}, \mathrm{Pr}$ ) giving rise to zeolites called rare earth $\mathrm{Y}$ zeolite (REY) which have shown their ability to work in this reaction at very low temperatures [46-47].

The key point of this process is to control the amount of 2-phenyl isomer in the final alkylate. Depending on the zeolite type it is possible to obtain different percentages of this isomer in the product. Large-pore zeolites with moderate shape selectivity allow the production of LABs with a 
low 2-phenyl content, similar to the distribution from a non-selective catalyst such as HF. However, zeolites with monodimensional channels such as Mordenite allow production of alkylates with a high amount of this isomer, thus increasing versatility in the process. The combination of these zeolites allows the desired amount of the isomer to be obtained [45].

The DETAL ${ }^{\mathrm{TM}}$ process launched by Cepsa and UOP in 1995 supposed a breakthrough in the state of the art, being the first benzene alkylation process to produce LAB with a solid bed configuration [48-49]. As an upgrade to this process, the DETAL Plus ${ }^{\mathrm{TM}}$ process employs an improved catalyst that allows to production of fewer by-products and a lower benzene/olefin ratio [5o].

\subsection{Xylenes isomerization}

The target of this process is mainly to maximize the production of $p$-xylene by isomerizing the xylenes to thermodynamic equilibrium. This also implies in some cases the transformation of ethylbenzene (as the reforming streams and pyrolysis gasoline contain from $15-55 \%$ of ethylbenzene, which is very expensive to separate). Typical temperatures for this process are between 200 and $500{ }^{\circ} \mathrm{C}$.

Mobil launched two processes based on ZSM-5. The MVPI (Mobil Vapor Phase Isomerization), besides isomerising xylenes, converts ethylbenzene via transalkylation to benzene and diethylbenzene. The small pore diameter of the ZSM- 5 facilitates the formation of $p$-xylene, which tends to diffuse rapidly minimising disproportionation reactions of xylenes (product and transition state shape selectivity). The process MHTI (Mobil HighTemperature Isomerization) works at higher temperatures with a catalyst based on a noble metal on ZSM-5. Under these conditions, ethylbenzene is dealkylated to yield benzene and ethane. Currently, ExxonMobil offers the $\mathrm{XyMax}^{\mathrm{SM}}$ and the Advanced MHAI (Mobil High-Activity Isomerization) processes that use a double catalytic bed system to optimize conversion of ethylbenzene, cracking of non-aromatics and isomerization of $o$-xylene and $m$-xylene to $p$-xylene [51]. The UOP ISOMAR ${ }^{\mathrm{TM}}$ process is also used to convert mixtures of xylenes. Two different types of catalysts can be used, one that converts the ethylbenzene to xylenes, and another one that converts the ethylbenzene to benzene [52]. AXENS also has a new generation catalyst for the isomerization of xylenes and ethylbenzene with the name OPARIS $^{\text {TM }}$ (Optimized Aromatics Isomerization), which minimizes the production of benzene [53]. 


\subsection{Toluene disproportionation}

The purpose of this process is to convert toluene to xylenes, particularly to $p$-xylene. The processes that maximize the production of $p$-xylene normally use ZSM-5, whose selectivity is increased by partial coking or by silanization. Mobil Selective Toluene Disproportionation (MSTDP) and Mobil Toluene Paraxylene (MTP) are two examples in which the catalysts combine the catalytic activity with the diffusional characteristics of the zeolite used. The catalyst can achieve $80 \% p$-xylene selectivity, whereas a mixture in equilibrium would result in $24 \%$ [54]. ExxonMobil has recently launched the Mobil Toluene Disproportionation Process (MTDP-3). Their third generation catalyst is based on a more selective and stable ZSM-5 zeolite, which allows cycles of more than seven years. The catalyst minimizes the production of $\mathrm{C}_{9}$ aromatics, which is one of the current limitations of this process [55]. UOP proposes for this process its PXPlus $^{\mathrm{TM}}$ technology, which selectively disproportionates toluene to xylenes and benzene [56]. Typical temperatures for this process are between $35^{\circ}$ and $500{ }^{\circ} \mathrm{C}$.

\subsection{Heavy aromatics and toluene transalkylation}

When the feeds are rich in aromatics of nine or more carbon atoms (pseudocumene, durene, etc.), disproportionation of toluene to benzene and xylenes can be combined with transalkylation reactions between toluene and higher aromatics to yield xylenes. Three types of reactions take place: transalkylation of $\mathrm{C}_{9}$ with toluene, toluene disproportionation, and alkylbenzene dealkylation. Typical temperatures for this process are between $35^{\circ}$ and $500{ }^{\circ} \mathrm{C}$.

Two types of catalysts are employed in this process, those having no shape selectivity and those, which increase selectivity to $p$-xylene. The former are based on Mordenite. The use of a large pore zeolite allows the disproportionation of toluene to benzene and xylenes and also the transalkylation reactions between toluene and other alkylaromatics. The latter are based on $\mathrm{ZSM}_{-5}$, which can be used in combination with the Mordenite-based catalyst [57]. In some patents, the use of metals such as Ni or Pd is described as improving the dealkylation of the heavy aromatics $\left[5^{8}\right]$.

There are several processes used for this purpose, one example being the Toray Advanced Catalyst (TAC-9*) process. The process can work with 
mixtures of toluene and aromatics $\mathrm{C}_{9^{+}}$or with pure $\mathrm{C}_{9^{+}}$. Ethyltoluene (one of the most abundant compounds in the $\mathrm{C}_{9^{+}}$fraction) is converted to toluene by dealkylation and can be further transalkylated to give xylenes [59]. Other industrial processes for the conversion of heavy aromatics are Tatoray* launched by UOP [6o] or TransPlus ${ }^{\mathrm{SM}}$ from ExxonMobil. The latter has been developed by the Chinese Petroleum Corporation (CPC) of Taiwan and uses a catalyst developed jointly with ExxonMobil. The great challenge of this process is to increase the selectivity to xylenes and to extend the catalyst lifetime. Licensors of all these processes claim to have significantly improved these drawbacks over the previous generations of catalysts [61].

\section{Zeolites in other processes related to the refining and petrochemical industry}

This section includes some processes related to the refining and petrochemical industry. The products of these processes are raw materials for the refining and petrochemical industry or materials derived from them. In Figure 6, gas conversion processes are shown.

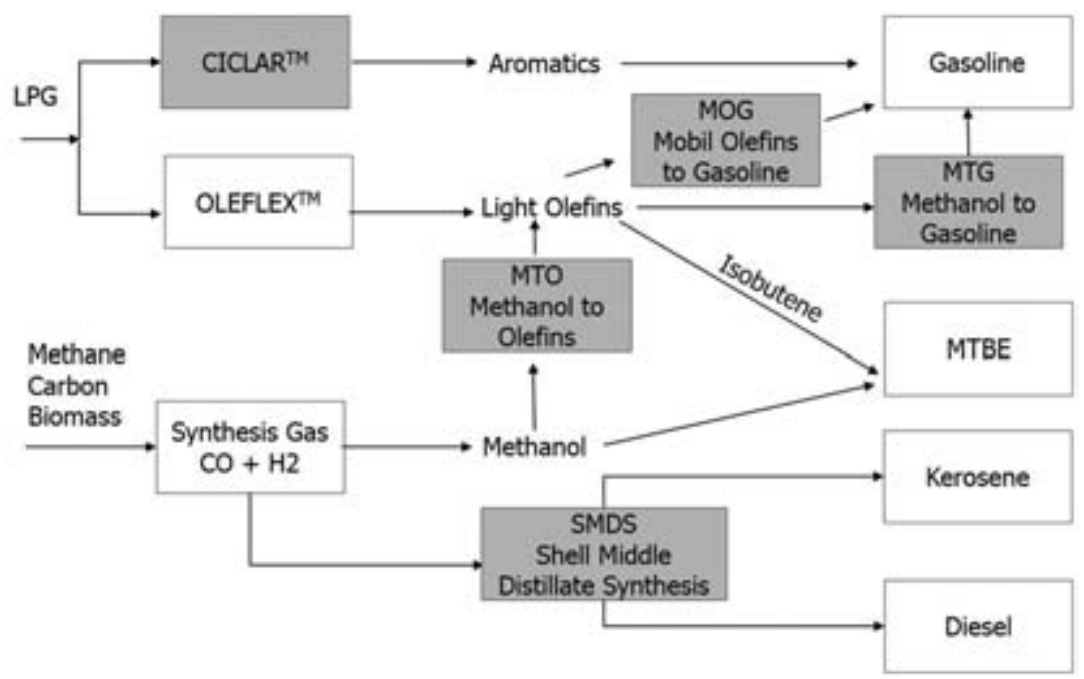

Figure 6. Gas conversion processes (zeolite-based processes are highlighted). 


\subsection{Conversion of light olefins}

Light olefin conversion to gasoline and middle distillates

Olefins from FCC, from the coker or from LPG dehydrogenation can be raw materials for gasoline production. ExxonMobil MOG process (Mobil Olefins to Gasoline) produces high octane gasoline through oligomerization, aromatization, and isomerization over ZSM-5 zeolite. Shell has developed a similar process called SPGK (Shell PolyGasoline and Kerosene) with $\mathrm{Ni}$-Mordenite for the gasoline production from $\mathrm{C}_{2}-\mathrm{C}_{5}$ olefins [62]. Ethylene, propylene and butene oligomerization are highly exothermic reactions. The reactions can be carried out with catalysts able to activate the olefin: in the homogeneous phase with metals ( $\mathrm{Ni}$ or $\mathrm{Ti}$ ) or in the heterogeneous phase with acidic supports ( such as zeolites or $\mathrm{Al}_{2} \mathrm{O}_{3}$ ). Operating temperature varies from 100 to $250^{\circ} \mathrm{C}$ in heterogeneous reactors or below $100{ }^{\circ} \mathrm{C}$ in homogeneous ones. Secondary reactions can occur on zeolites, (cracking and skeletal isomerization) and the reactivity of the different olefins increases with their molecular weight [63].

\section{Light olefin isomerization}

Isobutene is the raw material for the production of methyl tert-butyl ether (MTBE) and for poly-isobutylene. Several processes produce the skeletal isomerization of butenes to isobutylene. Mobil-BP-Kellogg developed ISOFIN $^{\mathrm{TM}}$, which uses a medium pore zeolite, while the Texaco (ISOTEX, Texaco olefin skeletal isomerization process) and the Shell processes use Ferrierite zeolite [64].

\subsection{Syngas to fuels through gas to liquids technology (GTL)}

Synthetic gas or syngas can be produced from many sources, including natural gas, coal, biomass, or virtually any hydrocarbon feedstock, by a reaction with steam or oxygen. Syngas is a crucial intermediate raw material for the production of hydrogen, ammonia, methanol, and synthetic hydrocarbon fuels. The syngas conversion to gasoline or other fuels or heavy streams constitutes the well-known Fischer-Tropsch (FT) process. The active phase is a composite of conventional catalysts and zeolites (ZSM-5 and/ or SAPO-11 and/or SAPO-12), which is used in a two-step process [65]. Shell Middle Distillates Synthesis (SMDS) is the world's first commercial project to employ gas in liquids (GTL) technology, which can be used to produce petroleum-like products, chemical products, waxes, and other materials 
from natural gas. The technology seems to have huge potential as an effective way to utilize natural gas. Mobil is another licensor that developed a two-stage slurry FT with ZSM-5 especially designed to produce high octane gasoline [66].

\subsection{Other processes}

\section{From LPG}

The most important process is the conversion of light paraffin $\left(\mathrm{C}_{3}\right.$ and $\left.\mathrm{C}_{4}\right)$ to aromatics using Ga on $\mathrm{ZSM}-5$. This $\mathrm{BP} / \mathrm{UOP}$ process is called CYCLAR ${ }^{\mathrm{TM}}$. The resulting gasoline has high octane due to the high benzene, toluene and xylenes (BTX) content [67].

Methanol to olefins (MTO) and to gasoline (MTG)

Methanol is produced from syngas, oil, coal or, increasingly, biomass. The methanol to hydrocarbons process was discovered by Mobil in 1977. This process is used to convert methanol to products such as olefins and gasoline. In the methanol to olefins (MTO) process, methanol is converted to olefins such as ethylene and propylene. The main catalysts applied are $\mathrm{ZSM}_{-5} 5$ and SAPO-34. The light olefins obtained can be used to produce polyolefins, which constitute many plastic materials. ZSM- 5 is used at temperatures of around $400^{\circ} \mathrm{C}$ in the methanol to gasoline (MTG) process, [68].

\section{Perspectives}

\subsection{The future of the refining and petrochemical industry}

Refining and petrochemistry are both mature sectors. The technologies we use today are the result of decades of operations and millions of processed tons. Any new technology aimed to overcome the mature ones will have to be proven not only technically but also in economic, sustainability and safety terms. So, what are the drivers for opportunities today? There are Three challenges:

- First of all, the oil and gas sector is facing the introduction of nonconventional crude oils. Today's operations are optimized for conventional crudes. Catalysts for refining are prepared to cope with some poisons at certain concentration levels. Non-conventional crudes are sour, dense, and present high levels of heavy metals, including unusual metals. This 


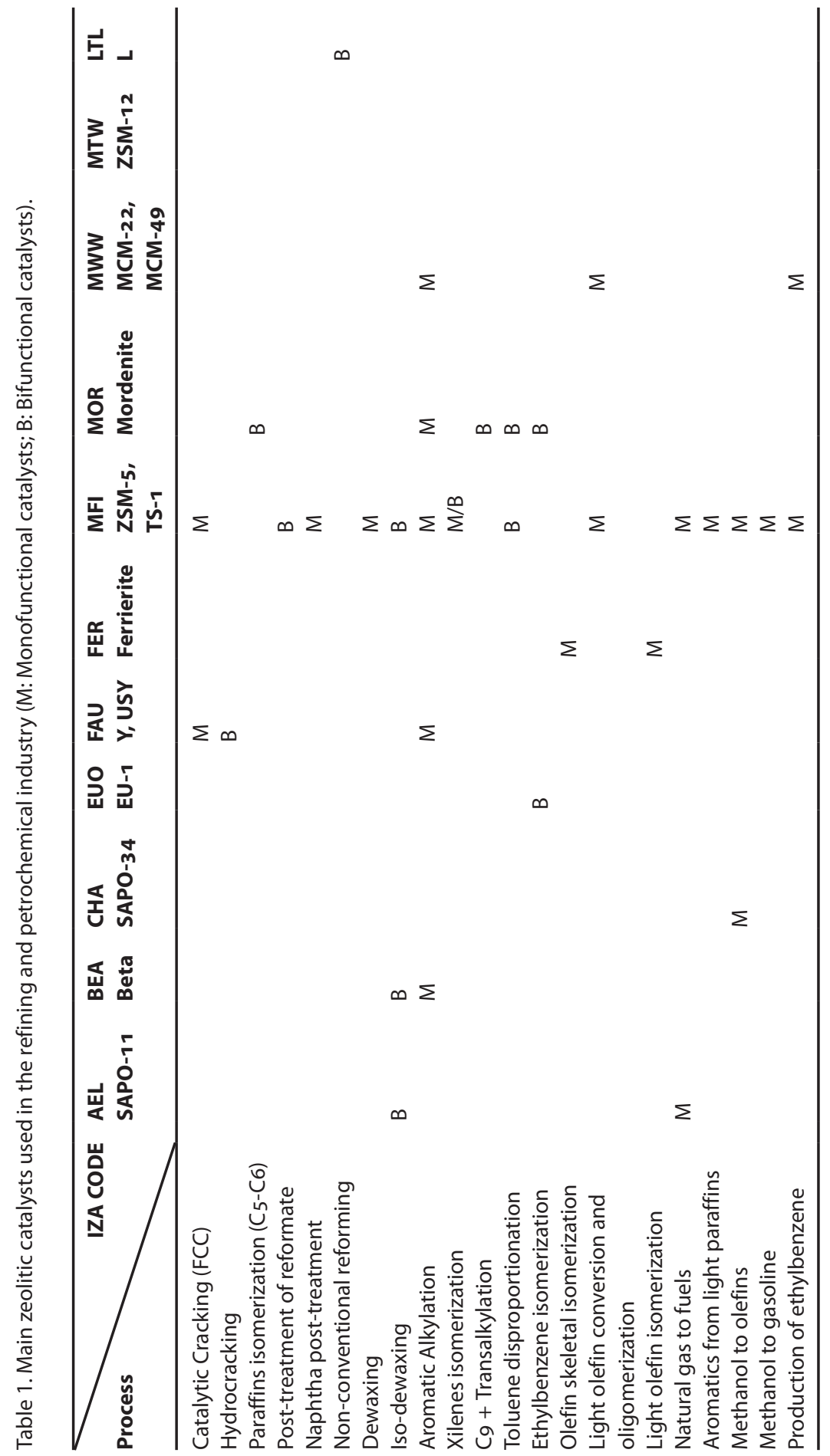




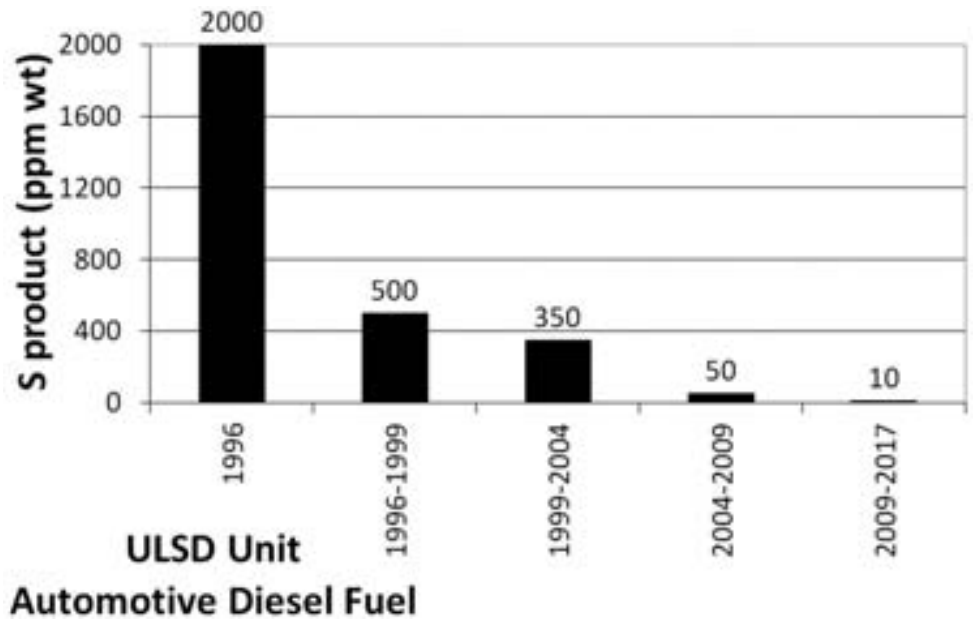

Figure 7. Evolution of the content of sulphur in diesel fuel.

is forcing a huge effort in $\mathrm{R} \& \mathrm{D}$ in pre-treatment processes - not only in catalysts but also in metal traps and guard beds.

- Secondly, legislation in Europe is posing more and more stringent specifications on fuels and emissions. The oil and gas industry has to implement and improve the finishing processes. A clear example is the new specification for marine fuel in terms of maximum sulphur. IMO's MARPOL Annex VI regulation will globally cap sulphur content to max. $0.5 \%$ in 2020 [69], thus posing a challenge for fuel oil manufacturers. Another example is sulphur in automotive fuels: in 21 years (from 1996 to 2017) sulphur in diesel has suffered a huge decrease due to European Regulations, Figure 7.

In the case of automotive fuels the European Commission is also putting pressure on refiners to include biofuels in the market [70]. Biofuels are liquid or gaseous transport fuels, such as biodiesel and bioethanol, which are made from biomass (see Chapter 6). By 2020, the EU aims to have $10 \%$ of the transport fuel in every country coming from renewable sources, such as biofuels. Fuel suppliers are also required to reduce the greenhouse gas intensity of the EU fuel mix by $6 \%$ by 2020 in comparison to 2010. Refining companies are coping with this in two different ways. It is possible to maintain the oil business as it is and acquire the biofuel for blending from third parties, or it could be possible to go towards the concept of biorefinery. That is, to introduce biomass in the production sites to co-process it along with the mineral feed. Cepsa's approach is the introduction of biomass in the current operations. This is another example of pressure on refiners. 


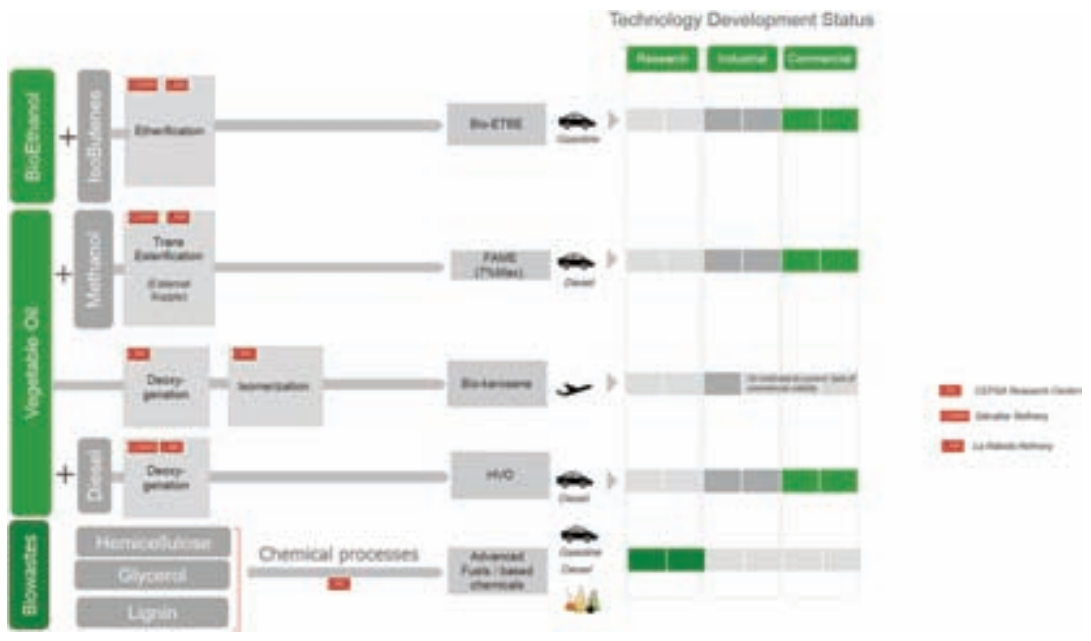

Figure 8. Biofuels R\&D Projects at Cepsa [71].

- Efficiency. Combustion motors are nowadays much more efficient than in the past. Less fuel is now required to cover the same distance. Refineries are facing this challenge by diversifying or looking at other markets or products in order to remain competitive. In the case of Cepsa, the integration of refining and petrochemical businesses is helping the company to face this challenge. Production schemes have to be flexible enough to optimize fuels or chemicals production depending on both demands.

There are other challenges but these three are certainly key in the oil and gas sector. What is Cepsa doing to face them? In relation to zeolites, in addition to refining and petrochemical projects dedicated to improving the present industrial operation, new materials and products are researched. These include the development of advanced fuel and biofuels. As can be seen in Figure 8, regarding the biofuels area, the technology readiness level (TRL) of the different studies range from lab-scale to industrial research, evidencing that there is room for improvement at every level.

\subsection{The future of zeolite and MOF materials in refining and petrochemistry}

As has been shown throughout this chapter, the fact that most relevant catalytic processes within refining and petrochemical industry are intended for conversion of certain crude oil fractions into higher value ones, future 
conversion trends will be aligned by a) optimizing economics of catalytic processes by maximizing the catalytic activity and selectivity under less severe conditions (temperature, LHSV, pressure, $\mathrm{H}_{2}$ demand, etc.), and b) increasing process flexibility to deal with changes in the economic environment. This aspect implies being able to manage cheaper and more complex raw materials (crude oil basket) while accomplishing newer and or stricter specifications of quality from demanded products (gasoline/diesel).

As for zeolites, the future challenges for each of the process' differ, although common goals include the improvement of selectivity, catalyst lifetime, and possibilities of regeneration. Zeolitic materials combine the advantages of having high specific surface areas (specific surface areas of $\left.300-800 \mathrm{~m}^{2} \mathrm{~g}^{-1}\right)[72]$ and therefore the possibility of spreading a high number of active sites per unit of mass, with a certain variety of pore and cage sizes and shapes. Apart from that, due to their chemical nature, it is easy to generate acid sites and modify their strength. Finally, zeolites have a high thermochemical resistance.

Sustainable chemistry for the future requires the improvement of catalyst selectivity and the production of fewer waste by-products. An example of this is the catalytic alkylation of aromatics with olefins. In both processes, the alkylation with short chain olefins to obtain cumene and in long chains to give LAB, the production of dialkylated compounds has to be avoided, as these have to be treated as by-products or subjected to processes of transalkylation to recover the desired monoalkylated product. The modification of the textural and catalytic properties of the zeolites making them more selective to the products of interest then becomes crucial [73].

It is desirable to reduce the cracking that produces light compounds and yields losses in transalkylation catalysts. In this case, research should be focused on modulating the acidity to increase the yield to the desired product without producing cracking.

The use of new zeolites, zeotypes or even MOFs could improve the selectivity, although the introduction in the market of new structures is not simple as a result of the strong implantation of the existing zeolites. The majority of the current zeolite catalysts are based on three main structures: Y, ZSM- 5 and Mordenite. It is true that other zeolites such as Beta or MCM22 have been applied, but about $95 \%$ of the volume of the zeolites used in catalysis is accounted for by $\mathrm{Y}$ zeolite for the FCC process.

Additionally, some possible new applications for zeolites can be expected, such as:

a) Methane steam reforming to produce hydrogen. Due to the fact that many refining and petrochemical processes require hydrogen, this conversion 
would be very useful and profitable. In the last few years, $\mathrm{Ru}-\mathrm{CeO}_{2} /$ Mordenite and $\mathrm{Ni}-\mathrm{La}_{2} \mathrm{O}_{3} / 5 \mathrm{~A}$, have been studied for $\mathrm{CH}_{4}$ reforming with $\mathrm{CO}_{2}[74-75]$. The catalyst with Mordenite as a support resulted in highly dispersed $\mathrm{Ru}$ and $\mathrm{CeO}_{2}$. Low coke formation and high activity were also reported, constituting a promising type of new steam reforming catalysts. b) Fine-tuning processes: developing new zeolites that work in a selective way to remove poisons or chemical species that are refractive to conversion. An example could be the removal of 4,6-dimethyldibenztiophene in hydrotreating processes [76].

In their turn, MOFs have different advantages. Due to the possibility of combining multiple metals with different oxidation levels and almost infinite organic ligands, the possibilities of tri-dimensional porous frameworks are huge compared to new potential zeolitic systems. However, most relevant refining and petrochemical processes require high temperature, making it difficult to envision MOFs as the catalytic phase for reactions like cracking, hydrocracking, etc., because of their low thermal stability [3-6]. The replacement of zeolites in the paraffins and xylenes separation processes seems difficult, too, since these processes are very well established and the adsorption process operates at moderately high temperatures.

A greater future for MOFs can be predicted in the case of adsorption and gas purification processes that usually work at moderate temperatures [77-78]. MOFs could be used in methane reforming (separation of $\mathrm{H}_{2}$ from $\mathrm{CO}_{2}$ stage) and hydrogen stream purification $\left(\mathrm{CO}_{x}\right.$ removal for vegetable oil co-processing) in gas streams. As for separation processes in a liquid phase, it is noted that several heterocompounds present in oil streams, such as some nitrogen containing molecules (quinolones, indoles...) poison some refining and petrochemical catalysts located downstream. MOF MIL-101 has shown a very good selectivity to remove nitrogen compounds in straight run gas oil or light cycle oil streams at the lab-scale [79]. These experiments could be a good starting point for combining purification units with current refining and petrochemical units [80-82].

In the petrochemical field, there are also oxidation reactions, which are currently carried out at low temperature without a catalyst that could be withstood by MOFs. The literature describes their application to redox reactions involving light molecules $[4,72]$. These include their use for the direct synthesis of phenol from benzene. In this field, MOFs have been used for cumene oxidation to yield cumene hydroperoxide, a precursor of phenol. This reaction is currently carried out by oxidation with air, without a catalyst, with residence times longer than 12 hours. Being that the 
temperatures are not very high $\left(8 \mathrm{o}-90^{\circ} \mathrm{C}\right)$, it could be a possible field of application for these products. In fact, applications of MOFs for this reaction can be found in the literature. In particular, MOF MIL-101 has been applied for hydroxylation-epoxidation reactions and for cumene oxidation to cumene hydroperoxide at the lab-scale [83-85]. The use of highly localized energy supply systems (e.g. microwaves) might be another possibility for temperature-sensitive materials, or to improve the efficiency of the catalysis in general [86-87].

Although there are many recognized and mature industrial applications for zeolites compared to MOFs, both fields have a long way to go in creating and, why not, to combining them for the development of novel, efficient applications. Research and development is a challenging task, in which every achievement will undoubtedly be of great interest to this industry and to our society.

\section{References}

[1] Chen, N.Y., Garwood, W.E., Dwyer, F.G. Shape Selective Catalysis in Industrial Applications, Marcel Dekker, 1989.

[2] Zeolite (Natural and Synthetic) Market for Catalysts, Adsorbents, Detergent Builders and Other Applications - Global Industry Perspective, Comprehensive Analysis, Size, Share, Growth, Segment, Trends and Forecast, 2014- 2020 Market Research Store.

[3] Unnikrishnan, P., Srinivas, D. Heterogeneous Catalysis. In Industrial Catalytic Processes for Fine and Specialty Chemicals, p. 41, Joshi, S.S., Ranade, V.V. (Eds.), Elsevier, 2016.

[4] Corma, A., García, H., Llabrés i Xamena, F.X. Chem. Rev. 2010, 110, 4606.

[5] Isaeva, V.I., Kustov, L.M. Metal-Organic Frameworks and Related Materials: Miles to Go. In Zeolites and Zeolites-like materials, p. 33, Sels, B., Kustov, L. (Eds.), Elsevier, 2016.

[6] Albero, J., García, H. Metal Organic Frameworks as Catalysts for Organic Reactions. In New Materials for catalytic applications, p. 13, Parvulescu, V.I., Kemnitz, K. (Eds.), Elsevier, 2016.

[7] Marcilly, C.R. Top. Catal. 2000, 13, 357.

[8] Wauquier, J.P. Petroleum Refining, Separation Processes, IFP Ed. Technip, 2000.

[9] Barrer, R.M.J. Soc. Chem. Ind. 1945, 44, 130.

[10] Eberly, P.E., Arey, W.F. US Patent 3126425, 1964.

[11] Rosback, D.H., Gillespie, J.M. US Patent 4283587 A, 1980.

[12] Broughtun, D.B. Adsorptive separation: liquids. In Kirk-Othmer Encyclopedia of Chemical Technology 3rd edition, p. 563, John Wiley, 1978. AICHE Symposium Series.

[13] Broughton, D.B. AICHE Symposium Series, 1984, 80, 233, 62.

[14] Hydrocarbon Process 1979, 58, 185 .

[15] Sie, S.T. Stud. Surf. Sci. Catal. 1994, 85, 587.

[16] Sadeghbeigi, R. Fluid Catalytic Cracking Handbook 3rd edition, Elsevier, 2012.

[17] Komvokis, V., Xin Lin Tan, L., Clough, M., Shaun Pan, S., Yilmaz, B. Zeolites in fluid catalytic cracking (FCC). In Zeolites in Sustainable Chemistry, p. 271, Feng-Shou Xiao, F.S., Meng, X. (Eds.), Springer, 2016.

[18] Furimsky, E. Stud. Surf. Sci. Catal. 2007, 169, 305. 
[19] Rigutto, M. in Zeolites and Catalysis: Synthesis, Reactions and Applications, p. 547, Čejka, J., Corma, A., Zones, S. (Eds.), Wiley-VCH, 2010.

[20] http://www.dilworthip.com/fluid-catalytic-cracking-patents-2015-catalyst-additives/

[21] http://www.dilworthip.com/review-2015-trends-fluid-catalytic-cracking-patents-partii-catalysts-zeolites/

[22] Scherzer, J., Gruia, A.J. Hydrocracking Science and Technology, p.174, CRC Press, 1996.

[23] Lloyd, L. in Handbook of Industrial Catalysts, $p$. 211, Springer.

[24] Degnan, T.F. Top. Catal. 2000, 13349.

[25] Valavarasu, G., Bhaskar, M., Balaraman, K.S. J. Petrol. Sci. Eng. 2003, 21, 1185.

[26] Morel, F., Kressmann, S., Harlé, V., Kasztelan, S. Processes and catalysts for hydrocracking of heavy oil residues. In Hydrotreating and Hydrocracking of Heavy Oil Fractions, $p$. 1, Froment, G.F., Delmon, D., Grange, P. (Eds.), Elsevier, 1997.

[27] Primo, A., García, H. Chem. Soc. Rev. 2014, 437548.

[28] Čejka, J., Pérez-Pariente, J., Roth, W.J. in Zeolites: From Model Materials to Industrial Catalysis, p. 357, Transworld Research Network.

[29] Lazaro, J., Corma, A., Frontela, J. US Patent 5057471, 1991.

[30] Antos, G.J., Moser, M.D., Lapinski, M.P. The New Generation of Commercial Catalytic Naphtha-Reforming Catalysts. In Catalytic Naphtha Reforming. Revised and Expanded, p. 344, Antos, G.J. (Ed.), Marcel Dekker, 2004.

[31] Babich, I.V., Moulijn, J.A. Fuel 2003, 82, 607.

[32] Bruneta, S., Meya, D., Pérot, G., Bouchyb, C. Diehlb, F. Appl. Catal., A 2005, 278, 143.

[33] Hughes, T.R., Buss, W.C., Tamm, R.W., Jacobson, R.L. Stud. Surf. Sci. Catal. 1986, 28, 725

[34] http://www.cpchem.com/en-us/rnt/licensing/aromaxtech/pages/history.aspx

[35] Lapinski, M., Baird, L. James, R. Catalytic Reforming. UOP platforming process. In Handbook of Petroleum Refining Processes, 3rd edition, p. 4, Meyers, R.A. (Ed.) Mc Graw Hill, 2006.

[36] Perego, C., Calenma, V., Pollesel, P. Naphtha Reforming and Upgrading of Diesel Fractions. In Zeolites and Catalysis: Synthesis, Reactions and Applications, p. 585, Čejka, J., Corma, A., Zones, S. (Eds.), Wiley-VCH, 2010.

[37] Sequeira Jr. A. Lubricant Base Oil and Wax Processing, Marcel Decker, 1994. 
[55] Cheng, N.Y., Kaeding, W.W., Dwyer, F.G.J. Am.Chem. Soc. 1979, 101, 6783

[56] Eur. Chem. News 1997, 67, 23.

[57] Levin, D., Ross, A.D., Beech, J.H. US Patent 8481443 B2, 2013.

[58] Nacamuli, G.J., Vogel, R.F., Zones, S.I. US Patent 5952536, 1999.

[59] Oil Gas Journal 1998, 96 (48), 35.

[6o] Hydrocarbon Processing International Edition, 1975, 54 (11), 115.

[61] Eur. Chem. News 1997, 68, 33.

[62] Blauwhoff, P.M.M., Gosselink, J.W., Kieffer, E.P., Sie, S.T., Stork, W.H.J. Zeolites as Catalysts in Industrial Processes. In Catalysis and Zeolites, p. 437, Weitkamp, J., Puppe, L. (Eds.), Springer, 1999.

[63] Martínez, C., Corma, A. Coord. Chem. Rev. 2011, 255, 1558.

[64] Sawicki, R.A. NPRA, vol 95, issue 45 of A.M: National Petroleum Refiners Association.

[65] Zwart, J., Vink, J. Appl. Catal. 1987, 33, 383.

[66] Kuo, J.C.W. Slurry Fischer-Tropsch/Mobil Two-Stage Process of Converting Syngas to High Octane Gasoline, Final Report, DOE Contract No. DE-AC22-80PC $30022,1983$.

[67] Zhou, L. Base aromatics production processes. In Handbook of Petroleum Refining Processes, $3^{\text {rd }}$ edition, 2.29, Meyers, R.A (Ed.), McGraw-Hill, 2003.

[68] Stöcker, M. Methanol to Olefins (MTO) and Methanol to Gasoline (MTG). In Zeolites and Catalysis: Synthesis, Reactions and Applications, p. 687, Čejka, J., Corma, A., Zones, S. (Eds.), Wiley-VCH, 2010.

[69] http://www.imo.org/en/OurWork/environment/pollutionprevention/airpollution/pages/ air-pollution.aspx

[70] Directive 2009/28/EC of the European Parliament and of the Council of 23 April 2009 on the promotion of the use of energy from renewable sources and amending and subsequently repealing Directives 2001/77/EC and 2003/30/ECOfficial Journal of the European Union, June, 5, 2009.

[71] Frontela, J. Cepsa Strategy for implementing Biofeedstocks into Downstream Operation, European Refining Technology Conference (ERTC), Lisbon, 2016.

[72] Czaja, U.A., Trukhanb, N., Muller, U. Chem. Soc. Rev., 2009, 38, 1284.

[73] Ceña-López, C., Peláez, J., Márquez-Álvarez, C., Franco, M.J., Lázaro, J.J., Sastre, E., XXV Congreso iberoamericano de catálisis, Montevideo (Uruguay).

[74] Luo, J., Gao, L., Ng, C., Au, C.T. Catal. Lett. 1999, 62, 153.

[75] Hashimoto, K., Watase, S., Toukai, N. Catal. Lett. 2002, 80, 147.

[76] Bej, S.K., Maity, S.K. Turaga, U.T. Energy Fuels 2004, 18, 1227.

[77] Yilmaz, B., Trukhan, N., Müller, U. Chinese J. Catal. 2012, 33, 1.

[78] Rangnekar, N., Mittal, N., Elyassi, B., Caro, J., Tsapatsis, M. Chem. Soc. Rev. 2015, 447128.

[79] Laredo, G.C., Vega-Merino, P.M., Trejo-Zárrega, F., Castillo, J. Fuel Process. Technol. 2013, 106, 21.

[8o] Achmann, S., Hagen, G., Hämmler, M., Malkowsky, I., Kiener, C., Moos, R. Chem. Eng. Technol. 2010, 33, 275 .

[81] Richter, I., Kiener, C., Malkowsky, I.M., Achmann, S., Hagen, G., Haemmerle, M., Moos, R. US Patent $20110138781 A 7,2011$.

[82] Mora, R.J., Montoya J.A., Laredo, G.C., Meneses, E., Castillo, J.J., Zapata, B. US Patent $20160332138 \mathrm{~A}$, 2016.

[83] Santiago-Portillo, A., Navalón, S., Cirujano, F.G., Llabrés i Xamena, F.X., Alvaro, M. García, H. ACS Catal. 2015, 5, 3216.

[84] Yang, H., Li, J., Wang, L., Dai, W., Lv, Y., Gao, S. Catal. Commun. 2013, 35, 101.

[85] Luz, I., León, A., Boronat, M., Llabrés I Xamena, F.X., Corma. A. Catal. Sci. Technol. 2013, 3, 371.

[86] Kustov, L.M., Sinev, I.M. Russ. J. Phys. Chem. A 2010, 84, 1676.

[87] Hakek, M. Chem. Commun. 1997, 62, 347. 


\title{
5. Biomass Transformation into Chemicals Using Zeolites and MOFs
}

\author{
Óscar Hernando Laguna Espita, ${ }^{1}$ Simona M. Coman, ${ }^{2}$ \\ Miguel Ángel Centeno Gallego ${ }^{1}$ and Vasile I. Pârvulescu ${ }^{2}$ \\ ${ }^{1}$ Institute of Materials Science of Seville, CSIC-University of \\ Seville, Américo Vespucio 49, 41092 Seville, Spain.
}

${ }^{2}$ Department of Organic Chemistry, Biochemistry and Catalysis, Faculty of Chemistry, University of Bucharest, 4-12 Regina Elisabeta Av., Bucharest, Romania.

Keywords: Cellulose, lignin, biorefinery, platform molecule

\section{Introduction}

Biomass is referred to as a renewable source of materials and energy, which it is most often associated with plants or plant-based materials that are not used for food or as a feed. From a chemical point of view, interest is focused on the valorization of the dry matter of biomass, commonly known as lignocellulosic biomass. Its total annual production exceeds 100,000 million tons of carbon, which corresponds to an associated energy of $1.5 \times 10^{3}$

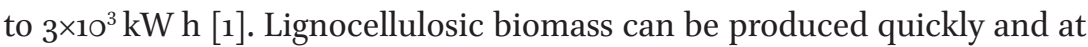
a low cost and is significantly cheaper than crude oil, generating both important economic and environmental advantages [2].

Lignocellulosic biomass is a tridimensional structure mainly composed of three polymers: cellulose, hemicellulose and lignin, together with small amounts of other components, like acetyl groups, minerals and phenolic substituents (Figure 1). Cellulose represents the main crystalline component of lignocellulosic biomass, whichisformed by repeating units of the disaccharide cellobiose. The second most abundant polymer is hemicellulose, which presents an amorphous structure composed of several heteropolymers, including xylan, galactomannan, glucuronoxylan, arabinoxylan, glucomannan, and xyloglucan. Lignin is the third main component, with a three dimensional structure of phenylpropanoid units including $p$-coumaryl alcohol, coniferyl alcohol, and sinapyl alcohol. 


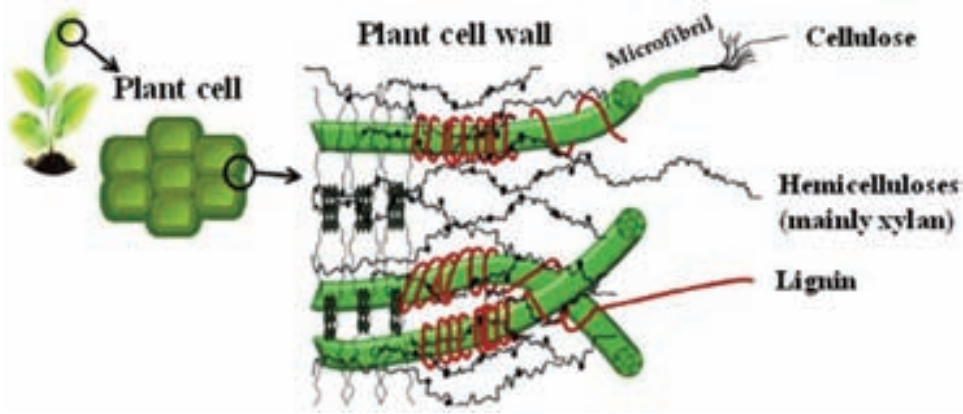

Figure 1. Lignocellulosic biomass generation and composition. By Khanok Ratanakhanokchai, Rattiya Waeonukul, Patthra Pason, Chakrit Tachaapaikoon, Khin Lay Kyu, Kazuo Sakka, Akihiko Kosugi and Yutaka Mori. CC BY-SA 3.0. DOI: 10.5772/51820.

All three components of lignocellulosic biomass have a large quantity of oxygen: almost half for cellulose, $\left(\mathrm{C}_{6} \mathrm{H}_{10} \mathrm{O}_{5}\right)_{\mathrm{n}}$, and around $30 \%$ for lignin, $\left(\mathrm{C}_{9} \mathrm{H}_{10} \mathrm{O}_{2}\right)_{\mathrm{n}}$. Nowadays, most feedstocks for the manufacture of commodity chemicals and polymers are obtained from fossil oil [3]. To produce them, an intermediate selective oxidation step is required in many cases. However, this step often has a low environmental (E-) factor and generates $\mathrm{CO}_{2}$, contributing to an increase in the greenhouse effect. Therefore, it appears wiser to valorize the oxygen already present in the lignocellulosic biomass for the production of commodity chemicals and polymers, and to devote fossil oil to the production of deoxygenated products.

The main step in chemical valorization of lignocellulosic resources involves, the depolymerization of the macromolecules. While for cellulose an efficient hydrolysis can directly generate monomers, lignin fragmentation is more difficult since the polymers are formed by connecting aromatic entities via both etheric and C-C bonds. Thus, the hydrolysis of cellulose is easier and has been reported by enzymatic [4-6], homogeneous [7] or heterogeneous catalysis [8]. The more complex fragmentation of lignin was carried using multiple approaches like fast pyrolysis $[9,10]$ or homogeneous and heterogeneous catalysis [11] followed by extraction [12]. In this case, the result is a cocktail of small aromatic molecules with different functionalities and masses. Figure 2 depicts the fragmentation of cellulose, along with further chemical valorization.

The solvent is very important in the chemical valorization of biomass. To date, achievements in conversion processes in both water and organic solvents have been reported, including the conversion of biomass to sugars, the conversion of sugars to furanic compounds, and the production of lignin monomers [13]. The solvent influences both conversion and product selectivity as it can affect the solubility of the biomass components, including 


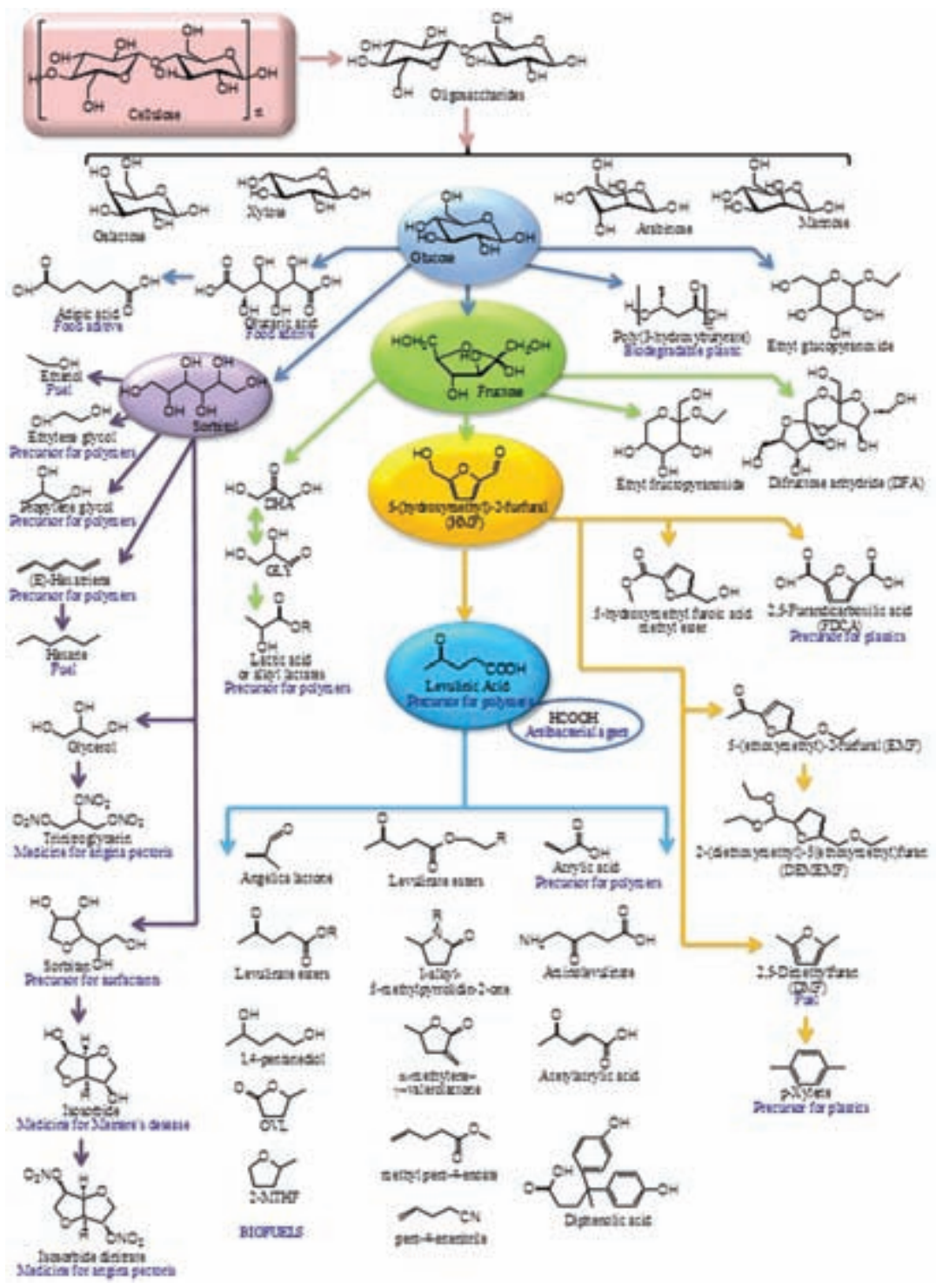

Figure 2. Fragmentation of cellulose and further transformations.

cellulose and lignin, and also affect the thermodynamics of reactants, intermediates, products, and/or catalysts. Studies carried in sub- and supercritical water revealed the advantages of working under supercritical conditions [14].

Overall, the aim of this chapter is to discuss the use of zeolites and metal-organic frameworks (MOFs) as catalysts for the fragmentation of cellulose and lignin and for the valorization of the resulting monomers 
by producing valuable chemicals. This approach is closely related to the concept of sustainability [15]. Both zeolites and MOFs present similar properties which make them perfect candidates for their application in these types of reactions, such as shape selectivity, microporosity, the simultaneous presence of Brønsted and Lewis acid sites, metallic active sites, and related crystal structures, which have been discussed in previous chapters. However, important differences exist between both families of materials in terms of diffusion, adsorption and stability aspects. Besides this, MOFs present a structural versatility that allows the tailored design of a huge variety of different MOF structures, exceeding the number of zeolite structures available. On top of this, zeolites are well-known materials applied since the 1960 os in different catalytic processes, while MOFs chemistry is just beginning and only a few families of materials have been employed so far. Thus, our chapter will describe firstly the use of zeolites in the main types of reactions for biomass transformation and, in the second part, the differences between and opportunities for using MOFs.

\section{Cellulose transformation into chemicals using zeolites}

The fragmentation of cellulose and hemicellulose carbohydrate polymers into their $\mathrm{C}_{5}$ and $\mathrm{C}_{6}$ constituent sugars and their subsequent transformation into biorefinery platform molecules for chemical commodities represents a unique means for the production of various oxygenates, such as furfural, 5-hydroxymethylfurfural, xylitol, sorbitol, mannitol, gluconic acid, etc. [16] The use of zeolites as market-accessible solid acid catalysts offers big opportunities.

Zeolites are microporous minerals commonly used as catalysts for acid catalysed reactions. They are also known as 'molecular sieves', mainly consisting of $\mathrm{Si}, \mathrm{Al}$ and $\mathrm{O}$, although the replacement of cations by metals such as $\mathrm{Ti}, \mathrm{Sn}$ or $\mathrm{Zn}$ is also possible. The concomitant presence of two cations in different oxidation states in the structure of these materials requires the compensation by a proton, which induces Brønsted acidity, or by an extra-framework cation, leading to Lewis acidity. Thanks to these properties, zeolites can replace inorganic acids in acid catalysed reactions, with the very important advantages of recyclability and the possibility of tuning the acid strength and type. Working with zeolites eliminates volatile and toxic solvents, and halogenated compounds such as alkylating agents. On this basis they are frequently used for the synthesis of fine chemicals [17], as will be presented in a later chapter, and, somehow related, for the 
production of chemicals from biomass [18-20]. This section presents examples of these advantages. They mainly refer to H-zeolites and Sn-Beta zeotype [21]. The processes analysed are depicted in Figure 2.

\subsection{Transformation of the primary products of the cellulose fragmentation: sucrose, glucose, fructose, xylose, arabinose, galactose, mannose}

The isomerization of glucose into fructose is a large-scale reaction typically performed by enzymes, which have as their main disadvantage deactivation and large reaction volumes [22]. Besides the production of syrups, this reaction is also a potential route for the production of chemicals from biomass. In this regard, recyclable catalysts are necessary.

Ca- and Ba-exchanged $\mathrm{A}, \mathrm{X}$ and $\mathrm{Y}$ zeolites were found less selective in this reaction, whereas those with milder basicity such as Na-X and K-X zeolites were found to achieve the isomerization of glucose into fructose with a selectivity of about 9o \%. [23]. However, this high selectivity to fructose is only obtained at low glucose conversions, below $25 \%$. Furthermore, it was found that a significant amount of the cation leached into water (around $15 \%$ in the case of monovalent ion exchanged $\mathrm{X}$ zeolites).

Large-pore zeolite containing tin (Sn-Beta) emerged as particularly effective to catalyse carbohydrate transformations. It is able to isomerize hexoses, pentoses and trioses by intramolecular hydride and carbon shift reactions in various solvents, including aqueous media, with high activity and selectivity. [24-27]. The properties of the large-pore zeolite have a dominant effect on these reactions. The reaction is very slow over Sn-MCM-41 or even does not proceed over a medium-pore zeolite like Sn-MFI [28].

\subsection{Hydrolytic hydrogenation of glucose to sugar alcohols or hexitols}

The hydrolytic hydrogenation of cellulose/glucose to sugar alcohols offers an alternative to petrochemicals and to the current environmental problems [29]. Such catalytic processes would provide promising platform molecules for oxygenates and liquid alkanes [30]. Sorbitol is an eloquent example. It can be used as a sweetener, dispensing agent and humectant in pharmaceuticals, cosmetics and textiles, as well as for the further chemical synthesis of surfactants [31]. To obtain it, the hydrolytic hydrogenation 
of cellulose in the presence of metallic (Ir, Ru, Rh, Pd)/ BEA bifunctional catalysts may produce sorbitol with yields of $55 \cdot 3-72.8 \%$ [32]. The selectivity to sorbitol correlated with the d-band width of the metal, i.e.: Ir > $\mathrm{Ru}>\mathrm{Rh}>\mathrm{Pd}$. However, besides the metal nature, both the acid groups of BEA zeolite and in the conversion of monosaccharides, the concentration of adsorbed hydrogen species on the zeolite surface played key roles in the formation of sorbitol. In fact, it was later shown that the rate of hexitol formation increases with the degree of zeolite dealumination [33]. The potential of bifunctional $\mathrm{Ru} / \mathrm{H}$-USY catalysts for the total conversion of cellulose to sugar alcohols or hexitols in hot liquid water (HLW) is limited by its physicochemical stability, i.e. the long-term catalytic performance of acid sites and noble metal functionality. Zeolite desilication is the main zeolite degradation mechanism in HLW, in which USY zeolite stability depends on two main parameters - framework and extra-framework aluminium content. The former protects the zeolite lattice by counteracting hydrolysis of framework bonds, and the latter, when located at the external crystal surface, prevents solubilization of the zeolite framework as a result of its low water-solubility.

\subsection{Production of HMF, furfural and 2,5-furandicarboxylic acid}

5-Hydroxymethylfurfural (HMF), furfural and 2,5-furandicarboxylic acid (FDCA) are derivatives of furan compounds, which were listed among the top 10 value-added bio-based chemicals by the US Department of Energy [34]. To obtain it from cellulose, from a mechanistic point of view, glucose needs to be isomerized to fructose, followed by dehydration to HMF. Accordingly, the process requires an isomerization catalyst for the transformation of glucose to fructose and a second component able to catalyse the dehydration [35].

Dehydration of fructose into HMF was found to depend on both acidic and structural properties of the zeolites used [36]. $\mathrm{H} / \mathrm{Y}$ faujasites and $\mathrm{H}$-Mordenites with $\mathrm{Si} / \mathrm{Al}$ ratios of 15 and 11 were used. Shape-selective properties and, particularly, the absence of cavities are very important, allowing selectivities over 9o-95\% for H-Mordenites [37].

Dehydration of D-xylose into furfural was also reported in liquidphase over various $\mathrm{H}$-zeolites (H-furrieries, H-Beta, H-ZSM-5, H-Y and $\mathrm{H}-$ Mordenite) with various $\mathrm{Si} / \mathrm{Al}$ molar ratios in different solvent systems: water, dimethyl sulfoxide (DMSO), and a mixture of water and toluene [38]. Conversion and furfural yield generally decreased with an increasing $\mathrm{Si} / \mathrm{Al}$ 
molar ratio, which was closely related to the accessibility of the acid sites, and depended on the solvent: water/toluene $>$ DMSO > water. Thus, H-Beta (25) showed the highest furfural selectivity in water and water/toluene, while H-Mordenite (20) showed the highest furfural selectivity in DMSO. Phase modifiers like DMSO and poly(1-vinyl-2-pyrrolidinone) also helped to suppress undesired side reactions [39]. However, the catalyst should also show high stability in highly acidic aqueous environments to allow coupling the isomerization with other acid-catalysed reactions, namely, the dehydration of the isomerized product. Sn-Beta zeotype has also been reported to catalyse the isomerization of xylose [40].

DFT calculations explained the role of $\mathrm{Sn}$ in these reactions [41], indicating that its main contribution is to promote the transformation of the acyclic glucose to a deprotonated fructose intermediate. The catalytic cycle is completed by the closure of the fructofuranose ring and the protonation of the anionic carbohydrate intermediate bound to the Sn site. These steps are facilitated by water molecules, which mediate the proton transfer reactions between the different oxygen-containing moieties of the carbohydrate. According to these calculations, the $\mathrm{Sn}(\mathrm{IV})$ tetrahedral lattice site and the hydroxylated $\mathrm{SnOH}$ species exhibit very similar reactivity.

The conversion of cellulose to HMF using a complex acid catalyst composed of $\mathrm{CrCl}_{2} /$ Zeolite/BMIMCl (ionic liquid 1-butyl-3-methylimidazolium chloride) was reported to be active at low catalyst loading and low temperature $\left(<120^{\circ} \mathrm{C}\right)[42]$. The role of zeolite in this process was to promote cellulose hydrolysis and to slow down the decomposition of the HMF product. Its effect varied in the order: $\mathrm{H}-\mathrm{Y}>\mathrm{H}-\mathrm{Beta}>\mathrm{H}-\mathrm{MOR}>\mathrm{H}-\mathrm{ZSM}-5$.

Mesoporous $\mathrm{Nb}_{\mathrm{x}} \mathrm{W}_{8_{-\mathrm{x}}}$ oxides synthesized by an evaporation-induced self-assembly method created large amounts of Lewis and Brønsted acid sites, which promoted the isomerization of glucose to fructose as well as its following dehydration to HMF. Mannose was also formed through the epimerization of glucose [43].

5-benzyl-2-furoic acid derivatives are important intermediates for the preparation of a variety of compounds with biological activity [44]. In the classic protocols they are produced by a Friedel-Crafts alkylation with chlorinated acid derivatives using $\mathrm{AlCl}_{3}$ or $\mathrm{ZnCl}_{2}$ as Lewis acid catalysts. To obtain methyl 5 -hydroxymethyl-2-furoate from HMF, a heterogeneous catalytic alternative comprises consecutive reactions using a two-component catalyst $\left(\mathrm{Au} / \mathrm{TiO}_{2}\right.$ and an acid catalyst). $\mathrm{Au} / \mathrm{TiO}_{2}$ catalyses the oxidative esterification of HMF, followed by its conversion into methyl 5-benzyl-2-furoate by alkylation of the benzene derivative of interest 


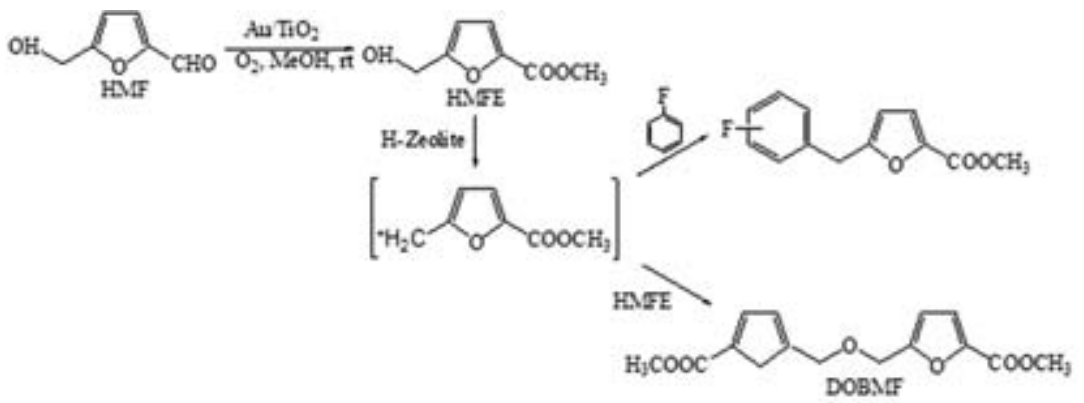

Figure 3. The production of 5-benzyl-2-furoate in the presence of a two-dimensional zeolite. (HMF = 5-hydroxymethylfurfural, HMFE = 5-hydroxymethyl furoic acid methyl ester, DOBMF = dimethyl 5,5'-(oxybis(methylene))bisfuran-2-carboxylate). Reprinted with permission from ref. [45]. Copyright 2016 American Chemical Society.

over the solid acid catalyst (Figure 3). For this last step, a number of zeolites with similar $\mathrm{Si} / \mathrm{Al}$ ratios including $\mathrm{H}-\mathrm{Beta}(\mathrm{Si} / \mathrm{Al}=12$ and 14), MCM-22 ( $\mathrm{Si} / \mathrm{Al}=15)$, USY $(\mathrm{Si} / \mathrm{Al}=12)$, 2D zeolite ITQ-2 $(\mathrm{Si} / \mathrm{Al}=15)$, and a hierarchical mesoporous zeolite USY-HYM zeolite ( $\mathrm{Si} / \mathrm{Al}=5 \cdot 3)$ were considered [44]. The catalytic results demonstrated the need for a balance between acidity and diffusional constraints. Large pore tridimensional zeolites, such as Beta and USY, showed an adequate acidity to carry out the reaction but they presented strong product diffusion limitations that led to rapid deactivation. On the other hand, MCM-41 had pores large enough to allow an easy product diffusion but exhibited a too low acidity for this reaction. Finally, delaminated ITQ-2 zeolite combined both requirements and thus allowed high reaction rates and selectivity.

\subsection{Cellulose to levulinic acid}

Levulinic acid (LA) represents a versatile bio-based chemical product of the valorization of cellulose and is an important feedstock for the production of many chemical commodities (Figure 4) [45]. It can be obtained by HMF transformation and the presence of a carbonyl and a carboxylic acid group confers to this molecule the properties of a building block for various chemicals [46]. On this basis, the catalytic conversion of carbohydrates to LA over solid catalysts has received much attention over the last few decades. Since these reactions require the presence of strong Brønsted acids, H-zeolites have been largely investigated. Zeolite 


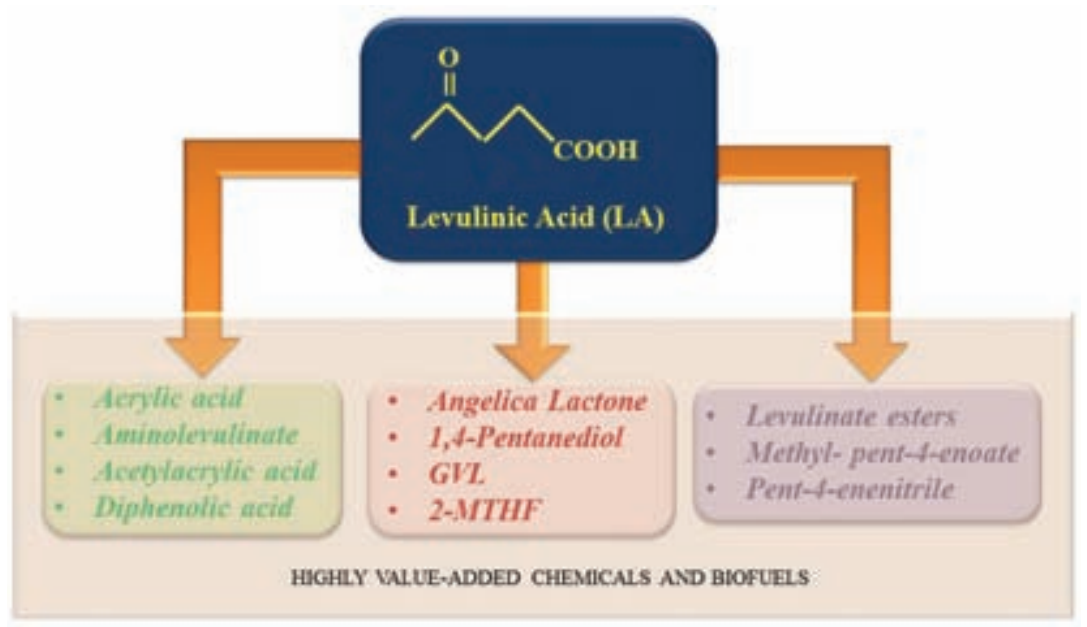

Figure 4. Chemical commodities produced from levulinic acid [45].

catalysts like $\mathrm{H}_{-\mathrm{ZSM}-5}$ have economic advantages over resin catalysts, in particular, they can be easily regenerated by a calcination treatment after they are deactivated [47]. However, employing these catalysts may result in significantly lower LA yields, especially when high LA concentrations are desired in the product stream. This disadvantage can be eliminated by utilising biphasic systems: an aqueous layer that contains the zeolite and an organic layer to extract the LA produced [47]. 2-sec-butylphenol, 4- $n$-hexylphenol and 4-propylguaiacol already demonstrated to be effective extracting agents for the production of furfural and LA in these biphasic systems [47]. Another advantage of the solvents is that they can be synthesized directly from biomass (i.e. lignin). Hybrid catalysts consisting of a homogeneous $\left(\mathrm{CrCl}_{3}\right)$ and a heterogeneous (zeolite $\mathrm{Y}$ ) catalyst have been indicated as effective in this reaction as well $[48,49]$.

The efficient production of levulinate esters is another important reaction in the valorization of biomass. Zeolites were largely investigated for this esterification because of their adjustable acidity, easy recyclability, and potential economic and environmental benefits. However, levulinic acid esterification with ethanol to ethyl levulinate over H-USY, H-BEA, H-ZSM-5, H-MOR indicated that they are not active enough to be considered in an industrial application [50]. Esterification on these materials occurred with smaller yields compared to other functionalized porous structures, like sulfonic-acid-functionalized SBA-15 catalyst [51]. 


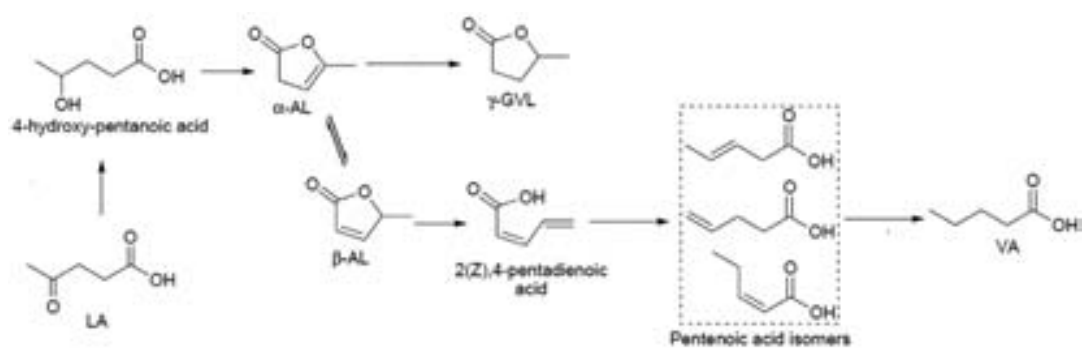

Figure 5. Reaction pathway for the microwave-assisted hydrogenation of LA in presence of FA using $\mathrm{Pd} / \mathrm{C}$ catalyst. Adapted from [53]. CC BY 4.0.

\subsection{Levulinic acid hydrogenation to $\gamma$-valerolactone}

Levulinic acid can be transformed to a series of other valuable chemicals. Among these, $\gamma$-valerolactone (GVL) is one of the most important. Several catalytic approaches to produce it have been reported. Qi and Horváth [52] demonstrated that working with a homogeneous catalyst with GVL as solvent it is possible to produce GVL directly from fructose having HMF and levulinic acid as the only intermediates.

As an alternative to noble metal catalysts, supported iron oxide nanoparticles on Al- and Zr-SBA-15 were investigated for the hydrogenation of levulinic acid to $\gamma$-valerolactone [53]. Reactions were carried out under continuous flow, with formic acid as the hydrogen donating agent to effectively replace high pressure H2. Since $\alpha$-angelica lactone can suffer an isomerization to $\beta$-angelica lactone leading to pentenoic acid isomers and valeric acid (Figure 5), the control of the selectivity is very important in this reaction.

\subsection{Oxidation of glucose to gluconic acid, glucuronic acid, tartaric acid, glycolic acid, and glyceric acid}

The use of redox zeolites and redox pillared clays for the valorization of biomass was suggested long ago [54]. The oxidation of D-glucose to gluconic acid, glucuronic acid, tartaric acid, glycolic acid and glyceric acid may also occur with hydrogen peroxide as oxidant over several zeolites and Ti-containing zeolites, like TS-1 or Ti-MCM-41 [55]. Using zeolites (ZSM-5, L, Y, Mordenite) gluconic acid is the main reaction product, having glucoronic and tartaric acids as main by-products. The presence of titanium preserves the high selectivity to gluconic acid but the oxidation by-products change to glycolic acid. 


\subsection{Production of lactic acid and methyl lactate}

Lactic acid is an important platform molecule in the synthesis of a wide range of chemicals $[56,57]$. Lactic acid fermentation is typically performed by lactic acid bacteria, which convert simple carbohydrates like glucose, sucrose, or galactose to lactic acid.

Lewis acidic Sn-Beta zeotype also allowed the direct formation of methyl lactate from common sugars [58]. The conversion of sucrose in methanol at $160^{\circ} \mathrm{C}$ afforded methyl lactate with a yield of $68 \%$. The conversion of pentoses and hexoses into methyl lactate over Sn-Beta showed a dependence on the nature of the saccharide. Pentoses are converted to methyl lactate in slightly lower yields $(\sim 40 \%)$ than those obtained from hexoses $(\sim 50 \%)$, but higher yields of glycolaldehyde dimethyl acetal are observed from pentoses. This finding agrees with a reaction pathway involving the retro aldol condensation of the sugars to yield triose and glycolaldehyde from pentoses, and two trioses from hexoses. When reacting glycolaldehyde (formally a $\mathrm{C}_{2}$-sugar) in the presence of Sn-Beta, aldol condensation occurs, leading to the formation of methyl lactate, methyl vinylglycolate and methyl 2-hydroxy-4-methoxybutanoate. By contrast, when converting the sugars in water at low temperature $\left(100^{\circ} \mathrm{C}\right)$, Sn-Beta catalyses the isomerization of sugars (ketose-aldose epimers) rather than the formation of lactates [59].

High selectivity to the desired lactate product was achieved with an ordered mesoporous material (OMM) Sn-MCM-41 [6o]. However, a more elaborated Lewis acid zeotype catalyst consisting of two components (MCM-41 filled with a porous polyaromatic hydrocarbon network) improved the performance in this reaction (Figure 6) [61]. Modification of the organic part with functional groups exhibiting mild Brønsted acidity and of the inorganic part by grafting Lewis acid $\mathrm{Sn}(\mathrm{IV})$ enabled a catalyst able to transform trioses and aldo- and ketohexoses (fructose) into lactic acid in aqueous medium $(\mathrm{R}=\mathrm{H})$ or into alkyl lactates $(\mathrm{A})$ in alcoholic solvents ( $\mathrm{R}=$ alkyl). Yields to lactate correlate with the length of the alcohol radical, octanol providing the highest values.

In aqueous solutions without alkali, the efficient preparation of lactic acid through the direct catalysis of sugars is hindered by the dehydration side reaction to 5 -hydroxymethylfurfural. This is due to Brønsted acidity, which originates from organic acids [62]. It was suggested that this could be avoided by using Zn-Sn-Beta prepared via solid-state ion exchange. In water under ambient air pressure, without a base, a sucrose conversion exceeding $99 \%$ with a lactic acid yield of $54 \%$ was achieved within 2 hours at $190{ }^{\circ} \mathrm{C}$. Studies of the acid and base properties of the $\mathrm{Zn}$-Sn-Beta zeolite 


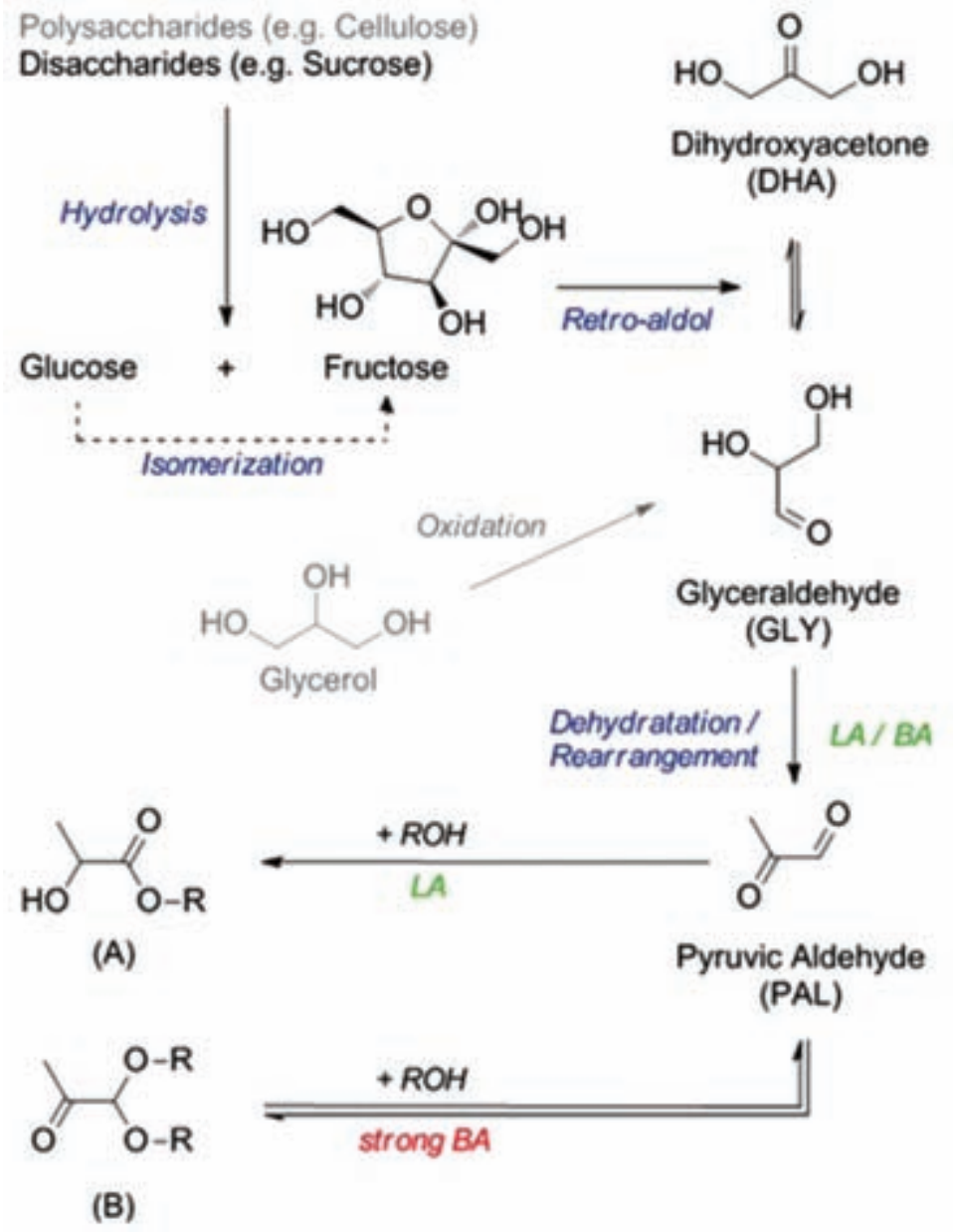

Figure 6. Conversion of monosaccharides like trioses and aldo- and ketohexoses (fructose) into lactic acid in aqueous medium $(R=H)$ or into alkyl lactates $(A)$ in alcoholic solvents $(R=a l k y l)$. The side reaction leading to the formation of pyruvic aldehyde dialkyl acetal $(B)$ is undesired $-\mathrm{LA}=$ Lewis acid; $\mathrm{BA}=$ Brønsted acid. Reprinted with permission from ref. [61]. Copyright 2012 American Chemical Society.

suggest that the introduction of Zn into the Sn-Beta zeolite enhanced both the Lewis acid and base sites. The base sites inhibited a series of side reactions related to fructose dehydration to 5-hydroxymethylfurfural and its subsequent decomposition.

Indeed, Sn-Beta zeolite was also found to be a highly active catalyst for the conversion of triose sugars (1,3-dihydixyacetone in Figure 6$)$ to lactic acid. In water, once they are produced, isomerization takes place to form 
lactic acid in very high yields at $125{ }^{\circ} \mathrm{C}[63]$. Methanol reaction at $80^{\circ} \mathrm{C}$, is also followed by esterification, leading to methyl lactate in quantitative yields. $\mathrm{H}-\mathrm{USY}(\mathrm{Si} / \mathrm{Al}=6)$ is also reasonably active in this reaction [64].

\subsection{Aldol condensation of fural aldehydes}

Zeolites have also been considered for aldol condensation of fural aldehydes. Nitrided zeolite Y was used in the aldol condensations of furaldehydes with acetone and propanal, showing comparable activity to $\mathrm{MgO}-\mathrm{ZrO}_{2}$ and much higher than that over $\mathrm{NaY}[65]$. However, under reaction conditions part of the nitrogen leached, resulting in the loss of catalytic activity [65]. $\mathrm{H}$-zeolites of different structural types also showed appreciable activity in the aldol condensation of furfural with acetone and resulted in the formation of products, which are usually obtained using basic catalysts [66,67]. However, the reaction on these zeolites can continue with the dimerization of the classical condensation products. Large-pore three-dimensional BEA and FAU zeolites afforded higher furfural conversion than medium-pore MFI or large-pore mono-dimensional MOR [66]. Another disadvantage of these catalysts is the quick formation of coke inside the zeolite pores, requiring regeneration of the catalysts.

\section{Lignin valorization}

Catalytic valorization of lignin for the production of renewable chemicals has been recently reviewed [11]. The participation of zeolites in this effort is merely associated with biomass fast-pyrolysis. Such technology upgrades solid biomass as it yields products with higher energy density, and is also more economical than the alternative technologies of gasification $[68,69]$. The composition of the produced bio-oil depends on the origin of the lignin. Softwood lignins yielded exclusively guaiacyl derivatives, coniferaldehyde and coniferyl alcohol, hardwood lignins gave rise to guaiacyl and syringyl derivatives, among which syringaldehyde, coniferyl alcohol and sinapyl alcohol were the most abundant. Grass lignins, represented by bamboo lignin, yielded $p$-vinylphenol as the main compound. In addition, other guaiacyl and syringyl pyrolysis products were identified, too [70].

In situ electron paramagnetic resonance (EPR) measurements and model compounds studies reveal two main decomposition routes in this process - firstly, the homolytic fission of the weakest bond-forming radicals 


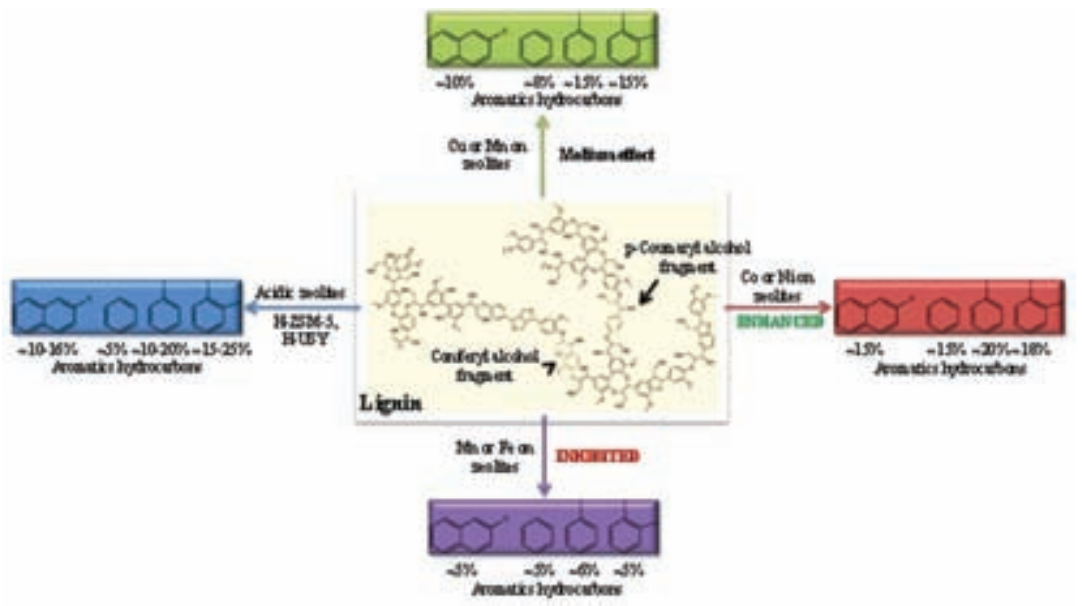

Figure 7. Zeolite transformation of the pyrolysis oil. Copyright $\odot$ Swiss Chemical Society: CHIMIA, ref. [71]. DOI: https://doi.org/10.2533/chimia.2015.597

and, secondly, the radical initiation leading to different radicals, which further rearrange or recombine depending on the nature of the radicals [71]. However, there is a significant potential to control the selectivity by varying catalysts and reaction conditions [72-74]. The formation of aromatic hydrocarbons is enhanced over zeolite-supported Co and $\mathrm{Ni}$ catalysts, (Figure 7). The intermediates are stabilized by adsorption on the pores in materials without acidity, such as silicalite, thus increasing the yield of liquid without changing the liquid products distribution. In the presence of strongly acidic zeolites, such as H-USY and H-ZSM5 of low Si/Al ratio, complex reactions, such as dehydration, decarboxylation, dealkylation, cracking, isomerization and oligomerization are involved.

To preserve the catalyst activity, the establishment of a step of pyrolytic lignin deposition at $400{ }^{\circ} \mathrm{C}$ avoids the blockage of the on-line catalytic bed and attenuates the deactivation of a HZSM- 5 zeolite based catalyst used for hydrocarbon production [75]. As mentioned above, the stability of the zeolites is a critical issue in these processes. One of the major concerns is their hydrothermal stability in the presence of steam or hot water. Therefore, given the high water content in pyrolysis oils and biomass as a whole, as well as in aqueous processes, the selection of H-USY and H-ZSM5 is reasonable. These materials, exhibit a good stability in liquid water between 150 and $200^{\circ} \mathrm{C}$ regardless of their $\mathrm{Si} / \mathrm{Al}$ ratio $[76]$.

Biomass pyrolysis oil contains oxygenate compounds that may be partially transformed to hydrocarbons on acid catalysts to obtain raw a material for the petrochemical industry (light olefins and benzene, toluene, 
and xylene). The utilization of a catalyst based on HZSM-5 zeolite led to a product distribution similar to that obtained from the transformation of light alcohols $[77,78]$.

\section{Metal-organic frameworks for the upgrading of biomass}

So far, we have demonstrated the use of zeolites in different reactions involved in the production of platform molecules derived from biomass. This is due to the structural properties of these porous materials that present a $3^{\mathrm{D}}$ framework with high surface areas and suitable porous structures, as well as chemical and thermal stabilities. The $3 \mathrm{D}$ structure leaves confined spaces with limited dimensions where only certain substrates, transition states or products are allowed, resulting in a control of the process called shape selectivity [79]. Moreover, zeolites, which have coordinatively unsaturated metal sites, present strong Lewis acidity and can also have Brønsted acidity, both of them useful for biomass reactions such as hydrolysis. Nevertheless, despite the advantages of zeolites and the wide number of possible structures [8o], higher chemical adaptability of the zeolite-based catalysts is required to improve yields and selectivities in biomass upgrading and to allow one-pot syntheses. Additionally, textural properties of zeolites also require a more specific design to control the transport phenomena during the catalytic reactions and to enhance the density of active sites. Therefore, a step forward is required in the design of catalysts for reactions such as hydrolysis, pyrolysis, condensation, isomerization, deoxygenation, hydrogenation, and oxidation, working under complex chemical environments with multiple functional groups and in the presence of water.

In this sense, a new type of porous materials, with a huge potential for catalysis, has been developed over the last 25 years. These are metal-organic frameworks (MOFs), also named porous coordination polymers (PCPs), which are composed of meal ions and various organic ligands [81-87]. MOFs can be classified into different families according to the inorganic framework, as was highlighted by Farrusseng et al. [87]. There are different systems, such as a) open-framework coordination polymers made from oD inorganic, hybrid or isolated metal clusters connected by bridging organic polytopic ligands, or organic-inorganic hybrid materials in which the inorganic moieties can be organized into b) $1 \mathrm{D}$ chains or c) $2 \mathrm{D}$ layers separated by organic pillars. These materials have demonstrated their high potential to outperform even zeolites in some reactions due to their tunability provided by the different compositions of organic ligands and metallic clusters [87]. 
Table 1. MOFs vs zeolites: a comparison of the main properties of both types of materials. Adapted from references $[79,80]$.

\begin{tabular}{|c|c|c|}
\hline Properties & MOFs & Zeolites \\
\hline $\begin{array}{l}\text { Thermal } \\
\text { stability }\end{array}$ & Stable below $300^{\circ} \mathrm{C}$ & Stable above $450^{\circ} \mathrm{C}$ \\
\hline $\begin{array}{l}\text { Chemical } \\
\text { stability }\end{array}$ & $\begin{array}{l}\text { Limited chemical stability } \\
\text { specially to } \mathrm{H}_{2} \mathrm{O} \text { in most cases }\end{array}$ & $\begin{array}{l}\text { Stable to solvents, acids, oxidizing } \\
\text { and reducing agents }\end{array}$ \\
\hline $\begin{array}{l}\text { BET surface } \\
\text { area }\end{array}$ & Around $1,000-10,000 \mathrm{~m}^{2} \mathrm{~g}^{-1}$ & Around $200-500 \mathrm{~m}^{2} \mathrm{~g}^{-1}$ \\
\hline Pore volume & Over $1 \mathrm{~cm}^{3}$ & $0.1-0.5 \mathrm{~cm}^{3}$ \\
\hline $\begin{array}{l}\text { Metal site } \\
\text { density }\end{array}$ & High & Low \\
\hline Lewis acidity & Accessible framework metal ions & Accessible framework metal ions \\
\hline $\begin{array}{l}\text { Brønsted } \\
\text { acidity }\end{array}$ & Introducible through organic linker & $\begin{array}{l}\text { Bridging } \mathrm{Si}(\mathrm{OH}) / \mathrm{Al} \text { hydroxyl } \\
\text { groups }\end{array}$ \\
\hline Basicity & Introducible through organic linker & From framework oxygen atoms \\
\hline $\begin{array}{l}\text { Active site } \\
\text { environment }\end{array}$ & $\begin{array}{l}\text { More hydrophobic, but linker- } \\
\text { dependent }\end{array}$ & $\begin{array}{l}\text { Mostly hydrophilic but can be } \\
\text { made hydrophobic }\end{array}$ \\
\hline $\begin{array}{l}\text { Additional } \\
\text { features }\end{array}$ & $\begin{array}{l}\text { Chiral functionalities, flexible and } \\
\text { stimuli responsive behaviour }\end{array}$ & - \\
\hline Reactivation & $\begin{array}{l}\text { Washing procedures; thermal } \\
\text { treatment not possible }\end{array}$ & By thermal treatment \\
\hline
\end{tabular}

The application of MOF catalysts is recent [8o], and the use of MOFs-based catalysts for the upgrading of lignocellulosic biomass is even more recent. In 2011, Akiyama et al. [88] presented one of the first works on the synthesis of a new PCP decorated with sulfonic acid functional groups for the hydrolysis of cellulose. Recently, Herbst and Janiak have presented a comprehensive review about the use of MOF catalysts in the transformation of biomass into valuable fine chemicals [80]. This includes the state of the art as well as the challenges and perspectives in this field of MOF applications. Furthermore, this review not only summarizes the few works devoted to this end but also deeply analyses the advantages and drawbacks of these types of materials and compares them to zeolites. Given the scope of this book, we have adapted some of the main relevant contrasts made by these authors in Table 1. 
The application of MOFs in the transformation of biomass is not easy since, although over 20,000 types have been reported in literature, only water-stable MOFs would be applicable in the sort of reactions cited above. Furthermore, certain thermal and chemical stabilities are required, thus reducing the number of suitable MOF-based catalysts considerably $[80,89,90]$. Thus, the stability of MOFs is an important issue that has to be controlled and carefully addressed, especially when reactions are carried out in the presence of water, which is a typical situation for the reactions involving biomass-derived feedstocks $[80,90]$. Concerning the relevance of the stability of MOFs for catalytic applications, Burtch et al. [91] have presented a comprehensive review defining some concepts on the water stability of MOFs, which can be considered during the design of MOF catalysts for the transformation of biomass. In this review, a broad classification of the huge number of MOFs that have been experimentally characterized after water exposure is presented, taking into account thermodynamic and kinetic stabilities. Thermodynamic stability considers chemical aspects, such as the metal-ligand bond strength or the lability in water and the possible variables that may affect them during the reactions. On the other hand, kinetic stability deals with aspects such as access to the active sites. From both, the hydrophobicity and the steric factors will determine if the MOF-based catalysts are suitable for reactions including water [91].

Another remarkable advantage of MOF catalysts is their high adsorption capacity and the possibility of achieving controlled pore sizes. In fact, to process the big molecules derived from the biomass deconstruction, materials with big pores are required to enhance the selectivity in the adsorption of reactants and in the desorption of products to allow one-pot procedures. In their review, Farrusseng et al. [87] presented the influence of the porous architecture on selectivity in different reactions. In this sense, the superior adsorption abilities of MOF catalysts over zeolites is demonstrated from results like those by Kruger et al. [92]. In this work, the pore sizes of different zeolite catalysts and the diameter of platform molecules were compared by means of simulations. While the zeolite structure with the largest pore sizes is $\mathrm{H}-\mathrm{Y}$ with $7 \cdot 5 \AA$, some of the most suitable MOFs systems for the upgrading of biomass present larger pore diameters. For instance, MIL-101Cr has pores of 29 and $34 \AA$ and pore windows of 12 to ${ }_{15} \AA$ [92], which made this system suitable for glucose recovery [93]. The unusual adsorption abilities of some MOFs has also been confirmed by Yabushita et al. [94], who observed that NU-10oo MOF adsorbs cellobiose and lactose dimers from aqueous solution in amounts exceeding $1250 \mathrm{mg} \mathrm{g}^{-1}$ while completely excluding adsorption of the glucose monomer. The high 
selectivity of the adsorption process in these materials, which results in a selective molecular recognition, seems to be due to the number of favourable $\mathrm{CH}-\pi$ interactions between the sugar with pyrene units and the studied MOF.

\subsection{Transformation of cellulose and hemicellulose carbohydrate polymers using MOF catalysts}

As remarked above, the work by Akiyama and coworkers [88] addressed the high potential of MOF catalysts for transforming biomass-derived products into biofuels or high added value chemicals. This will contribute to the development of biorefineries, aiming to transform residual biomass feedstocks rather than those derived from food crops so avoiding competition with the food supply. In this work, the authors modified the structure of a typical MIL-101 MOF introducing sulfonic acid groups on its pore surface for use in cellulose hydrolysis [88]. After comparing the catalytic performance of this system with that of different acid catalysts, such as Amberlyst-15, this MOF catalyst demonstrated clean catalytic activity and high Brønsted acidity but low yields to mono- and disaccharides due to the low solubility of crystalline cellulose in water. However, the MOF catalyst exhibited a considerable robustness in boiling water and strongly acidic conditions. Further studies from this research team about the use of functionalized MIL-101 catalysts have confirmed that the high stability and availability of its acid centres and the open metal sites resulted in the enhancement of glucose-to-fructose conversion [95]. In addition, the structural features in coordination of the chromium sites in this type of materials led to performances comparable to those of homogeneous catalysts, demonstrating the high specificity that can be achieved with these materials $[80,95]$.

Chen et al. presented a different approach to the conversion of cellulose, starting from a MIL-101 MOF but with different modifications [96]. In this case, water-tolerant phosphotungstic acid (PTA)/MOF-supported Ru catalysts are bifunctional (metal/acid) catalysts for obtaining cellulose- and cellobiose-derived alcohols. The metal/acid balance was studied in order to establish optimal conditions for the production of alcohols: selectivities to sorbitol of $57.9 \%$ and $95.1 \%$ were achieved in the conversion of cellulose and cellobiose, respectively [96].

Recently, Huang et al. [97] reported the use of a new 3D MOF/graphene catalyst prepared by in situ growth of zeolitic imidazolate framework (ZIF-8) 
nanoparticles inside the pores of a hydrogel. In this case, cellulose was dissolved in alkaline aqueous solution and consequently degraded over the catalyst under hydrothermal conditions, reaching total conversion with formic acid as the main product, along with oxalic and acetic acids.

Although the use of MOF catalysts for the transformation of biomass is an emerging topic, most works have focused on the transformation of molecules derived from cellulose and hemicellulose rather than lignin. This is due to the complex transformation of the lignin molecule, as a result of its complex structure and multiple possibilities of selective rupture in smaller molecules. Table 2 presents a comprehensive summary of works devoted to the transformation of cellulose/hemicellulose-derived molecules using MOF catalysts. It includes all the references cited by Herbst and Janiak [8o] and the newer works presented up until now. As can be inferred from the table, two families of MOFs have principally been studied as catalysts for these reactions: MIL-101(Cr) and UiO-66 - due to the thermal and water stability in both cases, as well as the contribution of strong and stable acid sites.

Lignin is also a potential source of renewable organics with high a content of oxygenated functional groups. However, the strong chemical bonds comprising this polymer make its transformation particularly difficult. Furthermore, the complexity of the obtained mixtures of products is also important because in such a multi-functional chemical environment oxygenated molecules may tend to re-polymerize. Despite this, the development of efficient heterogeneous catalysts for the selective cleavage of $\mathrm{C}-\mathrm{O}$ aromatic bonds in lignin has been recently demonstrated [98]. This verifies the first report by Stavila et al. [99] concerning the use of MOF-based catalysts for the hydrogenolysis of carbon-oxygen ether bonds, which are common linkages in oxygenated molecules derived from lignin.

\subsection{Transformation of lignin over MOF catalysts}

To date, only vanillin has been analysed as a model to explore the hydrogenation and deoxygenation routes of lignin. Recently, Zhang et al. [114] have studied the properties of $\mathrm{SO}_{3} \mathrm{H}-\mathrm{MIL}-1 \mathrm{Ol}(\mathrm{Cr})$, encapsulated $\mathrm{Pd}$ nanoparticles in the mesoporous cages of the catalysts and obtained an efficient catalyst for the hydrodeoxigenation of vanillin. In this case, the developed $\mathrm{Pd} / \mathrm{SO}_{3} \mathrm{H}-\mathrm{MIL}-1 \mathrm{O}(\mathrm{Cr})$ catalysts presented high catalytic activity in tandem hydrogenation-deoxygenation reactions, and could be recycled several times without any apparent loss in activity or selectivity. The high 


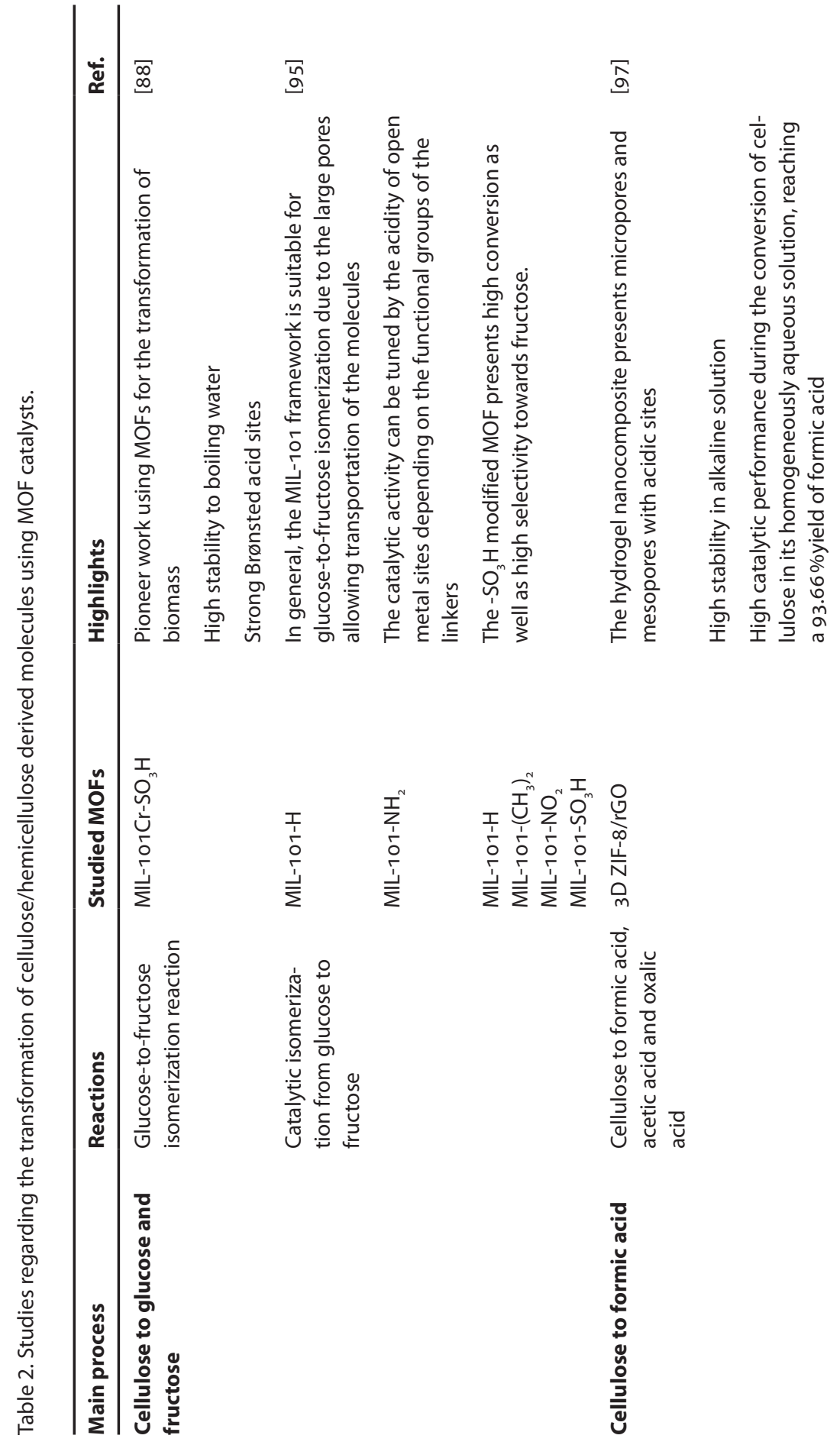




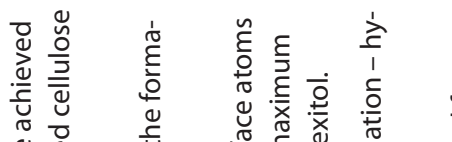

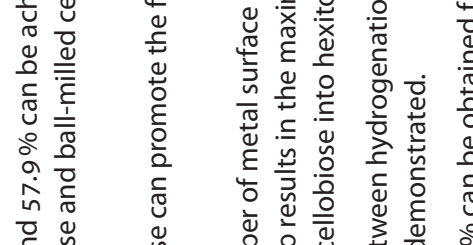

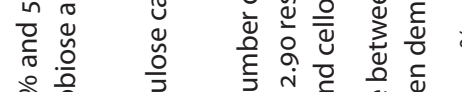

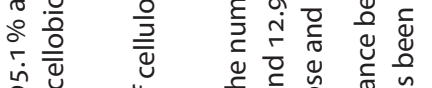

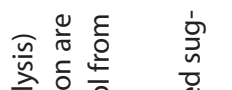

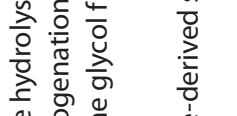

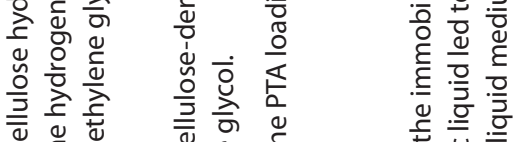

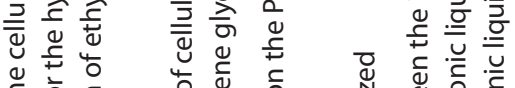

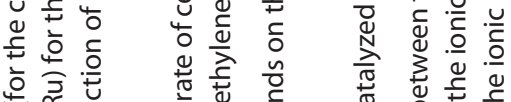

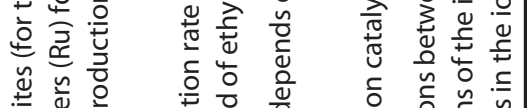

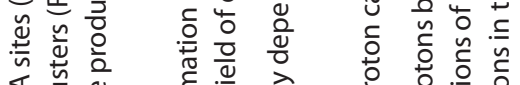

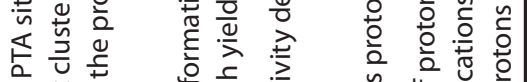

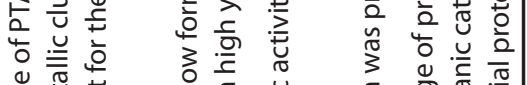
पु 㟧

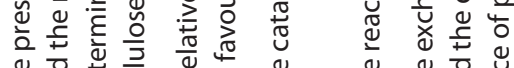

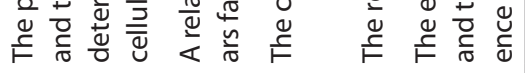

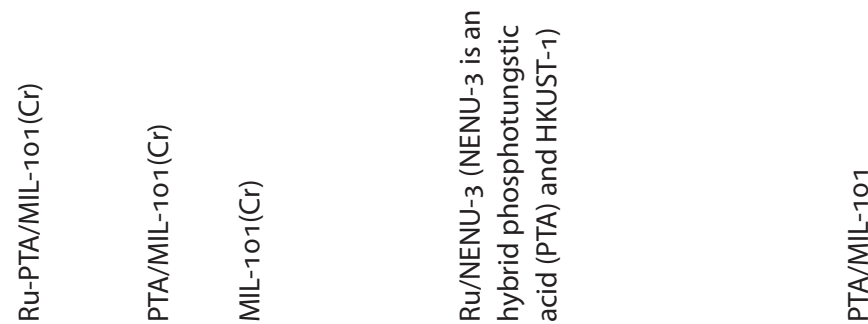
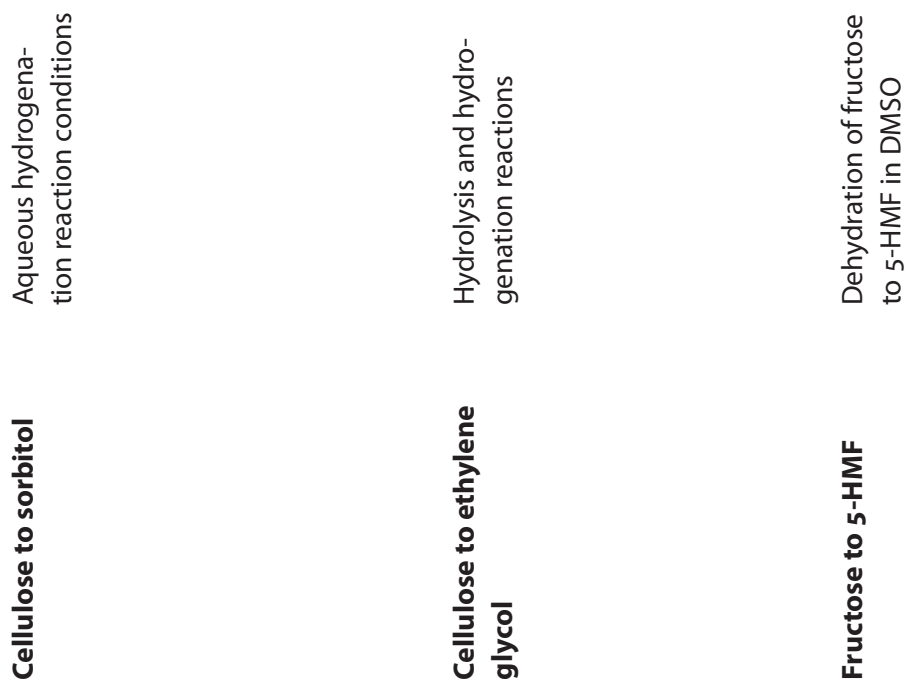


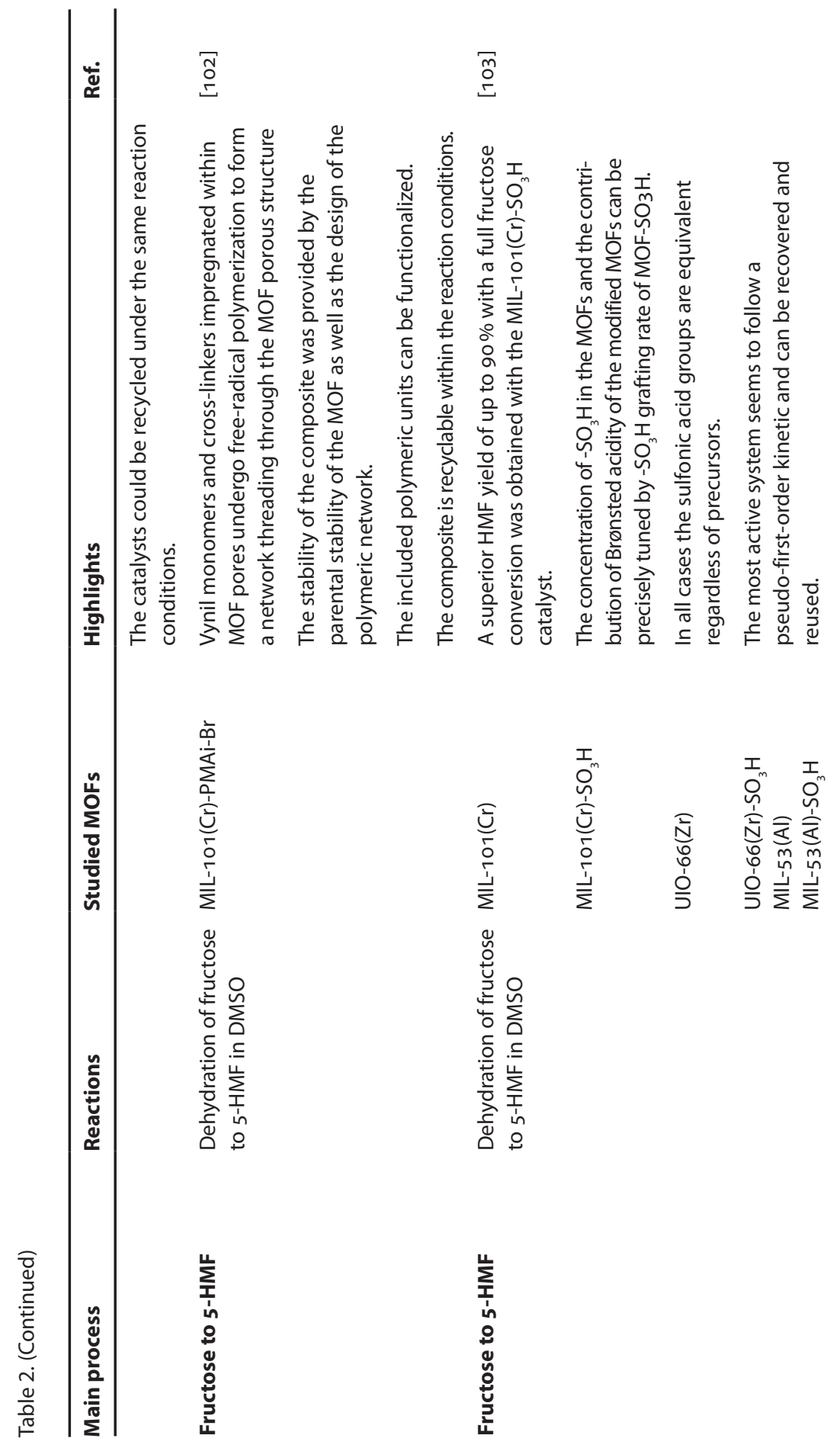




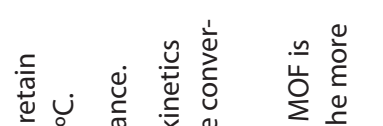

冚

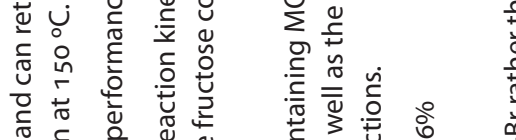

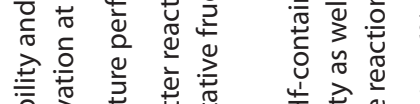

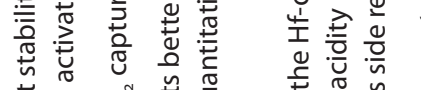

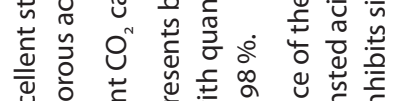

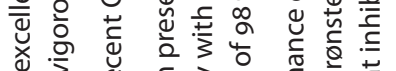

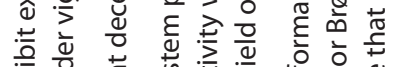

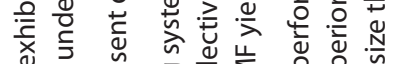

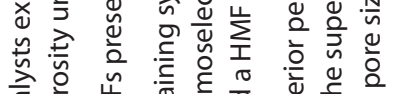

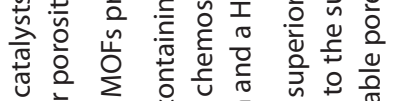

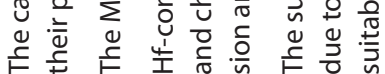

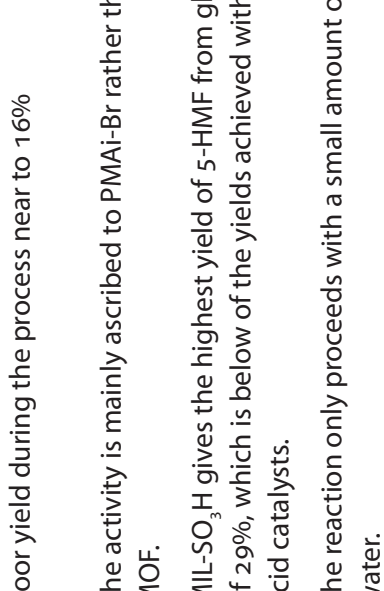

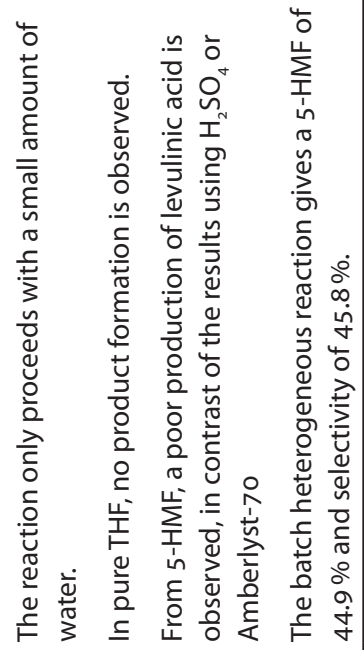

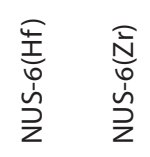

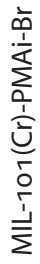

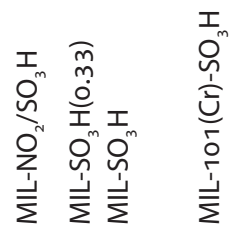

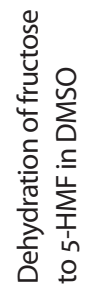

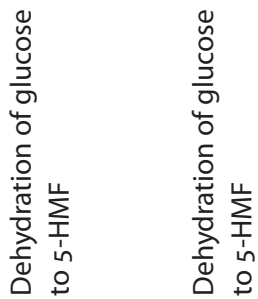

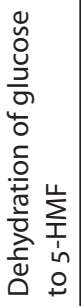

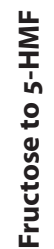

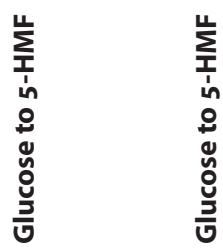

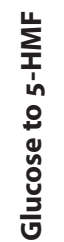




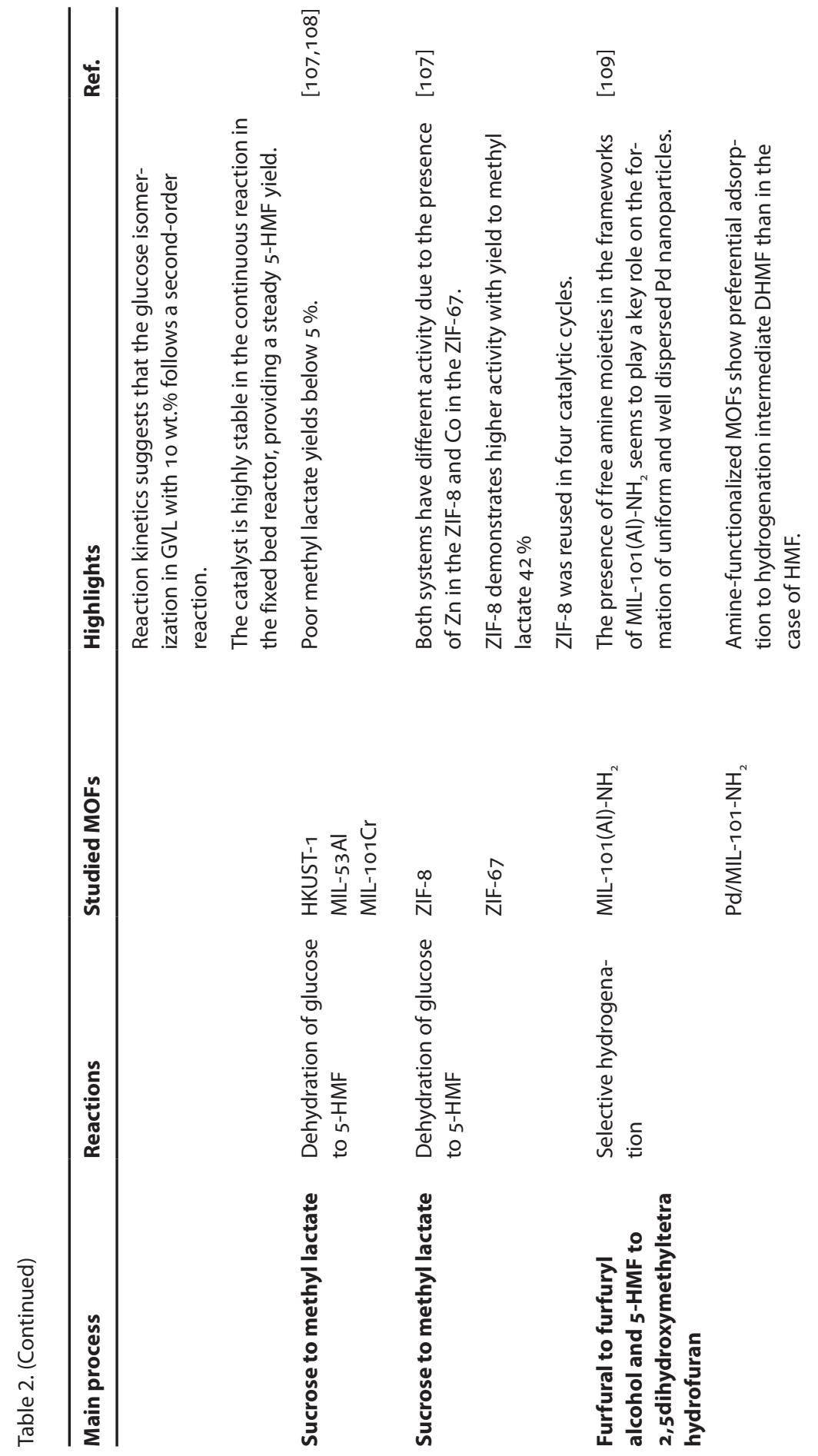


$\stackrel{ }{ }$

$\stackrel{F}{r}$

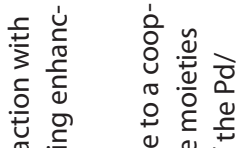

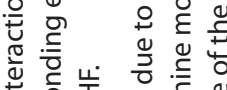

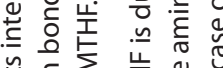

气 さ

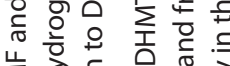

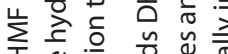

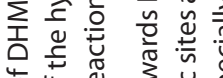

4

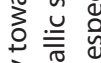

ڤั)

त苋

ฮั

㝴 응

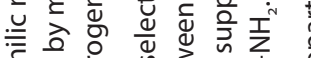

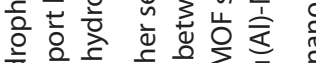

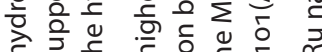

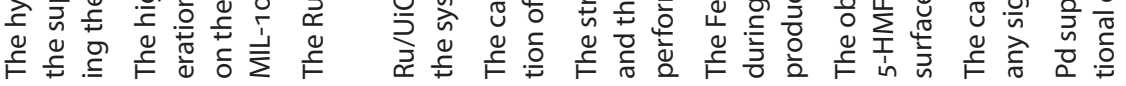

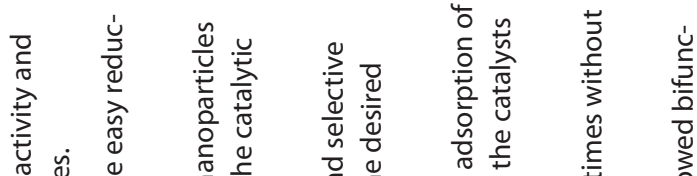

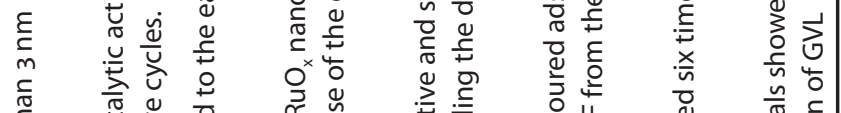

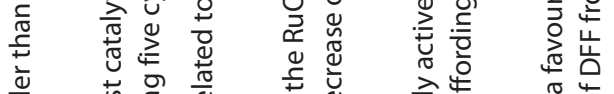

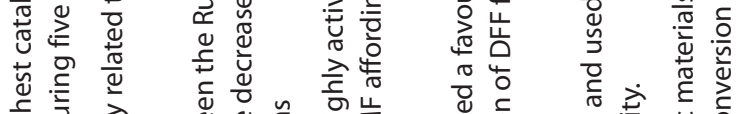

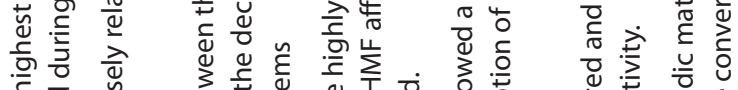

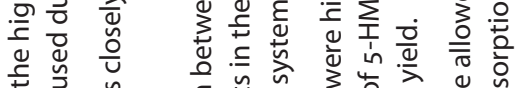

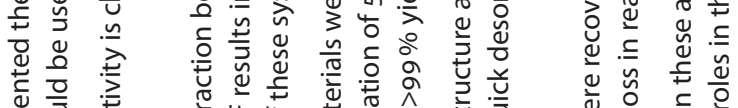
论

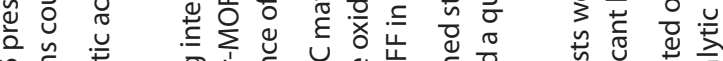

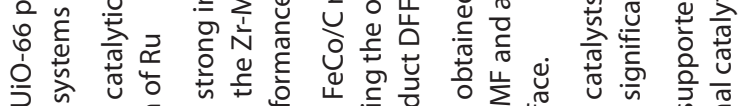

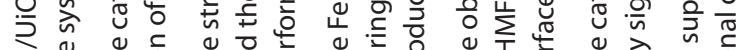
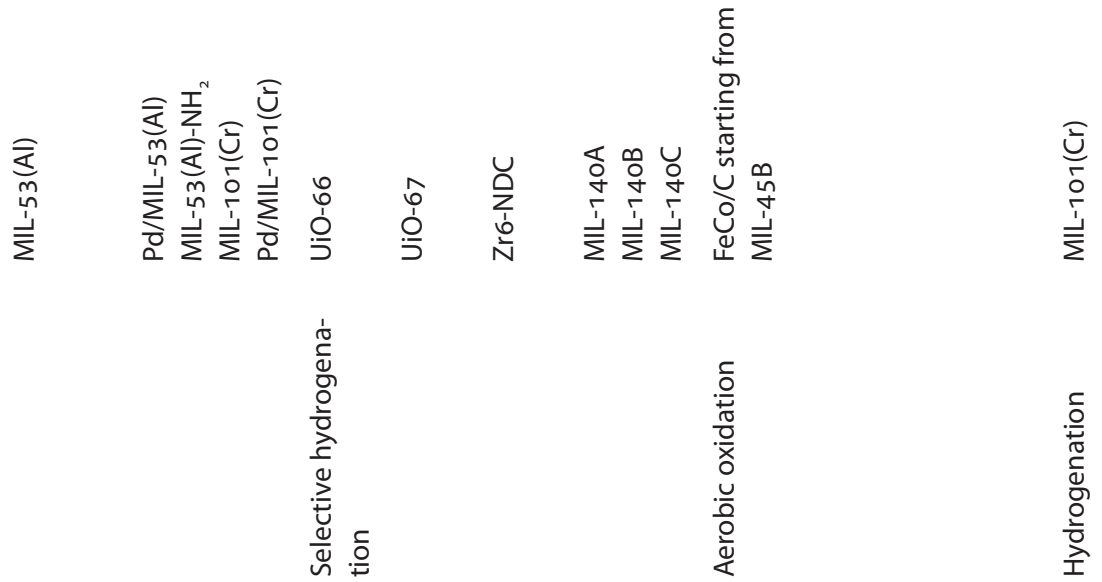

$\frac{5}{0}$
$\frac{0}{0}$
$\frac{0}{x}$
0
.$\frac{U}{0}$
$\frac{0}{0}$
$\frac{0}{4}$
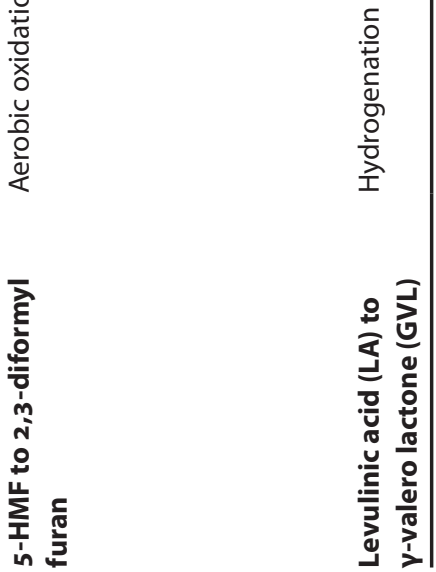


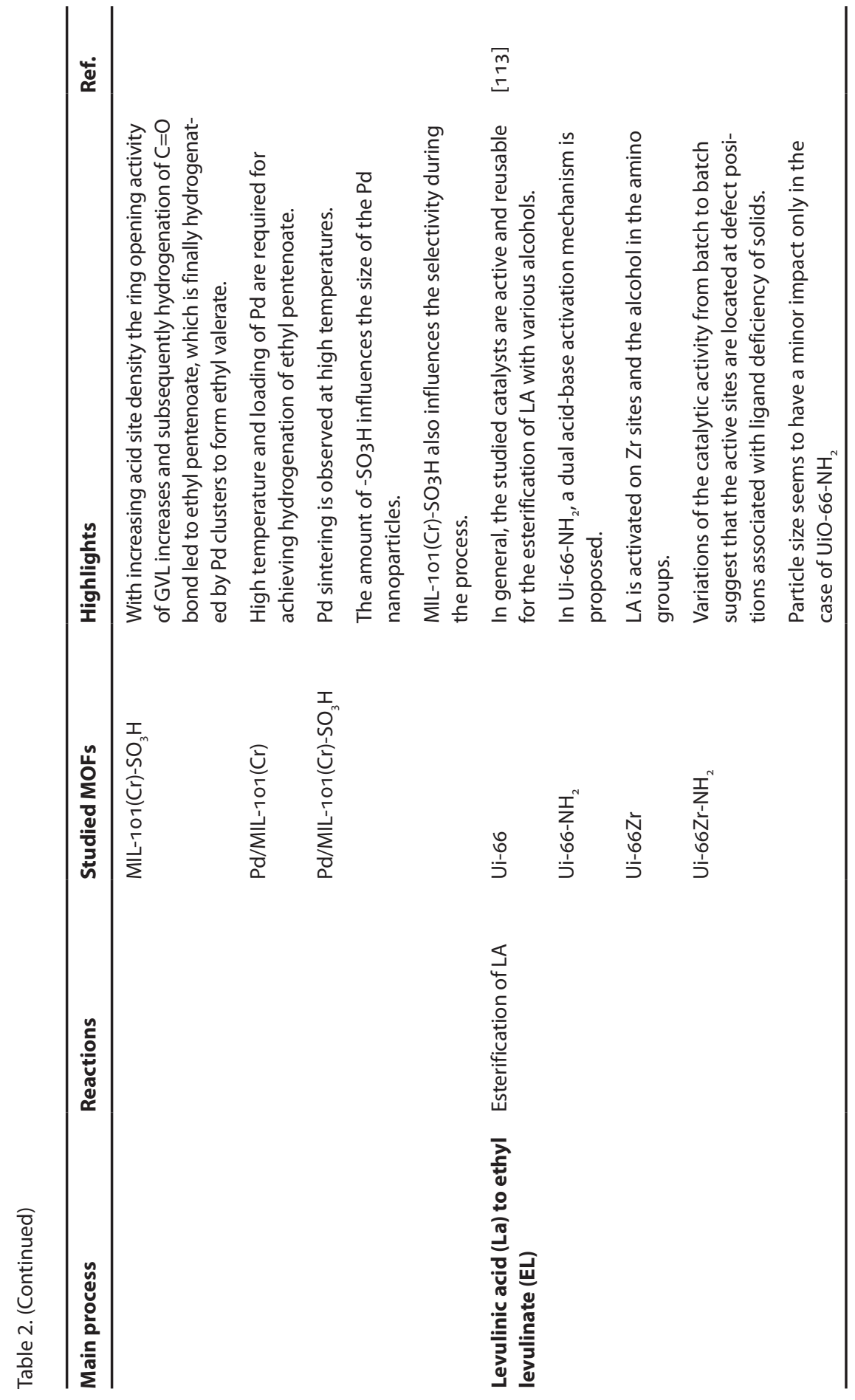


performance of the catalyst was attributed to the stability of the support, which maintained a high dispersion of the Pd nanoparticles. Additionally, the electronic activation of the reactants and the good dispersion of the catalyst in the water media were evidenced [114]. The same research team also reported the use of MOF catalysts based on the UiO-66 structure in the hydrodeoxygenation of vanillin [115]. The catalysts were prepared by introducing ultra-small Pd nanoparticles $\left(1.5^{-2.5} \mathrm{~nm}\right)$ into a highly porous and hydrothermally stable amine-functionalized UiO-66 $\left(\mathrm{NH}_{2}-\mathrm{UiO}-66\right)$. The stable and leaching-resistant dispersion of the Pd nanoparticles was attributed to the presence of free amine moieties in the framework of the catalyst. Consequently, the amine/Pd ratio should be optimized.

To the best of our knowledge, the most recent work devoted to the transformation of vanillin proposed the use of a hybrid support consisting of MOF crystals and partially reduced graphene oxide (PRGO) nanosheets to disperse metal nanoparticles. The Ce-based MOF was wrapped with thin PRGO nanolayers, and the obtained 5 wt.\% Pd/PRGO/Ce-MOF catalyst exhibited high catalytic activity in the tandem hydrogenation-deoxygenation reactions. Furthermore, it could be reused at least four times without any loss of activity or selectivity. The inclusion of PRGO seems to promote the selectivity of the process towards the production of 2-methoxy-4-methylphenol, which is an important phenol-type compound with high added value. In addition, PRGO provides stability and acidic sites that promote the hydrogenolysis reaction.

\section{Concluding remarks}

Zeolites and MOFs are very suitable materials to be used in reactions of biomass-to-chemicals. The chemical valorization of the lignocellulosic biomass involves a sequence of different catalytic processes, such as depolymerization, hydrolysis, hydrogenation, dehydration, isomerization, and oxidation reactions, among others. Thus, the high versatility of these materials, which extends to different porous structures (pore dimension and void volume), shape selectivity, number and nature of active sites (single or multiple), etc., makes them ideal for these applications by means of adjusting their structures to the desired reaction.

Literature reports have largely evidenced the strong capabilities of zeolites for the transformation of lignocellulosic biomass feedstocks, whereas the application of MOF-based catalysts goes almost unexplored. Few works on MOFs have been published so far, and only two MOF families (MIL-101 
and UiO-66) have demonstrated their stablilty under typical reaction conditions involving water and strongly acidic environments. Therefore, the design of novel MOFs is necessary, at the same time aiming to adapt the properties of the catalysts to the particular reaction under study. To this end, the tunability of Lewis and Brønsted acidity, as well as the nature of the open metal sites or the control of the hydrogen bonding interactions through the linkers are parameters amenable to modification. Furthermore, the hydrophilicity/hydrophobicity must be optimized to control the adsorption processes during the reactions [8o].

Apart from these considerations, the evolution of MOF-based catalysts for lignocellulosic biomass transformation must follow the principles of green chemistry [116]. One of these principles is the efficient utilization of raw materials. Thus, MOF catalysts need to be designed for the deconstruction of residual lignocellulosic biomass, which avoids competing with the food supply. However, this is a challenging task, since the processing of real residual biomass presents a huge complexity, including the presence of strongly deactivating species, such as sulphur- and nitrogen-containing compounds. Despite this, the success in using MOFs for this purpose would contribute both to the remediation of biomass waste and to the decreasing of the $\mathrm{CO}_{2}$ footprint as bio-derived fuels and fine chemicals would come from renewable carbon sources. Another approach that would improve the efficient use of resources would be the use of molecules derived from other transformations of lignocellulosic biomass in a biorefinery. This would also contribute to the decrease of $\mathrm{CO}_{2}$ emissions and would bring economic and environmental benefits.

\section{Acknowledgments}

The authors thank the support from the Spanish Ministerio de Economía y Competitividad (MINECO) (ENE2015-66975-C3-2-R and ENE2013-4788o$\mathrm{C}_{3}-2-\mathrm{R}$ ) co-financed by FEDER funds from the European Union. Financial support from the Junta de Andalucía to the Surface Chemistry and Catalysis research group (TEP106) is specifically acknowledged.

\section{References}

[1] Field, C.B., Behrenfeld, M.J., Randerson, J.T., Falkowski, P. Science 1998, 281, 237.

[2] Huber, G.W., Iborra, S., Corma, A. Chem. Rev. 2006, 106, 4044.

[3] Dodds, D.R., Gross, R.A. Science 2007, 318, $125^{0 .}$ 
[4] Baily, M., Enari, T.M., Linko, M., Halliwell, G. In SITRA Symposium on Enzymatic Hydrolysis of Cellulose, Finland, 1975.

[5] Fan, L.T., Gharpuray, M.M., Lee, Y.H. Cellulose Hydrolysis, Springer, 1987.

[6] Devendra, L.P., Kumar, K.M., Pandey, A. Biores. Technol. 2016, 213, 350.

[7] Serrano-Ruiz, J.C., Luque, R., Sepulveda-Escribano, A. Chem. Soc. Rev. 2011, 40, 5266.

[8] Lin, H., Strull, J., Liu, Y., Karmiol, Z., Plank, K., Miller, G., Guo, Z., Yang, L. Energy J. Environ. Sci. 2012, 5, 9773.

[9] Nowakowski, D.J., Bridgwater, A.V., Elliott, D.C., Meier, D., de Wild, P.J.J. Anal. Appl. Pyrolysis 2010, 88, 53 .

[10] Liu, C., Wang, H., Karim, A.M., Sun, J., Wang, Y. Chem. Soc. Rev. 2014, 43, 7594.

[11] Zakzeski, J., Bruijnincx, P.C.A., Jongerius, A.L., Weckhuysen, B.M. Chem. Rev. 2010, 110, 3552.

[12] Björkman, A. Sven. Papperstidn 1956, 59, 477.

[13] Shuai, L., Luterbacher, J. ChemSusChem 2016, 9, 133.

[14] Piqueras, C.M., Cabeza, A., Gallina, G., Cantero, D.A., Garcia-Serna, J., Cocero, M.J. Chem. Eng.J. 2017, 308, 110.

[15] Corma, A., Iborra, S., Velty, A. Chem. Rev. 2007, 107, 2411.

[16] Zhang, X., Wilson, K., Lee, A.F. Chem. Rev. 2016, 116, 12328.

[17] Climent, M.J., Corma, A., Iborra, S. Zeolites and Catalysis: Synthesis, Reactions and Applications, Wiley-VCH, 2010.

[18] Dapsens, P.Y., Mondelli, C., Perez-Ramirez, J. ACS Catal. 2012, 2, 1487.

[19] Ennaert, T., Van Aelst, J., Dijkmans, J., De Clercq, R., Schutyser, W., Dusselier, M., Verboekend, D., Sels, B.F. Chem. Soc. Rev. 2016, 45, 584.

[20] Li, H., Yang, S., Riisager, A., Pandey, A., Sangwan, R.S., Saravanamurugan, S., Luque, R. Green Chem. 2016, 18, 5701.

[21] Corma, A., Nemeth, L.T., Renz, M., Valencia, S. Nature 2001, 412, 423.

[22] Bhosale, S.H., Rao, M.B., Deshpande, V.V. Microbiol. Mol. Biol. Rev. 1996, 6o, 280.

[23] Moreau, C., Durand, R., Roux, A., Tichit, D. Appl. Catal., A 2000, 193, 257.

[24] Moliner, M., Roman-Leshkov, Y., Davis, M.E. PNAS 2010, 107, 6164.

[25] Roman-Leshkov, Y., Moliner, M., Labinger, J.A., Davis, M.E. Angew. Chem. Int. Ed. 2010, 49, 8954.

[26] Gunther, W.R., Wang, Y., Ji, Y., Michaelis, V.K., Hunt, S.T., Griffin, R.G., Roman-Leshkov, Y. Nat. Commun. 2012, 3.

[27] van der Graaff, W.N.P., Tempelman, C.H.L., Li, G., Mezari, B., Kosinov, N., Pidko, E.A., Hensen, E.J.M. ChemSusChem 2016, 9, 3145 .

[28] Lew, C.M., Rajabbeigi, N., Tsapatsis, M. Micropor. Mesopor. Mater. 2012, 153, 55.

[29] Kobayashi, H., Komanoya, T., Guha, S.K., Hara, K., Fukuoka, A. Appl. Catal., A 2011, 409, 13.

[30] Huber, G.W., Chheda, J.N., Barrett, C.J., Dumesic, J.A. Science 2005, 308, 1446.

[31] Deng, W., Tan, X., Fang, W., Zhang, Q., Wang, Y. Catal. Lett. 2009, 133, 167.

[32] Negoi, A., Triantafyllidis, K., Parvulescu, V.I., Coman, S.M. Catal. Today 2014, 223, 122.

[33] Ennaert, T., Geboers, J., Gobechiya, E., Courtin, C.M., Kurttepeli, M., Houthoofd, K., Kirschhock, C.E.A., Magusin, P.C.M.M., Bals, S., Jacobs, P.A., Sels, B.F. ACS Catal. 2015, 5, 754.

[34] Bozell, J.J., Petersen, G.R. Green Chem. 2010, 12, 539.

[35] Teong, S.P., Yi, G., Zhang, Y. Green Chem. 2014, 16, 2015.

[36] Moreau, C., Durand, R., Pourcheron, C., Razigade, S. Ind. Crops Prod. 1994, 3, 85.

[37] Moreau, C., Durand, R., Peyron, D., Duhamet, J., Rivalier, P. Ind. Crops Prod. 1998, 7, 95.

[38] Kim, S.B., You, S.J., Kim, Y.T., Lee, S., Lee, H., Park, K., Park, E.D. Korean J. Chem. Eng. 2011, 28,710 .

[39] Roman-Leshkov, Y., Chheda, J.N., Dumesic, J.A. Science 2006, 312, 1933.

[40] Choudhary, V., Pinar, A.B., Sandler, S.I., Vlachos, D.G., Lobo, R.F. ACS Catal. 2011, 1, 1724.

[41] Yang, G., Pidko, E.A., Hensen, E.J.M. ChemSusChem 2013, 6, 1688. 
[42] Tan, M., Zhao, L., Zhang, Y. Biomass Bioenergy 2011, 35, 1367.

[43] Guo, J., Zhu, S., Cen, Y., Qin, Z., Wang, J., Fan, W. Appl. Catal. B 2017, 200, 611.

[44] Arias, K.S., Climent, M.J., Corma, A., Iborra, S. ACS Sustain. Chem. Eng. 2016, 4, 6152.

[45] Yan, K., Jarvis, C., Gu, J., Yan, Y. Renew. Sustain. Energy Rev. 2015, 51, 986.

[46] Chen, H., Yu, B., Jin, S. Biores. Technol. 2011, 102, 3568.

[47] Guerbuez, E.I., Wettstein, S.G., Dumesic, J.A. ChemSusChem 2012, 5, 383.

[48] Ya'aini, N., Amin, N.A.S., Asmadi, M. Biores. Technol. 2012, 116, 58.

[49] Ya'aini, N., Amin, N.A.S., Endud, S. Micropor. Mesopor. Mater. 2013, 171, 14.

[50] Fernandes, D.R., Rocha, A.S., Mai, E.F., Mota, C.J.A., Teixeira da Silva, V. Appl. Catal., A 2012, 425-426, 199.

[51] Saravanamurugan, S., Riisager, A. Catal. Commun. 2012, 17, 71.

[52] Qi, L., Horvath, I.T. ACS Catal. 2012, 2, 2247.

[53] Yepez, A., De, S., Salud Climent, M., Romero, A.A., Luque, R. Appl. Sci. 2015, 5, 532.

[54] Sheldon, R.A. Stud. Surf. Sci. Catal. 1991, 59, 33.

[55] Velarde, A.M., Bartl, P., Nießen, T.E.W., Hoelderich, W.F.J.J. Mol. Catal. A Chem. 2000, 157, 225.

[56] Dusselier, M., Van Wouwe, P., Dewaele, A., Makshina, E., Sels, B.F. Energy Environ. Sci. 2013, 6,1415 .

[57] Maki-Arvela, P., Simakova, I.L., Salmi, T., Murzin, D.Y. Chem. Rev. 2014, 114, 1909.

[58] Holm, M.S., Saravanamurugan, S., Taarning, E. Science 2010, 328, 602.

[59] Holm, M.S., Pagan-Torres, Y.J., Saravanamurugan, S., Riisager, A., Dumesic, J.A., Taarning, E. Green Chem. 2012, 14, 702.

[6o] Li, L., Stroobants, C., Lin, K., Jacobs, P.A., Sels, B.F., Pescarmona, P.P. Green Chem. 2011, 13,1175 .

[61] de Clippel, F., Dusselier, M., Van Rompaey, R., Vanelderen, P., Dijkmans, J., Makshina, E., Giebeler, L., Oswald, S., Baron, G.V., Denayer, J.F.M., Pescarmona, P.P., Jacobs, P.A., Sels, B.F. J. Am. Chem. Soc. 2012, 134, 10089.

[62] Dong, W., Shen, Z., Peng, B., Gu, M., Zhou, X., Xiang, B., Zhang, Y. Sci. Rep. 2016, 6, 26713.

[63] Taarning, E., Saravanamurugan, S., Holm, M.S., Xiong, J., West, R.M., Christensen, C.H. ChemSusChem 2009, 2, 625 .

[64] West, R.M., Holm, M.S., Saravanamurugan, S., Xiong, J., Beversdorf, Z., Taarning, E., Christensen, C.H.J. Catal. 2010, 269, 122.

[65] Shen, W., Tompsett, G.A., Hammond, K.D., Xing, R., Dogan, F., Grey, C.P., Conner Jr, W.C., Auerbach, S.M., Huber, G.W. Appl. Catal., A 2011, 392, 57.

[66] Kikhtyanin, O., Kelbichová, V., Vitvarová, D., Kubů, M., Kubička, D. Catal. Today 2014, 227,154 .

[67] Kikhtyanin, O., Chlubna, P., Jindrova, T., Kubicka, D. Dalton Trans. 2014, 43, 10628.

[68] Kubička, D., Kikhtyanin, O. Catal. Today 2015, 243, 10.

[69] Kubicka, D., Kubickova, I., Cejka, J. Catal. Rev. Sci. Eng. 2013, 55, 1.

[70] Saiz-Jimenez, C., Deleeuw, J.W. Org. Geochem. 1986, 10, 869.

[71] Ma, Z., Custodis, V., Hemberger, P., Baehrle, C., Vogel, F., Jeschke, G., van Bokhoven, J.A. Chimia 2015, 69, 597.

[72] Ma, Z., Troussard, E., van Bokhoven, J.A. Appl. Catal., A 2012, 423-424, 130.

[73] Ma, Z., van Bokhoven, J.A. ChemCatChem 2012, 4, 2036.

[74] Ma, Z., Custodis, V., van Bokhoven, J.A. Catal. Sci. Technol. 2014, 4, 766.

[75] Gayubo, A.G., Valle, B., Aguayo, A.T., Olazar, M., Bilbao, J.J. Chem. Technol. Biotechnol. 2010, $85,132$.

[76] Hicks, J.C. J.J. Phys. Chem. Lett. 2011, 2, 2280.

[77] Gayubo, A.G., Aguayo, A.T., Atutxa, A., Prieto, R., Bilbao, J. Energy Fuels 2004, 18, 1640.

[78] Deng, S.M., Fan, M.H., Wang, T.J., Li, Q.X. Chinese J. Chem. Phys. 2014, 27, 361. 
[79] Dhakshinamoorthy, A., Alvaro, M., Corma, A., Garcia, H. Dalton Trans. 2011, 40, 6344.

[8o] Herbst, A., Janiak, C. Cryst. Eng. Comm. 2017.

[81] Kitagawa, S., Kitaura, R., Noro, S. Angew. Chem. Int. Ed. 2004, 43, 2334.

[82] Ferey, G. Chem. Soc. Rev. 2008, 37, 191.

[83] Bloch, E.D., Britt, D., Lee, C., Doonan, C.J., Uribe-Romo, F.J., Furukawa, H., Long, J.R., Yaghi, O.M.J. Am. Chem. Soc. 2010, 132, 14382.

[84] Deng, H., Doonan, C.J., Furukawa, H., Ferreira, R.B., Towne, J., Knobler, C.B., Wang, B., Yaghi, O.M. Science 2010, 327, 846.

[85] Volkringer, C., Cohen, S.M. Angew. Chem. Int. Ed. 2010, 49, 4644.

[86] Bernt, S., Guillerm, V., Serre, C., Stock, N. Chem.Commun. 2011, 47, 2838.

[87] Farrusseng, D., Aguado, S., Pinel, C. Angew.Chem. Int. Ed. 2009, 48, 7502.

[88] Akiyama, G., Matsuda, R., Sato, H., Takata, M., Kitagawa, S. Adv. Mater. 2011, 23, 3294.

[89] Furukawa, H., Cordova, K.E., O’Keeffe, M., Yaghi, O.M. Science 2013, 341, 1230444.

[9o] Qadir, N.U., Said, S.A.M., Bahaidarah, H.M. Micropor. Mesopor. Mater. 2015, 201, 61.

[91] Burtch, N.C., Jasuja, H., Walton, K.S. Chem. Rev. 2014, 114, 10575.

[92] Kruger, J.S., Nikolakis, V., Vlachos, D.G. Curr. Opin. Chem. Eng. 2012, 1, 312.

[93] Gupta, K.M., Zhang, K., Jiang, J.W. Sci. Rep. 2015, 5.

[94] Yabushita, M., Li, P., Bernales, V., Kobayashi, H., Fukuoka, A., Gagliardi, L., Farha, O.K., Katz, A. Chem. Commun. 2016, 52, 7094.

[95] Akiyama, G., Matsuda, R., Sato, H., Kitagawa, S. Chem. Asian J. 2014, 9, 2772.

[96] Chen, J., Wang, S., Huang, J., Chen, L., Ma, L., Huang, X. ChemSusChem 2013, 6, 1545.

[97] Huang, P., Yan, L.F. Chinese J. Chem. Phys. 2016, 29, 742.

[98] Allendorf, M.D., Stavila, V., Ramakrishnan, P., Davis, R. Sandia Report 2014, SAND201418259, prod.sandia.gov/techlib/access.

[99] Stavila, V., Parthasarathi, R., Davi, R.W., El Gabaly, F., Sale, K.L., Simmons, B.A., Singh, S., Allendorf, M.D. ACS Catal. 2016, 6, 55 .

[100] Wang, S., Chen, J., Chen, L. Catal. Lett. 2014, 144, 1728.

[101] Zhang, Y., Degirmenci, V., Li, C., Hensen, E.J.M. ChemSusChem 2011, 4, 59.

[102] Bromberg, L., Su, X., Hatton, T.A. Chem. Mater. 2014, 26, 6257.

[103] Chen, J., Li, K., Chen, L., Liu, R., Huang, X., Ye, D. Green Chem. 2014, 16, 2490.

[104] Hu, Z., Peng, Y., Gao, Y., Qian, Y., Ying, S., Yuan, D., Horike, S., Ogiwara, N., Babarao, R., Wang, Y., Yan, N., Zhao, D. Chem. Mater. 2016, 28, 2659.

[105] Herbst, A., Janiak, C. New J. Chem. 2016, 40, 7958.

[106] Su, Y., Chang, G., Zhang, Z., Xing, H., Su, B., Yang, Q., Ren, Q., Yang, Y., Bao, Z. AIChEJ. 2016, 62, 4403.

[107] Park, K.S., Ni, Z., Cote, A.P., Choi, J.Y., Huang, R., Uribe-Romo, F.J., Chae, H.K., O’Keeffe, M., Yaghi, O.M. PNAS 2006, 103, 10186.

[108] Murillo, B., Zornoza, B., de la Iglesia, O., Tellez, C., Coronas, J.J. Catal. 2016, 334, 6 o.

[109] Chen, J., Liu, R., Guo, Y., Chen, L., Gao, H. ACS Catal. 2015, 5, 722.

[110] Yuan, Q., Zhang, D., van Haandel, L., Ye, F., Xue, T., Hensen, E.J.M., Guan, Y.J. Mol. Catal. A Chem. 2015, 406, 58 .

[111] Fang, R., Luque, R., Li, Y. Green Chem. 2016, 18, 3152.

[112] Zhang, D., Ye, F., Guan, Y., Wang, Y., Hensen, E.J.M. RSC Adv. 2014, 4, 39558.

[113] Cirujano, F.G., Corma, A., Llabres i Xamena, F.X. Chem. Eng. Sci. 2015, 124, 52.

[114] Zhang, F., Jin, Y., Fu, Y., Zhong, Y., Zhu, W., Ibrahim, A.A., El-Shall, M.S. J. Mater. Chem. A 2015, 3, 17008.

[115] Zhang, F., Zheng, S., Xiao, Q., Zhong, Y., Zhu, W., Lin, A., El-Shall, M.S. Green Chem. 2016, 18, 2900.

[116] Sheldon, R.A. in Ionic Liquids in the Biorefinery Concept: Challenges and Perspectives, p 258, The Royal Society of Chemistry, 2016. 



\title{
6. Biocatalysis on Porous Materials
}

\author{
Isabel Díaz, Rosa María Blanco, Manuel Sánchez-Sánchez \\ and Carlos Márquez-Álvarez
}

Instituto de Catálisis y Petroleoquímica (CSIC), c/Marie Curie 2, 28049 Madrid, Spain.

Keywords: Enzymes, immobilization, porous solids, mesoporous materials, OMM, lipase, laccase

\section{Introduction}

The wide variety of reactions catalysed in nature in a selective fashion and under mild conditions makes enzymes the potentially ideal catalysts for industrial use. Enzyme application, however, is limited by their lability and solubility, and therefore the first step in achieving this goal is to make them insoluble. This can be achieved using several approaches (Figure 1), which include the formation of enzyme agglomerates by cross-linking using bifunctional linkers that react with amino acid residues, trapping of enzymes within polymer networks and binding of enzymes to solid supports. In the latter case, immobilization can be undertaken either by covalent attachment or by adsorption on porous solids, and materials of any imaginable origin or chemical nature have been used as supports [1-9].

Since the last decade, special attention has been paid to the material used as support, particularly leading to the development of Ordered Mesoporous Materials (OMM) $[10,11]$ as an alternative to the classical sol-gel entrapment. The advantageous features of the OMM over the previously available amorphous mesoporous silicas prepared by conventional sol-gel methods make them potentially ideal enzyme carriers. In particular, the optimal control of textural properties allows for designing and customising the mesostructure for specific applications. This possibility was explored early on, and since the pioneer works by Balkus et al. in 1996 [12], many different enzymes have been encapsulated in OMM [13]. 


\section{cross-linking}

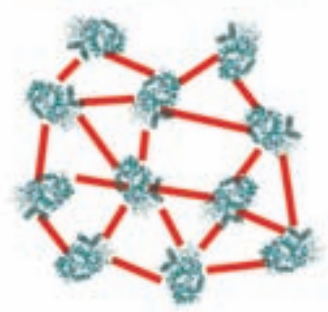

adsorption

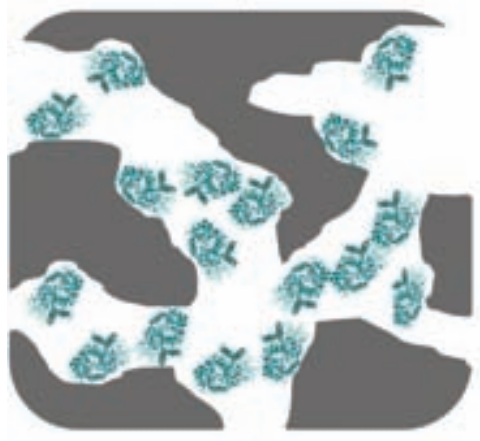

entrapment

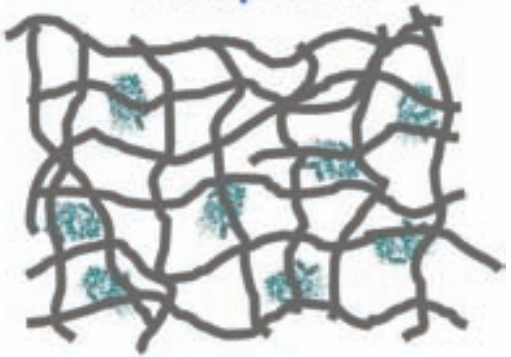

covalent bonding

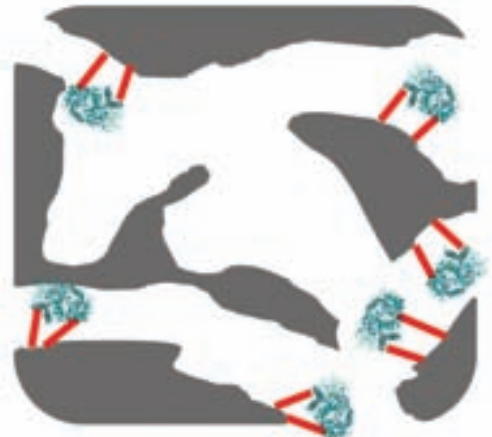

Figure 1. Different approaches employed to make enzymes insoluble.

\section{Zeolites versus Ordered Mesoporous Materials}

In biocatalysis, the catalytic active site is part of an enzyme, which is a macromolecule of large dimensions ranging from 100 to 500 amino acids long. Bearing this in mind, supporting enzymes in porous matrices to yield a heterogeneous catalyst requires the confinement of macromolecules within pores in the same size-range. In this scenario, zeolites, with pore sizes below the nanometer range, have been tested as supports of enzymes basically using the outer surface of the zeolite crystals. Some good reports can be found in which delaminated zeolites $[14,15]$ or pillared lamellar precursors of zeolites [16] were tested as biocatalyst supports. Delaminated zeolites become good enzyme supports due to their high surface area, to which the enzymes may be anchored via electrostatic interactions, or even covalent bonding. In this manner, supporting enzymes on zeolites potentially solves the drawbacks that are usually associated with encapsulation via sol-gel, such as enzyme aggregation and poor pore connectivity. However, this 
approach has brought limitations in leaching, conformation, and deactivation of the enzymes.

Nowadays, mesoporous silica structures can be fine-tuned controlling the synthesis conditions, using micelles of cationic, anionic or non-ionic surfactants as templates. The choice of the surfactant rules the $\mathrm{pH}$ of the synthesis gel in a way that further controls the hydrolysis and condensation of the silica source that will form the walls of the porous architecture. Cationic and anionic surfactants usually work well in forming mesoporous silica phases at high $\mathrm{pH}$, typically above 9 . At high $\mathrm{pH}$, silica would be negatively charged, creating electrostatic interactions with the cationic surfactant micelles and leading the formation of the amorphous pore walls. Thus, in early works, Ordered Mesoporous Materials were synthesized purely as aluminosilicates, using cationic surfactants in highly alkaline media [17]. Later on, anionic surfactants were employed and then aminosilane precursors had to be used in order to provide a positive charge at $\mathrm{pH}$ above 8 [18]. It could be said that, nowadays, the most widely reported OMM are those prepared using non-ionic block copolymer surfactants. These surfactants are commonly based on polyethylene and polypropylene chains. Hence, they aggregate efficiently in robust micelles only at very acidic $\mathrm{pH}$, even below $\mathrm{pH}=1$, at which the silica is positively charged and condensation takes place while assembling around the micelles via hydrogen bonds. The wide variety of synthesis conditions based on non-ionic surfactants has covered a full range of symmetries as well as pore sizes [19].

In any case, due to the sensitive nature of enzymes, the drastic synthesis conditions require immobilization to be limited only to a post-synthesis approach, i.e. the OMM has to be prepared in a first step, the surfactant has to be removed, and then the enzyme can be allocated inside the pores of the OMM. This approach was initially limited to (and tested with) enzymes that could fit in the obtained pore sizes, and those that could somehow interact with a pore surface made of amorphous silica. This strategy has now been reversed, and what we intend to show in the next few pages is how it is possible to design the right OMM support for a particular enzyme of a certain size and properties, using chemical affinities and confined space while maintaining biocatalytic activity. Furthermore, we have developed synthesis methods commonly employed for OMM in order to obtain this kind of heterogeneous biocatalysts by an in situ immobilization route [20].

The possibility of designing the solid matrix according to the characteristics of an enzyme is the key to enabling a step towards a second generation of enzyme supports, providing improved properties to the obtained biocatalysts $[11,21-23]$. Compared to the microporosity of zeolites, in which pore 


\section{IUPAC classification of pore sizes}

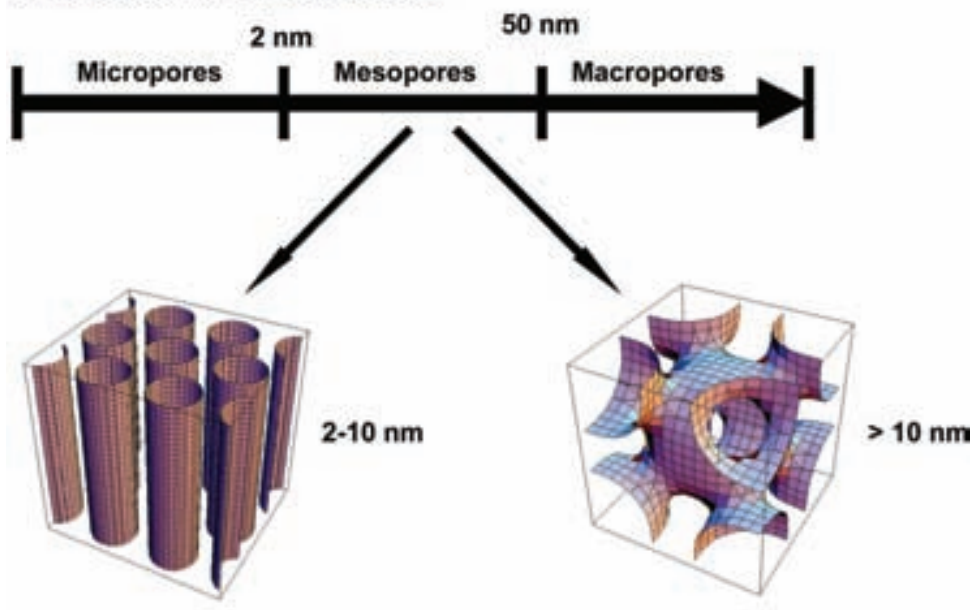

Channel like

Cage like

Figure 2. Classification of porous solids.

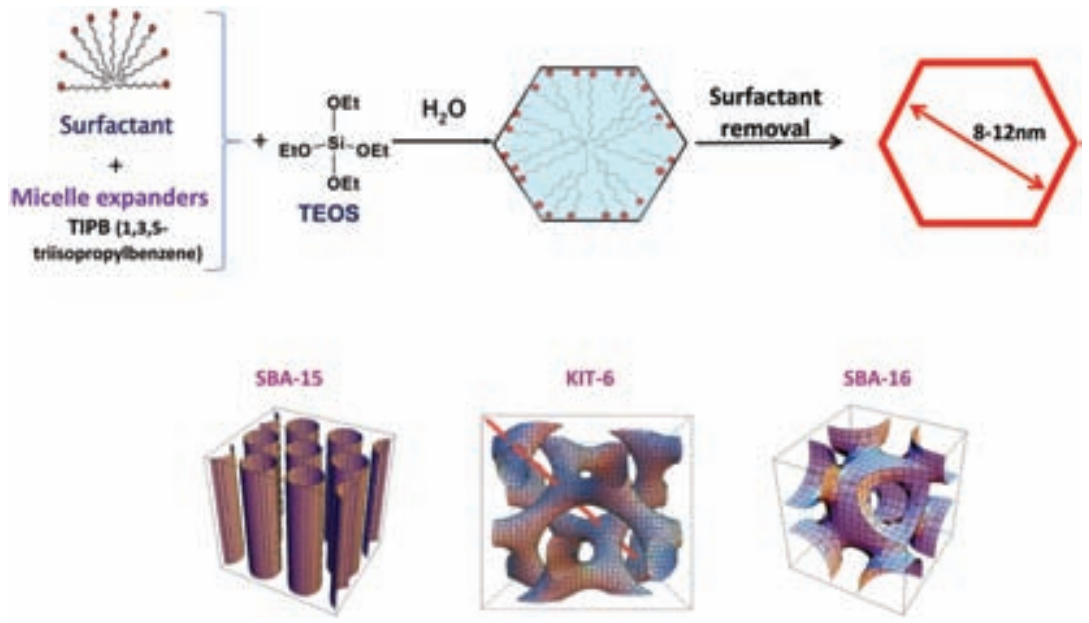

Figure 3. General synthesis procedure of Ordered Mesoporous Materials.

sizes are below $1.5 \mathrm{~nm}$, Ordered Mesoporous Materials show pores whose size ranges from 2.5 to $10 \mathrm{~nm}$ or more, by exploiting the use of surfactant micelles and micelle expanders as structure directing agents (Figure 2). Pore architectures formed by channels or cages can be easily designed during the synthesis process leading to tunable diffusion properties (Figure 3). 
Besides the pore size, the structure of OMM is characterized by the amorphous nature of the pore framework, as opposed to the crystallinity of zeolites, and a more flexible and versatile chemistry in these pore walls. The amorphous nature of the silicate framework leads to the presence of a high number of defects, i.e. non-condensed silanol groups, with an increased presence of these $\mathrm{OH}$ groups on the pore surface, which can be further used as anchoring points for functional groups. Surface functionalization has been widely reported. The versatility of alcoxysilane sources gives a wide range of functionalities as potential interactions for further immobilization of enzymes [24]. Two approaches could be used to include functional groups on the pore surface of OMM: grafting and co-condensation (Figure 4). Grafting refers to a two-step process in which the OMM is first synthesized to further react with an organosilane holding the desired functional group. The advantage of this method is the amount of functional groups that can be incorporated, which is mainly restricted by the number of $\mathrm{OH}$ available on the surface. However, the chemistry of this functionalization reveals that the process is very sensitive to the reaction conditions and pore size of the OMM. The alcoxysilane has to diffuse into the pores to anchor. Therefore, the distribution of functionalities along the pore surface may not be even or homogeneous. Finally, the grafting of functionalities implies a decrease in the final available pore size in this method. Alternatively, in the co-condensation approach the organosilane is incorporated in the material during the synthesis of the OMM. In this manner, the functionalities are already incorporated while interacting with the surfactant

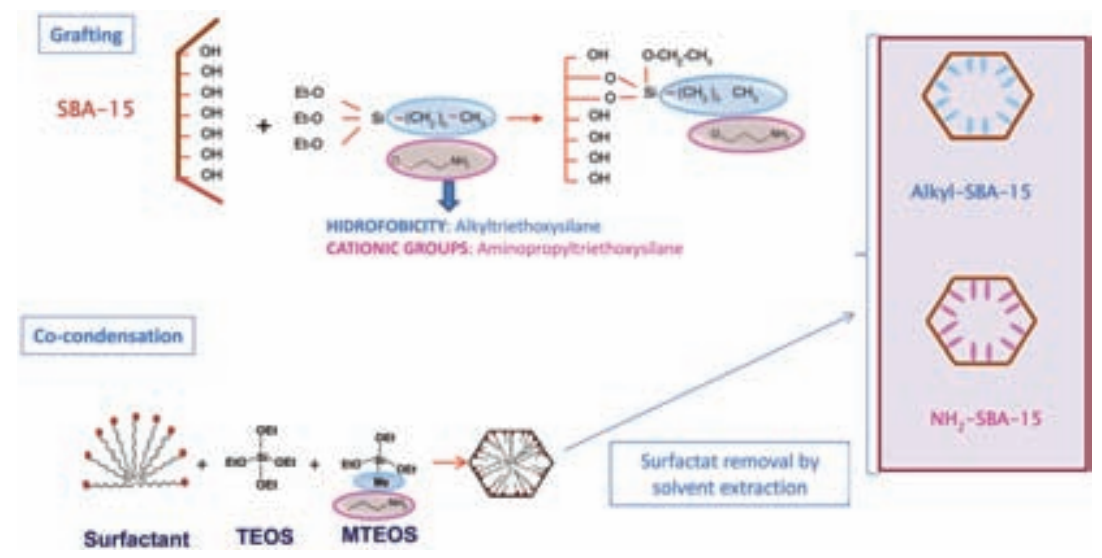

Figure 4. Two routes commonly employed in the functionalization of the pore surface of Ordered Mesoporous Materials. 
micelles, which are the pore directing agents. On the other hand, given the necessity of the silica-surfactant interaction to obtain a structured OMM, the number of functional groups should not be higher than $10 \%$ of the total number of silicon atoms. Furthermore, this type of functionalization is restricted to functional groups that do not alter the chemistry of the silica-surfactant interactions under the synthesis conditions $(\mathrm{pH}<1)$.

A third functionalization method in OMM should be described to further understand the possibilities of tailored design. Periodic Mesoporous Organosilicas (PMO) were introduced into the OMM community in 1999 [25] as a hybrid version of OMM in which a variety of organosilica sources actually form the pore walls, leading to very hydrophobic and very reactive pore walls [26]. These PMO are synthesized using $100 \%$ organodisilanes, which assemble around the surfactant micelles in a very weak manner via van der Waals forces. There are less $\mathrm{OH}$ groups remaining on the pore surface since the entire framework is built up from organic groups bridging $-\mathrm{Si}-(\mathrm{O}-\mathrm{Si}-)_{3}$ moieties. A more recent version of $\mathrm{PMO}$ is known as PMA (Periodic Mesoporous Aminosilicas), in which the same approach is used to introduce amino groups on the pore walls following the scheme in Figure 5 [27]. The synthesis conditions can be slightly altered to obtain even larger pores in which the two functionalities can be accommodated while producing large pores. In summary, depending on the enzyme to be used, the functional groups could be selected, and the functionalization method should be designed.

As mentioned before, the properties to be exploited from these supports include surface area, pore architecture, and functionalities. These properties are discussed and evaluated using $\mathrm{N}_{2}$ adsorption/desorption isotherms results as evidence of the adequate initial textural properties of the supports and how the pore volume decreases upon enzyme loading.

Periodic Mesoporous Organosilicas: PMO Periodic Mesoporous Aminosilicas: PMA

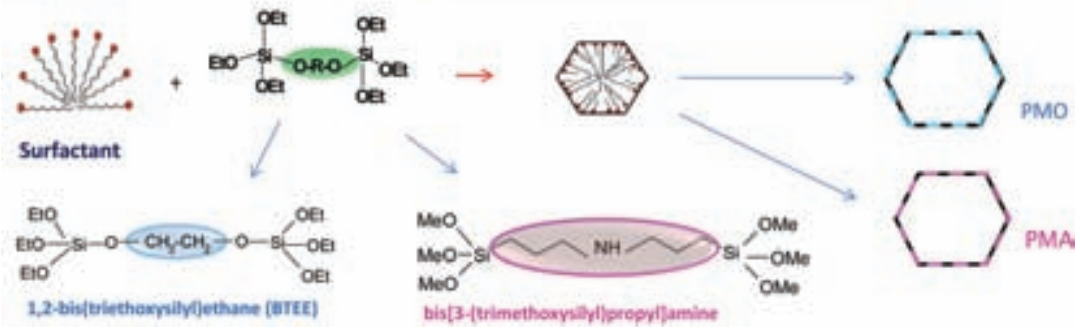

Figure 5. General synthesis scheme of Periodic Mesoporous Organosilicas. 
Thermogravimetry, chemical analysis, and solid state NMR enable the identification and quantification of the functionalities. Scanning electron microscopy (SEM) is used to evaluate the OMM particle size and morphology in those cases where we aim to control the particle growth to facilitate enzyme diffusion. Conventional transmission electron microscopy (TEM) allows identifying the pore architecture. However, given the low scattering power of carbon-based materials, it remains a challenge to locate the presence of enzymes inside the pores after immobilization. In this regard, we have devoted efforts to analyse the materials using advanced electron microscopy techniques such as scanning transmission electron microscopy (STEM) combined with a high-angle annular dark field (HAADF) detector, in which the contrast is related to the atomic number Z. With modern electron microscopes incorporating spherical aberration (Cs) correctors, probes down to sub-Angstrom resolution can be achieved, which facilitates an exhaustive analysis of the pores of the OMM. This, combined with electron energy loss spectroscopy (EELS), allows locating the enzymes inside the pores [28-30].

A high and permanent activity per unit weight or volume of the biocatalyst is the goal when designing the enzyme-support immobilization system. The support must provide high surface area to accommodate a high amount of protein molecules. This surface must contain residual groups to interact with side groups of the amino acids of the protein, either via covalent bonding or non-covalent interactions. The use of ordered mesoporous materials fulfils most of the requirements for an ideal enzyme-support system. In addition to the high surface area, these materials possess a uniform structure of the porous network and tuneable size and shape of the pores. Pores of just a few nanometers larger than the enzyme dimensions permit enzyme diffusion across them.

By means of covalent attachment, the enzyme is permanently linked to the support, so no leaching can happen. However, the enzyme molecules bonded at the external edges of the pores may act as a stopper, preventing the access of new molecules and decreasing the enzyme loading achievable. For this reason, non-covalent interaction is preferred for the immobilization of enzymes on OMM. Another drawback of covalent bonding is that it requires chemical modification of the protein, often leading to activity loss, as well as the impossibility of reusing the support after enzyme inactivation. Non-covalent link of the enzyme in OMM does not require chemical modification of the protein and the support can be reused after enzyme inactivation [31]. Exploiting the confined mesoporous space of OMM allows developing biocatalysts in which the close match between the 
sizes of the enzymes and the pores prevents leaching and preserves activity. Additionally, the narrow and uniform pores of OMM contribute to preventing the immobilization of enzyme aggregates and provide connectivity to favour a good diffusion of substrate and products.

Prior to describing enzyme-support immobilization systems with OMM, the parameters available to improve the biocatalysts are listed and described. These catalysts are defined by:

- Enzyme loading: miligrams of enzyme immobilized per gram of the siliceous material

- Time of immobilization: the time needed to complete the enzyme immobilization, i.e. when the activity of the supernatant is zero or decreases to a constant value.

- Catalytic activity: units of enzyme activity per gram of final catalyst (immobilized enzyme). A unit of enzyme activity is defined as the micromoles of substrate transformed by the enzyme per minute.

- Specific activity (also called catalytic efficiency): the ratio between catalytic activity and enzyme loading, this is, the units of activity per milligram of immobilized enzyme. This is a crucial parameter that serves to compare different catalysts (regardless of the enzyme loading of them) and indicates the activity preserved by the enzyme upon immobilization. Specific activity is the best way to gauge how efficient the immobilization system is.

\section{Hydrophobic porous supports for lipase immobilization}

Lipases are glycerol hydrolases: these enzymes catalyse the hydrolysis of ester bonds, particularly those between glycerol and fatty acids. This family of enzymes shares as a common feature the presence of a hydrophobic domain on the surface, which becomes part of their regulation mechanism in living beings. The hydrophobic amino acids interact among them acting as a lid, which is closed in aqueous medium preventing the access of substrates to the active centre. In the presence of hydrophobic interfaces a conformational change occurs and the lid opens. This is known as interfacial activation [32]. These amino acids establish new interactions with the hydrophobic interface, which gives access to the active centre. This phenomenon has been profusely exploited for the immobilization of lipases on hydrophobic surfaces of support materials. The particular enzyme in this case is lipase from Candida antarctica $\mathrm{B}$, which has a molecular weight of $35 \mathrm{kDa}$ and approximate molecular dimensions of $3 \mathrm{~nm} \times 4 \mathrm{~nm} \times 5 \mathrm{~nm}$. 
Although this enzyme lacks the lid, it has a hydrophobic domain on its surface. Siliceous OMM and functionalized OMM with surfaces chemically modified by alkyl groups were obtained to study how the size and shape of the pores may affect the parameters related to enzyme immobilization, namely, enzyme loading, activity, leaching, and stabilization of the enzyme [33].

Two purely siliceous supports with channel-like pore structure were obtained:SBA-15, consisting of parallel cylindrical pores in a $2 \mathrm{D}$ hexagonal arrangement, and KIT-6, which has two sets of interweaving, non-intersecting channels in a bicontinuous cubic symmetry (see Figure 3). To prevent an excessive decrease in the pore diameter, functionalization was performed with methyl groups. Methylation via co-condensation with methyltriethoxysilane produced the respective functionalized materials bearing pendant methyl groups (see Figure 4) Me-SBA-15 and Me-KIT-6. PMO, a hybrid organosilica with $2 \mathrm{D}$ hexagonal arrangement of parallel cylindrical pores, was synthesized as described above (see Figure 5), containing bridging ethylene groups as part of the surface of the pores.

The textural properties of supports are collected in Table 1 along with the immobilization results. The obtained pore sizes seem to be large enough for the dimensions of the CaLB. However, the enzyme loading in purely siliceous materials only reached moderate values and the corresponding methylated counterparts did not surpass these. The mild hydrophobicity provided by methyl groups does not seem to be enough to compensate the decrease in pore diameter when alkyl groups are anchored. The enzyme loading increased in PMO, in which ethylene groups are present in higher amounts than the methyl ones in Me-SBA-15 and Me-KIT-6 [34].

Table 1. Textural properties of supports, enzyme adsorption time and loading, and activity of the immobilized lipase in tributyrin hydrolysis.

\begin{tabular}{lcccccc}
\hline Material & Pd nm & $\mathbf{S}_{\text {BET }} \mathbf{m}^{2} / \mathbf{g}$ & $\mathbf{t} \mathbf{m i n}$ & $\begin{array}{l}\text { Enz Load } \\
\mathbf{m g} / \mathbf{g}\end{array}$ & $\begin{array}{l}\text { Cat Act } \\
\mathbf{U} / \mathbf{g}\end{array}$ & $\begin{array}{l}\text { Specif Act } \\
\mathbf{U} / \mathbf{m g}\end{array}$ \\
\hline SBA-15 & 8.8 & 890 & 120 & 44 & 2.647 & 60 \\
Me-SBA-15 & 7.9 & 794 & 200 & 23 & 2.009 & 88 \\
KIT-6 & 8.4 & 917 & 280 & 37 & 2.442 & 66 \\
Me-KIT-6 & 7.7 & 984 & 50 & 35 & 2.884 & 82 \\
PMO & 7.1 & 960 & 60 & 91 & 18.280 & 202 \\
AS & 27.9 & 305 & 120 & 45 & 4.552 & 100 \\
OAS & 23.6 & 294 & 60 & 200 & 13.000 & 83 \\
\hline
\end{tabular}

Free enzyme activity: $533 \mathrm{U} / \mathrm{mg}$ 


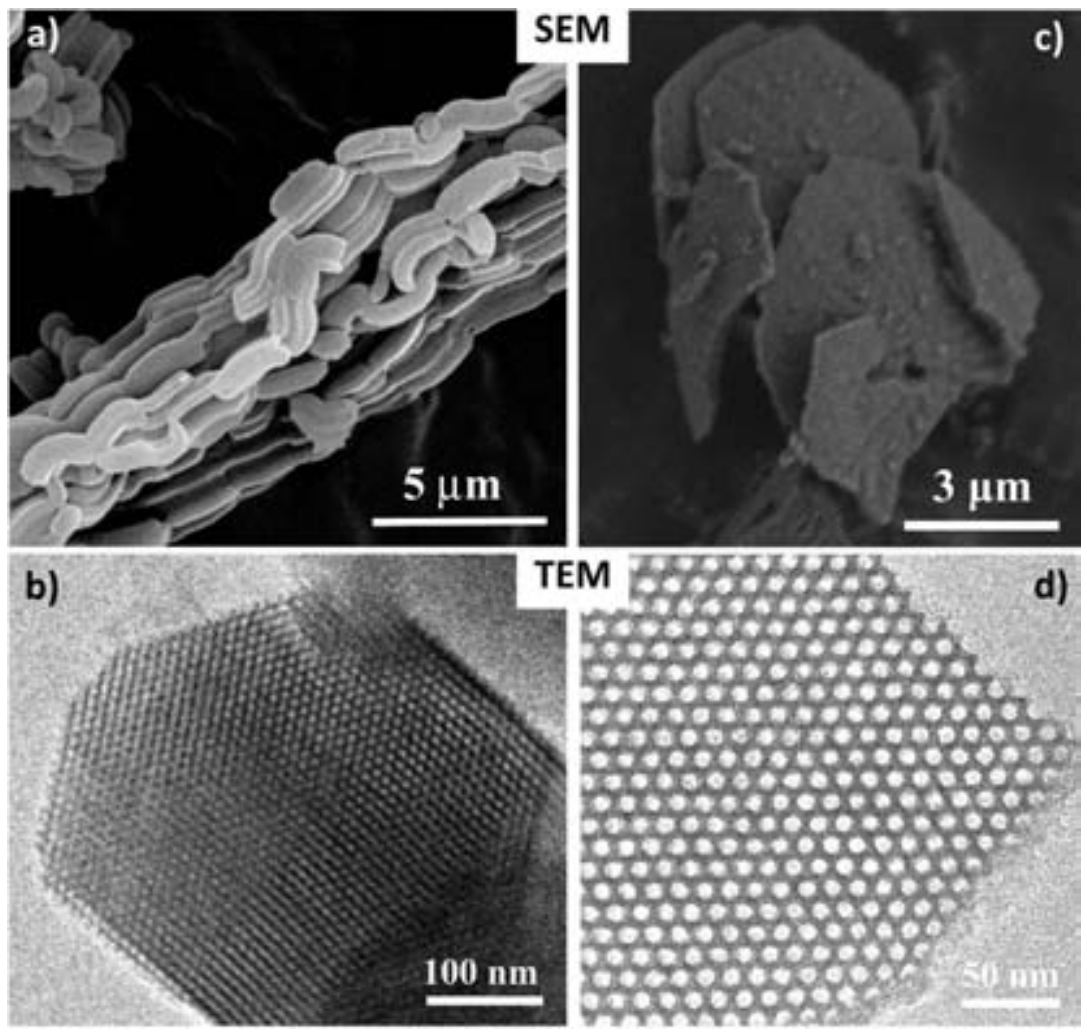

Figure 6. Scanning (SEM) and Transmission (TEM) Electron Microscopy of SBA-15 prepared under different synthesis conditions obtaining long (SBA-15-L) and short (SBA-15-S) channels.

Transmission electron microscopy studies allowed the porous structure to be revealed, along with the scanning of electron microscopy, which showed the morphology of the materials. Figures $6 \mathrm{a}$ and $6 \mathrm{~b}$ show the rodlike morphology of Me-SBA-15 (SBA-15-L in Figure 6) and the hexagonal arrangement of channels along the axis of the rods. Diffusion in such long fibres is predictably difficult. However, in the case of PMO, despite the presence of similar crystal morphology (Figure $7 \mathrm{a}$ ), the higher hydrophobicity of the pore walls seems to be the driving force for the lipase to diffuse in and load the channels. Advanced electron microscopy techniques in this case allowed the identification of the lipase macromolecules inside the channels of the hexagonal PMO structure. Figure $7 \mathrm{~b}$ shows a Cs-STEM image in which the carbon contrast is not observed due to its weak scattering power even though the Z-contrast mode was used (i.e. the High Angular Annular Dark Field Detector, HAADF). However, a careful exposure of the area to 

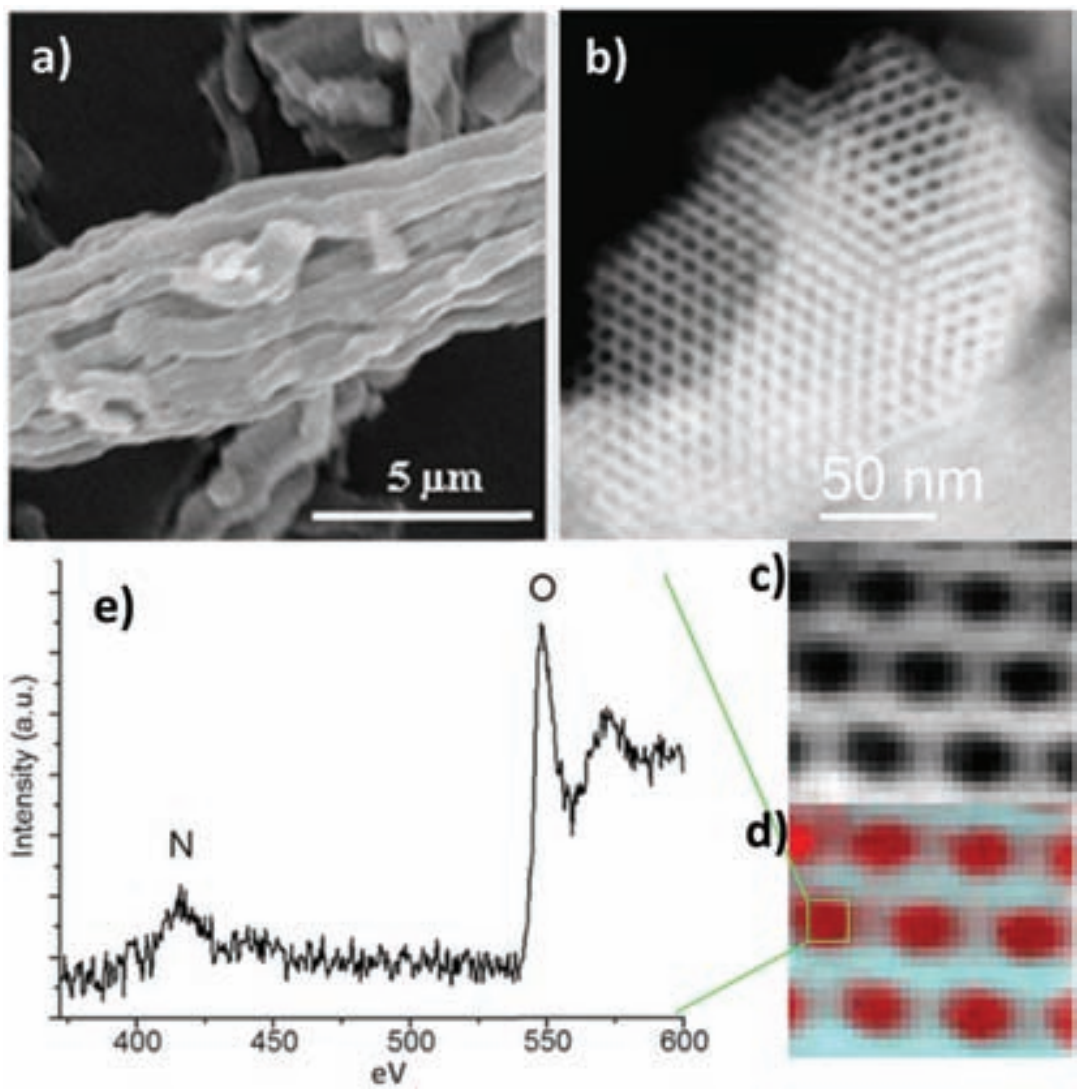

Figure 7. a) Morphology of PMO particles observed by SEM, b) Cs-corrected STEM-HAADF image of lipase/PMO, c) Cs-corrected STEM-HAADF image of interest, d) image in which red areas correspond to a higher content of carbon, e) extracted EELS signals from the inner area of the channels indicate that the areas with a high content in $\mathrm{C}$ also contain $\mathrm{N}$, which correspond to the enzymes.

the electron beam, controlling the electron dose and voltage, allowed obtaining the Electron Energy Loss Spectra (EELS) of a small area depicted in the inset of the image. Figure $7 \mathrm{c}$ shows a small region in which the red areas are those with a higher $\mathrm{C}$ content. At the same time, it is possible to confirm that the centre of a pore (in red) corresponds to the loading of the lipase by punctual EEL spectra (Figure $7 \mathrm{~d}$ ).

Enzyme leaching was also related to both the shape and chemical surface of the pores. The purely siliceous surface failed to retain the enzyme in the straight, parallel, cylindrical pores, but it succeeded in siliceous KIT- 6 because of the tortuosity of the pore network in this material (Figure 8). The materials containing hydrophobic organic moieties were efficient in preventing enzyme leaching in both pore structures. In all these cases, an 


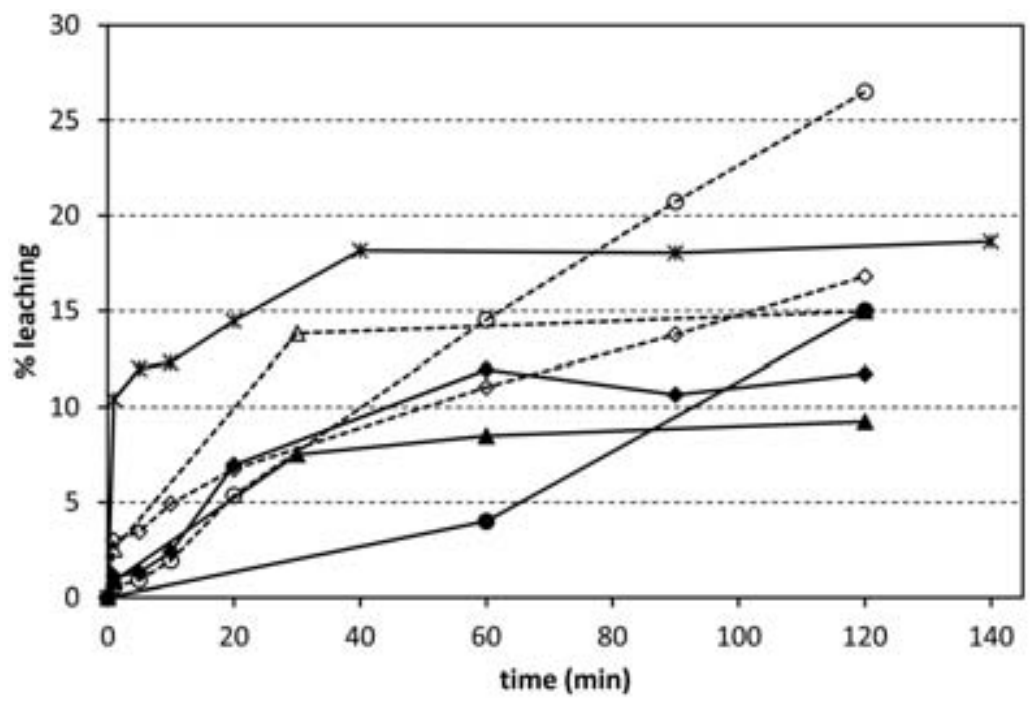

Figure 8. Leaching courses of lipase from OMM (channel pores) and amorphous silica materials, both in purely siliceous form and functionalized with hydrophobic groups. Empty rhombus: SBA-15. Filled rhombus: Me-SBA-15. Empty triangles: KIT-6. Filled triangles: Me-KIT-6. Empty circles: Amorphous silica. Filled circles: octyl-amorphous silica. Asterisk: PMO.

initial fast protein release was detected during the leaching tests. This was attributed to molecules immobilized on the external surfaces of the particles or at the external edges of the pores, less protected by the confinement provided by the pores. After this, the enzyme remained within pores of methylated SBA-15 and KIT-6, as well as in PMO.

Since the enzyme loading is different in each case, the activity of the catalysts is compared in terms of specific activity (i.e. per miligam of immobilized lipase). The activity is similar in the catalysts to methylated surfaces and higher than in the purely siliceous ones (see Table 1). PMO-lipase also retained more activity with values of catalytic activity and specific activity significantly higher than in the other systems. Therefore, even with the same type of pores, the higher hydrophobicity of PMO enabled an improvement in the obtained catalyst compared to Me-SBA-15 [35].

Cage-like structures were also evaluated as an alternative to retain the enzymes. Cages can lead to a higher activity because the enzyme has more free space to move and adjust its conformation to the reaction states. The idea was to provide some degree of hydrophobicity as in the channel-like materials. We prepared Me-SBA-16 via the co-condensation method, although we failed to prepare cage-like PMO type of materials. Instead, we tackled a more delicate synthesis method that implied a 

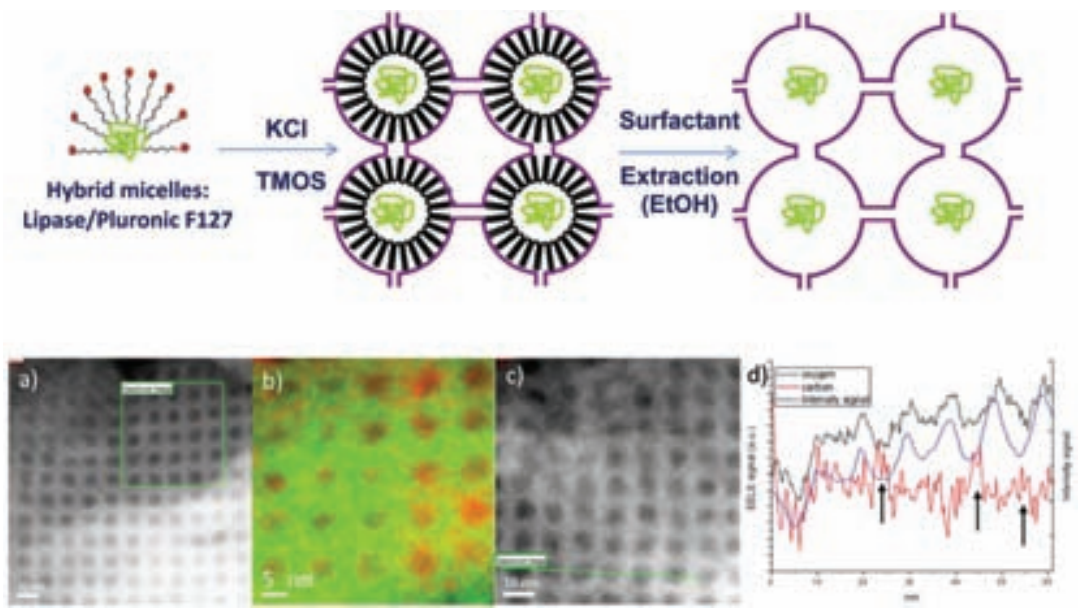

Figure 9. Synthesis scheme for the preparation of 'in situ' lipase/SBA-12, a cage-like OMM. In the lower part of the Figure: a) Cs-corrected STEM-HAADF, where the area of interest is marked by a green square. b). Spectrum image extracted by the carbon (in red) and oxygen (green) signals. c) Cs-corrected STEM-HAADF image with the spectrum profile marked as a green line. d) Extracted signals from the line profile: in blue the intensity signal, in red the carbon and in black the oxygen. The arrows indicate that the valleys in the blue and black lines, corresponding to the channels, match the peaks in the red line, indicating the presence of carbon filling the pores. Reprinted from [28] with permission from Elsevier.

ship-in-a-bottle scheme. The enzyme was used along with the surfactant as a structure directing agent of a cage-like hybrid material, as indicated in the top part of Figure 9. This sample is called 'in-situ'. We succeeded in finding the optimal synthesis conditions that allowed a combined micelle lipase-surfactant to form, while maintaining the activity of the enzyme. Furthermore, we managed to conduct the polymerization of the silica at $\mathrm{pH} 3.5$ yielding a cage-like OMM [20], further elimination of the surfactant finally allowed realization of the encapsulated hybrid biocatalyst. Table 2 collects the parameters corresponding to these cagelike samples. Figure 10 shows the SEM and TEM characterization of MeSBA- 6 that allowed the $3 \mathrm{D}$ reconstruction using Mathematica software. As a result, the sizes of pore widows and cages could be accurately calculated, as indicated in Table 2. For the 'in situ' sample, it was possible to characterize the ordered mesoporous structure, furthermore, advanced electron microscopy techniques allowed the location of the lipase in the cages [28]. The lower part of Figure 9 shows a spectrum image and a spectrum profile analysis performed on the [100] orientation of the 'in situ' lipase-SBA-12. 

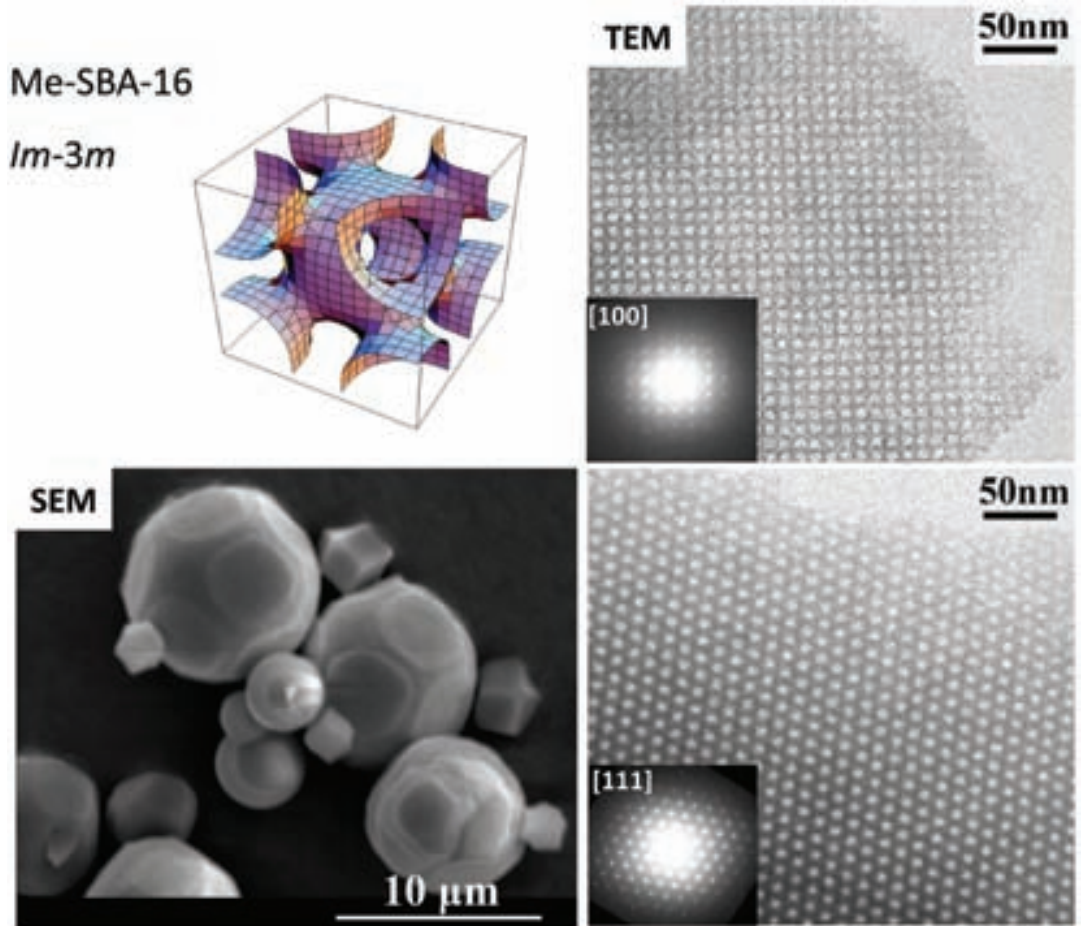

Figure 10. Me-SBA-16 structure 3D reconstruction based on the TEM results included as [100] and [111] zone axis, and SEM micrograph showing the faceted rounded particles.

Table 2. Textural properties of supports, enzyme loading and activity of immobilized lipase in tributyrin hydrolysis.

\begin{tabular}{lcccl}
\hline Material & Pd $\mathbf{n m}$ & $\mathbf{P d}_{3 \mathbf{D R}} \mathbf{n m}$ & Enz Load $\mathbf{~} \mathbf{g} / \mathbf{g}$ & Specif Act U/mg \\
\hline SBA-16 & 10.2 & $12.3(4.6)$ & 5 & 72.4 \\
Me-SBA-16 & 8.8 & $13.1(5.9)$ & 30 & 76.7 \\
In situ & & & 93 & 108 \\
\hline
\end{tabular}

In the case of Me-SBA-16, the immobilization is handicapped by restricted access of the enzyme across the narrow windows, as indicated by the long incubation times required and the low enzyme loadings achieved (Table 2), even in methylated materials. For this same reason, the enzyme release is also hindered and leaching profiles show saturation-like shapes (Figure 11). If the materials are synthesized in the presence of the enzyme ('in situ' sample), the enzyme loading increases while leaching is prevented 

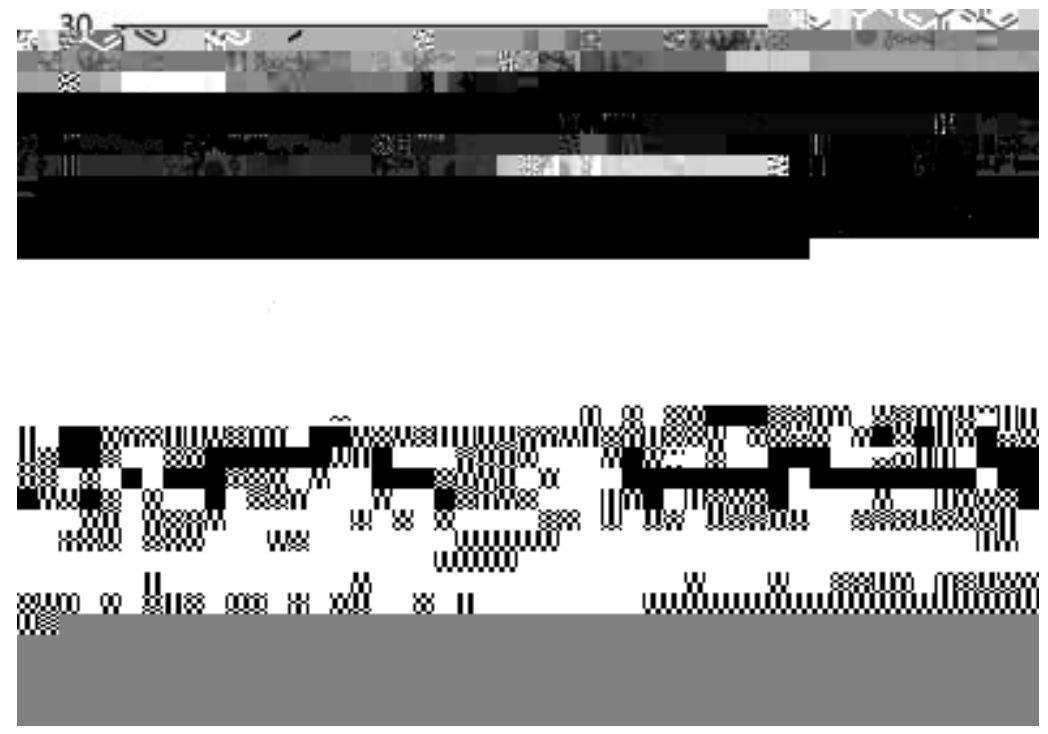

Figure 11. Leaching courses of lipase from the OMM (cage-window pores), both purely siliceous and functionalized with hydrophobic groups. Empty rhombus: SBA-16. Filled rhombus: Me-SBA-16. Triangles: In situ lipase.

[20]. The activity retained by the enzyme is higher than the values displayed by all the catalysts prepared through post-synthesis immobilization (with both pore structures: channel and cages).

\section{Amino-functionalized porous supports for laccase immobilization}

Laccases are a group of multicopper enzymes of industrial interest that catalyse the oxidation of phenolic compounds such as ortho- and para-diphenols to their corresponding quinones, with the concomitant reduction of oxygen to water [36,37]. The laccase used, from M. thermophila expressed in A. oryzae, has a low isoelectric point ( $\mathrm{pI}$ around 4), a molecular weight of around $89 \mathrm{kDa}$, and dimensions of approximately $6.1 \mathrm{~nm}$ x $5.0 \mathrm{~nm} \times 4.9 \mathrm{~nm}$. [38]. Having in mind these features, to carry out enzyme adsorption at $\mathrm{pH}$ values close to neutrality, the support material should contain positive charges in order to establish electrostatic interactions with the enzyme [10,39]. Therefore, functionalization of the siliceous supports was carried out with high pKa groups (amine) using aminopropyltriethoxysilane as the precursor. In this case, it is important to notice that pore size can be significantly decreased when the support is functionalized 

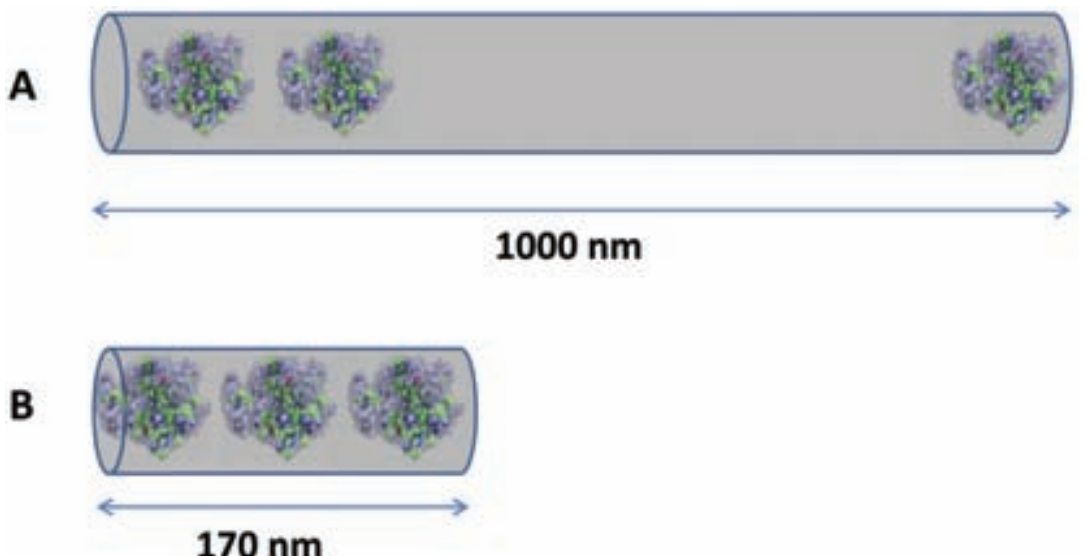

Figure 12. Schematic illustration of laccase immobilization on a) SBA-15-L mesochannels and b) SBA-15-S mesochannels.

with a large reagent such as aminopropyltriethoxysilane, which should be taken into account when designing the appropriate mesoporous support according to the dimensions of this enzyme.

First, immobilization on purely siliceous materials was studied through the synthesis of SBA-15. Two different strategies were used that yielded different particle morphology as shown in Figure 6: an SBA-15 sample with conventional rod type of particles, showing long channels (SBA-15-L) running parallel to the rod axis, and a second one grown as thin hexagonal plates containing shorter mesochannels aligned parallel to the short axis of the plates (SBA-15-S) [40]. Despite the smaller pore diameter and lower surface area of SBA-15-S, its shorter channels (of about $170 \mathrm{~nm}$ length) can accommodate more enzyme molecules per unit length than the longer ones of SBA-15-L (1000 nm length) where a part of the inner surface of the channel is not accessible to laccase molecules. Regarding specific activity, the lower value obtained for SBA-15-L indicates that the longer channels seem to hamper the diffusion of substrates and products more than the shorter ones (Figure 12).

PMA, or Periodic Mesoporous Aminosilica, was synthetized using bis[(3-trimethoxysilyl)propyl] amine and 1,2-bis(trimethoxysilyl)ethane as framework co-precursors following the scheme in Figure 5. Instead of being anchored to the pore surface as pendant groups, the amine groups in this material are integrated within the hybrid organosiliceous framework, and thus do not contribute to decreasing the pore diameter but are part of the pore surface itself. However, the enzyme loading achieved does not significantly increase compared to the purely siliceous SBA-15 materials 
Table 3. Textural properties of silica and hybrid amino-silica supports, enzyme loading and activity of immobilized laccase in ABTS oxidation.

\begin{tabular}{|c|c|c|c|c|c|c|}
\hline Material & Dp nm & $\mathrm{S}_{\mathrm{BET}} \mathrm{m}^{2} / \mathrm{g}$ & $\mathrm{Pv} \mathrm{cm}^{3} / \mathrm{g}$ & $\begin{array}{l}\text { Channel } \\
\text { length nm }\end{array}$ & $\begin{array}{l}\text { Enz load } \\
\mathrm{mg} / \mathrm{g}\end{array}$ & $\begin{array}{l}\text { Specif act } \\
\mathrm{U} / \mathrm{mg}\end{array}$ \\
\hline SBA-15-L & 7.8 & 609 & 1 & 1000 & 31.28 & 0.134 \\
\hline SBA-15-S & 6.4 & 550 & 0.7 & 170 & 38.41 & 0.159 \\
\hline PMA & 7.2 & 594 & 0.7 & - & 42 & 0.11 \\
\hline
\end{tabular}

Table 4. Textural properties of supports, nitrogen content (amino groups), enzyme loading and activity of immobilized laccase in ABTS oxidation.

\begin{tabular}{llllllll}
\hline Material & Dp nm & $\begin{array}{l}\mathbf{P v} \\
\mathbf{c m}^{3} / \mathbf{g}\end{array}$ & $\mathbf{S}_{\mathrm{BET}} \mathbf{m}^{2} / \mathbf{g}$ & $\begin{array}{l}\mathbf{N} \text { content } \\
\mathbf{m m o l} / \\
\mathbf{g S i O}\end{array}$ & $\begin{array}{l}\text { Enzload } \\
\mathbf{m g} / \mathbf{g}\end{array}$ & $\begin{array}{l}\text { Cat Act } \\
\mathbf{U} / \mathbf{g}\end{array}$ & $\begin{array}{l}\text { Specif } \\
\text { Act } \mathbf{~} / \\
\mathbf{m g}\end{array}$ \\
\hline NGOES & 11.2 & 1.0 & 339 & 1.2 & 170 & 50.7 & 0.30 \\
NCOES & 17.6 & 1.2 & 385 & 1.5 & 174 & 56 & 0.32 \\
E-PMA & 10.4 & 0.6 & 264 & 1 & 88 & 29 & 0.33 \\
AS & & 2.5 & 296 & - & 16 & 0.52 & 0.03 \\
NAS & & 2.1 & 236 & 1.8 & 187 & 169.5 & 0.91 \\
\hline
\end{tabular}

as indicated in Table 3. These results suggest that adsorption of laccase in these supports might be restricted due to the presence of too narrow pores. It seemed, therefore, necessary to synthesize supports with wider pore diameters.

A micelle expander was used in the synthesis of SBA-15 and PMA to obtain supports with wider pores following the general synthesis approach described in Figure 3 as well as Figure 5 . Thus, wide-pore silica SBA-15 and hybrid aminosilica PMA (sample E-PMA) were obtained using triisopropylbenzene (TIPB) as a swelling agent. Wide-pore SBA-15 silica was grafted with APTES for functionalization with amino groups (sample NGOES). Also, a wide-pore aminopropyl-SBA-15 support was obtained by a one-step method via co-condensation of TEOS and APTES (Figure 4) in the presence of the non-ionic surfactant and the swelling agent (sample NCOES). The textural properties and nitrogen content of these supports are reported in Table 4, along with the enzyme loading and activity of the biocatalysts obtained by adsorption of laccase on them.

Enzyme loading increased significantly in expanded SBA-15 materials containing amine groups and E-PMA compared to supports with narrower pores. The enzyme loading achieved in the mesoporous materials seems to 


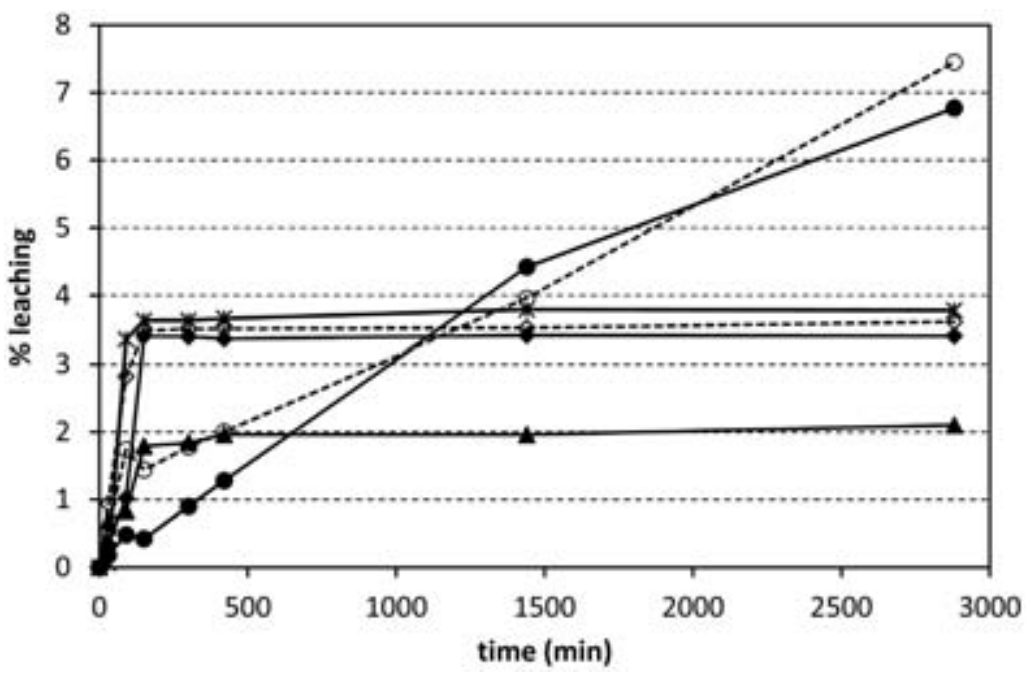

Figure 13. Leaching courses of laccase from the OMM purely siliceous and functionalized with amine groups. Empty rhombus: OES. Filled rhombus: NGOES. Triangles: NCOES. Asterisks: PMA. Empty circles: Amorphous silica. Filled circles: Propylamine-Amorphous silica.

be directly related to the nitrogen content in each one of them. Actually, the catalytic efficiency is very similar in the three samples [41]. An amorphous silica (AS) functionalized with APTES (sample NAS) used for comparison also followed this trend: higher laccase loading and higher $\mathrm{N}$ content. In this case, the catalytic efficiency was also higher. However, the enzyme leaching profiles repeated the same pattern as the lipase catalysts (Figure 13).

Similarly to the lipase in PMO, this study reveals that the close environment provided by the E-PMA material yields a maximum catalytic efficiency for the immobilized laccase. In this sample, we tried to locate and identify the presence of the laccase inside the channels using advanced transmission electron microscopy techniques. The highly regular porosity of the support achieved using micellar expanders was confirmed by the Cs-corrected STEM-HAADF images (Figure 14a). A selected region of the sample (green box in Figure 14a) was further analysed yielding the N-mapping (Figure 14b). In this case, chemical analysis by EELS indicated that nitrogen was evenly distributed within the framework that forms the pore walls. As shown in Figure 14b, nitrogen was also detected inside most of the pores, which could be attributed to adsorbed laccase molecules. However, the low scattering power of carbon-based molecules such as enzymes yields no contrast at all inside the pore spaces of the Cs-corrected STEM-HAADF images (Figure 14 a and c), which obstructs the direct observation of the protein molecules inside the pore channels. Nevertheless, the punctual analysis in the centre of 


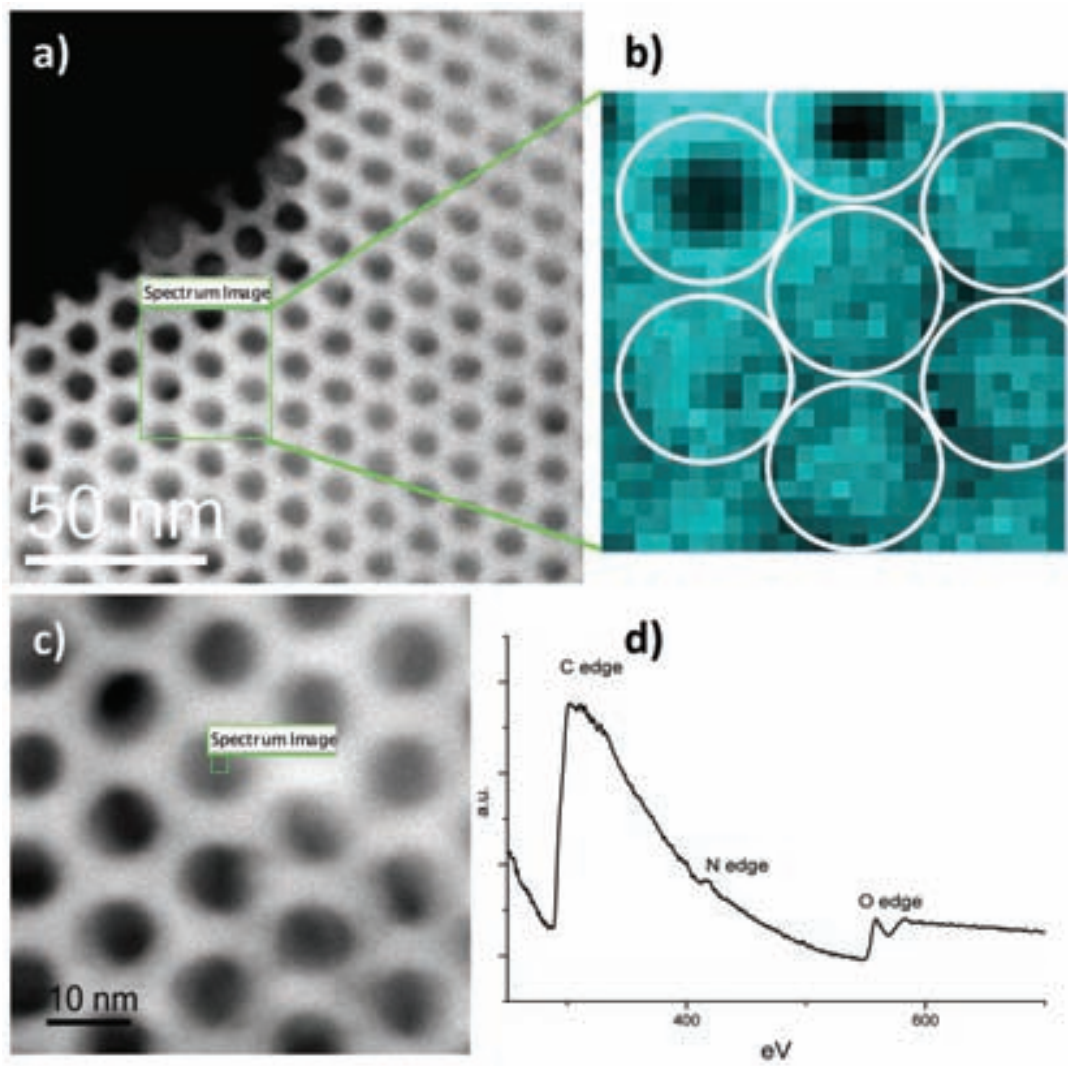

Figure 14. a) Cs-corrected STEM-HAADF image of laccase/E-PMA b) Image in which blue areas correspond to higher content of Nitrogen c) Cs-corrected STEM-HAADF image of interest d) Extracted EELS signals from the inner area of the channels indicating that the high content in $\mathrm{N}$ is associated with $\mathrm{C}$ and $\mathrm{O}$, which therefore corresponds to the enzyme molecules.

a channel (marked in Figure 14c) allows observation of the $\mathrm{C}, \mathrm{N}$ and $\mathrm{O}$ edges in the EELS profiles, corroborating the location of the laccase molecules.

\section{MOF as platforms to support enzymes}

Among the numerous potential applications of MOF materials, their use as supports for enzyme immobilization was evident due to their extraordinary compositional versatility and porous nature. Obviously, the most direct approach was to search MOF materials having pores large enough to encapsulate enzymes $[42,43]$. Unfortunately, just a few MOF materials possess big enough pores. In addition, such MOF materials are generally 
unstable and very difficult and expensive to prepare. More importantly, even those scarce large-pore-containing MOF materials can host only a limited number of enzymes.

In the last few years, these drawbacks have been solved by very different strategies, making MOFs practically universal supports for almost any enzyme. Some of the most innovative approaches are mentioned in this section. As a complement to the information given here, recent reviews covering in detail all described methodologies of enzyme immobilization on MOF materials can be found elsewhere [44-47].

The affinity between MOF materials and enzymes is so favoured that the simple contact between both entities somehow generates the immobilization, although the kind of interaction is not described $[48,49]$. The immobilization of enzymes on MOFs can also be achieved by in situ strategies [50-52] taking advantage of the possibility of preparing MOF materials under soft conditions compatible with the bioactivity of enzymes. Depending on the followed in situ strategy, enzymes can finally be embedded within the MOF crystal [50] or they can even become part of the MOF, acting as nodes (linkers) $\left[51,5^{2}\right]$. The first strategy may encounter serious diffusion problems with reactants and/or products, as they must go through the pores of the MOF materials, indeed the enzymatic activity of the biocatalysts prepared in this way was only probed in the degradation of $\mathrm{H}_{2} \mathrm{O}_{2}$, which has no diffusion problems [50]. In the second strategy, the enzymatic immobilization implies the covalent union to the support, which sometimes affects negatively the catalytic activity of the immobilized enzyme.

Our group developed a new methodology to prepare MOF materials under very soft conditions, which included the use of water as unique solvent, room temperature preparation, the absence of any corrosive reactant, and no energy input [53-56]. As an additional advantage, this method generally produces nanocrystalline MOF materials, with crystals that are aggregated or agglomerated, describing more or less ordered intercrystalline mesoporosity. Equally important, the experimental conditions (temperature, synthesis time, $\mathrm{pH}$, etc.) under which the MOF supports are prepared are friendly to the enzymatic activity. All of this allows carrying out a systematic comparison between the in situ and post-synthesis immobilization of enzymes on these nanocrystalline MOF materials following the scheme represented in Figure 15 [57,58].

In situ approaches generated solid biocatalysts with high enzyme loadings (at least $85 \%$ of the enzyme added to the synthesis media) and with lower enzyme leaching, whereas the intrinsic catalytic activity per enzyme molecule was higher in the post-synthesis biocatalysts. These 


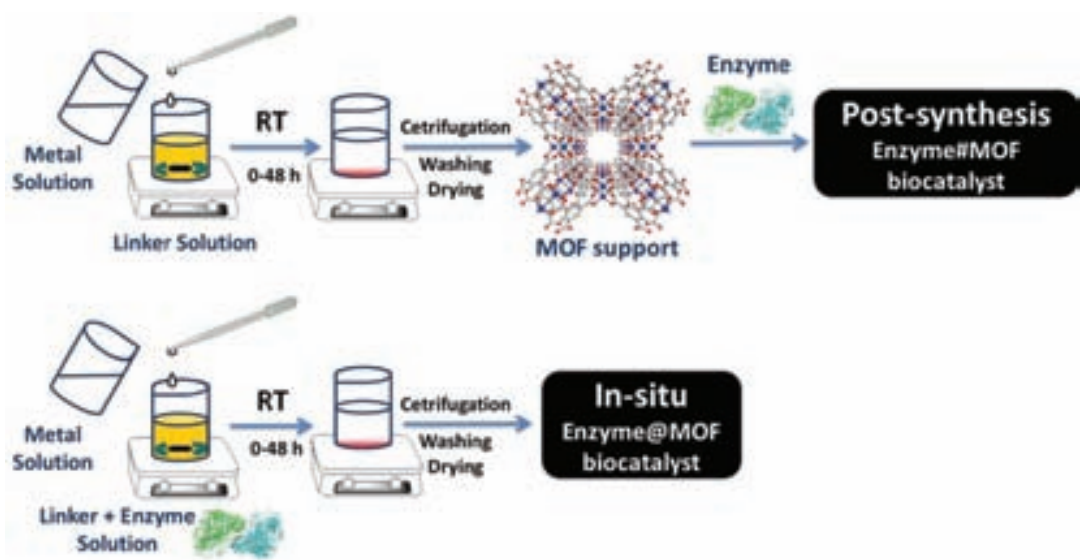

Figure 15. Visual diagram of the experimental procedures to prepare: i) post-synthesis Enzyme\#MOF biocatalysts, at the top, and ii) in situ (one step) Enzyme@MOF biocatalysts, at the bottom, developed by our group [57-59].

results are in agreement with the encapsulation of enzymes within the intercrystalline mesoporosity of the MOF materials [58]. The universality of this methodology was made clear by using different immobilization methodologies, different MOF supports, different enzymes and different synthesis media, including N,N-dimethylformamide, in which $\beta$-glucosidase immobilized on Mg-MOF-74 material kept certain activity for at least 24 hours, whereas the free enzyme was inactivated in a few minutes [57,58].

The method was particularly effective when the semi crystalline MOF material Fe-BTC (commercially known as Basolite F300) was the support. Such material, directly prepared by our group under sustainable conditions [55], efficiently immobilized the enzymes laccase, alcohol dehydrogenase, lipase, and glucose oxidase by an in situ procedure [59]. Table 5 shows the enzyme immobilization efficiency, the enzyme loading, and the enzymatic activity of different solid biocatalysts prepared by in situ immobilization of the mentioned enzymes on the Fe-BTC MOF material. Regardless of the nature of the immobilized enzyme, the efficiency of the immobilization is higher than $95 \%$, except for the experiment carried out at high lipase content $(71 \%)$. Enzyme loadings become as high as $100 \mathrm{mg} / \mathrm{g}$ in the case of alcohol dehydrogenase. Moreover, the catalytic activity of the biocatalysts Enzyme@Fe-BTC is maintained to some extent, even outperforming (2.5 higher!) that of the free enzyme in the case of the glucose oxidase.

The value of the enzyme immobilization methods on MOFs supports presented here goes beyond mere academic interest. Although the MOF 
supports are sometimes thought of as expensive or hard-to-prepare materials, the materials used in these approaches utilized inexpensive chemicals, water as solvent and room temperature. Moreover, the MOF materials can be considered as bioMOF, as they are composed of metals biocompatible even upon ingestion by human beings (such as Fe, $\mathrm{Zn}$ or $\mathrm{Mg}$ ), which makes them good candidates for any application related to food industry, biomedicine, etc.

\section{How do regular structures improve catalytic properties?}

In order to study and compare the possible effects of a regular structure of the support, lipase and laccase were immobilized on a commercial amorphous silica, with an average pore size close to $30 \mathrm{~nm}$, much wider than those of any OMM studied. Despite the lower surface area of this material, the enzyme loadings achieved were higher than those obtained with OMM, as can be seen in Tables 1 and 4. However, significant differences were found and serve to distinguish which features can be improved in the final catalyst via the regular structure of the solid matrix.

First of all, the confinement of the enzyme in pores with a uniform size that closely matches the size of the enzyme has a double effect. A first consequence is that enzyme leaching is prevented due to the joint effect of the confinement and the chemical affinity with the pore surfaces. In the leaching tests, after the initial release of enzyme discussed above, no more enzyme release was detected at long incubation times for enzymes immobilized on OMM. On the contrary, enzymes were not retained in the wide-pore amorphous material functionalized to provide its surface with the same chemical affinity with the respective enzyme. The second effect of confinement is to ensure that the enzyme is immobilized in a dispersed form, i.e. with no aggregation of enzyme molecules because of the restricted pore space where only one molecule of enzyme can fit. Thus, no loses of activity can be due to immobilization of aggregates. Thirdly, the high pore connectivity provided by the uniform shape and distribution of the porous network in OMM favours the diffusion of substrates and products. This effect, added to the lack of enzyme aggregation, contributes to obtaining higher specific activities than on the amorphous silica, especially in the case of lipase. Table 1 shows the lipase loading on amorphous silica (200 mg lipase/g of octyl-functionalized amorphous silica, OAS), whose 
pores have wide diameters and where the enzyme loading is much higher than over the OMM. However, the specific activity is less than half the one obtained with PMO.

Particle size and shape also play a fourth main role. Decreasing particle size contributes to reduce diffusional restrictions of substrates and products, and thus also to obtain better catalytic efficiencies thanks to the shortening of the length of pores. Furthermore, especially when the diameter of the pores is very close to the enzyme dimensions, diffusion of the enzyme during the immobilization by adsorption can be restricted, leading to lower enzyme loadings in supports with longer pore channels, as shown above for the immobilization of laccase on SBA-15 silica.

In summary, obtaining regular mesoporous materials, which can be tuned according to the requirements of each enzyme, allows separate study of each of the effects on the catalysts, and to improving their properties. Furthermore, the strong chemical affinity between MOFs and enzymes can be inferred by the high number of successful studies about enzyme immobilization on these supports carried out in the last two years by different groups and using different approaches, none of them requiring MOFs with structural mesopores [48-52,57-59]. Having been researched for so short, it is evident that many aspects (location of the enzymes, interaction enzyme-MOF, scale up to industrial level, etc.) of these strategies must be further investigated, but it is also evident that MOFs should definitely be considered as enzyme supports for future applications.

\section{References}

[1] Braun, S., Rappoport, S., Zusman, R., Avnir, D. Ottolenghi, M. Mater. Lett. 1990, 10, 1.

[2] Avnir, D., Braun, S., Lev, O., Ottolenghi, M. Chem. Mater 1994, 6, 1605.

[3] Gorton, L. Electroanal. 1995, 7, 23.

[4] Lev, O., Wu, Z., Bharathi, S., Glezer, V., Modestov, A., Gun, J., Ravinovich, L., Sampath, S. Chem. Mater. 1997, 9, 2345.

[5] Gill, I., Ballesteros, A.J. Am. Chem. Soc. 1998, 120, 8587.

[6] Livage, J., Coradin, T., Roux, C.J. Phys. Condens. Matter. 2001, 13, R673.

[7] Nassif, N., Bouvet, O., Rager, M.N., Roux, C., Coradin, T., Livage, J. Nat. Mater. 2002, 1, 42.

[8] Brennan, J.D., Benjamin, D., DiBattista, E., Gulcev, M.D. Chem. Mater. 2003, 15, 737

[9] Ferrer, M.L., Levy, D., Gomez-Lor, B., Iglesias, M.J. Mol. Catal. B Enzim. 2004, 27, 107.

[10] Yiu, H.P., Wright, P.A.J. Mat. Chem. 2005, 15, 3690.

[11] Hartmann, M. Chem. Mater. 2005, 17, 4577.

[12] Díaz, J.F., Balkus Jr. K.J.J. Mol. Catal. B Enzim. 1996, 2, 115.

[13] Zhou, Z., Hartmann, M. Chem. Soc. Rev. 2013, 42, 3894. 
[14] Corma, A., Fornes, V., Jordá, J.L., Rey, F., Fernández-Lafuente, R., Guisan, J.M., Mateo, C. Chem. Commun. 2001, 419.

[15] Corma, A., Fornes, V., Rey, F. Adv. Mater. 2002, 14, 71.

[16] Dumitriu, E., Secundo, F., Patarin, J., Fechete, I.J. Mol. Catal. B Enzym. 2003, 22, 119.

[17] Kresge, C.T., Leonowicz, M.E., Roth, W.J., Vartulli, J.C., Beck, J.S. Nature 1992, 359, 710.

[18] Zhao, D., Feng, J., Huo, Q., Melosh, N., Fredrickson, G.H., Chmelka, B.F., Stucky, G.D. Science 1998, 279, 548.

[19] Wan, Y., Zhao, D. Chem. Rev. 107, 2007, 2821.

[20] Urrego, S., Serra, E., Alfredsson, V., Blanco, R.M., Díaz. I. Microp. Mesop. Mater. 2010, 129, 173.

[21] Gaffney, D., Cooney, J., Magner, E. Top. Catal. 2012, 55, 1101.

[22] Magner, E. Chem. Soc. Rev. 2013, 42, 6213.

[23] Hartmann, M., Kostrov, X. Chem. Soc. Rev. 2013, 42, 6277.

[24] Wight, A.P., Davis, M.E. Chem. Rev. 2002, 102, 3589.

[25] Hoffmann, F., Cornelius, M., Morell, J., Fröba, M. Angew. Chem. Int. Ed. 2006, 45, 3216.

[26] Fujita, S., Inagaki, S. Chem. Mater. 2008, 20, 891.

[27] Shea, K.J., Loy, D.A. Chem. Mater. 2001, 13, 3306.

[28] Mayoral, A., Blanco, R.M., Díaz, I.J. Mol. Catal. B Enzym. 2013, 90, 23.

[29] Mayoral, A., Arenal, R., Gascón, V., Márquez-Álvarez, C., Blanco, R.M., Díaz I. ChemCatChem 2013, 5, 903 .

[3o] Mayoral, A., Gascón, V., Blanco, R.M., Márquez-Álvarez, C., Díaz, I. Appl. Mater. 2014, 2, 1133041.

[31] Gascón, V., Márquez-Álvarez, C., Díaz, I., Blanco, R.M. in Non-covalent Interactions in the Synthesis and Design of New Compounds, Chap. 19, Maharramov, A.M., Mahmudov, K.T., Kopylovich, M.N., Pombeiro, A.J.L. (Eds.) John Wiley \& Sons, 2016.

[32] Jaeger, K.E., Ransac, S., Dijkstra, B.W., Colson. C., van Heuvel. M., Misset, O. FEMS Microbiol. Rev. 1994, 15, 29.

[33] Serra, E., Mayoral, A., Sakamoto, Y., Blanco, R.M., Diaz, I. Microp. Mesop. Mater. 2008, 114, 201.

[34] Serra, E., Díez, E., Diaz, I., Blanco, R.M. Microp. Mesop. Mater. 2010, 132, 487.

[35] Gascón, V., Díaz, I., Blanco, R.M., Márquez-Álvarez, C. RSC Adv. 2014, 4, 34356.

[36] Mayer, A.M., Staples, R.C. Phytochem. Rev. 2002, 6o, 551.

[37] Baldrian, P. FEMS Microbiol. Rev. 2006, 30, 215.

[38] Berka, R.M., Schneider, P., Golightly, E.J., Brown, S.H., Madden, M., Brown, K.M., Halkier, T., Mondorf, K., Xu, F. Appl. Environ. Microbiol. 1997, 63, 3151.

[39] Tran, D.N., Balkus, K.J. ACS Catal. 2011, 1, 956.

[40] Gascón, V., Díaz, I., Márquez-Álvarez, C., Blanco, R.M. Molecules 2014, 19, 7057.

[41] Gascón, V., Márquez-Álvarez, C., Blanco, R.M. Appl. Catal., A 2014, 482, 116.

[42] Lykourinou, V., Chen, Y, Wang X.S., Meng L., Hoang, T., Ming L.J., Musselman R.L., Ma S. J. Am. Chem. Soc. 2011, 133, 10382.

[43] Deng, H., Grunder, S., Cordova, K.E., Valente C., Furukawa, H., Hmadeh, M., Gandara, F., Whalley, A.C., Liu, Z., Asahina, S., Kazumori, H., O’Keeffe, M., Terasaki, O., Stoddart, J.F., Yaghi, O.M. Science 2012, 336, 1018.

[44] Gkaniatsou, E., Sicard, C., Ricoux, R., Mahy, J.P., Steuno, N., Serre, C. Mater. Horizon. $2017,4,55$.

[45] Mehta, J., Bhardwaj, N., Bhardwaj, S.K., Kim, K.H. Coord. Chem. Rev. 2016, 322, 30.

[46] Wu, X., Hou, M., Ge, J. Catal. Sci. Technol. 2015, 5, 5077.

[47] Fried, D.I., Brieler, F.J., Froba, M. ChemCatChem 2013, 5, 862.

[48] Liu, W.L., Lo, S.H., Singco, B., Yang, C.C., Huang, H.Y., Lin, C.H.J. Mater. Chem. B 2013, 1, 928. 
[49] Liu, W.L., Yang, N.S., Chen, Y.T., Lirio, S., Wu, C.Y., Lin, C.H., Huang, H.Y. Chem. Eur. J. 2015, 21,115 .

[50] Shieh, F.K., Wang, S.C., Yen, C.I., Wu, C.C., Dutta, S., Chou, L.Y., Morabito, J.V., Hu, P., Hsu, M.H., Wu, K.C., Tsung, C.K.J. Am. Chem. Soc. 2015, 137, 4276.

[51] Sontz, P.A., Bailey, J.B., Ahn, S, Tezcan, F.A.J. Am. Chem. Soc. 2015, 137, 11598.

[52] Fujita, D., Fujita M. ACS Cent. Sci. 2015, 1, $35^{2}$.

[53] Sánchez-Sánchez, M., Díaz, I, Getachew, N., Chebude, Y. ES Patent P2012231968, 2012.

[54] Sánchez-Sánchez, M., Getachew, N., Díaz, K., Díaz-García, M., Chebude, Y, Díaz, I. Green Chem. 2015, 17, 1500.

[55] Sánchez-Sánchez, M., de Asua I., Ruano, D., Díaz, K. Cryst. Growth Des. 2015, 15, 4498.

[56] Guesh, K., Caiuby, C.A.D., Mayoral, A., Díaz-García, M., Díaz, I., Sánchez-Sánchez, M. Cryst. Growth Des. 2017, 17, 1806.

[57] Castro-Miguel, E., Gascón, V., Sánchez-Sánchez, M., Blanco, R.M., Díaz-García, M. WO Patent 2016193516, 2016.

[58] Gascón, V., Castro-Miguel, E., Diaz-Garcia M., Blanco, R.M., Sánchez-Sánchez, M. J. Chem. Technol. Biotechnol. 2017, 92, 2583.

[59] Gascón, V., Carucci, C., Jimenez, M.B., Blanco, R.M., Sánchez-Sánchez, M,, Magner, E. ChemCatChem 2017, 9, 1182 . 



\title{
7. Adsorption Processes on Zeolites and Metal-Organic Frameworks for Industrial and Environmental Applications
}

\author{
Elena López-Maya, ${ }^{1}$ Carmen Montoro, ${ }^{2}$ \\ L. Marleny Rodriguez-Albelo ${ }^{1}$ and Carmen R. Maldonado ${ }^{1}$ \\ ${ }^{1}$ Universidad de Granada, Departamento de Química Inorgánica, \\ Avda. Fuentenueva s/n, 18071 Granada, Spain. \\ ${ }^{2}$ Universidad Autónoma de Madrid, Departamento de Química \\ Inorgánica, C/ Tomás y Valiente $n^{\circ} 7,28049$ Madrid, Spain.
}

Keywords: Adsorption, gas storage, harmful gases, greenhouse gases, pollutant, wastewater

\section{Introduction}

This Chapter discusses, the impact that inorganic porous matrixes (namely, zeolites and metal-organic frameworks) have on industrial development and environmental remediation processes. In particular, the role of these materials as adequate adsorbents for gas storage, air purification, gas mixture separation or wastewater treatment is thoroughly analysed. In fact, zeolites and metal-organic frameworks (MOFs) exhibit exceptional features, mainly due to their high surface area and tunability, which make them ideal candidates for these types of application, outperforming, in some cases, the classic porous materials. Thus, the basic principles of the adsorption process, as well as the main strategies employed to enhance the adsorptive properties (capacity and selectivity) of these porous matrixes is presented. In particular, we focus attention on those methodologies capable of modifying the final characteristics (size and functionality) of the pores and/or frameworks and, therefore, able to adapt the adsorption properties and selectivity of the resulting materials to a specific application or adsorbate. The gas adsorption properties of zeolites and MOFs are outlined focusing on suitable applications of each gas. These include energy $\left(\mathrm{H}_{2}, \mathrm{CH}_{4}\right)$, environmental $\left(\mathrm{CO}_{2}, \mathrm{NOx}, \mathrm{SOx}\right.$, etc $)$ and 
other gases $\left(\mathrm{O}_{2}\right.$ and noble gases $)$ purification and separation. Finally, the adsorption capacity of these materials towards different pollutants present in water, such as heavy metals, radionuclides, dyes, pharmaceuticals and other toxic organic molecules, is also discussed, including the most representative examples found in recent literature.

\section{Principles of adsorption processes}

The term adsorption was introduced by Kaiser in 1881 to describe the

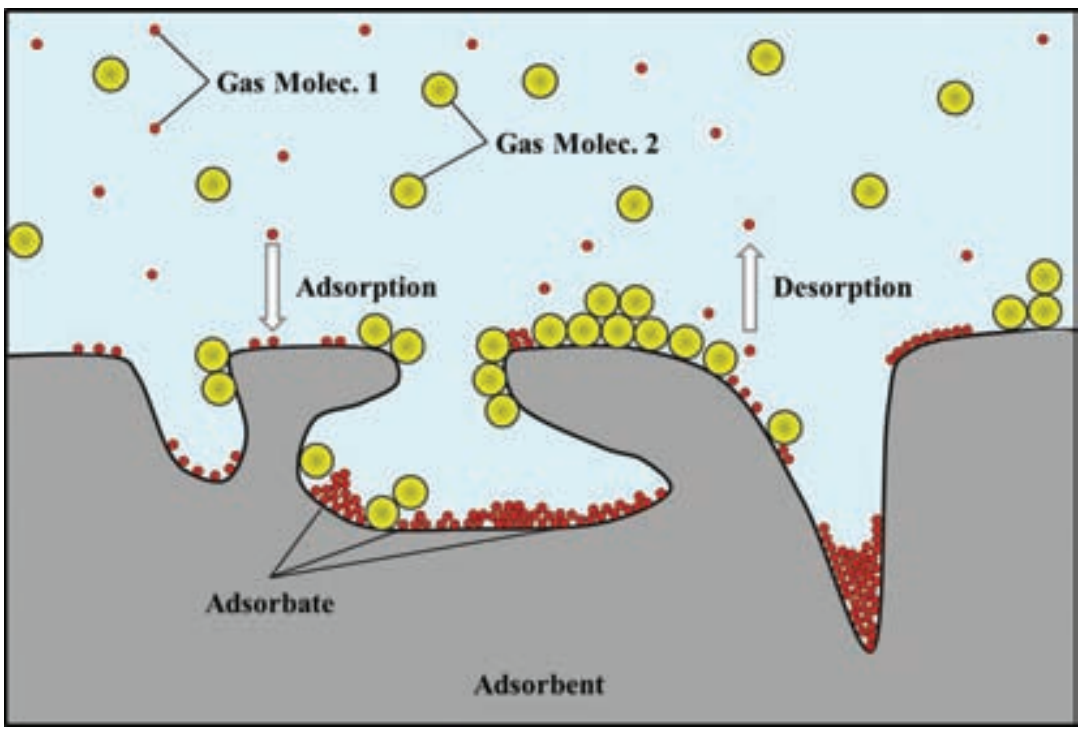

Figure 1. Schematic representation of a gas-solid adsorption process. 

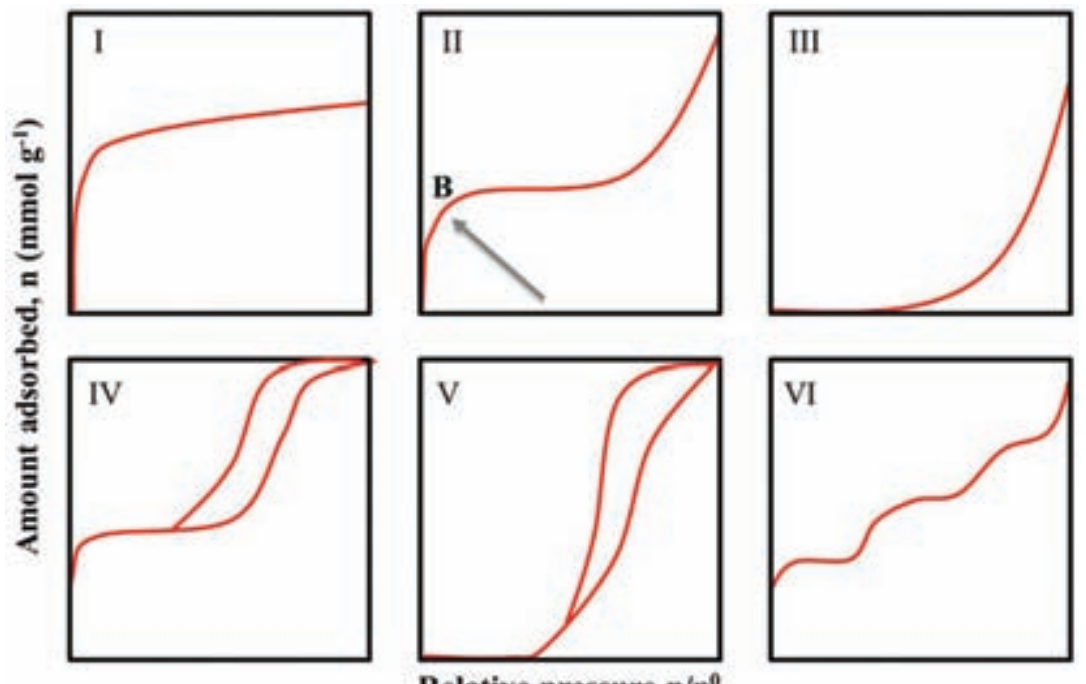

Relative pressure $\mathbf{p} / \mathbf{p}^{0}$

Figure 2. Types of adsorption isotherms according to IUPAC classification.

The interaction forces between both phases can be of a physical and chemical nature, giving rise to physisorption and chemisorption phenomena, respectively [1]. The main difference between both processes is that while physisorption is characterized by weak and reversible interactions, chemisorption is characterized by strong, generally irreversible bonds, and the formation of a new compound. In this chapter, we focus on the physisorption phenomena. In order to do this, it is very important to know different characteristic parameters of a porous matrix in order to show their potentialities in gas storage, capture, and separation processes.

One of these parameters is the quantification of the gas adsorbed on the surface of a solid at a given temperature by the measurement of its adsorption isotherm. This measurement represents the amount of gas adsorbed as a function of the gas pressure in equilibrium with the solid. Adsorption isotherms can be classified, according to the IUPAC recommendations, in six different types: I to VI (Figure 2) [1,2]. The first five types of the classification (I to V) were originally proposed by S. Brunauer, L. S. Deming, W. E. Deming and E. Teller, and constitute the classification BDDT, [3-5] also called the Brunauer classification.

The isotherms types are described as follows:

- Type I isotherm is characteristic of microporous materials, such as activated carbons, zeolites and MOFs, since it shows the filling of the 
micropores at very low relative pressures until reaching a limit value corresponding to the coating of the surface by a monolayer. The predominating interactions are adsorbate-adsorbent.

- Type II isotherm corresponds to the typical adsorption of macroporous solids, showing the formation at low pressures of a monolayer (near point B) and the subsequent formation of the multilayer, with a thickness increasing under pressure. When the pressure reaches saturation, the adsorbed layer corresponds to that of the liquid. The adsorbate-adsorbent interactions are stronger than the adsorbate-adsorbate interactions.

- Type III isotherm describes the adsorption processes of non-porous solids, like metal solids, where adsorbate-adsorbent interactions are weaker than adsorbate-adsorbate interactions.

- Type IV isotherm is characterized by the presence of hysteresis cycles (adsorption and desorption curves do not follow the same trajectory). It describes the adsorption behaviour of mesoporous materials, such as silica and alumina, which are filled by capillary condensation.

- Type V isotherms are uncommon and difficult to interpret. The affinity of the adsorbent (porous in this case, in contrast to type III) by the adsorbate is low and the hysteresis links with the filling of the pores. It is typical of the adsorption of water vapour at temperatures close to the environment.

- Type VI isotherm describes the adsorption in multilayer of noble gases on highly uniform surfaces. Each of the first layers is adsorbed within a certain range of pressures, each step corresponding to the filling of a monolayer, up to a total of two or three layers. These are cooperative adsorption processes that contribute to each layer, helping the formation of the next through lateral interactions of the molecules themselves.

Furthermore, prior to the measurement of the adsorption isotherm, an activation of the material is required. In particular, this process consists of the evacuation of the solvent molecules, coming from the synthesis or even from the atmosphere, by applying appropriate temperature and vacuum conditions. In this sense, the required temperature is determined by the corresponding thermogravimetric analysis.

Adsorption isotherms provide very important information about specific surface area, pore volume, and even about the distribution of pore sizes. The determination of these textural properties is performed through the analysis of the isotherm, by adjusting the experimental curve to a mathematical expression that represents an approximate model for the adsorption process of the molecules on the surface. The most widely used models for MOFs and zeolites are the Langmuir model and the Brunauer-Emmet-Teller 
(BET). The determination of the surface area of MOFs and zeolites is calculated by the measurement of the $\mathrm{N}_{2}$ adsorption isotherm at $77 \mathrm{~K}$ and is then interpreted with the BET model in the range of low relative pressures $\left(\mathrm{p}_{\mathrm{r}}=\mathrm{p} / \mathrm{p}^{\circ}, \mathrm{p}^{\circ}\right.$ being the saturation vapour pressure $)$, usually $0.05-0.30[6]$. In other cases, when the volume or adsorption capacity of the micropores is concerned, the $\mathrm{CO}_{2}$ adsorption isotherm is measured at $273 \mathrm{~K}$ and the Dubinin-Radushkevich method is used [5,7].

Another important parameter that must be known when establishing the possible application of a material is the enthalpy of adsorption. This magnitude provides information on the affinity of the pore surface for the adsorbate molecules and the energy required for its removal during the regeneration of the adsorbent. Generally, the enthalpy of adsorption is measured as an isosteric heat of adsorption (meaning that it is determined at a fixed amount of adsorbent, see below) and is a function of the amount of adsorbed adsorbate on the surface. Its determination can be carried out either statically, by isosteric methods or dynamically, by the inverse gas chromatography (IGC) technique [8-10], both methods use the ClausiusClapeyron equation (1) to determine the isosteric heat of adsorption.

$$
\Delta H_{a d s}=-R\left(\frac{\partial \ln p}{\partial\left(\frac{1}{T}\right)}\right)_{N}
$$

where $\Delta \mathrm{H}_{\text {ads }}$ is the enthalpy of adsorption, $\mathrm{p}$ is the adsorbate partial pressure, $\mathrm{T}$ is the temperature, $\mathrm{N}$ is the amount of adsorbed gas, and $\mathrm{R}$ is the gas constant.

\section{Isosteric method}

The isosteric method consists of the measurement of at least two adsorption isotherms at different temperatures (Figure 3, where $\mathrm{T}_{1}>\mathrm{T}_{2}$ ) and the application of the Clausius-Clapeyron equation. This method assumes that the adsorbed phase remains unchanged and in thermodynamic equilibrium, at the temperature range studied. Linearizing equation (1) to an Arrhenius equation, equation (2) is obtained, from which we can calculate the value of $\Delta \mathrm{H}_{\text {ads }}$, which is the slope of the line representing the natural logarithm of the partial pressure $\ln \mathrm{p}$, for a constant value of the amount of gas adsorbed, versus the inverse of the temperature $1 / \mathrm{T}$. 


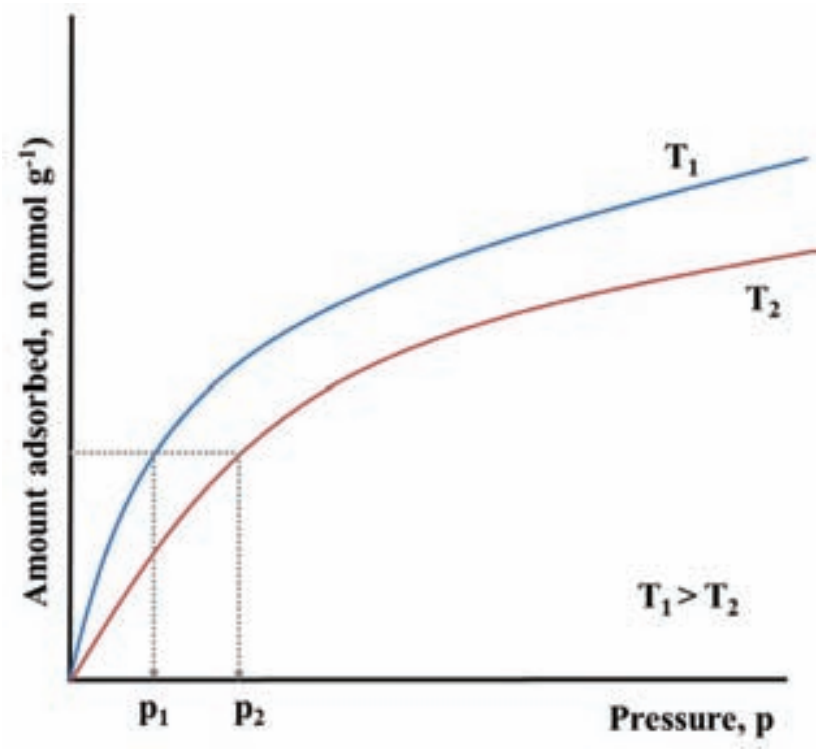

Figure 3. Determination of adsorption heat by isosteric methods.

$$
(\ln p)_{N}=-\frac{\Delta H_{a d s}}{R T}+C
$$

\section{Chromatographic methods}

As mentioned earlier, it is considered that the adsorption under dynamic conditions and at temperature is more realistic and it generally differs from the adsorption at ambient temperature [11-13]. Inverse Gas Chromatography (IGC) is essentially analogous to conventional gas chromatography with the exclusive difference, and hence the inverse term, that the object of interest is the stationary phase formed by the porous material in the form of a pellet to avoid pressure drops (Figure 4) $[12,14]$. IGC is based on an adsorption process at infinite dilution (or zero coverage), that is, at very low adsorbate vapour partial pressures. Under these conditions, the solute concentration is very low, the adsorbate-adsorbate side interactions are negligible, and the adsorption enthalpy depends only on the adsorbate-adsorbent interactions. IGC also allows establishing, for each adsorbate, the thermodynamic parameters of the adsorption process, that is, the Henry constants, $\mathrm{K}_{\mathrm{H}}$, and adsorption heats, $\Delta \mathrm{H}_{\text {ads }}$ as well as the partition coefficients, $\alpha_{x / y}$, which will determine the selectivity of the material towards a given adsorbate, $\mathrm{X}$, compared to another one, $\mathrm{Y}$. 


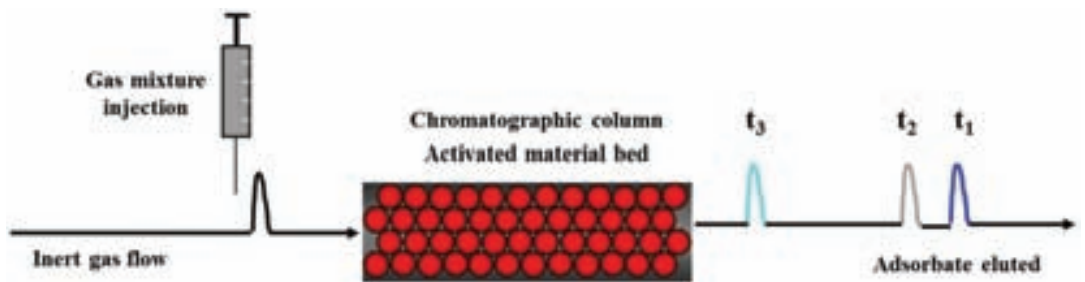

Figure 4. Scheme of the Inverse Gas Chromatography method.

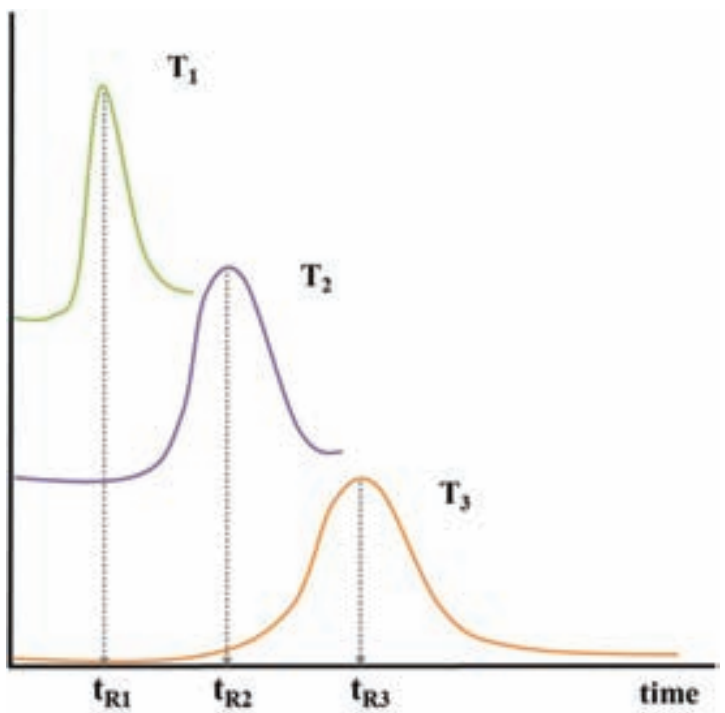

Figure 5. Effect of temperature variation on the shape and position of the peak of a chromatogram.

After the introduction of the probe molecules into the system, adsorptiondesorption processes take place and are represented in the chromatogram by means of peaks (Figure 5) [15]. Each peak corresponds to an adsorbate eluted from the column at a certain time, known as retention time $\left(t_{\mathrm{R}}\right)$, and at a different temperature (Figure 5 , where $\mathrm{T}_{1}>\mathrm{T}_{2}>\mathrm{T}_{3}$ ).

The independence of the retention time with the volume of adsorbate injected ensures that the adsorption process takes place in the region where Henry's Law is satisfied (zero coverage surface). This region involves chromatographic peaks being symmetric or with a small asymmetry, regardless of the amount injected. Then, the equilibrium constant of the adsorption process or Henry constant $\mathrm{c}$ is defined as the net retention volume per unit area $\left(\mathrm{V}_{\mathrm{s}}\right)$, which can be determined by the equation (3): 


$$
V_{s}=\frac{V_{R}}{S_{B E T}} m
$$

where $S_{B E T}$ is the specific surface area of the material, $m$ is the dry weight of the adsorbent sample inside the column, and $V_{R}$ is the retention volume, which is determined from the retention time and flow of the carrier gas $[16,17]$. The adsorption heat, $\Delta \mathrm{H}_{\text {ads }}$, can be calculated by applying the Clausius-Clapeyron equation (4) to this region.

$$
\Delta H_{a d s}=-R\left(\frac{\partial \ln V_{s}}{\partial\left(\frac{1}{T}\right)}\right)_{N}
$$

This equation can be linearized to obtain (5) from which the graphical representation calculates $\Delta \mathrm{H}_{\text {ads }}$ more easily.

$$
\ln V_{s}=-\frac{\Delta H_{a d s}}{R T}+C
$$

Finally, with knowledge of the values of the Henry constants for each adsorbate, the partition coefficient can also be estimated, Equation (6) shows $\alpha_{\mathrm{x} / \mathrm{y}}$ as a measure of the degree of separation expected for a binary mixture.

$$
\ln \alpha_{x / y}=\frac{\left(\Delta H_{a d s 1}-\Delta H_{a d s 2}\right)}{R T}
$$

\section{Strategies to enhance adsorption processes}

Different methodologies can be applied to modify the adsorption properties of MOFs and zeolites. For example, post-synthetic modification (PSM) of the structure of porous materials is a widely used technique for the improvement of the adsorption capacity and selectivity of different gases. It should be noted that adsorption is not only important for gas adsorption and separation processes but is also essential for many other applications, such as catalysis, water purification, etc.

In the field of zeolites, it is important to highlight a process known as ion exchange, which has been widely used for many years. This process refers to the exchange of ions between a liquid and a solid phase [18]. As is well known, the lattice of zeolites is built by $\mathrm{SiO}_{4}$ and $\mathrm{AlO}_{4}$ tetrahedra. Since aluminium is trivalent, the lattice carries a negative electric charge. This charge must be balanced by cations, which can be exchanged for other cations from a liquid phase as they diffuse along the channels of the zeolite [19]. 


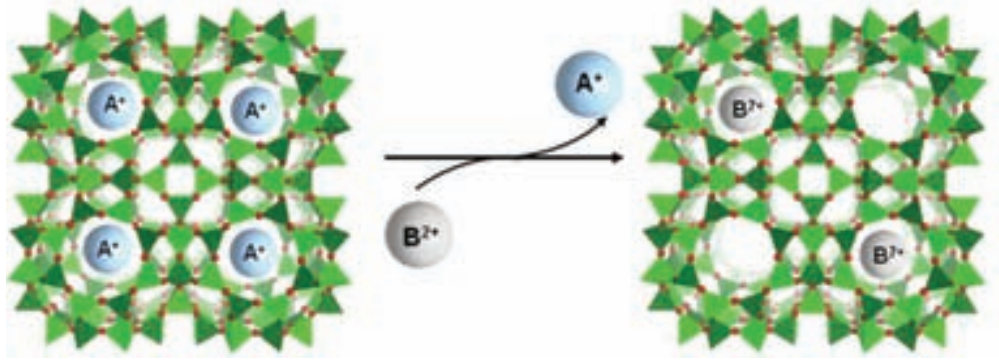

Figure 6. Example of ion exchange process in LTA zeolite.

Thus, when a zeolite containing a generic cation $\mathrm{A}^{+}$is immersed in a solution containing $\mathrm{B}^{+}$, a diffusion process is established in which $\mathrm{B}^{+}$diffuses into the porous structure and replaces $\mathrm{A}^{+}$, which diffuses out from the zeolite crystal (Figure 6).

In this context, the incorporation of different cations in the structure of some zeolites can improve the adsorption capacity of different gases [20]. For example, the ion exchange method has been researched for the purposes of eliminating sulphur compounds $\left(\mathrm{H}_{2} \mathrm{~S}\right)$ from diesel and gasoline. Hoguet et al. synthesized different ion exchanged zeolites, namely $\mathrm{Nd}-$ $\mathrm{NaY}, \mathrm{La}-\mathrm{NaY}$ and $\mathrm{Ce}-\mathrm{NaY}$, through the liquid ion exchange method from the commercial precursor zeolite $\mathrm{NaY}$. The $\mathrm{La}-\mathrm{NaY}$ and $\mathrm{Ce}-\mathrm{NaY}$ zeolites improved the $\mathrm{H}_{2} \mathrm{~S}$ adsorption capacity significantly compared to the commercial zeolite [21].

In addition, in recent decades post-synthesis treatments of parent zeolites have also been studied in order to alter the Si/Al ratio $[22,23]$. The framework $\mathrm{Si} / \mathrm{Al}$ atomic ratio of zeolites is an important parameter that exerts a strong influence on properties such as the maximum ion exchange capacity, thermal and hydrothermal stability, hydrophobicity, and acidic properties. A variation in the $\mathrm{Si} / \mathrm{Al}$ ratio can also create a certain extra porosity due to the presence of defect sites in the zeolite framework upon post-treatment [24]. For instance, silicon extraction through treatment in an aqueous alkaline solution, known as desilication, has proven to create extra porosity in different types of zeolites [25-27]. Furthermore, the selective removal of aluminium from the zeolite framework or dealumination, is generally achieved through steam treatment at relatively high temperatures by means of acid leaching or alkaline treatments [28-30].

However, PSM in zeolites has not been very widely used due to the limitations of these materials, such as the rigidity of their porous skeleton or the difficulty in functionalizing the porous surface. Nevertheless, a few 


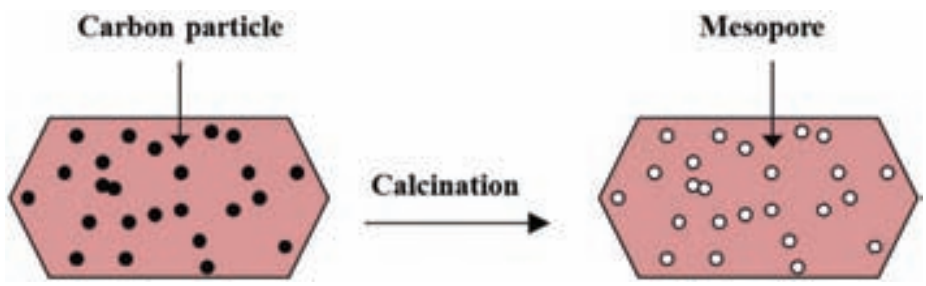

Figure 7. Synthesis of hierarchical zeolite by carbon nanoparticles.

examples of zeolite surface modifications have been reported and will be described below. For this reason, other methods have been developed in order to improve the porosity and adsorption properties of zeolites. In this sense, over the last decade studies regarding hierarchical zeolites have increased dramatically [31-33]. The term hierarchical zeolites, refers to zeolites featuring at least one additional level of porosity in addition to their intrinsic micropore system [34]. Nevertheless, in practice, the expression hierarchical zeolites, is nearly always used to refer to zeolites containing mesopores (pores with diameters in the range of $2-50 \mathrm{~nm}$ ).

In this context, the most commonly used synthesis method for increasing porosity in zeolites is by templates. The templating route involves the use of different materials such as carbon nanoparticles [35], carbon aerogels [36], micelles [37], surfactants [38], polymers [39], etc. Carbonaceous materials were the first systems studied as templates due to their properties including chemical inertness, structural diversity and ease of removal by combustion [40]. In this case, the hierarchical zeolites are produced when the growing zeolite crystals encapsulate the carbon matrix during synthesis, featuring a zeolite embedded with carbon material after the zeolite crystallization. Afterwards, the carbon matrix is removed by combustion, resulting in a material with mesopores (Figure 7) [32].

On the other hand, the enormous versatility of MOFs has allowed the development of different methodologies with the intention of modifying their structures and, consequently, improving their adsorption properties. The ability to modify the physical environment of the pores and cavities within MOFs would allow the tuning of interactions with guest species. In this sense, two different strategies have been employed to achieve MOF functionalization.

The first is a pre-synthetic methodology. This strategy involves the use of a functionalized ligand $[41,42]$ or metalloligands [43] as the organic linker. For instance, the isoreticular metal-organic framework (IRMOF) series of materials described by Yaghi et al. were synthesized with many different 
functional groups (amine, nitro, chlorine, bromine, etc.) [44]. However, this methodology presents some limitations. For example, under solvothermal conditions, the ligands cannot contain thermally labile groups. For this reason, the range of chemical functionality found in IRMOFs is rather limited. Functional groups such as alcohols, aldehydes, carboxylic acids, nitriles, azides, alkylamines, thiols, phosphines, and many others are absent from the list of ligands that have been used.

Fortunately, PSM can overcome the limitations present in prefunctionalized methods. In this sense, modified materials with a wide variety of functional groups can be obtained as long as the original MOFs are sufficiently robust and porous allowing transformations in a final step. The materials are thus able to conserve their structural integrity. Post-synthetic modification of MOFs can be also described as the chemical derivatization of MOFs after their formation [45]. The advantages offered by the PSM approach to functionalizing MOFs are the following: $i$ ) it is possible to include a more diverse range of functional groups, ii) purification and isolation of modified products is easy because the chemical functionalization is performed directly on crystalline solids, iii) the same MOF structure can be functionalized with different groups, thereby yielding a high number of topologically identical but functionally diverse MOFs, iv) control over both the type of functional group and the degree of modification allows for the optimization and improvement of MOF adsorption properties.

In the last decade, many different PSM strategies related to MOFs have been developed. Regarding gas adsorption, modification of secondary building units (SBUs) [46], ion exchange [47] and formation of defects [48] have been the most widely studied.

\section{Modification of secondary building units (SBUs)}

As we have seen in previous chapters, secondary building units are the inorganic parts of a MOF. There are many MOFs containing labile ligands, which are often coordinated solvent molecules, attached to the secondarybuilding units. These ligands can be removed by heating to generate a porous material with open coordination sites, thus allowing for the substitution of these labile ligands with another ligand, often leading to enhanced adsorption properties [49-51].

For example, post-synthetic modifications of HKUST-1 $\left[\mathrm{Cu}_{3}(\mathrm{btc})_{2}\left(\mathrm{H}_{2} \mathrm{O}\right)_{3}\right]$ (btc = benzene-1,3,5-tricarboxylate) have been widely studied due to the easy generation of unsaturated metal sites in its structure. HKUST-1 


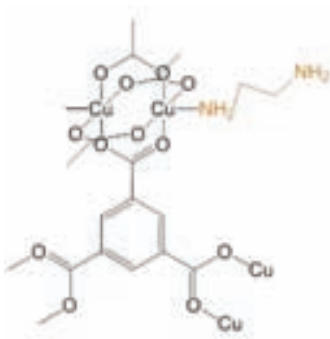

Ethylenediamine

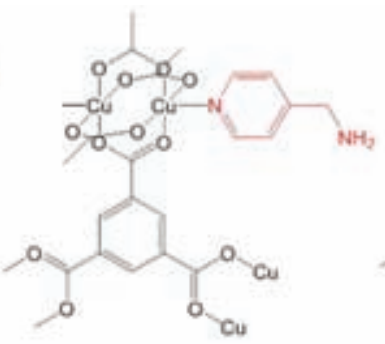

4- picolylamine

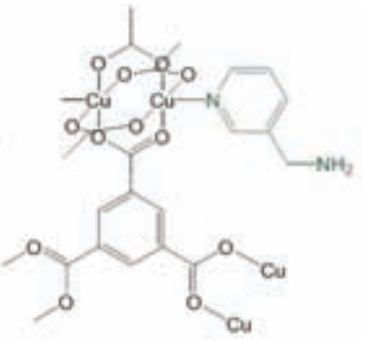

4- picolylamine

Figure 8. Scheme of functionalization of HKUST-1 with ethylenediamine, 4-picolylamine and 3-picolylamine.

contains coordinated water molecules at $\mathrm{Cu}$ (II) centres which can be easily removed by heating and consequently, replaced by another ligand. In this sense, Navarro et al. have carried out the functionalization of HKUST-1 with different bifunctional amines (Figure 8). It is interesting to note that the material modified with 3-picolylamine significantly improved $\mathrm{CO}_{2}$ adsorption compared to pristine HKUST-1 [52].

\section{Formation of defects}

Defects can be defined as structural disorders and heterogeneities that break the periodic arrangement of atoms. In this context, the structure of MOFs contains a considerable density of structural irregularities or defects. These inherent defects are formed during crystal growth in the form of missing metal nodes or linkers (either the entire molecule or as a result of stacking faults or dislocations) [48].

Nevertheless, in recent years, many studies have demonstrated that the presence of defects in MOFs produces an improvement in adsorption and catalytic properties [53-57]. Therefore, the possibility of controlling the form, size, and number of defects through post-synthetic modification is very interesting and several methodologies have been developed to this end. For instance, acid/base post-synthetic treatment has been proven to be an efficient strategy for introducing defects into pre-formed MOFs [58]. Recently, thermal treatment of $\left[\mathrm{Zn}_{4} \mathrm{O}(\mathrm{BDC})_{3}\right](\mathrm{BDC}=1,4$-benzene dicarboxylate) MOF- 5 has been studied. This study reveals that temperatures below the decomposition point of the material but above the conventional solvent evacuation temperature induce the partial decomposition of the 
carboxylate ligands, generating a local defective vacancy structure. The vacancy defect led to an improvement in $\mathrm{CO}_{2}$ adsorption capacity due to the presence of active sites at the framework linker and the unsaturated metal sites [59].

\section{Ion exchange}

It is possible to carry out cation exchange in MOFs in a similar way to zeolites. Although there are not many examples of ionic MOFs [6o-62], ionic sites can be introduced to neutral frameworks by means of postsynthetic functionalization treatments, including the removal of some of the linkers, among others [47]. In this context, cation exchange in the material $\mathrm{K}\left[\mathrm{Ni}_{8}(\mathrm{OH})_{6}\left(\mathrm{H}_{2} \mathrm{O}\right)_{2}(\mathrm{BDP})_{5.5}\right](\mathrm{BDP}=1,4$-bis(pyrazol-4-yl) benzene) has recently been studied. In this study, extra-framework $\mathrm{Ba}(\mathrm{II})$ ions were introduced into the porous structure through an exchange process with $\mathrm{K}(\mathrm{I})$ cations. It was found that the post-synthetically modified MOF material enhanced $\mathrm{SO}_{2}$ adsorptive properties [63].

\section{Gas adsorption on zeolites and metal-organic frameworks}

As already mentioned, adsorption capacity is one of the main factors that determine the possible application of a porous material. In this sense, different gas adsorption capabilities in MOFs and zeolites have been tested, mainly focused on specific potential applications related to energy, environment remediation, gas separation, and sensors, among the most important ones. The following discussion is divided into three sections with the objective of highlighting the gas adsorptive properties of zeolites and MOFs according to their applications: energy, $\left(\mathrm{H}_{2}\right.$ and $\left.\mathrm{CH}_{4}\right)$, environmental remediation $\left(\mathrm{CO}_{2}, \mathrm{NOx}\right.$ and $\left.\mathrm{SO}_{2}\right)$, and other applications for gas adsorption.

\subsection{Applications in energy}

\section{Hydrogen}

In the last few decades, a demand for clean and efficient energy sources has grown due to the decreasing availability of fossil fuel resources. The chemistry community has focused research on the exploration of alternative sources of clean energy, hydrogen being a promising means for these purposes [64]. 
Hydrogen adsorption is governed by framework topologies and exchangeable extraframework cations in zeolites. For example, Langmi et al. reported the hydrogen adsorption capacities in zeolites A, X, Y and RHO, and diverse results were obtained depending on the adsorption temperature, pore size and metal ions exchanged on each zeolite [65]. In another work Na-LEV, H-OFF, Na-MAZ and Li-ABW zeolites were examined with similar concluding remarks referred to the large volume of micropore cations, as a key factor for enhancing hydrogen uptake capacities [66].

On the other hand, MOFs with extremely high surfaces areas (up to $7100 \mathrm{~m}^{2} \mathrm{~g}^{-1}$ ) [67] have been widely researched for hydrogen adsorption. However, the relatively low gravimetric and volumetric densities of hydrogen adsorbed on MOFs under ambient conditions remains a challenge to be overcome. This is especially so in view of the US Department of Energy (DOE) goals for 2020 [68], by which hydrogen on-board vehicle storage systems should achieve densities of $5.5 \mathrm{wt} . \%$ and $40 \mathrm{~g} \mathrm{l}^{-1}$.

Several strategies have been adopted to enhance hydrogen adsorptive properties on MOFs [69]. These are mainly focused on improving the interactions between adsorbed hydrogen molecules and the adsorbent by means of higher surface areas and porosities (achievable by catenation and interpenetration), interactions with metal centres (open metal sites, extra-framework metal species, etc.) and with pore walls and/or ligands (van der Waals, functional groups, etc.).

Different authors have studied and reviewed works on MOFs for hydrogen storage applications [70-72], underlining the features needed in these materials for improved hydrogen adsorption at room temperatures. It is worth mentioning materials like MOF-5 $\left(\mathrm{Zn}_{4} \mathrm{O}(\mathrm{BDC})_{3}\right)$ [73], on which Yaghi et al. studied the rotational transitions of hydrogen molecules adsorbed on binding sites by inelastic neutron scattering microscopy, or the higher hydrogen uptake showed by the isoreticular IRMOF- 6 and -8. In another work, the same authors followed the study of the isoreticular series from which it was assumed that the stronger influence of catenation in hydrogen adsorption behaviour compared with the introduction of functional groups like $-\mathrm{Br}$, $-\mathrm{NH}_{2}$ and $-\mathrm{C}_{2} \mathrm{H}_{4}$ and additionally, a substantial hydrogen uptake was noted when open metal sites, like in HKUST-1 and MOF-74 [74], were presented.

Dincă et al. studied the interactions of metal cations $\mathrm{Li}^{+}, \mathrm{Cu}^{+}, \mathrm{Fe}^{2+}, \mathrm{Co}^{2+}$, $\mathrm{Ni}^{2+}, \mathrm{Cu}^{2+}$ and $\mathrm{Zn}^{2+}$ in exchanged $\mathrm{Mn}_{3}\left[\left(\mathrm{Mn}_{4} \mathrm{Cl}\right)_{3}(\mathrm{BTT})_{8}\left(\mathrm{CH}_{3} \mathrm{OH}\right)_{10}\right]_{2}(\mathrm{BTT}=$ 1,3,5-tris(tetrazol-5-yl)benzene) MOF with adsorbed hydrogen molecules $[75,76]$. The same approach was taken by Schroder et al. [77], who used $\mathrm{Li}^{+}$as a counter-ion in the framework to enhance the adsorptive properties 
of the indium-containing MOF studied. Hydrogen adsorption on a series of $\mathrm{Cu}$ (II) tetracarboxylate MOFs (namely NOTT-10o-109) was studied by Schroder et al., supporting the central role of exposed metal centers, pore size and ligands functionalities in the adsorption process [74].

In contrast, a study on a rare earth metal MOF by powder neutron diffraction revealed the preferred interactions of hydrogen molecules with the pore walls rather that with the open metal sites [75]. Chen and colleagues, studied the hydrogen adsorption properties in an interpenetrated, framework, $\mathrm{Zn}(\mathrm{NDC})\left(4,4^{\prime} \mathrm{Bpe}\right)_{0.5}(\mathrm{NDC}=2,6$-naphthalenedicarboxylate, Bpe = 4,4'-trans-bis(4-pyridyl)-ethylene), showing features of dynamic MOFs hydrogen adsorption at room temperature and high pressures [79].

Some examples of zeolites and MOFs are summarized in Table 1, highlighting their adsorption capacities and measurement conditions [77-83].

\section{Methane}

Methane is considered an alternative source of transport fuel, since there are large natural reserves and it has a high research octane number $(\mathrm{RON}=107)$ due to its low carbon content. In addition, the current compressed natural gas technologies require the use of containers at high pressure and extreme safety conditions. Therefore, natural gas adsorption technologies are a highly desirable -and also challenging- choice. For practical uses in transport, it is important to clearly differentiate the terms used for methane, the deliverable capacity being the most important one, compared to the adsorption capacity. The former is related to the working capacity of the adsorbent releasing methane between high pressure to 5 bar from the adsorbent container [88,89].

The Advanced Research Projects Agency-Energy (ARPA-E) of the US Department of Energy (DOE) has set the new target for methane volumetric storage capacity to $350 \mathrm{~cm}^{3}$ (STP) $\mathrm{cm}^{-3}$, and for gravimetric capacity to $0.5 \mathrm{~g}$ $\left(\mathrm{CH}_{4}\right) \mathrm{g}^{-1}$, at ambient temperature, for automobile ANG (Adsorbed Natural Gas) applications [9o].

Over zeolites, due to the ionic nature of the pore surface and their hydrophillicity, methane adsorption is not especially favoured [91,92]. However, this handicap has been overcome and different zeolites have been tested for methane storage [93], and even a methane storage system has been patented using zeolites as adsorbents [94]. Similarly, the combination of MOFs and zeolites in a device named zeolite adsorbent-MOF layered nanovalve led to the improvement of their $\mathrm{CH}_{4}$ storage capacities. For example, an Al-MOF/zeolite $5 \mathrm{~A}$ composite nanovalve can store $33.6 \mathrm{l}$ (STP) $\mathrm{l}^{-1}$ at pressures $<1$ bar [95]. 


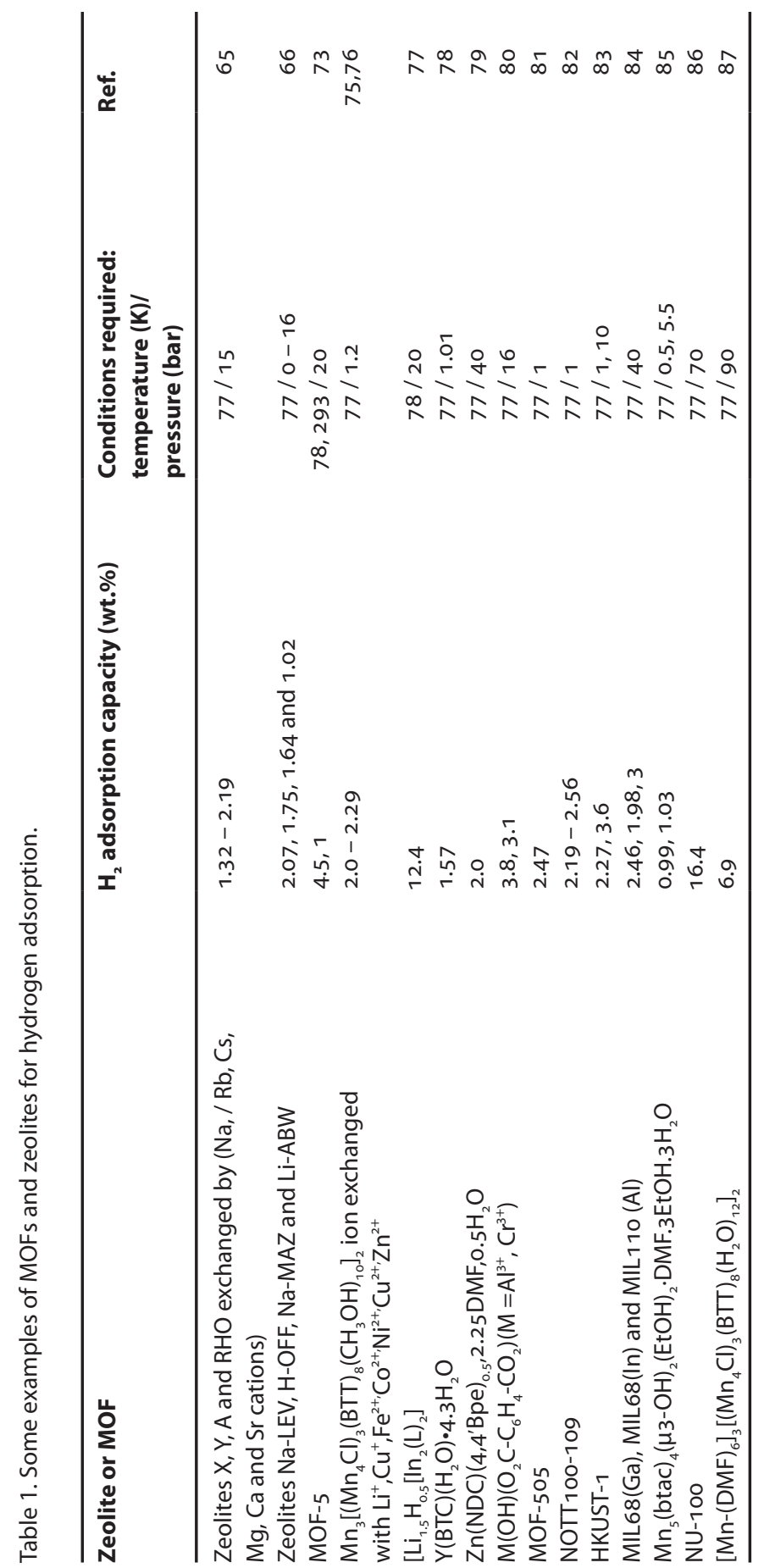


Table 2. Some examples of methane storage capacities and conditions of adsorption in MOFs and zeolites.

\begin{tabular}{|c|c|c|c|}
\hline Zeolite or MOF & $\begin{array}{l}\mathrm{CH}_{4} \text { volumetric } \\
\text { uptake }\left(\mathrm{cm}^{3} \mathrm{~cm}^{-3}\right) / \\
\text { working capacity } \\
\left(\mathrm{cm}^{3} \mathrm{~cm}^{-3}\right)\end{array}$ & $\begin{array}{l}\text { Conditions required: } \\
\text { temperature }(\mathrm{K}) / \\
\text { pressure (bar) }\end{array}$ & Ref. \\
\hline $5 \mathrm{~A}$ & $104 /-$ & $298 / 35$ & 88 \\
\hline $13 X$ & $106 /-$ & $298 / 35$ & 93 \\
\hline CaX zeolite & $150 /-$ & $276 / 9$ & 94 \\
\hline HKUST-1 & $225 / 200$ & $298 / 35$ & 101 \\
\hline $\mathrm{Co}(\mathrm{BDP})$ & $-/ 197$ & $298 / 65$ & 102 \\
\hline NOTT-108a & $247 / 186$ & $298 / 65$ & 99 \\
\hline NOTT-101a & $237 / 188$ & $298 / 65$ & 105 \\
\hline$\left[\mathrm{Zn}_{3}(\mathrm{pypz})_{3}(\mathrm{btc})\right] \cdot$ guest & 263 & $298 / 65$ & 106 \\
\hline UTSA-20 $\left[\mathrm{Cu}_{3}(\mathrm{BHB})\right]$ & 195 & $300 / 35$ & 107 \\
\hline UTSA-76a & $260 / 200$ & $298 / 65$ & 97 \\
\hline UTSA-88a & $248 / 185$ & $298 / 65$ & 111 \\
\hline MOF-74-Ni & $251 / 129$ & $298 / 65$ & 109 \\
\hline PCN-14 & $230 / 157$ & $298 / 65$ & 109 \\
\hline $\mathrm{NU}-125$ & $232 / 183$ & $298 / 65$ & 103 \\
\hline $\mathrm{NU}-111$ & $206 / 179$ & $298 / 65$ & 109 \\
\hline MOF-905 & $228 / 203$ & $298 / 80$ & 104 \\
\hline MOF-950 & $209 / 174$ & $298 / 80$ & 104 \\
\hline ZJNU-30a & $179.3 / 151$ & $298 / 65$ & 112 \\
\hline ZJNU-31a & $137.7 / 115$ & $298 / 65$ & 112 \\
\hline ZJNU-53 & $241 / 190$ & $298 / 65$ & 113 \\
\hline NJU-Baizo & $186 /-$ & $298 / 65$ & 114 \\
\hline NJU-Bai31 & $158 /-$ & $298 / 65$ & 114 \\
\hline
\end{tabular}

Taking into consideration the high surface areas and tunabilities of pore surface in MOFs, they are the candidates of choice compared to other porous adsorbents such as zeolites or porous carbons. In MOFs, the study of adsorption and release capacity of methane has allowed to identify two favourable binding interactions of methane: i) the Coulomb interactions of methane with open metal sites, and ii) the van der Waals interactions with small cages/channels [96]. However, more recently, new findings on improved methane adsorption have been found in MOFs with nitrogen-containing $[97,98]$ or fluorinated $[99,100]$ ligands.

Some benchmark materials for methane storage, like HKUST-1 [101], Co(BDP) [102], UTSA-76a [97] and Al-soc-MOF-1 [103], with working capacities close to DOE target values (Table 2) have been reported. Recently, other MOFs with also high volumetric storage capacities have been evaluated, such as 
MOF-905 and MOF-950 (with working capacities of 203 and $174 \mathrm{~cm}^{3}$ (STP) $\mathrm{cm}^{-3}$, respectively, at $298 \mathrm{~K}$ and 8o bar) [104], or NOTT-108a [99] and NOTT-101a [105].

It is worth noting a very recent paper where $\left[\mathrm{Zn}_{3}(\mathrm{pypz})_{3}(\mathrm{btc})\right]$.guest.MOF $(\mathrm{MAF}-38)(\mathrm{pypz}=4-(1 \mathrm{H}-$ pyrazol-4-yl $)$ pyridine $)$ affords a high methane uptake $(263 \mathrm{v} / \mathrm{v}$ at $298 \mathrm{~K}$ and 65 bar $)$ and adsorption enthalpies $\left(21.6 \mathrm{~kJ} \mathrm{~mol}^{-1}\right)$ close to the highest storage MOFs available, like HKUST-1, with open metal sites as the preferred binding sites for methane adsorption in the framework [106]. Another good example of high methane uptake at room temperature is UTSA-20 that reaches a storage density of $0.22 \mathrm{~g} \mathrm{~cm}^{3}$ resulted the highest density of open copper sites in a MOF [107]. Other authors have illustrated the methane storage capacity of other benchmark MOFs, the possibilities to have improved capacities in the future MOFs, and the influence of packing density of MOFs on experimental working capacities [101,108-110]. Some reviews also highlight the methane storage capacities of adsorbent or porous materials like MOFs, COFs (covalent organic frameworks), zeolites and carbons [100]. In Table 2, a few examples of zeolites and MOFs are summarized for a better comparison of their capacities [111-114].

\subsection{Environmental remediation}

\section{Carbon dioxide}

In recent decades, our dependence on fossil fuels for energy generation has led to an increase in the $\mathrm{CO}_{2}$ emissions released to the atmosphere. As a consequence, the temperature of the Earth surface has increased by about $0.85^{\circ} \mathrm{C}$ since pre-industrial levels. The temperature is expected to increase by the end of the 21 st century by $4{ }^{\circ} \mathrm{C}$ if greenhouse gas emissions are not reduced. According to a report published by the Intergovernmental Panel on Climate Change (ICPP) [115], in order to reduce the adverse effects of climate change, by 2100 the Earth's surface temperature must not increase beyond a threshold of $2{ }^{\circ} \mathrm{C}$. To achieve this, greenhouse gas emissions must be reduced by between 40 and $70 \%$ globally between 2010 and 2050 , and be reduced to zero by 2100 . With this in mind, many zeolites and metalorganic frameworks have been studied for their ability to capture $\mathrm{CO}_{2}$.

In the case of zeolites, a robust nature, coupled with their low cost and well-developed structural chemistry, makes these materials an attractive target for use in $\mathrm{CO}_{2}$ capture applications. Moreover, the presence of cations such as $\mathrm{Na}(\mathrm{I}), \mathrm{Ca}(\mathrm{II})$, or $\mathrm{Li}(\mathrm{I})$ influences the adsorption capacity of $\mathrm{CO}_{2}$ due to the interaction between alkali ions and $\mathrm{CO}_{2}$ molecules [116]. The high quadrupole moment of $\mathrm{CO}_{2}\left(14.2910^{-40} \mathrm{C} \mathrm{m}^{2}\right)$ interacts strongly with the electric 
Table 3. Some examples of carbon dioxide storage capacities and conditions of adsorption in zeolites.

\begin{tabular}{lclr}
\hline Zeolite & $\begin{array}{l}\mathrm{CO}_{2} \text { adsorption } \\
\text { capacity (wt\%) }\end{array}$ & $\begin{array}{l}\text { Conditions required: } \\
\text { temperature (K) / pressure (bar) }\end{array}$ & \multicolumn{1}{l}{ Ref. } \\
\hline 13X & $4,20.5$ & $393 /-, 298 / 1.01$ & 124,118 \\
$13 \mathrm{X}-\mathrm{MEA}$ & 14 & $393 /-$ & 124 \\
$\mathrm{NaX}$ & 25.1 & $305 / 1.01$ & 119 \\
$\mathrm{NaY}$ & 24.2 & $305 / 1.01$ & 119 \\
$\mathrm{ZSM}-5$ & 1.3 & $313 / 0.1$ & 120 \\
$5 \mathrm{~A}$ & 23.3 & $298 / 14$ & 121 \\
\hline
\end{tabular}

field created by the structural cations of zeolites, increasing their adsorption capacity [117]. Because of this, zeolites have shown promising results with regard to the separation of $\mathrm{CO}_{2}$ from gas streams. In this sense, there are many studies concerning the $\mathrm{CO}_{2}$ adsorption capacity of both natural and synthetic zeolites. Table 3 summarizes some different zeolites that have been studied for $\mathrm{CO}_{2}$ adsorption and separation from gas mixtures [118].

However, $\mathrm{CO}_{2}$ adsorption capacity in zeolite materials decreases significantly at high temperatures and/or by the moisture present in flue gases [122]. Nevertheless, it is possible to avoid these limitations by means of post-synthetic modification of zeolites. For example, Zhang et al. showed a cation exchange strategy for chabazite $(\mathrm{CHA})$ zeolites $(\mathrm{Si} / \mathrm{Al}<2.5)$ with several ions, including $\mathrm{Li}(\mathrm{II}), \mathrm{Na}(\mathrm{I})$ and $\mathrm{Mg}(\mathrm{II})$, among others. In the study, the Na-CHA and Ca-CHA materials presented advantages in $\mathrm{CO}_{2}$ separation at high temperatures [123]. Furthermore, it is important to highlight PSM use through the incorporation of amine groups. In this sense, Jadhav et al. studied the modified zeolite ${ }_{13} \mathrm{X}$ via monoethanol amine (MEA) impregnation to improve its $\mathrm{CO}_{2}$ adsorption capacity (Table 3). The aminated zeolites showed a higher capacity than the pristine zeolites at $120^{\circ} \mathrm{C}[124]$. Su et al reported a similar conclusion. In this case, the Y-type zeolite was functionalized with tetraethylenepentamine [125].

On the other hand, MOFs are also excellent candidates for $\mathrm{CO}_{2}$ adsorption due to their remarkably high surface area, controllable pore structures, and tunable pore surface properties. MOF- 5 was the first MOF reported for $\mathrm{CO}_{2}$ capture [126]. Since then, the amount of studies published has increased dramatically. Table 4 summarizes the $\mathrm{CO}_{2}$ adsorption capacity of the most common MOFs for $\mathrm{CO}_{2}$ capture [127-130]. It is interesting to note that MOF210 presents an excellent $\mathrm{CO}_{2}$ adsorption capacity due to the ultrahigh porosity of this MOF (Table 4) [131]. 
Table 4. Some examples of carbon dioxide storage capacities and conditions of adsorption in MOFs.

\begin{tabular}{llll}
\hline MOF & $\begin{array}{l}\text { CO } \text { adsorption } \\
\text { capacity (wt.\%) }\end{array}$ & $\begin{array}{l}\text { Conditions required: } \\
\text { temperature (K)/ } \\
\text { pressure (bar) }\end{array}$ & Ref. \\
\hline MOF-5 & 50 & $298 / 14$ & 121 \\
MOF-177 & 39.7 & $298 / 1.01$ & 121 \\
MOF-74-Ni & 23.9 & $298 / 1$ & 128 \\
MOF-74-Mg & 27.5 & $298 / 1$ & 129 \\
ED-MOF-74-Mg & 38.2 & $298 / 1$ & 132 \\
MOF-74-Co & 24.9 & $298 / 1$ & 130 \\
MOF-74-Zn & 19.8 & $298 / 1$ & 128 \\
HKUST-1 & 19.8 & $298 / 1$ & 127 \\
MOF-210 & 240 & $298 / 50$ & 131 \\
\hline
\end{tabular}

As discussed earlier, PSM in metal-organic frameworks is a very useful tool for improving $\mathrm{CO}_{2}$ adsorption capacity due to the versatility of these materials. As with zeolites, the presence of amino groups in MOFs enhances $\mathrm{CO}_{2}$ adsorption. However, it is interesting to note that the incorporation of amines into MOFs is not achieved through the impregnation method as it is in zeolites. As it was mentioned in the previous section, in MOF materials it is possible to functionalize the coordinatively unsaturated metal sites. For example, MOF-74-Mg has been widely studied in $\mathrm{CO}_{2}$ adsorption and their functionalization with ethylenediamine (ED) improves significantly its adsorption capacity [132].

\section{Toxic gases}

In addition to $\mathrm{CO}_{2}$, fossil fuel combustion also emits toxic gases such as $\mathrm{NO}_{\mathrm{x}}$ and $\mathrm{SO}_{\mathrm{x}}$ into the atmosphere. Among various sulphur and nitrogen oxide species, $\mathrm{NO}_{2}, \mathrm{NO}$, and $\mathrm{SO}_{2}$ are considered the most toxic and harmful gases. These acidic gases generate serious environmental problems such as photochemical smog and acid rain.

In the field of zeolites, adsorptive removal of $\mathrm{NO}$ and $\mathrm{NO}_{2}$ has been studied using zeolites at relatively low temperatures. These gases can be adsorbed through the reversible binding of nitrogen with framework cations. In this case, the gas is physically adsorbed and can be easily removed by slightly decreasing the pressure. In addition, NO can be chemically adsorbed by an irreversible bond to the surface, forming nitrosyl complexes. Notably, this kind of bonding of NO provides the opportunity for its storage [133]. 
Zeolites have also been tested for $\mathrm{SO}_{2}$ capture. The $\mathrm{SO}_{2}$ adsorption process in zeolites may be favoured due to the strong ion-dipole interaction between $\mathrm{SO}_{2}$ molecules and the cations of the zeolite. However, due to the variety of bonding modes of $\mathrm{SO}_{2}$ molecules, they can also interact with the Lewis basic sites of zeolites (the bridging oxygen atom in $\mathrm{Si}-\mathrm{O}-\mathrm{Al}$ ). Oxygen can form a bond with the sulphur atom through donor-acceptor interaction. In addition, in some zeolites $\mathrm{SO}_{2}$ is chemisorbed and a formation of $\mathrm{HSO}_{3}^{-}$or $\mathrm{SO}_{4}^{2-}$ is observed [134]. Table 5 shows $\mathrm{SO}_{2}$ and NO adsorption capacity for ${ }_{13} \mathrm{X}$ and $5 \mathrm{~A}$ zeolites.

On the other hand, the presence of open coordinatively unsaturated metal centres in MOFs opens the way to functionalization with basic groups or to incorporation of cations with affinity for $\mathrm{SO}_{2}$ and $\mathrm{NO}_{\mathrm{x}}$ adsorbates. Moreover, the adsorption process in MOFs can be carried out by coordination bonds, acid-base/electrostatic interactions, H-bonding formation, etc. [135] Many articles discussing this process have been published in the last decade. Table 5 shows the adsorption capacity of these toxic gases in different MOFs [136-143]. In addition, other articles have

Table 5. Some examples of sulphur dioxide, nitrogen oxide and nitrogen dioxide storage capacities and conditions of adsorption in zeolites and MOFs.

\begin{tabular}{lllll}
\hline Gas & $\begin{array}{l}\text { Zeolite or } \\
\text { MOF }\end{array}$ & $\begin{array}{l}\text { Adsorption } \\
\text { capacity } \\
\left.\mathbf{( m m o l ~ g}^{-1}\right)\end{array}$ & $\begin{array}{l}\text { Conditions required: } \\
\text { temperature }(\mathbf{K}) / \text { pressure } \\
\text { (bar) or [concentration] }\end{array}$ & Ref. \\
\hline $\mathrm{SO}_{2}$ & $13 \mathrm{X}$ & 2.5 & $323 / 0.5$ & \\
& $5 \mathrm{~A}$ & 1.5 & $323 / 0.5$ & 136 \\
& MOF-5 & 1 & $298 /[1 \%]$ & 136 \\
& MOF-177 & $<1$ & $298 /[1 \%]$ & 137 \\
& HKUST-1/Ba & 2.4 & $473 /[50 \mathrm{ppm}]$ & 137 \\
& IRMOF-3 & 6 & $298 /[1 \%]$ & 138 \\
& MOF-74-Zn & 3 & $298 /[1 \%]$ & 137 \\
\hline NO & 13X & 0.1 & $323 / 0.5$ & 137 \\
& 5A & 0.1 & $323 / 0.5$ & 136 \\
& MOF-74-Ni & 7 & $298 / 1$ & 136 \\
& HKUST-1 & 3 & $298 / 1$ & 139 \\
& MIL-88A & 7.1 & $303 / 1$ & 140 \\
& UiO-66 & 1.1 & $298 /[1000 \mathrm{ppm}]$ & 141 \\
\hline $\mathrm{NO}_{2}$ & UiO-67 & 1.7 & $298 /[1000 \mathrm{ppm}]$ & 142 \\
& HKUST-1 & 1.7 & $298 /[1000 \mathrm{ppm}]$ & 143 \\
& & & 143 \\
\hline
\end{tabular}

${ }^{a}$ gas toxic concentration in gas mixture 
been published for MOFs concerning other toxic gases adsorption, such as chemical warfare agents $[144,145]$.

\subsection{Other applications for gas adsorption}

Adsorption of other gases on zeolites and MOFs, such as oxygen, noble gases, water vapour and volatile organic compounds (VOCs), has been studied with interesting results. Some examples of adsorption of the abovementioned gases, is described below.

Since oxygen needs to be stored in high-pressure containers for medical, industrial and aerospace applications, its adsorptive properties on different materials like, zeolites, porous carbons and MOFs, have been evaluated. For instance, zeolites have been widely used for adsorption and enrichment of $\mathrm{O}_{2}$ from air, obtaining concentrations of up to $99.5 \%$ pure $\mathrm{O}_{2}$ using AgLiLSX zeolite [146]. Other zeolites used for similar purposes are zeolite 5 A [147] and LiLSX zeolite [148,149]. However, oxygen adsorption in MOFs has been less explored. An excellent review has been published by DeCoste et al. [150] where a computational screening of 10,000 MOFs for oxygen storage is presented. Benchmark MOFs, such as HKUST-1, NU-125 and UiO-66, are experimentally tested and their structural features and properties that can stimulate the $\mathrm{O}_{2}$ adsorption are also studied. In another work, MOF Al-soc-MOF-1 showed an absolute gravimetric $\mathrm{O}_{2}$ uptake of $29 \mathrm{mmol} \mathrm{g}^{-1}$, being among the highest uptakes reported so far [103]. Additionally, other MOFs have been reported for the adsorption and storage of oxygen, highlighting the framework properties that stimulate the $\mathrm{O}_{2}$ adsorption processes [151-154].

As fossil fuel-based energy sources are in a constant decline, other alternative sources like nuclear energy are used instead, with the problem in this case of dangerous radioactive waste being generated. In addition, xenon and krypton are evolved as radionuclides from nuclear fuel reprocessing facilities. The high cost of cryogenic techniques enforces the search for less expensive solutions, such as porous materials, for the selective adsorption and separation of these gases [155]. The use of zeolites in devices like membranes was assessed experimentally and computationally by Forster et al. They concluded that channel and pore dimensions are key factors in the adsorption and separation of Xe/Kr mixtures [156]. Additionally, other zeolites membranes have been tested for this application [157]. Furthermore, MOFs were experimentally and computationally screened for $\mathrm{Xe} / \mathrm{Kr}$ adsorption and separation SBMOF-1 being the most selective to 
Xe gas. Benchmark MOFs like UiO-66(Zr), MIL-10o(Fe), MIL-101(Cr) [158], and ultramicroporous MOFs [159] have also been used for these purposes with interesting results.

Adsorption in porous materials opens the way to other applications such as separations and sensors [16o]. In this sense, the separation capacities of gases and VOCs have been the subject of several works concerning both zeolites and MOFs. Actually, many authors have published the use of zeolites incorporated into membranes or filters for separation and purification technologies of gases and light hydrocarbons [161-163]. Excellent reviews have been published for separations in MOFs by Li et al. [164,165], and also the adsorption and separation processes in densified MOFs was outlined by Nune et al. [166] Moreover, MOFs have been incorporated into membranes to improve their performance, as reported by different authors [167-169], and separations of hydrocarbons and their isomers [170-172] as well as the separation of acetylene from $\mathrm{CO}_{2}$ have also been extensively studied [173-175].

\section{Adsorption of molecules of environmental interest in solution}

Although most of the examples shown in this chapter imply the adsorption of gas molecules into the cavities of a solid porous matrix (namely, zeolites or MOFs), there is also an increasing interest in developing advanced materials, which are able to efficiently adsorb toxic molecules in solution or sludges. In this sense, most of the examples reported in the literature so far have been focused on aqueous solutions, such as water purification, which has a tremendous impact of the health and welfare of society. In particular, great efforts are being directed towards the development of low-cost but selective adsorbent materials capable of retaining harmful substances such as, heavy metals, ionic species (ammonium, nitrate, phosphate, etc.), radionuclides, dyes and other organic molecules, as well as pharmaceuticals and personal care products, in their cavities [176].

In the last decades, zeolites have been explored as adsorbents in wastewater treatments due to their abundance in nature or low-cost preparation in this context. In particular, they exhibit high performance towards adsorption of cations (mainly, ammonium and heavy metals), based on their excellent ion exchange properties [177,178]. Moreover, different modifications, such as acid/base treatments or surfactant functionalizations [179], have been carried out in order to improve their selectivity towards adsorption of non-polar organic molecules or anions. In this sense, the use of 
metal-organic frameworks allows a more specific control over the final properties of the resulting materials, becoming more suitable candidates for the increasingly demanding needs in terms of water policy [180]. In fact, these 'laboratory materials' can be easily scaled-up and designed or functionalized according to the requirements of each application (tunable pore geometries, flexible skeletons, presence of reactive open metal sites, etc.) [181,182].

Some of the most relevant pollutants involved in water contamination are briefly discussed below, emphasizing the role that zeolites and MOFs have played in this context to date.

\section{Removal of heavy metals and ionic species}

As is well known, heavy metals are not biodegradable and tend to bioaccumulate in humans and other living organisms, resulting in severe diseases and disorders [183]. For this reason, the removal of these toxic species, mainly from wastewater and acid mine drainage, is of crucial importance. Both natural and synthetic zeolites have been extensively used in this field, as they exhibit excellent ion exchange properties and are inexpensive materials. In fact, they have been employed in the purification of aqueous solutions containing, among others, $\mathrm{Cr}(\mathrm{III}), \mathrm{Ni}(\mathrm{II}), \mathrm{Cu}(\mathrm{II}), \mathrm{Zn}(\mathrm{II}), \mathrm{Cd}(\mathrm{II})$ and $\mathrm{Pb}(\mathrm{II})$ [184-189]. Moreover, it has been demonstrated that a simple surfactant-modification of natural zeolites (e.g. Mordenite, clinoptilolite and chabazite-Ca) can significantly improve their adsorption capacity [190,191].

Metal-organic frameworks, have also been explored as trapping agents for different toxic cations (e.g. $\mathrm{Cu}(\mathrm{II}), \mathrm{Cd}(\mathrm{II}), \mathrm{Hg}(\mathrm{II})$ and $\mathrm{Pb}(\mathrm{II}))$ [192,193], while post-synthetic modifications of their skeletons, including thiol functionalization of HKUST-1 [194], thiourea functionalization of UiO-66- $\mathrm{NH}_{2}$ [195], and metalation of a Zn-based MOF containing a flexible tetracarboxylate linker [196] have been carried out in order to increase their adsorption properties in these toxic species.

Furthermore, some of these metal pollutants, such as $\mathrm{AsO}_{4}^{-}$or $\mathrm{CrO}_{4}^{-}$in their oxo-hydroxo anionic forms, occur in water. Indeed, some studies have been focused on the removal of different arsenic species by the zeolitic imidazole framework ZIF-8 [197,198], or different synthetic zeolites (e.g. Y, ZSM-5, Beta, Ferrierite, H-MFI-24 and H-MFI-9o in their $\mathrm{NH}_{4}^{+}$or $\mathrm{H}^{+}$forms) $[199,200]$, while chromate has been selectively trapped in the cavities of cationic metal-organic solid solutions [201].

Finally, adsorbent composites, based on zeolites or MOFs and other materials (cellulose acetate, nanoscale zero-valent iron, PVA polymer, 
magnetic nanoparticles, ceramic membrane ultrafiltration, etc.), are currently under development aiming to create hybrid systems with improved properties [202-207].

On the other hand, the discharge of effluents containing ionic species, such as ammonium, nitrates and phosphates, from domestic or industrial sources, can result in eutrophication of receiving water bodies, with a consequent environmental impact and ecosystem alteration. In this context, inorganic porous materials have been proposed as containers to keep these inorganic species trapped [208-209]. For example, phosphate ions, which are responsible for the algal bloom, have been selectively removed by using the synthetic zeolites HSZ 330 HUD and its $\mathrm{Al}^{3+}$ activated form [210], and the zirconium-based MOFs UiO-66 and UiO-66- $\mathrm{NH}_{2}$ [211].

\section{Removal of radionuclides and fission products}

The fast development of nuclear energy since the middle of the last century has also led to the accumulation of radionuclides and fission products in waste streams, becoming a serious concern with regard to environmental and public health. In this sense, zeolites and MOFs have emerged as promising candidates to satisfactorily handle these radioactive residues. Uranium, which is usually found in its hexavalent form, has been shown to be strongly adsorbed over different inorganic porous matrixes such as manganese oxide coated zeolites [212] and metal-organic frameworks exhibiting UiO-68 network topology [213]. Likewise, the remediation of aqueous solutions containing long-lived fission products (e.g. Tc-99 or Cs-135) is also needed. In fact, several studies have been carried out to date, employing four zeolites minerals (natural clinoptilolite, natural chabazite, natural Mordenite and synthetic Mordenite) to remove cesium from low-level radioactive liquid waste [214] and the efficient and selective uptake of pertechnetate ion $\left(\mathrm{TcO}_{4}^{-}\right)$by a cationic metal-organic framework material exhibiting open $\mathrm{Ag}^{+}$sites [215].

\section{Removal of dyes from the textile industry}

Dyes are also non-biodegradable pollutants commonly present in effluents from textile, paper, printing and other industries. The discharge of dye molecules into the environment generates serious problems not only from an aesthetic but also a toxicological point of view. Although 
commercial activated carbon is widely used as an adsorbent for colour removal, its relatively high cost and regeneration problems have encouraged the development of more profitable materials. Among them, zeolites are attractive adsorbents because of their low cost and easy availability. In fact, numerous studies have already demonstrated their adsorption capacity towards several cationic dyes (e.g. methylene blue, crystal violet or rhodamine B) in aqueous solution [216-218]. In addition, the use of modified zeolites (e.g. using hexamethylenediamine as surfactant) has allowed the successful removal of reactive azo dyes, such as Reactive Red 239 and Reactive Blue 250, which constitute over $50 \%$ of all textile dyes used in the industry [219].

The first example of a MOF being used as a dye adsorbent was published in 2010 [220]. In this work, two highly porous Cr-based MOFs were selected for the adsorptive removal of methyl orange from aqueous solutions. Since then, several examples using different framework topologies have been reported in the literature [221].

Moreover, recent studies have demonstrated that composites comprising a metal-organic framework (e.g. MIL-101, HKUST-1, ZIF-8, UiO-66, etc.) and other species (e.g. polyoxometalates, graphite oxide, polymers, etc.) are also promising candidates in terms of dye capture efficiency, outperforming in some cases the behaviour of commercial activated carbons [222-224]. Composites based on zeolites (e.g. natural clinoptilolite, NaZMS-5, etc.) loaded with titanium oxide are a new class of hybrid materials able to absorb and decompose efficiently different organic dyes by irradiation with light $\left[225^{-227}\right]$.

\section{Removal of pharmaceuticals and personal care products}

Finally, pharmaceuticals and personal care products (PPCPs) are considered emerging contaminants due to their widespread development and use in the last decades. Unfortunately, conventional wastewater treatment plants lack adequate purification processes to remove these organic contaminants. As result, pharmaceuticals and other related organic molecules are currently present, in detectable levels, in surface and ground water around industrial and residential communities, generating, among others, antibiotic-resistance issues. In this context, the research community and industry are currently working together in order to develop purification strategies able to remove these harmful substances from water supplies. For example, adsorption materials, including zeolites and metal-organic 
frameworks, can be considered a feasible solution as they are efficient, simple to design, and inexpensive. Indeed, different zeolites (such as, Y, Mordenite and ZSM-5) have already been tested, showing positive results as absorbents of different pharmaceutically active compounds [228-229]. In addition, Zr-based MOFs have recently been used for the detection and removal of antibiotics [230], while well-known HKUST-1 proved to be a suitable sorbent for different parabens [231].

In summary, inorganic porous materials, namely zeolites and metalorganic frameworks, have attracted a great deal of attention for wastewater treatment mainly due to their high surface areas and versatility. On the one hand, zeolites seem to be promising candidates in those applications that require easy availability and low-cost materials. On the other hand, the possibility of designing and preparing MOFs 'à la carte', could become a clear advantage in more specific applications with higher budgets.

\section{Conclusion}

In recent years both zeolites and metal-organic frameworks have attracted a great deal of attention due to their exceptional properties (high porosity, high stability, tunable porosity and customizable skeletons), which make them promising candidates for industrial and environmental applications. In fact, different synthetic methodologies, including pre- and post-synthetic modifications, ion exchange processes, dealumination, desilication, controlled introduction of defects or metal cluster doping, have been recently developed in order to achieve increasingly demanding environmental requirements.

In this chapter, some of the most representative examples of adsorption in environmental and industrial applications have been compiled, including studies in both gas and liquid phase. On the one hand, the gas adsorption capability of both materials (zeolites and MOFs) has been discussed, trying to evaluate their potential as adsorbents in energy applications and/ or environment remediation processes $\left(\mathrm{CO}_{2}\right.$ capture and removal of toxic gases). On the other hand, these porous materials have also shown promising results regarding the adsorption of toxic molecules in solutions. In fact, some zeolites have already exhibited similar adsorption properties to those of commercial activated carbons, without the regeneration problems associated with these. In addition, MOFs, which can be easily designed and functionalized, are becoming suitable adsorbent materials in those water treatment processes that require the removal of more specific/difficult adsorbates. 
In conclusion, the excellent adsorption properties of zeolites and MOFs, linked to their ability to be designed in a rational way, make them appropriate candidates exhibiting promising perspectives towards this type of application.

\section{References}

[1] Atkins, P.W. The Elements of Physical Chemistry, Oxford University Press, 1994.

[2] IUPAC Compendium of Chemical Terminology, 2nd Ed., 1997.

[3] Sing, K.S.W, Gregg, S.J. Adsorption, Surface Area and Porosity, 2nd Ed., Academic Press, 1982.

[4] Brunauer, S., Deming, L.S., Deming, W.E., Teller, E.J. Am. Chem. Soc. 1940, 62, 1723.

[5] Reinoso, F.R. Chemistry and Physics of Carbon, Marcel Dekker, 1989.

[6] Brunauer, S., Emmett, P.H., Teller, E. J. Am. Chem. Soc. 1938, 6o, 309.

[7] Dubinin, M.M. Carbon 1985, 23, 373.

[8] Thielmann, F. J. Chromatogr. A 2004, 1037, 115.

[9] Rückriem, M., Enke, D., Hahn, T. Microp. Mesop. Mater. 2015, 209, 99.

[10] Mohammadi-Jam, S., Waters, K.E. Adv. Colloid Interface Sci. 2014, 212, 21.

[11] Conder, J.R., Young, C.L. Physicochemical Measurements by Gas Chromatography, Wiley, 1979 .

[12] Papirer, E., Brendle, E., Ozil, F. Balard, H, Carbon 1999, 37, 1265.

[13] Papirer, E., Li, S., Balard, H., Jagiello, J, Carbon 1991, 29, 1135.

[14] Díaz, E., Ordóñez, S., Vega, A. J. Colloid Interface Sci. 2007, 305, 7.

[15] Voelkel, A., Strzemiecka, B, Adamska, K,, Milczewska, K. J. Chromatogr. A 2009, 1216, 1551.

[16] Garzón, F.J.L., García, M.D. Adsorption on New and Modified Inorganic Sorbents, Elsevier, 1996.

[17] Neumann, M.G.J. Chem. Educ. 1976, 53, 708.

[18] Helfferich, F. Ion Exchange, Dover Publications, 1995.

[19] Inglezakis, V.J.J. Colloid Interface Sci. 2005, 281, 68.

[20] Ozekmekci, M., Salkic, G., Fellah, M.F. Fuel Process. Technol. 2015, 139, 49.

[21] Hoguet, J.C., Karagiannakis, G.P., Valla, J.A., Agrafiotis, C.C., Konstandopoulos, A.G. Int. J. Hydrogen Energy 2009, 34, 4953.

[22] Groen, J.C., Moulijn, J.A., Perez-Ramirez, J.J. Mater. Chem. 2006, 16, 2121.

[23] Groen, J.C., Peffer, L.A.A., Pérez-Ramírez, J. Microp. Mesop. Mater. 2003, 6o, 1.

[24] Beyer, H. in Post-Synthesis Modification I, Molecular Sieves Vol. 3, Chap. 3, Springer, 2002.

[25] Ogura, M., Shinomiya, S.Y., Tateno, J., Nara, Y., Nomura, M., Kikuchi, E., Matsukata, M. Appl. Catal., A 2001, 219, 33 .

[26] Suzuki, T., Okuhara, T. Microp. Mesop. Mater. 2001, 43, 83.

[27] Su, L., Liu, L., Zhuang, J., Wang, H., Li, Y., Shen, W., Xu, Y., Bao, X. Catal. Lett. 2003, 91, 155.

[28] Motz, J.L., Heinichen, H., Hölderich, W.F. J. Mol. Catal. A Chem. 1998, 136, 175.

[29] Janssen, A.H., Koster, A.J., de Jong, K.P. Angew. Chem. Int. Ed. 2001, 113, 1136.

[30] van Donk, S., Janssen, A.H., Bitter, J.H., de Jong, K.P. Catal. Rev. 2003, 45, 297.

[31] Pérez-Ramírez, J., Christensen, C.H., Egeblad, K., Christensen, C.H., Groen, J.C. Chem. Soc. Rev. 2008, 37, 2530.

[32] Egeblad, K., Christensen, C.H., Kustova, M., Christensen, C.H. Chem. Mater. 2008, 20, 946.

[33] Tao, Y., Kanoh, H., Abrams, L., Kaneko, K. Chem. Rev. 2006, 106, 896.

[34] Holm, M.S., Taarning, E., Egeblad, K., Christensen, C.H. Catal. Today 2011, 168, 3. 
[35] Schmidt, I., Madsen, C., Jacobsen. C.J.H. Inorg. Chem. 2000, 39, 2279.

[36] Tao, Y., Kanoh, H., Kaneko, K. Langmuir 2005, 21, 504.

[37] Schacht, S., Huo, Q., Voigt-Martin, I.G., Stucky, G.D., Schuth, F. Science 1996, 273, 768.

[38] Bagshaw, S.A., Baxter, N.I., Brew, D.R.M., Hosie, C.F., Yuntong, N., Jaenicke, S., Khuan, C.G. J. Mater. Chem. 2006, 16, 2235.

[39] Xiao, F.S., Wang, L., Yin, C., Lin, K., Di, Y., Li, J., Xu, R., Su, D.S., Schlögl, R., Yokoi, T. Angew. Chem. Int. Ed. 2006, 45, 3090.

[40] Wei, Y., Parmentier, T.E., de Jong, K.P., Zečević, J. Chem. Soc. Rev. 2015, 44, 7234.

[41] Colombo, V., Montoro, C., Maspero, A., Palmisano, G., Masciocchi, N., Galli, S., Barea, E., Navarro, J.A.R. J. Am. Chem. Soc. 2012, 134, 12830.

[42] Colombo, V., Montoro, C., Maspero, A., Palmisano, G., Masciocchi, N., Galli, S., Barea, E., Navarro, J.A.R.J. Am. Chem. Soc. 2012, 134, 12630.

[43] Wei, Z., Yuan, D., Zhao, X., Sun, D., Zhou, H.C. Sci. China Chem. 2013, 56, 418.

[44] Deng, H., Doonan, C.J., Furukawa, H., Ferreira, R.B., Towne, J., Knobler, C.B., Wang, B., Yaghi, O.M. Science, 2010, 327, 846.

[45] Wang, Z., Cohen, S.M. Chem. Soc. Rev. 2009, 38, 1315.

[46] Chui, S.S.Y., Lo, S.M.F., Charmant, J.P.H., Orpen, A.G., Williams, I.D. Science 1999, 283, 1148.

[47] López-Maya, E., Montoro, C., Colombo, V., Barea, E., Navarro, J.A.R. Adv. Funct. Mater. 2014, 24, 6130 .

[48] Fang, Z., Bueken, B., De Vos, D.E., Fischer, R.A. Angew. Chem. Int. Ed. 2015, 54, 7234.

[49] Bae, Y.S., Farha, O.K., Hupp, J.T., Snurr, R.Q.J. Mater. Chem. 2009, 19, 2131.

[50] Demessence, A., D’Alessandro, D.M., Foo, M.L., Long, J.R. J. Am. Chem. Soc. 2009, 131, 8784.

[51] McDonald, T.M., Mason, J.A., Kong, X., Bloch, E.D., Gygi, D., Dani, A., Crocellà, V., Giordanino, F., Odoh, S.O., Drisdell, W.S., et al. Nature, 2015, 519, 303.

[52] Montoro, C., Garcia, E., Calero, S., Perez-Fernandez, M.A., Lopez, A.L., Barea, E., Navarro, J.A.R.J. Mater. Chem. 2012, 22, 10155 .

[53] Gutov, O.V., Hevia, M.G., Escudero-Adán, E.C., Shafir, A. Inorg. Chem. 2015, 54, 8696.

[54] Wu, H., Chua, Y.S., Krungleviciute, V., Tyagi, M., Chen, P., Yildirim, T., Zhou, W. J. Am. Chem. Soc. 2013, 135, 10525.

[55] Shearer, G.C., Chavan, S., Ethiraj, J., Vitillo, J.G., Svelle, S., Olsbye, U., Lamberti, C., Bordiga, S., Lillerud, K.P. Chem. Mater. 2014, 26, 4068.

[56] Cliffe, M.J., Wan, W., Zou, X., Chater, P.A., Kleppe, A.K., Tucker, M.G., Wilhelm, H., Funnell, N.P., Coudert, F.X., Goodwin, A.L. Nat. Commun. 2014, 5, 4176.

[57] López-Maya, E., Montoro, C., Rodríguez-Albelo, L.M., Aznar Cervantes, S.D., Lozano-Pérez, A., Cenís, J.A.L., Barea, E., Navarro, J.A.R. Angew. Chem. Int. Ed. 2015, 54, 6790.

[58] Vermoortele, F., Ameloot, R., Alaerts, L., Matthessen, R., Carlier, B., Fernandez, E.V.R., Gascon, J., Kapteijn, F., De Vos, D.E.J. Mater. Chem. 2012, 22, 10313.

[59] Gadipelli, S., Guo, Z. Chem. Mater. 2014, 23, 6333.

[6o] Banerjee, D., Kim, S.J., Wu, H., Xu, W., Borkowski, L., Li, J., Parise, J.B. Inorg. Chem. 2011, 50, 208.

[61] An, J., Geib, S.J., Rosi, N.L.J. Am. Chem. Soc. 2009, 131, 8376.

[62] Procopio, E.Q., Linares, F., Montoro, C., Colombo, V., Maspero, A., Barea, E., Navarro, J.A.R. Angew. Chem. Int. Ed. 2010, 49, 7308.

[63] Rodríguez-Albelo, L.M., López-Maya, E., Hamad, S., Ruiz-Salvador, A.R., Calero, S., Navarro, J.A.R. Nat. Commun. 2017, 8, 14457.

[64] He, T., Pachfule, P., Wu, H., Xu, Q., Chen, P. Nat. Rev. Mater. 2016, 1, 16059.

[65] Langmi, H.W., Walton, A., Al-Mamouri, M.M., Johnson, S.R., Book, D., Speight, J.D., Edwards, P.P., Gameson, I., Anderson, P.A., Harris, I.R.J. Alloys Compd. 2003, 356, 710.

[66] Dong, J., Wang, X., Xu, H., Zhao, Q., Li, J. Int. J. Hydrogen Energy 2007, 32, 4998. 
[67] Farha, O.K., Hupp, J.T., Wilmer, C.E., Eryazici, I., Snurr, R.Q., Gomez-Gualdron, D.A., Borah, B. US Patent 9216939, 2015.

[68] https://energy.gov/sites

[69] Rowsell, J.L.C., Yaghi, O.M. Angew. Chem. Int. Ed. 2005, 44, 4670.

[70] Ren, J., Musyoka, N.M., Langmi, H.W., Mathe, M., Liao, S. Int.J. Hydrogen Energy 2016, 42, 289.

[71] Thomas, K.M. Catal. Today 2007, 120, 389 .

[72] Thomas, K.M. Dalton Trans. 2009, 1487.

[73] Rosi, N.L., Eckert, J., Eddaoudi, M., Vodak, D.T., Kim, J., O’Keeffe, M., Yaghi, O.M. Science 2003, 300, 1127.

[74] Rowsell, J.L.C., Yaghi, O.M.J. Am. Chem. Soc. 2006, 128, 1304.

[75] Dincǎ, M., Han, W.S., Liu, Y., Dailly, A., Brown, C.M., Long, J.R. Angew. Chem. Int. Ed. 2007, 46,1419 .

[76] Dincă, M., Long, J.R.J. Am. Chem. Soc. 2007, 129, 11172.

[77] Yang, S., Lin, X., Blake, A.J., Walker, G.S., Hubberstey, P., Champness, N.R., Schröder, M. Nat. Chem. 2009, 1, 487.

[78] Luo, J., Xu, H., Liu, Y., Zhao, Y., Daemen, L.L., Brown, C.J. Am. Chem. Soc. 2008, 130, 9626.

[79] Chen, B., Ma, S., Zapata, F., Lobkovsky, E.B., Yang, J., Arbor, A. Inorg. Chem. 2006, 45, 5718.

[80] Férey, G., Latroche, M., Serre, C., Millange, F., Loiseau, T., Percheron-Guégan, A. Chem. Commun. 2003, 2976.

[81] Chen, B., Ockwig, N.W., Millward, A.R., Contreras, D.S., Yaghi, O.M. Angew. Chem. Int. Ed. 2005, 44, 4745.

[82] Lin, X., Telepeni, I., Blake, A.J., Dailly, A., Brown, C.M., Simmons, J.M., Zoppi, M., Walker, G.S., Thomas, K.M., Mays, T.J., et al.J. Am. Chem. Soc. 2009, 131, 2159.

[83] Xiao, B., Wheatley, P.S., Zhao, X., Fletcher, A.J., Fox, S., Rossi, A.G., Megson, I.L., Bordiga, S., Regli, L., Thomas, K.M., et al.J. Am. Chem. Soc. 2007, 129, 1203.

[84] Volkringer, C., Meddouri, M., Loiseau, T., Guillou, N., Haouas, M., Taulelle, F., Audebrand, N., Latroche, M. Inorg. Chem. 2008, 47, 11892.

[85] Han, Z.B., Lu, R.Y., Liang, Y.F., Zhou, Y.L., Chen, Q., Zeng, M.H. Inorg. Chem. 2012, 51, 674.

[86] Farha, O.K., Yazaydın, Ö., Eryazici, I., Malliakas, C.D., Hauser, B.G., Kanatzidis, M.G., Nguyen, S.T., Snurr, R.Q., Hupp, J.T. Nat. Chem. 2010, 2, 944.

[87] Dincă, M., Dailly, A., Liu, Y., Brown, C.M., Neumann, D.A., Long, J.R.J. Am. Chem. Soc. 20o6, 128,16876 .

[88] He, Y., Zhou, W., Qian, G., Chen, B. Chem. Soc. Rev. 2014, 43, 5657.

[89] Lin, Y., Kong, C., Zhang, Q., Chen, L. Adv. Energy Mater. 2016, 1601296.

[9o] https://arpa-E.energy.gov/q=arpa-E-Programs/move

[91] Makal, T.A., Li, J.R., Lu, W., Zhou, H.C. Chem. Soc. Rev. 2012, 41, 7761.

[92] Rolniak, P.D., Kobayashi, R. AIChEJ. 1980, 26, 616.

[93] Menon, V.C., Komarneni, S. J. Porous Mater. 1998, 5, 43.

[94] Stockmeyer, R. US Patent 44959oo, 1985.

[95] Tate, K.L., Li, S., Yu, M., Carreon, M.A. Adsorption 2016, 23, 1.

[96] Wu, H., Simmons, J.M., Liu, Y., Brown, C.M., Wang, X. Sen, Shengqian, M., Peterson, V.K., Southon, P.D., Kepert, C.J., Zhou, H.C., et al. Chem. A Eur.J. 2010, 16, 5205.

[97] Li, B., Wen, H., Wang, H., Wu, H., Tyagi, M., Yildirim, T., Zhou, W., Chen, B.J. Am. Chem. Soc. 2014, 136, 6207 .

[98] Li, B., Wen, H.M., Wang, H., Wu, H., Yildirim, T., Zhou, W., Chen, B. Energy Environ. Sci. 2015, 8,2504 .

[99] Chang, G., Wen, H., Li, B., Zhou, W., Wang, H., Alfooty, K., Bao, Z., Chen, B. Cryst. Growth Des. 2016, 16, 3395 .

[100] Liu, J., Zou, R., Zhao, Y. Tetrahedron Lett. 2016, 57, 4873. 
[101] Mason, J.A., Veenstra, M., Long, J.R. Chem. Sci. 2014, 5, 32.

[102] Mason, J.A., Oktawiec, J., Taylor, M.K., Hudson, M.R., Rodriguez, J., Bachman, J.E., Gonzalez, M.I., Cervellino, A., Guagliardi, A., Brown, C.M., et al. Nature 2015, 357 .

[103] Alezi, D., Belmabkhout, Y., Suyetin, M., Bhatt, P.M., Weseliński, L.J., Solovyeva, V., Adil, K., Spanopoulos, I., Trikalitis, P.N., Emwas, A.H., et al.J. Am. Chem. Soc. 2015, 137, 13308.

[104] Jiang, J., Furukawa, H., Zhang, Y.B., Yaghi, O.M. J. Am. Chem. Soc. 2016, 138, 10244.

[105] Li, B., Wen, H.M., Cui, Y., Zhou, W., Qian, G., Chen, B. Adv. Mater. 2016, 28, 8819.

[106] Lin, J.M., He, C.T., Liu, Y., Liao, P.Q., Zhou, D.D., Zhang, J.P., Chen, X.M. Angew. Chem. Int. Ed. 2016, 55,4674 .

[107] Guo, Z., Wu, H., Srinivas, G., Zhou, Y., Xiang, S., Chen, Z., Yang, Y., Zhou, W., O'Keeffe, M., Chen, B. Angew. Chem. Int. Ed. 2011, 50, 3178.

[108] Zhou, W. Chem. Rec. 2010, 10, 200.

[109] Peng, Y., Krungleviciute, V., Eryazici, I., Hupp, J.T., Farha, O.K., Yildirim, T.J. Am. Chem. Soc. 2013, 135, 11887 .

[110] Li, B., Wen, H., Zhou, W., Xu, J.Q., Chen, B. Chem 2016, 1, 557.

[111] Chang, G., Li, B., Wang, H., Bao, Z., Yildirim, T., Yao, Z.Z., Xiang, S., Zhou, W., Chen, B. Chem. Commun. 2015, 51, 14789 .

[112] Liu, H., Chen, F., Bai, D., Jiao, J., Zhou, W., Yildirim, T., He, Y. Cryst. Growth Des. 2017, 17, 248.

[113] Song, C., Liu, H., Jiao, J., Bai, D., Zhou, W., Yildirim, T., He, Y. Dalton Trans. 2016, 45, 7559.

[114] Wang, Q., Song, X., Zhang, M., Liu, W., Bai, J. Cryst. Growth Des. 2016, 16, 6156.

[115] Intergovernmental Panel on Climate Change (IPCC). Climate Change, 2014.

[116] Dunne, J.A., Rao, M., Sircar, S., Gorte, R.J., Myers, A.L. Langmuir, 1996, 12, 5896.

[117] Coriani, S., Halkier, A., Rizzo, A., Ruud, K. Chem. Phys. Lett. 2000, 326, 269.

[118] Cavenati, S., Grande, C.A., Rodrigues, A.E.J. Chem. Eng. Data 2004, 49, 1095.

[119] Choudhary, V.R., Mayadevi, S. Langmuir, 1995, 12, 980.

[120] Harlick, P.J.E., Tezel, F.H. Sep. Purif. Technol. 2003, 33, 199.

[121] Saha, D., Bao, Z., Jia, F., Deng, S. Environ. Sci. Technol. 2010, 44, 1820.

[122] Samanta, A., Zhao, A., Shimizu, G.K.H., Sarkar, P., Gupta, R. Ind. Eng. Chem. Res. 2012, 51, 1438 .

[123] Zhang, J., Singh, R., Webley, P.A. Microp. Mesop. Mater. 2008, 111, 478.

[124] Jadhav, P.D., Chatti, R.V., Biniwale, R.B., Labhsetwar, N.K., Devotta, S., Rayalu, S.S. Energy Fuels 2007, 21, 3555 .

[125] Su, F., Lu, C., Kuo, S.C., Zeng, W. Energy Fuels 2010, 24, 1441.

[126] Walton, K.S., Millward, A.R., Dubbeldam, D., Frost, H., Low, J.J., Yaghi, O.M., Snurr, R.Q. J. Am. Chem. Soc. 2008, 130, 406.

[127] Aprea, P., Caputo, D., Gargiulo, N., Iucolano, F., Pepe, F. J. Chem. Eng. Data 2010, 55, 3655.

[128] Yazaydin, O., Snurr, R.Q., Park, T.H., Koh, K., Liu, J., Levan, M.D., Benin, A.I., Jakubczak, P., Lanuza, M., Galloway, D.B., et al.J. Am. Chem. Soc. 2009, 131, 18198.

[129] Mason, J.A., Sumida, K., Herm, Z.R., Krishna, R., Long, J.R. Energy Environ. Sci. 2011, 4, 3030.

[130] Caskey, S.R., Wong-Foy, A.G., Matzger, A.J.J. Am. Chem. Soc. 2008, 130, 10870.

[131] Furukawa, H., Ko, N., Go, Y.B., Aratani, N., Choi, S.B., Choi, E., Yazaydin, A.O., Snurr, R.Q., O'Keeffe, M., Kim, J., et al. Science 2010, 329, 424.

[132] Choi, S., Watanabe, T., Bae, T.H., Sholl, D.S., Jones, C.W.J. Phys. Chem. Lett. 2012, 3, 1136.

[133] Rezaei, F., Rownaghi, A.A., Monjezi, S., Lively, R.P., Jones, C.W. Energy Fuels 2015, 29, 1.

[134] Liu, Y., Bisson, T.M., Yang, H., Xu, Z. Fuel Process. Technol. 2010, 91, 1175.

[135] Barea, E., Montoro, C., Navarro, J.A.R. Chem. Soc. Rev. 2014, 43, 5419.

[136] Deng, H., Yi, H., Tang, X., Yu, Q., Ning, P., Yang, L. Chem. Eng.J. 2012, 188, 77.

[137] Britt, D., Tranchemontagne, D., Yaghi, O.M. PNAS 2008, 105, 11623.

[138] Dathe, H., Haider, P., Jentys, A., Lercher, J.A.J. Phys. Chem. B 2006, 110, 26024. 
[139] McKinlay, A.C., Xiao, B., Wragg, D.S., Wheatley, P.S., Megson, I.L., Morris, R.E.J. Am. Chem. Soc. $2008,130,10440$.

[140] Xiao, B., Wheatley, P.S., Zhao, X., Fletcher, A.J., Fox, S., Rossi, A.G., Megson, I.L., Bordiga, S., Regli, L., Thomas, K.M., et al.J. Am. Chem. Soc. 2007, 129, 1203.

[141] McKinlay, A.C., Eubank, J.F., Wuttke, S., Xiao, B., Wheatley, P.S., Bazin, P., Lavalley, J.C., Daturi, M., Vimont, A., De Weireld, G., et al. Chem. Mater. 2013, 25, 1592.

[142] Ebrahim, A.M., Levasseur, B., Bandosz, T.J. Langmuir 2013, 29, 168.

[143] Petit, C., Bandosz, T.J. Dalton Trans. 2012, 41, 4027.

[144] Montoro, C., Linares, F., Quartapelle Procopio, E., Senkovska, I., Kaskel, S., Galli, S., Masciocchi, N., Barea, E., Navarro, J.A.R.J. Am. Chem. Soc. 2011, 133, 11888.

[145] Padial, N.M., Quartapelle Procopio, E., Montoro, C., López, E., Oltra, J.E., Colombo, V., Maspero, A., Masciocchi, N., Galli, S., Senkovska, I., et al. Angew. Chem. Int. Ed. 2013, 52, 8290.

[146] Ferreira, D., Boaventura, M., Bárcia, P., Whitley, R.D., Mendes, A. Ind. Eng. Chem. Res. 2016, 55,722 .

[147] Hamed, H.H. Int.J. Sci. Eng. Res. 2015, 6, 597.

[148] Wu, C.W., Vemula, R.R., Kothare, M.V., Sircar, S. Ind. Eng. Chem. Res. 2016, 55, 4676.

[149] Wu, C., Kothare, M.V, Sircar, S. Ind. Eng. Chem. Res. 2014, 53, 12428.

[150] DeCoste, J.B., Weston, M.H., Fuller, P.E., Tovar, T.M., Peterson, G.W., LeVan, M.D., Farha, O.K. Angew. Chem. Int. Ed. 2014, 53, 14092.

[151] Bloch, E.D., Queen, W.L., Hudson, M.R., Mason, J.A., Xiao, D.J., Murray, L.J., Flacau, R., Brown, C.M., Long, J.R. Angew. Chem. Int. Ed. 2016, 55, 8605.

[152] Wang, C.Y., Wang, L., Belnick, A., Wang, H., Li, J., Lueking, A.D. Chem. Eng. Sci. 2017, 165, 122.

[153] Xiao, D.J., Gonzalez, M.I., Darago, L.E., Vogiatzis, K.D., Haldoupis, E., Gagliardi, L., Long, J.R. J. Am. Chem. Soc. 2016, 138, 7161.

[154] Zhang, W., Banerjee, D., Liu, J., Schaef, H.T., Crum, J.V., Fernandez, C.A., Kukkadapu, R.K., Nie, Z., Nune, S.K., Motkuri, R.K., et al. Adv. Mater. 2016, 28, 3572.

[155] Banerjee, D., Simon, C.M., Plonka, A.M., Motkuri, R.K., Liu, J., Chen, X., Smit, B., Parise, J.B., Haranczyk, M., Thallapally, P.K. Nat. Commun. 2016, 7, 11831.

[156] Lawler, K.V., Sharma, A., Alagappan, B., Forster, P.M. Microp. Mesop. Mater. 2016, 222, 104.

[157] Feng, X., Zong, Z., Elsaidi, S.K., Jasinski, J.B., Krishna, R., Thallapally, P.K., Carreon, M.A. J. Am. Chem. Soc. 2016, 138, 9791.

[158] Lee, S.J., Yoon, T.U., Kim, A.R., Kim, S.Y., Cho, K.H., Hwang, Y.K., Yeon, J.W., Bae, Y.S. J. Hazard. Mater. 2016, 320, 513 .

[159] Mohamed, M.H., Elsaidi, S.K., Pham, T., Forrest, K.A., Schaef, H.T., Hogan, A., Wojtas, L., Xu, W., Space, B., Zaworotko, M.J., et al. Angew. Chem. Int. Ed. 2016, 55, 8285.

[160] Yi, F.Y., Chen, D., Wu, M.K., Han, L., Jiang, H.L. ChemPlusChem 2016, 81, 675.

[161] Kosinov, N., Gascon, J., Kapteijn, F., Hensen, E.J.M.J. Membrane Sci. 2016, 499, 65.

[162] Bellussi, G., Millini, R., Pollesel, P., Perego, C. New J. Chem. 2016, 40, 4061.

[163] Khulbe, K.C., Matsuura, T., Feng, C.Y., Ismail, A.F., Himeno, S., Tomita, T., Shujiki, K., Yoshida, S., Burton, A., Sherman, J.D., et al. RSC Adv. 2016, 6, 42943.

[164] Li, J.R., Kuppler, R.J., Zhou, H.C. Chem. Soc. Rev. 2009, 38, 1477.

[165] Li, J., Sculley, J., Zhou, H. Chem. Rev. 2012, 112, 869.

[166] Nandasiri, M.I., Jambovane, S.R., McGrail, B.P., Schaef, H.T., Nune, S.K. Coord. Chem. Rev. 2016, 311, 38 .

[167] Qiu, S., Xue, M., Zhu, G. Chem. Soc. Rev. 2014, 43, 6116.

[168] Melgar, V.M.A., Kim, J., Othman, M.R.J. Ind. Eng. Chem. 2015, 28, 1.

[169] Kang, Z., Fan, L., Sun, D. J. Mater. Chem. A 2017.

[170] Cadiau, A., Adil, K., Bhatt, P.M., Belmabkhout, Y., Eddaoudi, M. Science, 2016, 353, 137. 
[171] He, Y.P., Zhou, N., Tan, Y.X., Wang, F., Zhang, J.J. Solid State Chem. 2016, 238, 241.

[172] Liu, H., He, Y., Jiao, J., Bai, D., Chen, D.L., Krishna, R., Chen, B. Chem. Eur. J. 2016, 22, 14988.

[173] Chang, G., Li, B., Wang, H., Hu, T., Bao, Z., Chen, B. Chem. Commun. 2016, 52, 3494.

[174] Foo, M.L., Matsuda, R., Hijikata, Y., Krishna, R., Sato, H., Horike, S., Hori, A., Duan, J., Sato, Y., Kubota, Y., et al.J. Am. Chem. Soc. 2016, 138, 3022.

[175] Luo, F., Yan, C., Dang, L., Krishna, R., Zhou, W., Wu, H., Dong, X., Han, Y., Hu, T.L., O’Keeffe, M., et al.J. Am. Chem. Soc. 2016, 138, 5678.

[176] Gupta, V.K., Carrott, P.J.M., Ribeiro Carrott, M.M.L., Suhas. Crit. Rev. Environ. Sci. Technol. 2009, 39, 783 .

[177] Wang, S., Peng, Y. Chem. Eng.J. 2010, 156, 11.

[178] Misaelides, P. Microp. Mesop. Mater. 2011, 144, 15.

[179] Bowman, R.S. Microp. Mesop. Mater. 2003, 61, 43.

[180] Khan, N.A., Hasan, Z., Jhung, S.H. J. Hazard. Mater. 2013, 244, 444.

[181] Kitagawa, S., Kitaura, R., Noro, S. Angew. Chem. Int. Ed. 2004, 43, 2334.

[182] Rowsell, J.L.C., Yaghi, O.M. Microp. Mesop. Mater. 2004, 73, 3.

[183] Jaishankar, M., Tseten, T., Anbalagan, N., Mathew, B.B., Beeregowda, K.N. Interdiscip. Toxicol. 2014, 7, 60 .

[184] Álvarez-Ayuso, E., García-Sánchez, A., Querol, X. Water Res. 2003, 37, 4855.

[185] Babel, S., Kurniawan, T.A.J. Hazard. Mater. 2003, 97, 219.

[186] Perić, J., Trgo, M., Vukojević Medvidović, N. Water Res. 2004, 38, 1893.

[187] Erdem, E., Karapinar, N., Donat, R. J. Colloid Interface Sci. 2004, 280, 309.

[188] Günay, A., Arslankaya, E., Tosun, I. J. Hazard. Mater. 2007, 146, 362.

[189] Motsi, T., Rowson, N.A., Simmons, M.J.H. Int. J. Miner. Process. 2009, 92, 42.

[19o] Chutia, P., Kato, S., Kojima, T., Satokawa, S.J. Hazard. Mater. 2009, 162, 204.

[191] Zeng, Y., Woo, H., Lee, G., Park, J. Microp. Mesop. Mater. 2010, 130, 83.

[192] Jamali, A., Tehrani, A.A., Shemirani, F., Morsali, A. Dalton Trans. 2016, 45, 9193.

[193] Fang, Q.R., Yuan, D.Q., Sculley, J., Li, J.R., Han, Z.B., Zhou, H.C. Inorg. Chem. 2010, 49, 11637.

[194] Ke, F., Qiu, L.G., Yuan, Y.P., Peng, F.M., Jiang, X., Xie, A.J., Shen, Y.H., Zhu, J.F. J. Hazard. Mater. 2011, 196, 36 .

[195] Saleem, H., Rafique, U., Davies, R.P. Microp. Mesop. Mater. 2016, 221, 238.

[196] Chakraborty, A., Bhattacharyya, S., Hazra, A., Ghosh, A.C., Maji, T.K. Chem. Commun. 2016, $5^{2,1 .}$

[197] Wu, Y., Zhou, M., Zhang, B., Wu, B., Li, J., Qiao, J., Guan, X., Li, F. Nanoscale 2014, 6, 1105.

[198] Jian, M., Liu, B., Zhang, G., Liu, R., Zhang, X. Colloids Surf. A 2015, 465, 67.

[199] Shevade, S., Ford, R.G. Water Res. 2004, 38, 3197.

[200] Chutia, P., Kato, S., Kojima, T., Satokawa, S.J. Hazard. Mater. 2009, 162, 440.

[201] Fei, H., Han, C.S., Robins, J.C., Oliver, S.R.J. Chem. Mater. 2013, 25, 647.

[202] Ji, F., Li, C., Tang, B., Xu, J., Lu, G., Liu, P. Chem. Eng. J. 2012, 209, 325.

[203] Kim, S.A., Kamala-Kannan, S., Lee, K.J., Park, Y.J., Shea, P.J., Lee, W.H., Kim, H.M., Oh, B.T. Chem. Eng. J. 2013, 217, 54.

[204] Rad, L.R., Momeni, A., Ghazani, B.F., Irani, M., Mahmoudi, M., Noghreh, B. Chem. Eng. J. 2014, 256, 119 .

[205] Ricco, R., Konstas, K., Styles, M.J., Richardson, J.J., Babarao, R., Suzuki, K., Scopece, P., Falcaro, P. J. Mater. Chem. A 2015, 3, 19822.

[206] Ghorbani-Kalhor, E. Microchim. Acta 2016, 183, 2639.

[207] Yin, N., Wang, K., Wang, L., Li, Z. Chem. Eng.J. 2016, 306, 619.

[208] Huang, H., Xiao, X., Yan, B., Yang, L.J. Hazard. Mater. 2010, 175, 247.

[209] Bhatnagar, A., Sillanpää, M.A. Chem. Eng.J. 2011, 168, 493.

[210] Onyango, M.S., Kuchar, D., Kubota, M., Matsuda, H. Ind. Eng. Chem. Res. 2007, 46, 894. 
[211] Lin, K.Y.A., Chen, S.Y., Jochems, A.P. Mater. Chem. Phys. 2015, 16o, 168.

[212] Han, R., Zou, W., Wang, Y., Zhu, L. J. Environ. Radioact. 2007, 93, 127.

[213] Carboni, M., Abney, C.W., Liu, S., Lin, W. Chem. Sci. 2013, 4, 2396.

[214] Borai, E.H., Harjula, R., Malinen, L., Paajanen, A.J. Hazard. Mater. 2009, 172, 416.

[215] Sheng, D., Zhu, L., Xu, C., Xiao, C., Wang, Y., Wang, Y., Chen, L., Diwu, J., Chen, J., Chai, Z., et al. Environ. Sci. Technol. 2017, 51, 3471.

[216] Wang, S., Li, H., Xu, L. J. Colloid Interface Sci. 2006, 295, 71.

[217] Wang, S., Zhu, Z.H.J. Hazard. Mater. 2006, 136, 946.

[218] Han, R., Zhang, J., Han, P., Wang, Y., Zhao, Z., Tang, M. Chem. Eng.J. 2009, 145, 496.

[219] Alver, E., Metin, A.Ü. Chem. Eng.J. 2012, 200, 59.

[220] Haque, E., Lee, J.E., Jang, I.T., Hwang, Y.K., Chang, J.S., Jegal, J., Jhung, S.H.J. Hazard. Mater. 2010, 181, 535 .

[221] Hasan, Z., Jhung, S.H.J. Hazard. Mater. 2015, 283, 329.

[222] Li, L., Liu, X.L., Geng, H.Y., Hu, B., Song, G.W., Xu, Z.S. J. Mater. Chem. A 2013, 1, 10292.

[223] Huo, J., Marcello, M., Garai, A., Bradshaw, D. Adv. Mater. 2013, 25, 2717.

[224] Yan, A.X., Yao, S., Li, Y.G., Zhang, Z.M., Lu, Y., Chen, W.L., Wang, E.B. Chem. Eur. J. 2014, 20, 6927.

[225] Huang, M., Xu, C., Wu, Z., Huang, Y., Lin, J., Wu, J. Dye. Pigment. 2008, 77, 327.

[226] Nikazar, M., Gholivand, K., Mahanpoor, K. Desalination 2008, 219, 293.

[227] Zhang, W., Wang, K., Yu, Y., He, H. Chem. Eng.J. 2010, 163, 62.

[228] Martucci, A., Pasti, L., Marchetti, N., Cavazzini, A., Dondi, F., Alberti, A. Microp. Mesop. Mater. 2012, 148, 174.

[229] Blasioli, S., Martucci, A., Paul, G., Gigli, L., Cossi, M., Johnston, C.T., Marchese, L., Braschi, I. J. Colloid Interface Sci. 2014, 419, 148.

[230] Wang, B., Lv, X.L., Feng, D., Xie, L.H., Zhang, J., Li, M., Xie, Y., Li, J.R., Zhou, H.C.J. Am. Chem. Soc. 2016, 138, 6204 .

[231] Rocío-Bautista, P., Martínez-Benito, C., Pino, V., Pasán, J., Ayala, J.H., Ruiz-Pérez, C., Afonso, A.M. Talanta 2015, 139, 13. 


\title{
8. Membrane Technology: how, where, and why
}

\author{
Motomu Sakai, Masahiro Seshimo and Masahiko Matsukata \\ Department of Applied Chemistry, Waseda University
}

Keywords: Permeability, seeding, crystallization, separation, dehydration, membrane reactor

\section{Introduction}

Thin films of inorganic porous crystals, zeolites and metal-organic frameworks (MOFs), have been developed for use as sensors [1-5], electronic materials [6-9], micro-reactors [10-13], and separation membranes. In particular, zeolite membranes have been attracting intense research interest as separation materials in the past decades. MOFs have also been studied in the field of membrane research in recent years. This chapter deals with zeolite and MOF membranes as separation materials.

Membrane separations using polymeric membranes have become widespread worldwide for seawater desalination [14-16], waste water treatment $[17,18]$, and clarification $[19,20]$. These membrane separation processes have considerably contributed to reducing energy consumption. For example, reverse osmosis ( $\mathrm{RO}$ ) for seawater desalination consumes less than about $20 \%$ of the energy required in thermal desalination processes [14,15]. Consequently, seawater $\mathrm{RO}$ has exponentially expanded its throughput in recent decades. More than 40 million cubic meters of desalinated water were produced in 2008, and more than 100 million cubic meters desalinated water production were projected for 2016 [16]. The polymeric membranes used in water treatment, however, are difficult to apply in energy production and chemical industry processes because of their insufficient thermal, chemical, and pressure resistance. Accordingly, the development of inorganic materials-based membranes usable under such conditions, for example elevated temperature and pressure, is expected.

There are several kinds of inorganic membranes competitive with zeolites and MOFs. Other types of inorganic-based membrane materials, like metal [21-23], carbon $[24,25]$, silica $[26,27]$ and organosilica [28], have been 


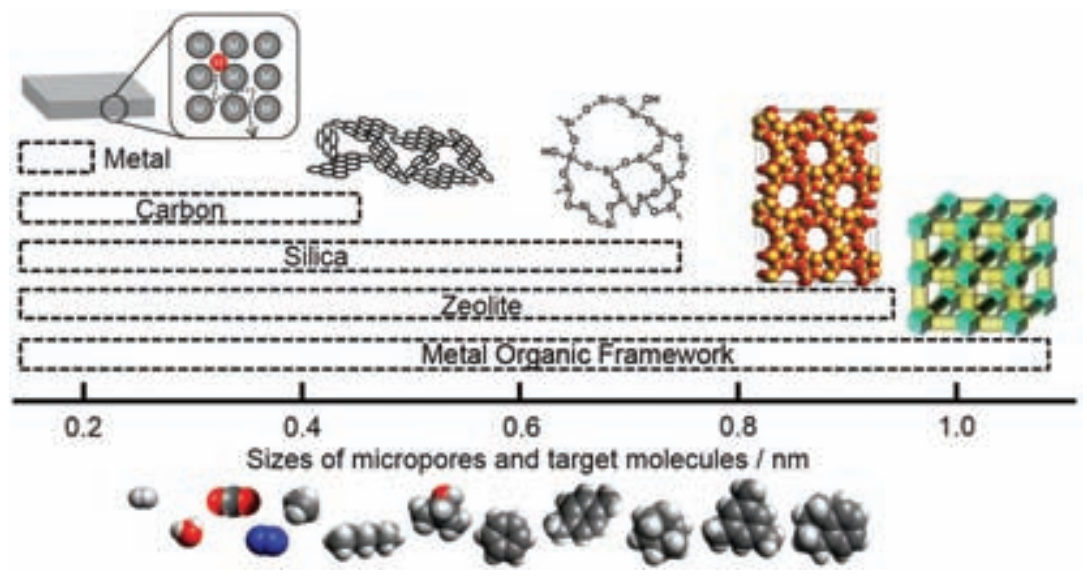

Figure 1. The variety of inorganic porous membranes.

studied. Figure 1 shows the typical characteristics and uses of these membranes. Although Pd and Pd-Ag alloy membranes exhibit superior permeability and extremely high selectivity, they can only be applied to hydrogen separation. While having good chemical and thermal stability, carbon membranes are better suited to separation of small gas molecules, such as hydrogen, because of their small pore sizes. The separation properties and stabilities of silica and organosilica membranes are controlled by synthesis methods. Silica-based membranes have been widely studied for gas separation, not only that of hydrogen, but also gas mixtures of light hydrocarbon [26-28].

Compared to the membrane materials mentioned above, the greatest features of zeolites as membrane materials are their rigid uniform micropore channels and unique adsorption properties. By selecting an appropriate framework topology, zeolite membranes can be applied to diverse separations, like hydrogen, olefin gases and hydrocarbons such as alkanes or xylenes. The flexibility available for designing the pore size, pore-shape, and adsorption properties is the strongest point of MOFs as membrane materials thanks to the number and diversity of MOF structures, which far exceeds the number of zeolite frameworks available.

Separation and purification processes account for as much as $40 \%$ of energy consumption in the chemical industry. In other words, innovation in separation process is almost as significant as innovation in the chemical industry or in large-scale energy production. It is expected that the inorganic materials-based membranes mentioned above can contribute to drastically reducing current energy consumption. Moreover, a downsizing of equipment is expected upon introduction of novel membrane separation 
units to replace conventional separation processes. Economic advantages based on energy-savings and downsizing, are strong motivations to pursue their practical realization in the chemical industry.

In this chapter, the preparation methods, uses, applications, and separation principles of zeolite and MOF membranes are presented. Additionally, membrane reactors are described as a major potential application of zeolite membranes.

\section{How to prepare zeolite and MOF membranes}

\subsection{The variety of zeolite and MOF membranes}

The three major properties required for separation by membranes are their separation selectivity, permeability, and lifetime. Permeance is defined as permeation flux/pressure difference, while permeability is defined as permance/membrane thickness. The majority of studies about zeolite and MOF membranes have targeted the improvement of separation and/or permeation properties. Since both zeolite and MOF are crystals, these membranes have structures in which crystals are accumulated. Thus, one can assume two kinds of pathways across the membrane, that is, intracrystalline and inter-crystalline pathways [29]. The intra-crystalline and inter-crystalline pathways are micropores in crystals and defects in membranes, such as cracks and voids between crystals. Figure 2 provides a schematic illustration of these two kinds of pathways. A very small amount of inter-crystalline pathway can easily spoil the separation properties. Improving the separation properties of zeolite and MOF membranes critically depends on how much the inter-crystalline pathways, or more specifically the defects between crystals, are reduced. On the other hand, the way of

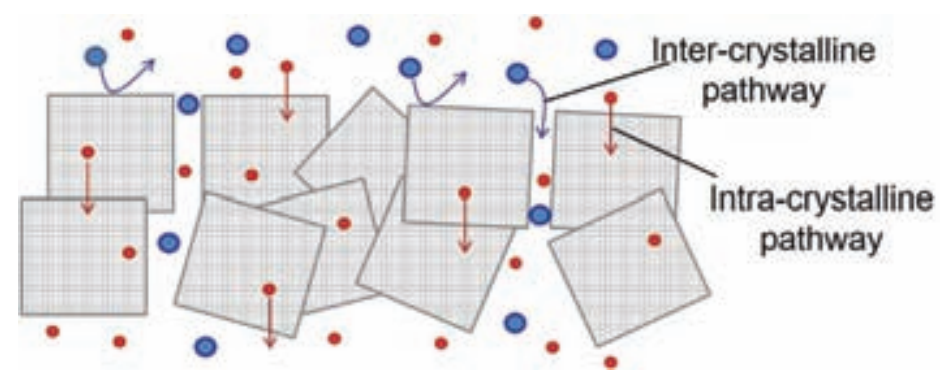

Figure 2. Model of intra-crystalline and inter-crystalline pathways. 
improving the permeation property should focus on improving permeability through intra-crystalline pathways.

Although over 230 zeolite frameworks have been synthesized, few have been successfully prepared as membranes, namely AEI [30,31], CHA [32-35], LTA [36-47], DDR [38,39], MFI [40-43], FAU [44-46], and MOR $[47,48]$. To develop membranes with good separation properties, avoiding the formation of inter-crystalline pathways by controlling the nucleation and crystal growth is absolutely imperative. Crystal size and morphology control is one of the preferred ways of preparing high quality membranes with few defects. The knowledge of basic synthesis studies is extremely important for membrane synthesis. Preparing zeolite membranes with a novel topology is a really hard challenge and, therefore, the development of zeolite membrane requires the knowledge of synthesis procedures and crystallization behaviours, which has so far been generated mostly in research on adsorbents and catalysts.

More than 20,000 types of MOFs, a hundred times more than zeolite topologies, have been reported [49]. However, most of them are unstable in the presence of water or humidity $\left[5^{\circ}, 5_{1}\right]$. Some MOFs, namely ZIF and MIL series, which have sufficient water and thermal stability, are frequently chosen as membrane materials. For examples, ZIF-7 [52,53], ZIF-8 [54-56], ZIF-9o [57,58], MIL-53 [59,60] and HKUST-1 [61,62] membranes have been developed for $\mathrm{H}_{2}, \mathrm{CO}_{2}$ and propylene separations. MOFs offer great possibilities for designing their pore size, adsorption properties, and stability. For this reason, MOF membranes can be used in various applications by optimizing the MOF species for each separation. As in the case of zeolites, studies on MOF membranes cannot develop without advancement in basic studies about design and synthesis. Although many studies on MOF synthesis are reported in the literature and the research on MOF membranes has grown much over the past decade, this field is still immature and the performance of MOF membranes is still low.

\subsection{Material and shape of membrane supports}

Since self-supporting membranes are thick and lack mechanical strength, almost all zeolite and MOF membranes are prepared on or in a porous support. Porous silica, alumina, mullite, titania, zirconia, and stainless steel are used as inorganic supports of membranes. Polymers are also occasionally used as supports. Polymeric membrane in which zeolite and/or MOF crystals are dispersed are called mixed matrix membranes, MMM. Polymeric 
supports have the advantage of a high membrane surface area and moldability common to general polymeric membranes. For MMMs, the compatibility between dispersion and polymer phase is important $[63,64]$ because microgaps between these phases reduce the separation selectivity. In addition, since using polymeric supports may limit the chemical and thermal resistance of the membrane, inorganic porous supports are preferred for relatively stable zeolites and MOFs.

In addition to sufficient mechanical strength, inorganic porous supports require high stability, high permeability, and appropriate surface properties such as roughness and pore size. Permeability of porous support depends on pore size, thickness, and tortuosity. Highly permeable supports need large pore size, small thickness and low tortuosity. However, these factors pose a trade-off with mechanical strength and suitability for membrane preparation. Some of porous supports have asymmetric structures, e.g. a surface layer with small pores on top of large pore support [65].

The shape of the porous support is a main factor not only for membrane preparation but also for membrane module design. Plate-like [40,42], tubular [30-32], hollow fiber [66-68], and monolith-type [69-70] supports have been proposed so far. Tubular supports have a strong advantage of mechanical strength, which favours their use in high-pressure conditions. Most zeolite membranes used commercially are tubular-type membranes $[71,72]$ and the module can be designed with almost the same concept as a multitubular heat exchanger. Plate-like and hollow fiber supports have high packing density (i.e. membrane area/module volume). For example, hollow fiber supports with a diameter of $4 \mathrm{~mm}$ yield packing densities as large as $1000 \mathrm{~m}^{2} \mathrm{~m}^{-3}$, more than 10 times larger than those of tubular supports [66]. However, hollow fibre membranes have some problems for their use in a module, such as low mechanical strength, high-pressure loss, and sealing method. Monolith-type supports show both high mechanical strength and packing density, whereas suitability for membrane preparation is enormously poor [69].

\subsection{Membrane preparation procedure}

Zeolite and MOF membranes are prepared by in situ or seed-assisted hydrothermal or solvothermal synthesis methods. In situ synthesis, just heating the support in a synthesis solution in which nucleation and crystal growth occur, is the simplest way to obtain membranes. This method was employed in the early stage of membrane synthesis studies because 


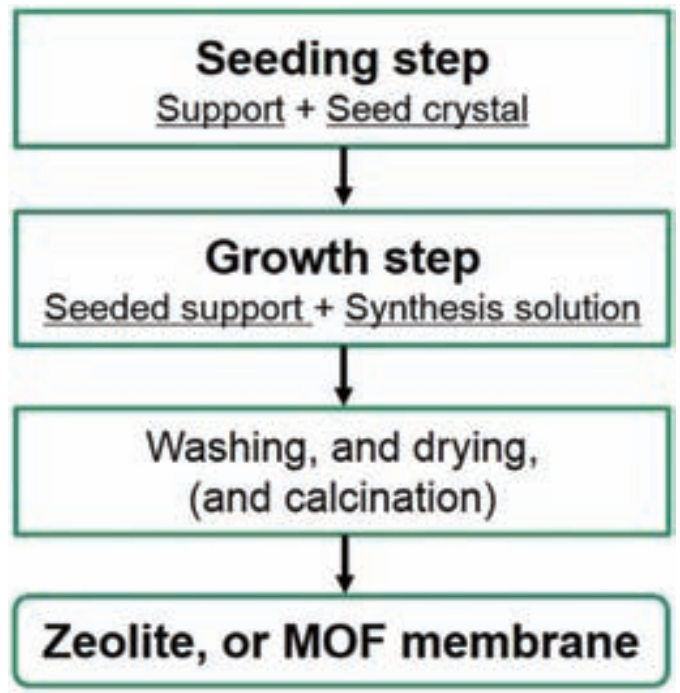

Figure 3. Typical preparation procedure for porous crystal membrane.

of its ease. However, it is difficult to control where nucleation and crystal growth occur using the situ synthesis method. As a result, the membranes obtained by this method tended to be thick and uncompact, and situ synthesis has hardly been used in recent years. In order to solve the problems of the in situ method, seed-assisted synthesis, usually called 'secondary growth', was developed. Figure 3 shows a typical procedure of membrane preparation by a seed-assisted method. Seed crystals are placed on a support prior to a growth step, mainly a hydro, or solvothermal treatment. A thin and compact membrane can be obtained by the seed-assisted method because nucleation and/or crystal growth are stimulated to occur nearby the seed crystals on the surface of a support. At present, seed-assisted syntheses is commonly used for zeolite and MOF membranes. The details of the seed-assisted method are described in the following section.

In addition, some synthesis methods have been reported besides the hydrothermal synthesis mentioned above. Zeolite membranes can be synthesized by the vapour phase transport (VPT) method [73-76]. A general procedure of membrane preparation by the VPT method is as follows. The porous support is soaked in a parent aluminosilicate gel and dried. The support coated with dry gel is treated by heating in the presence of water and organic structure-directing agent vapour for crystallization of the dry gel layer. It is noted that membrane can be prepared on a support having intricate shapes by the VPT method. On the other hand, this method has the 
disadvantage that the thickness of obtained membrane is relatively large. MOF membranes can be obtained by the counter diffusion method $[77,78]$ or by the interfacial reaction method $[67,79]$. The metal ion solution and the organic ligand solution are separated by a porous support and then, MOF crystals are generated at the interface at which metal ions and ligands encounter and react. Alternatively, the support is soaked in the metal ion solution and then immersed into the organic ligand solution. In both these methods thin MOF membranes can be prepared without overgrowth because the reaction stops by the formation of a compact crystal layer.

\subsection{Seeding techniques}

The role of seed crystals in the seed-assisted method is quite important and the obtained membrane performances are strongly influenced by the seeding conditions before the growth step. Various techniques to control seeding have been extensively developed. These include the amount, size, location, and orientation of the seedcrystals.Rubbing [30-32,80], dip-coating [35,37,42], hot dip-coating [81-84], spin-coating [85], Langmuir-Blodgett [86], filtration seeding [87], vacuum seeding [88], spray-coating [89,9o], electrophoresis [91], etc. have been used to prepare seed layers on supports. Figure 4 gives a schematic diagram of different seeding techniques.

One of the simplest ways of seeding is the rubbing method. Seed crystals with or without a small amount of dispersion media are rubbed on the surface of the support, in many cases by fingers or hands. The rubbing method is widely employed at the laboratory scale. However, the quality of the seeded
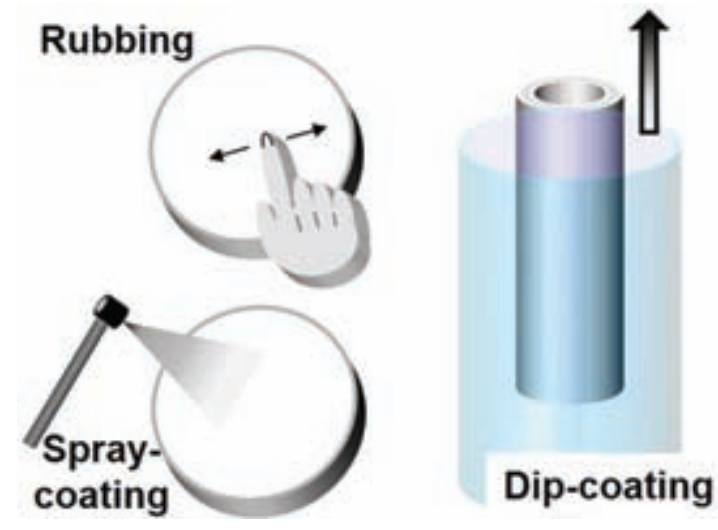

Figure 4. Schematic diagrams of seeding techniques. 
layer prepared depends on the experimenter's skill, while the quality of the seed layer is difficult to control among membranes. In addition, how to scale up the membrane area by the rubbing method is also a severe problem.

The dip-coating method is one of the easiest ways and it is easy to scale up. The general procedure of the dip-coating method is as follows. The support is immersed in a slurry into which seed crystals are dispersed. After drawing up and drying the support from the slurry, the seeded support is obtained. Capillary and gravitation forces govern the formation of a seeding layer here. This method is suitable for commercialization because the quality of the seeded layer is not influenced by hand skills and, in addition, it is economically efficient because of the reusability of the seed slurry and the use of simple equipment. However, the seed crystal distribution is often not uniform because of the negative influence of gravitation force during drawing up and drying steps. A larger amount of seed crystals tend to attach to the lower side of support than the upper side. Additionally, a combination method of rubbing and dip-coating was reported [92]. It has been reported that rubbing and dip-coating are suitable for rough and flat surfaces, respectively, after which the combined approach can produce high performance membranes with high reproducibility.

In the filtration seeding and vacuum seeding methods, a slurry of seed crystals is also used. Seed crystals larger than the pore size of the porous support are dispersed in the slurry. After removing the dispersion media by filtration, strained seed crystals remain on the surface of the support. Although seeding behaviour is influenced by the capillary and gravitation forces in the vacuum seeding, the negative effect of gravitation force can be reduced by the assistance of a vacuum rather than the dip-coating method [88]. Furthermore, hot dip-coating, a combination of dip-coating and vacuum seeding has also been reported [81-84]. In this method, the porous support is preheated prior to dip-coating. Capillary and vacuum forces work simultaneously through the immersion of the preheated support into the seed slurry. As a result, a uniform seed layer can be obtained as in the case of filtration and vacuum seeding.

Electrostatic interactions can contribute to the seeding behaviour. In the case of the preparation of a thin MFI-type zeolite membrane, seed crystals electrostatically adsorbed on the surface support were coated with cationic polymer molecules [93]. Seed crystals can be deposited on a conductive support such as stainless steel by electrophoresis under an electric field. In addition, spin-coating and spray-coating methods are applied to seeding in lab-scale. The spin-coating method can only be applied to the seeding on relatively small plate-like supports with a smooth surface. 
Physically supported seed crystals often detach from support surface and are unlikely to contribute to the formation of membrane in the growth step. When the grown layer weakly adheres to the support, the crystal layer readily peels off through friction. Thus, preventing the detachment of seed crystals from the support surface is also of great importance. For zeolite membrane preparation, chemical bonding between seed and support can be formed by dehydration condensation of hydroxyl groups [94]. Dehydration condensation between hydroxyl groups on the surface of zeolite and on a support such as alumina or titania occur upon heating, leading to strong adhesion. In addition, steam-assisted conversion seeding was reported as another way to immobilize the seed layer [95]. Seeds-containing synthesis paste was rubbed on the support and then steam-assisted conversion (heat treatment in the presence of steam) was carried out. A well-interlocked seed layer was generated on the support surface using this steam-assisted conversion seeding. On the other hand, chemical modification was reported to improve the interaction between MOF and support in MOF membrane preparation [96]. Some functional groups, such as amino and carboxyl groups produced by modification, combine with MOF linkers. These functional groups can contribute to heterogeneous nucleation on the support surface as well.

\subsection{Role of seed crystals in the growth step}

In seed-assisted synthesis, a hydrothermal or solvothermal treatment of the seeded support is carried out to grow the zeolite and MOF layers. The role of the crystal seeds on crystal growth in the synthesis solution has been energetically studied. In fact, it has been one of the most interesting topics in not only membrane but also in the whole zeolite and MOF synthesis fields for some years. A portion of it is has been clarified and some of it is still unclear. Some related discussion was already presented in Chapter 2. The role of seed crystals contributing to form compact zeolite membranes have been reported. For example, they work as an agent in crystal growth $[40,97]$, are the origin of heterogeneous nucleation [42,98,99], structuredirecting agents [100], and directing crystal orientation [40,97]. Figure 5 shows examples of seed roles in synthesis solution through growth step. Some examples are introduced here with a focus on their role in formating a thin crystal layer.

The seed crystals sometimes act as the origin of crystal growth, particularly in silicalite-1 (pure silica MFI-type zeolite) membrane synthesis. 


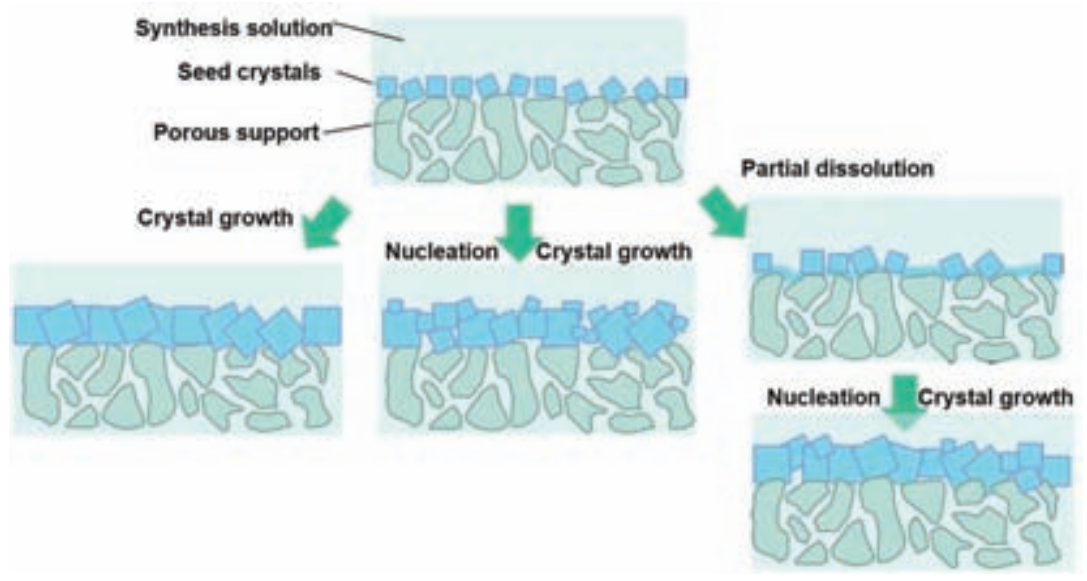

Figure 5. Roles of seed crystals during the growth step of membrane formation.

The seed crystals play a role in the dilute synthesis solution such that secondary growth of seed occurs without homogeneous and heterogeneous nucleation. It is noted that the silicalite-1 membrane formed without nucleation sometimes retains the crystal orientation of seed layer [40]. In particular, the $b$-oriented silicalite-1 membrane had been obtained by secondary growth of $b$-oriented seed layer in dilute solutions. As another specific example of secondary growth, the heteroepitaxial growth of zeolite membrane has been reported [101]. ETS-10, a kind of titanosilicate zeolite, was grown from ETS-4 which is a structurally related material with ETS-10. In this case, ETS-10 crystals were grown epitaxially on the (hoo) face of ETS-4.

Homo- and heterogeneous nucleation and crystal growth often occur simultaneously during the growth step in the seed-assisted method. In such cases, it is difficult for the crystal layer to uniformly cover the whole support surface unless seed crystals are previously used to cover the support. Additional new crystals are generated by homogeneous nucleation in the bulk synthesis solution and/or by heterogeneous nucleation on the support. Then, the already supported seed and the fresh crystals formed in situ grow in the solution. Both the growth of seed crystals and the heterogeneous nucleation on the support contribute to the formation of a compact membrane layer.

Controlling the zeolite topology formed from a synthesis solution by using seed crystals has been widely reported for powder synthesis. Seed crystals often act as a substrate to determine the topology of the zeolite formed in the membrane preparation as well as in powder synthesis. For 
example, both MOR and ZSM-5 zeolite membranes can be synthesized under the same synthesis conditions by using different seed crystals [100]. Pure MOR and ZSM- 5 zeolite membranes can be obtained using MOR seed and ZSM- 5 seed crystals, respectively. *BEA zeolite membrane can be obtained by using *BEA seed in the synthesis solution, whereas nucleation and growth of MOR zeolite occurs without the seed crystals [102]. In this report, *BEA membrane was prepared by a seed-assisted method in the absence of an organic structure-directing agent as an example.

\subsection{Scale-up of the membrane preparation method}

Quality control is one of the important issues for the development of commercial membranes. Several companies have commercialized tubular types of zeolite membranes since 1998 [103-106]. The length of the membranes is one meter or so with diameters of $10-16 \mathrm{~mm}$. This is about ten times longer than the membranes synthesized in laboratories. For scaling-up zeolite and MOF membranes on such long tubular supports, uniformity of seed crystals applied to the tube surface and distributions of temperature and concentration in synthesis solution throughout the growth step are often problems. In addition, the qualities of porous supports are important as well.

In general, hundreds, or more, membranes are bundled in a module, and the separation performance of the module can be easily spoiled by a single poor-quality membrane. The simplest but most burdensome solution in this case is checking the separation performance of all the membranes. The total inspection can be applied to up to a few hundred membranes in a relatively small process. However, it is naturally an unsuitable control method for a larger process, which requires over tens of thousands of tubes. Therefore, inexpensive and effective approaches of quality check are required in the future.

\section{Where are membranes used?}

As shown above, zeolite and MOF membranes have great advantages in mechanical strength, thermal and chemical resistance compared to the polymeric membranes currently used. They are now expected to be applied to processes running under more severe conditions. This section describes four kinds of applications: gas separation, dehydration of organic solvents, 
hydrocarbon separation, and food manufacturing. Typical applications drawing attention as targets of zeolite and MOF membrane are introduced below. Figure 6 shows typical applications of nanoporous membranes.

\subsection{Gas separation}

There are numerous reports about gas separation by zeolite and MOF membranes such as $\mathrm{CO}_{2}$ recovery, $\mathrm{H}_{2}$ purification, natural gas purification and air separation. In addition, demonstration tests of $\mathrm{CO}_{2}$ recovery from natural gas using zeolite membrane have been started [107].

$\mathrm{CO}_{2}$ recovery from gas mixtures with $\mathrm{N}_{2}, \mathrm{O}_{2}, \mathrm{H}_{2}, \mathrm{H}_{2} \mathrm{O}$, and $\mathrm{CH}_{4}$, etc. is one of the hottest topics of zeolite and MOF membranes as a measure against global warming $[30,31,33,34,38,39,6 \circ, 63,69] \cdot \mathrm{CO}_{2}$ recovery from air and light hydrocarbon gas mixtures is required in the treatment of exhaust gases from thermal power plants, and $\mathrm{CO}_{2}$ separation from $\mathrm{CH}_{4}$ is a step in natural gas upgrading. Such $\mathrm{CO}_{2}$ recoveries from exhaust gas and natural gas treatments are carried out at relatively high pressure ( $\sim 7 \mathrm{MPa})$ and/ or temperature $(\sim 473 \mathrm{~K})$. In addition, a high $\mathrm{CO}_{2}$ concentration causes the plasticization of polymeric membranes and thus inorganic membranes are required for such applications. Low $\mathrm{CO}_{2}$ purity in the permeation side of membranes is so far allowed because recovered $\mathrm{CO}_{2}$ is not always utilized as a feedstock. On the other hand, since the amount of mixture gas to treat is huge in both processes, an important property of $\mathrm{CO}_{2}$ separation membrane has been permeability rather than selectivity. For this application membranes have been developed with a focus on small pore zeolites and MOFs, such as CHA, AEI, DDR and ZIF-8, which have a strong interaction with $\mathrm{CO}_{2}$ molecules. Mitsubishi Chemical Corp. is conducting a demonstration test for natural gas upgrading by CHA-type zeolite membrane at Kurosaki, a northern part of Fukuoka prefecture in Japan, since 2016 [107].

$\mathrm{H}_{2}$ separation from organic hydrides has been investigated by using small pore zeolite as in the case of $\mathrm{CO}_{2}$ separation. Since the polymer electrolyte fuel cell needs $\mathrm{H}_{2}$ with a high purity $(>99.97 \%)$, the membrane should possess an excellent separation property. However, the selectivity to $\mathrm{H}_{2}$ through the zeolite and MOF membrane is at a magnitude lower than those of carbon and metal membranes mentioned above. The relatively wide working window of temperature is a strong positive point of zeolite and MOF membranes: for example, $\mathrm{H}_{2}$ separation from propane and propylene through SAPO-34 membrane from room temperature up 
to $923 \mathrm{~K}$ was reported for the development of propane dehydrogenation process [108].

\subsection{Dehydration of organic solvents}

Dehydration of organic solvents through zeolite membranes for recycle use is relatively straightforward. The process size is generally small and the recycle system does not generally cause critical problems. Therefore, zeolite membranes for dehydration and dehydration systems using zeolite membranes have been commercialized. For the dehydration of organic solvents, hydrophilic zeolites such as LTA, CHA, and FAU are used. The first large-scale plant using 16 modules with 125 pieces of LTA tubular membrane has been operated for the dehydration of ethanol since 1999 [71]. $531 \mathrm{~L} \mathrm{~h}^{-1}$ of $99.8 \mathrm{wt} . \%$ ethanol is successfully produced from $6 \circ 5 \mathrm{~L} \mathrm{~h}^{-1}$ of $90 \mathrm{wt} . \%$ ethanol solution by the membrane separation system. In addition, a commercial plant of isopropyl alcohol (IPA) purification for lenses cleaning by using LTA membrane has been operating since 1999 as well [109]. In this operation, $8.6 \mathrm{~L} \mathrm{~h}^{-1}$ of $99.65 \mathrm{wt} . \%$ IPA is successfully obtained from $10.5 \mathrm{~L} \mathrm{~h}^{-1}$ of $88.8 \mathrm{wt} . \%$ IPA solution by using 24 pieces of membrane. Recently, there have been some reports on dehydration from acidic or basic solutions using MOR or MFI-type zeolite membrane [47,110].

\subsection{Hydrocarbon separation}

Hydrocarbon separation is one of the largest and most promising targets for zeolite and MOF membranes. Hydrocarbon separation falls roughly into two categories, isomer separation and saturated/unsaturated hydrocarbon separation.

As typical isomer separation, separations of butane isomers [41-43,93], hexane isomers $[41,93]$, and xylene isomers separations have been attempted for decades $[40,41,93,111,112]$. The general principle is the molecular sieving effect that enables us to separate mixtures of molecules by their sizes.

Xylene isomers are some of the most important basic chemicals produced from petroleum. $o$-Xylene is the feedstock of phthalic anhydride and $p$-xylene is used as a raw material for plasticizing agents and colorants. The demand of $p$-xylene as the feedstock of polyesters is particularly larger than that of the other isomers. $p$-Xylene is produced by isomerization of xylene isomers, by disproportionation of toluene or by transalkylation. However, 
$p$-xylene selectivity in these reactions is limited by thermodynamic equilibria. For example, $p$-xylene selectivity in isomerization reaction is about $20 \%$ under common reaction conditions. This is the reason why $p$-xylene has to be separated from large amounts of other xylene isomers. MFI-type zeolite has been studied as a $p$-xylene selective membrane as it is also used as a $p$-xylene selective catalyst based on its shape selectivity for xylene isomerization and toluene disproportionation. Although excellent MFI membranes and preparation methods were previously reported [40,93], the permeabilities of $p$-xylene through these membranes were still insufficient for practical use.

Propylene/propane and ethylene/ethane separations are representative examples of unsaturated/saturated hydrocarbon separations $[54-56,78,113,114]$. ZIF-8 having a pore size between propylene and propane molecules has extensively been studied as a propylene-selective membrane material based on the molecular sieving effect [55,56]. Additionally, FAU-type zeolite membrane containing Ag cation was found to consitute a membrane selective to olefins [113]. The Ag cation has a strong affinity with olefins, so that olefins preferentially adsorb and penetrate through the micropores of Ag-zeolite membranes. Similarly, benzene/cyclohexane separation by FAU zeolite was reported [114].

Issues such as a relatively elevated operation temperature, the existence of impurities, and the large throughput required make it difficult to use membrane separations in petroleum refinery and petrochemistry. Nevertheless, one can expect great profits for paraffin/olefin separation using gas separation membranes because enormous amounts of power consumption are inevitable in current cryogenic separations.

\subsection{Food manufacturing}

Zeolite membranes have started to be used in food manufacturing. A novel Japanese sake (rice wine) has been produced by the dehydration of conventional Japanese sake using CHA-type zeolite membrane [115]. The alcohol content in Sake is increased from the conventional $20 \%$ to $30 \%$ by membrane dehydration. With the help of membranes sake can be concentrated without losing molecules such as flavours and tastes in contrast to fermentation and distillation processes. The new Japanese Sake was served at the $42^{\text {nd }} \mathrm{G}_{7}$ summit held at the Mie prefecture in Japan in 2016. Membrane separation processes are expected to continue to create high added value products in food manufacturing in the future. 


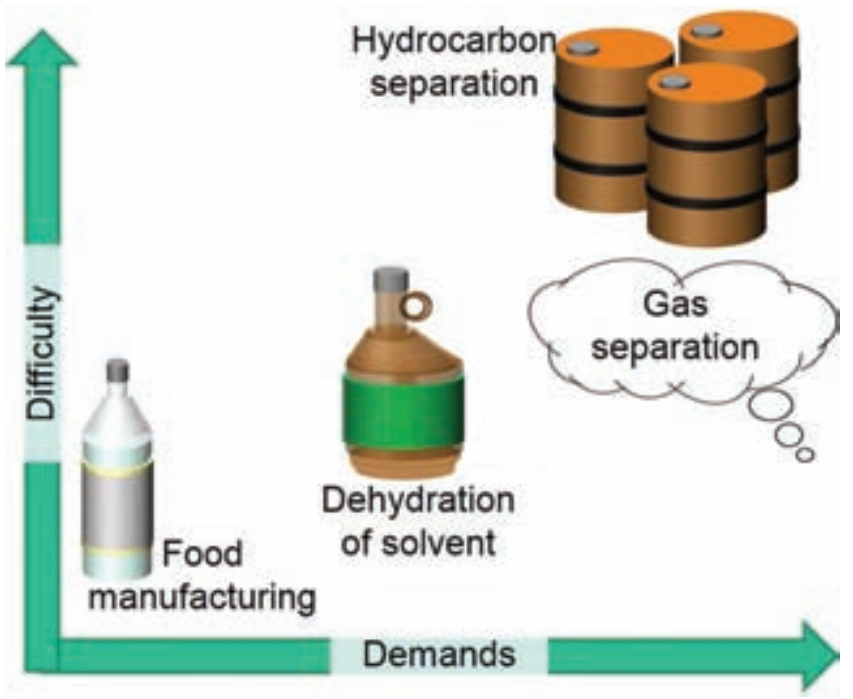

Figure 6. Typical fields of nanoporous membrane applications.

\section{Why do nanoporous crystal membranes separate mixtures?}

The principles of separation with nanoporous crystal membranes are described here. Although a perfectly compact porous crystal membrane with no defects should have only intra-crystalline pathways, actual membranes generally have both inter- and intra-crystalline pathways. Thus, it is necessary to consider the effects of both pathways on the permeation properties. In general, selective permeation occurs through intra-crystalline pathways and non-selective permeation occurs through inter-crystalline voids and generally reduces the separation selectivity. Selective permeation through inter-crystalline pathways only occurs exceptionally.

Permeation phenomena through intra-crystalline pathway can be divided into three steps as follows. The first step is the adsorption from the gas or liquid phase on the micropores of zeolite and MOF in the feed side. The second step is the diffusion along the micropores through the membrane. The last step is the desorption from the membrane to gas or liquid phase in the permeate side. A mixture is separated through zeolite and MOF membranes when specific molecules in the mixture preferentially proceed across the membrane through these three steps. In many cases, mixtures are separated by preferential adsorption and/or faster diffusion in the first and second step. Details are shown in the following part with some examples. Further insights on the adsorption and diffusion processes can also be obtained from Chapters 7 and 10 in this book. 


\subsection{Molecular sieving}

Separation by molecular sieving is based on the difference in molecular sizes as the term suggests. In other words, the molecules are separated by the difference in their diffusion rates along the membrane micropores. Because diffusivity in micropores tends to decrease with increasing molecular size, smaller molecules permeate preferentially through the membrane. When the molecular size is obviously larger than the pore size and unable to enter the micropore, separation selectivity could theoretically be perfect (infinite). It is noted that molecular sieving membranes certainly exhibit selectivity to smaller molecules. Propylene/propane separation by ZIF-8 membrane and xylene isomer separation by MFI zeolite membrane are typical examples of separation based on the molecular sieving effect. In the case of propylene/propane separation by ZIF- 8 membrane, it was reported that the diffusivity of propylene through ZIF-8 membrane was 9-23 times larger than that of propane [56]. Because the diffusion coefficient of $p$-xylene in MFI zeolite is $c a$. 100 and 1000 times higher than those of $o$ - and $m$-xylene, MFI zeolite has been used as selective membrane material for $p$-xylene separation [116].

To separate mixtures by the molecular sieving effect, the selection of the types of zeolite and MOF with an appropriate size of pore for a specific separation target is important. In addition, methods for pore size control by ion exchange and post-treatment have been studied to obtain a membrane with an optimum pore size. Ion exchange of SAPO-34 membrane from $\mathrm{H}^{+}$to larger cations for $\mathrm{CO}_{2} / \mathrm{CH}_{4}$ separation was reported [117]. The ion exchange decreased the permeance of both $\mathrm{CO}_{2}$ and $\mathrm{CH}_{4}$, and with larger cations exchanged, their permeances were further reduced because of steric hindrance. $\mathrm{CO}_{2}$ selectivity increased in all cases by cation exchange from $\mathrm{H}^{+}$to $\mathrm{Li}^{+}, \mathrm{Na}^{+}, \mathrm{Cu}^{2+}$, and $\mathrm{NH}_{4}^{+}$. Control of pore size with pyrolytic carbon was also reported [118].

Hydrocarbon vapour was thermally deposited on silicalite-1 membrane at $973 \mathrm{~K}$, and then the pore size of membrane was narrowed by deposited carbon. As a result, $n$-hexane selectivity for 2-methylpentane increased from 15 to 5 o by the pyrolytic carbon treatment.

\subsection{Selective permeation because of affinity}

Not only the difference of molecular sizes but also the affinity between molecules and membrane material is utilized for separation. Molecules 
that have a strong interaction with membrane material preferentially adsorb in the micropores of membranes and penetrate through them. Adsorption properties due to heteroatoms and cations in zeolites and metal sites in MOFs can play important roles in such separation depending on the affinity. Propylene/propane separation by Ag-FAU membrane and benzene/cyclohexane separation by Na-FAU mentioned above are examples of separation based on affinity. Strong interactions between propylene and Ag cation and between benzene and Na cation contribute to the selective permeations of propylene and benzene in each system. In separation systems based on affinity differences, the permeation of molecules that have a relatively weak interaction with membrane material is often inhibited by preferentially adsorbed molecules. In fact, the propane permeance through Ag-FAU membrane was drastically decreased by the coexistence of propylene $[113,119]$. The activation energy of cyclohexane permeation through Na-FAU membrane increased by the coexistence of benzene [111].

Additionally, it should be noted that larger molecule selective membranes can be obtained in the case of separations using affinity. For example, it was reported that Na-MFI membrane exhibited a high methanol selectivity for methanol $/ \mathrm{H}_{2}$ mixture, indicating that larger methanol molecules strongly adsorbed on Na cation occluded in the micropores of zeolite blocked the permeation of $\mathrm{H}_{2}$ in the system [120].

Selective permeations by both the molecular sieving effect and affinity described above occur through intra-crystalline pores. On the other hand, selective permeation can occur even through inter-crystalline pathways when there is great intermolecularly interaction in addition to affinity with membrane. In the dehydration of organic solvents, there are cases where capillary condensation of water molecules in the inter-crystalline pathways such as defects and voids in a membrane occurs, and then condensed water blocks permeation of organic molecule [35]. As a result, water selectively penetrates through both intra- and inter-crystalline pathways.

\subsection{Evaluation method for inter-crystalline pathways in a membrane}

Reducing inter-crystalline pathways, voids and cracks is the first step in obtaining a membrane exhibiting excellent separation properties because non-selective permeation through a very small amount of inter-crystalline readily disables selectivity. For example, it has been reported that only o.19\% defect area of the total membrane area is enough to depress selectivity for $\mathrm{CO}_{2} / \mathrm{H}_{2}$ through MFI membrane [121]. Therefore, characterization for 
inter-crystalline pathway is essentially important for membrane development, and various evaluation methods for pore size distribution and position of defects have been reported [122-128].

Pore size distribution is often evaluated by using nano-permporometry [122-124]. In this method, a mixture of gas with a given partial pressure of condensable vapour is fed to a membrane and gas permeance is measured. Usually, $\mathrm{He}$ or $\mathrm{N}_{2}$ is used for gas, and steam or hexane is used as a condensable vapour. The partial pressure of vapour is raised in a stepwise manner during measurement. Pores in a membrane are plugged with condensed vapour in the order small to large, and then gas permeance decreases with increasing vapour partial pressure. Because there is relationship between vapour partial pressure and pore size plugged with condensed vapour at a given partial pressure, pore size distribution can be evaluated from gas permeation at each vapour partial pressure. The pore size distribution in the range of $0.5^{-} 3^{\circ} \mathrm{nm}$ is evaluated based on the Kelvin equation [124].

To locate nano-sized defects, permeation tests using a capillary assembly were proposed $[125,126]$. Feed and permeate areas were limited by using probe needles and permeances could be measured with the resolution of ca. $1 \mathrm{~mm}$. The locations of defects in TS-1 and SAPO-34 membrane were visually mapped using this method. As another way to visualize intercrystalline pathways, a fluorescence confocal optical microscopy was developed $[127,128]$. Inter-crystalline pathways in MFI membrane were filled with a fluorescent dye by impregnation and the dye was unable to enter intra-crystalline pathways because of its bulky size. As a result, it was possible to observe the three-dimensional network of inter-crystalline pathways.

\subsection{Membrane reactors}

In recent years, membrane reactors have received much attention from the viewpoint of saving energy, saving space, and highly efficient production. When the membrane reactors could be applied into practice, drastic and innovative change would occur in chemical processes. Membrane reactors are categorized into three types, namely, extractor, distributor and active contactor, as shown in Figure 7. Membrane reactors using nanoporous membranes often refer to the extractor type. Researchers have extensively studied the extractor-type membrane reactors applied to reactions in which attainable conversion levels are limited by equilibrium, such as dehydrogenation, steam reforming, and esterification. In this type of reactor, reaction and separation occur simultaneously. In accordance with Le 
Chatelier's principle, equilibrium values shift by removing products from a reaction system, allowing conversion levels and product yields in the membrane reactor to exceed those in conventional reactors.

Both organopolymeric and inorganic membranes can be applied to membrane reactors. Inorganic membranes are advantageous compared with organopolymeric membranes for high temperature and/or highpressure applications. Among inorganic membranes, palladium alloy, amorphous silica and zeolite have so far been used for membrane reactors. Whereas, palladium and palladium alloy membranes in particular, were developed for the membrane reactor with hydrogen extraction, there were problems such as hydrogen embrittlement and high cost. Thus, development of amorphous silica and zeolite membrane for membrane reactor for dehydrogenation with hydrogen extraction has progressed in recent years. In this case, lower reaction temperature is desirable from the view point of equipment deterioration and energy cost, because conventional hydrogen production reactions such as steam reforming of naphtha and methane need a reaction temperature above $1100 \mathrm{~K}$. Compared to the conventional packed bed reactor, the membrane reactor can be operated at a lower reaction temperature due to removal of hydrogen from the reaction system, inhibiting the formation of methane below $1100 \mathrm{~K}$. Zeolite membrane showed relatively high hydrogen permeance and selectivity [108,129-131]. The improvement of the stability and selectivity of zeolite membranes at higher temperature is still an open question. Reports of better permselective performance in the membrane reactor of dehydrogenation with amorphous silica membrane in comparison to zeolite membranes have been published. Permselectivity is a general term used to refer to both permeance and selectivity. It is supposed that cations occluded in zeolite framework inhibit the permeation of hydrogen molecules. Membranes of aluminophosphate $\left(\mathrm{AlPO}_{4}-\mathrm{n}\right)$ or silicoaluminophosphate (SAPO-n), which have less or no ion exchange sites possibly blocking permeation paths in the zeolitic micropores, could be applied in dehydrogenation membrane reactors in the future.

Many researchers have developed membrane reactors for esterification using zeolite membranes. Compared with conventional reactors, these membrane reactors also lead to higher conversion and yield owing to the removal of water, as a product of the esterification, from the reaction system. Zeolite membranes with a hydrophilic nature are expected to show a high water flux and permselectivity in the esterification reaction system. Zeolite A (LTA) has Si/Al ratio = 1 in its framework and thus develops strong hydrophilicity, resulting in the LTA membrane showing a high water flux [132-135]. Since esterification reaction is generally operated 
under an acidic atmosphere, the membrane is required to be acid resistant. Comparing the acid resistance of zeolite A with that of MOR membrane, MOR membrane showed better acid resistance in the membrane reactor tests $[136,137]$. Zeolite A is much more hydrophilic owing to its lowest $\mathrm{Si} / \mathrm{Al}$ ratio (=1) among zeolites and, thus, the water flux through MOR membrane is smaller than that through zeolite A membrane.

Zeolite T membrane was also developed for the membrane esterification reactor with experimental and simulation approaches. Simulation results of vapour permeation-aided esterification of lactic acid with ethanol, using a simple model incorporated second-order reversible reaction with separation, showed good agreement with experimental results [138]. In addition, CHA zeolite membranes were successfully applied to the membrane reactor for the esterification of adipic acid with isopropyl alcohol and the yield of diisopropyl adipate was increased compared to the esterification without CHA-type zeolite membrane [139]. The water flux through CHA zeolite membrane was decreased after being used ten times in the esterification. In this paper, the authors claimed that a great decrease of water flux took place because of the adhesion of sublimed adipic acid rather than by problem of acid resistance.

Xylene isomerization is also limited by thermodynamic equilibrium. When an extractor-type membrane reactor using MFI-type zeolite membrane that shows $p$-xylene permselective performance is applied for this reaction, $p$-xylene selectivity can be enhanced by the removal of $p$-xylene from the product. This type of membrane reactor is considered to show full use of the unique characteristics of zeolite membranes having a uniform pore size derived from their crystal structures. Compared with the conventional packed bed reactor, the membrane reactor using MFI membrane showed the enhancement of $p$-xylene selectivity and yield [140-142]. However, the practical use of membrane reactor for the xylene isomerization still suffers from insufficient flux and selectivity through MFI membrane. Development of a membrane showing high permselective performance is the key issue for this type of membrane reactor. On the other hand, MOFs membranes have been rarely used for membrane reactors, although these membranes can be expected in the future.

\section{Summary}

Separation by zeolite membrane has started to be used in relatively small-scale processes such as the recycling of organic solvents and food 


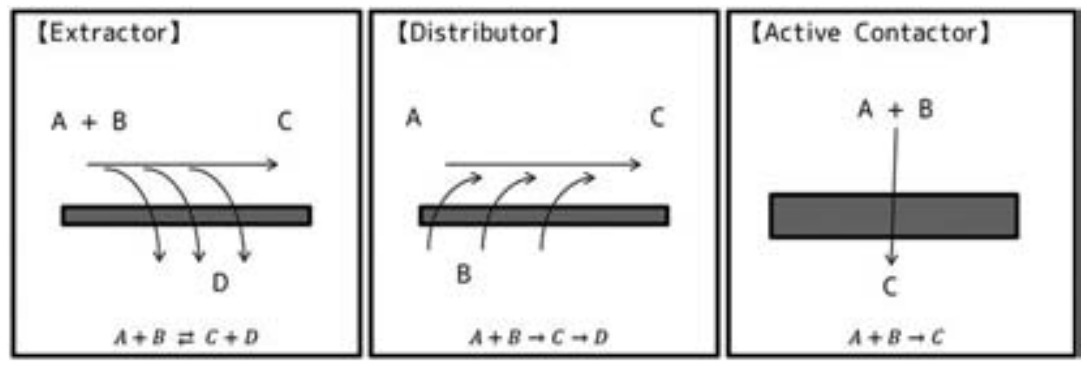

Figure 7. Schematic diagram of extractor, distributor and active contactor-type membrane reactors.

manufacturing since the late 1990s. MOF membranes are expected to find practical applications in the near future as well. However, there are issues that need to be solved when applying these porous crystal membranes to large-scale processes like gas separation or hydrocarbon separation.

Innovative improvement of permeability through the membrane needs to be developed in lab-scale studies. To this end, studies about not only membrane preparation but also membrane support and module are important. The achievement and experience of small processes in industry will be of great help in expanding future applications.

The combination of separation units of membrane, distillation, and adsorption are realistic solutions for innovating separation in large-scale processes. Design and optimization for such hybridized processes are seriously required. Similarly, membrane reactors, combinations of reaction and separation, also face the problems of process design and optimization. In addition, process dynamics is subject to future developments such as startup and shutdown. Separation using porous crystal membranes will become more popular when such issues are solved by the endeavour of researchers.

\section{References}

[1] Bein, T. Brown, K. Frye, G.C Brinker, C.J.J. Am. Chem. Soc. 1989, 111, 7640.

[2] Meier, B. Werner, T. Klimant, I. Wolfbeis, O.S. Sens. Actuators B Chem. 1995, 29, 240.

[3] Urbiztondo, M.A. Pellejero, I. Villarroya, M. Sese, J. Pina, M.P. Dufour, I. Santamaria, J. Sens. Actuators B Chem. 2009, 137, 608.

[4] Liu, J., Sun, F., Zhang, F., Wang, Z., Zhang, R., Wang, C., Qui, S. J. Mater. Chem. 2011, 21, 3775.

[5] Kung, C.W., Chang, T.H., Chou, L.Y., Hupp, J.T., Farha, O.K., Ho, K.C. Electrochem. Commun. $2015,58,51$.

[6] Wang, Z., Wang, H., Mitra, A., Huang, L., Yan, Y. Adv. Mater. 2001, 13, 746.

[7] Eslava, S., Baklanov, M.R., Neimark, A.V., Iacopi, F., Kirschhock, C.E.A., Maex, K., Martens, J.A. Adv. Mater. 2008, 20, 3110. 
[8] Jahan, M., Bao, Q., Loh, K.P.J. Am. Chem. Soc. 2012, 134, 6707.

[9] Alvaro, M., Carbonell, E., Ferrer, B., Llabrés i Xamena, F.X., Garcia, H. Chem. Eur. J. 2007, 13, 5106 .

[10] Zhang, X., Lai, E.S.M., Martin-Aranda, R., Yeung, K.L. Appl. Catal., A 2004, 261, 109.

[11] Rebrov, E.V., Seijger, G.B.F., Calis, H.P.A., de Croon, M.H.J.M., van den Bleek, C.M., Schouten, J.C. Appl. Catal., A 2001, 206, 125 .

[12] Wan, Y.S.S., Chau, J.L.H., Gavriilidis, A., Yeung, K.L. Microp. Mesop. Mater. 2001, 42, 157.

[13] Huo, J., Aguilera-Sigalat, J., El-Hankari, S., Bradshaw, D. Chem. Sci. 2015, 6, 1938.

[14] Elimelech, M., Phillip, W.A. Science 2011, 333, 712.

[15] Avlonitis, S.A., Kouroumbas, K., Vlachakis, N. Desalination 2003, 157, 151.

[16] Schiermeier, Q. Nature 2008, 452, 260.

[17] Le-Clech, P., Chen, V., Fane, T.A.G.J. Membrane Sci. 2006, 284, 17.

[18] Meng, F., Chae, S.R., Drews, A., Kraume, M., Shin, H.S., Yang, F. Water Res. 2009, 43, 1489.

[19] Riedl, K., Girard, B., Lencki, R.W.J. Membrane Sci. 1998, 139, 155.

[20] Rektor, A., Pap, N., Kokai, Z., Szabo, R., Vatai, G., Bekassy-Molnar, E. Desalination 2004, 162,271 .

[21] Itoh, N., Akiha, T., Sato, T. Catal. Today 2005, 104, 231.

[22] Uemiya, S., Sato, N., Ando, H., Kude, Y., Matsuda, T., Kikuchi, E. J. Membrane Sci. 1991, 56,303 .

[23] Barbieri, G., Violante, V., Maio, F.P.D., Criscuoli, A., Drioli, E. Ind. Eng. Chem. Res. 1997, 36,3369 .

[24] Kiyono, M., Williams, P.J., Koros, W.J.J. Membrane Sci. 2010, 359, 2.

[25] Yoshimune, M., Fujikawa, I., Haraya, K. Carbon 2007, 45, 553.

[26] Gopalakrishnan, S., da Costa, J.C.D. J. Membrane Sci. 2008, 323, 144.

[27] Nomura, M., Ono, K., Gopalakrishnan, S., Sugawara, T., Nakao, S. J. Membrane Sci. 2005, 251,151 .

[28] Kanezashi, M., Yada, K., Yoshioka, T., Tsuru, T.J. Membrane Sci. 2010, 348, 310.

[29] Nomura, M., Yamaguchi, T., Nakao, S. J. Membrane Sci. 2001, 187, 203.

[30] Carreon, M.L., Li, S., Carreon, M.A. Chem. Commun. 2012, 48, 2310.

[31] Wu, T., Wang, B., Lu, Z., Zhou, R., Chen X.J. Membrane Sci. 2014, 471, 338.

[32] Hasegawa, Y., Hotta, H., Sato, K., Nagase, T., Mizukami, F. J. Membrane Sci. 2010, 347, 193.

[33] Li, S., Falconer, J.L., Noble, R.D. J. Membrane Sci. 2004, 241, 121.

[34] Tian, Y., Fan, L., Wang, Z., Qiu, S., Zhu, G. J. Mater. Chem. 2009, 19, 7698.

[35] Hirota, Y., Watanabe, K., Uchida, Y., Egashira, Y., Yoshida, K., Sasaki, Y., Nishiyama, N. J. Membrane Sci. 2014, 415, 176.

[36] Aoki, K., Kusakabe, K., Morooka, S. J. Membrane Sci. 1998, 141, 197.

[37] Okamoto, K., Kita, H., Horii, K., Tanaka, K. Ind. Eng. Chem. Res. 2001, 40, 163.

[38] Tomita, T., Nakayama, K., Sakai, H. Microp. Mesop. Mater. 2004, 68, 71.

[39] van den Bergh, J., Tihaya, A., Kapteijn, F. Microp. Mesop. Mater. 2010, 132, 137.

[40] Lai, Z., Tsapatsis, M., Nicolich, J.P. Adv. Funct. Mater. 2004, 14, 716.

[41] Hedlund, J., Sterte, J., Anthonis, M., Bons, A.J., Carstensen, B., Corcoran, N., Cox, D., Deckman, H., Gijnst, W.D., de Moor, P.P., et al. Microp. Mesop. Mater. 2002, 52, 179.

[42] Li, G., Kikuchi, E., Matsukata, M. Microp. Mesop. Mater. 2003, 60, 225.

[43] Coronas, J., Falconer, J.L., Noble, R.D. AIChE J. 1997, 43, 1797.

[44] Kumakiri, I., Yamaguchi, T., Nakao, S. Ind. Eng. Chem. Res. 1999, 38, 4682.

[45] Zhu, G., Li, Y., Zhou, H., Liu, J., Yang, W. J. Membrane Sci. 2009, 337, 47.

[46] Sato, K., Sugimoto, K., Sekine, Y., Takada, M., Matsukata, M., Nakane, T. Microp. Mesop. Mater. 2007, 101, 312.

[47] Li, G., Kikuchi, E., Matsukata, M. Sep. Purif. Technol. 2003, 32, 199. 
[48] Zhang, Y., Xu, Z., Chen, Q.J. Membrane Sci. 2002, 210, 361.

[49] Furukawa, H., Cordova, K.E., O’Keeffe, M., Yaghi, O.M. Science, 341, 1.

[50] Gul-E-Noor, F., Jee, B., Poppl, A., Hartmann, M., Himsl, D., Bertmer, M. Phys. Chem. Chem. Phys. 2011, 13, 7783 .

[51] Cychosz, K.A., Matzger, A.J. Langmuir 2010, 26, 17198.

[52] Li, Y.S., Bux, H., Feldhoff, A., Li, G.L., Yang, W.S., Caro, J. Adv. Mater. 2010, 22, 3322.

[53] Kang, C.H., Lin, Y.F., Huang, Y.S., Tung, K.L., Chang, K.S., Chen, J.T., Hung, W.S., Lee, K.R., Lai, J.Y.J. Membrane Sci. 2013, 438, 105.

[54] Bux, H., Chmelik, C., Krishna, R., Caro, J.J. Membrane Sci. 2011, 369, 284.

[55] Pan, Y. Li, T., Lestari, G., Lai, Z. J. Membrane Sci. 2012, 390-391, 93-98.

[56] Hara, N., Yoshimune, M., Negishi, H., Haraya, K., Hara, S., Yamaguchi, T. J. Membrane Sci. 2014, 450, 215.

[57] Yang, T., Chung, T.S.J. Mater. Chem. A 2013, 1, 6081.

[58] Huang, A., Dou, W., Caro, J.J. Am. Chem. Soc. 2010, 132, 15562.

[59] Hu, Y., Dong, X., Nan, J., Jin, W., Ren, X., Xu, N., Lee, Y.M. Chem. Commun. 2011, 47, 737.

[6o] Basu, S., Cano-Odena, A., Vankelecom, I.F.J. Sep. Purif. Technol. 2011, 81, 31.

[61] Guerrero, V.V., Yoo, Y., McCarthy, M.C., Jeong, H.K.J. Mater. Chem. 2010, 20, 3938.

[62] Nan, J., Dong, X., Wang, W., Jin, W., Xu, N. Langmuir 2011, 27, 4309.

[63] Zornoza, B., Martinez-Joaristi, A., Serra-Crespo, P., Tellez, C., Coronas, J., Gascon, J., Kapteijn, F. Chem. Commun. 2011, 47, 9522.

[64] Lin. R., Ge, L., Hou, L., Strounina, E., Rudolph, V., Zhu, Z. ACS Appl. Mater. Interfaces 2014, 6,5609 .

[65] Sato, K., Sugimoto, K., Nakane, T.J. Membrane Sci. 2008, 307, 181.

[66] Wang, Z., Shao, Q.G.J., Yan, Y.J. Am. Chem. Soc. 2009, 131, 6910.

[67] Brown, A.J., Brunelli, N.A., Eum, K., Rashidi, F., Johnson, J.R., Koros, W.J., Jones, C.W., Nair, S. Science 2014, 345, 72 .

[68] Liu, X., Demir, N.K., Wu, Z., Li, K.J. Am. Chem. Soc. 2015, 137, 6999.

[69] Ping, E.W., Zhou, R., Funke, H.H., Falconer, J.L., Nobel, R.D. J. Membrane Sci. 2012, 415, 7705.

[70] Kalipcilar, H., Gade, S.K., Nobel, R.D., Falconer, J.L.J. Membrane Sci. 2002, 210, 113.

[71] Morigami, Y., Kondo, M., Abe, J., Kita, H., Okamoto, K. Sep. Purif. Technol. 2001, 25, 251.

[72] Kondo, M., Komori, M., Kita, H., Okamoto, K. J. Membrane Sci. 1997, 133, 133.

[73] Nishiyama, N., Ueyama, K., Matsukata, M. Microp. Mater. 1996, 7, 299.

[74] Matsufuji, T., Nakagawa, S., Nishiyama, N., Matsukata, M., Ueyama, K. Microp. Mesop. Mater. 2000, 38, 43 .

[75] Kikuchi, E., Yamashita, K., Hiromoto, S., Ueyama, K., Matsukata, M. Microp. Mater. 1997, 11,107 .

[76] Matsufuji, T., Nishiyama, N., Matsukata, M., Ueyama, K.J. Membrane Sci. 2000, 178, 25.

[77] Hara, N., Yoshimune, M., Negishi, H., Haraya, K., Hara, S., Yamaguchi, T. J. Chem. Eng. Jpn. 2014, 47, 770 .

[78] Kwon, H.T., Jeong, H.K.J. Am. Chem. Soc. 2013, 135, 10763.

[79] Li, Y., Wee, L.H., Volodin, A., Martiens, J.A., Vankelecom, I.F.J. Chem. Commun. 2015, 51, 918.

[8o] Kusakabe, K., Kuroda, T., Murata, A., Morooka, S. Ind. Eng. Chem. Res. 1997, 36, 649.

[81] Tao, K., Cao, L., Lin, Y., Kong, C., Chen, L.J. Mater. Chem. A 2013, 1, 13046.

[82] Xiao, W., Chen, Z., Zhou, L., Yang, J., Lu, J., Wang, J. Microp. Mesop. Mater. 2011, 142, 154.

[83] Yang, J., Li, H., Xu, J., Wang, J., Meng, X., Bai, K., Lu, J., Zhang, Y., Yin, D. Microp. Mesop. Mater. 2014, 192, 60 .

[84] Chen, X., Wang, J., Yin, D., Yang, J., Lu, J., Zhang, Y., Chen, Z. AIChEJ. 2013, 59, 936.

[85] Mintova, S., Bein, T. Adv. Mater. 2001, 13, 1880. 
[86] Tosheva, L., Mihailova, B., Wee, L.K., Gasharova, B., Garbev, K., Doyle, A.M. Angew. Chem. Int. Ed. 2008, 47, 8650.

[87] Algieri, C., Bernardo, P., Barbieri, G., Drioli, E. Microp. Mesop. Mater. 2009, 119, 129.

[88] Huang, A., Lin, Y.S., Yang, W.J. Membrane Sci. 2004, 245, 41.

[89] Ji, C., Tian, Y., Li, Y., Lin, Y.S. Microp. Mesop. Mater. 2014, 186, 80.

[9o] Melgar, V.M.A., Kwon, H.T., Kim, J. J. Membrane Sci. 2014, 459, 190.

[91] Seike, T., Matsuda, M., Miyake, M.J. Mater. Chem. 2002, 12, 366.

[92] Liu, Y., Yang, Z., Yu, C., Gu, X., Xu, N. Microp. Mesop. Mater. 2011, 143, 348.

[93] Hedlund, J., Jareman, F., Bons, A.J., Anthonis, M. J. Membrane Sci. 2003, 222, 163.

[94] Pan, M., Lin, Y.S. Microp. Mesop. Mater. 2001, 43, 319.

[95] Zhou, L., Yang, J., Li, G., Wang, J., Zhang, Y., Lu, J., Yin, D. Int. J. Hydrogen Energy 2014, 39, 14949 .

[96] Bradshaw, D., Garai, A., Huo, J. Chem. Soc. Rev. 2012, 41, 2344.

[97] Lai, Z., Bonilla, G., Diaz, I., Nery, J.G., Sujaoti, K., Amat, M.A., Kokkoli, E., Terasaki, O., Thompson, R.W., Tsapatsis, M., Vlachos, D.G. Science 2003, 300, 456.

[98] Boudreau, L.C., Kuck, J.A., Tsapatsis, M.J. Membrane Sci. 1999, 152, 41.

[99] Lai, R., Gavalas, G.R. Ind. Eng. Chem. Res. 1998, 37, 4275.

[100] Li, G., Kikuchi, E., Matsukata, M. Microp. Mesop. Mater. 2003, 62, 211.

[101] Jeong, H.K., Krohn, J., Sujaoti, K., Tsapatsis, M.J. Am. Chem. Soc. 2002, 124, 12966.

[102] Sakai, M., Oshima, Y., Matsukata, M. Proceeding of $18^{\text {th }}$ International Zeolite Conference 2016, 441 .

[103] Membrane Technol. 2016, 10, 3.

[104] Membrane Technol. 2011, 7, 3.

[105] Urtiaga, A., Gorri, E.D., Casado, C., Ortiz, I. Sep. Purif. Technol. 2003, 32, 207.

[106] Hoof. V.V., Dotremont, C., Buekenhoudt, A. Sep. Purif. Technol. 20o6, 48, 304.

[107] http://asia.nikkei.com/magazine/20160512-WEALTHIER-UNHEALTHIER/Tech-Science/ Mitsubishi-Chemical-strains-out-profit-with-zeolites

[108] Kim, S.J., Liu, Y., Moore, J.S., Dixit, R.S., Pendergast Jr., J.G., Sholl, D., Jones, C.W., Nair, S. Chem. Mater. 2016, 28, 4397.

[109] Kondo, M., Yamamura, T., Yukitake, T., Matsuo, Y., Kita, H., Okamoto, K. Sep. Purif. Technol. $2003,32,191$.

[110] Nomura, M., Uchida, M.JP 2017018848, 2017.

[111] Matsukata, M., Seshimo, M., Sakai, M., Kaneko, T., Hamamatsu, T., Kimura, N. JP Patent 2016073956, 2016.

[112] Matsukata, M., Sakai, M., Kaneko, T., Araki, Y., Hamamatsu, T., Kimura, N. WO Patent $2016084651,2016$.

[113] Matsukata, M., Seshimo, M., Sakai, M., Kimura, N., Adachi, M., Waku, T. WO Patent 2015141686, 2015 .

[114] Kita, H., Fuchida, K., Horita, T., Asamura, H., Okamoto, K. Sep. Purif. Technol. 2001, 25, 261.

[115] Schmitt, M., Christmann, M., Ueno, N., Kyotani, T., Kakiuchi, H. BIO web Conferences, $39^{\text {th }}$ World Congress of Vine and Wine, 2016, 7 .

[116] Mirth, G., Cejka, J., Lercher, J.A.J. Catal. 1993, 139, 24.

[117] Hong, M., Li, S., Funke, H.H., Falconer, J.L., Noble, R.D. Microp. Mesop. Mater. 2007, 106, 140.

[118] Sakai, M., Tomaki, K., Matsukata, M. Proceeding of 14th International Conference on Inorganic Membranes 2016, P1.

[119] Matsukata, M., Sasaki, Y., Sakai, M., Tomono, T. Proceeding of International Symposium on Zeolite and Microporous Crystals, 2015, P1.

[120] Sandstorm, L., Lindmark, J., Hedlund, J.J. Membrane Sci. 2010, 36o, 265. 
[121] Karimi, S., Korelskiy, D., Yu, L., Mouzon, J., Khodadadi, A.A., Mortazavi, Y., Esmaeili, M., Hedlund, J.J. Membrane Sci. 2015, 489, 270.

[122] Caro, J., Noack, M., Kolsch, P. Adsorption 2005, 11, 215.

[123] Hedlund, J., Korelskiy, D., Sandstorm, L., Lindmark, J.J. Membrane Sci. 2009, 345, 276.

[124] Tsuru, T., Hino, T., Yoshioka, T., Asaeda, M.J. Membrane Sci. 2001, 186, 257.

[125] Kumakiri, I., Stange, M., Peters, T.A., Klette, H., Kita, H., Bredesen, R. Microp. Mesop. Mater. $2008,115,33$.

[126] Funke, H.H., Tokay, B., Zhou, R., Ping, E.W., Zhang, Y., Falconer, J.L., Noble, R.D.J. Membrane Sci. 2012, 409, 212.

[127] Bonilla, G., Tsapatsis, M., Vlachos, D.G., Xomeritakis, G. J. Membrane Sci. 2001, 182, 103.

[128] Snyder, M.A., Lai, Z., Tsapatsis, M., Vlachos, D.G. Microp. Mesop. Mater. 2004, 76, 29.

[129] van den Bergh, J., Gucuyener, C., Gascon, J., Kapteijn, F. Chem. Eng. J. 2011, 166, 368.

[130] Illgen, D., Schafer, R., Noack M., Kolsch, P., Kuhnle, A., Caro, J. Catal. Commun. 2001, 2, 11.

[131] Ciavarella, P., Casanave, D., Moueddeb, H., Miachon, S., Fiaty, K., Dalmon, J.A. Catal. Today 2001, 67, 177 .

[132] Jafar, J.J., Budd, P.M., Hughes, R.J. Membrane Sci. 2002, 199, 117.

[133] Peng, X., Lei, Q., Lv, G., Zhang, X. Sep. Purif. Technol. 2012, 89, 84.

[134] Li, W., Liu, W., Xing, W., Xu, N. Ind. Eng. Chem. Res. 2013, 52, 6336.

[135] Han, Y., Lv. E., Ma, L., Lu, J., Chen, K., Ding, J. Energy Convers. Manag. 2015, 106, 1379.

[136] de la Iglesia, O., Mallada, R., Menendez, M., Coronas, J. Chem. Eng.J. 2007, 131, 35.

[137] Tanaka, K., Yoshikawa, R., Ying, C., Kita, H., Okamoto, K. Chem. Eng. Sci. 2002, 57, 1577.

[138] Zhang, W., Na, S., Li, W., Xing, W. Ind. Eng. Chem. Res. 2015, 54, 4940.

[139] Hasegawa, Y., Abe, C., Mizukami, F., Kowata, Y., Hanaoka, T.J. Membrane Sci. 2012, 415, 368.

[140] van Dyk, L., Lorenzen, L., Miachon, S., Dalmon, J.A. Catal. Today 2005, 104, 274.

[141] Zhang, C., Hong, Z., Gu, X., Zhong, Z., Jin, W., Xu, N. Ind. Eng. Chem. Res. 2009, 48, 4293.

[142] Daramola, M.O., Burger, A.J., Giroir-Fendler, A., Miachon, S., Lorenzen, L. Appl. Catal., A 2010, 386, 109 . 



\title{
9. Computational Chemistry Experiment Possibilities
}

\author{
Barttomiej M. Szyja ${ }^{1}$ and Danny Vanpoucke $e^{2,3}$ \\ ${ }^{1}$ Wroclaw University of Science and Technology, Faculty of \\ Chemistry, Division of Fuels Chemistry and Technology, \\ ul. Gdańska 7/9, 50-344 Wrocław, Poland. \\ ${ }^{2}$ UHasselt - Hasselt University, Institute for Materials Research \\ (IMO), 3590 Diepenbeek, Belgium. \\ ${ }^{3}$ IMOMEC, IMEC vzw, 3590 Diepenbeek, Belgium.
}

Keywords: Force fields, density functional theory, potential energy surface, molecular dynamics

\section{Introduction}

Thanks to a rapid increase in the computational power of modern CPUs, computational methods have become a standard tool for the investigation of physico-chemical phenomena in many areas of chemistry and technology. The area of porous frameworks, such as zeolites, metalorganic frameworks (MOFs) and covalent-organic frameworks (COFs), is not different. Computer simulations make it possible, not only to verify the results of the experiments, but even to predict previously inexistent materials that will present the desired experimental properties. Furthermore, computational research of materials provides the tools necessary to obtain fundamental insight into details that are often not accessible to physical experiments.

The methodology used in these simulations is quite specific because of the special character of the materials themselves. However, within the field of porous frameworks, density functional theory (DFT) and force fields (FF) are the main actors. These methods form the basis of most computational studies, since they allow the evaluation of the potential energy surface (PES) of the system. 


\section{Energy calculations}

\section{Force fields}

The simplest, and consequently least computationally demanding, way of calculating the energy of a system is through the force field method, sometimes referred to as Molecular Mechanics (MM). This method evaluates the energy of the system by making use of classical mechanics concepts and formulations. The specific energy expression is given by a sum of energy terms. These can be single particle terms (the kinetic energy terms) or multi-particle terms (the potential energy terms), the latter are often parameterized. For example, bond vibrations can be described using the harmonic oscillator. In this case, the energy expression would take the form:

$$
\mathrm{E}={ }_{1 / 2} \mathrm{kx}^{2}
$$

where $\mathrm{E}$ is the potential energy of the system, $\mathrm{k}$ is the force constant and $\mathrm{x}$ is the displacement from the optimal bond length. This approach uses only one parameter: $\mathrm{k}$. The harmonic oscillator approximates the energy of the system reasonably well for displacements close to the equilibrium position; however, far from the equilibrium deviations become large. As a result, it fails to describe the dissociation of a bond, which is not captured at all by the parabolic curve of the harmonic oscillator.

In contrast, the Morse potential is a much better approximation of the energy profile of a bond (Figure 1), but it has a slightly more complex energy expression with three parameters. The Morse potential has the following form:

$$
\mathrm{E}=\mathrm{D}\left(1-\mathrm{e}^{-\mathrm{a}(\mathrm{ro}-\mathrm{r})}\right)
$$

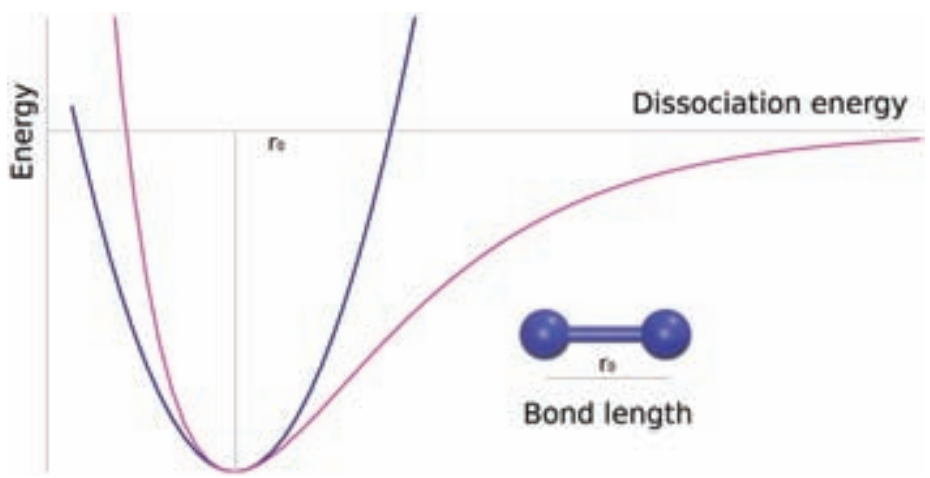

Figure 1. Harmonic oscillator (blue) and Morse (pink) potential energy curves. $r_{0}$ represents the optimal bond length of a diatomic molecule, the minimum of the potential energy. 
where $\mathrm{D}$ is the dissociation energy, $\mathrm{r}_{\mathrm{o}}$ gives the equilibrium position, and a controls the width of the potential well.

Although the Morse potential only describes diatomic molecules, it has the right shape to describe any bond between two atoms. As such, similar terms can be added to describe bonds in case of more complex geometries. Because any given atom of a system undergoes several interactions at the same time, a typical force field has an energy expression consisting of several terms representing the different types of interactions:

$$
\mathrm{E}=\mathrm{E}_{\text {bond }}+\mathrm{E}_{\text {angle }}+\mathrm{E}_{\text {dihedral }}+\mathrm{E}_{\mathrm{vdW}}+\mathrm{E}_{\text {coulomb }}
$$

The first three terms describe the bonding or intramolecular interactions, namely the energies related to the stretching of the covalent bonds, to the bending of the angles formed by three atoms, and to the twisting of the dihedral angles along the bonds. These interactions are usually described with the energy expression of the harmonic oscillator (equation 1). The last two terms describe the non-bonding or intermolecular interactions. The interactions of the van der Waals type are usually described by the Lennard-Jones potential:

$$
\mathrm{E}_{\mathrm{vdW}}=\mathrm{A} / \mathrm{r}^{12}-\mathrm{B} / \mathrm{r}^{6}
$$

where A and B are the specific parameters for the interaction.

The Coulomb -or electrostatic- interactions depend on the charges $q_{1}$ and $\mathrm{q}_{2}$ in the system:

$$
\mathrm{E}_{\text {Coulomb }}=\left(\mathrm{q}_{1} \cdot \mathrm{q}_{2}\right) /(\varepsilon \cdot \mathrm{r})
$$

The parametrization of a force field is a very complex and difficult procedure. In almost all cases, the parameters need to match one another, and even changing the atomic charge calculation procedure is not something that should be taken lightly. Instead, one usually wants to use the force field assigned charges.

Several force fields have been developed for the study of porous frameworks. One example of a zeolite force field is proposed by van Beest, Kramer and van Santen (BFS force field) [1]. It is used to model the properties of aluminosilicates, such as pore sizes [2]. While this force field can only be applied to model the intrinsic properties of the solid, other force fields are specialized in the host-guest interactions, such as the YAFF force field used to study the interaction between $\mathrm{CO}_{2}$ molecules and differently functionalized MIL-47(V) MOFs [3]. 
Besides the obvious advantage of simplicity, force field methods have important limitations as well. These result from neglecting any type of electronic interactions. For example, this method cannot be used to investigate optical effects. More importantly, bond formation and cleavage, which are the result of electronic interactions, render force fields useless for the investigation of catalytic phenomena or chemisorption.

\section{Density Functional Theory}

Electronic phenomena can, however, be investigated using Quantum Mechanical methods. These methods are often referred to as ab-initio (meaning 'from the beginning' or 'first principles') ${ }^{1}$ because, unlike force fields, they only require physical constants and involve no fitting to other data. In the case of porous periodic frameworks, the vast majority of quantum mechanical calculations are performed using Density Functional Theory (DFT). Such calculations are far more complex, time- and resourcesdemanding than force field calculations. This puts a limit on the available size of the computational model, and leads to so-called "model" vs. "method" accuracy, which will be discussed later on.

DFT is the current gold standard in the description of chemical reactivity and, along with ab-initio methods such as Hartree-Fock or post-HartreeFock, it is capable of describing the electronic state of the system. Contrary to ab-initio methods, DFT does not involve solving the Schrödinger equation to find the many-electron wave function of the system, but it solves instead a different set of equations (the Hohenberg-Kohn-Sham equations) which relate the total energy of the system with the electron density.

One of the attractive features of the DFT method is the fact that the electron density can be directly and experimentally measured (e.g. through $\mathrm{X}$-ray diffraction) and is therefore much easier to understand than the concept of wave function, which is not a quantum mechanical observable. Moreover, the electron density is only a function of position, thus it only depends on three parameters: the spatial coordinates. This makes it much easier to treat mathematically.

The foundations of the DFT method were provided by the work of Hohenberg, Kohn and Sham [4,5], who demonstrated that the properties of the system are a functional of the electron density. Furthermore, the total energy can be decomposed into the kinetic energy of the electrons and the potential energy of nuclear-electron and electron-electron interactions. The motion of the nuclei can be neglected - this is called 
the Born-Oppenheimer approximation and is based on the fact that nuclei are much heavier than electrons. As a result, the nuclear motion is orders of magnitude slower than the electronic motion, such that the former can be considered static on the timescales relevant for electron interactions.

As a result, the expression for the total energy of the system can be written as a sum of four terms:

$$
E=T_{\circ}+\int v(r) \rho(r) d r+J[\rho]+E_{x c}[\rho]
$$

where $T_{o}$ is the kinetic energy of the electrons, the second term describes the interactions between the nuclei and the electrons, and $\mathrm{J}[\mathrm{q}]$ is the term for the static Coulombic self-interaction of the electron cloud. These terms can be calculated directly from fundamental physical expressions. This is not the case for the exchange-correlation term, $\mathrm{E}_{\mathrm{xc}}$. This term is needed because the $\mathrm{J}$ term does not take into account the repulsion of the electrons caused by the correlation of their motion or the repulsion of the electrons with the same spin due to the Pauli exclusion principle. The XC subscript in this term represents these two factors: eXchange and Correlation. Although DFT is in theory an exact method, its practical implementation is approximate because no general analytic formulation of the $\mathrm{E}_{\mathrm{xc}}$ functional is known. As a result of the never ending quest for this functional, many differently defined exchange-correlation functionals have seen the light of day.

The most simple and least accurate approaches rely only on the value of the electron density in the given spatial position, and are known as LDA (Local Density Approximation). A better solution is the GGA (Generalized Gradient Approximation), which, in addition to the electron density at the given point, also takes into account its gradient (the first derivative of the density). This results in an improvement of the accuracy of the method but, obviously, at the expense of a (slight) increase in the computational effort. With this in mind, it was proposed that by adding another term, the second derivative of the density, accuracy could be improved even further. This led to so called meta-GGA functionals, which often offered an improvement although more limited than that of GGA functionals over LDA ones.

Another approach to improve the $\mathrm{E}_{\mathrm{xc}}$ functional is through the inclusion of the exact Hartree-Fock exchange energy in the $\mathrm{E}_{\mathrm{xc}}$ term in addition to the approximated DFT exchange-correlation energy. This gives a hybrid functional, in which the amount of the HF exchange is defined 
by a mixing coefficient, which is fitted to a set of test systems. An important difference between LDA and GGA, on the one hand, and hybrid functionals, on the other, is that the former are local functionals only requiring information on the electron density at the position where the functional is calculated, while the latter are non-local functionals, where information on the electron density in the entire system is needed. This increases the computational cost of hybrid functionals by several orders of magnitude.

Despite the fact that high level or hybrid functionals often predict electronic properties that are in very good agreement with the experiment, the DFT method still suffers from two important shortcomings. One is the poor description of dispersion interactions. As such, when van der Waals interactions are of interest, one needs to extend DFT to account for the dispersion interactions. A simple and computationally inexpensive extension dubbed DFT-D, was devised by Grimme and coworkers, with currently two parametrization sets available in many DFT codes: $\mathrm{D}_{2}$ and $\mathrm{D}_{3}[6,7]$. The second issue is more fundamental: the electron density is a ground state property and the excited states are usually beyond a reasonable description. This issue can be addressed by so called time-dependent DFT but even then the accuracy is not perfect.

\section{'Partial' solutions}

There are two approaches that attempt to combine the advantages of force fields and first principles methods in a single solution. The first one is QM/ MM (Quantum Mechanics/Molecular Mechanics) [8]. This method allows a separatation of the computational model into two (or more) zones one zone is described by the force field (MM) and the other by quantummechanics $(\mathrm{QM})$. The advantage of this method is that the direct vicinity of the reactive site is described by the method that is fully aware of the electronic effects, and as such allows for investigations of chemical reactions. The rest of the model is described by the force field, and provides a simplified description of the framework (Figure 2).

Although QM/MM methods are generally very efficient compared to purely QM described systems, they are still rather tricky to handle. The regions simulated by the QM need to be carefully selected, because moving the region's boundaries can influence the results as well as the computing time. The choice of the size of the QM region also depends strongly on the range of interactions present and can require sizeable QM regions to get atomic properties converged in the presence of active sites [9]. 


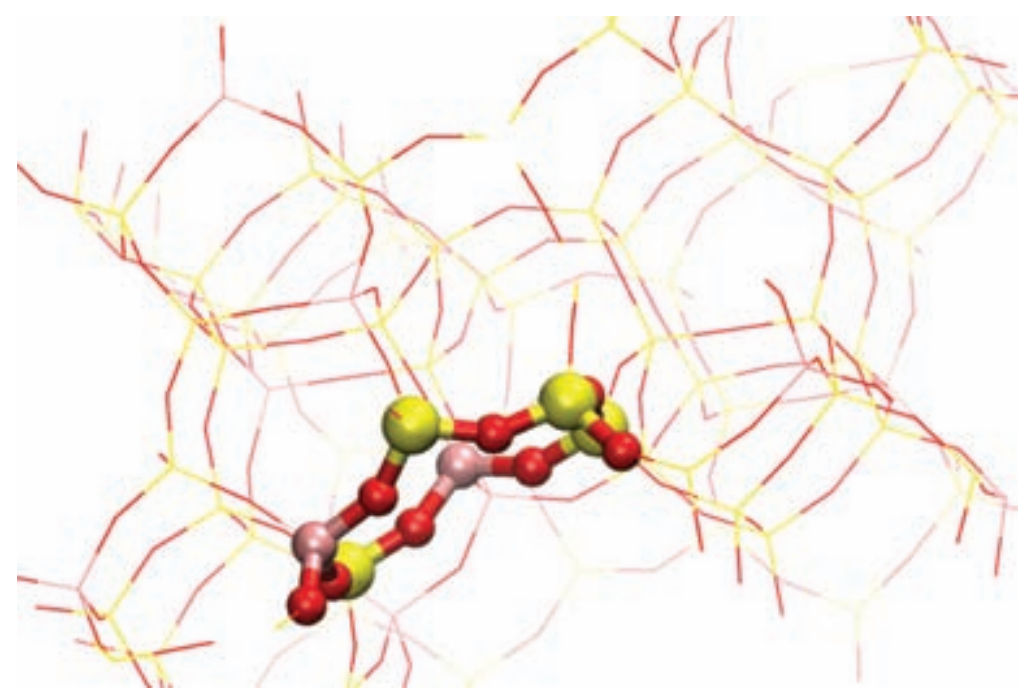

Figure 2. Example of QM/MM regions in TS-1 system, which is isostructural with the MFI framework. The titanium, silicon and oxygen atoms are shown in pink, yellow and red, respectively. The energy of the framework is described by MM, and represented by thin lines, the QM region (the 6-membered ring) is shown as balls and sticks.

Another approach is the reactive force field, ReaxFF, developed by Adri van Duin [10]. This method does not split the system into parts, but treats all the atoms on the same level of theory. The calculations are less resource-intensive, because they do not rely on a calculation of the electronic state of the system. Instead, the extensive set of parameters combined with a quite complex energy expression makes it possible to describe different kinds of interactions between the atoms. The most important feature is obviously the formation and cleavage of the chemical bonds. With a proper set of parameters, the reactivity of the system can be investigated with good accuracy. In addition, depending on the direct vicinity of the particular atom, the bonding between it and its neighbour might change - as in singly, doubly or triply bonded $C$ atoms with different number of hydrogens bound to them. The description of the energy of a system using the reactive force field is much more complex than that of classical force fields, although these simulations are still much more efficient that any QM method, which allows investigation of much larger systems.

\section{The Potential Energy Surface (PES)}

Having the model constructed and the method selected, one can proceed to investigate the reactivity of the system. The reaction pathways run via specific points on the potential energy surface (PES), called stationary 
points. These points in the potential energy surface are localminima (all the gradients are zero) and they reflect stable geometries of the system. Small displacement of the atoms in any direction from the optimal positions increases the potential energy of the system or, in other words, the gradients next to the minimum become positive, which leads to a force pulling them back to their optimal position. Finding the geometries of the systems in those specific points is called geometry optimization or energy minimization. This is the most basic technique in most areas of computational chemistry and solid-state physics.

Finding a minimum of a function of many variables (coordinates of the atoms) is a non-trivial task and for problems related to real-life materials it cannot be done analytically. Instead, numerical methods are used. The optimization starts with an evaluation of the initial energy of the system and gradients with respect to the position of the atoms. Next, the atoms are displaced according to the gradients, which allow a decrease in the potential energy of the system. This is followed by the evaluation of the potential energy and a calculation of the gradients in the new coordinates. The whole procedure is repeated until the convergence is achieved.

There are different methods for finding the minimum and they generally differ in the way they move towards the minimum (Figure 3). The steepest descent method finds the largest negative gradient in each iteration and follows that direction. This usually leads to oscillations in the followed direction, resulting in more steps being needed. The conjugate gradients (CG) method and the methods derived thereof make a correction in the current gradient based on the previous step(s). This usually allows a reduction of the total number of steps taken. The Newton-Raphson method finds the direction to the minimum based on the calculated curvature of the PES close to the initial point.

As it is the case in almost all areas of computational research, a compromise is often necessary. The CG and Broyden-Fletcher-GoldfarbShanno (BFGS) methods have become the current gold standards. Both of them keep track of the previously taken steps and adaptively change the direction. At the same time they keep the computational demand for each iteration relatively small, especially in the limited-memory version of BFGS.

Finding the local minima corresponding to the desired geometries allows one to estimate the thermodynamics of the process. Transition from one minimum to another on the PES corresponds to the change in geometry of the system, where some bonds might be broken or formed, i.e. the 

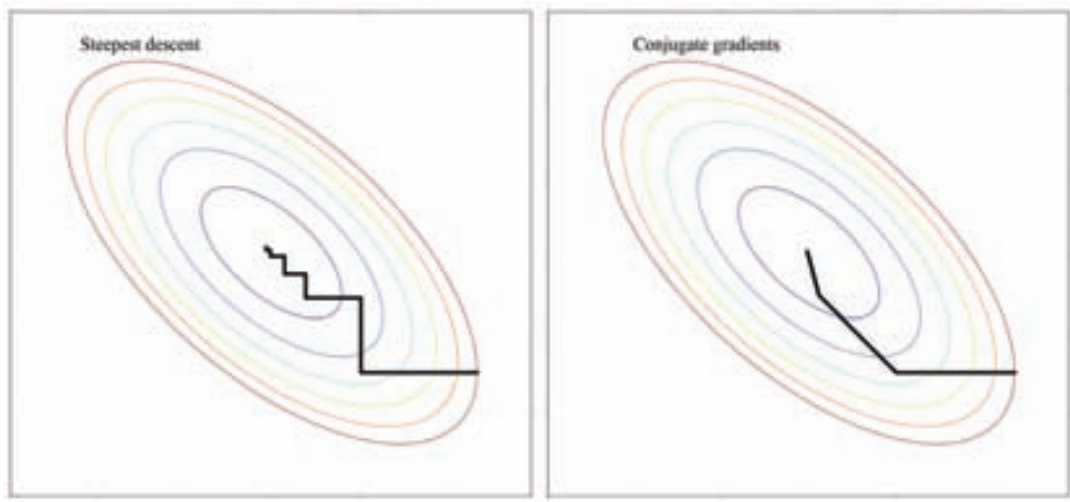

Figure 3. Example of Steepest Descent (left) and Conjugate Gradient (right) method of finding the minimum of a second order polynomial.

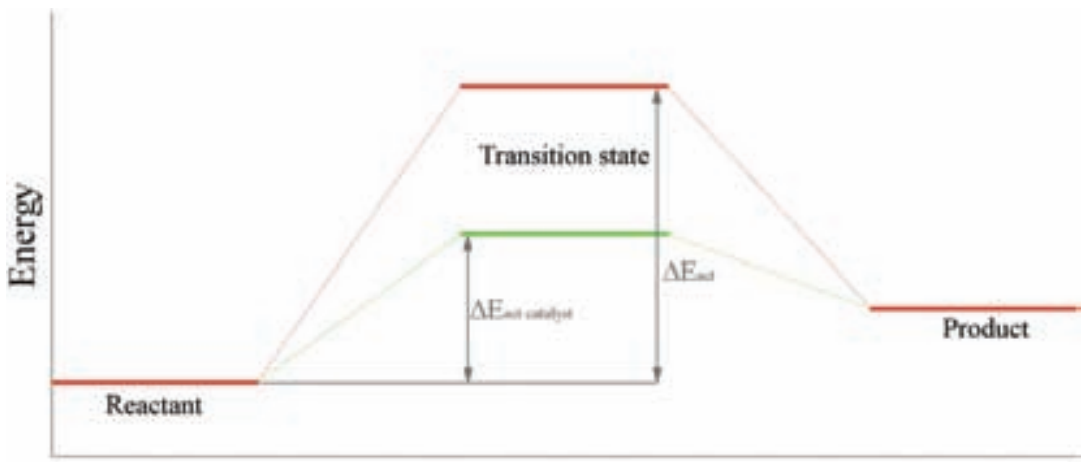

\section{Reaction coordinate}

Figure 4. Schematic representation of the transition state stabilization. The non-catalysed path is shown in red, while the catalysed path is shown in green. The presence of the catalyst does not influence the reactant or product energy but it lowers the activation energy.

chemical reaction has occurred. The change in the energy of the system determines if the reaction is exo- or endothermic.

This, however, is not a full or sufficient description of the reactivity, because it is not only thermodynamics that determines the possible reaction pathways. Kinetics is represented on the PES as the height of the barrier in the transition from one minimum to another. These points are extremely important in the studies of catalytic effects. A catalyst increases the rate of the reaction without influencing its thermodynamics because it lowers the activation energy (i.e. the barrier height). This effect is based on the formation of an active complex of the reactant and the catalyst, which is more stable than the transition state in the absence of a catalyst (Figure 4). 
The activation barriers correspond to other types of points on the PES, often referred to as saddle points or transition states (TS). Simple geometry optimizations are insufficient to find these points, and other algorithms are needed. Depending on the model used, different approaches are available.

For cluster models (section 6) the Berny algorithm of 'eigenvector following' is usually the method of choice [11]. It is based on the optimization of a candidate for the TS, which might be either guessed at or found from a series of constrained optimizations (e.g. a particular structural parameter related to the reaction coordinate is varied to locate the structure of maximum energy within this trajectory). In the Berny algorithm, the calculation starts with the evaluation of the Hessian matrix (the matrix of second derivatives of the energy, which describes the local curvature of the PES). Then, a structure optimization is carried out in which the algorithm follows the eigenvector corresponding to most negative eigenvalue of the hessian matrix. As a result, the structure will evolve on a path on the PES, which goes through the TS.

Because the calculation of the Hessian in a periodic model is highly time-consuming, another approach is needed. A method used frequently is called the Nudged Elastic Band (NEB) method [12]. This method does not rely on the Hessian matrix. Instead, the pathway leading from the reactant to the product is divided into several steps. The geometries at these steps, called images, are generated by means of interpolation of the atomic positions. The energy of each image is calculated, which results in an energy profile along the path. Subsequently the structure of the images is optimized in parallel. An artificial spring force between the images, similar to springs between beads, is used to keep the images equally spaced. This makes the NEB method especially well-suited for tracking the transition states along the reaction pathway [13]. Another advantage besides avoiding the calculation of the Hessian is that the NEB method does not need a good initial guess. Unfortunately, there are also two main disadvantages: the first is that most of the computation time is spent on calculations of energies and gradients of images other than the TS candidate. The second is that the exact saddle point might be missed, because the energy of the images is minimized and images tend to be fit along the pathway initially interpolated.

An improvement on the NEB method is the Climbing Image variant. This method neglects the forces from the virtual springs on the image with the highest energy. The image is then optimized to maximize its energy along the path defined by the other images (i.e. the reaction coordinate) and to minimize its energy in the other directions. The principle of the NEB method is shown in Figure 5 . 

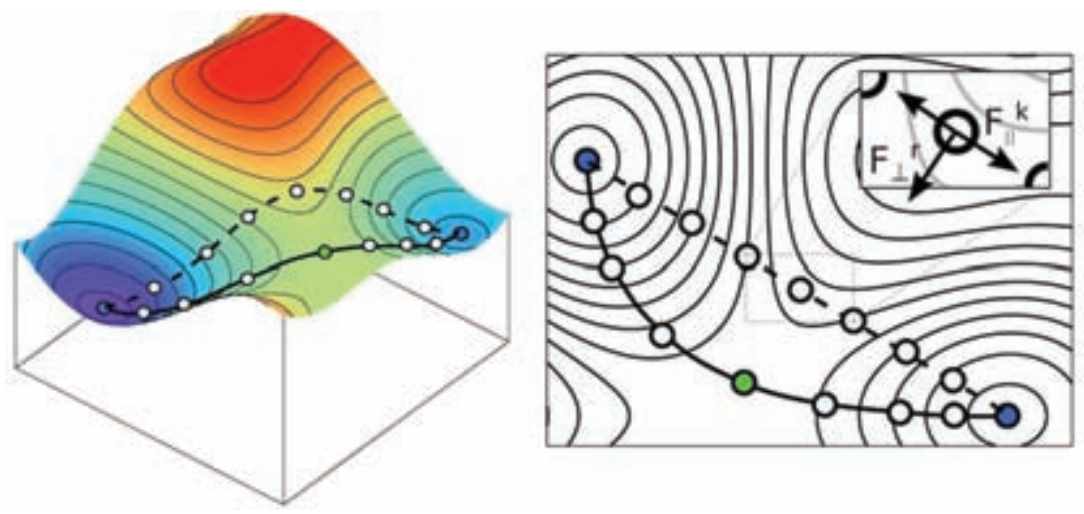

Figure 5. The principle of the NEB method of finding the saddle point between two minima. The climbing image is shown as a green point. Figure by Antoine Kraych. Reprinted with permission from [14].

Regardless of the objective, the optimization procedure is iterative, and as such it never ends exactly on a stationary point but rather approaches it. Hence, a condition is needed upon which the procedure will be terminated. Generally, one needs to calculate the energy of the system and in this case the procedure should be terminated when the energy changes are below a certain threshold. The accuracy of the DFT method is in the order of magnitude of $10 \mathrm{~kJ} \mathrm{~mol}^{-1}$, thus an energy difference of $1 \mathrm{~kJ} \mathrm{~mol}^{-1}$ is more than sufficient. However, when vibrational frequencies are desired, this criterion might not be sufficient. In this case, it is useful to use additional criteria, such as the maximum force acting on the atom not exceeding $0.01 \mathrm{eV} / \AA$.

\section{Molecular Dynamics}

In contrast to the static calculations of the stationary points on the PES, molecular dynamics (MD) allows a study of the motion of particles.

\section{Diffusion of guest molecules}

In the case of favourable reaction kinetics, diffusion limitations might play a role in the efficiency of the overall process. This is especially so in small pore size zeolites, in which much effort is devoted to the synthesis of mesoporous or hierarchical materials to alleviate this problem, as discussed in previous chapters of this book. Diffusion of guest molecules is 
also important when porous membranes are used, for instance, in the separation of gas mixtures.

Diffusion is a mass transport process and it cannot be investigated by static calculations as is possible for chemical reactions. The motion of the atoms and molecules needs to be considered explicitly. This can be accomplished by a method called Molecular Dynamics. This method calculates the evolution of the system over time by solving Newton's equation of motion:

$$
\mathrm{F}=\mathrm{ma}
$$

where $\mathrm{F}$ is the force acting on a particular atom, $\mathrm{m}$ represents its mass, and a its acceleration. There are many different methods to integrate Newton's equation of motion over time in molecular dynamics and all of them rely on the iterative calculation of the properties of the system in consecutive time steps. Assuming that the time interval between the steps is short, the acceleration can be safely assumed to be constant in this interval, and the equation of motion can be expressed as a Taylor's expansion:

$$
\begin{gathered}
\mathrm{r}(\mathrm{t}+\Delta \mathrm{t})=\mathrm{r}(\mathrm{t})+v(\mathrm{t}) \Delta \mathrm{t}+{ }_{1 / 2} \mathrm{a}(\mathrm{t}) \Delta \mathrm{t}^{2} \\
v(\mathrm{t}+\Delta \mathrm{t})=v(\mathrm{t})+{ }_{1 / 2}[\mathrm{a}(\mathrm{t})+\mathrm{a}(\mathrm{t}+\Delta \mathrm{t})] \Delta \mathrm{t}
\end{gathered}
$$

where $r(t)$ and $r(t+\Delta t)$ represent the spatial coordinates of the given atom at a given time $(\mathrm{t})$ and after a timestep $(\mathrm{t}+\Delta \mathrm{t})$, respectively. Analogously $\mathrm{v}(\mathrm{t})$ and $\mathrm{v}(\mathrm{t}+\Delta \mathrm{t})$, and $\mathrm{a}(\mathrm{t})$ and $\mathrm{a}(\mathrm{t}+\Delta \mathrm{t})$ stand for the velocity and the acceleration at these times. These equations are known as the Velocity Verlet algorithm [15].

Repeating these steps iteratively to cover the desired timespan generates a sequence of geometries vs. time, called a trajectory. These points represent different conformations of the same system but they all belong to the same ensemble. ${ }^{2}$ The course of the MD simulation is schemed in Figure 6.

Two ensembles are mostly used for simulations in solid frameworks: NVE and NVT. The former represents a micro-canonical ensemble, corresponding to an isolated system with a constant number of particles $(\mathrm{N})$, volume (V), and total energy (E). In this ensemble, neither mass nor energy can be exchanged with the environment. The latter is the canonical ensemble, with temperature (T) held constant by means of a thermostat. This ensemble is better suited, for example, to simulations where adsorption takes place or energy change is expected for another reason. Upon adsorption, energy related to the interactions between the components of the system is released and can be dissipated by the external thermostat. 
Simplified schematic of the molecular dynamics algorithm

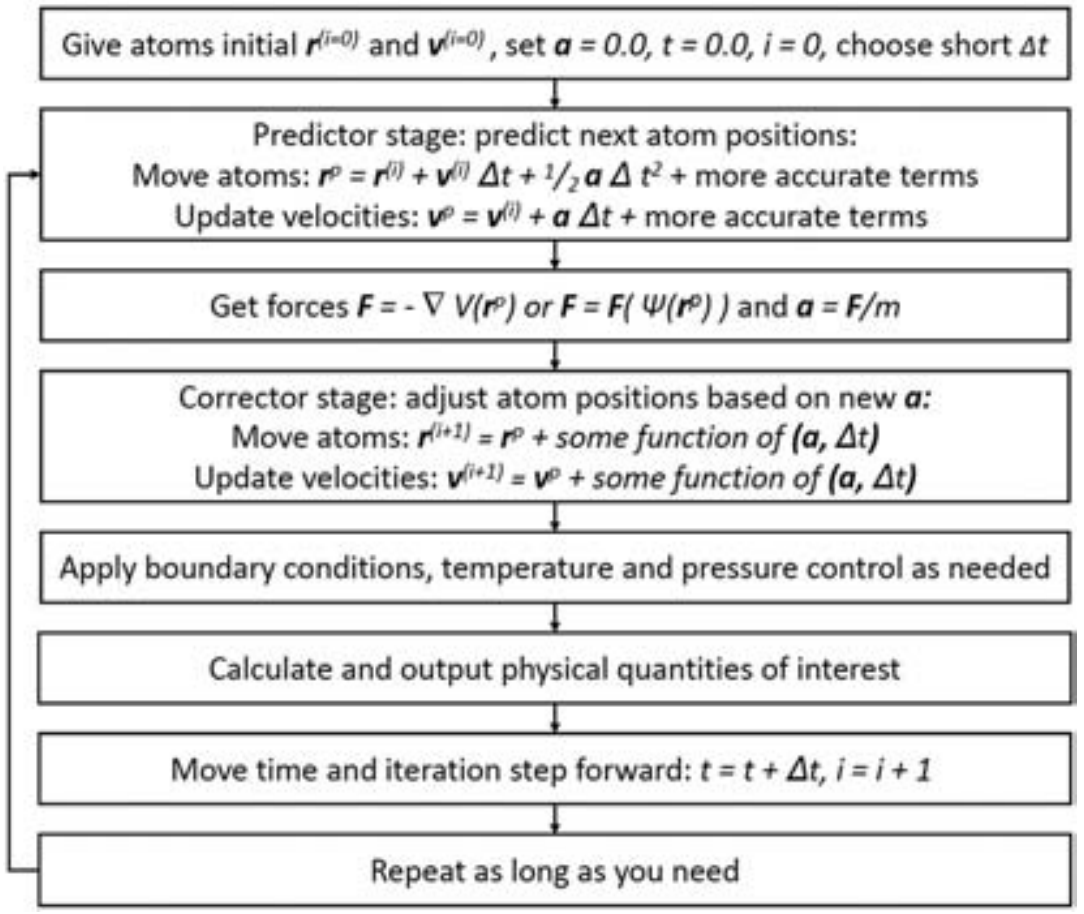

Figure 6. The algorithm for Molecular Dynamics simulation. By Knordlun, CC BY-SA 3.0, https://commons.wikimedia.org/w/index.php?curid=41419953.

The NPH and NPT ensembles with constant number of particles (N), pressure $(\mathrm{P})$, enthalpy $(\mathrm{H})$ and temperature $(\mathrm{T})$ represent systems that are in contact with a barostat. These are rarely used in simulation of zeolites because the crystalline structure of these materials is generally rigid.

The proper initiation of an MD simulation is important, because at the beginning the velocities of the atoms are unknown. Typically, initial velocities are assigned randomly but special conditions have to be met. Firstly, the magnitude of the velocity vectors needs to match the desired temperature, especially when the NVE ensemble is used. Secondly, the directions of the velocity vectors must not be aligned otherwise, the centre of mass of the system would translate.

The random numbers used in the initialization of each MD run, obviously, do not depend on physical interactions between the atoms. However, the further the simulation proceeds, the less important they are, because each consecutive iteration of the MD run relies directly on the previous one, and new positions and velocities are obtained by integration of 
Newton's equation of motion. Thus, the first part of the trajectory is only used to equilibrate the system in the desired state, and is usually characterized by large fluctuations of temperature or energy. The equilibration run is followed by the data collection (or production) run, for which calculated physical quantities can be trusted.

The length of the run should be sufficiently long to be able to observe the phenomenon of interest. In the case of calculating diffusion coefficients, the length of the simulations should be in the order of nanoseconds. On the other hand, the timestep taken in the run should be small enough to properly sample the vibrations of the atoms. That means that if a timestep is $0.5 \mathrm{fs}, 2$ million iterations are needed to observe the evolution of the system in 1 ns. Depending on the system size and computational power available, a compromise often has to be made.

Assuming that the MD run was sufficiently long, the diffusion coefficient can be calculated from the mean square displacement (MSD) of the atoms by using Einstein's formula. MSD is defined as follows:

$$
M S D=\frac{1}{N} \sum_{n=1}^{N}\left(x_{n}(t)-x_{n}(\mathrm{o})\right)^{2}
$$

where $\mathrm{N}$ is the total number of particles, $x_{n}(t)$ and $x_{n}(o)$ are the positions of the $n$-th particle at time $t$ and o, respectively. The plot of the MSD as a function of time should be a straight line, the slope being proportional to the diffusion coefficient. If the MSD has a concave curvature, then the motion of the particles is impeded: they cannot diffuse away from its starting point. It has to be stressed that in micro- or mesoporous materials the diffusion of guest molecules is always impeded due to more (zeolites) or less (MOFs) rigid surfaces of the pores. Zeolites of the MOR topology have a large, 12-membered ring pore in z-direction, which allows for relatively easy diffusion. These channels intersect with smaller channels, formed by 8-membered rings, where the diffusion is limited, especially for bulkier molecules. This would be visible as the lines of different slopes when MSD is calculated only along the particular direction.

On the contrary, if the MSD increases faster than at linear rate, then another mechanism of transport takes place. This effect is called superdiffusion, and is usually a result of specific interactions of the guest molecules with the environment, being either the pore walls or other guest molecules. Superdiffusion in zeolites and MOFs is frequently associated with the confined space effects, where the geometry (i.e. size and shape) of the guest molecule closely matches the geometry of the channel. 


\section{IR spectra}

Another useful application of the MD is predicting infrared spectra for a given model. In the most basic approach, the calculation of the IR spectra can be performed for a harmonic oscillator model with the assumption that the PES behaves like the harmonic potential in the direct neighbourhood of the minimum. This involves the calculation of vibrational frequencies directly from the derivatives of the potential energy. This method, however, neglects anharmonicity and, in order to improve the results, empirical scaling factors need to be used. In addition, the presence of the solvent can also greatly influence the spectra, especially when hydrogen bonds between the solvent and the analysed species are formed [16].

The MD allows for the proper simulation of the bulk phase together with the solvent, where different configurations, such as the arrangement of the hydrogen bonds and the orientation of the solvent molecules, can easily be sampled. Carrying out the MD simulation on the DFT level ensures the proper electronic structure to be maintained during the run.

The procedure is quite straightforward: to calculate the IR spectra, it is necessary to obtain the dipole information for the investigated system and to localize the molecular orbitals to obtain the Wannier centres. The length of the simulation needed to obtain reasonable results varies with the system type. A system with explicit solvent molecules generally needs more time to sample the phase space, while a solid system can be studied in a much shorter time. If the Wannier centres are written to the trajectory every $5^{\text {th }}$ step, approximately 50000 trajectory steps should be sufficient.

The IR spectra can then be obtained from the Fourier transformation of the dipole moment autocorrelation function:

$$
I(\omega)=\int\langle M(t) \cdot M(\mathrm{o})\rangle \cos (\omega t) d t
$$

where $\mathrm{I}$ is the intensity $\mathrm{M}(\mathrm{t})$ is the total dipole moment in time $\mathrm{t}$ and $\omega$ is the vibrational frequency.

\section{Monte Carlo methods}

While MD simulations are of deterministic nature (i.e. given exactly the same initial positions and velocities of the atoms, identical results are obtained), ${ }^{3}$ Monte Carlo (MC) methods represent a stochastic approach. 
These methods rely on a random number generator to evaluate the probability of a particular state of the system.

Perhaps the most important variant of the MC method is the Grand Canonical Monte Carlo (GCMC). The simulations are carried out in the $\mu \mathrm{VT}$ ensemble, in which, in addition to volume $(\mathrm{V})$ and temperature $(\mathrm{T})$, the chemical potential $(\mu)$ is also kept constant. This represents the system coupled to an infinite reservoir with which it can exchange particles. It is a system where not only heat but also mass is exchanged.

Having an initial state of the system, with atoms in the desired positions, a new state of the system is then generated in one of the following ways: (1) a particle is added to a randomly generated location, (2) an existing particle is removed, (3) an existing particle is translated or rotated. Such a newly generated state is either accepted as one of the probable states in the phase space, or rejected as an unlikely one. The probability of this acceptance is based on the change of the total energy of the system.

This procedure needs to be repeated a statistically meaningful number of times after which the system can be considered in thermodynamic equilibrium. This can be exploited to obtain, for example, the adsorption isotherms if the simulations are carried out at varying pressures but at the same temperature, see section 10.7.

\section{Models}

Zeolites and MOFs are crystalline solids with a well-defined porosity, which is the result of the interconnection of distinct building units (Figure 7). In the case of zeolites, a single class of building units, generally silica or alumina tetrahedra, referred to as T-sites, is used. MOFs, on the other hand, consist of two classes of building units. In this case the framework is formed by metal(-oxide) nodes which are connected through organic linkers (in the case of COFs the metal(-oxide) nodes are replaced by organic nodes). The periodic crystalline nature of these porous frameworks has implications in virtually all aspects of modelling: from the diffusion of small molecules through the boundaries of the periodic cell, to the Ewald method for charge summation or the Plane-Wave basis set in the description of molecular orbitals.

For both zeolites and MOFs two types of models are generally available: cluster models and periodic models, each with its own strengths and limitations. As a result, one should choose the type of model depending on the particular phenomenon to be investigated. 

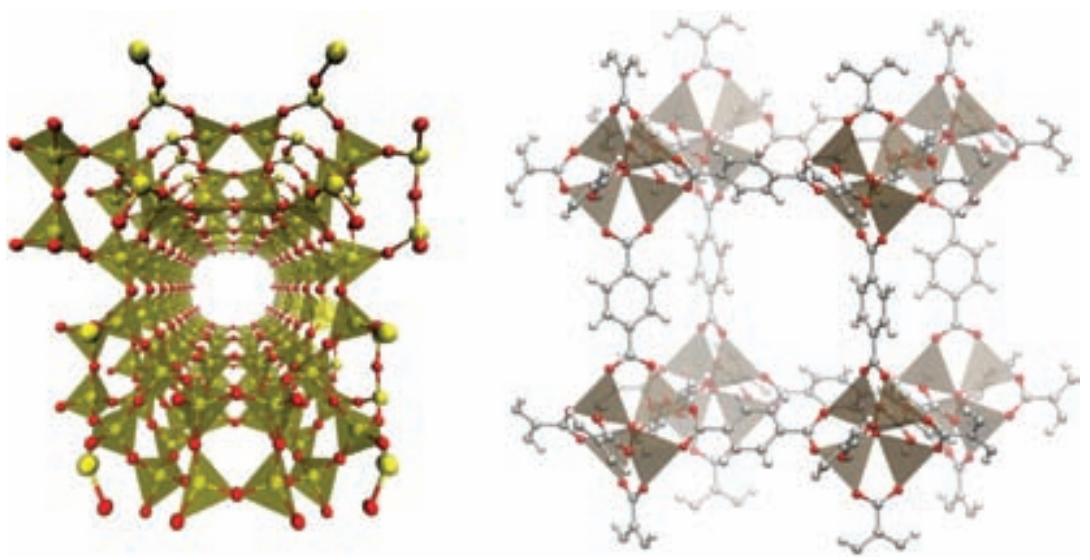

Figure 7. Left: structure of MOR framework. Silicon atoms (shown as yellow spheres) are located in the centres of the tetrahedra (T-sites). Oxygen atoms are shown as red spheres. Right: structure of MOF-5. ZnO nodes represented as thetrahedra, which are interconnected by the terephthalate linkers.

\section{Cluster models}

A cluster model is obtained by cutting a finite piece out of the crystalline periodic structure. This piece is centred on the specific atoms or sites of interest. Their finite nature makes cluster models simpler and easier to handle within a computational setup. However, they do not represent the full crystalline structure and may suffer from finite size and boundary effects. In order to deal with the cleaved bonds at the edges of the cluster, the uncoordinated sites need to be passivated with hydrogens or hydroxyls. Cluster models are typically used to study the reactivity of the active sites of zeolites (for instance, Brønsted acid sites, BAS) or MOFs (interactions with a node or linker of the framework).

The main advantage of cluster models is their reduced size. Depending on the particular reaction of interest, the model can contain from a dozen to a few hundreds of atoms. This allows the application of accurate methods to describe the electronic structure of the system and, consequently, the study of effects resulting from this electronic structure. In addition, finding TS is much easier on clusters than it is in periodic systems.

Figure 8 shows a model used to study the effect of substituting Si by $\mathrm{Al}$ in the zeolite framework on the propylene protonation reaction [17]. The cluster model consists of three T-sites, out of which two are Si and one is Al. A proton, coordinated with one of the bridging oxygen atoms compensates the charge imbalance and the dangling bonds are saturated by hydrogen atoms. 

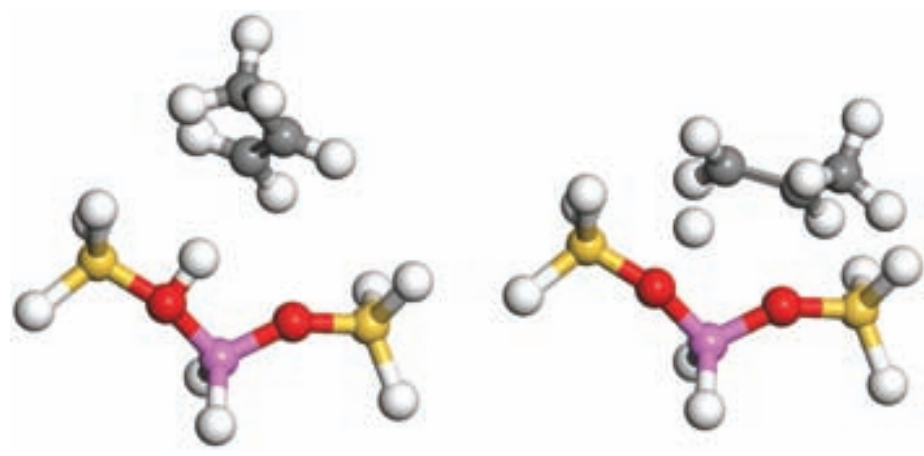

Figure 8. The cluster model of the small section of the ZSM-5 framework used in the simulation of the propylene protonation reaction [17].

This model can be considered extreme on the scale of accuracy. While its relatively small size allows one to use a high level method (such as MP2 or CCSD) to obtain accurate results, the model itself has too many limitations preventing the achievement of a qualitatively accurate picture. In the given example, the small cluster model does not take into account interactions with the other parts of the zeolite framework, especially the longrange electrostatic interactions. A larger cluster model would improve the qualitative picture but would limit the level of methodology that could be used given the same computational resources available (time, memory, and CPU-power). This effect is called the method vs. model accuracy trade-off.

\section{Periodic models}

In contrast to a cluster model, a periodic model represents a perfect infinitely repeating crystal. Because of its periodicity, it is possible to represent such a system considering only a single finite unit cell, which is infinitely duplicated in every direction. Periodic boundary conditions prevent atoms from exiting the system and rule out the existence of boundary effects. The use of a periodic model also has an important consequence when calculating the total energy of a system. For a simple system, like a molecule or a cluster, the energy contribution to the total energy coming from the electrons is simply the sum of the energies of these electrons. The latter are obtained by solving the Schrödinger equation, which provides a set of possible energy levels the electrons may occupy. Although the same is still true for a periodic system, the calculation of the energies is more complicated as the energies are also a function of the wavevector $k$ of the 
electron. This wavevector $\mathrm{k}$ gives the periodicity in three dimensions of the specific energy state (cf. section on electronic structure). Consequently, the energies or states, which are just single valued numbers in the case of molecules, become three-dimensional surfaces in a four-dimensional space ( 1 dimension for energy and 3 dimensions for the wavevector), in what is called reciprocal space, for solids and other periodic systems. The energy of a single state is now obtained through integration of this surface. Looking back at a molecule, one could say that for a molecule only a single wavevector k exists: $\mathrm{k}=(\mathrm{O}, \mathrm{O}, \mathrm{O})$ or no periodicity in any direction. This special point is called the $\Gamma$-point. This means that for a molecule (or a cluster) the reciprocal space is reduced to a single point. In contrast, the finite size of a periodic unit cell gives rise to a reciprocal space with a finite size. As a result, the energy of an electron needs to be calculated for each point in the reciprocal space. Luckily, the periodicity of our system allows one to limit these calculations to only a part of the reciprocal space, called the first Brillouin zone. Assume that the energy surface (imagine it as a function $f(x, y, z)$ ) in the first Brillouin zone can be accurately described by a grid of 10 points in each direction, then one would have to solve the 'molecular' Schrödinger equation, we started with, 1000 times. This makes periodic calculations computationally much more demanding, since each point in the reciprocal space needs to be considered. Fortunately, the large size of zeolite and MOF unit cells means that their corresponding reciprocal space is relatively small, making it possible to reasonably sample the reciprocal space by only considering the $\Gamma$-point, as is done by many authors. However, if very accurate results are required, such a sampling turns out to be insufficient, as was shown in an accuracy study of the flexible MOF: MIL-47(V) [18].

Because periodic models give access to the full reciprocal space of a crystalline material, they are ideally suited to a detailed investigation of the electronic structure of porous frameworks. Since long-range effects are included in a qualitatively accurate manner, it is also easier to investigate interactions of the spin of unpaired electrons with neighbouring metal nodes. This, however, all comes at a significant computational cost. Fortunately, the vast increase in computational resources in recent decades and the steady improvement of methodologies and algorithms in computational materials science have made it possible to handle larger and larger models in routine simulations, including zeolite reactivity or the breathing behaviour of flexible MOFs. Nowadays, it is possible to perform high quality studies of porous frameworks using fully periodic models. Two examples which will be discussed further in this section are: (1) the study of the $\mathrm{CO}_{2}$ hydrogenation by the FAU zeolite and $\mathrm{Ir}_{4}$ cluster supported on it, and 
(2) the electronic structure of the breathing of MIL-47(V) MOF and how this can be linked back to the experiments.

\section{Electronic structure of porous frameworks}

A central topic in the first principles investigation of periodic crystals is the study of their electronic structure. This can be done by solving either the Schrödinger equation or the HKS equations of the system. In both cases, this means solving an eigenvalue problem, where the eigenvalues are the possible energies of the particles in the system, i.e. the electrons. ${ }^{4}$ In contrast to a molecule or cluster, electrons in a solid experience a periodic potential, and their movement can be described using a 'Bloch wave'. Such a Bloch wave consists of two parts: a periodic function $\mathrm{u}(\mathrm{r})$ with the same periodicity as the crystal, and a plane wave:

$$
\varphi_{\text {nk }}(r)=u_{n k}(r) e^{i k . r}
$$

where $\mathrm{u}_{\mathrm{nk}}$ is a periodic function in real space, $\mathrm{n}$ is the band index (similar to the one found when solving the eigenvalue problem for a molecule), and $\mathrm{k}$ is the wave vector related to the momentum of the electron in the crystal. The wave vector $\mathrm{k}$ gives the periodicity of the specific state and can take any value within the reciprocal space of the simulation cell. As a result, varying the crystal momentum $(p=\hbar k)$ will lead to a change in the energy of the electron. While for electrons in a molecule the energy states form a discrete $1 \mathrm{D}$ spectrum, each energy state of an electron in a periodic solid gives rise to a $4 \mathrm{D}$ energy surface. To visualize the latter, one can plot the energy states/bands as a function of their position in reciprocal space, following a path along the high symmetry lines of the system. Since each electron gives rise to a single energy band, such a representation quickly starts to look like spaghetti. Figure 9 shows the example of diamond, which contains 2 atoms in its unit cell.

In the case of a MOF or a zeolite, with a hundred or more atoms in their primitive unit cell, the band structure picture contains too much information. A simplified picture can be obtained in the density of states (DOS). The density of states shows the density of bands in the reciprocal space as a function of the energy (i.e. it integrates the number of bands over the first Brillouin zone) presenting a picture comparable to the energy spectrum obtained for a molecule. In addition, by projecting the energy bands of a system onto the atomic orbitals, it is possible to obtain a so-called 


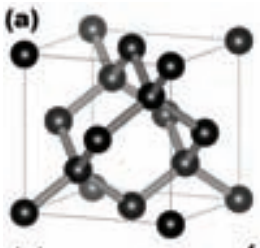

(b)
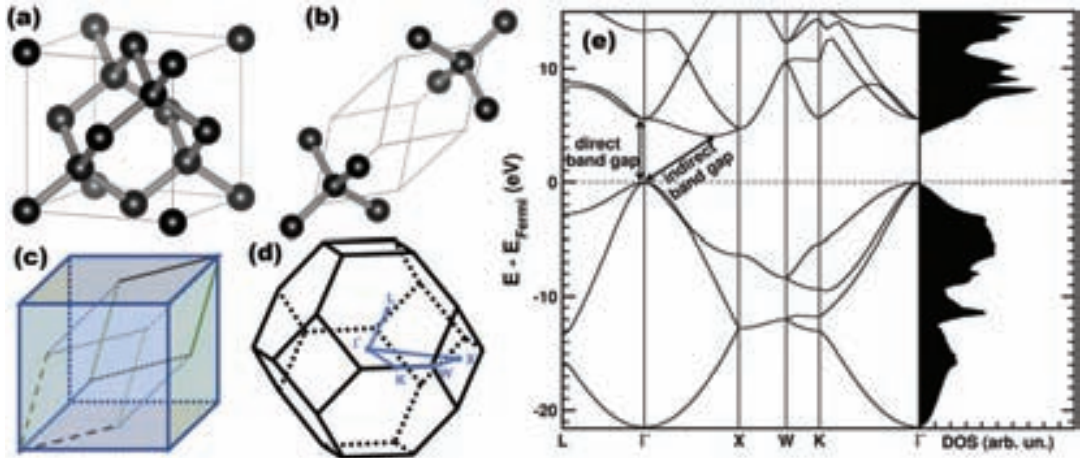

Figure 9. a) Ball-and-stick representation of a conventional unit cell of diamond. b) Ball-and-stick representation of the primitive unit cell of diamond. Note that only the centre atoms of the two clusters are inside the cell, the other atoms belong to neighbour cells (they were added to clarify the local environment of the $\mathrm{C}$ atoms in the primitive unit cell). c) Relationship between the conventional and primitive unit cells, in case of diamond, or any other material with face-centred cubic symmetry, such as for example the UiO-66 MOF. d) First Brillouin zone of a system with face-centred cubic symmetry. Some high symmetry lines are indicated. e) The electronic band structure and density of states of diamond, using a combined representation typical in solid-state literature.

local-DOS or LDOS. This teaches us which atoms or even atomic orbitals give rise to the specific peaks seen in the DOS. As such it can be used to discover which states make up the edges of the band gap, and are thus relevant for excitations and luminescence. Figure 10 shows an example of a DOS and LDOS for the luminescent and breathing MOF COK-69(Ti) [19]. The two different spin states ('up and down' or 'majority and minority' in the context of solid state physics) are indicated in black and red, respectively, with one spin state flipped down. The specific MOF mentioned here presented very clear luminescent behaviour, turning bright blue upon irradiation. This can be understood in terms of the protonation of the oxogroup of the Ti clusters. To retain charge balance upon this protonation, one of the $\mathrm{Ti}$ atoms needs to undergo a valence change from $\mathrm{Ti}^{\mathrm{IV}}$ to $\mathrm{Ti}^{\mathrm{III}}$. This is clearly visible in the electronic structure through the appearance of a gap state. Using the LDOS of the different Ti centres this gap state could be attributed to the $\mathrm{Ti}^{\mathrm{III}}$ atoms.

The above approach presents a way to investigate the electron distribution as a function of their energy. However, sometimes it is also interesting to investigate the spatial distribution of the electrons. This can be done through the direct visualization of the electron density or through density difference plots. It is also possible to partition the electron density between the atoms in an atoms-in-molecules (AIM) type of fashion providing atomic charges (useful for constructing a force field). This partitioning can 

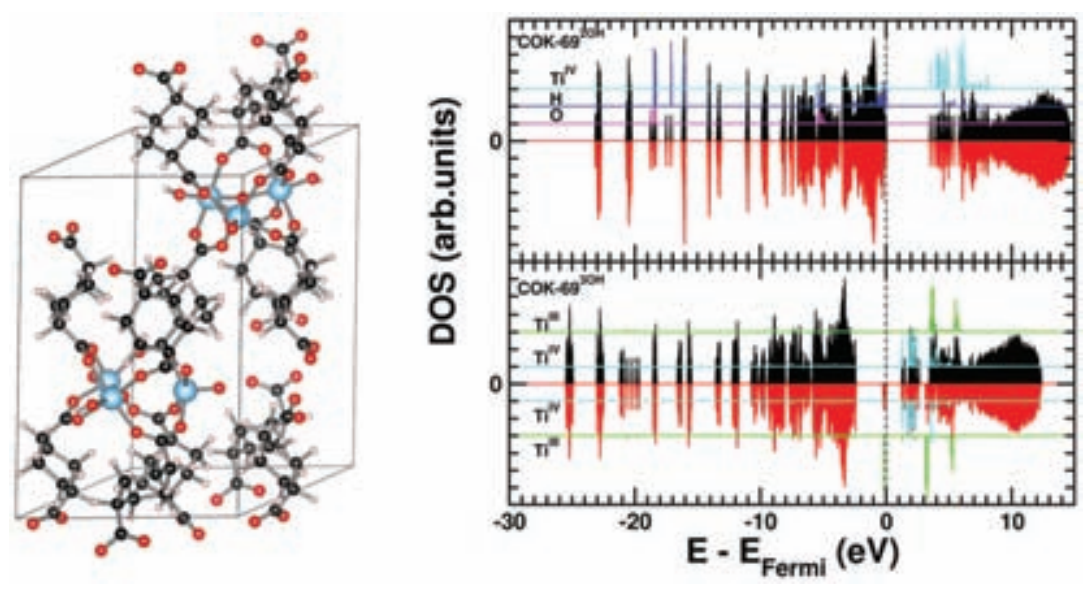

Figure 10. Left: ball-and-stick representation of the primitive unit cell of the COK-69(Ti) MOF. Atomic positions of $\mathrm{Ti}$ are indicated in blue. Right: spin polarized DOS of the COK-69(Ti) with different titanium clusters. In the case of $\mathrm{COK}-692 \mathrm{OH}$, each Ti-cluster has two hydroxy groups, leading to three $\mathrm{Ti}^{\mathrm{iV}}$ atoms, while the Ti-clusters of $\mathrm{COK}-693 \mathrm{OH}$ have three hydroxy groups, giving rise to a single $\mathrm{Ti}$ II in each cluster. This Ti"l gives rise to a gap state and luminescent behaviour.

be done in several different ways: Bader's QTAIM, Mulliken charges, Hirshfeld charges, etc. Each of these methods comes with its own strengths and weaknesses, and one has to be careful when comparing their results [20]. The main problem observed by Hamad and coworkers was the fact that, upon geometry deformation, such as in case of breathing, some schemes may give rise to significant changes in the calculated charges, making them less suitable. In this regard, the Hirshfeld-I partitioning scheme performs remarkably well [21]. Tests performed by one of the present authors showed no significant variations in the obtained charges over the full volume range covered by the breathing MIL-47 MOF [18].

As noted earlier, DFT is a ground state theory. One important consequence of that is the well-known band gap problem of local functionals, which boils down to a general underestimation of the band gap width for all semiconductors and insulators. Luckily, it mainly entails a downward shift of the conduction bands, meaning that the shape of those bands is qualitatively accurate. Several solutions are available in the solid-state community, varying from scissor-operators, $\mathrm{DFT}+\mathrm{U}$, the use of hybrid functionals or Green's function approaches. Despite this limitation, DFT generally provides very good atomic structures for periodic systems (in the case of porous frameworks it is often useful to include van der Waals contributions via a corrective scheme such as DFT-D). This means that one can use local DFT calculations, to obtain optimized structures, in tandem with hybrid 
functional calculations, to obtain high quality electronic structures. Using such an approach the computational cost is kept in check while results are obtained in good agreement with experimental measurements [22].

\section{Practical investigations of porous frameworks using DFT}

\section{Example 1: $\mathrm{CO}_{2}$ hydrogenation on $\mathrm{Ir}_{4} /$ FAU system}

This example shows the importance of the fully periodic model of the zeolite lattice [23]. The model used is a combination of the cluster model of the $\mathrm{Ir}_{4}[24]$ and a realistic model of FAU with a reduced cell size [25]. The cluster is supported in one of the 6-membered rings in the faujasite cage while the surrounding lattice provides the reaction environment. The $\mathrm{Ir}_{4}$ cluster is able to generate hydrides, while the BAS of the FAU framework provides the protons, both hydrogen species are needed in the reaction.

The reaction starts with the $\mathrm{CO}_{2}$ activation on the $\mathrm{Ir}_{4}$ nanoparticle, which is partially hydrogenated while the top site remains available to bind a $\mathrm{CO}_{2}$ molecule. The first step in the process is the hydrogen transfer to the oxygen atom of $\mathrm{CO}_{2}$. This can be accomplished in two ways: either by an attack of the hydride from the $\mathrm{Ir}_{4}$ cluster, or by a proton from the Brønsted acid site (BAS). The former is accompanied by a high barrier of $143 \mathrm{~kJ} \mathrm{~mol}^{-1}$ because the hydrogen atom, formally a hydride, is used to protonate the oxygen. The latter, however, requires a water assist because the BAS is located farther from the $\mathrm{CO}_{2}$, but in such case the barrier is only $98 \mathrm{~kJ} \mathrm{~mol}^{-1}$. Such an observation could not have been made if the cluster model had been used in the simulation and the presence of distant BAS had been neglected.

The result of the first step is the carboxyl bound to the $\mathrm{Ir}_{4}$ cluster. The second step can lead, either to formic acid (FA) by hydrogenation of the $\mathrm{C}$ atom, or to carbon monoxide by protonation of the hydroxyl $\mathrm{O}$. These pathways are shown in Figure 11. The pathway to FA is kinetically preferred because it occurs via the [8-9] transition state, which is associated with the barrier of $114 \mathrm{~kJ} \mathrm{~mol}^{-1}$, compared to the [8-11] transition state, associated with a barrier of $146 \mathrm{~kJ} \mathrm{~mol}^{-1}$. On the other hand, the latter is thermodynamically preferred because it leads to a strongly bound carbonyl complex, more stable than FA by $86 \mathrm{~kJ} \mathrm{~mol}^{-1}$. This complex should be considered undesired, because the release of the $\mathrm{CO}$ from the $\mathrm{Ir}_{4}$ cluster requires as much as $246 \mathrm{~kJ} \mathrm{~mol}^{-1}$, which is a prohibitive amount. The $\mathrm{CO}$ thus poisons the active site by blocking it for adsorption of the reactants. 


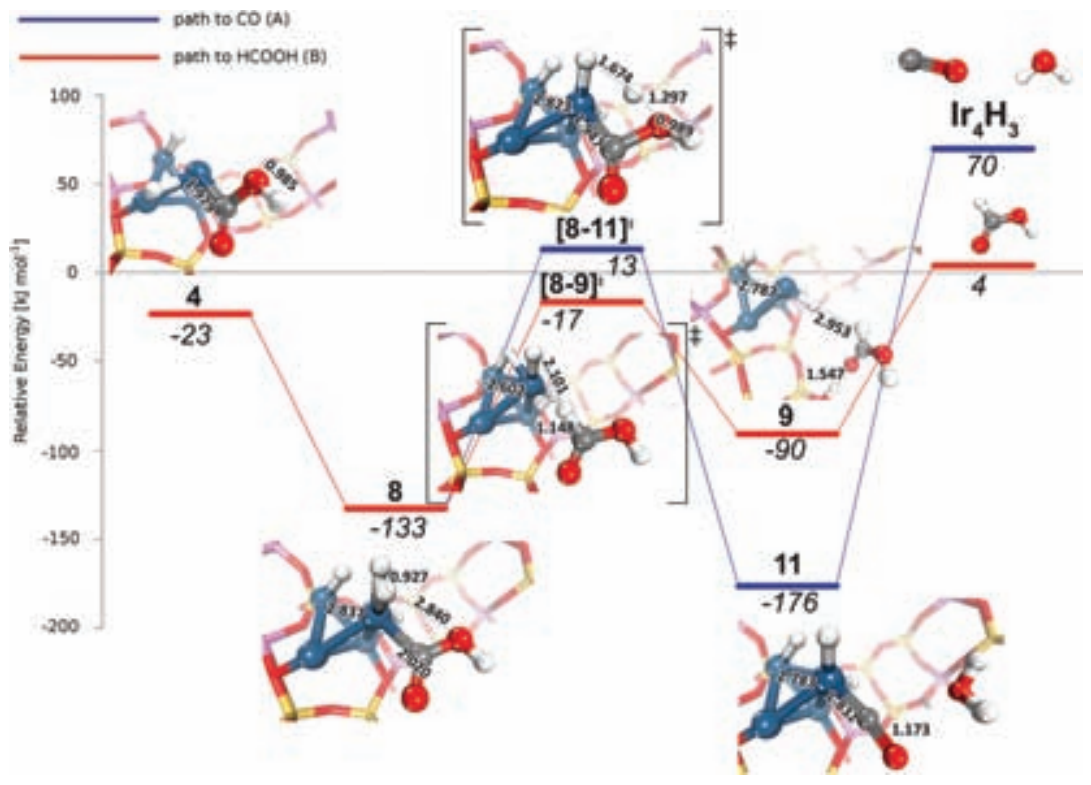

Figure 11. Reaction pathways for the CO (blue) or FA (red) from the Ir-bound carboxyl.

\section{Example 2: Electronic structure of breathing MOFs}

\section{Structure optimization and breathing}

Porous frameworks exist in all possible shapes and forms, and their modular nature gives rise to an almost infinite number of possible systems. In the case of MOFs, one class of MOFs attracted attention due to its breathing behaviour. Breathing is observed when a material can undergo reversible volume changes under the influence of an external stimulus. This can be a temperature or pressure change, or the presence of a specific gas. In breathing MOFs, these volume changes are large (easily $30 \%$ or more). Many MOFs with MIL-47/53 topology are known to present breathing behaviour. Of these, MIL- $47(\mathrm{~V})$ is considered a rigid example as it only shows breathing under application of an external pressure of about $100 \mathrm{MPa}$ (or $1 \mathrm{kbar}$ ). This flexibility makes structure optimization a difficult exercise, since a poor computational setting may push it over the barrier between large and narrow pore geometry. The Pulay stress, an effect, which has no relevant size in modern day calculations on standard solids, can be large enough in MOFs to do the trick [18]. To circumvent this problem, structures can be optimized under the constraint of a fixed volume. The equilibrium volume is then obtained through an equation of state fit performed on a set of calculated structures with (slightly) different volumes. Fitting the parameters 
Table 1. Effect of the magnetic configuration of the MIL-47(V) MOF on its mechanical properties.

\begin{tabular}{lclc}
\hline Magnetic configuration & $\mathbf{P}_{\mathrm{t}}(\mathbf{M P a})$ & $\mathbf{B}_{\mathrm{o}}(\mathbf{G P a})$ & $\mathbf{E}_{\mathrm{f}}$ (meV/V atom) \\
\hline $\mathrm{AF}$ & 124 & 8.12 & 0 \\
$\mathrm{AF}+\mathrm{FM}$ & 102 & 7.17 & 36 \\
$\mathrm{FM}$ & 83 & 5.95 & 70 \\
\hline
\end{tabular}

of the state equation also has the advantage of allowing a calculation of the transition pressure $\mathrm{P}_{\mathrm{t}}$ in a breathing MOF. An important feature of the MIL-47(V) MOF not mentioned before is the unpaired electron present on each of the $\mathrm{V}$ centres. The four unpaired electrons in the unit cell of MIL$47(\mathrm{~V})$ can have different orientations. This gives rise to ferromagnetic (FM) or antiferromagnetic (AF) configurations on the vanadyl chains. Calculations show the AF configuration to be the most stable one, as can be seen from the relative energies, $E_{f}$ shown in the Table. Furthermore, calculations also show that these spin configurations have a significant influence on the mechanical properties (bulk modulus, $\mathrm{B}_{\mathrm{o}^{\prime}}$ and transition pressure, $\mathrm{P}_{\mathrm{t}}$ ) of the MOF, as shown in Table 1 [26].

From single crystal experiments, the ground state configuration is expected to be AF, which is corroborated by the calculations [27]. However, linking the calculated transition pressures to the results obtained from mercury intrusion experiments that indicated an FM configuration was generally present [28]. These apparently contradictory results can also be elucidated making use of high accuracy computational data. Starting from optimized geometries for the two spin configurations, Bogaerts and coworkers simulated and fitted X-ray diffraction patterns for the MIL-47(V) MOF. The simulated results were compared to experimental spectra from single crystal and powders, leading to the conclusion that single crystal samples were best represented by the AF configuration, while powder samples were best represented by the FM configuration [29].

\section{Electronic structure: from spin coupling to splitting orbitals}

In the previous section, it was noted that spin configuration plays a crucial role in the mechanical properties of MIL- $47(\mathrm{~V})$. As the vanadyl chains are linked via relatively long benzenedicarboxylate (BDC) linkers, one may wonder how strongly the spins of different chains are coupled. Making use 


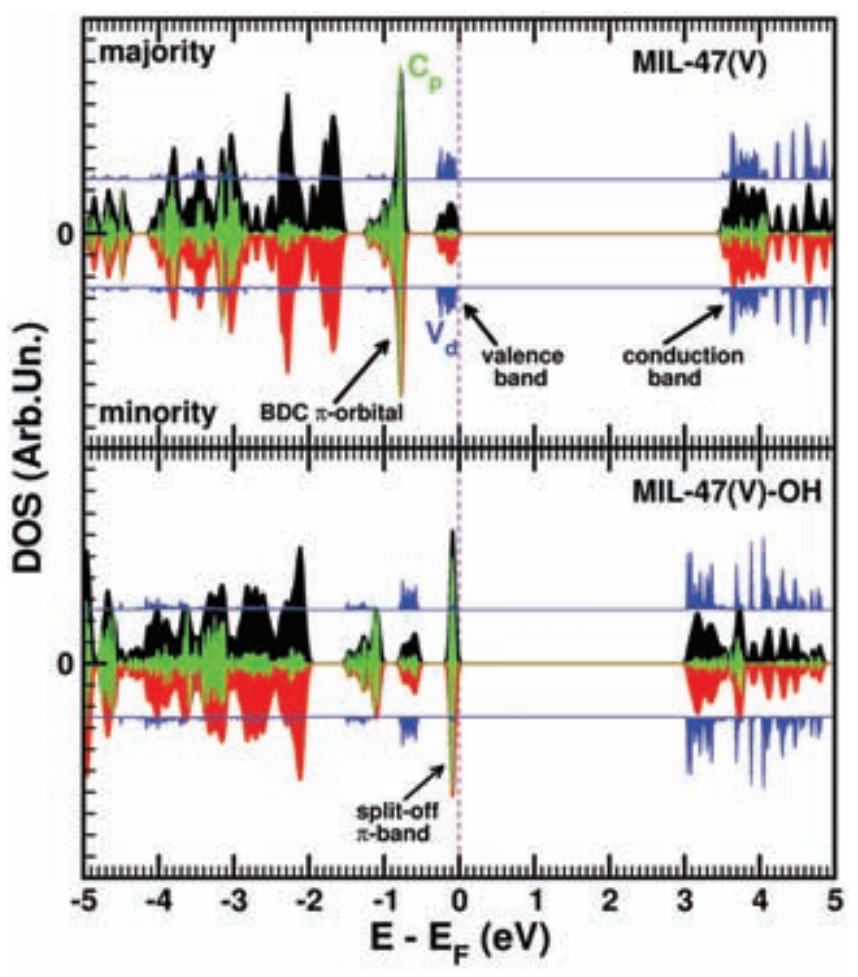

Figure 12. DOS and LDOS for the non-functionalized MIL-47(V) (top) and the hydroxy-functionalized MIL-47(V) (bottom).

of a simple spin Hamiltonian, it is possible to calculate the intra- and inter-chain coupling. Although the obtained values are quite sensitive to the function used, the qualitative picture is constant. For the MIL- $47(\mathrm{~V})$ the inter-chain coupling is about 2 orders of magnitude weaker than the intra-chain coupling, making the vanadyl chains quasi-1D systems.

The different spin configurations also have an influence on the electronic band structure. However, things get more interesting upon functionalization of the BDC linker. Looking at the band structure of the MIL-47 $(\mathrm{V})$ host material (Figure 12), one finds that the band gap edges are dominated by states originating from the $\mathrm{V}$ centres. The $\pi$-orbital associated with the BDC linker is located about $1 \mathrm{eV}$ below the Fermi level. However, upon functionalization, this orbital splits and the split-off $\pi$ orbital is shifted upward toward the Fermi level. The upward shift depends on the functional group and the number of functional groups on the linker. In the hydroxy group, the upward shift becomes so big that the split-off $\pi$ orbital moves above the $\mathrm{V}$ valence band, effectively reducing the band gap [30]. This 
behaviour is not unique for the MIL-47 $(\mathrm{V})$ and has also been observed for the linker-functionalized UiO-66(Zr).

\section{Example 3: Separation of $\mathrm{CO}_{2}$ and $\mathrm{CH}_{4}$ in UIO-66}

Microporous solids such as zeolites and MOFs are often referred to as molecular sieves, because of their well-defined porosity that matches the dimensions of individual molecules. The frameworks interact with molecules of particular species differently, which enables the separation of gas mixtures. In this example, the separation of $\mathrm{CO}_{2}$ and $\mathrm{CH}_{4}$ molecules by UIO-66 will be discussed.

GCMC simulations were carried out on a model of UIO-66 consisting of only one cubic unit cell of dimensions of $a=b=c=20.75 \AA$. The interactions between the host and guest molecules was described by the Force Field based on the parameters by Prakash et al. [31] Only van der Waals and Coulomb type interactions were taken into account because the structure of both MOF and guest molecules remained rigid during simulations, meaning that the bonding interactions were always exactly the same. Figure 13 shows the adsorption isotherms for both types of guest molecules in the pressure range up to $1000 \mathrm{kPa}$. It can be seen that the amount of adsorbed $\mathrm{CO}_{2}$ is much higher than that of $\mathrm{CH}_{4}$. In addition, the temperature does not seem to have an influence on the adsorption of $\mathrm{CH}_{4}$, while it

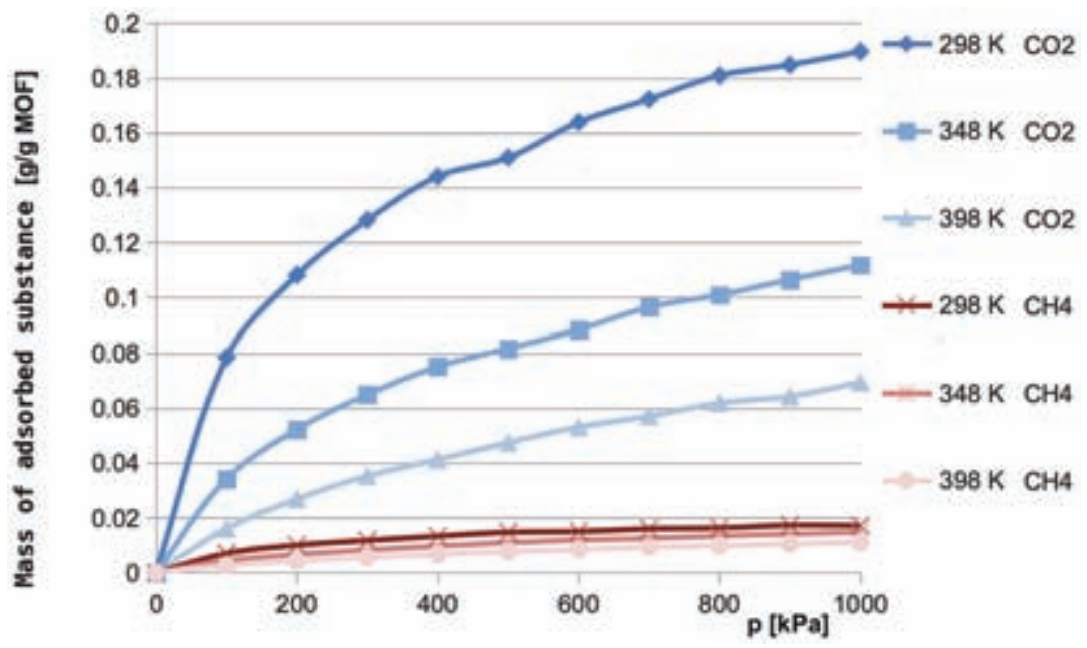

Figure 13. Adsorption isotherms for the mixture of $\mathrm{CO}_{2}$ and $\mathrm{CH}_{4}$ in UIO-66 in 298-398 K temperature range. 

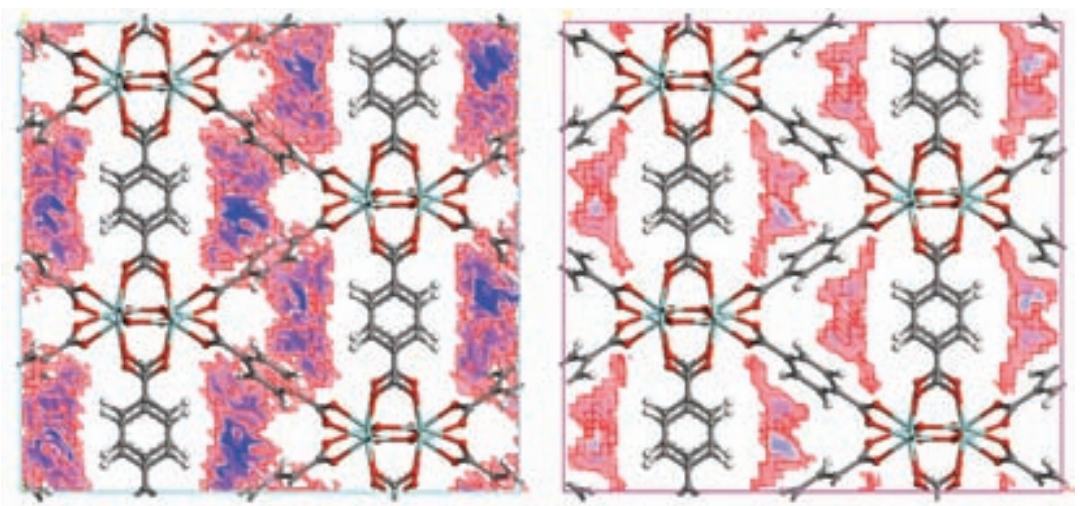

Energy Distribution

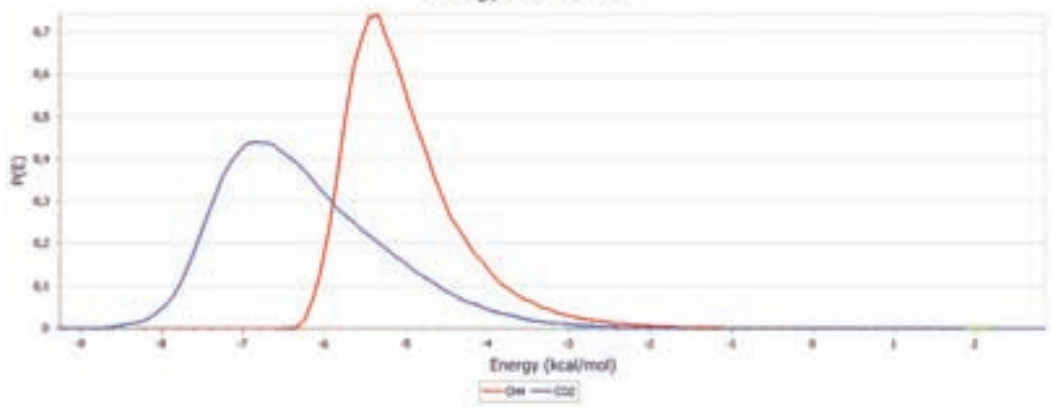

Figure 14. a) Distribution of $\mathrm{CH}_{4}$ (left) and $\mathrm{CO}_{2}$ (right) molecules inside the UIO-66 MOF resulting from GCMC simulation. The colour represents the relative interaction energy - blue, white and red represent the strongest, intermediate and weakest interactions, being related to the probability of the guest molecules occupying that location. b) Energy distribution profile for $\mathrm{CH}_{4}$ and $\mathrm{CO}_{2}$ in UIO-66.

significantly affects the isotherms for $\mathrm{CO}_{2}$. The best separation properties are thus observed at the highest pressure and the lowest temperature.

Figure 14 a) shows the distribution of the guest molecules inside the host pores. It shows that the $\mathrm{CO}_{2}$ molecules form a more diffuse 'cloud', which suggests that they fit into the thin pores slightly better. On the contrary, methane, which is a bulkier molecule, is located only in specific areas. This observation is also consistent with the slight influence of the temperature or pressure on the adsorption of $\mathrm{CH}_{4}$. The properties of the methane molecules allow them to occupy only some 'spots' and neither a temperature decrease nor a pressure increase allows the accommodation of more guests. The situation seems different for $\mathrm{CO}_{2}$ molecules, which have a linear shape, allowing them to adjust better to the pore sizes and shapes of the MOF and making other locations available for adsorption. Interestingly, sites available for $\mathrm{CH}_{4}$ adsorption are mostly coincident with those for $\mathrm{CO}_{2}$, which suggests 
the competition for these locations might take place. Finally, the energy histogram shows the probability of finding a molecule with a particular interaction energy inside the pores of UIO-66, Figure 14 b). It shows one peak for each of the species, which corresponds to the preferred adsorption site. The peak for $\mathrm{CH}_{4}$ is sharper, suggesting a better localization of this adsorption site. In contrast, the peak in the $\mathrm{CO}_{2}$ curve is smaller and broader. This means that the molecules are not limited to one particular location and can 'spill' over a larger area in the pores. The separation abilities of the UIO-66 in the given mixture illustrated by the isotherms above is the result of a stronger interaction of the framework with the $\mathrm{CO}_{2}$ molecules. The peaks of the curves are separated by approximately $2 \mathrm{kcal} \mathrm{mol}^{-1}$ in the figure, meaning that the interaction with $\mathrm{CO}_{2}$ is stronger and the presence of this species leads to a stronger stabilization of the system than the presence of $\mathrm{CH}_{4}$.

\section{References}

[1] van Beest, B.W., Kramer, G.J., van Santen, R.A. Phys. Rev. Lett. 1990, 64, 1955.

[2] Shi, H., Migues, A.N., Auerbach, S.M. Green Chem. 2014, 16, 875 .

[3] Llewellyn, P.L., Bourrelly, S., Vagner, C., Heymans, N., Leclerc, H., Ghoufi, A., Bazin, P., Vimont, A., Daturi, M., Devic, T., Serre, C., De Weireld, G., Maurin, G.J. Phys. Chem. C, 2013, 117, 962.

[4] Hohenberg, P., Kohn, W. Phys. Rev. 1964, 136, B864.

[5] Kohn, W., Sham, L.J. Phys. Rev. 1965, 140, A1133.

[6] Grimme, S.J. Comput. Chem. 2006, 27, 1787.

[7] Grimme, S., Antony, J., Ehrlich, S., Krieg, H.J. Chem. Phys. 2010, 132, 154104.

[8] Warshel, A., Levitt, M.J. Mol. Biol. 1976, 103, 227.

[9] Vanpoucke, D.E.P., Oláh, J., De Proft, F., Van Speybroeck, V., Roos, G.J. Chem. Inf. Model. 2015, 55,564 .

[10] van Duin, A.C.T., Dasgupta, S., Lorant, F., Goddard, W.A.J. Phys. Chem. A 2001, 105, 9396.

[11] Li, X., Frisch, M.J.J. Chem. Theory Comput. 2006, 2, 835.

[12] Mills, G., Jónsson, H. Phys. Rev. Lett. 1994, 72, 1124.

[13] Henkelman, G., Jónsson, H.J. Chem. Phys. 2000, 113, 9978.

[14] http://www.rheoman.eu/

[15] Allen, M.P., Tildesley, D.J. Computer Simulation of Liquids, Oxford University Press, 1989.

[16] Thomas, M., Brehm, M., Fligg, R., Vöhringer, P., Kirchner, B. Phys. Chem. Chem. Phys. 2013, 15, 6608.

[17] Dziekoński, P., Sokalski, W.A., Szyja, B., Leszczynski, J. Chem. Phys. Lett. 2002, 364, 133.

[18] Vanpoucke, D.E.P., Lejaeghere, K., Van Speybroeck, V., Waroquier, M., Ghysels, A. J. Phys. Chem. C 2015, 119, $2375^{2}$.

[19] Bueken, B., Vermoortele, F., Vanpoucke, D.E.P., Reinsch, H., Tsou, C.C., Valvekens, P., De Baerdemaeker, T., Ameloot, R., Kirschhock, C.E.A, Van Speybroeck, V., Mayer, J.M., De Vos, D. Angew. Chem. Int. Ed. 2015, 54, 13912.

[20] Hamad, S., Balestra, S.R.G., Bueno-Perez R., Calero, S., Ruiz-Salvador, A.R. J. Solid State Chem. 2015, 223, 144.

[21] Vanpoucke, D.E.P., Bultinck, P., Van Driessche, I.J. Comput. Chem. 2013, 34, 405. 
[22] Hendrickx, K., Vanpoucke, D.E.P., Leus, K., Lejaeghere, K., Van Yperen-De Deyne, A., Van Speybroeck, V., Van Der Voort, P., Hemelsoet, K. Inorg. Chem. 2015, 4, 10701.

[23] Szyja, B.M., Smykowski, D., Szczygieł, J., Hensen, E.J.M., Pidko, E.A. ChemCatChem, 2016, 8, 2500.

[24] Ferrari, A.M., Neyman, K.M., Mayer, M., Staufer, M., Gates, B.C., Rösch, N. J. Phys. Chem. B 1999, 103, 5311.

[25] Pidko, E.A., Mignon, P., Geerlings, P., Schoonheydt, R.A., van Santen, R.A. J. Phys. Chem. C 2008, 112, 5510.

[26] Vanpoucke, D.E.P., Jaeken, J.W., De Baerdemacker, S., Lejaeghere, K., Van Speybroeck, V. Beilstein, J. Nanotechnol. 2014, 5, 1738.

[27] Barthelet, K., Marrot, J., Riou, D., Férey, G. Angew. Chem. Int. Ed. 2002, 41, 281.

[28] Yot, P.G., Ma, Q., Haines, J., Yang, Q., Ghoufi, A., Devic, T., Serre, C., Dmitriev, V., Férey, G., Zhong, C., Maurin, G. Chem. Sci. 2012, 3, 1100.

[29] Bogaerts, T., Vanduyfhuys, L., Vanpoucke, D.E.P., Wieme, J., Waroquier, M., Van Der Voort, P., Van Speybroeck, V. Cryst. Eng. Comm. 2015, 17, 8612.

[30] Vanpoucke, D.E.P.J. Phys. Chem. C 2017, 121, 8014.

[31] Prakash, M., Jobic, H., Ramsahye, N.A., Nouar, F., Damasceno-Borges, D., Serre, C., Maurin, G. J. Phys. Chem. C 2015, 119, 23978.

\section{Notes}

1 The use of the term "ab-initio" can be debated as hybrid functionals (such as $B_{3} L Y P$ ) and $\mathrm{DFT}+\mathrm{U}$ do contain a fitting component. In addition, due to historical reasons the IUPAC definition of 'ab-initio', aimed at distinguishing QM and empirical methods, also excludes any non-wave function based method, as it was inconceivable in the early days of Quantum Chemistry that a non-wave-function based description could exist. To accommodate this difference between physics and chemistry-oriented views, DFT is often referred to as 'first principles'. In this chapter, we will follow the IUPAC definition of ab-initio referring to wave function based implementations of QM, in contrast to the density-based implementation used in DFT.

2 The ensemble is defined as the collection of all possible systems that have different microscopic states but the same macroscopic or thermodynamic state.

3 Strictly speaking the results might still differ due to the accumulation of the round-off errors or a different precision of numbers stored in a memory/ disk. This is known as the butterfly effect.

4 In the HKS-equations this is not exactly true, however, it is possible to relate the obtained states to the electron states. So, for practical purposes, we can continue as if they are. 


\title{
10. Zeolites and Metal-Organic Frameworks as Biomedical Nanodevices
}

\author{
Alejandro Cabrera-García, ${ }^{1}$ Zeneida Díaz-Betancor ${ }^{2}$ and \\ Eva Rivero-Buceta ${ }^{1}$ \\ ${ }^{1}$ Instituto de Tecnología Química, Universitat Politècnica de \\ València-Consejo Superior de Investigaciones Científicas, 46022 \\ Valencia, Spain. \\ ${ }^{2}$ Instituto Interuniversitario de Reconocimiento Molecular y \\ Desarrollo Tecnológico, Universitat Politècnica de València, 46022 \\ Valencia, Spain.
}

Keywords: Nanomedicine, cell viability, diagnostic, theranostic, drug delivery, stimuli-responsive, magnetic resonance imaging, combination therapy

\section{From nanotechnology to biomedicine}

Nowadays, there are a wide variety of treatments for all kinds of illnesses. However, many of them are not specifically used to combat the illness for which they were designed. A new field has emerged within nanotechnology to solve this situation. Nanomedicine is responsible for designing, creating and optimizing new pharmacological vectors, which improve the specificity and radius of actuation of current clinical treatments, with the aim of avoiding or reducing side effects during therapy. Nanomedicine is a very broad study area at the present time, mostly focused on the treatment of complicated diseases, such as different types of cancer. For that purpose, the use of nanomaterials takes advantage of the enhanced permeability and retention (EPR) effect, which directs the accumulation of nanomedicines preferentially towards the tumour instead of remaining in plasma or other organs, thus increasing its plasma half-life. Vascular tumours possess poorly aligned, defective endothelial cells with broad fenestrations and no muscular tissue or innervations and a relatively wide lumen. Their receptor function appears altered for vasoactive mediators, especially angiotensin II, and lack functional lymphatics, which greatly contributes to their hyperpermeability. They also show a hyperproduction of vascular mediators 
such as the vascular endothelial growth factor, bradykinin, nitric oxide peroxynitrite, prostaglandins and matrix metalloproteinases [1].

The use of solid drug containers for biomedical purposes requires an accurate control of their dimensions because particles larger than $200 \mathrm{~nm}$ are more easily removed by the splenic filtration system and particles smaller than $10 \mathrm{~nm}$ are likewise cleared through the kidney filtering system. For this reason, the most useful size range is from 10 to $100 \mathrm{~nm}$, which would allow entering the cell by endocytosis, whereas bigger particles or aggregates usually enter the cell by phagocytosis. For in vivo applications of nanoparticles (NPs), their surface needs to be modified in order to protect them from the environment and prolong blood circulation time. Polyethylene glycol (PEG) attachment, mostly known as surface PEGylation, shields the NPs with a hydrophilic coat, which enhances these properties and increases dispersibility whilst avoiding particle aggregation [2].

Porous materials offer many features, which make them especially suitable for biomedical applications. Zeolites and MOFs designed with a biologically friendly composition are potential nanocarriers able to act in different ways to improve human health. Metal-organic frameworks and zeolites can be customized according to the required compositions because of their easy compositional and structural tunability as well as to their size and chemical properties. They possess highly porous structures, which are useful for loading large amounts of therapeutic and imaging agents. The inherent biodegradability of MOFs caused by their relatively labile metal-ligand bonds, in comparison to the high stability of zeolites, increases their applications in this field. So metal-organic frameworks offer a greater variety of biomedical applications than zeolites and that is the reason why we will be citing more examples of MOFs than zeolites throughout the body of the present chapter [3].

\section{Zeolites and MOFs as drug delivery devices}

Drug delivery is the method or process for administering pharmaceutical compounds to achieve a therapeutic effect in humans or animals for the treatment of human diseases [4]. Drug encapsulation and biodegradation are contemplated when developing new drug delivery systems (DDS) and devising potential biomedical applications for MOFs, zeolites and other nanomaterials. Drug loading, which can be carried out by covalent or non-covalent binding methods, are made possible because of their controlrelease properties as well as the highly porous structure of these materials. 
Whereas therapeutic compounds require protection, drugs from vector biodegradation visibly minimize their toxicity and increase their efficiency and half-life in blood. The main objective of drug delivery is to encapsulate molecules of different structures and sizes and release them slowly over several days from the nanocarrier. Nanomaterials are versatile. It is possible to modulate drug delivery by tuning the host-guest interactions through the introduction of various polar or apolar functional groups, or by changing the structure of the solid (interconnectivity, pore size, flexibility) in order to control diffusion through the porous structure. In brief, drug delivery in nanomaterials is controlled by different factors such as the vector degradation in different biological conditions, drug diffusion level through the pores and drug-matrix interactions. The carrier degradation may be affected by complex variables - the carrier solubility, the quality of its surface and others. When the material is very stable in water or other biological environments, drug diffusion mainly depends on the NP porosity and the drug size $[2]$.

In order to demonstrate the utility of synthetic zeolites as drug delivery systems, Rimoli et al. [5] encapsulated the non-steroidal anti-inflammatory drug (NSAID) ketoprofen inside Vegobond ${ }_{13} \mathrm{X}$, a pure zeolite $\mathrm{X}$ with a pore volume of $0.377 \mathrm{~cm}^{3} \mathrm{~g}^{-1}$ and a crystal size of around $2 \mu \mathrm{M}$. Vegobond AX was also used, a mixed phase zeolite with zeolite A in the centre of the crystals and zeolite $\mathrm{X}$, the main component, covering zeolite A. Vegobond AX has a pore volume of $0.374 \mathrm{~cm}^{3} \mathrm{~g}^{-1}$ and the crystal sizes vary from 3 to $8 \mu \mathrm{m}$. Both zeolites accepted the same ketoprofen amount loading of $28.5 \%$. The drug release experiment was carried out at different $\mathrm{pH}$ levels and time periods with the aim of simulating the course of the DDS through the gastrointestinal tract. At an early stage, the loaded zeolites were maintained at $\mathrm{pH} 1$ for an hour and a half, after which the $\mathrm{pH}$ value was increased to 5 and kept at this value for another hour,and the $\mathrm{pH}$ was finally raised to 6.8. The drug platform showed that less than $10 \%$ of the drug was released under acid $\mathrm{pH}$ conditions. However, once the $\mathrm{pH}$ level was increased, the drug loading gradually started to leave the zeolites until its complete delivery. Ketoprofen became thus protected by the zeolite and extended its half-life and bioavailability, which makes it a good choice for treating inflammatory gastrointestinal tract diseases.

Colorectal carcinoma (CRC) is a common type of cancer at present, mostly affecting the male population. In order to fight against CRC, Vilança et al. [6] designed a new drug delivery system hosting the anticancer medicine 5-fluorouracil (5-FU), currently used for the treatment of CRC, stomach, breast, head and neck cancers. 5-FU was loaded into two different 
zeolites - faujasite in the sodium form with two different particle sizes $\mathrm{NaY}$ $(700 \mathrm{~nm})$ and nanoNaY $(150 \mathrm{~nm})$ and Linde type $\mathrm{L}$ in the potassium form (LTL) with a particle size of $80 \mathrm{~nm}$. Both kinds of zeolites possess a similar pore diameter of around ${ }_{7} \AA \AA$, which allowed 5 -FU loading. The study showed a better effectiveness of 5 -FU loading into $\mathrm{NaY}$ with $71 \%$ than for the nanosized materials with around $55 \%$. The drug release assay was carried out in simulated physiological conditions (phosphate buffer solution, PBS, at $\mathrm{pH}=7.4$ and $37^{\circ} \mathrm{C}$ ) showing $80 \%$ for $\mathrm{NaY}$ and with $90 \%$ released after 48 hours for nanosized materials. In vitro tests were also carried out with these platforms and naked zeolites showed no significant toxicity in the human colorectal carcinoma cell lines tested, HCT-15 and RKO. However, 5-FU loaded zeolites produced a significant decrease in viability in both cell lines and an increase in the effect of 5 -FU within the zeolites when acting freely. In this way, zeolites loaded with 5 -FU are presented as a possible alternative to the administration of this drug in the fight against cancer.

MOFs have also been widely studied as drug carriers. Liu and coworkers [7] designed an interesting system based on hollow nanospheres of the highly stable MOF ZIF-8, which is composed of $\mathrm{Zn}^{2+}$ cations and a 2-methylimidazole ligand. This framework was loaded with $51 \%$ of 5 -FU and finally coated with polymer layers of the developed FA-CHI-5-FAM. The FA-CHI-5-FAM polymer was composed of folic acid (FA) as a targeting molecule, able to bond to the folate receptors overexpressed on many cancer cell surfaces, the chitosan (CHI) linker, which facilitates the polymer formation and its attachment to the nanoparticle surface and, finally, the imaging agent 5 -carboxylfluorescein $(5$-FAM) for monitoring the controlled drug release process. The synthesized nanodevice ZIF-8/5-FU@FA-CHI-5FAM had a size distribution of approximately $400 \mathrm{~nm}$. The lack of toxicity of the non-loaded system was also checked on MGC-803 cells. However, $200 \mu \mathrm{g} \mathrm{mL} \mathrm{m}^{-1}$ of ZIF-8/5-FU@FA-CHI-5-FAM produced the death of $55 \%$ of the cells. Its powerful targeting and signalling effect was also demonstrated by following the strong green fluorescence of the material incubated in MGC-803 cells. Finally, the authors showed the sustained 5-FU release from the nanomaterial in physiological conditions (PBS, $37^{\circ} \mathrm{C}$ ) and in acidic conditions $(\mathrm{pH}=5)$, which favour the dissolution of CHI and ZIF-8. The complete delivery was carried out along 45 and 21 hours respectively. Finally, ZIF-8/5-FU@FA-CHI-5-FAM was presented as a very useful high-loading drug carrier for killing cancer cells controllably.

Here, we give a brief description of another kind of trigger mechanism in a combined therapy system, which we will delve into further in this chapter. It is about directing the drug release through $\mathrm{pH}$ and temperature. 


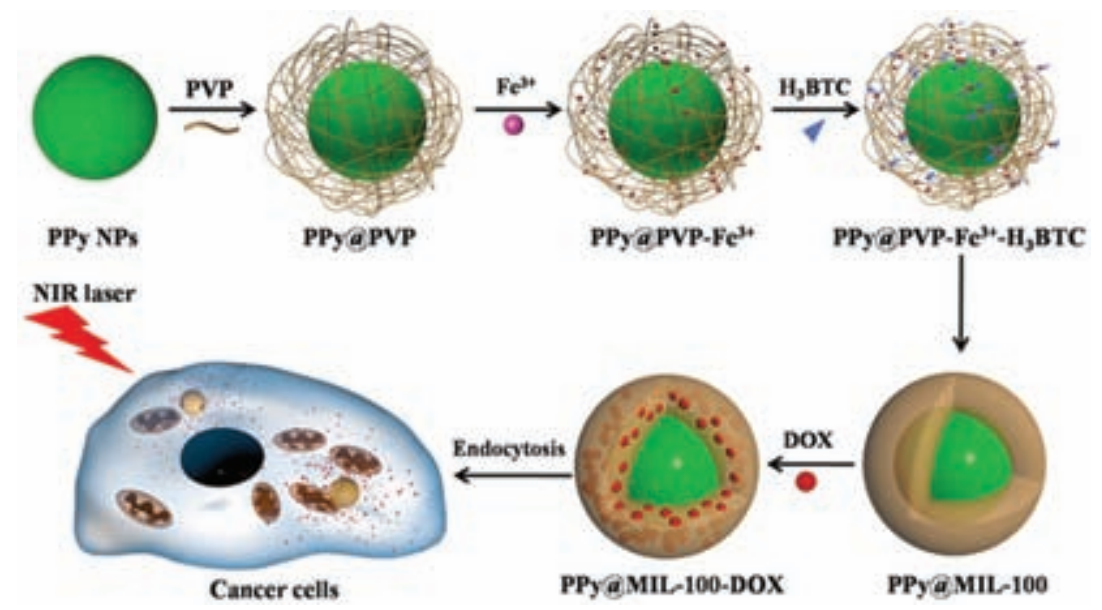

Figure 1. Schematic illustration of multifunctional NPs consisting of a PPy core and a mesoporous MIL-100 shell, designed for simultaneous PTT and chemotherapy of cancer cells. Reprinted with permission from ref. 8. Copyright 2016 American Chemical Society.

In this field, Zhang and coworkers [8] have engineered core-shell nanoparticles composed of organic polypyrrole nanospheres (PPy) in its core, later coated with poly(vinylpyrrolidone) (PVP). This polymer layer made it easier to build a mesoporous MOF shell based on MIL-101(Fe), thus facilitating the coupling between iron (III) cations, bezene-1,3,5-trycarboxylate (BTC) ligands and the PPy NPs surface. The high porosity of MIL-101(Fe) shell was also taken advantage of, in order to load the anticancer drug doxorubicin hydrochloride (DOX). PPy@MIL-10o-DOX NPs of around $107 \mathrm{~nm}$ in size, which were able to accept a $12.8 \%$ DOX loading. The PPy core is able to raise its temperature under near-infrared light (NIR) (Figure 1). This property has proved useful in killing cancer tissue and accelerating the drug delivery process. The nanoplatform was able to release $31.9 \%$ of its DOX load at $\mathrm{pH}=7.4$ and $49.1 \%$ at $\mathrm{pH}=5$ after 120 minutes in the dark because of the MIL-101(Fe) degradation in acidic conditions. However, whenever the system was treated under NIR irradiation for the same amount of time, such release reached $53.2 \%$ at $\mathrm{pH}=7.4$ and $70.4 \%$ at $\mathrm{pH}=5$. These results demonstrate that the rise in temperature facilitates the DOX delivery thanks to the increase in the molecular mobility of the drug and the movement of the crystalline framework. This effect was checked in HeLa cells in an in vitro assay demonstrating that the DOX free system is non-toxic and that the best results were achieved when incubating the HeLa cells with PPy@MIL-10o-DOX NPs following the NIR laser irradiation, which provoked a faster DOX release and the thermal ablation of 
the cancer cells. This combined system brings us a little closer to directed therapy.

\section{Zeolites and metal-organic frameworks in other biomedical modalities}

Zeolites and MOFs can be very useful in other fields of biomedicine thanks to their excellent properties. They can serve as contrast agents in magnetic resonance imaging (MRI) or in other kinds of diagnostic modalities or therapies. Magnetic resonance imaging is one of the most powerful clinical diagnostic tools due to its non-invasive nature, high spatial resolution, reliance on non-radioactive contrast agents (CAs), infinite penetration depth and anatomic resolution. However, the intrinsically low sensitivity of MRI requires the use of CAs, which accelerate the relaxation rates of surrounded water protons and generate an improvement of sensitivity in the obtained signal. Hence, CAs are often administered in high doses. Based on their relaxation mechanisms, CAs can be classified as $T_{1}$-positive agents modifying the longitudinal relaxation rate, which appears enhanced in brightness or positive contrast when computerized, or as $T_{2}$-negative agents affecting the transversal relaxation rate, thus generating a darkening of the obtained image or negative contrast. As regards this property, it is well known that paramagnetic nanomaterials can often work at very low concentration levels with a remarkable enhancement of the MRI signal. $\mathrm{Gd}^{3+}$ and $\mathrm{Fe}^{3+}$ have the ability of increasing the longitudinal $\left(r_{1}\right)$ and transversal $\left(r_{2}\right)$ water proton relaxation rates in human tissues, thus improving clinical MR images. Those cations, as part of a stable framework, avoid the undesirable side effects of soluble chelates and allow large payloads of paramagnetic metal ions. They also extend the half-lives of the circulating plasma and provide good targeting and accumulation at tumour sites [9]. Hatakeyama et al. [10] studied the influence of particle size of two gadolinium MOFs with 1,4-benzenedicarboxylic acid (1,4-BDC) or 1,2,4-benzenetricarboxylic acid (1,2,4-BTC) over the relaxivity of water protons for MRI. Using controlled micelles, they synthesized Gd MOFs in different shapes and sizes, ranging from $24 \mathrm{~nm}$ to larger than $1 \mathrm{mi}-$ cron (Figure 2). Their magnetic properties were also analysed under a $4.7 \mathrm{~T}$ magnetic field. They found that the particles with a greater area available for the interaction of $\mathrm{Gd}^{3+}$ cations with surrounding water molecules produced greater relaxation rates than larger and coarser particles, which had less surface area. Furthermore, the highest longitudinal relaxivity of $83.9 \mathrm{mM}^{-1} \mathrm{~s}^{-1}$ was shown by the Gd MOF with the highest surface area of $4 \cdot 5 \cdot 10^{19} \mathrm{~nm}^{2}$, and 
(a)

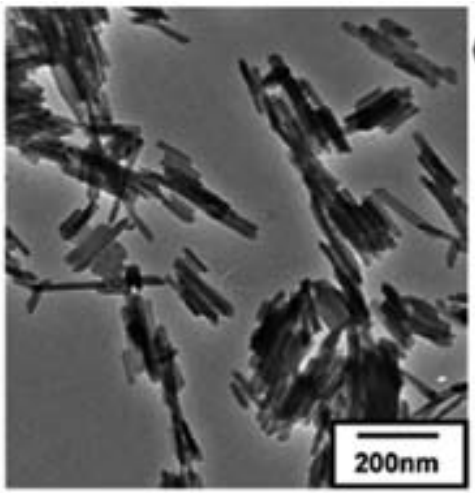

(c)

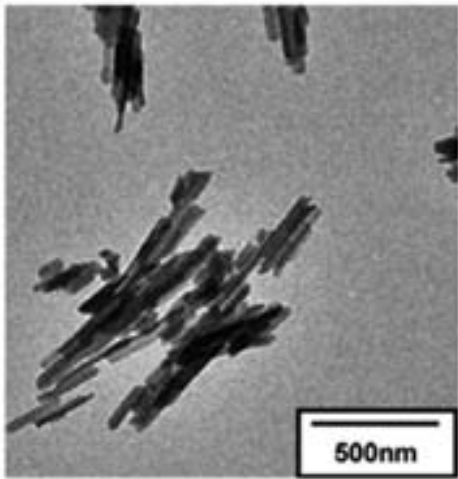

(b)

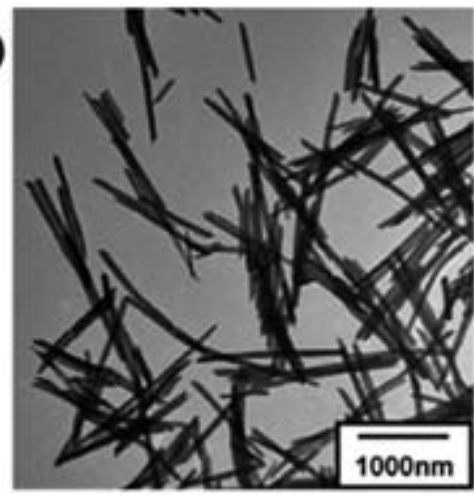

(d)

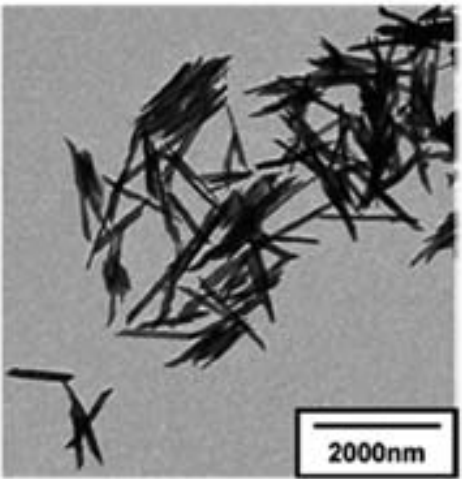

Figure 2. Transmission electron microscopy of 1,4-BDC and $\mathrm{Gd}^{3+}$ MOFs with different shapes and sizes. Reprinted with permission from ref. 10. Copyright 2011 American Chemical Society.

the lowest $r_{1}=17.8 \mathrm{mM}^{-1} \mathrm{~s}^{-1}$ was shown by the largest Gd MOF, with a surface area of $0.893 \cdot 10^{19} \mathrm{~nm}^{2}$. These results demonstrated that the best relaxivity enhancing did not depend on the Gd loaded inside the Gd MOF nanoparticles but on their availability to interact with the surrounding water molecules.

Prussian Blue (PB) and its analogues (PBAs) are a family of hexacyanometallate compounds where two identical or different metal ions are linked by the cyanide ligand, which gives these compounds very interesting properties. Because PB and PBAs are solid frameworks formed by metal ions and organic linkers, they can be considered as the oldest MOFs. Indeed, $\mathrm{PB}$, with a general formula including two different valence iron cations in $\mathrm{Fe}^{\mathrm{III}}{ }_{4}\left[\mathrm{Fe}^{\mathrm{II}}(\mathrm{CN})_{6}\right]_{3} \cdot \mathrm{nH}_{2} \mathrm{O}$, has been used extensively in the pigment industry since 1704 [11]. These compounds are non-toxic and have demonstrated a lot of applications in the biomedical field as biosensors [12], MRI contrast agents [13] and drug delivery devices [14] amongst others. Furthermore, the 
United States Food and Drug Administration (FDA) approved the clinical treatments with the Prussian Blue nanoparticles marketed as Radiogarda$\mathrm{se}^{\mathrm{TM}}$ from HEYL Chemisch-pharmazeutische Fabrik GmbH \& Co. KG as a treatment for internal contamination with radioactive cesium and radioactive or nonradioactive thallium [11]. Recently, Fu et al. [15] reported the application of PB as a photothermal ablation agent for cancer therapy. Photothermal therapy (PTT) is aimed at removing cancer cells through heat generated by photothermal agents, which absorb light energy and convert it into heat. Useful photothermal agents have to work in the near-infrared (NIR) region because the absorption of NIR laser in biological tissues is minimal and the penetration depth is optimal. The authors demonstrated that PB nanocubes with $42 \mathrm{~nm}$ of average diameter were able to increase the temperature in a $500 \mathrm{ppm}$ water suspension to more than $60^{\circ} \mathrm{C}$ when exposed to an $808 \mathrm{~nm}$ laser. They also checked that 3 minutes was enough to reach a temperature of $43 \mathrm{C}$, enough to kill cancer cells. Even though the material suspension suffered some thermal cycles, it remained unaltered and showed good photothermal stability. Finally, the material activity and toxicity of the naked nanovector in HeLa cells was checked to prove that the material did not cause relevant damage in the cell cultures until they were exposed to an $808 \mathrm{~nm}$ laser. The good photothermal activity of the PB nanoparticles was proven because less than $10 \%$ of cell viability was recorded after the treatment with NIR irradiation in the presence of only 16 ppm.

Computed tomography (CT) is a quick and cheap clinical diagnostic tool whereby an X-ray beam is passed through the body of the patient against a movable detector for data collection from various directions and angles. Out of these data, pictures in different directions of the target area will be obtained, which can be used to build up a tomogram in a computer, containing $3 \mathrm{D}$ information about the relevant part of the patient's body. Acting as CAs for CT, the heaviest elements produce a strong scattering of X-rays, so the introduction of these elements will decrease the data collection by the detector in the accumulation areas of these heavy elements. Then, if the heavy elements are well located around the affected tissue, the contrast between normal and diseased tissues will be enhanced in the tomogram, thus contributing a conclusion for the state of the disease [16]. Dekrafft et al. [17] introduced iodine as a heavy element constituent of a MOF able to attenuate X-rays from the irradiated sample, allowing for the construction of images with high spatial resolution. Five nanoscale metal-organic frameworks were synthesized with the bridging ligand 2,3,5,6-tetraiodo-1, 4-benzenedicarboxylic acid $\left(\mathrm{I}_{4}-\mathrm{BDC}\right)$ and $\mathrm{Cu}^{2+}$ or $\mathrm{Zn}^{2+}$ to function as the 
metal cations building up the MOFs. Such NMOFs demonstrated the high $\mathrm{X}$-ray attenuation displayed in phantom studies, which makes them potential CAs for X-ray-based computed tomography. These nanoplatforms showed good biodegradability with a half-life in simulated biological conditions (phosphate buffer saline, $\mathrm{pH}=7.4,37^{\circ} \mathrm{C}$ ) of 1.5 hours. In this period of time prior to its complete dissolution, the new materials can be used as a powerful diagnostic tool in CT.

MOFs can also be used as sensitizing agents in photodynamic therapy (PDT). This treatment uses chemical species like porphyrins, which can be a part of the framework and are able to be excited by light and transfer the energy excess to other molecules like $\mathrm{O}_{2}$ yielding the very unstable form singlet oxygen: ${ }^{1} \mathrm{O}_{2}$. This species can interact with the macromolecules necessary for cell life, killing the cell, or with water, producing reactive oxygen species (ROS), such as superoxide, $\mathrm{O}_{2}^{-}$, hydroxyl radical, $\mathrm{OH}$; or hydrogen peroxide, $\mathrm{H}_{2} \mathrm{O}_{2}$. These very reactive agents can also damage cells. Tissues where singlet oxygen or ROS have been formed by the action of light over a photosensitizer will be killed in a controlled area. The most important limitation of this therapy is that the penetration depth is very short so it is not very useful for deep tumours [16]. Zhang et al. [18] prepared a new system for this purpose by including cationic ruthenium complexes (RCs) able to catalyse ${ }^{1} \mathrm{O}_{2}$ production into the anionic metal-organic framework bio-MOF-1, composed of zinc, adenine (Ad) and biphenyl dicarboxylic acid (BPDC), with the chemical formula $\mathrm{Zn}_{8}(\mathrm{Ad})_{4}(\mathrm{BPDC})_{6} \mathrm{O} \cdot 2 \mathrm{Me}_{2} \mathrm{NH}_{2}$, through an ion-exchange method. Three different sized RCs, $\left(\left[\mathrm{Ru}(\mathrm{bpy})_{3}\right]^{2+}\right.$, bpy = 2,2-bipyridine, the smallest one; $\left[\mathrm{Ru}(\mathrm{phen})_{3}\right]^{2+}$, phen $=1,10$-phenanthroline, and $\left[\mathrm{Ru}(\text { phen })_{2} \text { hipp }\right]^{2+}$, hipp $=2-(1 H$-imidazo $[5,5-f][1,10]-$ phenanthrolin-2-yl)phenol, the largest one) were inserted into the bio-MOF-1, and the composed materials were named bio-MOF-1\&RCs by the authors (Figure 3). Evidently, the RC that entered the framework in the greatest quantity was $\left[\mathrm{Ru}(\mathrm{bpy})_{3}\right]^{2+}$, whereas $\left[\mathrm{Ru}(\mathrm{phen})_{2} \mathrm{hipp}\right]^{2+}$ entered in the lowest amount due to size restrictions. When these materials were irradiated in a single-photon experiment with $490 \mathrm{~nm}$ light, the highest efficiency of ${ }^{1} \mathrm{O}_{2}$ generation was shown by bio-MOF- $1 \&\left[\mathrm{Ru}(\text { phen })_{3}\right]^{2+}$ with a singlet oxygen quantum yield $\left(\varphi_{\Delta}\right)$ of 0.32 . These systems were also assayed for the ${ }^{1} \mathrm{O}_{2}$ generation via two-photon excitation absorption at $800 \mathrm{~nm}$, showing greater singlet oxygen yields thanks to the high electron delocalization of bio-MOF- 1 and the energy transfer from the MOF to RCs. For these reasons, this approach was proposed as a PDT sensitizer by the authors.

Another interesting approach to zeolites is their use as hemostatic agents to prevent uncontrolled bleeding after an open injury, which can 


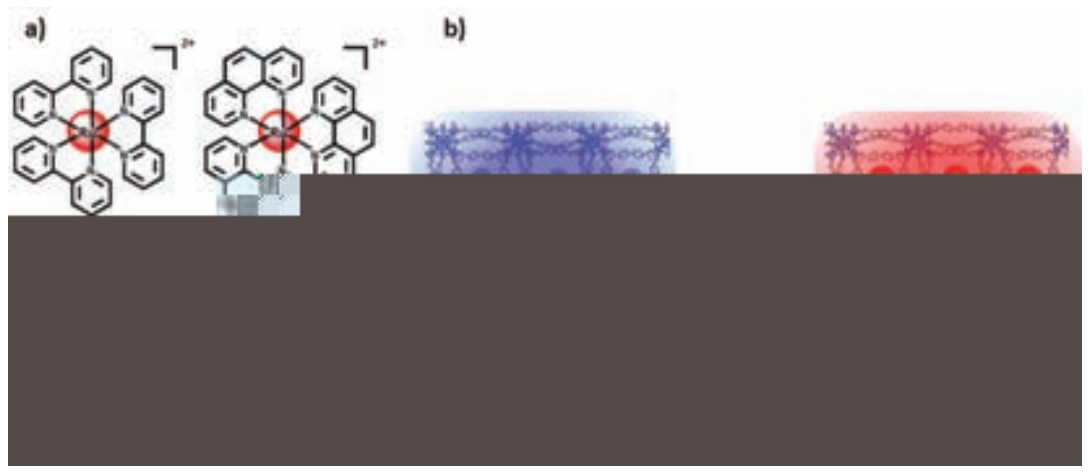

Figure 3. a) Chemical structures of complexes $\left[R u(b p y)_{3}\right]^{2+}$, $\left[R u-(p h e n)_{3}\right]^{2+}$, and $\left[R u(p h e n)_{2} h i p p\right]^{2+}$.

b) Encapsulation of cationic ruthenium(II) complexes [RuL $]^{2+}$ into the nanospace of bio-MOF-1 (L stands for 2,2'-bipyridine(bpy), 1,10-phenanthroline(phen), or 2-(1H-imidazo[5,5-f][1,10]phenanthrolin-2-yl)phenol(hipp)). Reprinted with permission from ref. 18. Copyright 2016 American Chemical Society.

cause death in many cases. Taeb and coworkers [19] demonstrated that their patent CoolClot, a mixture of bentonite and zeolite minerals, was able to produce an effective decrease in the bleeding time of dogs and in human blood samples - the clotting time in human blood samples was reduced from 253.4 to 143.4 seconds. Zeolites are able to absorb water from the wound tissues by concentrating the biological blood coagulating agents. However, this reaction is exothermic and can cause burns through contact with the affected tissues. The presence of bentonite in CoolClot prevents the rise in temperature and protects the skin in contact with CoolClot from additional damage. Therefore CoolClot stands as a cheap and safe effective hemostatic agent to prevent complications in sports, civil or military accidents.

Due to the extensively adaptable properties of MOFs, they have been proposed for many different sensing applications. Here we will briefly remark on their function as biosensors, that is, a self-sufficient integrated device which is capable of providing specific quantitative or semi-quantitative analytical information using a biological recognition element which is maintained in direct spatial contact with a transduction element [12]. In this sense, Wang et al. [20] developed a label-free colorimetric sensor based on the MOF Fe-MIL-88A, which was built up with iron and fumaric acid. This MOF was able to catalyse the oxidation of 3,3,5,5'-tetramethylbenzidine (TMB) with a very distinct colour change in an aqueous solution. With the introduction of a target biomolecule and its corresponding aptamer, the binding between the target/aptamer and MOF inhibited the catalytic effect of Fe-MIL-88A on TMB, which was used for the sensor design. Their 
sensitive and selective response in complex samples such as human serum was demonstrated by the detection of thrombin, which offered a range of linear detection from o.8 $\mathrm{nM}$ to 8 o $\mathrm{nM}$. Determination could be performed with bare eyes and without absorbance measurements starting at $10 \mathrm{nM}$. This system presents a promising new MOF-based biomolecule detection tool in health care.

\section{Zeolites and MOFs as multifunctional biomedical devices}

Zeolites and MOFs nanocrystals present some valuable properties, which enable them to be nanoplatforms for different kinds of nanomedicinal approaches. Furthermore, due to their compositional and structural tunability, they are able to combine more than one therapeutic or diagnostic modality in the same nanodevice. Here, we combine nanomedicine approaches with various synergistic biomedical effects in the same material. We consider nanomaterials for combination therapy, meaning the use of more than one therapeutic agent or modality on the same platform, multimodal diagnostic imaging and theranostic nanodevices, which integrate diagnosis and disease therapy. This type of nanomedicine brings us closer and closer to personalized medicine.

\section{Combination Therapy}

Combination therapies involve a rational co-delivery of different drugs with synergistic therapeutic effects aimed at improving the single-drug action with different mechanisms of action in the multiple therapeutic modalities and their capacities for hitting multiple targets and overcoming cross-resistance [21]. Many different nanomedical devices have been developed to that purpose. Lin and coworkers [22] prepared a new core-shell $\mathrm{Zn}^{2+}$ bisphosphonate nanoscale coordination polymer (NCP) containing the cisplatin prodrug cis,cis,trans- $\left[\mathrm{Pt}-\left(\mathrm{NH}_{3}\right)_{2} \mathrm{Cl}_{2}\left(\mathrm{OCONHP}(\mathrm{O})(\mathrm{OH})_{2}\right)_{2}\right]$. It was covered with a Polyethylene Glycol (PEG) layer, covalently bounded by a disulfide linkage. These particles were subsequently shielded with cholesterol. The referred nanoplatforms had a spherical shape with a diameter of $105.3 \pm 6.2 \mathrm{~nm}$ and were able to combine two therapies - the chemotherapy of cisplatin or cisplatin and gemcitabine and the gene therapy of siRNA by systemic injection. The researchers found a multiple therapeutic vehicle useful for the eradication of cisplatin-resistant ovarian cancer in mouse models. 
Zeolites have also played a biomedical role in this field. De Cola and coworkers [23] designed a zeolitic co-delivery system of DNA and drugs. They used Zeolite L nanocrystals of $50 \mathrm{~nm}$ as nanocontainers for the blue fluorescent model drug 4,6-diamidino-2-phenylindole (DAPI) for changing the strong negative surface charge due to $\mathrm{OH}$ groups in the zeolite surface. They functionalized the material surface with (3-aminopropyl)triethoxysilane in order to obtain free amino groups on the surface, which were protonated in physiological conditions and allowed an electrostatic anchoring of a DNA oligonucleotide labelled with the red emissive fluorescent dye cyanine5 (Cy5). The authors thereby demonstrated the potential application for this approach in living cells, as it was possible to delay and follow the release of the oligonucleotide as well as the molecules protected inside the nanocontainers.

At times, chemotherapeutics can be combined with photodynamic therapy (PDT), which involves the administration of a tumour-localizing photosensitizer (PS), which is irradiated to generate highly cytotoxic reactive oxygen species (ROS), like singlet oxygen $\left({ }^{1} \mathrm{O}_{2}\right)$, which in turn is able to produce cell apoptosis and necrosis [21]. Here, He et al. [24] combined PDT, chemotherapy and anti-PD-L1 (the PD-1/PD-L1 pathway inhibits immune activation by suppressing effector T-cell function and is upregulated in many tumours to cause apoptosis of tumour-specific cytotoxic T-limphocytes and transmit an antiapoptotic signal to tumour cells) in the same nanomedicine. A NCP was prepared using $\mathrm{Zn}^{2+}$ and oxaliplatin, a prodrug with two phosphate metal donor groups as a core. These nanoparticles were later shielded using phosphate interactions with 1,2-dioleoyl-sn-glycero-3-phosphate (DOPA). The authors took advantage of the hydrophobic interactions between DOPA and other lipid molecules and coated the particles with 1-palmitoyl-2-hydroxy-sn-glycero3-phosphocholine, which had been previously modified with the photosensitizer pyropheophorbide-a via ester bonds. These nanodevices were uniformly spherical with a size of $55 \cdot 3 \pm 0.2 \mathrm{~nm}$ and showed prolonged half-life in blood as well as favourable tumour accumulation after systemic administration. This new system provided a combinational therapy for metastatic colorectal cancer including oxaliplatin as a chemotherapeutic agent and a lipid shell carrying a photosensitizer for PDT and a PD-L1 checkpoint blockade, all three acting as a combined cancer therapy agent, which showed successful results in mice models. PDT can also be combined with checkpoint blockade immunotherapy. Lu et al. [25] have rationally built new nanorod chlorin-based metal-organic framework particles from hafnium and 5,10,15,20-tetra( $p$-benzoato)chlorin. This ligand was chosen 


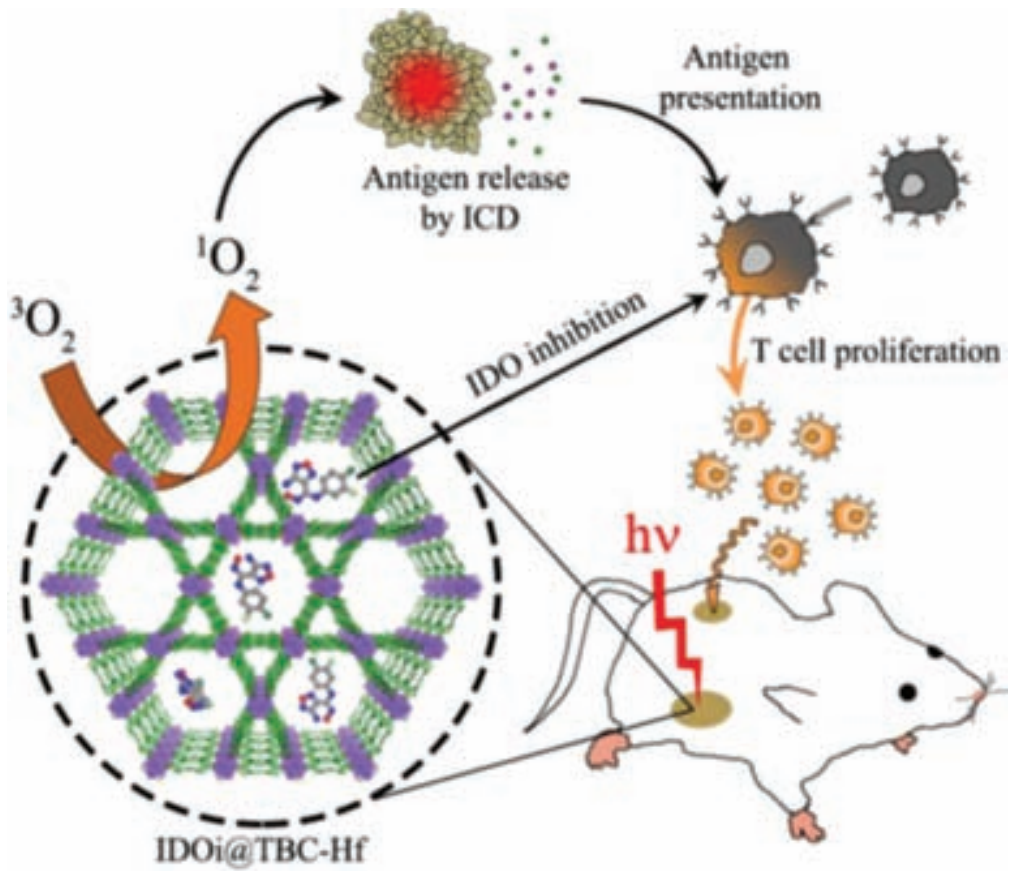

Figure 4. Schematic representation of combined PDT and immunotherapy by IDOi@TBC-Hf. Local injection of IDOi@TBC-Hf and light irradiation generate reactive oxygen species, causing immunogenic cell death (ICD) and releasing tumour-associated antigens, which are presented to T cells. Meanwhile, the IDO inhibitor released from IDOi@TBC-Hf modulates tryptophan/kynurenine catabolism to activate the immunosuppressive tumour microenvironment. The combination of antigen presentation from PDT and checkpoint blockade by IDO inhibition causes T cell proliferation and infiltration, leading not only to eradication of local, treated tumours but also a rejection of distant, untreated tumours. Reprinted with permission from ref. 25. Copyright 2016 American Chemical Society.

on account of its ability to generate highly porous frameworks and because it is an efficient photosensitizer for PDT of cancer. The huge channels allowed loading of small molecules aimed at the inhibition of indoleamine 2, 3-dioxygenase. Such molecules are effective in blocking the expression of this enzyme in cancer processes and enhancing the ROS generation, diffusion and action, especially ${ }^{1} \mathrm{O}_{2}$ after light absorption. The engineered nanomedicine applied to this purpose showed successful results in mice models (Figure 4). This treatment was proposed by the authors as a new way to fight metastatic processes.

Combination therapy also addresses different cancer treatments such as PDT and radiotherapy (RT), which uses X-ray or other sorts of ionizing irradiation to destroy cancer cells. In this sense, Liu and coworkers [26] have designed hafnium MOF nanoparticles. Hafnium is a high-Z element 
functioning as a radio-sensitizer. Photo/auger electrons are generated when the ionizing radiation interacts with these kinds of elements and as a result, the free radicals become able to destroy cancer cells. The ligand was tetrakis(4-carboxyphenyl) porphyrin (TCPP), a photosensitizer for PDT. The material surface was protected with PEG showing an average hydrodynamic diameter of $130 \mathrm{~nm}$ and extremely good stability in physiological conditions. The authors demonstrated the availability of this combined treatment in mice and therefore injected the PEGylated material into tumour-bearing mice, where the material showed long half-life in blood. With no apparent toxicity, the material became rapidly degraded, excreted and showed excellent tumour accumulation levels. They took advantage of this scenario in order to proceed with the RT doses and 8 hours later, so that the tumour oxygenation would be recovered, the tumour was irradiated with $661 \mathrm{~nm}$ light for PDT. Following this combined treatment, they achieved an effective inhibition of the tumour growth.

\section{Multimodal Diagnostic Imaging}

Biomedical imaging is a very important tool in many diagnostics. Due to their compositional tunability, nanoscale metal-organic frameworks can be useful to function as different kinds of contrast agents (CAs) in clinical imaging diagnostic techniques. Furthermore, they can combine the ability to enhance the signal of different imaging techniques in the same material. Rieter et al. [27] chose gadolinium (III) ions as metal nodes to build the framework because its high electronic spin value $(S=7 / 2)$ and slow electronic relaxation rate allows for a decrease in the longitudinal water protons relaxation time, thus enhancing the MRI bright signal around $\mathrm{Gd}^{3+}$. 1,4-benzenedicarboxylate (BDC) was chosen as a binding ligand, yielding $\mathrm{Gd}(\mathrm{BDC})_{1.5}\left(\mathrm{H}_{2} \mathrm{O}\right)_{2}$ nanorods of $100 \mathrm{~nm}$ in length. The authors also doped the NMOFs with $5 \%$ terbium(III) or $5 \%$ europium(III) generating $\mathrm{Gd}_{\text {o.95 }}(\mathrm{BDC})$ $\left(\mathrm{H}_{2} \mathrm{O}\right)_{2}: \mathrm{Eu}_{0.05}$ and $\mathrm{Gd}_{0.95}(\mathrm{BDC})\left(\mathrm{H}_{2} \mathrm{O}\right)_{2}: \mathrm{Tb}_{0.05}$ with red and green luminescence respectively. These materials showed large relaxivity values $\left(r_{1}=35.8, r_{2}\right.$ $=55.6 \mathrm{mM}^{-1} \mathrm{~s}^{-1}$ under a 3.0 Tesla magnetic field) and an intense luminescence so they were proposed as a new class of bimodal imaging agents. The same group [28] also chose benzenehexacarboxylate (bhc) ligands because they are able to form stable Gd NMOFs and to carry a high payload of $\mathrm{Gd}^{3+}$ ions. Block-like nanoparticles of $\left[\mathrm{Gd}_{2}(\mathrm{bhc})\left(\mathrm{H}_{2} \mathrm{O}\right)_{6}\right]$ were synthesized in sizes between 25 and $100 \mathrm{~nm}$ which showed low longitudinal relaxivity 


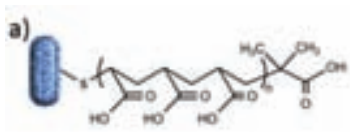

d)

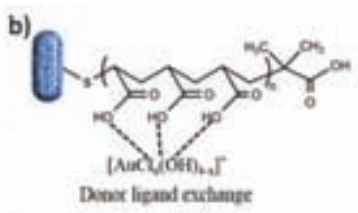

Dener lipand exhante
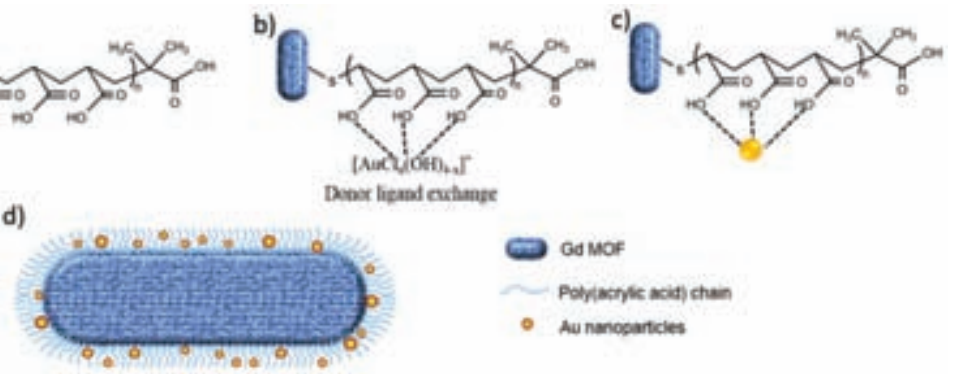

Figure 5. Synthesis layout of GdMOF-PAA-Au nanoparticles a) after deposition of PAA onto GdMOF nanostructures, b) loading of Au ions onto PAA-modified GdMOF nanostructures, followed by c) reduction of the Au ions to produce AuNPs entrapped in the surface immobilized PAA. d) A schematic representation of the structure of hybrid GdMOF-PAA-Au nanostructures. The GdMOF core is shown in blue, the PAA chains as blue chains and the AuNPs in gold. Reprinted with permission from ref. 29. Copyright 2015 American Chemical Society.

$\left(r_{1}=1.5 \mathrm{mM}^{-1} \mathrm{~s}^{-1}\right)$ and very high transversal relaxivity $\left(r_{2}=122.6 \mathrm{mM}^{-1} \mathrm{~s}^{-1}\right)$ under a 9.4 T magnetic field. This large $r_{2}$ rate makes the material a valuable CA for $\mathrm{T}_{2}$-weighted MRI. This material was also doped with $5 \%$ of europium(III) or terbium(III) to make the materials luminescent (green for $\mathrm{Tb}$ and red for $\mathrm{Eu}$ ) and useful as CAs for optical imaging in combination with MRI.

Tian et al. [29] developed a new nanocomposite suitable for MRI and $\mathrm{X}$-ray-based computed tomography (CT). CT is a very important diagnostic modality due to its cost effectiveness, wide availability and anatomical imaging ability. It is however, limited to soft tissue resolutions so it would prove more effective when used in combination with MRI as it takes advantage of the Gd-MOFs magnetic properties. Since gold nanoparticles (AuNPs) have a high atomic number and a high X-ray attenuation coefficient, they are very valuable materials for X-ray-based CT imaging. Accordingly, the authors synthesized $\mathrm{Gd}(\mathrm{BDC})_{1.5}\left(\mathrm{H}_{2} \mathrm{O}\right)_{2}$ in nanorod shapes of $155 \mathrm{~nm}$ in length. The vacant orbitals on the $\mathrm{Gd}^{3+}$ ions were used for coordinating the thiol-modified poly(acrylic acid) (PAA) through the thiolate ending groups. The PAA were therefore able to attach $\mathrm{HAuCl}_{4}$ as a $4 \mathrm{~nm}$ AuNPs precursor after the reduction reaction (Figure 5). The nanocomposite GdMOF-PAA-Au was tested for MRI and displayed good brightness enhancement in $T_{1}$-weighted MR images with an $r_{1}$ value of $4.9 \mathrm{mM}^{-1} \mathrm{~s}^{-1}$ at a $4.7 \mathrm{~T}$ magnetic field. The nanocomposite also improved the contrast of CT imaging remarkably even at low gold concentrations and emerged as a promising multimodal diagnostic tool able to enhance the quality of MR and CT images. 


\section{Theranostic Nanoplatforms}

Current trends in nanomedicine are focused on nanoplatforms, which can be loaded with interesting drugs and later tracked by clinical imaging techniques. Theranostics is a word combination of therapy and diagnosis, mostly focused on cancer treatment. These materials open the door to personalized patient treatments because of the possibilities they offer for designing smart systems for controlled drug release. They are also continuously detected from the time they are administered until they are excreted. This enables us to discover the best way to treat different diseases in every different case. Horcajada et al. [30] tested the ability of different biocompatible porous iron carboxylate nanoMOFs (NMOFs) to encapsulate huge amounts of different antitumor, antiviral drugs and cosmetic agents with different polarities, sizes and functional groups. For that purpose, the materials were immersed in concentrated solutions of the product to be entrapped. The authors described the behaviour of the material as 'molecular sponges'. Those NMOFs showed a progressive molecular release with no burst effect in a phosphate buffer solution at $37^{\circ} \mathrm{C}$. The effects of the nanoMOF MIL-10o(Fe) loaded with the anti-HIV (anti-Human Immunodeficiency Virus) drug 3'-Azido-2',3'-dideoxythymidine-5'-Triphosphate (AZT-TP) were proved in vitro in human peripheral blood mononuclear cells infected by HIV-1-LAI and approximately $90 \%$ inhibition of HIV replication was achieved. The non-toxicity of the empty material was also tested. The iron cations in the framework structure can also offer a water proton transversal relaxivity rate increase useful for $T_{2}$-weighted MRI diagnosis. In fact, this property was also tested and $r_{2}$ values greater than $50 \mathrm{~s}^{-1} \mathrm{mM}^{-1}$ under a $9.4 \mathrm{~T}$ magnetic field were obtained. PEGylated iron-carboxylate NMOF MIL-88A $\left(r_{2}=95 \mathrm{~s}^{-1} \mathrm{mM}^{-1}\right)$ was used in an in vivo assay as a $T_{2}$ contrast agent for MRI in rats. Darkening in the accumulation tissues (liver and spleen) in the MR images was produced by the CA effect, generating good images for clinical diagnostics.

Similarly, Chen and coworkers [31] developed a new core-shell nanoplatform composed of the Prussian blue analogue $\mathrm{Mn}_{3}\left[\mathrm{Co}(\mathrm{CN})_{6}\right]_{2}$, synthesized through a simple coprecipitation method in which manganese acetate and potassium cobaltocyanide were mixed, and a silica wall generated by tetraethylorthosilicate alkaline polymerization. Finally, they attached $12 \mathrm{~nm}$ silver nanoparticles to the silica surface trough S-Ag coordination bond. For that purpose, $\mathrm{Mn}_{3}\left[\mathrm{Co}(\mathrm{CN})_{6}\right]_{2} @ \mathrm{SiO}_{2}$ nanoparticles were treated with mercaptopropyltrimethoxysilane (MPTMS) dissolved into a silver nitrate solution and reduced with $\mathrm{NaBH}_{4}$. The final material $\mathrm{Mn}_{3}\left[\mathrm{Co}(\mathrm{CN})_{6}\right]_{2} @ \mathrm{SiO}_{2} @ \mathrm{Ag}$ 
presented an average size of $190 \mathrm{~nm}$ and was loaded with doxorubicin (DOX) at a high quantity of $600 \mathrm{mgg}^{-1}$. The release study of DOX was carried out in the dark, where, after $100 \mathrm{~h}$, only $39 \%$ of DOX was released. However, under $808 \mathrm{~nm}$ NIR laser irradiation, the system underwent an additional release of the DOX content of $31 \%$ within the same time interval. This phenomenon was due to Ag nanoparticles converting the laser light irradiation into thermal energy thus accelerating the DOX release. The presence of $\mathrm{Mn}^{2+}$ and $\mathrm{Co}^{3+}$ in the framework is able to accelerate the longitudinal and transversal relaxation rates of the surrounding water protons, which is necessary to achieve efficient CAs for MRI. The recorded values for longitudinal rates were $r_{1}=4.7716 \mathrm{mM}^{-1} \mathrm{~s}^{-1}$ for $\mathrm{Mn}_{3}\left[\mathrm{Co}(\mathrm{CN})_{6}\right]_{2} @ \mathrm{SiO}_{2}$ and $r_{1}=2.4224$ $\mathrm{mM}^{-1} \mathrm{~s}^{-1}$ for $\mathrm{Mn}_{3}\left[\mathrm{Co}(\mathrm{CN})_{6}\right]_{2} @ \mathrm{SiO}_{2} @ \mathrm{Ag}$. For transversal rates, the values were $r_{2}=166.0791 \mathrm{mM}^{-1} \mathrm{~s}^{-1}$ for $\mathrm{Mn}_{3}\left[\mathrm{Co}(\mathrm{CN})_{6}\right]_{2} @ \mathrm{SiO}_{2}$ and $r_{2}=75.5520 \mathrm{mM}^{-1} \mathrm{~s}^{-1}$ for $\mathrm{Mn}_{3}\left[\mathrm{Co}(\mathrm{CN})_{6}\right]_{2} @ \mathrm{SiO}_{2} @ \mathrm{Ag}$ under $3 \mathrm{~T}$ magnetic field. The authors attributed the reduction after Ag nanoparticles loading to the decrease of the connection area between water and $\mathrm{Mn}_{3}\left[\mathrm{Co}(\mathrm{CN})_{6}\right]_{2}$ inner structure. Besides, $-\mathrm{C} \equiv \mathrm{N}$ ligands in the structure are useful to enhance the fluorescent signal of the material incubated in A549 human lung cells in confocal laser scanning microscopy (CLSM), yielding blue, yellow-green and red fluorescence when the sample is excited with different single-photon laser wavelengths 403, 488 and $543 \mathrm{~nm}$ respectively. Furthermore, this material is also useful to improve two-photon fluorescence imaging (TPFI). This microscopy is more interesting than traditional confocal microscopy because autofluorescence is low and deep penetration and spatial resolution in three dimensions are achieved. The best results were shown by $\mathrm{Mn}_{3}\left[\mathrm{Co}(\mathrm{CN})_{6}\right]_{2} @$ $\mathrm{SiO}_{2} @$ Ag due to the coupling of plasmon resonance of silver nanoparticles and $\mathrm{Mn}_{3}\left[\mathrm{Co}(\mathrm{CN})_{6}\right]_{2}$, which also showed an enhancement in the intensity of TPFI. Finally, the PTT effect was tested showing that $0.05 \mathrm{mgmL}^{-1}$ treated with $808 \mathrm{~nm}$ laser radiation for 10 minutes was enough to reach $43{ }^{\circ} \mathrm{C}$, the critical temperature for this kind of therapy. The material also showed excellent photothermal stability with no change in its morphology. A cell viability study apparently showed no toxicity of the material and $80 \%$ of the HeLa and HepG2 cells survived, even at concentrations of $100 \mu \mathrm{g} \mathrm{mL}$. At a second stage, this study was carried out using NIR irradiation and cell viability was reduced to $16.92 \%$. This approach included the light to heat conversion process, which is able to kill cells through a DOX release effect accelerated by the thermal effect. This material is an ideal example of combination therapy and diagnosis CA nanoplatform. All along the procedure, the progress of the cancer treatment could be monitored through noninvasive clinical diagnosis techniques. 
The same group [32] have also developed a new theranostic nanopatform composed of Prussian blue nanocubes coated with the biodegradable MIL-101(Fe) metal-organic framework, in a dual-MOFs (d-MOFs) system. The nanoagents were prepared to an average size of $190 \mathrm{~nm}$ and loaded with an $85 \%$ of artemisin (ART), a traditional anticancer substance in Chinese medicine. The system is very similar to the one mentioned above - the ART release process was very slow but it accelerated as the $\mathrm{pH}$ level decreased or the temperature increased when the d-MOFs were exposed to the $808 \mathrm{~nm}$ laser light because of the presence of PB mentioned above. The synergy between ART chemotherapy and PTT was successfully tested in vitro in HeLa cells with a cell viability of $20.47 \%$ after the treatment. D-MOFs nanomedicine was also useful as a biological fluorescence marker for multicolour and two-photon bioimaging, as discussed in the previous example - this property was confirmed using human cervical carcinoma HeLa cells. The authors took advantage of the iron cations in the structure in order to shorten water proton relaxation rates $\left(r_{1}=1.313 \mathrm{mM}^{-1} \mathrm{~s}^{-1}, r_{2}=22.258 \mathrm{mM}^{-1} \mathrm{~s}^{-1}\right.$ at $\left.3 \mathrm{~T}\right)$ and checked the MRI enhancement of darkening and brightening in the material accumulation zones after I.V. injection of d-MOFs (100 $\mu \mathrm{L}, 5 \mathrm{mg} / \mathrm{mL})$ into HeLa tumour-bearing mice, which facilitated the mice tumour study. Finally, chemo-photothermal therapy was studied using these d-MOFs, which were able to produce a high tumour inhibition ratio. In view of the results, these systems were positioned as promising nanomedicines for the treatment of cancer in personalized therapies.

\section{Stimuli-Responsive Systems for Drug Delivery}

In recent years, stimuli-responsive nanodevices have emerged as promising drug delivery vehicles (DDSs) due to their capacity for transporting therapeutic agents to the target tissues with no previous release. This effect is possible due to the fact that these vehicles are able to control the release of the drug through a specific stimulus and to minimize undesired side effects. The stimuli responsible for activating the liberation of the therapeutic agent can be classified in a number of ways. In particular, we classify DDSs depending on the stimulus, (endogenous or exogenous) which activates the drug release mechanism [33-35]. An overview of the different types of endogenous/exogenous has been compiled in Figure 6.

Whereas there are various examples of stimuli-responsive MOFs, which can be used for drug delivery in the literature, it is more complicated to find 


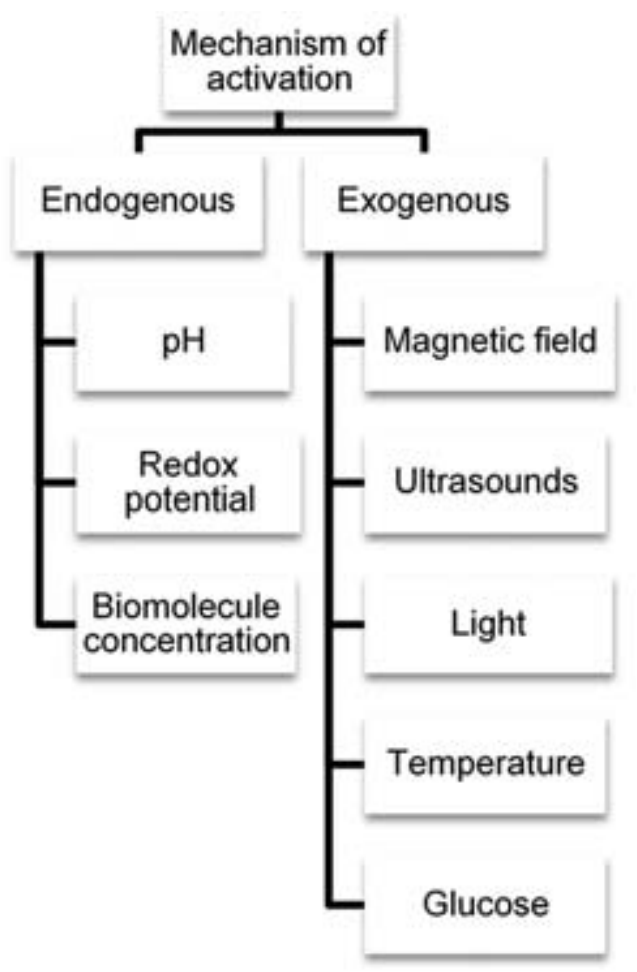

Figure 6. Different types of endogenous/exogenous stimulus.

examples of zeolites. In this section, we summarize the most important systems.

In a pioneering work, Nunzio and coworkers [36] developed a nanoMOF to deliver Topotecan, (TPT) which resembles a 'ship in a bottle' structure (Figure 7). In this work, the authors developed a MIL-10o nanoMOF by coordination self-assembly of $\mathrm{Fe}^{3+}$ octahedral trimers and trimesic acid (BTC) into hybrid supertetrehedra, (ST) which would assemble giving rise to a rigid micromesoporous zeotypic-like structure containing interconnected mesoporous small cages (SC) and large cages (LC) delimited by pentagonal and hexagonal openings respectively. Subsequently, TPT was filled into the pores by means of several consecutive impregnations of MIL-10o in the anticancer agent solutions. The entrapment mechanism could explain the ability of the drug monomers to penetrate into the hexagonal openings but not into the pentagonal windows and the molecules could be loaded in the large cages. Moreover, inside the MOF, the molecules of the monomer were able to aggregate in the large cages through stacking interactions. 

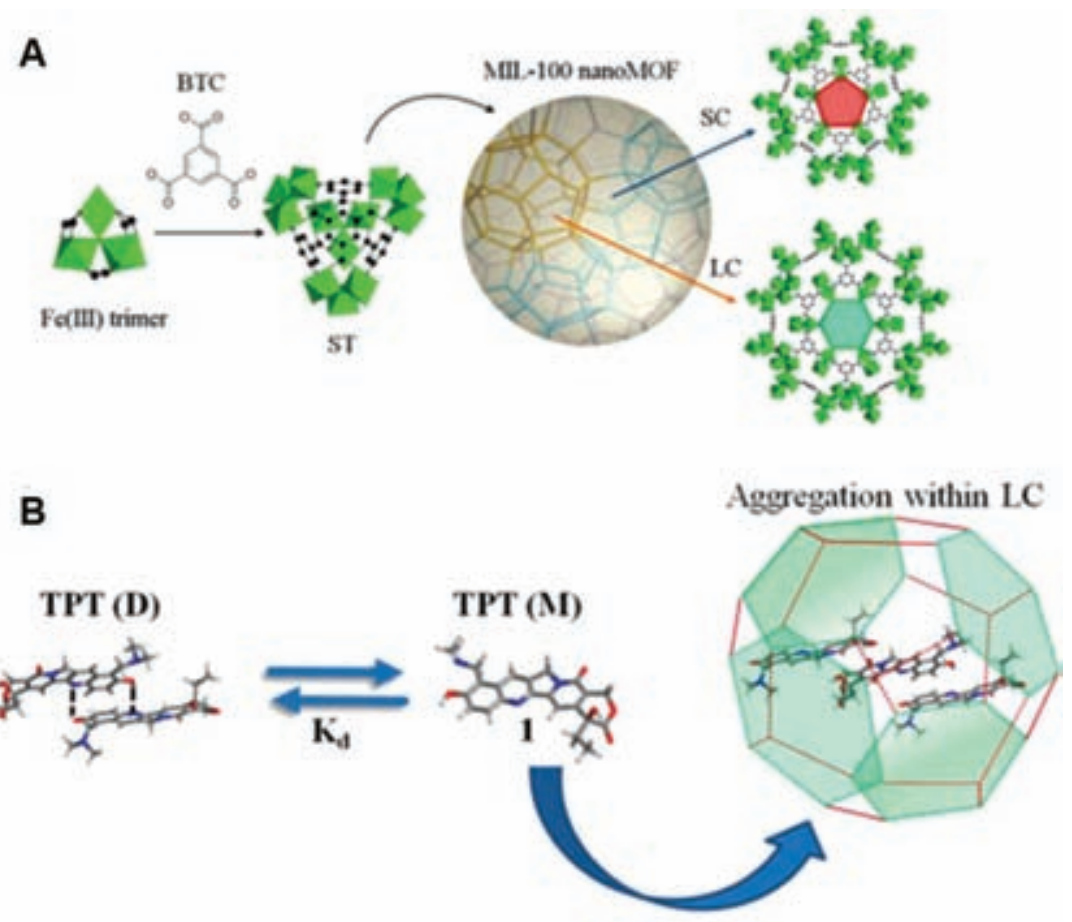

Hexagonal windows crossing

Figure 7. Schematic illustration of the synthesis and structure of MIL-100 nanoMOF structure. A) The nanoMOF possess small cages (SC) and large cages (LC). B) Schematic representation of the hypothesized entrapment mechanism of TPT inside of nanoMOF. Reprinted with permission from ref. 36. Copyright 2014 American Chemical Society.

This new DDS was based upon the one- (OPA) and two-photon absorption (TPA) mechanism to trigger and control the release of the cargo. This concept allows using the near-infrared region (NIR) instead of UV/Vis light as it leads to better tissue penetration because UV/Vis irradiation can damage cells and make it more difficult for the light to penetrate the tissue. In vitro efficacy assays demonstrated that MOFs with no TPT load did not affect the biological activity against A549 alveolar adenocarcinoma and $\mathrm{MiaPaCa} 2$ human pancreatic cell lines whereas the nanoMOF loaded with TPT induced cell death with half maximal inhibitory concentration $\left(\mathrm{IC}_{50}\right)$, which were values higher than those observed for the naked drug against the PANCı human pancreatic cell line. One hypothesis to explain the previous effect could point towards the interaction between the nanoMOF and cells, which allows the drug to be transported inside the cells in order to exert the antitumor activity. 


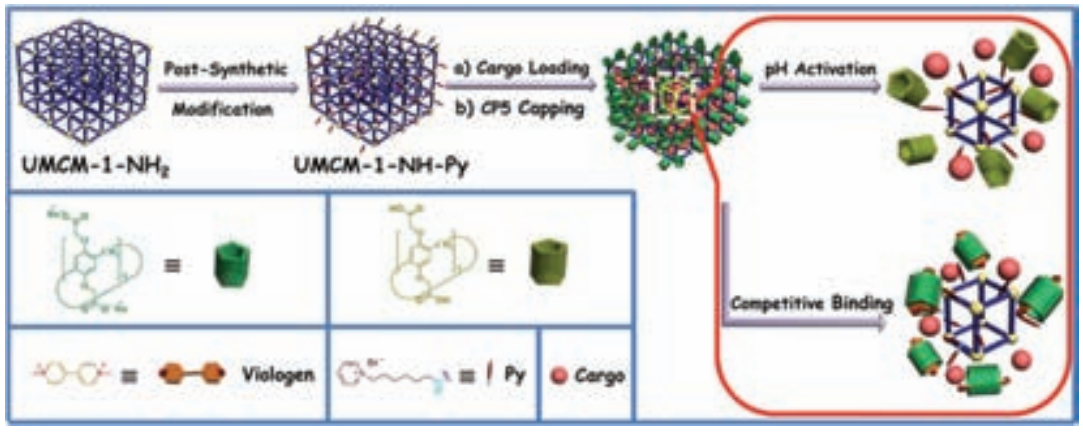

Figure 8. Schematic representation of the stimuli-responsive system based on UMCM-1-NH $\mathrm{Nod}_{2}$ ified with positively-charged pyridinium (Py) stalks and CP5 on the surface. This system, under pH or competitive binding can regulate the release of cargo molecules. [37] - Published by The Royal Society of Chemistry.

On the other hand, $\mathrm{pH}$-responsive MOFs have been prepared by combining post-synthetic modification (PSM) with stimuli-responsive host-guest chemistry. These MOFs were obtained via post-synthetic modification (PSM), due to the fact that the positively charged pyridinium stalks (py) were tethered onto the surface of UMCM-1- $\mathrm{NH}_{2}$ [37]. Then, rhodamine $6 \mathrm{G}$ was loaded into the nanopores, followed by introduction of the negativelycharged carboxylatepillar[5]arene $\left(\mathrm{CP}_{5}\right)$ via host-guest complexation so as to cap the surface of the nanoMOF (Figure 8). Rh6G release was compared at different $\mathrm{pH}$ values, concluding that nanoMOFs presented a much faster drug release at acid $\mathrm{pH}$ because when the $\mathrm{pH}$ level changed, the pores of the structure opened. Moreover, the study of the release of the cargo through the addition of a competitive binding agent, such as methyl viologen, shows that the release of Rh6G depends on the amount of methyl viologen, which dethreaded the $\mathrm{CP}_{5}$ rings from the Py, causing the drug to release more easily. In vitro cytotoxicity assays in human embryonic kidney cells 293 demonstrated that these materials possessed negligible toxicity.

Recently, a novel water-stable zirconium MOF (Zr-MOF) functionalized with azobenzene moieties was developed as an exclusive structure for on-command drug delivery, which can be activated by different types of external stimuli [38]. To study the release of the cargo, these light-triggered MOFs were loaded with Rhodamine $\mathrm{B}(\mathrm{RhB})$ in the pore interiors and the surface was functionalized with $\beta$-cyclodextrin $(\beta-C D)$ rings, which were threaded onto the azobenzene stalks so as to cap the nanopores and stop the release of the cargo. Upon irradiation with UV light, the isomerization of azobenzene molecule from trans- to cis-form caused the dissociation of the $\beta$-CD ring from the azobenzene stalks. The cis-isomer shows a decreased 
affinity for the $\beta$-CD rings in comparison to trans- isomer, allowing the opening of the nanopores and releasing the cargo. In another experiment, the authors studied the release of the cargo through the addition of a competitive binding agent, such as amantadine. In this case, the RhB release mechanism is based on the competition between amantadine and azobenzene for binding $\beta$-CD rings. Amantadine binds strongly to $\beta$-cyclodextrin, inducing the disruption of the $\beta-\mathrm{CD}$ rings from the azobenzene stalks. Moreover, the combination of UV irradiation after the addition of amantadine can improve the amount of RhB released, due to the fact that the trans- to cis- isomerization impels an extra discharge of RhB outwards of the nanopores.

\section{Remarks on the Future}

MOFs are hybrid porous solids formed by the self-assembly between metal ions or clusters and organic ligands. These structures present a number of advantages such as tunable pore size and shape, large surface areas, compositional and structural diversity and biodegradability as a result of the relatively labile metal-ligand bonds. The main characteristic of MOFs is their large pore size, which allows loading a high amount of therapeutic agents. Another advantage of these vehicles is the variety of low-toxic metal ions and organic ligands. All of which are elected to improve biocompatibility and control the stability of the material matrix and the degradation of the structure.

Although more efforts and improvements are needed to test in vitro the behavior of MOFs in physiological conditions and simulated body fluids in order to identify potential hazards associated with their components and it is necessary to evaluate in vivo their degradation mechanisms, biocompatibility and toxicity, their characteristics make them an exceptional tool for future application in the field of nanomedicine.

Zeolites on the other hand, are crystalline inorganic solid materials that possess a three dimensional structure with channels and cavities of uniform size. These structures present a number of properties, such as tunable pore size and shape, large surface areas, bi-modal porosity, diverse morphology and stability in suspension among others. They can serve as transport for therapeutic agents inside the pores or on their surface. Another important characteristic is their low cytotoxicity, which has been reported in the literature. 
In this chapter, we have tried to reflect some of the most interesting characteristics and advantages of zeolites and MOFs, which make them attractive as the object of further investigation in the field of biomedicine on the grounds of the improvements that these kinds of materials can contribute to nanomedicine.

\section{Acknowledgments}

The authors would like to thank the support of Prof. Joan Ripoll-Moragón in developing this chapter. A. Cabrera-García expresses his thankfulness to La Caixa Foundation for granting him a Ph.D. scholarship, Z. Díaz-Betancor is deeply grateful to the Spanish Ministry of Economy and Competitiveness for her scholarship, Dr. E.M. Rivero-Buceta would like to express her gratitude to the Cursol Foundation for her post-doctoral scholarship. Finally, all of us owe a special debt to Dr. Pablo Botella, whose teachings have made it possible for the entire team to develop this project.

\section{References}

[1] Grobmyer, S.R., Moudgil, B.M. (Eds.) Cancer Nanotechnology, Methods and Protocols, ch.3, Springer, 2010.

[2] Giménez-Marqués, M., Hidalgo, T., Serre, C., Horcajada, P. Coord. Chem. Rev. 2016, 307, 342.

[3] He, C., Liu, D., Lin. W. Chem. Rev. 2015, 115, 11079.

[4] Tiwari, G., Tiwari, R., Sriwastawa, B., Bhati, L., Pandey, S., Pandey, P., Bannerjee, S.K. Int. J. Pharm. Investig. 2012, 2, 2.

[5] Rimoli, M.G., Rabaioli, M.R., Melisi, D., Curcio, A., Mondello, S., Mirabelli, R., Abignente, E.J. Biomed. Mater. Res. A 2007, 156.

[6] Vilança, N., Amorim, R., Machado, A.F., Parpot, P., Pereira, M.F.R., Sardo, M., Rocha, J., Fonseca, A.M., Neves, I.C., Baltazar, F. Colloids Surf. B Biointerfaces 2013, 112, 237.

[7] Gao, X., Hai, X., Baigude, H., Guan, W., Liu, Z. Sci. Rep. 2016, 6, 37705.

[8] Zhu, Y.D., Chen, S.P., Zhao, H., Yang, Y., Chen, X.Q., Sun, J., Fan, H.S., Zhang, X.D. ACS Appl. Mater. Interfaces 2016, 8, 34209.

[9] Cabrera-García, A., Vidal-Moya, A., Bernabeu, Á., Pacheco-Torres, J., Checa-Chavarria, E., Fernández, E., Botella, P. Nanomaterials 2016, 6, 109.

[10] Hatakeyama, W., Sanchez, T.J., Rowe, M.D., Serkova, N.J., Liberatore, M.W., Boyes, S.G. ACS Appl. Mater. Interfaces 2011, 3, 1502.

[11] Huang, S.D., Basu, S., Khitrin, A.K., Shokouhimehr, M., Soehnlen. E.S. US Patent 20100215587, 2010.

[12] Tiwari, A., Nordin, A.N. (Eds.) Advanced Biomaterials and Biodevices, ch. 12, John Wiley \& Sons, 2014.

[13] Guari, Y., Larionova, J., Corti, M., Lascialfari, A., Marione, M., Poletti, G., Molvinger, K., Guérin. C. Dalton Trans. 2008, 3658. 
[14] Roy, X., Hui, J.K.H., Rabnawaz, M., Liu, G., MacLachlan, M.J.J. Am. Chem. Soc. 2011, 133, 8420.

[15] Fu, G., Liu, W., Feng, S., Yue, X. Chem. Commun. 2012, 48, 11567.

[16] Dabrowiak, J.C. Metals in Medicine, Ch. 9, John Wiley \& Sons, 2009.

[17] Dekrafft, K.E., Xie, Z.G., Cao, G.H., Tran, S., Ma, L.Q., Zhou, O.Z., Lin, W. Angew. Chem. Int. Ed. 2009, 48, 9901.

[18] Zhang, W., Li, B., Ma, H., Zhang, L., Guan, Y., Zhang, Y., Zhang, X., Jing, P., Yue, S. Acs Appl. Mater. Interfaces 2016, 8, 21465.

[19] Mortazavi, S.M.J., Tavasoli, A., Atefi, M., Tanide, N., Radpey, N., Roshan-shomal, P., Moradi, H., Taeb, S. World J. Emerg. Med. 2013, 4, 123.

[20] Wang, Y., Zhu, Y., Binyam, A., Liu, M., Wu, Y., Li, F. Biosens. Bioelectron. 2016, 86, 432.

[21] He, C., Lu, J., Lin. W.J. Control. Release 2015, 219, 224.

[22] He, C., Poon, C., Chan, C., Yamada, S.D. Lin, W.J. Am. Chem. Soc. 2016, 138, 6010.

[23] Lülf, H., Bertucci, A., Septiadi, D., Corradini, R., De Cola, L. Chem. Eur. J. 2014, 20, 10900.

[24] He, C., Duan, X., Guo, N., Chan, C., Poon, C., Weichselbaum, R.R., Lin, W. Nat. Commun. 2016, 7, 12499 .

[25] Lu, K., He, C., Guo, N., Chan, C., Ni, K., Weichselbaum, R.R., Lin, W.J. Am. Chem. Soc. 2016, $138,12502$.

[26] Liu, J., Yang, Y., Zhu, W., Yi, X., Dong, Z., Xu, X., Chen, M., Yang, K., Lu, G., Jiang, L., Liu, Z. Biomaterials 2016, 97, 1.

[27] Rieter, W.J., Taylor, K.M.L., An, H., Lin, W., Lin, W.J. Am. Chem. Soc. 2006, 128, 9024.

[28] Taylor, K.M.L., Jin, A., Lin, W. Angew. Chem. Int. Ed. 2008, 47, 7722.

[29] Tian, C., Zhu, L., Lin, F., Boyes. S.G. ACS Appl. Mater. Interfaces 2015, 7, 17765.

[3o] Horcajada, P., Chalati, T., Serre, C., Gillet, B., Sebrie, C., Baati, T., Eubank, J.F.E., Heurtaux, D., Clayette, P., Kreuz, C., Chang, J.S., Hwang, Y.K., Marsaud, V., Bories, P.N., Cynober, L., Gil, S., Férey, G., Couvreur, P., Gref. R. 2010, 9, 172.

[31] Wang, D., Guo, Z., Zhou, J., Chen, J., Zhao, G., Chen, R., He, M., Liu, Z., Wang, H., Chen, Q. Small 2015, 11, 5956 .

[32] Wang, D., Zhou, J., Chen, R., Shi, R., Zhao, G., Xia, G., Li, R., Liu, Z., Tian, J., Wang, H., Guo, Z., Wang, H., Chen, Q. Biomaterials 2016, 100, 27.

[33] Ganta, S., Devalapally, H., Shahiwala, A., Amiji, M.J. Control. Release 2008, 126, 187.

[34] Botella, P., Rivero-Buceta, E.J. Control. Release 2017, 247, 28.

[35] Mura, S., Nicolas, J., Couvreur, P. Nat. Mater. 2013, 12, 991.

[36] Nunzio, M.R., Agostini, V., Cohen, B., Gref, R., Douhal A.J. Med. Chem. 2014, 57, 411.

[37] Tan, L.L, Li, H., Qiu, Y.C., Chen, D.X., Wang, X., Pan, R.Y., Wang, Y., Zhang, S.X.A., Wang, B., Yang, Y.W. Chem. Sci. 2015, 6, 1640.

[38] Meng, X., Gui, B., Yuan, D., Zeller, M., Wang, C. Sci. Adv. 2016, 2, e160048o. 


\title{
11. Zeolites and MOFs as Catalysts in Fine Chemical Reactions
}

\author{
Francisco G. Cirujano ${ }^{1}$ and Anna Nowacka ${ }^{2}$ \\ ${ }^{1}$ Centre for Surface Chemistry and Catalysis, KU Leuven. \\ ${ }^{2}$ Instituto de Tecnología Química, Universitat Politècnica de \\ València-Consejo Superior de Investigaciones Científicas.
}

Keywords: Turnover frequency (TOF), stereoselectivity, isomerization, selective oxidation, reduction

\section{Introduction}

During the last centuries, many people did not survive diseases that can be easily treated today by the use of pharmaceuticals such as antibiotics. On the contrary, this century developed countries face an aging revolution, suffering from neurodegenerative diseases and a higher proliferation of different types of cancers. The industrial synthesis of fine chemicals such as pharmaceuticals, perfumes, cosmetics and flavourings is a trillion dollar market with the aim of finding solution to such health problems. Although every year new drugs appear in order to better treat this problems, the high complexity of the pharmacologically active compounds requires expensive and hazarous chemical processes to produce them, which has a negative environmental impact. Due to the large volume of production and, more importantly, the high cost of the processes, it is necessary to develop more efficient catalysts able to increase the yield of the desired product. In relation to the low efficiency of the synthesis of pharmaceuticals, it is estimated that the kilograms of residue generated per kilogram of pharmaceutical component is between 20 and 100 (see $\mathrm{E}$ factor later on this chapter). Obviously it is contradictory that in order to solve health problems, we are affecting our health by the way we are doing those chemical processes.

If we observe nature, we find that bioactive compounds are naturally produced by plants, using just water and minerals from the soil and $\mathrm{CO}_{2}$ from the air. Their chemical synthesis under the most efficient and clean imaginable ways is possible by microscopic machines present in all cells 


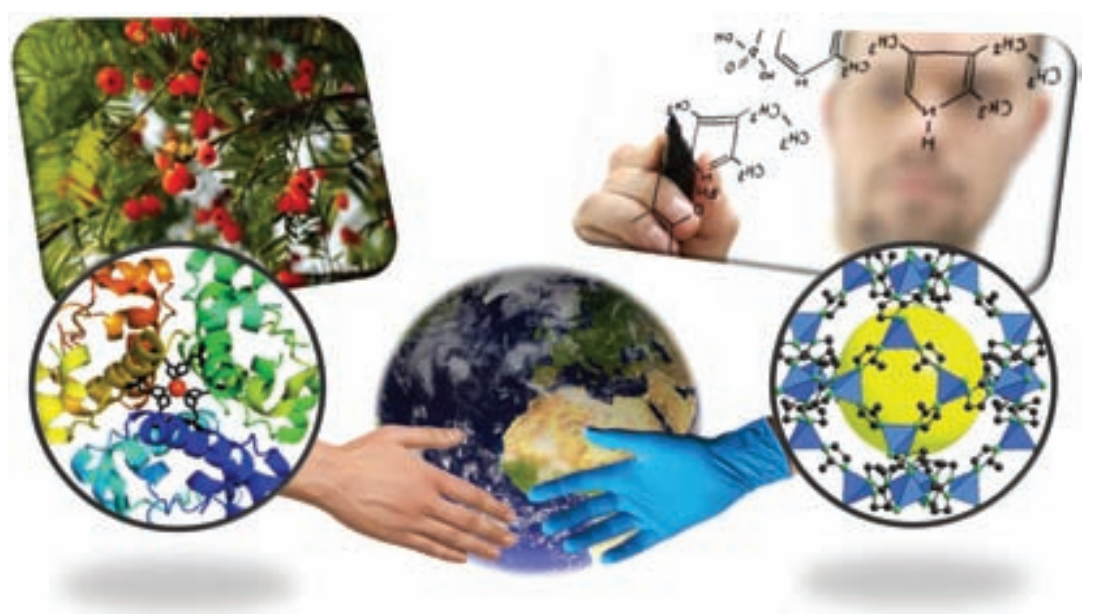

Figure 1. Sustainable synthesis of pharmaceuticals and fine chemicals through the use of porous materials designed and synthesized with structures and functionalities similar to those found in natural enzymes. Adapted with permission from Acc. Chem. Res., 2010, 43, 58. Copyright 2010 American Chemical Society.

of living creatures. These naturally evolved materials, known as enzymes, are able to perform the synthesis of complex molecules that find application as pharmaceuticals. By bonding together different building blocks in a porous solid, we use them as catalysts to promote the synthesis of pharmaceutically active compounds by clean chemical reactions under mild conditions. The materials have a sponge like porous structure that absorb and concentrate the pharmaceutical precursors. This favours the synthetic chemical reactions inside the material pores, which act as microscopic cages with dimensions similar to that of the desired pharmaceutical molecule. The final goal is to be as efficient as natural enzymes using more robust materials that withstand a broader range of reaction conditions, minimizing the environmental impact of the chemical transformations employed to produce affordable pharmaceutical treatments for future generations (Figure 1).

Therefore, selective catalysts are used to minimize the amount of by-products, behaving in a similar way to natural enzymes. Traditionally, homogeneous catalysts have been used in order to obtain the desired product in a reasonable amount of time and with a high selectivity. In this sense, homogeneous acids/bases and oxidants/reductants have been used in stoichiometric or catalytic amounts in multiple synthetic processes involving isomerization, condensation, oxidation or hydrogenation steps. However, when using these homogeneous catalysts, the costly separation of the 
catalyst and the neutralization-purification steps of the desired compound decrease the efficiency of the process due to waste formation. R. Sheldon defines waste as "everything but the desired product", with the exception of water. One way to quantify the amount of waste produced in a chemical synthesis is by the $\mathrm{E}$ (from environmental) factor as the mass of waste divided by the mass of product [1]. Once the waste produced is known, the $\mathrm{E}$ factor can be minimized using heterogeneous catalysis. Although the amount of chemicals produced in the fine chemical and pharmaceutical industries are not relatively high (compared with bulk chemicals), the E factor is about 1 or 2 orders of magnitude higher. To achieve sustainable fine chemicals and pharmaceuticals manufacture, the use of stoichiometric (mainly inorganic) reagents in organic synthesis needs to be substituted by (preferably heterogeneous) catalysts. However, due to the small volumes involved and to the use of the least expensive reagent, the implementation of waste reduction has not been as extensive as in the bulk chemicals industry. Nevertheless, in the last two decades more emphasis has been placed on the use of catalytic methods in organic synthesis. The particular use of MOFs and zeolites as heterogeneous catalysts for this type of organic transformations in order to improve their sustainability is illustrated in this chapter.

\section{MOFs and zeolites in acid catalysed reactions}

The main problem of typical homogeneous Lewis acid-catalysed reactions is the difficult handling of the catalysts due to the hazardous nature and high reactivity of such soluble compounds. Moreover, they have to be decomposed before their isolation from the reaction mixture in order to purify the product, which makes it impossible to reuse it and produces undesired wastes. Therefore, there is an urgent necessity to develop heterogeneous solid acid catalyst, to make the process more cost-effective with the possibility of reuse, operation in a continuous mode and to reduce the environmental impact. Porous solid materials like MOFs or Zeolites are ideal candidates for use as catalysts in this type of reaction, not only because of the presence of active Lewis and Brønsted acid sites in their structures, but also for the possibility of tuning them for the desired application. In the following, we provide examples of materials containing Lewis and Brønsted acids sites focusing on their catalytic application in fine chemistry. This chapter serves as a general introduction to the topic, readers are referred to reviews and references for a more detailed overview $[2,3-6]$. 


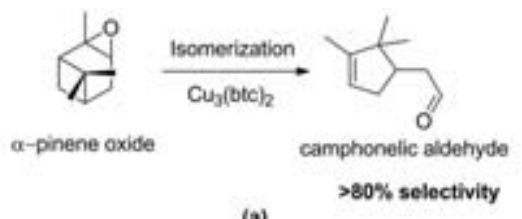

(a)

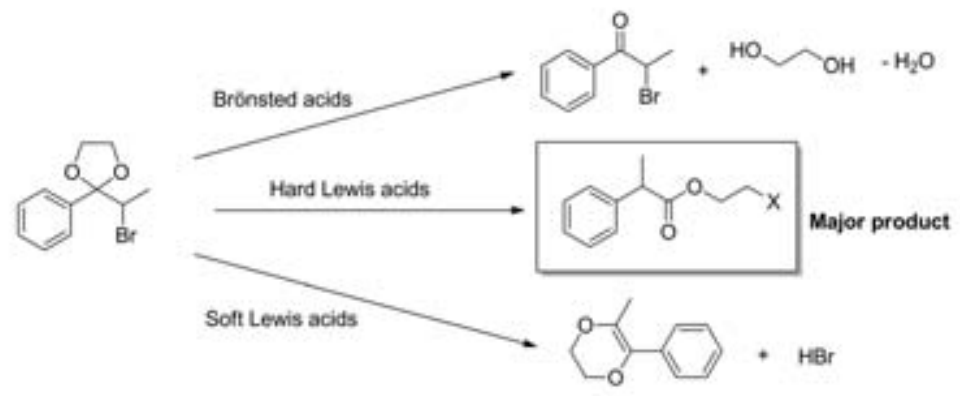

(b)

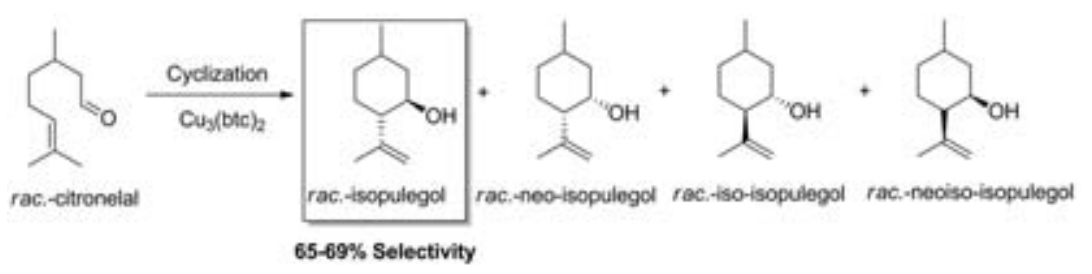

(c)

Figure 2. a) Isomerization of a-pinene oxide to campholenic aldehyde as the main product in the presence of Lewis acid sites of CUBTC, b) citronellal isomerization or cyclization towards the four different pulegols isomers (isopulegol as the main product) and c) reaction of 2-bromopropiophenone as a test to the nature and strength of the acid sites.

Typical reactions catalysed by Lewis and/or Brønsted acids sites are isomerizations. These are processes in which one molecule is transformed into another molecule, with exactly the same atoms, but a different arrangement (Figure 2, part a and c). There are multiple examples of isomerizations taking place in organic synthesis of fine chemicals such as fragrances or pharmaceuticals, especially in the terpene fraction of the biomass. For example, $\alpha$-pinene oxide or citronellal isomerization are well-studied reactions, that produce important fragrances. They are also popular test reactions to estimate the Brønsted or Lewis acid nature of the sites present in a solid catalyst. The strength of those Lewis acids can be estimated by using a cyclic acetal as a test substrate in the presence of the 
Lewis acids. The isomerization of $\alpha$-pinene oxide (Figure 2a) is a conventional way of producing campholenic aldehyde, which is an important intermediate used in the fragrance and pharmaceutical industry. Moreover, this reaction is a sensitive probe for the Lewis/Brønsted acid nature of the catalysts [7]. Briefly, Lewis acid sites catalyse the formation of campholenic aldehyde while the presence of Brønsted acidity lowers the selectivity towards this product. Dirk de Vos et al., [8] extended the study of the acid properties of $\mathrm{CuBTC}(\mathrm{BTC}=1,3,5$-benzenetricarboxylate) by determining which type of acid centres were present (Brønsted or Lewis, hard or soft). High selectivities in the isomerizations of $\alpha$-pinene oxide and citronellal clearly identify $\mathrm{Cu}_{3}(\mathrm{BTC})_{2}$ as a material with Lewis acid sites. By using the cyclic acetal of 2-bromopropiophenone as a test substrate, it was demonstrated that these sites are hard Lewis acid sites (Figure 2b). Results obtained for isomerization of $\alpha$-pinene oxide over some zeolites USY [9] and Ti-Beta [10] generates a $75 \%$ and $94 \%$ selectivity towards campholenic aldehyde at $100 \%$ and $95 \%$ conversion, respectively. A similar observation was made by Vermoortele et al., who used post-synthetic acid treatment (either $\mathrm{HClO}_{4}$ or $\mathrm{CF}_{3} \mathrm{COOH}$ ), to activate MIL-10o(Fe) [11] and test their catalytic performance in the $\alpha$-pinene oxide isomerization. Defect creation in acid modified materials, results in an increase of the Lewis and Brønsted acidity. During the reaction both, conversion and selectivity to campholenic aldehyde decrease was observed. This can be attributed to the increased amout of protonated carboxylate ligands inside cages of MOF, which might serves as weak Brønsted acid sites.

Cyclization of (+)-citronellal to (-)-isopulegol (Figure 2c) is an important intermediate reaction in (-)-menthol production. [12] Menthol is a naturally occurring compound in the essential oil of mint leaves, which is used for its medicinal, sensory and fragrant properties due to its action within the central nervous system. The generation of different diastereoisomeric products require not only active, but also diastereoselective heterogeneous catalyst. Citronellal cyclization to isopulegol is catalysed by both strong Lewis and weak Brønsted acid sites. Sn-Beta was employed for the first time in a reaction that involves carbon-carbon bond formation. The catalytic performance of the Sn Lewis acid sites in the zeolite matrix is much superior to conventional heterogeneous catalysts used for this reaction (Table 1). Moreover, this catalyst does not require the usual precautions against humidity needed for normal Lewis acids. The stability of the Sn-Beta zeolite makes the catalyst suitable for applications in a fixed bed continuous reactor [13] The superiority of the tin over other Lewis acids introduced into the zeolite framework is shown in Table 1 , where Ti-Beta gave lower conversion 
Table 1. Catalytic isomerization of citronellal using zeolites and MOFs.

\begin{tabular}{llccc}
\hline & Catalyst & Conversion & S $_{\text {isopulegol }}$ & TOF $\left(\mathbf{h}^{-1}\right)$ \\
\hline 1 & Sn-Beta & $99 \%(1 \mathrm{~h})$ & $83 \%$ & 4575 \\
2 & Al-Beta & $50 \%(1 \mathrm{~h})$ & $53 \%$ & 73 \\
3 & Ti-Beta & $35 \%(1 \mathrm{~h})$ & $66 \%$ & 112 \\
4 & Cr-MIL-101 & $100 \%(18 \mathrm{~h})$ & $74 \%$ & $>1$ \\
5 & Pure Silica Beta & $0 \%(1 \mathrm{~h})$ & - & - \\
6 & 20\%HPW/MCM-41 & & \\
\hline
\end{tabular}

(entry 3). Additionally, the diastereoselectivity with respect to the isopulegol is much lower for the titanium containing material (compare entries 1 and 3). Brønsted-acidic Beta zeolite with a comparable hydrophobicity as Sn-Beta in the same reaction conditions also gave only cyclization without any side-products. However, the diastereoselectivity towards the desired product was again lower (entry 2 ) and the conversion was only $50 \%$ vs. full conversion for Sn-Beta. The origin of the activity in the Sn-Beta can be attributed to the tin since the all-silica Beta sample has no activity (entry 5).

Chuah and coworkers investigated the catalytic activity of Zr-zeolite Beta in this reaction [15] showing good activity for isopulegols formation, with more than $97 \%$ selectivity and high diastereoselectivity for isopulegol $(\sim 93 \%)$. The presence of Lewis and Brønsted acid sites, involved in the reaction mechanism was confirmed also by pyridine IR studies. MOF materials with Lewis acid sites, like MIL-101 [16] and $\mathrm{Cu}_{3}(\mathrm{BTC})_{2}[17]$ also have been tested. However they presented lower activity than Lewis acid zeolites (entry 4) or encapsulated heteropolyacids (entry 6) [14]. When the authors used the acid modified MIL-10o( $\mathrm{Fe}$ ) to catalyse the isomerization of citronellal to isopulegol, they observed that the selectivity to isopulegol increased as more Brønsted acid sites were created, and attributed this observation to a dual Lewis-Brønsted acid site mechanism. However, lower diastereoselectivity for this material was achieved, compared to Zr- or Sn-beta zeolites.

\section{MOFs and zeolites in base catalyzed reactions}

As mentioned in the introduction, the pharmaceutical and chemical industries are continuously searching for processes with a lower environmental impact, not only for the use of greener solvents and reactants but also to limit the number of steps while safeguarding the purity and safety of high-quality but affordable therapeutic molecules. For most of the $\mathrm{C}-\mathrm{C}$ forming reactions 
that generate important fine chemicals and pharmaceuticals, stoichiometric amounts of base are normally required. However, the soluble base used as a catalyst (normally alkali, alkali earth metals, ammonium or organic basic salts) requires additional synthetic steps of neutralization and separation from the reaction mixture. Therefore, the use of heterogeneous catalysts simplifies the purification of the product and removal of the homogeneous catalyst counterpart. In fact, a deep knowledge has been achieved on the design of heterogeneous basic catalysts for carbon-carbon bond formation through condensation reactions [18-25]. One interesting example is the synthesis of jasminaldehyde perfume by the aldol condensation between benzaldehyde and heptanal. Vermoortele et al. [26] have described the use of the zirconium MOF UiO-66- $\mathrm{NH}_{2}$ as a bifunctional acid-base catalyst for the cross-aldol condensation between benzaldehyde and heptanal to produce jasminaldehyde. The amino groups of the ligand were proposed as the basic sites, while the acid sites were created in situ by controlled dehydroxylation of the material, creating coordination vacancies shared by three $\mathrm{Zr}^{4+}$ cations in the triangular faces of the inorganic cluster. The yields and conversions obtained with the UiO-66 dehydroxylated samples (Table 2, entries 1-4) were lower than those of bifunctional AlPOs [27] and various hydrotalcites [28] previously described (entries 5 and 6).

Condensation reactions, such as the aldol condensation just mentioned, are able to make $\mathrm{C}$ - $\mathrm{C}$ bonds in the synthesis of complex fine chemicals by the use of basic catalysts. The most frequently applied reaction for testing basic catalysts is a $\mathrm{C}-\mathrm{C}$ bond forming reaction known as the Knoevenagel reaction.

Table 2. Aldol condensation between benzaldehyde and heptanal performed on different catalysts.

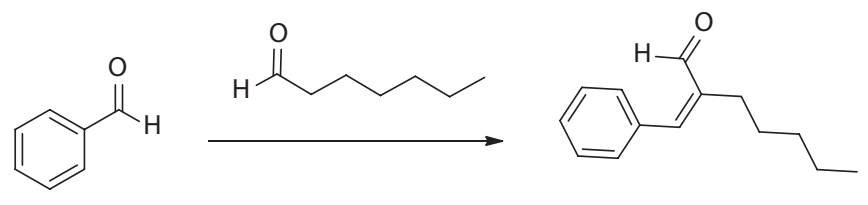

Jasminaldehyde

\begin{tabular}{llcll}
\hline & Catalyst & Conversion & $\mathbf{S}_{\text {Jasminaldehyde }}$ & TOF $\left(\mathbf{h}^{-1}\right)$ \\
\hline 1 & UiO-66 Treated at 423 K in air & $30 \%(1 \mathrm{~h})$ & 85 & 0.5 \\
2 & UiO-66 Treated at 573 K in vacuum & $42 \%(1 \mathrm{~h})$ & 80 & 0.7 \\
3 & UiO-66- $\mathrm{NH}_{2}$ Treated at 423 K in air & $67 \%(1 \mathrm{~h})$ & 92 & 1.1 \\
4 & UiO-66- $\mathrm{NH}_{2}$ Treated at 473 K in air & $38 \%(1 \mathrm{~h})$ & 92 & 0.6 \\
5 & ALPO & $96 \%(3 \mathrm{~h})$ & 83 & 1.5 \\
6 & Hydrotalcite & $98 \%(8 \mathrm{~h})$ & 84 & 1 \\
\hline
\end{tabular}


The results of Corma and coworkers indicate that on AlPO surface, weak acid and basic sites coexist in adequate proportion in such way it should be possible that an acid-base bifunctional catalysis was operating [20]. Gascón et al. [29] demonstrated that MOFs with non-coordinated amino groups, such as IRMOF-3 can be use as solid basic catalysts for the Knoevenagel condensation of ethyl cyanoacetate with benzaldehyde. The results obtained with this material were good, attaining a yield of $99 \%$ after $2 \mathrm{~h}$, comparable with other MOF catalysts reported so far. However, the activity of inorganic solids with mobile $\mathrm{OH}^{-}$ions is much higher due to the favoured abstraction of the proton in alpha to the carbonyl group in ethyl cyanoacetate $[30,31]$.

\section{MOFs and zeolites in oxidation reactions}

Selective oxidation reactions are important processes in the fine chemical industry due to the possibility of functionalizing $\mathrm{C}$-C bonds. Metal-catalysed oxidation processes can proceed through several kinds of mechanisms. In general, they can be simplified into two types: homolytic and heterolytic ones [32]. In homolytic oxidations, metal is oxidized outside the coordination sphere via a radical chain mechanism. This one-electron redox step set the role of catalyst as generator of organic radicals form substrate that react with molecular oxygen by an 'auto-oxidation' mechanism [33], forming peroxo radicals, able to initiate a new radical chain via the abstraction of hydrogen atom forming next substrate molecule (Figure 3). Heterolytic oxidations, involve substrate/oxidizing reagent activation for the nucleophilic attack, by coordination to the metal clusters. This is a two-electron redox change, where metal acts as a Lewis acid. In this section, we present examples of MOFs and Zeolites used in different oxidation reactions. Special attention is given to the selectivity and reusability of materials, and use of green oxidants like air, molecular oxygen and peroxides.

Thanks to the diversity of MOFs, many transition metals i.e. $\mathrm{Cr}, \mathrm{Mn}$, $\mathrm{Fe}, \mathrm{Co}, \mathrm{Cu}$ and $\mathrm{Ti}$ are usually used as oxidation catalyst once incorporated in the framework structure. This research is reflected by various reviews [34-36], where MOFs have been used to promote oxidation. Asefa and $\mathrm{Li}$ [37], have reported cobalt based MOF $\left[\mathrm{Co}(\mathrm{OBA})_{2}\left(\mathrm{H}_{2} \mathrm{O}\right)_{2}\right]\left(\mathrm{H}_{2} \mathrm{OBA}=\right.$ 4,4'-oxydibenzoic acid) as a highly active catalyst, for olefin epoxidation reactions. The material consists of a flexible $2 \mathrm{D}$ layered structure, which makes all of the active metal sites easily accessible for reactants. Water in the structure can be easily thermally removed, to give highly reactive open $\mathrm{Co}(\mathrm{II})$ centers (Lewis acid sites). The activity of dehydrated MOF was 
tested in solvent-free conditions and different aromatic olefins, with TBHP (tert-butyl hydroperoxide) as oxidant. In the case of styrene, the catalyst yielded $96 \%$ conversion and $96 \%$ of selectivity to styrene oxide at $75^{\circ} \mathrm{C}$. The reactivity of the epoxide ring makes these compounds important intermediates in the synthesis of fine chemicals and pharmaceuticals. To confirm the heterogeneous nature of this catalyst, the MOF catalyst was recovered and reused four times. A hot filtration experiment was also provided (in 4 cycles). Repeated reaction cycles gave similar conversions but slightly lower selectivity and some leaching of $\mathrm{Co}^{2+}$ was observed in the filtrate.

MIL-101(Cr) was reported for the first time by G. Férey [38] and is characterized by big pore size and surface area. It has a zeo-type cubic structure with very large cell volume (702,000 cubic angstroms). It is also claimed, to be one of the most stable MOFs. All these features, make it very promising material for liquid-phase oxidation reactions $[39,40,36]$, where big pore size can be helpful, to avoid diffusion control during the process. H. Garcia and coworkers reported the MOF MIL-101(Cr) as an active catalyst in benzylic oxidation of hydrocarbons [41]. In this experiment, indane was used as a model compound for oxidation, with molecular oxygen as the only oxidizing agent. The indanone product is part of the steroid biosynthesis and extensive studies on bioactivity of 1-indanone derivatives open up more and more new possibilities of their applications as pharmaceutically active ingredients. Selectivity, towards the alcohol/ketone mixture was higher for MIL-101(Cr) (87\% selectivity, 30\% conversion), compared to the same reaction on MIL-101(Fe) (71\% selectivity at $30 \%$ conversion) at $120^{\circ} \mathrm{C}$. The catalytic activity and crystallinity of this material, remains stable for up to four reuses. In the reaction mechanism, the metal nodes of the lattice interact with oxygen, forming metalloperoxides able to abstract a hydrogen

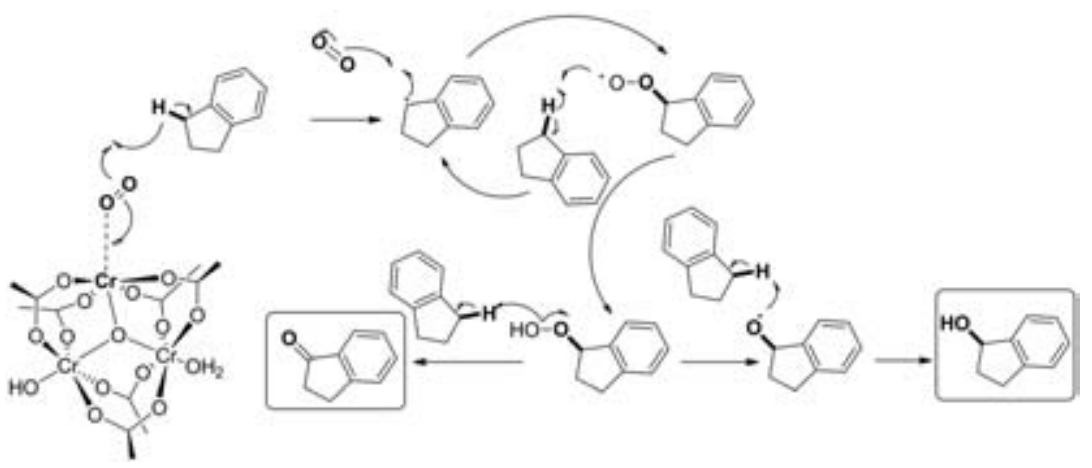

Figure 3. Mechanism proposed for the selective oxidation of alkenes to alcohols and ketones in the presence of oxygen and the $\mathrm{Fe}(\mathrm{Cr})-\mathrm{MIL}-101$ catalyst. 
atom form the benzylic position (Figure 3). High selectivity in this process can be explained, by reaction taking place inside the MOF pores, which favours adsorption of indane, and desorption of more polar products like indanol and indanone.

A copper-based MOF was reported by Pombeiro and coworkers, for selective oxidation of cyclopentane and cyclohexane [42]. The reaction was carried out at room temperature and atmospheric pressure, using hydrogen peroxide (in a slightly acidic medium) as oxidant. It has been shown, that MOF catalyst proceeds more efficiently in the presence of nitric acid (Table 3). This phenomenon can be explained, by the role of acid, in activations of catalyst by promoting unsaturated metal sites, which improves their oxidative properties. Increasing the amount of catalyst and hydrogen peroxide increased the percentage of conversion, leading (in the best case) to cyclohexanol $(25.1 \%)$ and cyclohexanone $(2.8 \%)$ as the only product of reaction.

Among zeolites, Titanium-Silicate-1 (TS-1) is the most widely used as a shape selective catalyst in oxidation reactions [43]. This material has hydrophobic properties, and its titanium active sites are tetrahedrally coordinated in MFI-lattice. Weak residual acidity properties make it a very selective catalyst in epoxidation reaction. However, TS-1 has medium pore size (5.5 ̊), which limits the scope of reaction to epoxidation of linear alkenes. For this reason many research groups have dedicated their work to incorporation of $\mathrm{Ti}$ into more open zeolite frameworks. $[44,45]$ The one-pot carbon-templating method was successfully used by Yang and Li et al., on synthesized mesoporous zeolite with transition-metal substitution, based on TS-1. To show the role of mesopores in the catalytic performance, TS-1 (meso-TS-1) and conventional microporous TS-1 were compared in a two model reaction phenol hydroxylation and methyl ethyl ketone ammoxiation [46] using $\mathrm{H}_{2} \mathrm{O}_{2}$ as an oxidant in both cases. No difference in selectivity was observed, which is strongly related to similar microenvironment of titanium species in meso-TS-1 and TS-1 zeolite. However, a difference in catalytic performance

Table 3. Acid-to-Catalyst molar ratio effect in the oxidation of cyclohexane over the Cu MOF.

\begin{tabular}{lcc}
\hline $\mathbf{n}\left(\mathrm{HNO}_{3}\right) \mathbf{n}($ catalyst $)$ & Cyclohexanol Yield (\%) & Cyclohexanone Yield (\%) \\
\hline 5 & 6.1 & 0.3 \\
10 & 25.1 & 2.8 \\
30 & 13.7 & 15.3 \\
\hline
\end{tabular}


is related to textural properties, by the authors. Detail analysis by ${ }^{129} \mathrm{Xe}$ NMR spectroscopy combined with TEM and $\mathrm{N}_{2}$ sorption results proved the existence of mesopores in meso-TS-1 and their good connectivity with micropores, which can increase the diffusion rate significantly, and in consequence lead to better catalytic activity of meso-TS-1 compared to TS-1.

Incorporation of Bi into the framework of mesoporous material has been reported by Qi et al. Mesoporous Bi-MCM-41 was synthesized under strongly acidic conditions. Catalytic activity was tested in cyclohexane oxidation [47] To make the system environmentally friendly, the reaction was carried out in a solvent-free system, using oxygen as an oxidant $(1 \mathrm{MPa})$ at $15 \mathrm{O}^{\circ} \mathrm{C}$. To prove the true active role of bismuth ions, blank reaction was carried out as catalyst on Si-MCM-41, with no observed catalytic activity. Samples with different bismuth contents were tested, showing that $1.4 \mathrm{wt} \%$ metal content is the optimum for the best catalytic performance. Increasing bismuth content does not improve oxidation rate due to change in the kind of bismuth species or distribution of this metal in the catalyst. No leaching of metal was detected, indicating good stability of this catalyst, in up to three runs, compared to Au/MCM-41 [48] and Au/ZSM-5 [49]. This can be explained by a good distribution of metal in the Bi-MCM-41 internal surface, showed by XPS analysis.

\section{MOFs and zeolites in reduction reactions}

An important reaction in the perfume, flavouring, agrochemical and pharmaceutical industries is the selective reduction of the carbonyl group of an $\alpha, \beta$-unsaturated ketone. The resulting allylic alcohols find a wide range of applications, for example as intermediates in the synthesis of fine chemicals [50-53] and for the production of the chemically very versatile oxiranes, as described in the last section [54-57]. To reduce the carbonyl group of an $\alpha, \beta$-unsaturated ketone to a hydroxy group, without affecting the conjugated $\mathrm{C}-\mathrm{C}$ double bond, different methods have been developed [58-61]. Besides catalytic hydrogenolysis with hydrogen as the reducing agent [62-68] an alternative method is Meerwein-Ponndorf-Verley (MPV) reduction, which traditionally employs an alcohol as a hydride source and aluminum alkoxides as a catalyst [69,70]. The choice of Lewis acid is the key to improving the reactions in terms of chemical selectivity, reaction temperature, reaction rate, the amount of catalyst needed, the reusability of the catalyst and the process of product isolation. In respect to the latter two aspects, heterogeneous catalysis is the most appealing. $\mathrm{Zr}$ or Hf 
functionalized mesoporous silica [71-73], Sn containing zeolites $[74,75]$ and metal-organic frameworks $[76,77]$ have been reported as a substitute for the MPV reduction. Metal-organic frameworks (MOFs) have recently attracted interest as heterogeneous catalysts due to their structural diversity, high surface area and different types of active sites [3,11,29,78-84]. MOFs are composed of metal (oxide) nodes interlinked by organic ligands that form three-dimensional periodic structures with well-defined micropores and pore channels [85-88]. However, there are a few reports on the selective reduction of unsaturated carbonyl compounds with $\mathrm{H} 2$ and MOF as support for noble metal nanoparticles [66,67], due to the low selectivity towards the reduction of the carbonyl group. Recently, the Zr MOFs UiO-66 and MOF-808 were shown to catalyse the hydrogen transfer from secondary alcohols to $\alpha, \beta$-unsaturated carbonyl compounds in a Meerwein-PonndorfVerley reduction, reducing the carbonyl group without affecting the $\mathrm{C}=\mathrm{C}$ double bond $[89,90]$. However, the performance of $\mathrm{Zr}-$ or Sn-Beta is much better in terms of activity.

Catalytic reduction has been applied to obtain $\gamma$-valerolactone, a high value-added levulinic acid derivative in fine chemistry. Gamma valerolactone (GVL) could be considered as a sustainable liquid for global storage/transportation and a renewable hydrocarbon resource for energy and carbon-based consumer products as described elsewhere [91]. The production of gamma valerolactone (also known as 5-methylbutyrolactone or 5 -valerolactone) as a renewable feedstock is interesting due to a much lower vapor pressure, (compared to methanol, ethanol, and methyl and ethyl tert-butyl ethers) which reduces volatile emissions and facilitates safe storage. On the other hand GVL has a high chemical stability, which avoids hydrolization to the acid form and the formation of peroxides under pH-neutral conditions. Moreover, because GVL does not form an azeotrope with water, less energy is needed to recover it through distillation. These properties enhance its general use as fuel additive to produce cleanerburning fuels [91], or as intermediate in the production of chemicals such as adipic acid [92], valeric acid [93,94], 5-nonanone [95], in the composition of biocides [96] and as a solvent [97]. There are multiple pathways to produce GVL, as described elsewhere [98]. Among them, the majority of the processes make use of levulinic acid (LA) as starting material [99]. The interest of using LA as a precursor of GVL is because of the huge availability of such a renewable compound, which proceeds from the deconstruction of biomass. An alternative to the use of hydrogen gas or formic acid in the synthesis of GVL takes place in the chemoselective reduction of the keto group of LA by hydrogen transfer from a secondary alcohol as a hydrogen source through a 
Table 4. MPV reduction of levulinic acid by various catalysts at moderate reaction temperatures [100].

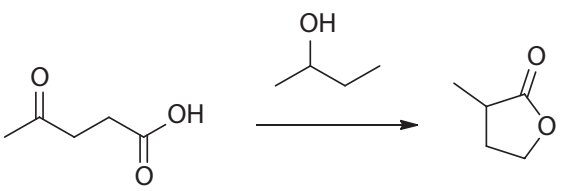

\begin{tabular}{|c|c|c|c|c|c|}
\hline & Catalyst & Temp. $\left({ }^{\circ} \mathrm{C}\right)$ & Conversion (\%) & $\mathbf{S}_{\mathrm{GVL}}(\%)$ & TOF $\left(\mathbf{h}^{-1}\right)$ \\
\hline 1 & MOF-808 & 130 & 100 & 85 & 7.7 \\
\hline 2 & UiO-66(Zr) & 130 & 43.3 & 18 & 0.8 \\
\hline 3 & $\mathrm{ZrO}_{2}$ & 130 & 36.5 & 60 & 0.2 \\
\hline 4 & Zr-Beta & 118 & 88 & 94 & 3.7 \\
\hline
\end{tabular}

Meerwein-Ponndorf-Verley (MPV) reaction. The use of the zirconium MOFs with MPV catalytic activity in the MPV reduction of LA to GVL by using secondary alcohols as hydrogen source has recently been described.

On the other hand, bimetallic $\mathrm{Zr}(\mathrm{Ti})$-NDC based metal-organic frameworks (MOFs) have been prepared by incorporation of titanium(IV) into zirconium(IV)-NDC-MOFs (UiO family). The resulting materials maintain thermal (up to $500^{\circ} \mathrm{C}$ ), chemical and structural stability with respect to parent Zr-MOFs as can be deduced from XRD, $\mathrm{N}_{2}$ adsorption, FTIR and thermal analysis. The materials have been studied in Lewis acid catalysed reactions, such as domino Meerwein-Ponndorf-Verley (MPV) reduction-etherification of $p$-methoxybenzaldehyde with butanol. In general, for activity in the MPV reaction, coordinative unsaturated (Lewis acid) Zr sites, which can react with an alcohol to form an activated alkoxide specie, are required. Zeolite $\mathrm{Zr}$ - and Sn-Beta is an excellent catalyst for the MPV due to the high activity with respect to the MOFs (entry 4 of Table 5 and entries 2 and 3 of Table 5 ). This indicates stronger Lewis sites than in the case of the $\mathrm{Zr}$ oxo-clusters of the UiO MOF, although the MOF-808 shows promising Lewis acid sites (entry 1 of Table 4 ).

\section{Conclusions and outlook}

This chapter has shown multiple applications of MOFs and zeolites in the transformation of different functional groups present in organic molecules. The potential catalytic sites present in the structure of these porous solids 
Table 5. MPV reduction-etherification of benzaldehyde with butanol preformed over different catalysts [101].

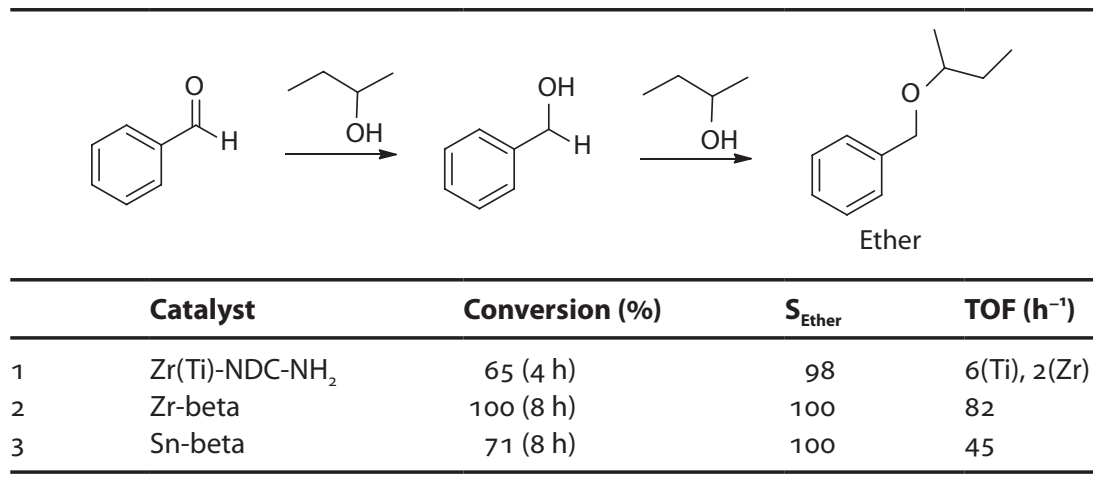

are able to selectively transform the desired functionality into a new one of industrial interest. Examples of active and selective crystalline and porous solids in isomerization, condensation, oxidation or hydrogenation using clean oxygen and hydrogen sources has been highlighted. The advantages of the solid catalyst, preferably in low amounts, are its non-toxicity, simple handling and separation from the reaction mixture. This fact allows up-scaling of the fine chemical and pharmaceutical synthesis from laboratory batch to a continuous industrial process. Relevant compounds that are obtained by classical organic chemistry reactions with the use of MOFs and zeolites as heterogeneous catalysts are fragances and pharmaceutically active compounds. Due to the relatively low attention the heterogeneous catalysis community has paid to synthetic organic transformations, the use of porous solids in organic and medicinal chemistry is currently a promising field of research with industrial applications. Therefore, in the coming years, the collaboration between solid state and organic chemists will produce synergic effects in the development of cleaner and more efficient organic transformations. This will eventually reduce the cost of the synthesis of complex molecules, as nature has done since life began on the planet, thanks to highly optimized enzymes than contain similar functions and structures to those present in the solids we prepare in the laboratory. Although we still far from imitate metabolic routes in animal and plant cells, silicates (zeolites and mesoporous silicas) and metal-organic frameworks are more robust and economic than natural enzymes, which enable us to work under a broad range of conditions. In the near future, we will be able to accurately mimic the active sites of most of the enzymes and then it 
will be possible to optimize the organic transformations for the synthesis of fine chemicals and pharmaceuticals and minimize health and environmental impact with the use of finely tuned supramolecular catalysts designed and synthetized by chemists.

\section{References}

[1] Sheldon, R.A. Green Chem. 2017, 9, 903.

[2] Corma, A., García, H., Llabrés i Xamena, F.X. Chem. Rev. 2010, 110, 4606.

[3] Jiang, J., Yaghi, O.M. Chem. Rev. 2015, 6966.

[4] Corma, A., García, H. Chem. Rev. 2003, 103, 4307.

[5] Hu, Z., Zhao, D. Cryst. Eng. Comm. 2017, 19, 4066.

[6] Rabo, J.A., Gajda, G.J. Catal. Rev. 1989, 31, 385.

[7] Rachwalik, R., Olejniczak, Z., Jiao, J., Huang, J., Hunger, M., Sulikowski, B. J. Catal. 2007, 252,161 .

[8] Alaerts, L., Séguin, E., Poelman, H., Thibault-Starzyk, F., Jacobs, P.A., De Vos, D.E. Chem. Eur. J. 2006, 12, 7353 .

[9] Hölderich, W.F., Röseler, J., Heitmann, G., Liebens, A.T. Catal. Today 1997, 37, 353.

[10] Kunkeler, P.J., van der Waal, J.C., Bremmer, J., Zuurdeeg, B.J., Downing, R.S., van Bekkum, H. Catal. Lett. 1998, 53, 135 .

[11] Vermoortele, F., Ameloot, R., Alaerts, L., Matthessen, R., Carlier, B., Fernandez, E.V.R., Gascon, J., Kapteijn, F., De Vos, D.E. J. Mater. Chem. 2012, 22, 10313.

[12] Gotz, N., Friedrich, M., Ebel, K. ES Patent 2352346, 2011.

[13] Corma, A., Renz, M. Chem. Commun. 2004, 5, 550.

[14] Braga, P.R.S., Costa, A.A., De Freitas, E.F., Rocha, R.O., De MacEdo, JL., Araujo, A.S., Dias, J.A., Dias, S.C.L.J. Mol. Catal. A Chem. 2012, 358, 99.

[15] Yongzhong, Z., Yuntong, N., Jaenicke, S., Chuah, G.K.J. Catal. 2005, 229, 404.

[16] Cirujano, F.G., Llabrés i Xamena, F.X., Corma, A. Dalton Trans. 2012, 41, 4249.

[17] Vandichel, M., Vermoortele, F., Cottenie, S., De Vos, D.E., Waroquier, M., Van Speybroeck, V. J. Catal. 2013, 305, 118 .

[18] Akutu, K., Kabashima, H., Seki, T., Hattori, H. Appl. Catal., A 2003, 247, 65.

[19] Corma, A., Martín-Aranda, R.M., Sánchez, F.J. Catal. 1990, 126, 192.

[20] Climent, M.J., Corma, A., Iborra, S., Velty, A.J. Mol. Catal. A Chem. 2002, 182, 327.

[21] Corma, A., Fornes, V., Martin-Aranda, R.M., Garcia, H., Primo, J. Appl. Catal. 1990, 59, 237.

[22] Climent, M.J., Corma, A., Fornés, V., Guil-López, R., Iborra, S. Adv. Synth. Catal. 2002, 344, 1090.

[23] Gianotti, E., Diaz, U., Velty, A., Corma, A. Catal. Sci. Technol. 2013, 3, 2677.

[24] Shylesh, S., Wagener, A., Seifert, A., Ernst, S., Thiel, W.R. Angew. Chem. Int. Ed. 2010, 49, 184.

[25] Corma, A., Garcia, H. Adv. Synth. Catal. 2006, 348, 1391.

[26] Vermoortele, F., Ameloot, R., Vimont, A., Serre, C., De Vos, D. Chem. Commun. 2011, 47, 1521.

[27] Climent, M.J., Corma, A., Garcia, H., Guil-Lopez, R., Iborra, S., Fornés, V.J. Catal. 2001, 197, 385.

[28] Sharma, S.K., Parikh, P.A., Jasra, R.V.J. Mol. Catal. A Chem. 2008, 286, 55.

[29] Gascon, J., Aktay, U., Hernandez-Alonso, M., Vanklink, G., Kapteijn, F. J. Catal. 2009, 261, 75.

[30] Climent, M.J., Corma, A., Iborra, S., Epping, K., Velty, A.J. Catal. 2004, 225, 316.

[31] Rodriguez, I., Iborra, S., Rey, F., Corma, A. Appl. Catal., A 2000, 194, 241.

[32] Sheldon, R.A., Kochi, J.K. Met. Oxidations Org. Compd. 1981, 1.

[33] Hsu, Y.F., Cheng, C.P.J. Mol. Catal. A Chem. 1998, 136, 1. 
[34] Dhakshinamoorthy, A., Alvaro, M., Garcia, H. Catal. Sci. Technol. 2011, 1, 856.

[35] Dhakshinamoorthy, A., Asiri, A.M., Garcia, H. Chem. Eur. J. 2016, 22, 8012.

[36] Kholdeeva, O.A. Catal. Today 2016, 278, 22.

[37] Zhang, J., Biradar, A.V, Pramanik, S., Emge, T.J., Asefa, T., Li, J. Chem. Commun. 2012, 48, 6541.

[38] Férey, G., Mellot-Draznieks, C., Serre, C., Millange, F., Dutour, J., Surblé, S., Margiolaki, I. Science 2005, 309, 2040.

[39] Skobelev, I.Y., Sorokin, A.B., Kovalenko, K.A., Fedin, V.P., Kholdeeva, O.A.J. Catal. 2013, 298, 61.

[40] Kholdeeva, O.A., Skobelev, I.Y., Ivanchikova, I.D., Kovalenko, K.A., Fedin, V.P., Sorokin, A.B. Catal. Today 2014, 238, 54 .

[41] Santiago-Portillo, A., Navalón, S., Cirujano, F.G., Llabrés i Xamena, F.X., Alvaro, M., Garcia, H. ACS Catal. 2015, 5, 3216.

[42] Nicola, C. Di, Karabach, Y.Y., Kirillov, A.M., Monari, M., Pandolfo, L., Pettinari, C., Pombeiro, A.J.L. Inorg. Chem. 2007, 46, 221.

[43] Clerici, M.J. Catal. 1993, 71.

[44] Wu, P., Tatsumi, T.J. Phys. Chem. B 2002, 106, 748.

[45] Wu, P., Tatsumi, T.J. Catal. 2003, 214, 317.

[46] Xin, H., Zhao, J., Xu, S., Li, J., Zhang, W., Guo, X., Hensen, E.J.M., Yang, Q., Li, C.J. Phys. Chem. C 2010, 114, 6553 .

[47] Qian, G., Ji, D., Lu, G.M., Zhao, R., Qi, Y.X., Suo, J.S.J. Catal. 2005, 232, 378.

[48] Lu, G.M., Zhao, R., Qian, G., Qi, Y.X., Wang, X.L., Suo, J.S. Catal. Lett. 2004, 97, 115.

[49] Zhao, R., Ji, D., Lv, G., Qian, G., Yan, L., Wang, X., Suo, J. Chem. Commun. 2004, 904.

[50] Saudan, L.A. Acc. Chem. Res. 2007, 40, 1309.

[51] Chapuis, C., Jacoby, D. Appl. Catal., A 2001, 221, 93.

[52] Cherkaoui, H., Soufiaoui, M., Grée, R. Tetrahedron 2001, 57, 2379.

[53] Kurosu, M., Marcin, L.R., Grinsteiner, T.J., Kishi, Y.J. Am. Chem. Soc. 1998, 120, 6627.

[54] Bisogno, F.R., Orden, A.A., Pranzoni, C.A., Cifuente, D.A., Giordano, O.S., Kurina Sanz, M. Steroids 2007, 72, 643 .

[55] Chen, C., Yuan, H., Wang, H., Yao, Y., Ma, W., Chen, J., Hou, Z. ACS Catal. 2016, 6, 3354.

[56] Hussain, M.M., Walsh, P.J. Acc. Chem. Res. 2008, 41, 889.

[57] Hanson, R.M., Sharpless, K.B.J. Org. Chem. 1986, 51, 1922.

[58] Walker, E.R.H. Chem. Soc. Rev. 1976, 5, 23.

[59] Ide, M.S., Hao, B., Neurock, M., Davis, R.J. ACS Catal. 2012, 2, 671.

[6o] Lumbroso, A., Cooke, M.L., Breit, B. Angew. Chem. Int. Ed. 2013, 52, 1890.

[61] Sakaguchi, S., Yamaga, T., Ishii, Y.J. Org. Chem. 2001, 66, 4710.

[62] Milone, C., Ingoglia, R., Tropeano, M.L., Neri, G., Galvagno, S. Chem. Commun. 2003, 7, 868.

[63] Mertens, P.G.N., Vandezande, P., Ye, X., Poelman, H., Vankelecom, I.F.J., De Vos, D.E. Appl. Catal., A 2009, 355, 176.

[64] Santori, G.F., Moglioni, A.G., Vetere, V., Iglesias, G.Y.M., Casella, M.L., Ferretti, O.A. Appl. Catal., A 2004, 269, 215.

[65] De bruyn, M., Coman, S., Bota, R., Parvulescu, V.I., De Vos, D.E., Jacobs, P.A. Angew. Chem. Int. Ed. 2003, 42, 5333 .

[66] Guo, Z., Xiao, C., Maligal-Ganesh, R.V., Zhou, L., Goh, T.W., Li, X., Tesfagaber, D., Thiel, A., Huang, W. ACS Catal. 2014, 4, 1340.

[67] Liu, H., Li, Z., Li, Y. Ind. Eng. Chem. Res. 2015, 54, 1487.

[68] Zhao, M., Yuan, K., Wang, Y., Li, G., Guo, J., Gu, L., Hu, W., Zhao, H., Tang, Z. Nature 2016, 539, 76 .

[69] Cha, J.S. Org. Process Res. Dev. 2006, 10, 1032.

[70] Ruiz, J., Jimenez-Sanchidrian, C. Curr. Org. Chem. 2007, 11, 1113.

[71] De bruyn, M., De Vos, D.E., Jacobs, P.A. Adv. Synth. Catal. 2002, 344, 1120. 
[72] Zhu, Y., Jaenicke, S., Chuah, G.J. Catal. 2003, 218, 396.

[73] Iglesias, J., Melero, J., Morales, G., Moreno, J., Segura, Y., Paniagua, M., Cambra, A., Hernández, B. Catalysts 2015, 5, 1911.

[74] Corma, A.J. Catal. 2003, 215, 294.

[75] Corma, A., Domine, M.E., Nemeth, L., Valencia, S.J. Am. Chem. Soc. 2002, 124, 3194.

[76] Rasero-Almansa, A.M., Iglesias, M., Sánchez, F. RSC Adv. 2016, 6, 106790.

[77] Valekar, A.H., Cho, K.H., Chitale, S.K., Hong, D.Y., Cha, G.Y., Lee, U.H., Hwang, D.W., Serre, C., Chang, J.S., Hwang, Y.K. Green Chem. 2016, 18, 4542.

[78] Choi, K.M., Na, K., Somorjai, G.A., Yaghi, O.M.J. Am. Chem. Soc. 2015, 137, 7810.

[79] Goesten, M.G., Juan-Alcañiz, J., Ramos-Fernandez, E.V., Sai Sankar Gupta, K.B., Stavitski, E., van Bekkum, H., Gascon, J., Kapteijn, F. J. Catal. 2011, 281, 177.

[8o] Rösler, C., Fischer, R.A. Cryst. Eng. Comm. 2015, 17, 199.

[81] Valvekens, P., Vermoortele, F., De Vos, D. Catal. Sci. Technol. 2013, 3, 1435.

[82] Chen, Y.Z., Jiang, H.L. Chem. Mater. 2016, 28, 6698.

[83] Li, Y., Zhou, Y.X., Ma, X., Jiang, H.L. Chem. Commun. 2016, 52, 4199.

[84] Wee, L.H., Lescouet, T., Fritsch, J., Bonino, F., Rose, M., Sui, Z., Garrier, E., Packet, D., Bordiga, S., Kaskel, S., et al. Catal. Lett. 2013, 143, 356.

[85] Canivet, J., Fateeva, A., Guo, Y., Coasne, B., Farrusseng, D. Chem. Soc. Rev. 2014, 5594.

[86] Jiang, J., Zhao, Y., Yaghi, O.M. J. Am. Chem. Soc. 2016, 138, 3255.

[87] Goesten, M.G., Kapteijn, F., Gascon, J. Cryst. Eng. Comm. 2013, 15, 9249.

[88] Hirschle, P., Preiß, T., Auras, F., Pick, A., Völkner, J., Valdepérez, D., Witte, G., Parak, W.J., Rädler, J.O., Wuttke, S. Cryst. Eng. Comm. 2016, 18, 4359.

[89] Plessers, E., De Vos, D.E., Roeffaers, M.B.J.J. Catal. 2016, 340, 136.

[9o] Plessers, E., Fu, G., Tan, C., De Vos, D., Roeffaers, M. Catalysts 2016, 6, 104.

[91] Horváth, I.T., Mehdi, H., Fábos, V., Boda, L., Mika, L.T. Green Chem. 2008, 10, 28.

[92] Castelijns, A.M.C.F., Janssen, M.C.C., Vaessen, H.W.L.M. WO2012175439 Al, 2012.

[93] Palkovits, R. Angew. Chem. Int. Ed. 2010, 49, 4336.

[94] Lange, J.P., Price, R., Ayoub, P.M., Louis, J., Petrus, L., Clarke, L., Gosselink, H. Angew. Chem. Int. Ed. 2010, 49, 4479 .

[95] Serrano-Ruiz, J.C., Wang, D., Dumesic, J.A. Green Chem. 2010, $12,574$.

[96] Merlet, S., Scherer, M. EP Patent 236459oAt, 2011.

[97] Fegyverneki, D., Orha, L., Láng, G., Horváth, I.T. Tetrahedron 2010, 66, 1078.

[98] Serrano-Ruiz, J.C., West, R.M., Dumesic, J.A. Annu. Rev. Chem. Biomol. Eng. 2010, 1, 79.

[99] Wright, W.R.H., Palkovits, R. ChemSusChem 2012, 5, 1657.

[100] Wang, J., Jaenicke, S., Chuah, G.K. RSC Adv. 2014, 4, 13481.

[101] Corma, A., Renz, M.A. Angew. Chem. Int. Ed. 2007, 46, 298. 



\title{
12. The Future of Zeolite and MOF Materials
}

\author{
Eduardo Falabella Sousa-Aguiar, ${ }^{1}$ Pedro Augusto Arroyo, ${ }^{2}$ \\ Maria Angélica Simões Dornellas de Barros ${ }^{2}$ and \\ Jussara Lopes de Miranda ${ }^{3}$
}

${ }^{1}$ Federal University of Rio de Janeiro (UFRJ), School of Chemistry, Department of Organic Processes, Centro de Tecnologia, Bloco E, Av. Athos da Silveira Ramos, 149 - Cidade Universitária, 21941-909, Rio de Janeiro-RJ, Brazil.

${ }^{2}$ State University of Maringá (UEM), Department of Chemical Engineering, Centro de Tecnologia, Bloco D9o, Av. Colombo, 5790, - Jd. Universitário, 87020-900, Maringá - Paraná, Brazil.

${ }^{3}$ Federal University of Rio de Janeiro (UFRJ), Institute of Chemistry, Centro de Tecnologia, Bloco A, Av. Athos da Silveira Ramos, 149 - Cidade Universitária, 21941-909, Rio de Janeiro, Brazil.

Keywords: Adsorbent, technical catalyst, hydrogen storage, cracking, devices, commercial

\section{Introduction}

Zeolites were discovered in 1756 by the Swedish mineralogist Axel Friedrik Cronstedt [1], who recognized in the mineral stilbite (the first reported zeolite) a new class of hydrated aluminosilicates of the alkali and alkaline earths. He observed that upon rapidly heating stilbite produced large amounts of steam from water that had been adsorbed by the material. Hence, Cronstedt called the mineral a 'zeolite' (derived from two Greek words, meaning boiling stone). From that date on, several authors have described the properties of zeolite minerals, including their adsorption properties and reversible cation exchange and dehydration. In 1932 McBain coined the term 'molecular sieve', defining porous solid materials that act as sieves on a molecular scale [2]. Richard M. Barrer [3-5] began his pioneering work in zeolite adsorption and synthesis in the decade from 1930 
to 1940. He presented the first classification of zeolites based on molecular size considerations in 1945. In 1948, he reported the first definitive synthesis of zeolites.

The work of Robert Milton [6] and Donald Breck [7] of the Linde Division of Union Carbide Corporation deserves special attention. Between 1949 and 1954 they developed several zeolites, such as types A, X and Y, which had commercial applications in separation and purification. Mobil performed one of the first commercial applications of synthetic zeolites as catalysts, using $\mathrm{X}$ zeolite as the main component of cracking catalyst, in 1962. Moreover, in 1967, Mobil published the synthesis of new zeolites (beta and ZSM-5), which had a rather high silica-to-alumina ratio. The work carried out by Grace cannot be disregarded. In 1969, Grace introduced the first modification of $\mathrm{Y}$ zeolite, which underwent steaming to produce the well-known ultrastable Y zeolite, USY. It must be borne in mind that such zeolites are still being used in commercial cracking processes.

Later, in 1974, zeolite NaA produced by Henkel and Degussa was tested as a component of commercial detergents, opening a new window for zeolites' application. In fact, A zeolite currently accounts for more than twothirds of the total world consumption of synthetic zeolites.

Last but not least, due to the importance thereof in terms of consumption, natural zeolites have to be mentioned. Natural zeolites account for almost $60 \%$ of the total world consumption of zeolites. The main natural zeolites include minerals capable of ion exchange, such as clinoptilolite, chabazite, and Mordenite. China is the largest producer of natural zeolites, reaching almost $70 \%$ of global production in 2016; South Korea, Turkey, and Japan follow. The main use for natural zeolites is as a cement additive, to produce a slower-hardening and stronger product. Other major applications are as a nutrient-release agent in soil conditioners, in animal and poultry nutrition, and as an odour control agent in animal litter.

Synthetic zeolites are used mainly as detergent builders, catalysts, and absorbents/desiccants. As previously mentioned, the largest-volume use for synthetic zeolites is as a builder in home laundry detergent, accounting for almost $70 \%$ of the total. FCC catalysts are the second-largest synthetic zeolite use, with about $18 \%$ of the total volume. Consumption for adsorbent/desiccant applications represents the smallest share, but will be the fastest-growing market, driven by the recovery of construction markets use (as a desiccant in insulated multiglazed windows), the natural gas market (as a drying agent), and environmental applications (trapping volatile organic compounds, VOCs, to prevent release into the environment). 
The importance of zeolites has progressively increased since the pioneer publications of Barrer about synthesis and adsorption in the 193os. Since then, catalytic applications, ion exchange as well as adsorption spread all over the scientific and industrial community. Around the 80's SAPO, MeAPO, TS1, MCM and UOP-Norsk Hydro structures were synthesized for catalytic purposes. Big industries such as Chevron and Union Carbide are relevant protagonists in this process. In the following decade mesoporous zeolitic frameworks found their role in the catalytic processes [8]. It is worth mentioning that the past fifty years have seen a tremendous progression in the generation of innovative molecular sieve materials. Indeed, since the discovery of traditional aluminosilicate zeolites, advances have been made towards the synthesis [9] of microporous silica polymorphs, microporous aluminophosphate-based polymorphs, metallosilicate and metallophosphate compositions, octahedral-tetrahedral frameworks, mesoporous molecular sieves, and, most recently, hybrid metal-organic frameworks (MOFs). Nevertheless, most of these very interesting new materials still call for industrial applications.

There are several reasons why zeolites have become the preferred catalyst for many reactions of industrial importance, as well as undefeatable adsorbents and ion-exchangers. The following characteristics must be depicted $[10,11]$ :

a) Thermal stability - surely, this is the paramount reason for the selection of zeolites as catalysts for high temperature reactions. Most of the zeolites remain unaffected by temperatures as high as $65^{\circ}{ }^{\circ} \mathrm{C}$. For zeolites presenting high silica-to-alumina ratios, structural collapse becomes significant only at temperatures as high as $1000{ }^{\circ} \mathrm{C}$.

b) Acidity - zeolites exhibit much higher acidity than the earlier amorphous silica-alumina catalysts. Zeolites also present both Brønsted and Lewis acid sites. The density of these acid sites is related to the silica-to-alumina ratio (SAR) in the zeolite framework. In general, the following parameters affect the overall acidity of a zeolite:

- nature or type of the acid sites

- density or concentration of the acid sites

- strength distribution of the acid sites

- location of the acid sites within the zeolite framework

- geometric distribution of the acid sites over the zeolite crystals.

c) Shape selectivity - pore dimensions within the zeolite frameworks are often similar to the sizes of the molecules participating in the reactions, 
which they catalyse, generating a strong influence on the selectivity of such reactions.

d) Concentration effects - concentration effects are related to the concepts of hydrophilicity or hydrophobicity of the zeolites, affecting the reaction selectivity. Such properties may be fine-tuned when zeolites are being synthesized, producing specific zeolites for a given purpose.

e) Ion-exchange characteristics - zeolites spontaneously undergo cation exchange. Such property makes zeolites undefeatable materials for water treatment.

f) High specific surface area - zeolites may present a rather high surface area, and are therefore excellent adsorbents and desiccants.

What is the future of zeolites and zeolite-like materials as far as industrial and commercial applications are concerned? This rather difficult question is the main target of this chapter, in which we discuss the potential uses of these spectacular materials, bearing in mind that zeolites are considered inert materials and not harmful to the environment. Hence, in a world in which environmental protection is a major concern, one may forecast a brilliant future for zeolite-type materials. Besides the use as catalysts, such solids have also increased their importance in the field of adsorption and ion exchange processes. It is difficult to separate them, as most processes involve, somehow, both phenomena. This chapter firstly describes this traditional use. Then, novel applications are presented. Last but not least, some comments about the future of these still potentially unexplored materials are made.

\section{Zeolites and zeolite-type materials as adsorbents and ion exchangers}

Zeolites are well-known adsorbents and ion exchangers. Nobody doubts it. It is possible to find zeolites in a wide range of applications. As well highlighted [12], zeolites find application in animal nutrition, wastewater treatment, soil fertilization and zeoponic cultivation, construction materials, cellulosic and sugar industrial uses, etc. As already mentioned, this is a consequence of their thermal stability, acidity, shape selectivity and high surface area. Today, more than 230 different zeolite frameworks have been synthesized and many more will be in the near future. Zeolites are molecular sieves and their frameworks are decisive in separation process, as such they are a major driving force for research in this area. 

to the aluminosilicate framework. Nevertheless, its adsorption capacity is incredibly strengthened in silver impregnated zeolites. This happens due to the chemical affinity of silver to mercury, forming an amalgam. Besides removing mercury in elemental form, the saturated silver-zeolite adsorbent may be regenerated through heating to $400^{\circ} \mathrm{C}[15]$, which aggregates tremendous advantages in continuous systems.

Other wastes that deserve attention are those rich in radionuclides. Such an effluent is commonly generated in nuclear power plants, nuclear weapons testing, medical examination or mining waters. ${ }^{134} \mathrm{Cs}$ and ${ }^{137} \mathrm{Cs}$, as well as ${ }^{226} \mathrm{Ra}$ and ${ }^{228} \mathrm{Ra}$, are some of the radionuclide elements commonly found in these wastewaters, which are easily treated through ion exchange in zeolites. It should be emphasized that these solids have an additional, important characteristic - resistance to radiation. It means that the final disposal of the radionuclide-containing zeolite will not harm the environment. This was the case when zeolites mitigated the impact of the accident at the Chernobyl nuclear power plant in Ukraine in 1986. The cationic form of radionuclides is successfully exchanged by the charge balancing sodium cation, generally present after the zeolite synthesis. Natural zeolites may be also used as cation exchangers. In order to improve removal efficiency, the zeolite surface may be modified. Some examples include the surface modification of $\mathrm{Y}$ zeolite with n-octadecyltrichlorosilane to remove ${ }^{137} \mathrm{Cs}$ and ${ }^{90} \mathrm{Sr}$ from aqueous to organic phases. In another tailored modification the balancing cation was replaced by different quaternary alkylammonium ions with promising results in the removal of ${ }^{121} \mathrm{I}$ and ${ }^{131} \mathrm{I}$ ions [16].

Natural zeolites can be applied in the remediation of soils rich in heavy metals. In addition to their high ion exchange capacity and low price, they exhibit significant selectivity even at low temperatures, releasing nontoxic metals such as $\mathrm{K}^{+}, \mathrm{Na}^{+}, \mathrm{Ca}^{2+}$ and $\mathrm{Mg}^{2+}$. Such features provide some extra advantages. This is the case when clinoptilolite is used in the sewage sludge to remove toxic metals with successful results [17]. It may supply alkalinity to the soil with the consequent precipitation of insoluble phases and the enhancement of metal sorption via surface complexation. Moreover, the ion exchanging process occurs regardless of the soil $\mathrm{pH}$. There is no need to emphasize the importance of more studies in this area, as leaching may be in some cases a drawback to sustainable recycling [18]. Ammonium can also be retained through ion exchange in natural zeolites. When removal of ammonium is considered, the released $\mathrm{Ca}^{2+}$ ions are used to precipitate phosphorous, which, along with ammonium, is also found in fertilizers [19]. It should be emphasized that phosphorous deserves special attention because its presence in soil or water is a result of 
the use of fertilizers, mostly added without adequate control, thus accelerating undesired eutrophication.

Removal of ammonium may also be important to improving wastewater treatment. In this case, ion exchange is not the main purpose of the process. Free ammonia released in anaerobic reactors inhibits methanogenic microorganisms. Thus, ammonia removal indirectly improves methane production [20]. Nevertheless, ammonia retained in the zeolite is also useful because its desorption provides enrichment in systems were nitrogen is needed. Then, the desorbed ammonia previously removed from wastewater acts exactly as the nitrogen source [21]. These new promising processes are astonishing! It means that adsorption and ion exchange properties have no limits and make zeolites the connexion for a sustainable world.

The ion exchange property of zeolites is not only limited to cation removal. In fact, tailored processes have been investigated to produce modified zeolites able to remove anions from the fluid phase. Some examples are related to the removal of the toxic anions arsenite, arsenate, chromate, cyanide and radioactive iodide. This is the case of zeolites treated with large cationic surfactants such as hexadecyltrimethylammonium amine (HDTMA). The resultant organo-zeolites have a wide range of applications because they can not only adsorb anions, but also adsorb or exchange cations in the remaining original sites [22]. It is also possible to tailor zeolites to multi-functional purposes (like adding magnetic properties to the support) or even redox purposes. These technologies are now in a pilot phase with the focus on environmentally friendly processing [23].

Curious uses involving hydrophilic properties, such as medicines to control diarrhea, are fundamental [24]. Moreover, it is already known that pharmaceuticals such as sulfa drugs and their metabolites are excreted in urine and feces. These low biodegradable compounds are not completely removed through conventional wastewater treatment and in most cases reach bodies of water. In this case, synthetic zeolites, such as HSZ-385, can adsorb molecules as long as the solution $\mathrm{pH}$ and $\mathrm{pK}_{\mathrm{a}}$ of each molecule favours the process, since the adsorption mechanism is based on the acid-base equilibria of the sulfa drugs and on hydrophobic interactions [25].

Zeolites can also be a support for drug release, such as non-steroidal anti-inflammatory compounds (NSAIDs). The surface of the modified zeolite releasing drugs through anion exchange as the sodic form of NSAIDs acts as the bound salt [26]. It means that the ion exchange capacity is not limited to cations, which opens a broad pathway for new discoveries. In fact, synthetic or even purified natural zeolites can be used as gastric 
antacid, anti-diarrheic, anti-hyperglycemic, hypocholesterolemic agents, also useful in the release of organic molecules, such as the anti-parasitic drug metronidazole or the antibiotic sulfamethoxazole, based on a strong dependence on the $\mathrm{pH}[27]$.

The application of zeolites as adsorbents is not restricted to liquid phases. In fact, separation of gases is one of the most important uses of zeolites. Indeed, our knowledge and the number of patents on this subject have increased dramatically over the years. This is a consequence of the flexibility of the design of adsorption systems. The successful use of zeolites in gas systems is due to the molecular sieve effect, whereby only small compounds are allowed to diffuse into the zeolite channels. Thermodynamic selectivity for each component in the gas mixture and even the kinetic selectivity, related to how rapidly the component diffuses into the channels, should be taken into account as well [28].

Zeolites act as adsorbents mainly in the removal of dilute impurities, which includes the classical use in gas drying, desulphurization or removal of organic compounds. Zeolites may be used to separate gases when increased purity and recovery are sought, or as a low-cost separation alternative. In these cases, a packed bed with one or several layers of zeolites is operated in PSA (pressure swing adsorption), TSA (temperature swing adsorption) or other mixed operation modes. The fundamentals of this wellknown technology have been extensively reported. In gas systems, pressure is of utmost importance and the contribution of zeolite-gas or gas-gas interactions should be carefully investigated. Synthetic zeolites are more appropriate to gas applications, although some studies related to natural zeolites have been reported [29].

As an application example, long stay patients at hospitals sometimes need medical oxygen for rehabilitation. Moreover, oxygen is absolutely necessary for astronauts, on space trips. Such pure gas may be generated through cryogenic distillation, water electrolysis or adsorption. The last one is, by far, the simplest technology. Of course, zeolites are used for such processes, such as LiLSX (Li-exchanged low silica X [30]). The light or noble gases may be separated with $3 \mathrm{~A}, 4 \mathrm{~A}, 5 \mathrm{~A}$ or $13 \mathrm{X}$ zeolites [31]. Zeolites are of huge importance in natural gas storage or decontamination [32]. Finally, zeolites may be applied in gas chromatography as well.

These are some of the current application of zeolites directly linked to ion exchange and adsorption. New challenges keep being overcome with the help of such materials, from single ion removal to health care, and new possibilities continue to appear, in this research area thus remaining far from exhausted. 


\subsection{Novel applications of zeolites as adsorbents and ion exchangers}

The main objective of novel applications is not directly related to the sorption process, although it is the way in which they are achieved. This is the case when zeolites, coupled with carbons, are used as bactericides, in hybrid membranes, electrodes, capacitors, and sensors or even in energy storage processes.

Ion-exchange systems have been improved combining zeolite with different adsorbents such as activated carbons through extrusion and calcination. The silicate-carbon modified zeolite (SCMZ) is an example of this trend. Such a combined system yields advantages related to higher removal efficiency and easier cycle regeneration [33].

As an example, natural zeolite containing $\mathrm{Cu}^{2+}, \mathrm{Zn}^{2+}$ or $\mathrm{Ni}^{+2}$ may be used as a bactericide to pathogenic Gram-negative Bacteria Escherichia coli and Gram-positive Bacteria Staphylococcus aureus. This is a novel application in the tertiary stage of wastewater treatment [34]. Silver ion exchanged zeolites may also be applied as bactericide of Escherichia coli, Vibrio harveyi, Vibrio cholerae and Vibrio parahaemolyticus, commonly found shrimp pathogenic bacteria, providing an adequate and eco-friendly shrimp aquaculture [35]. In fact, silver is recognized by its bactericidal activity, influenced by the oxygen content $[36,37]$.

Bactericidal systems can also incorporate zeolites mixed with membranes, where the zeolite acts indirectly in the process, preventing fouling. One example is Linde type L (LTL) zeolite nanoparticles, which may be embedded in polysulphone ultrafiltration (UF) membranes. Such hybrid systems provide high anti-adhesion efficiency to both Escherichia coli and P. aeruginosa precisely because of the presence of the zeolite, although no bactericidal property was seen [38]. This is obviously a technological advance because bio-fouling is always a challenge to be overcome when dealing with membranes.

The zeolite-membrane system may provide more advantageous uses. Reverse osmosis is a widely used technology for desalination that however, has low resistance to oxidizing agents such as chlorine and low water permeability. These factors may promote operational problems that add to its high cost. Nevertheless, osmosis membranes containing zeolite nanoparticles seem to be a possible solution for such problems. Indeed, poly(arylene ether sulphone) reverse osmosis hybrid membrane containing EMT-type zeolite clearly enhanced chlorination resistance and improved desalination performance. It happens because zeolite nanoparticles modify membrane structure generating a high degree of cross-linking. As a 
consequence, a decrease in salt rejection and an increase in water permeation were observed [39]. Such advantages were also observed in thin filmnanocomposite membranes in which $\mathrm{NaY}$ zeolite was incorporated [40].

Natural zeolites particles may also be incorporated into polysulphone polymer membranes. Likewise, this combined system is efficient for ammonium removal with negligible leaching [41]. Membrane water treatments may separate even toxic ions. Unfortunately, the rejected ions are present in high concentrations and need further treatments to be recovered. This fact motivated investigations related to hybrid systems where membranes are combined with nanoparticles of zeolites. Then, the nanoparticleimpregnated membranes may efficiently remove and easily recover toxic metals. Advantages of this technology include high removal efficiency and the improvement in the hydraulic permeability of the membranes. Promising results for $\mathrm{Pb}^{2+}$ and $\mathrm{Ni}^{2+}$ removal in $\mathrm{NaX}$ nanoparticle impregnated polysulphone membrane have been reported [42].

The ion exchange membranes provided by zeolite-membrane hybrid systems have applications in electrodialysis for desalting brackish waters, simple production of table salt, or even reconcentrating brine from seawater. This is the case of PVC based-co-zeolite membranes. The zeolite enhances membrane electrical conductivity and ionic flux, which is undeniably a huge advantage [43].

Zeolites are such versatile solids that new discoveries have been published in many previously unexpected research areas. These aluminosilicates have been involved in electrochemistry, giving rise to a wide range of applications including electrocatalysis or its use in electrodes for analytical purposes. Zeolite-modified electrodes combine the intrinsic properties of a modifier with the specific electrochemical reaction. Such technology was introduced in the 8o's and its immediate popularity was a result of combining specific charge-transfer reactions with the zeolite ion exchange properties. Nowadays, zeolite-modified electrodes are seen as biosensors or even toxic metal electrodes in a simple device where size, shape or charge selectivity are considered [44]. In this context, Beta and L zeolites are successfully applied as glucose and acetylcholine biosensors [45] and zeolite $\mathrm{NH}_{4}$-Y modified carbon paste electrode (ZYMCPE) has been used in the voltammetric detection of toxic metals in ground waters or wastewaters [46].

Zeolites are also used in energy applications. When an electrical doublelayer occurs between a charge in a pore and the framework, an electrical, double-layer capacitive electrical energy storage is achieved using ultracapacitors. The zeolite-templated carbon has been studied with extremely positive results in the capacitor performance. Moreover, storing solar energy in zeolitic 
systems is a reality. It is based on simple principles based on their hydrophilic properties. When water is adsorbed, heat energy in the zeolite is released. On the other hand, when desorption occurs, heat energy is stored in it. This is of course an important application related to renewable energies. Zeolite hydrophilic properties are also applied in the measurement of humidity. Capacitors coated with zeolite films may detect humidity through the electrical properties of this material, which is a function of the Si/Al ratio [47].

These are some of the novel applications of zeolites. Indeed, the ion exchange and adsorption properties of such materials generate infinite possibilities. It may be surprising that although these materials have been known for almost three centuries, it was only in the second half of the twentieth century that scientists started to investigate zeolite hybrid systems. From now on, many more applications are expected.

\subsection{The future of zeolites as adsorbents}

Zeolites are undoubtedly versatile solids. They are unique because of their ability to combine ion exchange and adsorption properties, as well as the possibility of modulating properties by different post-synthesis treatments or synthesis modifications, in the desired molecular sieve for a specific process. Consequently, the more zeolites are investigated, the more applications are reported even in their traditional uses. The examples discussed above represent only a small contribution of the benefits zeolites can bring to the environment. Unfortunately, moving forward from laboratory research to commercially obtained technologies is a big step.

Frequently, effluents contain a large number of contaminants, with different structures, charges and toxicity. In many cases, a comprehensive knowledge of the molecule is not easily accessible. This is the case of dyes or other organics produced by trade secret processes. Moreover, toxic metals are also found in real effluents. Indeed, zeolites have a potential use in all types of wastewater treatment. Nevertheless, the development of new ion exchange/adsorption units is still hampered by the complexity of real effluents. These contain a wide diversity of molecules competing both with each other and with inorganic toxic metal ions for the zeolite sites. In this context, hybrid zeolitic systems are an important field to be explored. Mixing zeolites with activated carbons or membranes produces more selective removal with higher efficiency with commercial and technological acceptance.

The future of zeolites is not restricted to wastewater treatment. For instance, in drug delivery, a large variety of pharmaceutically active 
compounds (PhACs) may be desorbed from zeolites in the adequate doses. This is another research field with large potentiality as zeolites are completely harmless when ingested.

The importance of zeolite in gas phase adsorption mainly in the removal of contaminants $\mathrm{CO}_{2}$ and $\mathrm{H}_{2} \mathrm{~S}$ present in the natural gas and biogas as well as $\mathrm{CO}_{2}$ capture is also noteworthy. In recent years, this research field has received great attention as an alternative technology, ss shown previously in this book.

Of course, the presence of zeolites in electrodes, capacitors or any other system where an electric field is involved is a new technological branch, and should not be overlooked. One point that should especially be taken into account is related to energy storage, which is of great concern to the modern world.

Therefore, zeolites may be present in such a wide range of possibilities that restriction of their use in only one research field is deemed completely unacceptable. In any investigation where ion exchange or adsorption is required, the use of zeolites should never be discarded at the outset.

\section{Zeolites as catalysts}

It is worth highlighting that zeolites will continue to hold a strong position offering innovative solutions in catalytic processes. However, characteristics of the product slate required by the market will determine the use of traditional or new zeolites. In general, the following features concerning hydrocarbons, are to be considered [48]:

- light olefins from $\mathrm{C}_{3}$ to $\mathrm{C}_{5}$ as raw materials for petrochemicals or for the production of clean, good quality fuels

- highly branched paraffins from $\mathrm{C}_{5}$ to $\mathrm{C}_{12}$ for the gasoline pool, or longer and slightly branched paraffins for the kerosene and gas oil pools

- specific molecules such as first and/or second generation intermediaries in petrochemicals: alkylmonoaromatics in particular. The development of processes of inter-transformation of these aromatics can certainly be expected.

\subsection{Cracking catalysts}

The first commercial use of catalysts for cracking occurred in 1915 when A. M. McAfee developed a batch process using aluminium chloride (which 
was a traditional Friedel Crafts catalyst known since 1877) to promote the cracking of heavy petroleum oils. Nevertheless, the prohibitive cost of the catalyst prevented the widespread use of McAfee's process at that time. Since then, several catalysts have been used for cracking, being historically subdivided into the following three main groups:

- Natural catalysts - clays, such as bentonite or montmorillonite, would undergo acid treatment to remove $\mathrm{Na}$ and $\mathrm{Mg}$, thereby increasing their acidity,

- amorphous catalysts - these were gels of silica and alumina, resulting from the reaction of $\mathrm{Na}_{2} \mathrm{SiO}_{3}$ and $\mathrm{Al}_{2}\left(\mathrm{SO}_{4}\right)_{3}$. There were two types of such catalysts, low alumina (10 to $15 \mathrm{wt} . \%$ of $\mathrm{Al}_{2} \mathrm{O}_{3}$ ) and high alumina (2O to 30 wt. $\%$ of $\mathrm{Al}_{2} \mathrm{O}_{3}$ ).,

- crystalline catalysts - micro-spheres $(40-150 \mu \mathrm{m})$, having a crystalline zeolite as the main component.

Indeed, Houdry was the first one to use acid-treated bentonites as cracking catalysts, back in 1936. In the 1940s, silica-alumina catalysts were synthesized, showing a great improvement in performance when compared with clay-based catalysts. Early synthetic amorphous silica-alumina catalysts contained about 13 wt.\% of $\mathrm{Al}_{2} \mathrm{O}_{3}$ (low alumina), however, in 1955, the content of $\mathrm{Al}_{2} \mathrm{O}_{3}$ had increased to about $25 \mathrm{wt} . \%$.

In 1962, the most important catalytic breakthrough in the FCC process took place, when a component, known as zeolite $\mathrm{Y}$, was added to the active alumina catalyst. By adding small amounts of zeolite into the matrix of the traditional silica-alumina catalyst, a new catalyst was produced. This catalyst had an outstanding performance, much better than any catalyst before. The zeolite catalyst greatly improved gasoline yield (Table 1). The first commercial zeolite catalysts were introduced in 1964, and zeolite catalysts continue to be used today. However, that gasoline produced via the pristine zeolite-containing catalysts presented a lower Research Octane Number (RON). This was later related to the fact that zeolites, having a very high density of acid sites, were increasing the Hydrogen Transfer Reaction (HTR), as described next [49]:

$$
3 \text { Olefin }+ \text { Naphthene } \rightarrow \text { Aromatic }+3 \text { Paraffin }
$$

Such reaction is detrimental to the gasoline quality since it reduces the RON of the products. For that reason, zeolites have undergone several types of modification, which aim at reducing hydrogen transfer, thereby increasing the gasoline RON. 
Table 1. Main properties of the first zeolite-containing catalysts.

\begin{tabular}{ll}
\hline Type & Main feature \\
\hline Coke formation & Zeolite-containing catalysts reduced coke formation \\
Gasoline yield & Zeolite-containing catalysts increased gasoline yield \\
RON & Zeolite-containing catalysts reduced RON due to \\
& higher HTR \\
\hline
\end{tabular}

Among current FCC catalysts, a very high number of formulations may be found. However, regardless of their formulation the catalyst components may be classified as follows [11]:

a) Active component - responsible for most of the catalyst activity, are usually faujasites (Y zeolites). Currently, another zeolite has also been used (ZSM-5).

b) Matrices - promote bottoms conversion, increasing the overall activity of the catalyst. They must present a certain tolerance to $\mathrm{Ni}$ and $\mathrm{V}$, to nitrogen-containing compounds and, whenever possible, promote DESOX effects. They must also present binding properties.

- Active matrix - normally, the active matrix is an alumina, which is acidic, although less acidic than the zeolite. On the other hand, aluminas display much larger pores than zeolites, therefore they promote pre-cracking of heavier molecules,

- Inert matrix - normally, the inert matrix is a clay, such as kaolin, used to confer physical properties.,

- Synthetic matrix - the synthetic matrix is actually a binder that keeps all other components bound together in the final catalyst.

c) Functional ingredients - ingredients added to the catalyst to perform a specific function (i.e. traps to capture vanadium compounds).

\subsubsection{The zeolite component}

As previously mentioned, the zeolite component is the most important component in the FCC catalyst, being able to provide the catalyst with both convenient activity and selectivity. In FCC catalysts, the zeolite employed is faujasite (FAU). The $\mathrm{Si} / \mathrm{Al}$ ratio of the prepared Y zeolite is an important feature of the parent zeolite, since the higher $\mathrm{Si} / \mathrm{Al}$ ratio, the more stable it is against acid and hydrothermal dealumination. 
The zeolite is synthesized in its Na-form, which is non-active, since acidic sites (Brønsted acid sites), responsible for the formation of carbocations and hence cracking reactions, are not present. Thus, the NaY zeolite must undergo ion exchange with either ammonium or rare earth ions to prepare the ammonium/RE form of the zeolite, which will generate the acidic form HY upon calcination.

\section{RE-Yzeolites}

The introduction of rare earth elements (RE) in the zeolitic component, via ion exchange, followed by a calcination step, is one of the most important modifications carried out in the process of FCC catalyst preparation, since it increases both the stability of the zeolite and the overall activity of the catalyst. Furthermore, calcination promotes framework dealumination, changing acidic and textural characteristics in the zeolite.

Since RE do increase Brønsted acidity [50], RE affect both activity and selectivity. The higher the ionic radius of the RE atom, the higher the acidity generated in the zeolite. Since HT increases with the increasing zeolite acidity, the higher the atomic radius of the RE atom, the higher the HT. Generally, RE favour HT, lowering RON by reducing olefin concentration in the gasoline. Nevertheless, since RE also affect the zeolite stability [51], sometimes evaluation may be rather misleading. It must also be borne in mind that industrial results show that RE seem to have little influence on the gasoline MON. Finally, rare earths also play an important role in preventing deactivation by metals [52] as they are a very effective vanadium trap.

Rare earths are facing a terrible problem of increasing price and decreasing availability. Thus, the removal of these ingredients from the FCC catalyst composition has been a constant effort. However, the total removal of rare earth elements from the zeolite component of an FCC catalyst would result in a considerably detrimental effect in most FCC operations due to the lower activity and worsened product yield slate, not to mention a problem of higher vanadium deactivation. For that reason, other elements such as $\mathrm{Ca}$ and $\mathrm{Cr}$ have been tried, though without encouraging results.

\section{$\mathrm{NH}_{4}-$ Y zeolites}

$\mathrm{NH}_{4} \mathrm{Y}$ zeolites must also undergo thermal treatments, in order to generate Brønsted sites upon calcination. Calcined ammonium-exchanged Y-zeolites are often known as USY (ultrastable Y zeolites). Calcination is, however, a very complex step, since several reactions may take place. Furthermore, zeolites undergo structural modifications upon calcination. Calcination generates acidic sites, but also promotes dealumination, 
generating aluminium species known as EFAL (extra framework aluminium). The removal of framework aluminium changes the unit cell size of the zeolite. Calcination also generates mesoporosity, probably because part of the framework is destroyed as dealumination proceeds.

Several factors affect the characteristics of the zeolite after calcination. Among them, the following deserve attention:

- Si/Al ratio of the parent zeolite,

- Na content of the exchanged zeolite,

- steam partial pressure during calcination,

- temperature of calcination,

- calciner geometry (deep bed versus shallow bed).

\section{Hierarchical zeolites and the concept of accessibility}

The characteristics of current feedstocks, in which one may observe a growing content of heavier molecules, has drawn the attention of the zeolite community to the concept of accessibility. Recent literature has shown the importance of accessibility in cracking catalysts [53-6o]. Accessibility is a parameter related to the mass-transfer characteristics of an FCC catalyst, which may be a selectivity-determining step. Indeed, the geometric disposition of components in the final catalyst does affect selectivity in the cracking process. In fact, reducing diffusional effects improves gasoline selectivity.

As far as increased accessibility of the zeolite component is concerned, the development of methods to increase mesosurface area, via the following methods was the strategy adopted to cope with cracking of heavier molecules:

a) the synthesis of zeolites with larger micropores,

b) reduction of the crystal size,

c) introduction of additional pores of larger sizes (mesopores).

Such methods may be divided into two categories: bottom-up or primary syntheses, and top-down or post-synthetic modification. In both cases, hierarchical zeolites are generated. Notably [21], the term 'hierarchical zeolite' encompasses any zeolite with at least a secondary pore-structure system and, therefore, 'mesoporous zeolite' should be considered as a subclass of the former because it defines the size of the additional porosity as falling within mesopore range, that is, between 2 and $50 \mathrm{~nm}$.

Amid the characteristics that might have an effect on the accessibility of zeolites, the crystallite size, which affects the external surface area 
directly, deserves special attention. Thus, papers concerning the importance of small-crystallite zeolites on the cracking of bulky molecules are being revisited [58-6o].

A new commercial technology [61] (Rive's technology) to increase the accessibility of the zeolite component has been launched. It improves the characteristics of zeolite $\mathrm{Y}$ by creating a network of intermediate sized ( 2 to $6 \mathrm{~nm}$ ) mesopores throughout the crystals of zeolite Y. Performance benefits resulting from Rive's zeolite were reduced coke formation, improved bottoms upgrading, and increased olefinicity of the cracked products.

\section{Zeolites as functional ingredients}

The demand for light olefins such as ethylene and propylene has increased a lot in the last decade. Steam cracking of hydrocarbons has been the major source of light olefins for more than half a century. Nevertheless, recent studies have reported that ethylene and propylene can also be commercially produced through the cracking of hydrocarbons over modified ZSM-5 zeolites [62]. In this case, ZSM-5 would behave as a functional ingredient in the FCC process, or rather, an ingredient added with a specific function. As a functional ingredient, $\mathrm{ZSM}-5$ could be embedded in the final catalyst or used as a separate particle to be blended with the traditional catalyst.

ZSM- 5 has been used as an FCC additive for boosting gasoline octane number. Interestingly, the use of ZSM- 5 also increases $\mathrm{C}_{3}$ and $\mathrm{C}_{4}$ LPG selectivity with a concurrent decrease in gasoline yield. The yielded LPG consists predominantly of olefins, whereas there is essentially no change in dry gas (C2-) or coke selectivity.

However, a significant change in gasoline composition takes place.

Both the decrease in gasoline molecular weight and the increase in $\mathrm{C}_{5}$ hydrocarbons are important for an increase in the gasoline octane number. Since the total volume of gasoline is reduced due to the decrease in $\mathrm{C}_{7+}$ aliphatics, the concentration of aromatics and naphthenes in the gasoline is higher, which helps boost the gasoline octane number.

Regarding chemical modifications, treatment of ZSM- 5 with phosphorus compounds $[63,64]$ seems to be an interesting route to improving selectivity to light olefins in cracking reactions. After phosphorus treatment, the strong acid sites of the original zeolite are replaced by an increased number of weaker acid sites, with a concentration, which increases upon steam treatment. Interestingly, the combined treatment of phosphorus/ rare earths [65] results in an improvement in both stability and activity. Apparently, rare earths reduce aromatics formation on the external surface 
area of the zeolite, whereas phosphorus reduces the loss in activity caused by dealumination.

\subsubsection{The future of zeolites in cracking}

Vogt and Weckhuysen [66] have recently published an interesting review paper concerning FCC catalysts. It is clear that the role of zeolites as the most important component in FCC catalysts is certainly undisputable. Although new materials have been synthesized with several properties similar to those displayed by zeolites, such as acidity and selectivity, none is capable of replacing Y zeolites in the cracking process. Nevertheless, the characteristics of the refining industry demand modifications in the zeolite component in order to cope with several requirements concerning, for instance, heavier feedstocks or more environmentally friendly products. Among the improvements and modifications that could be carried out, the following deserve special attention:

a) Increased silica-to-alumina ratio (SAR) of the NaY zeolite - innovative routes of synthesis of $\mathrm{Y}$ zeolite with a higher silica-to-alumina ratio (higher than 6) must be proposed. The current routes of synthesis of high SAR NaY zeolites are very time-consuming, causing a decrease in capacity in the industrial plant.

b) Increased accessibility - synthesis of small crystallite zeolites, with high external surface area, is an alternative bottom-up method to increase accessibility. Such zeolites are, however, more unstable, therefore requiring a higher SAR. Moreover, the synthesis of hierarchical Y zeolites is also an excellent option for higher accessibility.

c) RE substitution - RE are becoming scarce and expensive. New options to replace RE elements in the formulation of the zeolite must be discovered. It should be born in mind, however, that the role of RE is rather complex, since they increase both activity and stability.

d) Zeolites in the FCC particle - It is important to bear in mind that the zeolite is a component of a rather complex system, that is to say, the FCC catalyst. Hence, interactions between the zeolite and other components should not be disregarded. Zeolite/binder interactions are often responsible for a deep loss in activity, since the binder causes micropore blocking. For that reason, new systems, or rather, the concept of compounding has to be taken into account. Zeolites are not to be considered as a single particle, but a component of a complex system in which several interactions do take place. The future calls for new technologies of FCC particle generation in which the properties of Y zeolite may be fully employed. 


\subsection{Hydrocracking/hydroisomerization catalysts}

In the next decade, clean fuels requirements will have a major impact on the way in which refineries are configured $[67,68]$. The Fluid Catalytic Cracking Unit (FCCU), in particular, will require more extensive integration with hydroprocessing units to produce low sulphur fuels, while meeting refinery emissions regulations. In fact, not only are cleaner products requested, there is also a significant switch from gasoline towards gasoil/diesel worldwide, with decreased fuel oil demand. Hence, hydrocracking and hydroisomerization processes are gaining much interest. Furthermore, the rebirth of gas-to-liquids/coal-to-liquids processes, which require an upgrade step, will also put the focus on hydroprocessing technologies.

Indeed, new mild hydrocracking (MHC) technologies will enable the optimization of FCCU and hydroprocessing refinery assets to produce high-quality-clean-fuels at lower costs. Innovative process allows the refiner to produce clean diesel and improve the quality of the FCCU feedstock. New UOP technology, for instance, features a unique flexibility to produce ULSD, improve cetane number and diesel cold-flow properties by the simple expedient of a catalyst change-out in the same reactor.

\subsubsection{Hydrocracking}

It has been known for a long time that hydrocracking involves a bifunctional mechanism where a 'metal' function is responsible for hydrogenation/dehydrogenation reactions (although in many hydrocracking catalysts the metal function is actually given by a transition metal sulphide phase), whereas an acidic function is responsible for isomerization and cracking reactions. A proper balance between 'metal' and acidic functions must exist in the ideal hydrocracking catalyst. The hydrogenation/dehydrogenation function must be strong enough to adequately supply the acidic sites with olefin molecules for carbenium ion production and quickly hydrogenate the product olefin to avoid secondary cracking [69].

Bouchy et al. [69] have observed that the potential secondary cracking augments with an increased average residence time of olefinic intermediates in the vicinity of acid sites. Therefore, any diffusional limitation or confinement effect resulting in a too strong adsorption of the intermediates should be minimized. For this reason, amorphous mesoporous supports, like silica-aluminas $\left[7^{\circ}-72\right]$ have been more frequently used than zeolite supports and, when these are used, a zeolite with little shape selectivity, such as USY, is the usual choice. 
Ultrastable Y zeolite (USY) and Beta zeolites are relatively wide-pore zeolites, not displaying shape selectivity in hydrocracking. This leads to a very wide product distribution [73]. Therefore, the search for alternative zeolites is a must.

The use of zeolites with straight parallel narrow pores, such as ZSM-22, leads to the phenomenon known as pore mouth catalysis [74-80], which brings about a high selectivity for isomerization near the extremity of the hydrocarbon chain but also undesirable light hydrocarbons production.

In a very interesting contribution, Thybaut et al.[73] speculated whether there could be some zeolite pore structure in which the adsorption of both extremities of the linear hydrocarbon chain would occur inside narrow straight pores, whereas the middle part would be located within wide cavities, where branching reactions might take place, eventually leading to cracking at the desired middle part of the chain to maximize the production of valuable hydrocarbons. A hierarchical zeolite combining Y-zeolite supercages with ZSM-22 segments would present such outstanding properties. As a matter of fact, a simulation of the reaction of n-dodecane in this type of structure using a single-event microkinetic model has been carried out. The results indicated that with this type of structure the percentage of C6 products obtained by central cracking in the chain can be increased from $25 \%$ with non-shape-selective Y zeolite, to up to $93 \%$. It has been then proposed that this is a promising approach in the development of zeolite catalysts for the selective hydrocracking of waxes into middle distillates. Synthesis of such materials is certainly the future in this area.

\subsubsection{Hydroisomerization/dewaxing}

These processes aim to produce good base oils for lubricants. The most important properties of lubricating oils are sulphur content, pour-point, cloud-point, oxidation stability and viscosity index. Viscosity index (VI) is a standard empirical measure, widely accepted by the lubrication industry, inversely related to the change in oil viscosity with temperature. Normal grade base oils (groups I and II, according to API classification) have VI's in the 80 to 119 range. High-grade group III oils are produced by modern hydroprocessing technology in petroleum refineries, including hydroisomerization, and have VIs above 120. Proper design of catalysts and process conditions for hydroisomerization/dewaxing has to take into account the molecular characteristics desired for obtaining proper cold-flow properties without compromising VI. It is often accepted that increasing the degree of alkane branching decreases the VI of the oil [81]. Miller et al. [82] suggested that minimizing overall branching while maximizing the branching 
towards the middle of the lubricant base oil molecules provides fluids with a high VI and low pour points.

It is therefore reasonable to admit that HIDW catalysts should be designed in order to promote branching of the largest possible fraction of the $n$-paraffin molecules present in the feed, but limiting as much as possible the number of the branches. The occurrence of hydrocracking reactions should also be avoided.

Hence, catalysts that have a high hydrogenation activity and a low acidity are indicated for maximizing hydroisomerization versus hydrocracking [83]. Platinum or palladium, are generally found to be the most appropriate metallic phase for hydroisomerization/dewaxing catalysts, rather than mixed-sulphides or a base metal such as nickel.

Regarding the zeolite component, it must be selective for adsorption of linear alkanes and the pores should be small enough to limit the occurrence of branching reactions inside such pores. The formation of multibranched hydrocarbons would be deleterious for the VI. Such molecules are precursors of hydrocracking reactions. Medium pore zeolites and, especially, those with parallel straight pores and ten-membered ring pore openings. ZSM-22, ZSM-23, ZSM-48 as well as SAPO-11 have been shown to be excellent acidic components for HIDW catalysts for long-chain n-paraffins, as reviewed by Bouchy et al.[69]. In fact, the geometry of the pores thereof induces the pore mouth mechanism for short-chain paraffins. However, a second effect named key-lock mechanism [69], appears in long chain hydrocarbons. In this last type of mechanism, both extremities of the hydrocarbon chain penetrate neighbouring pores emerging at the zeolite crystal surface and the branching occurs in the central part of the chain by reaction on acidic sites at the external surface of the zeolite between pore openings.

Indeed, studies regarding the hydroisomerization of n-octadecane in a series of closely related zeolites of the ZSM-48 family have been performed [69]. Maximal isomer yields of up to $77 \%$ at conversions approaching $100 \%$ were obtained in some cases and significant selectivity differences were observed between the different but related zeolites. This fact clearly indicates that even subtle differences in the arrangement of pore openings at the crystal surfaces, detailed topology of the zeolite channels, and concentration and position of aluminium atoms strongly influence the catalyst activity and selectivity.

It is therefore clear that the future of zeolites for hydroisomerization/ dewaxing lies in the search for innovative materials capable of providing outstanding performances based on both pore mouth and key-lock mechanisms. 


\section{Metal-Organic Frameworks}

MOF is the abbreviation for metal-organic framework, a designation coined by Yaghi [84] in 1995 to name a class of hybrid porous robust materials in which coordinated metal or metal clusters are connected/coordinated by bridged organic linkers, forming a crystalline welldefined range of frameworks. They have attracted great attention due their huge pore dimensions and tremendous superficial area associated with a chemical versatility, causing a fast development of the synthesis of new compounds. Several research groups have given different names to them, as can be seen in Table 2. The French group, Férey named new MOFs following the convention of the zeolite community, using three letters (generally associated with the geographic origin of the material) accompanied by a number, as in the series of MIL-n, for Materials of Institute of Lavoisier [85]. MOFs are also referred to as coordination polymers, although for some authors this terminology may cause confusion since it has its origin in the first hybrid open frameworks formed by isolated polyhedra or small metal clusters, which are unlike the extended dimensionality of the inorganic parts, able to give rise to channels, layers or 3 D frameworks [86].

Since then, there have been many books, reviews and articles on these compounds and their applications, which spread over storage energy, gas storage, $\mathrm{CO}_{2}$ capture, catalysis, supercapacitors, magnetic devices, luminescent compounds, drug delivery, water photolysis, water detoxification

Table 2. Some designations for hybrid metal-organic frameworks.

\begin{tabular}{ll}
\hline Abbreviation & Name \\
\hline MOF & Metal-organic Frameworks \\
IRMOF & Isoreticular Metal-organic Frameworks \\
ZIF & Zeolite Imidazol Frameworks \\
MIL-n & Materials of Institute of Lavoisier \\
UiO & University of Oslo \\
JUC-n & Jilin of University China \\
HKUST-n & Hong Kong University of Science and Technology \\
NOTT-n & University of Nottingham \\
ITQMOF-n & Institute of Chemical Technology (Spain) - Metal-organic Framework \\
BIFs & Boron Imidazolate Frameworks \\
ZMOF & Zeolite-like MOF \\
MOPs & Metal-Organic Polyhedra \\
POMOFs & Polyoxometalate-based metal-organic frameworks \\
\hline
\end{tabular}


and even compounds for disabling chemical weapons [87]. Some of these important applications of MOFs have already been discussed in previous chapters of this book. This contribution points out some of the challenges and outlooks of MOFs. To the readers, two main questions are suggested: 1) Have the researchers of MOFs fully dominated the tailoring of the construction of porous framework materials aiming for the functionality or final application of them? Or in informal language, have they finally dominated the Chemical tailoring land as it were the Legoland of our childhood? And 2) What is the future of MOFs?

\subsection{From modular chemistry to magic number ratios}

The initial conceptual design of MOFs [87] has proposed three challenges to be faced: 1 ) the control of the orientation and stereochemistry of building blocks in solid state to guarantee the targeted molecular architecture, 2) the synthesis of crystalline materials that can be fully characterized by single-crystal X-ray diffraction, and 3) reversible access to the pores with maintenance of the framework avoiding interpenetration even after the removal or replacement of the guest molecules.

Some strategies have been chosen in order to design and assemble MOFs based on what was initially called Modular Chemistry [87] and afterwards Reticular Synthesis [88]. These are:synthetic routes for achieving porous and crystalline modular networks using solvothermal, diffusion and gel techniques, the development of methods to obtain rigid non-interpenetrated structures containing pores with reversible access for guest molecules without affecting their structural integrity, and the discovery of new applications and properties of porous solid frameworks derived from tailoring their construction with the building blocks [87]. The schematic construction of these porous frameworks involves the inorganic part - the metal ion centers or nodes, and the organic part - the organic linkers that act as coordinator agents directing the assembly of a framework. An analogous terminology of zeolites has been adopted $[89,90]$ using the term Secondary Building Units (SBUs) for the units or entities which were not employed in the synthesis but formed in situ and are composed of molecular complexes and clusters connected by organic linkers [89-92]. The building blocks, on the contrary, were employed as synthetic units.

In order to obtain extended high porous frameworks, a careful selection of the organic linker and metal coordination environment should be done as well as a foresight of the topology derived from their connectivity. The 
<smiles>O=C(O)c1ccc(C(=O)O)c(Cl)c1</smiles>

a)

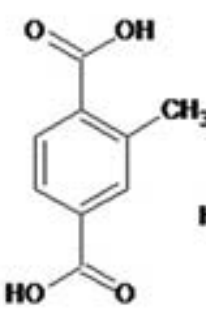

e)

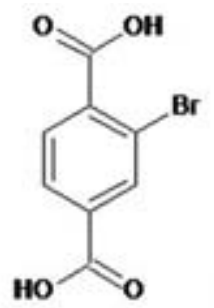

b)

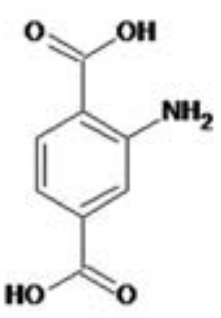

c)<smiles>O=C(O)c1ccc(C(=O)O)c([N+](=O)[O-])c1</smiles>

d)

Figure 1. Some of the dicarboxylic ligands employed in MOFs: a) 2-chloro-benzene-1,4-dicarboxylic acid, b) 2-bromo-benzene-1,4-dicarboxylic acid c) 2-amino-benzene-1,4-dicarboxylic acid,

d) 2-nitro- benzene-1,4-dicarboxylic acid, e) 2-methyl- benzene-1,4-dicarboxylic acid,

f) 2,5-dimethyl- benzene-1,4-dicarboxylic acid, g) benzene-1,2,4-tricarboxylic acid, h) benzene-

1,2,4,5-tetracarboxyic acid.

organic linker should present a rigid disk-like conformation able to coordinate to the metal ion in a multidentate mode, easily acting as bridges that form the voids in the structure and enhance their thermal stability and robustness. Examples of organic linkers range from dicarboxylate ligands (Figure 1) to bypiridines, imidazoles and porphyrins [93]. The molar metal/ organic linker ratio should be high to help the multidentate coordination necessary for the framework construction.

The guest molecules, on the other hand, play an important role in preventing the interpenetration of the structures and also contribute to tailor the shape and size of the pores. These molecules should help in deprotonating the organic linkers and should not coordinate to the metal ions, being easily evacuated to render the formation of the pores and the metal vacancy coordination site [87]. Examples of these guest molecules are triethylamine $(\mathrm{pKa}=11.01)$, dimethyl formamide, alcohols and even water. It is interesting to notice that it was observed an isostructure and an identical X-ray powder 
diffraction for the very first synthesized MOFs of the $\mathrm{M}_{2}(\mathrm{BTC}) \cdot 12 \mathrm{H}_{2} \mathrm{O}$ series, where BTC is 1,3,5-benzenetricarboxylate and $\mathrm{M}$ can be Co, Ni and $\mathrm{Zn}$. The inclusion process of aromatic guest molecules was governed by electronic interactions rather than only shape or size [87].

Following the tailoring design, an immense variety of new MOFs combining the building blocks have been synthesized with the already reported number reaching up or more than 20,00o [94]. Curiously, in 1998, Yaghi et al. anticipated their application in removal of undesired substances from industrial water and in low-temperature catalysis and sensor developments. Nowadays, regarding the great variety of MOFs, not only Yaghi's predictions were fulfilled but also far extended.

By choosing the metal nodes (metal ion centers or clusters) and the organic linker, a tremendous variety of MOFs can be designed, making them a special class of porous and crystalline materials of a high degree of structural and functional tenability [95], properties that overcome other materials such as zeolites and activate carbons [85]. The great tunability of MOFs can explain the wide range of their applications that embrace very different areas including the initial and most known uses in capture, separation and storage of gases $[96,97]$, as well as catalysis [98], chemical sensors, optical luminescence, ionic conductivity, and non-linear optical behaviour [99].

\subsubsection{Post-synthetic modification}

The wish to choose different molecular building units may unfortunately, be restricted in synthesis, in some cases, due to limited organic linker solubility, chemical stability, thermal stability, functional group compatibility or even undesired polymorphs of crystalline MOFs [100].

In those cases, however, a post-synthetic modification (can be made by heterogeneous chemical reactions that functionalize MOF structures previously assembled [99] through two different approaches: either by modifying a) the organic linker or b) the central metal nodes.

a) Modification of organic linker - can be made by making specific changes in the covalent interactions involving the linkers or in their functionality or charge compensation, with electron or ion addition [100].

b) Modification of the central metal node - can be made through inclusion of a non-framework ligand to the metal ion [100] incorporation of alkyl or silyl groups to oxygen coordinated atoms or addition [101] of metals at node oxygen atoms [102].

Notwithstanding the post-synthetic modification, we can go beyond that. Farah et al. [103] proposed a replacement in the building block without 
disruption of the metal-linker bonds, which involves solvent-assisted linker exchange, non-bridging ligand replacement, and transmetalation. This approach allows the design of new MOFs, using parent MOFs as templates via single crystal transformations.

\section{The supermolecular building approach for new MOFs}

Several researches have worked on a different approach for designing new MOFs: the modification and expansion of their topology components. One target of that approach is to produce zeolite-like metal-organic frameworks (ZMOF) based on, and expanding, zeolitic networks. A recent review [104] reported the strategies for design and synthesis of ZMOFs, which can be done through:

a) expansion of the edge (edge-expansion) of traditional zeolites,

b) assembling from hierarchically superior building units, such as metalorganic cubes,

c) enlarging the tetrahedral building units,

d) building via organic tetrahedral nodes.

To continue the focus on topology, there are two recent approaches for design and construction of new MOFs: supermolecular building block (SBB) and supermolecular building layer (SBL).

The already familiar approach of molecular building blocks (MBB) for MOFs allows the introduction of desired properties and functionalities prior to the assembly process and synthesis by the previous selection of specific building blocks, but for what is called simple topologies, i.e. with a number of connectivity that ranges up to 8 or 10-12. Producing MOFs employing the MBB approach with connectivities higher than 12 becomes harder due to the difficulty in achieving the proper reaction conditions [104]. For this reason, Eddaoudi's group employed the SBB and SBL approaches, enlarging the number of the connectivities among polyhedral and layer structures, respectively, constructing more elaborate building units and elucidating the relationship between them and minimal edge-transitive 3-periodic nets [105]. This opens a new pathway for the design and construction of desired and yet hypothetical 3-periodic metal-organic polyhedral or layer MOFs.

\subsubsection{Magic number ratios: order at heterogeneity?}

The functionalization of MOFs has not been restricted to adding functional groups to the organic linkers. Multiple organic functionalities can be added to MOFs on a nanoscale producing new assembles with different applications. The insertion of nanoparticles into materials is not new, it can 
and is being done with MOFs. The question posed by Yaghi himself was how to control the resulting combination of heterogeneity and order when producing assemblies or composites by adding different multiple organic components to MOFs? [105]

The challenge is to obtain a framework with a heterogeneity component controlled even with a change of the environment in its structure. Although two different organic struts of rigid bones (one of a crown ether- $\mathrm{L}_{\mathrm{r}}$ structure and the other of a catenane- $\mathrm{L}_{\mathrm{b}}$ ) were added in a nanoscale to zinc nitrate in very different $\mathrm{L}_{\mathrm{r}} / \mathrm{L}_{\mathrm{b}}$ ratios. Both produced MOF-20oo $\left(\mathrm{Zn}_{4} \mathrm{O}\left(\mathrm{CO}_{2}\right)_{6}\right)$ with a 2:1 ratio. This magic number ratio of 2:1 between the two organic components, $\mathrm{L}_{\mathrm{r}}$ and $\mathrm{L}_{\mathrm{b}}$, was amazingly observed in MOF-20oo crystals, sometimes in different ratios (8:1, 6:1, 4:1, 2:1, 1:1, 1:2, 1:4, 1:6 to 1:8), even if the composition of the parent solution was varied by an order of magnitude in the synthesis. To explain this magic number, standard Monte Carlo simulations were done and revealed that this specific ratio is not derived from thermodynamically restriction but instead suggested that the two components were kinetically trapped during crystal growth [105]. Moreover, the calculations showed how to obtain different but controlled arrangements of component types by varying their non-covalent interactions or the topology in which they are added [105].

The idea of this Magic Number and the control of order in the heterogeneity opens an interesting new window: a new prediction that we may, in the future, combine the heterogeneity of biological materials with the robust properties of the synthetic compounds by arranging and controlling multicomponent types in solid frameworks.

\subsection{Expanding the MOFs Family: MOF composites and MOF derivatives}

Besides MOFs, even considering the huge variety of them, functionalized or not, composites and derivatives of MOFs have also been researched and synthesized enlarging their applications to a great extent.

MOF composites can be produced by integrating functional materials in order to obtain multifunctional compounds and very new technologicallyadvanced materials. In this perspective, MOFs may act as support substrates to assemble different kinds of compounds like graphene, carbon nanotubes (CNTs), nanorods, nanoparticles, metals, metal oxides, metal sulphides, complexes, and even enzymes, forming composites with very interesting performances in catalysis. These include $\mathrm{CO}_{2}$ conversion, 
photo-induced $\mathrm{H}_{2}$ generation, proton conduction, and magnetic behaviour. It is interesting to note that these multifunctional MOF composites not only present a behaviour that involves synergistically in all the components but may also have new properties [106].

On the other hand, MOFs can also be templates as sacrificial precursors to direct the formation of higher porous and organized nanoporous carbon materials via pyrolysis in a process called MOF-to-carbon (MTC). Graphene nanoribbons with excellent supercapacitor performance [106] have already been obtained employing MTC process.

\subsection{MOF devices \& applications: the MOF-based technology}

One of the hottest areas involving MOFs is the investigation in micro- and nanofabrication methods for MOF-based devices for a wide range of applications.

Due to their high degree of organization and porosity, associated with their self-assembly in ordered lattices, MOFs are being employed in device fabrication through different approaches for a great diversity of area. These include sensing, microelectronics, optics, micro-motors, molecular rotors, pollutant sequestration, energy storage and production, bioreactors, diagnostics and controlled drug release [107], some of which have been dealt with in detail in previous chapters of this book. As it is the case for zeolites, there are two different patterns to fabricate MOF-based devices as seen in Figure 2: the bottom-up, in which MOF crystals are grown in pre-defined locations, and the top-down, in which the transfer or removal of pre-existing MOF crystals is done in the material [107].

The prerequisite and challenge for the development of MOF-based technology is the spatial control over the location of MOF materials, regarded as a determinant step for design and fabrication of their devices. To face this challenge, a great variety of techniques are being pursued and employed for the fabrication of MOF-based devices [107]. In Figure 3 a pictorial sample of the world of MOF applications is shown.

At this point, the reader should think about the question first proposed in the introduction. Have the researchers of MOFs fully dominated the tailoring of the construction of porous framework materials aimed at functionality or the final application of them?

The diversity of the fabrication of metal-organic frameworks has not ended yet and neither has that of MOF-based devices. Indeed, the combination of MOFs with other functional materials producing new ones by tailoring 


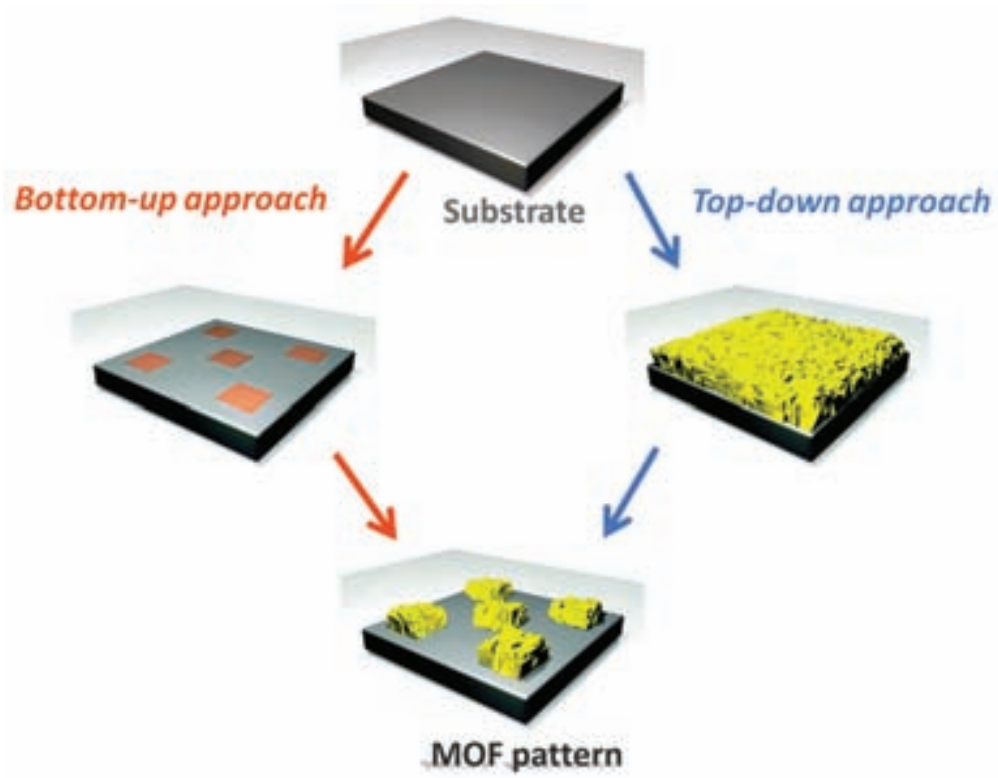

Figure 2. Scheme illustrating the bottom-up and top-down patterning approaches. Bottom-up patterning is defined here as any protocol, which grows MOF crystals in pre-identified locations, whereas top-down patterning is defined as the transfer or removal of pre-existing MOF crystals. Chem. Soc. Rev. vol. 43, p.5513-5560, 2014 - Published by The Royal Society of Chemistry.

and pre-designing them is certainly a hot area both in research and in technology. Thus, MOFs are excellent examples of a very fast transfer process from research knowledge to industry and final use [99,107]. Moreover, the integration of MOFs with functional materials in a miniaturized scale will certainly contribute to producing portable multiuse devices suitable for use in cell phones, electronic equipment, drug delivery compounds and especially, in new ways to store energy. There are, however, some key challenges that should be overcome to make these devices affordable for industrial, medicinal, and biotechnological applications. A remarkable example of these challenges has been delved in Chapter 10: the design and development of MOFs or MOF-based material with specific stimuli-responsive characteristics for drug delivery, which can be trigged by an external agent like light or temperature, or a biological condition of $\mathrm{pH}$ or concentration fluid.

\section{Zeolites and MOFs: from lab to industry}

Porous materials like zeolites and MOFs have attracted enormous attention from industry due to the great variety of technological applications that they 


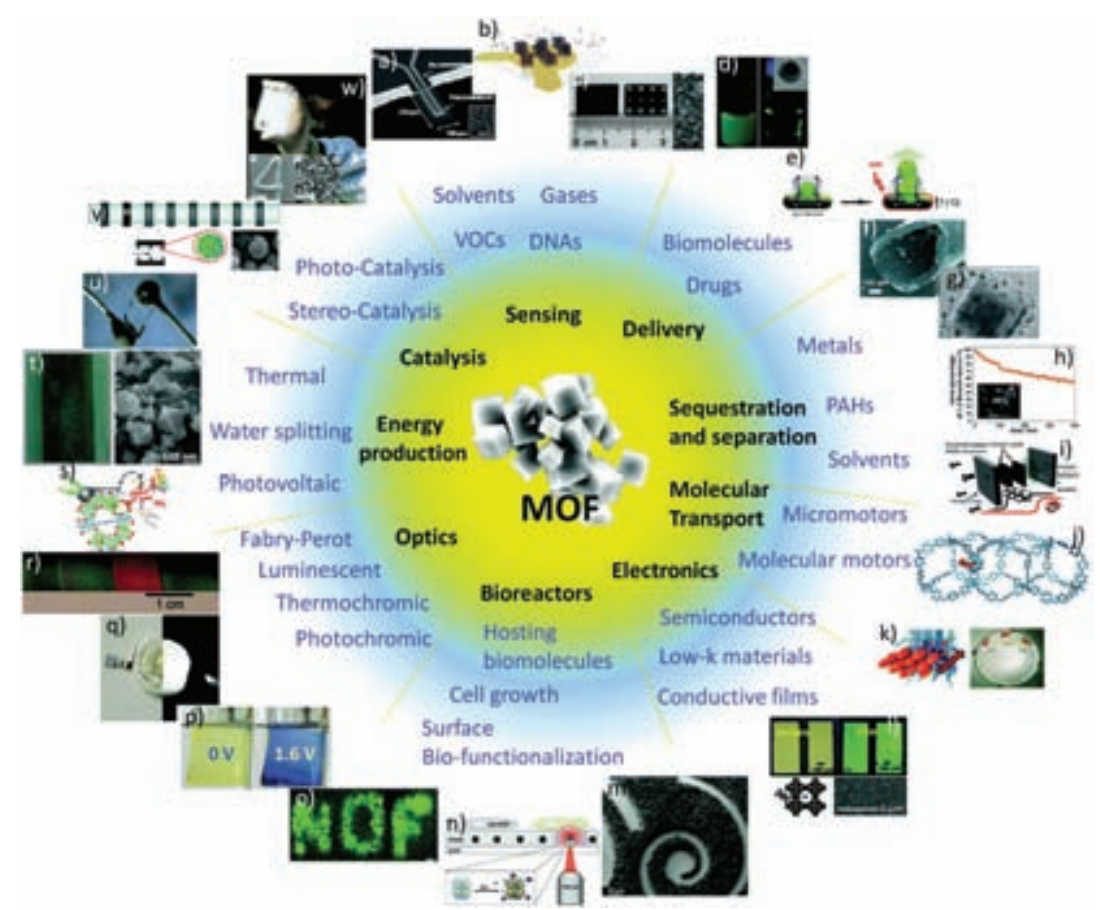

Figure 3. MOF-based devices a) MOF-based piezoresistive microcantilever, b) MOF-deposited gold QCM electrodes, c) HKUST-1 film on aluminium electrodes, d) magnetic manipulation of $\mathrm{Fe}_{3} \mathrm{O}_{4^{-}}$ loaded ZIF-8, e) near infrared-induced drug release from gold nanorod-loaded MOF, f) MOF-5coated fused-silica capillary, g) HKUST-1-coated silica magnetic bead, h) MOF-5 magnetic framework composite and the uptake of benzanthracene, i) HKUST-1 incorporated micro-separator assembly, j) porous aromatic framework structure with a highlighted $p$-phenylene rotor, $\mathrm{k}$ ) projection of a MOF-peptide 'boat' around the Petri-dish, I) TCNQ molecule entering a HKUST-1 film devices, m) MOF patterns created by UV lithography, n) photosensitive MOFs for cell activation, o) cell activation by irradiation-induced selective release of NO from MOF devices, p) MOF thin film-based reversible electrochromic device, q) MOF-based white emitting LED, r) ZIF-8-based Fabry-Perot interferometer, s) photoexcited organic linker induced electron transfer from the linker-to-cluster charge-transfer mechanism, t) MOF-coated doctor bladed $\mathrm{TiO}_{2}-$ MWCNTs composite, $\mathrm{u}$ ) gold electrodes attached to a single MOF crystal, v) magnetic framework composite immobilized into a microfluidic catalytic system, w) HKUST-1 films on a photolithographed copper plate. Chem. Soc. Rev. 2014, 43, 5513-5560, and references therein. Published by The Royal Society of Chemistry.

already present. Both zeolites and MOFs have presented a considerable increase in the number of patents issued in the last decades - especially MOFs, which are more recent materials. However, these two kinds of materials exhibit different stages of maturity regarding their transfer to industry [99,108]. Whereas the worldwide consumption of zeolites ranges in the order of 5 million metric tons per year [108], MOFs are just beginning to be produced in large and commercialized scale in the last years, initially by BASF in association with Sigma Aldrich, followed by MOF Technologies and Strem [99]. 
Throughout the last decades, zeolites assumed a prominent role in industry and society in general, being used as detergents $(73 \%)$, adsorbents (10\%), and catalysts (17\%), especially for the petrochemical industry [109]. The expected tendency for zeolites is an increased demand in the catalysis sector, in important conversion processes such as methanol-to-olefins (MTO), methanol-to-gasoline (MTG), methanol-to-propylene (MTP) and, more recently, methane-to-benzene [109]. Nowadays, zeolite catalysts are being investigated, for instance, for their use in processes for the conversion of renewable resources and biodegradable wastes to valuable chemicals such as ethanol $[110,111]$. There is a closer relationship between the implementation of new processes in industry and the development and use of new or modified/existing zeolites due to some of their intrinsic properties of great porosity, selectivity, very high thermal stability, chemical stability, and an established route for large scale synthesis.

It is worth noting that, rather than competing with zeolites, MOFs take their place in some strategic and technological applications that do not demand severe conditions since they are, on average, stable up to 400$45^{\circ}{ }^{\circ} \mathrm{C}$ under air. Another issue to be considered when choosing MOFs or zeolites is their water resistance. Most MOFs cannot withstand water and, therefore, gases containing water have to undergo dehumidification. Nonetheless, MOFs such as Al-MOFs, commercially available as Basolite A-52O (Al(III)/fumaric acid) or Basolite A1oo (Al(III)/terephtahlic acid) [112], are extremely stable under vapor conditions, and, zeolitic imidazolate frameworks in particular, present high thermal and chemical stability [113].

The peculiar properties of MOFs with their extremely high adsorption capacity, polyfunctional architecture, tailor-made synthesis, high molecular selectivity, and fine arrangement of active sites have made them eligible for applications in a vast range of technologies. MOFs applications for industry comprise energy storage, gas storage and separation, fine organic synthesis, enantioselective synthesis and catalytic processes under mild conditions. Hydrogen and methane storage can be enhanced with the use of MOFs to an extent that makes hybrid hydrogen vehicles and higher autonomy methane cars a reachable and more environmental friendly reality. For hydrogen storage, Basolite Z377 (Zn(II)-benzene tribenzoate: MOF-177) has demonstrated the best result for prototype tank equipment with $32 \mathrm{~g}$ $\left(\mathrm{H}_{2}\right) \mathrm{L}^{-1}$ at $-196^{\circ} \mathrm{C}$ and 50 bar [109]. Regarding methane storage, MOFs adsorbent, namely Basolite $\mathrm{C}_{3} \mathrm{Oo}$, has already been used at room temperature in a Volkswagen Caddy Ecofuel using natural gas in a test tour from Berlin, to Bangkok, in 2007 [99]. The use of the MOF enhanced the tank capacity by $30 \%$, increasing the fuel autonomy by approximately $20 \%$ 
and reducing $\mathrm{CO}_{2}$ emission by 1.3 tons (compared to a $1.6 \mathrm{~L}$ gasoline car) [99]. Still, regarding energy and environmental applications, $\mathrm{CO}_{2}$ capture and separation have prominent roles [114] due to climate change and the $2{ }^{\circ} \mathrm{C}$ limit for global temperature increase established by the Paris Agreement [115]. In a comparative study employing MOFs, zeolitic MOFs and zeolites [116], MgMOF-74 was the best adsorbent for post-combustion $\mathrm{CO}_{2}$ capture. Indeed, several recent articles on $\mathrm{CO}_{2}$ capture by MOFs and MOFs composites [99,109,114,117-123] have already been published, and others are probably in progress. In addition, at the recent International Petroleum Exhibition \& Conference held in Abu Dhabi, UAE, in 2016, Mg-MOF-74 was confirmed as a promising $\mathrm{CO}_{2}$ material in pressure swing adsorption (PSA) and temperature swing adsorption (TSA), although zeolite ${ }_{13} \mathrm{X}(\mathrm{NaX})$ was regarded as preferential for the vacuum industrial process (VSA-vacuum swing adsorption), taking into consideration the current large-scale availability and the cost of both compounds [124].

Beyond gas storage and separation, catalytic processes and innovative MOF-based devices are becoming commercially available. In 2016, MOF Technologies in association with Queens University Belfast launched, a device called TruPick, which can be employed as a post-harvest freshness management tool for fruits and vegetables, that is already being used in the USA and is approved in Turkey [125]. Another ready-to-use product has been produced by NuMat Technologies in association with Northwestern University, who delivered a complete MOF-integrated product ready for the market instead of preparing only MOFs. They have fabricated ION-X cylinders, claimed to be the world's first commercial product to integrate Metal-Organic Frameworks for the storage and release of highly hazardous gases like arsine and phosphine used in the semiconductor industry [126]. NuMat executives have also reported that MOF-based products can also reach the end consumer, broadening the application for society, through their use in portable oxygen cylinders for people with chronic respiratory diseases [126]. In contrast to the petrochemical and environmental industry sector, which in many cases can preferably use zeolites, in the pharmaceutical industry MOFs can have paradigm-breaking uses in sensing, protecting enzymes and delivering drugs, taking into consideration their flexibility and compatibility to biological molecules.

In short, zeolites and MOFs are not competitors. Zeolites are in their prime, having been commercially used for decades. Many are stable at high temperatures and some can withstand water and drastic conditions. However, MOFs possess outstanding properties not only related to adsorption capacity but also to chemical flexibility. Technological advances will define 
the choice between zeolites and MOFs. In either case, challenges will have to be overcome to make them both economically accessible and properly tuned for large-scale use in industry and society, certainly, in many different applications.

\section{References}

[1] Gusenius, E.M. Trans. Kansas Academy of Science, 1969, 72, 476.

[2] McBain, J.W. The Sorption of Gases and Vapors by Solids, G. Routledge \& Sons, 1932.

[3] Barrer, R.M.J. Soc. Chem. Ind. 1945, 64, 13 .

[4] Barrer, R.M.J. Chem. Soc. 1948, 2158.

[5] Barrer, R.M.J. Chem. Soc. 1948, 33, 127.

[6] Milton, R.M. in Zeolite Synthesis, ACS Symposium Series 398, 1, Occelli, M.L., Robson, H.E. (Eds.) Am. Chem. Soc. 1989 .

[7] Reed, T.B., Breck, D.W.J. Chem. Soc. 1956, 78, 5972.

[8] Guisnet, M., Ribeiro, F.R. Zeólitos um nanomundo ao serviço da catálise, Fundação Calouste Gulbekian, 2004 (in Portuguese).

[9] Flanigen, E.M., Broach R.W. Wilson, S.T. in Zeolites in Industrial Separation and Catalysis, ch. 1, Kulprathipanja, S. (Ed.), Wiley-VCH, 2010.

[10] Sousa-Aguiar, E.F., Trigueiro, F., Zotin, F. Catal. Today 2013, 218, 115.

[11] Sousa-Aguiar, E.F. in Zeolites and Zeolite-Like Materials, p. 265, Kustov, L., Sels, B. (Eds.), Elsevier, 2016.

[12] Pace, G.G., Rendón, A.M., Fuentes, G.R. Zeolitas Características, Propiedades Y Aplicaciones Industriales, EdiT, 2000 (in Spanish).

[13] Breck, D.W. Zeolite Molecular Sieves, Krieger, 1984.

[14] Erdem, E., Karapinar, N., Donata, N. J. Colloid Interface Sci. 2004, 280, 309.

[15] Carocci, A., Rovito, N., Sinicropi, M.S., Genchi, G. in Reviews of Environmental Contamination and Toxicology, vol. 229, ch. 1, Whitacre, D.M. (Ed.), 2013.

[16] Wdowin, M., Franus, W. In Zeolites - Useful Minerals, ch. 4, Belviso, C. (Ed.), InTech, 2016.

[17] Zorpas, A.A., Constantinides, T., Vlyssides, A.G., Haralambous, I., Loizidou, M. Biores. Technol. $2000,72,113$.

[18] Shi, W.Y., Shao, H.B., Li, H., Shao, M.A., Du, S. J. Hazard. Mater. 2009, 170, 1.

[19] Lin, L., Wan, C., Lee, D.J., Lei, Z., Liu, X. Sep. Purif. Technol. 2014, 133, 351.

[20] Ho, L., Ho, G. Water Res. 2012, 46, 4339.

[21] Markou, G., Vandamme, D., Muylaert, K. Biores. Technol. 2014, 155, 373.

[22] Rhodes, C.J. Sci. Prog. 2010, 93, 1.

[23] Barczyk, K., Mozgawa, W., Krói, M. Spectrochim. Acta A 2014, 133, 876.

[24] Sadeghi, A.A., Shawrang, P. Livest. Sci. 2008, 113, 307.

[25] Fukahori, S., Fujiwara, T., Ito, R., Funamizu, N. Desalination 2011, 275, 237.

[26] Pasquino, R., Di Domenico, M., Izzo, F., Gaudino, D., Vanzanella, V., Grizzuti, N., de Gennaro, B. Colloid Surf. B 2016, 146, 938.

[27] Farıas, T., Ruiz-Salvador, A.R., Rivera, A. Microp. Mesop. Mater. 2003, 61, 117.

[28] Sircar, S., Myers, A.L. in Handbook of Zeolite Science and Technology, ch. 22, Auberbach, S.M., Carrado, K.A., Dutta, P.K. (Eds.), Marcel Dekker, 2003.

[29] Ackley, M.W., Rege, S.U., Saxena, H. Microp. Mesop. Mater. 2003, 61, 25.

[30] Mitchell, L. Adsorption of Light Gases and Gas Mixtures on Zeolites and Nanoporous Carbons, Ph.D. thesis, Vanderbilt University, 2014. 
[31] Vermesse, J., Vidal, D, Malbrunot, P. Langmuir, 1996, 12, 419 o.

[32] Sapag, K., Vallone, A., Blanco, A.G., Solar, C. in Natural Gas, ch. 10, Potocnik, P. (Ed.) Intech, 2010.

[33] Li, M., Zhu, X., Zhu, F., Ren, G., Cao, G., Song, L. Desalination, 2011, 271, 295.

[34] Hrenovic, J., Ivnkovic, T., Rajiic, N. J. Hazard. Mater. 2012, 201, 260.

[35] Krishnani, K.K., Zhang, Y., Xiong, L., Yan, Y., Boopathy, R., Mulchandani, A. Biores. Technol. $2012,117,86$.

[36] Inoue, Y., Hoshino, M., Takahashi, H., Noguchi, T., Murata, T., Kanzaki, Y., Hamashima, H., Sasatsu, M.J. Inorg. Biochem. 2002, 92, 37.

[37] Matsumura, Y., Yoshikata, K., Kunisaki, S.I., Tsuchido, T. Appl. Environ. Microb. 2003, 69, 4278 .

[38] Dong, L.X., Yang, H.W., Liu, S.T., Wang, X.M., Xie, Y.F. Desalination, 2015, 365, 70.

[39] Kim, S.G., Hyeon, D.H., Chun, J.H., Chun, B.H., Kim, S.H. J. Membrane Sci. 2013, 443, 10.

[40] Dong, H., Zhao, L., Zhang, L., Chen, H., Gao, C., Winston Ho, W.S. J. Membrane Sci. 2015, 476, 373 .

[41] Ahmadiannamini, P., Eswaranandam, S., Wickramasinghe, R., Qian, X. J. Membrane Sci. 2017, 526, 147 .

[42] Yurekli, Y.J. Hazard. Mater. 2016, 309, 53.

[43] Hosseini, S.M., Rafiei, S., Hamidi, A.R., Moghadassi, A.R., Madaeni, S.S. Desalination, 2014, $351,138$.

[44] Muresan, L.M. Pure Appl. Chem. 2011, 83, 325 .

[45] Kucherenko, I., Soldatkin, O., Kasap, B.O., Kirdeciler, S.K., Kurc, B.A., Jaffrezic-Renault, N., Soldatkin, A., Lagarde, F., Dzyadevych, S. Nanoscale Res. Lett. 2015, 10, 1.

[46] Senthilkumar, S., Saraswathi, R. Sens. Actuators B Chem. 20o9, 141, 65.

[47] Urbiztondo, M., Pellejero, I., Rodriguez, A., Pinaa, M.P., Santamaria, J. Sens. Actuators B Chem. 2011, 157, 45o.

[48] Marcilly, C. Oil Gas Sci. Technol. 2001, 56, 499.

[49] Sedran, U. Catal. Rev. 1994, 36, 405.

[50] Sousa-Aguiar, E.F., Camorim, V., Zotin, F., Santos, R. Microp. Mesop. Mater. 1998, 25, 25.

[51] Sousa-Aguiar, E.F., Monteiro, D., Trigueiro, F., Zotin, F.J. Alloy. Compd. 2002, 344, 337.

[52] Sousa-Aguiar, E.F., Baugis, G., Castro, F.R., Oliveira, W., Brito, H. Microp. Mesop. Mater. 2001, 49, 179 .

[53] Jonker, R.J., O'Connor, P., Nicolaas, H., Wijngaards, J. US Patent 6828153 B2, 2004.

[54] O'Connor, P., Olthof, F.P., Smeink, R., Coopmans, J. in Fluid Cracking Catalysts, Ch. 6, Occelli, O'Connor (Eds.), Marcel Dekker, 1998.

[55] Fletcher, R., Hakuli, A., Imhof, P. Oil Gas J. 2002, 100, 54.

[56] Rainer, D., Rautiainen, E., Nelissen, B., Imhof, P., Vadovic, C. Stud. Surf. Sci. Catal. 2004, 149, 165.

[57] O'Connor P., Berends, E. US Patent 6930067, 2005.

[58] Sousa-Aguiar, E.F., Murta-Valle, M.L., Sobrinho, E.V., Cardoso, D. Stud. Surf. Sci. Catal. 1995, 97,417 .

[59] Al-Khattaf, S., Atias, J.A., Jarosch, K., de Lasa, H. Chem. Eng. Sci. 2002, 57, 4909.

[6o] Costa, A.F., Cerqueira, H.S., Sousa-Aguiar, E.F., Ludvig, M.M. Stud. Surf. Sci. Catal. 2004, 154-C, 2296.

[61] Li, K., Valla, J., Garcia-Martinez, J. ChemCatChem, 2014, 6, 46.

[62] Rahim, N., Karimzadeh, R. Appl. Catal., A 2011, 398, 1.

[63] Blasco, T., Corma, A., Martínez-Triguero, J.J. Catal. 20o6, 237, 267.

[64] Lee, J., Hong, U.G., Hwang, S., Youn, M.H., Song, I.K. Fuel Process. Technol. 2013, 109, 189.

[65] Wang, Q. EP Patent o801027 A7, 1997. 
[66] Vogt, E., Weckhuysen, B. Chem. Soc. Rev. 2015, 44, 7342.

[67] Sousa-Aguiar, E.F., Bellot Noronha, F., Faro Jr., A. Catal. Sci. Technol. 2011, 1, 698.

[68] Prada Silvy, R., Santos, A.C.B., Sousa-Aguiar, E.F. Oil Gas J. 2010, 108, 5170498171.

[69] Bouchy, C., Hastoy, G., Guillon, E., Martens, J.A. Oil Gas Sci. Technol. 2009, 64, 91.

[70] Leckel, D. Energy Fuel. 2009, 23, 2370.

[71] Dupain, X., Krul, R., Schaverien, C., Makkee, M., Moulijn, J. Appl. Catal., B 2006, 63, 277.

[72] Calemma, V., Gambaro, C., Parker Jr., W.O., Carbone, R., Giardino, R., Scorletti, P. Catal. Today, 2010, 149, 40.

[73] Thybaut, J.W., Choudhury, I.R., Denayer, J.F., Baron, G.V., Jacobs, P.A., Martens, J.A. Marin, G.B. Top. Catal. 2009, 52, 1251.

[74] Arroyo, J.A.M., Martens, G.G., Froment, G.F., Marin, G.B., Jacobs, P.A., Martens, J.A. Appl. Catal., A 2000, 192, 9.

[75] Denayer, J., Baron, G.V., Vanbutsele, G., Jacobs, P.A., Martens, J.A. Chem. Eng. Sci. 1999, 54, 3553.

[76] Denayer, J.F., Ocakoglu, A.R., Huybrechts, W., Martens, J.A., Thybaut, J.W., Marin, G.B., Baron, G.V. Chem. Commun. 2003, 15, 188o.

[77] Denayer, J.F., Souverijns, W., Jacobs, P.A., Martens, J.A., Baron, G.V.J. Phys. Chem. B 1998, 102, 4588.

[78] Laxmi Narasimhan, C.S., Thybaut, J.W., Marin, G.B., Martens, J.A., Denayer, J.F., Baron, G.V. J. Catal. 2003, 218, 135 .

[79] Martens, J.A., Vanbutsele, G., Jacobs, P.A., Denayer, J., Ocakoglu, R., Baron, G.V., Muñoz Arroyo, J.A., Thybaut, J., Marin, G B., Catal. Today, 2001, 65, 111.

[8o] Souverijns, W., Martens, J.A., Froment, G.F., Jacobs, P.A. J. Catal. 1998, 174, 177.

[81] Verdier, S., Coutinho, J.A.P., Silva, A.M.S., Alkilde, O.F., Hansen, J.A. Fuel, 20o9, 88, 2199.

[82] Miller, S.J., O'rear, D.J., Rosembaum, J.M. WO Patent 2005037963 A2, 2005.

[83] Deldari, H. Appl. Catal., A 2005, 293, 1.

[84] Yaghi, O.M., Li, H.J. Am. Chem. Soc. 1995, 117, 10401.

[85] Férey, G. Chem. Soc. Rev. 2008, 37, 191.

[86] Kuppler, R.J., Timmons, D.J., Fang, Q.R., Li, J.R., Makal, T.A., Young, M.D., Yuan, D., Zhao, D., Zhuang, W., Zhou, H.C. Coord. Chem. Rev. 2009, 253, 3042.

[87] Yaghi, O.M., Davis, H., Li, C., Richardson, D., Groy, T.L. Acc. Chem. Res. 1998, 31, 474.

[88] Yaghi, O.M., O'Keeffe, M., Ockwig, N.W., Chae, H.K., Eddaoudi, M., Kim, J. Nature 2003, 423, 705 .

[89] Rowsell, J.L.C., Yaghi, O.M. Microp. Mesop. Mater. 2004, 73, 3.

[90] Meier, W.M., Olson, D.H. Atlas of Zeolite Framework Types, Elsevier, 2001.

[91] O’Keeffe, M., Eddaoudi M., Hailian Li, Reineke, T., Yaghi, O.M. J. Solid State Chem. 2ooo, 152,3 .

[92] Férey, G.J. Solid State Chem. 2000, 152, 37.

[93] Motoyama, S., Makiura, R., Sakata, O., Kitagawa, H.J. Am. Chem. Soc. 2011, 133, 5640.

[94] Furukawa, H., Cordova, K.E., O'Keeffe, M., Yaghi, O.M. Science, 2013, 341, 974.

[95] Lu, W., Wei, Z., Gu, Z.Y., Liu, T.F., Park, J., Park, J., Tian, J., Zhang, M., Zhang, Q., Gentle 3rd, T., Bosch, M., Zhou, H.C. Chem. Soc. Rev. 2014, 43, 5561.

[96] Alezi, D., Belmabkhout, Y., Suyetin, M., Bhatt, P.M., Weseliński, Ł.J., Solovyeva, V, Adil, K., Spanopoulos, I., Trikalitis, P.N., Emwas, A.H., Eddaoudi, M. J. Am. Chem. Soc. 2015, 137, 13308.

[97] Ribeiro, J.S., Costa, E.S., Hatimondi, S.A., Miranda, J.L. Revista Virtual da Química, 2014, 6, 1172.

[98] Bhattacharjee, S., Chenab, C., Ahn, W.S. RSC Adv. 2014, 4, 52500.

[99] Silva, P., Vilela, S.M.F., Tomé, J.P.C., Paz, F.A.A. Chem. Soc. Rev. 2015, 44, 6774.

[10o] Li, J.R., Timmons, D.J., Zhou, H.C. J. Am. Chem. Soc. 2009, 131, 6368. 
[101] Meilikhov, M., Yusenko K., Fischer, R.A.J. Am. Chem. Soc. 20o9, 131, 9644.

[102] Mondloch, J.E., Bury, W., Fairen-Jimenez, D., Kwon, S., de Marco, E.J., Weston, M.H., Sarjeant, A.A., Nguyen, S.T., Stair, P.C., Snurr, R.Q., Farha, O.K., Hupp, J.T.J. Am. Chem. Soc. 2013, 135, 10294.

[103] Deria, P., Mondloch, J.E., Karagiaridi, O., Bury, W., Hupp, J.T., Farha, O.K. Chem. Soc. Rev. 2014, 43, 5896 .

[104] Eddaoudi, M., Sava, D.F., Eubank, J.F., Adila, K., Guillerm, V. Chem. Soc. Rev. 2015, 44, 228.

[105] Sue, A.C.H., Mannige, R.V., Deng, H., Cao, D., Wang, C., Gándara, F., Stoddart, J.F., Whitelam, S., Yaghi, O.M. PNAS 2015, 112, 5591.

[106] Wang, H., Zhu, Q.L., Zou, R., Xu, Q. Chem 2017, 2, $5^{2}$.

[107] Falcaro, P., Ricco, R., Doherty, C.M., Liang, K., Hill, A.J., Styles, M.J. Chem. Soc. Rev. 2014, 43, 5513.

[108] Davis S, Inoguchi Y. In Zeolites - Chemical Economics Handbook, ch. 6, SRI Consulting, 2009.

[109] Yilmaz, B., Trukhan, N., Müller, U. Chinese J. Catal. 2012, 33, 3.

[110] Taarning, E., Osmundsen, C.M., Yang, X., Voss, B., Andersen, S.I., Christensen, C.H. Energy Environ. Sci. 2011, 4 (3), 793.

[111] Messina, L.I.G., Bonelli, P.R., Cukierman, A.L. Fuel Process. Technol. 2017, 159, 160.

[112] Leung, E., Müller, U., Cox, G., Mattenheimer, H., Blei, S. EP Patent 10183283.o, 2010.

[113] Park, K.S., Ni, Z., Côte, A.P., Choi, J.Y., Huang, R., Uribe-Romo, F.J., Chae, H.K., O'Keeffee, M., Yaghi, O.M. PNAS 2006, 103, 10186.

[114] Sumida, K., Rogow, D.L., Mason, J.A., McDonald, T.M., Bloch, E.D., Herm, Z.R., Bae, T.H., Long, J.R. Chem. Rev. 2012, 112, 724.

[115] https://unfccc.int/resource/docs/2015/cop21/eng/lo9

[116] Krishna, R., van Baten, J.M. Sep. Purif. Technol. 2012, 87, 120.

[117] Wang, H.H., Shi, W.J., Hou, L., Li, G.P., Zhu, Z., Wang, Y.Y. Chem. Eur. J. 2015, 21, 16525.

[118] Chen, D.M., Tian, J.M., Chen, M., Liu, S., Du, M.C. ACS Appl. Mater. Inter. 2016, 8, 18043 .

[119] Ye, Y., Xiong, S., Wu, X., Zhang, L., Li, Z., Wang, L., Ma, X., Chen, Q.H., Zhang, Z., Xiang, S. Inorg. Chem. 2016, 55, 292.

[120] Li, S., Chung, Y.G., Snurr, R.Q. Langmuir, 2016, 32, 10368.

[121] Nandi, S., Collins, S., Chakraborty, D., Banerjee, D., Thallapally, P.K., Woo, T.K., Vaidhyanathan, R.J. Am. Chem. Soc. 2017, 139, 1734.

[122] Sargazi, G., Afzali, D., Mostafavi, A., Ebrahimipour, S.Y.J. Solid State Chem. 2017, 25०, 32.

[123] Zhu, C.J. Mol. Struct. 2017, 1136, 140.

[124] Vega, L.F., Bahamon, D. Comparative Study of MOFs and Zeolites For CO Capture and Separation at Process Conditions, Abu Dhabi International Petroleum Exhibition \& Conference, 7-10 November, 2016.

[125] Editorial, Nat. Chem. 2016, 8, 987 .

[126] Faust, T. Nat. Chem., 2016, 8, 990. 